\title{
Electroacoustics for Characterization of Particulates and Suspensions
}

Proceedings of Workshop held at the

National Institute of Standards and Technology

February 3-4, 1993

Gaithersburg, Maryland

Subhas G. Malghan, Editor

QC 
$T$

he National Institute of Standards and Technology was established in 1988 by Congress to "assist industry in the development of technology ... needed to improve product quality, to modernize manufacturing processes, to ensure product reliability ... and to facilitate rapid commercialization ... of products based on new scientific discoveries."

NIST, originally founded as the National Bureau of Standards in 1901, works to strengthen U.S. industry's competitiveness; advance science and engineering; and improve public health, safety, and the environment. One of the agency's basic functions is to develop, maintain, and retain custody of the national standards of measurement, and provide the means and methods for comparing standards used in science, engineering, manufacturing, commerce, industry, and education with the standards adopted or recognized by the Federal Government.

As an agency of the U.S. Commerce Department's Technology Administration, NIST conducts basic and applied research in the physical sciences and engineering and performs related services. The Institute does generic and precompetitive work on new and advanced technologies. NIST's research facilities are located at Gaithersburg, MD 20899, and at Boulder, CO 80303. Major technical operating units and their principal activities are listed below. For more information contact the Public Inquiries Desk, 301-975-3058.

\section{Technology Services}

- Manufacturing Technology Centers Program

- Standards Services

- Technology Commercialization

- Measurement Services

- Technology Evaluation and Assessment

- Information Services

\section{Electronics and Electrical Engineering} Laboratory

- Microelectronics

- Law Enforcement Standards

- Electricity

- Semiconductor Electronics

- Electromagnetic Fields ${ }^{1}$

- Electromagnetic Technology ${ }^{1}$

\section{Chemical Science and Technology}

Laboratory

- Biotechnology

- Chemical Engineering

- Chemical Kinetics and Thermodynamics

- Inorganic Analytical Research

- Organic Analytical Research

- Process Measurements

- Surface and Microanalysis Science

- Thermophysics ${ }^{2}$

Physics Laboratory

- Electron and Optical Physics

- Atomic Physics

- Molecular Physics

- Radiometric Physics

- Quantum Metrology

- Ionizing Radiation

- Time and Frequency ${ }^{1}$

- Quantum Physics ${ }^{1}$
Manufacturing Engineering Laboratory

- Precision Engineering

- Automated Production Technology

- Robot Systems

- Factory Automation

- Fabrication Technology

Materials Science and Engineering Laboratory

- Intelligent Processing of Materials

- Ceramics

- Materials Reliability ${ }^{1}$

- Polymers

- Metallurgy

- Reactor Radiation

Building and Fire Research Laboratory

- Structures

- Building Materials

- Building Environment

- Fire Science and Engineering

- Fire Measurement and Research

Computer Systems Laboratory

- Information Systems Engineering

- Systems and Software Technology

- Computer Security

- Systems and Network Architecture

- Advanced Systems

Computing and Applied Mathematics Laboratory

- Applied and Computational Mathematics

- Statistical Engineering ${ }^{2}$

- Scientific Computing Environments ${ }^{2}$

- Computer Services ${ }^{2}$

- Computer Systems and Communications ${ }^{2}$

- Information Systems

${ }^{1}$ At Boulder, CO 80303.

${ }^{2}$ Some elements at Boulder, CO 80303. 


\section{Electroacoustics for Characterization of Particulates and Suspensions}

Proceedings of Workshop held at the National Institute of Standards and Technology

February 3-4, 1993

Gaithersburg, Maryland

Subhas G. Malghan, Editor

Ceramics Division

Materials Science and Engineering Laboratory

National Institute of Standards and Technology

Gaithersburg, MD 20899

Issued September 1993

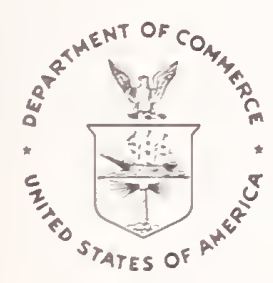

U.S. Department of Commerce

Ronald H. Brown, Secretary

Technology Administration

Mary L. Good, Under Secretary for Technology

National Institute of Standards and Technology

Arati Prabhakar, Director 
National Institute of Standards and Technology Special Publication 856

Natl. Inst. Stand. Technol.

Spec. Publ. 856

308 pages (Sept. 1993)

CODEN: NSPUE2
U.S. Government Printing Office

Washington: 1993
For sale by the Superintendent of Documents

U.S. Government Printing Office

Washington, DC 20402 


\section{PREFACE}

Traditionally, interface chemistry of particulates and colloidal stability in liquids has been characterized by techniques based on microelectrophoresis. Since the conception of the zeta potential, colloid scientists have utilized microelectrophoresis and moving boundary electrophoresis extensively for the measurement of electrophoretic mobilities. Significant progress has been made in developing understanding and scientific data on many particulates of commercial and technical interests. Some of the major drawbacks of these methods are the need for use of low concentrations of particulates in the suspensions, the requirement of optical opacity of particulates, and the exclusive restriction to colloidal size particulates.

The electroacoustic technique, based on physical effects described by Debye, is relatively new and the laboratory instrumentation has become available only in the last decade. This new instrumentation by Matec Applied Sciences and Pen Kem Inc. is based on the application of either electrical fields or ultrasound as external fields to the particles and measurement of the resulting electrical or ultrasonic response. These instruments, while addressing the primary drawbacks of electrophoresis instruments, allow the determination of signals that are related to the electrokinetic zeta potential for colloidal suspensions in polar and nonpolar media. In recent years, the electroacoustic technique has been extensively utilized for the characterization of a variety of particulates in dense suspensions of polar and nonpolar liquids with great success. As a result of this surge in activity, a number of technical issues have surfaced.

In order to discuss these issues and develop a common understanding of the fundamentals of electroacoustics, a workshop was organized at the suggestion of the user community. "Electroacoustics for Characterization of Particulates in Suspensions" is the first workshop on this subject organized by the National Institute of Standards and Technology (NIST). This workshop was held on February 3-4, 1993 at NIST, Gaithersburg, MD. The primary purpose of this workshop was to bring together scientists and engineers working in this field to address relevant scientific and technical issues on the measurement of electroacoustic parameters in the study of particulates. The participants included leading scientists, manufacturers of electroacoustics, and researchers from minerals, ceramics, pharmaceutical, inks, paints, cement and paper industries. The technical content of this workshop included theoretical understanding of electroacoustics, latest developments in equipment, application of electroacoustics to different industrial problems, methods and procedures in the measurement of electroacoustic parameters, interpretation of these measurement parameters, and major issues in the measurements.

The participants received a compilation of presentation viewgraphs after the meeting. This proceedings of the workshop has been possible due to the efforts of a number of my colleagues who not only presented their results but also provided their papers. Without their dedication, this would not have been possible. This volume contains all but two papers presented at the workshop. The papers were reviewed by the participants of the workshop. 
In addition, the session chairs prepared the attached notes based on the discussions held under different groups.

This report is divided into six sections based on the sessions held at the workshop. The first session constituted papers on theoretical aspects and new data on electroacoustics phenomenon, followed by application of this theory to the development of new instrumentation. Prof. Hunter described the results of verification of electrophoretic mobility and dynamic mobility for mono and polydisperse particles. In addition, data on measurements with the Matec Applied Science's new prototype version of Acoustosizer were presented to illustrate the accuracy of determination of magnitude and phase of the dynamic mobility over a frequency range of 0.3 to $11.1 \mathrm{MHz}$. Prof. Pendse presented the analysis of high densicy suspensions by analyzing attenuation coefficient spectra over 1.0 to $100 \mathrm{MHz}$ frequency range to determine particle size distribution. In the area of new instrumentation, both theory and experimental results of Matec Applied Sciences and Pen Kem Inc. were presented by Messrs. D.W. Cannon and P. Goetz, respectively. The second generation of these instruments have the capability to measure both particle size distribution and zeta potential in dense suspensions.

In the second session, papers related to applications and procedural aspects of using electroacoustics were presented. Dr. R. O. James discussed the calibration of Electrokinetic Sonic Amplitude instrumentation using lattices and oxide colloids as a function of volume fraction. Excellent precision of the ESA technique in comparison with the classical electrophoresis techniques was demonstrated. The application of ESA technique to concentrated suspensions of caroon black in apolar liquids was used to study charging mechanisms and influence of dispersants by Dr. R. E. Kornbrekke, I. D. Morrison and T. Oja. Their results showed that particle charging was due to a combination of selective acidbase type interaction coupled with ion-pair dissociation. In a paper by Dr. Valdes, the application of the ESA technique to the characterization of colloidal diamond particles as applied to the development of an electrophoretic coating process for selected area nucleation and patterning of diamond thin films was presented. Background electrolyte correction ir the measurement of ESA was discussed by Dr. F. N. Desai. Under high ionic concentrations, these effects become significant. If not corrected for, the results might indicate an apparent shift in the iso-electric point.

In the third session, two papers were presented by Drs. J. Pollinger and V. A. Hackley on the application of ESA measurements for silicon nitride powder processing. The powder behavior in aqueous environment was studied by Dr. Pollinger to evaluate aging of powders in slurries, characterization of dispersion of powders, development of multiple powder dispersions, and correlation of ESA measurements with other suspension characterization methods. The primary focus of Dr. Hackley's paper was on the role played by solution ionic species in modifying surface charge behavior and the ionic contribution to the ESA signal of the silicon nitride particles. 
The fourth session was devoted to the application of ESA measurements to minerals such as clays, coal, and cements. Dr. N. D. Sanders presented the results of ESA measurements on precipitated calcium carbonate for surface charge measurements. These results were supported by calorimetric and rheological measurements in improving the understanding of the coating behavior of this material. Prof. Chander discussed the role of ESA measurements in studying the electrokinetic behavior of coal particulates. The ESA measurements were used in the interpretation of surface charge and strength of adsorption of species from an aqueous environment. In the study of portland cement, Dr. Darwin presented a comparative data on electroacoustics, microelectrophoresis, and streaming potential in the development of superplasticizers. Lack of strong agreement between the data from these methods and difficulties in the determination of true zeta potential in such complex systems was discussed. Prof. Moudgil presented the results of the application of ESA measurements in mineral systems where solid-liquid or liquid-liquid interface chemistry data are required.

The primary theme of the fifth session was coatings, though two related papers were presented in other sessions. Dr. Krishnan's paper (not presented at the workshop) deals with the application of the ESA technique in improving the performance of solvent based gravure inks. A maximum value of ESA corresponded to a minimum in the particle size distribution, thus providing a measure to control the coating process. The application of ESA measurements was employed in the characterization of phosphors by Dr. A. Dutta. This study involved the investigation of electrokinetic behavior of phosphors in the presence of non-luminescent additives, surface coatings, and polyelectrolytes. An investigation on mechanisms of charging of liquid toner by ESA were presented by Dr. J. R. Larson. Besides presenting a model for micelle ionization and particle charging in these systems, the ESA technique was used in the study of liquid toner particles.

The sixth session was devoted to discussions and identification of issues. Based on the group discussions, the session chairs were responsible for the development of the issues identified in the last chapter of this report

We are pleased that Ceramics Division at the National Institute of Standards and Technology sponsored this workshop. Our sincere thanks to Stephen W. Freiman, Division Chief, for his support and encouragement. Special thanks to Margaret Robinson who was responsible for workshop arrangements and preparation of the workshop proceedings. Also, thanks to Vincent A. Hackley for his assistance in organizing the workshop and reviewing papers. Most of all, our thanks go to Lori Phillips and her colleagues at NIST for the excellent arrangements of the workshop and help with registration.

Subhas G. Malghan

National Institute of Standards and Technology 


\section{DISCLAIMER}

Information on product names, manufacturers, or suppliers are included in this report for clarification. This does not imply endorsement of the products or services by the National Institute of Standards and Technology. 
TABLE OF CONTENTS

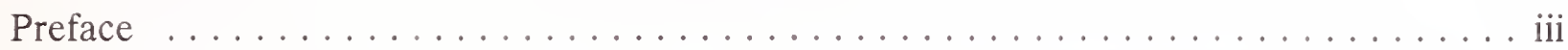

Session I. Fundamentals of Electroacoustics and Instrumentation

Chairman: R. O. James, Eastman Kodak Co.

Determining Charge and Size with the AcoustoSizer ................. 1

Richard W. O’Brien, William N. Rowlands and Robert J. Hunter

University of Sydney

Theoretical Considerations in Acoustophoretic Analysis of Concentrated

Colloids ........................................ 23

Hemant P. Pendse, Terry A. Strout and Arvind Shanna

University of Maine

New Developments in Electroacoustic Methods and Instrumentation . . . . . . . . . . 40

David W. Cannon, Matec Applied Sciences

Session II. Applications and Procedures

Chairman: H. P. Pendse, University of Maine

Calibration of Electroacoustic (ESA) Apparatus Using Lattices and Oxide

Colloidal . . . . . . . . . . . . . . . . . . . . . . . . . . . . . . 67

R. O. James, Eastman Kodak Company

Electrokinetic Measurements of Concentrated Suspensions in Apolar

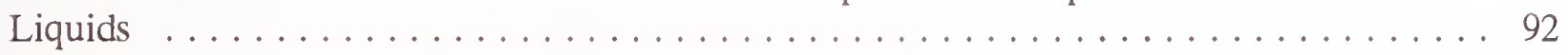

R. E. Kornbrekke, I. D. Morrison and T. Oja, Lubrizol Corp.

Acoustophoretic Characterization of Colloidal Diamond Particles ............ 111

J. L. Valdes, AT\&T Bell Laboratories

Session III. Advanced Ceramics Applications

Chairman: R. Mountain, NIST

ESA Measurements for Silica and Alumina: Background Electrolyte Corrections

F. N. Desai, H. R. Hammad and K. F. Hayes

Department of Civil and Environmental Engineering

Oxide and Non-Oxide Powder Processing Applications Using Electroacoustic

Characterization .

J. P. Pollinger, Garrett Ceramic Company 
Investigation of Parameters and Secondary Components Affecting the

Electroacoustic Analysis of Silicon Nitride Powders . . . . . . . . . . . . . . . . 161

Vincent A. Hackley and Subhas G. Malghan, NIST

\section{Session IV. Clays, Cements and Coal}

\section{Chairman: B. M. Moudgil, University of Florida}

The Impact of Surface Chemistry on Particle Electrostatic Charging and

Viscoelasticity of Precipitated Calcium Carbonate Slurries . . . . . . . . . . . . . . . 180

Yan C. Huang, Nigel D. Sanders, Frederick M. Fowkes and Thomas B.

Lloyd

Lehigh University and Specialty Minerals Inc.

Characterization of Coal Slurries by Electrokinetic Sonic Amplitude

(ESA) Technique . . . . . . . . . . . . . . . . . . . . . . . 200

H. Polat, M. Polat and S. Chander, Penn State University

Surface Charge Characterization of Minerals by Electroacoustic

Measurements .................................. 219

B.M. Moudgil and R. Damodaran, University of Florida

Surface Charge Characterization of Portland Cement in the Presence of

Superplasticizers . . . . . . . . . . . . . . . . . . . . . 238

Company

David C. Darwin, Roger Y. Leung and Trevor Taylor, W.R. Grace \&

\section{Session V. Coatings}

Chairman: N. Sanders, Minerals Inc.

Role of Particle Size and Electrokinetic Sonic Amplitude in Performance of Solvent Based Gravure Inks . . . . . . . . . . . . . . . . . . . . . . . . . . 263

R. Krishnan, Sun Chemical Corp.

Electrokinetics of Phosphors . . . . . . . . . . . . . . . . . . . . 274

Arunava Dutta, Osram Sylvania Inc.

Advances in Liquid Toners Charging Mechanism: Electroacoustic

Measurement of Liquid Toner Charge . . . . . . . . . . . . . . . . . . 301

James R. Larson, Xerox Corporation

Session VI. Subgroups Discussions and Identification of Issues

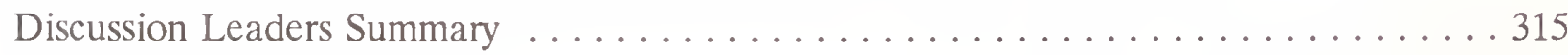

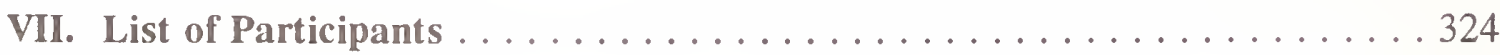




\title{
SESSION I
}

\section{FUNDAMENTALS OF ELECTROACOUSTICS AND INSTRUMENTATION}

\author{
Session Chairman
}

\author{
R. O. James \\ Eastman Kodak Company
}





\title{
DETERMINING CHARGE AND SIZE WITH THE ACOUSTOSIZER
}

\author{
Richard W. O'Brien, William N. Rowlands and Robert J. Hunter \\ School of Chemistry \\ University of Sydney \\ NSW 2006 AUSTRALIA
}

\section{ABSTRACT}

The electrokinetic sonic amplitude (ESA) effect can be measured using the Matec ESA8000 . The measured signal can be used to estimate the magnitude of the dynamic mobility at the measuring frequency (normally $1 \mathrm{MHz}$ ). Provided the particle size is known, this can be related to the conventional zeta potential or electrophoretic mobility of the particles, using the theory developed by O'Brien. We have verified the correlation between electrophoretic (d.c.) mobility and dynamic mobility with suspensions of monodisperse smooth spherical particles of inorganic colloids, and have shown that it can be extended as well to nonspherical and polydisperse systems by using the mass average particle size to characterize the system. Even if the system is undergoing coagulation, it is possible to correlate the measurements of d.c. and high frequency mobility, provided one can estimate the effective mass average particle size using, for example, a light scattering technique.

We have also made accurate determinations of both the magnitude and the phase of the dynamic mobility, over the frequency range 0.3 to $11.1 \mathrm{MHz}$, using a prototype version of the Matec AcoustoSizer. This makes it possible to determine the size independently as well as the zeta potential or kinetic charge. The software provided with the instrument assumes that the particles have a log-normal distribution of particle sizes and estimates the median particle size and gives a measure of the spread. Good agreement is found between these estimates at concentrations up to about $5 \%$ and those found using other methods of sizing. Unstable systems lead to some deposition of particles on the electrode surfaces and this contributes its own signal to the measured value. This may be sufficiently large to vitiate the measurement at low particle concentrations, but should be less of a problem for the higher particle concentrations for which the instrument is likely to find most use. In any case the final version of the AcoustoSizer will incorporate arrangements which should eliminate the worst effects of particle deposition on the electrodes. The main advantage of the AcoustoSizer is that it is able to measure quite concentrated systems without the need for dilution. 


\section{INITIAL EXPERIMENTS IN ELECTROACOUSTICS}

$\mathrm{O}^{\prime}$ Brien et al. ${ }^{1}$, Scales and Jones ${ }^{2}$ and James et $a l_{.}{ }^{3}$ have recently shown the value of the electroacoustic method for the determination of electrokinetic properties. More recent work in our laboratory has extended the application of the electro-acoustic method to the study of adsorption of surfactants ${ }^{4}$ and hydrolysable metal ions ${ }^{5}$ onto clay mineral particles. We have also examined in some detail, the conductance and electrokinetic behaviour of a latex system which exhibits anomalous surface conductance ${ }^{6}$. All of these studies were conducted using the standard electroacoustic apparatus (ESA-8000, Matec Applied Science, Hopkinton, MA). Since much of that material has not yet appeared I shall describe some of it here, along with the more recent results we have obtained with the Matec AcoustoSizer.

Our first step ${ }^{1}$ was to establish that a monodisperse suspension of a colloid consisting of smooth spherical particles gave an electroacoustic signal which was in quantitative agreement with the predictions made by O'Brien's theory ${ }^{7}$. The theory describes how electroacoustics can be used to determine a quantity called the dynamic mobility, $\mu_{\mathrm{d}}$, of the colloidal particles. This quantity is analogous to the d.c. mobility $\mu_{\mathrm{E}}$, as measured by electrophoresis, but is a function of the frequency of the applied field. Since the motion of the particles lags behind the applied field, $\mu_{\mathrm{d}}$ is actually a complex quantity, with a magnitude which depends mainly on the particle charge and a phase lag which depends on the size of the particles. It turns out that $\mu_{\mathrm{d}}$ is equal in magnitude to $\mu_{\mathrm{E}}$ and has no phase lag at the lowest frequencies, but with increase in frequency (in the $\mathrm{MHz}$ region) the magnitude decreases and the phase lag increases.

Establishing the validity of O'Brien's theory proved more difficult than had been anticipated because of the difficulty of obtaining sufficient material to prepare a few hundred mls of sample at the required particle concentration (at least $1 \%$ ). We were limited here by our suspicions about the behaviour of latex systems in electrokinetic experiments. Our earlier studies ${ }^{8}$ had suggested that lattices were unusual in that they appeared to exhibit a phenomenon which we have called anomalous surface conduction; that is, the surface charge which sits between the particle surface and the plane of shear appears to be able to conduct 
an electric current. Such behaviour is not expected and is not included in the normal treatment of the electrokinetic phenomena. We decided that in view of the uncertainties connected with this phenomenon it would be unwise to predicate our examination of the theory on such systerns. Our first experiments were therefore done using a cobalt phosphate sol and they gave very satisfactory agreement with the theory when the sol was highly dispersed. The dynamic mobility, $\mu_{\mathrm{d}}$ could in that case be related to the normal electrophoretic mobility, $\mu_{\mathrm{E}}$ by the formula

$$
\mu_{d}=\mu_{E} \cdot G\left(\omega a^{2} / \nu\right)
$$

where the function $G$ measures the inertia of the particle, $\omega$ is the measuring frequency, $\nu$ is the kinematic viscosity and $a$ is the particle radius. [The theoretical expression for $G$ is described in Ref. 3]. In these experiments the particle radius, $a$, was known with high presision so $G$ could be accurately estimated.

When the suspension was treated with a reagent which reduced the effective charge to a point where the sol became unstable ${ }^{1}$, the measured ESA signal decreased below the value expected from the electrophoretic mobility. It appeared then, that coagulation reduced the mobility of the particles in the high frequency $(1 \mathrm{MHz})$ field, and that was hardly surprising.

We next examined the behaviour of less idealized systems like alumina and silicon nitride dispersions ${ }^{3}$ with a range of particle sizes and some variation in shape. It turned out that, if one could estimate the mass average particle size from light scattering data, then this value could be used to determine the effective value of the inertial correction, $G$ for a polydisperse sol. Since $G$ is a complex function, the proper procedure for taking it into account is a little complicated; it is described in Ref 3.

We were also able to show in that work that, even when the sol was undergoing coagulation, it was still possible to measure the zeta potential, and get good agreement with the value obtained from the electrophoretic method, if one could determine the effective particle size during the coagulation. This we were able to do by withdrawing a sample of the sol and diluting it rapidly with a solution with approximately the same composition as the background electrolyte. The mass average particle size, estimated by light scattering, then 
provided a suitable correction to bring the high frequency mobility into line with the static value, as indicated by equation [1]. It would seem then that the electroacoustic effect and the light scattering process both respond in the same way to a group of aggregating particles. [It is important to note that the particle size which is measured in this way is not the size of the aggregates but some augmented size for the primary particles. It is as though the particles are somewhat restricted in their movement in the electric field and so they behave like larger particles but the aggregate does not move as a single entity].

At this time we were already developing the AcoustoSizer, which could measure both size and charge. It was the latter observation which encouraged us to believe that, in addition to providing a means of measuring the particle size distribution in highly dispersed samples, it might be possible to use the AcoustoSizer to determine some sort of effective particle size, even in coagulating systems, and to use this to estimate the zeta potential and charge. That may ultimately prove to be possible, but unfortunately, our preliminary attacks on coagulating systems have run into some difficulties which will be discussed below.

We next set about using the ESA-8000 to look at some interesting systems in a more qualitative way. We were able to follow the adsorption of cetyl pyridinium chloride (CPC) onto the surface of kaolinite particles at different $\mathrm{pH}$ values ${ }^{4}$. One could readily identify the point at which the particle surface was covered with the surfactant, and hence estimate the surface area of the solid from the known area per molecule of the adsorbate. The isoelectric point of the clay also seemed to correlate well with the cation exchange capacity of the clay, although one would need to repeat the experiment with a variety of clays to determine whether the method was general. Kaolinite particles are flat discs and the edges of the disc display a different sort of adsorption behaviour to the faces. It seemed to be possible to identify the differences in adsorption and so to measure, rather crudely, the relative area of the edges and faces, and hence to estimate the aspect ratio of the mineral crystals (i.e. the ratio of width to thickness). That is an important determinant in the flow behaviour of such systems and is not readily accessible by any other technique.

In another study on kaolinite ${ }^{5}$, we could readily identify the adsorption of hydrolysable metal ions onto the surface as a function of $\mathrm{pH}$. Rather surprisingly, divalent ions like $\mathrm{Co}^{2+}$, $\mathrm{Cu}^{2+}$ and $\mathrm{Cd}^{2+}$ show no special adsorption behaviour on the kaolinite surface under acid 
conditions. As the $\mathrm{pH}$ is raised from acid values, a point is reached at which the metal ion evidently becomes strongly adsorbed on the clay mineral surface, since the normally negative surface becomes much less so. At sufficiently high concentrations of dissolved metal ion, the underlying particle may become positive.

According to Healy and James ${ }^{9}$, who studied such adsorption processes on the silica surface, the divalent ion is not adsorbed because its hydration energy is too large. It becomes adsorbable when it is converted at higher $\mathrm{pH}$ to a partially hydroxylated form: $\mathrm{M}(\mathrm{OH})^{+}$, which has a lower hydration energy. On adsorption, that ion can interact with the surface through hydrogen bonds to the surface oxygens. At still higher $\mathrm{pH}$ values, the zeta potential goes through a maximum positive value and then may decrease to become negative at a $\mathrm{pH}$ which correlates with the isoelectric point of the adsorbed metal oxide. In effect, the particle behaves as though coated by a layer of the metal ion oxide or hydroxide, assuming the latter is insoluble.

The ESA-8000 is very suitable for studying such adsorption processes for it can readily evaluate the exact $\mathrm{pH}$ at which the electrokinetic potential changes from negative to positive and back again. It can also measure, more or less quantitatively, the magnitudes of the zeta potential, although there will be some error engendered by the fact that the particles undergo some degree of coagulation as they pass through those iep values. This is again an area where the AcoustoSizer may prove capable of rendering more quantitative information, if it can measure the particle size, even in coagulating suspensions. An important feature of the apparatus is the ease with which measurements can be made as a function of $\mathrm{pH}$ over the whole range of interest in a single titration experiment.

The detailed study of the polymer latex ${ }^{6}$ depended on measurements of both the complex conductivity and the electroacoustic behaviour of a very well characterized system. In this case we also had detailed potentiometric titration data, which gave us a measure of the surface charge, and d.c. electrophoresis data which gave us the standard zeta potential value. The story is too complicated to reproduce here but the upshot was that this latex system did exhibit anomalous surface conduction. (That is to say, when placed in an electric field, there is some ionic conduction which occurs in the region between the surface of the particle and the shear plane. Normally that is considered to be a layer of solvent and ions which move 
wholly with the particle). This is an area of active investigation at the moment. It may prove to be of far-reaching importance, for it is possible that this anomalous surface conduction is more prevalent than we had thought. Wherever it occurs it must be properly taken into account if one is to obtain reliable zeta potential data from electrokinetics.

\section{THE ACOUSTOSIZER}

This is a new instrument which is about to be released onto the general market. Our company, Colloidal Dynamics, is involved in the development of the instrument and so had access to the first prototype and now has the first example of what is expected to be the production model.

The instrument is based on O'Brien's (1992) patent ${ }^{10}$. A sinusoidal voltage is applied to a pair of gold plated disc electrodes immersed in the colloidal suspension (Fig. 1). The electric field causes the particles to oscillate back and forwards due to their charge and in so doing they generate a sound wave. This is referred to as the Electrokinetic Sonic Amplitude (ESA)

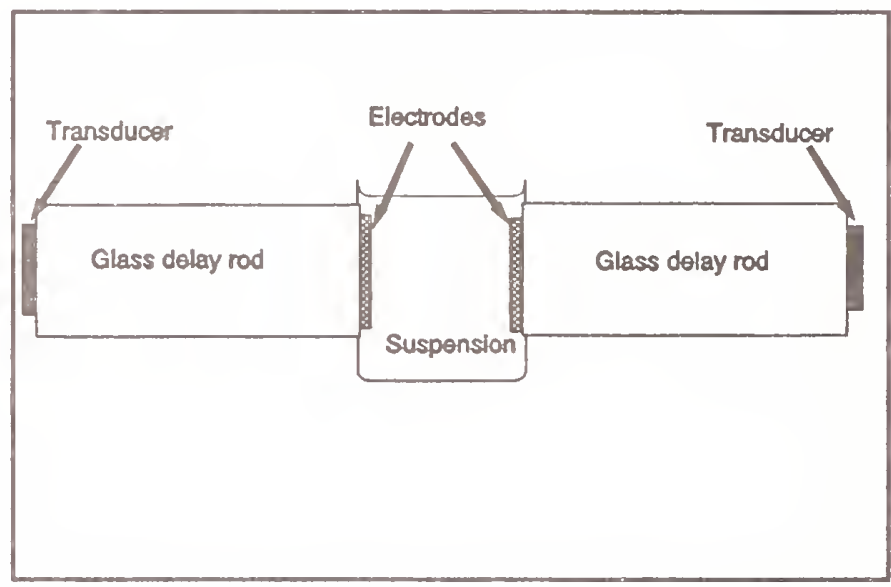

Figure 1. The AcoustoSizer cell and electrode system

effect. The sound wave travels down a glass delay line and is detected by a transducer attached to the end of the rod. The magnitude of the sound signal can be related to the charge on the particles (since this determines the amount of movement which is induced by a given applied field). The particle motion in the AcoustoSizer is driven by the electric force which is balanced by the hydrodynamic drag and the inertial force on the particle. At very low frequency the inertial force is insignificant and the dynamic mobility is equal to the d.c. (electrophoretic) mobility (i.e. $G=1$ in eqn.[1]). As the frequency of the applied electric field is increased, the inertial force on the particle becomes more significant, causing the particle motion to lag behind the applied field. Since the inertial effect also increases with 
particle size, the frequency-dependent phase lag provides information about the size distribution of the particles. As the frequency increases, the magnitude of the measured signal also decreases more rapidly for particles of larger size, since their inertia effect is larger. Both the magnitude and the phase of the measured signal can therefore be used to obtain the best estimate of the particle size distribution and the particle charge. In the AcoustoSizer the frequency range has been chosen to size particles ranging from 0.1 to 10 $\mu \mathrm{m}$.

Since the electroacoustic technique measures sound, it should be possible to determine the electrokinetic potential and size of colloidal particles in opaque or photosensitive suspensions at a much higher particle concentration (ranging from a few percent up to $40 \%$ by volume) than is possible using typical light scattering methods.

Attempts to extend the measurements up to very high volume fraction encounter another problem: the acoustic properties of the suspension, and hence the response, become dependent on the particle concentration. In particular, the acoustic impedance will change with concentration and this affects the magnitude of the resulting signals. [The acoustic impedance, $Z$, is defined as follows. If the boundary of a system is caused to move with a sinusoidal motion characterized by a velocity, $V \exp (i \omega t)$, then the pressure, $p$, required to produce this motion can be written $p=Z V$. For a homogeneous material, $Z=\rho c$ where $\rho$ is the density and $c$ is the velocity of sound in the material. For non-homogeneous systems, like colloidal suspensions, $Z$ can be complex (if there is absorption occurring) but we have found it to be real, and proportional to particle concentration, over the frequency range we have studied].

The second electrode and rod are used to provide a reference signal to which the signal from the first electrode can be compared, and this corrects for any drift in the electronics, or change in the acoustic impedance of the suspension, or small shifts in phase due to temperature effects.

The frequency of the applied field varies from 0.3 to $11.1 \mathrm{MHz}$ in 13 steps and the magnitude and phase of the signal is determined at each of those frequencies. From the spectrum of magnitudes and phases, both the charge and the size of the particles can be 
determined, at least for the case where the particles are smooth and spherical. Non-spherical particles, (disc shaped or cigar shaped spheroids) have been treated theoretically (subject to certain restrictions discussed below) and are expected to behave like spheres with a modified radius.

\section{Instrument Output}

In normal operation the computer associated with the instrument takes the spectrum of the ESA magnitudes and phases and compares them with the reference signals to deduce the dynamic mobility spectrum. The electrokinetic or zeta potential, the median size and standard deviation, assuming the particles have a log-normal distribution, are then adjusted to obtain the best fit to the mobility spectrum. Fig. 2 shows the fit obtained between theory and experiment for a monodisperse silica sol of radius $1.5 \mu \mathrm{m}$. Normally the user is unaware of the nature and treatment of the spectrum; only the charge and size are provided as output.

There is a further complication to consider when the particle concentrations at which we are working are towards the bottom end of the range for which the instrument is designed. Under such conditions it is usually advisable to correct for the effect of the ESA signal from the background electrolyte; this has to be subtracted (vectorially) from the measured signal. Fortunately it turns out that even for the most dilute systems studied this effect usually has only a very minor influence on the results and the correction must be applied only if one is striving for maximum precision.

The instrument can also perform a $\mathrm{pH}$ titration, plotting $\mathrm{pH}$ as a function of added base or acid and from this we can determine the magnitude of the titration charge, which is usually regarded as the total surface charge. The charge derived from the electroacoustic measurement is, on the other hand, a kinetic or effective charge, related to that part of the charge which is in the diffuse part of the electrical double layer around the particle. It is generally accepted that this latter charge gives a better indication of the likely coagulation behaviour of the colloid. 


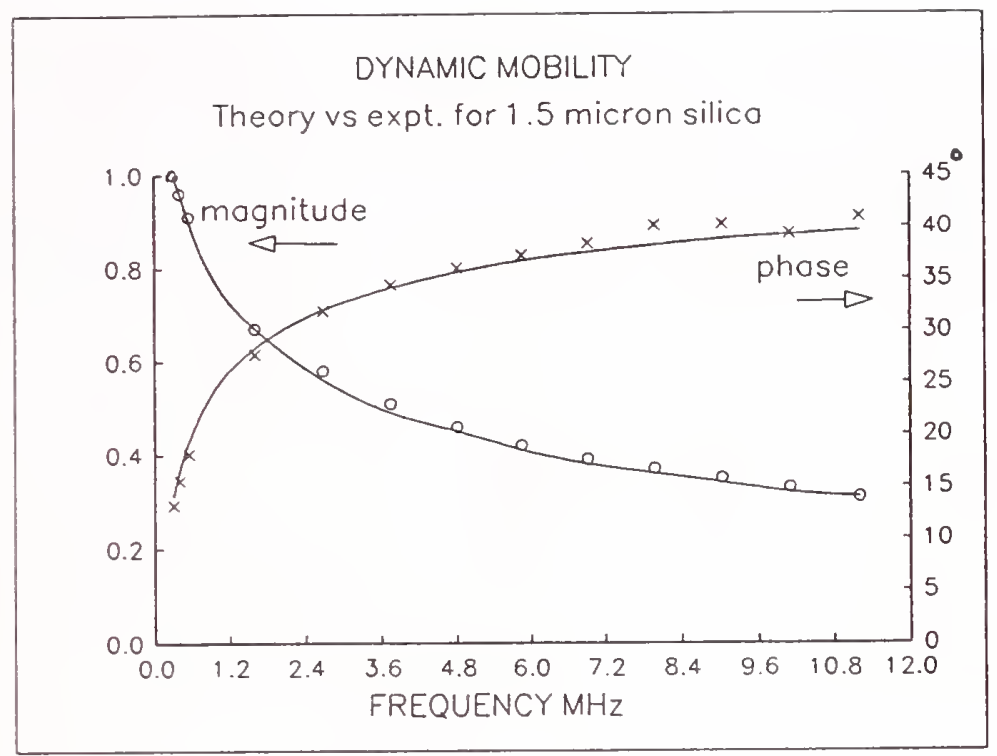

Figure 2: The magnitude is measured in relative units and the phase angle is given in degrees.

Although the theoretical analysis of the electroacoustic behaviour ${ }^{7}$ assumes the suspension to consist of a dilute collection of smooth spherical particles, we know from our more recent theoretical work ${ }^{11}$ that for spheroidal particles the behaviour is qualitatively similar. The result which is calculated for a system of anisometric particles is, therefore, an equivalent electroacoustic radius, in much the same way as the sedimentation radius in such cases is the equivalent settling radius.

\section{THEORY OF THE ELECTROACOUSTIC EFFECT}

$\mathrm{O}^{\prime} \mathrm{Brien}^{7}$ has shown that the ESA signal is related to the dynamic mobility, $\mu_{\mathrm{d}}$ of the particles by the following equation:

$$
E S A=C(\Omega) \cdot \phi\left(\frac{\Delta \rho}{\rho}\right) \cdot \mu_{d} \cdot \frac{Z_{g} Z_{s}}{Z_{g}+Z_{s}}
$$

where $C(\Omega)$ is an instrument constant which does not depend on the properties of the suspension, $\phi$ is the volume fraction of the particles and $\Delta \rho$ is the difference between the density of the particles, $\rho_{\mathrm{p}}$ and that of the medium, $\rho . Z$ is the acoustic impedance and subscripts $g$ and $s$ refer to the glass rods and the suspension. This expression is valid for 
suspensions of arbitrary concentration. The dynamic mobility is a complex quantity which depends on the measurement frequency.

The theoretical analysis of dilute suspensions of spheres shows that the dynamic mobility can, in that case, be related to the radius, $a$, and zeta potential, $\zeta$, of the particles through the equations

$$
\mu_{d}=\frac{2 \varepsilon \zeta}{3 \eta} G\left[\frac{\omega a^{2}}{\nu}\right)(1+f)
$$

where

$$
G(q)=\left[1-\frac{i q[3+2(\Delta \rho / \rho)]}{9[1+(1-i) \sqrt{ }(q / 2)]}\right]^{-1}
$$

and $\omega$ is the measuring frequency and $\nu$ is the kinematic viscosity. $G(q)$ measures the inertia effect and causes the magnitude of the mobility to decrease monotonically from its d.c. value $\left(\mu_{\mathrm{B}}\right)$ to about half that value at $q=10$, provided $f$ is constant. The value of $q\left(=\omega a^{2} / \nu\right)$ in water at $25^{\circ} \mathrm{C}$ is $7.00 a^{2}$ for $a$ in $\mu \mathrm{m}$ and a frequency of $1 \mathrm{MHz}$. [ $q=6.26 a^{2}$ at $\left.20^{\circ} \mathrm{C}\right]$.

The function $(1+f)$ is proportional to the tangential component of the electric field at the particle surface and it, too, is complex:

$$
1+f=\frac{3\left(1-i \omega^{\prime}\right)}{2\left(1-i \omega^{\prime}\right)+\left(2 \lambda-i \omega^{\prime} \varepsilon_{p} / \varepsilon\right)}
$$

where $\lambda=K^{3} / K^{\infty} a$ is called the surface conductance parameter and $\varepsilon_{\mathrm{p}}$ is the particle permittivity; $\omega^{\prime}=\omega \varepsilon / K^{\infty}$ is the frequency divided by the relaxation frequency for ion movement in the double layer (a few $\mathrm{MHz}$ in ordinary electrolytes). For many systems it turns out that the second term in the denominator is much smaller than the first and so we can take $f=1 / 2$ and equation [3] reduces to eqn [1]. This is the relation which is used in the software associated with the Matec ESA-8000. The AcoustoSizer makes a more accurate assessment by allowing $\lambda$ to vary as the estimated zeta potential varies. In this way the new instrument is able to take account of 'surface conduction effects' provided that they are 'normal', i.e. the system exhibits the amount of double layer conduction which would be 
expected from the magnitude of zeta, assuming that the ions in the diffuse double layer have their normal conductivity values and conduction occurs only outside the slipping plane where zeta is measured.

\section{Expected Behaviour of a Colloidal System}

The predicted behaviour of a system of spherical particles exhibiting small surface conduction can be seen from Fig. 2. Note that the magnitude of the dynamic mobility decreases monotonically and the phase angle increases from zero to a limiting value as the frequency increases. Bigger particles show bigger effects in both cases but the limiting value for the phase angle is $45^{\circ}$.

The effect of size on phase angle is independent of the charge so the size and charge can be uniquely determined. The output we obtain from the AcoustoSizer is the dimensionless mobility, $E_{\mathrm{d}}$, which is related to the dynamic mobility by:

$$
E_{d} \cdot \frac{2 \varepsilon k T}{3 \eta e}=\mu_{d}
$$

so $\mu_{\mathrm{d}}=1.17 \times 10^{-8} E_{\mathrm{d}}$ in SI units at $20^{\circ} \mathrm{C}$.

\section{The Sizing Algorithm}

The mobility spectrum is determined by a series of steps: (1) the raw ESA signal is compared in magnitude with the reference signal taken from the second electrode; (2) the background electrolyte signal is subtracted (in the case of dilute suspensions) and (3) the resulting set of complex numbers is divided by the corresponding set for a system with the same or similar conductivity and a known dynamic mobility. This last comparison takes account of the effects of electrolyte conductance on the shape of the electric field inside the cell. That calibration is the most crucial step in the process if one wants absolute values rather than qualitative comparisons. We are still refining the values used by crossreferencing data obtained on different known systems. 
Estimation of the size and charge of a system is based on obtaining a best fit to the entire spectrum of mobility data both with respect to magnitude and phase. This is best understood in terms of Figure 3 in which the anticipated values of the mobility are plotted for different sized particles. The plot is done on an Argand diagram which shows the values of the magnitude of the mobility $\left(\left|\mu_{\mathrm{d}}\right|\right)$ and its phase, $\theta$, where $\mu_{\mathrm{d}}=\mathrm{X}+\mathrm{iY}$ and

$$
\left|\mu_{\mathrm{d}}\right|=\left(\mathrm{X}^{2}+\mathrm{Y}^{2}\right)^{1 / 2} ; \quad \tan \theta=(\mathrm{Y} / \mathrm{X}) \text {. }
$$

Fig. 3(a) illustrates equation (7). Fig. 3(b) shows how the spectrum of mobility values for a particular system is expected to look for different sized particles. The successive points represent the values of the complex quantity, $\mu_{\mathrm{d}}$, at different frequencies

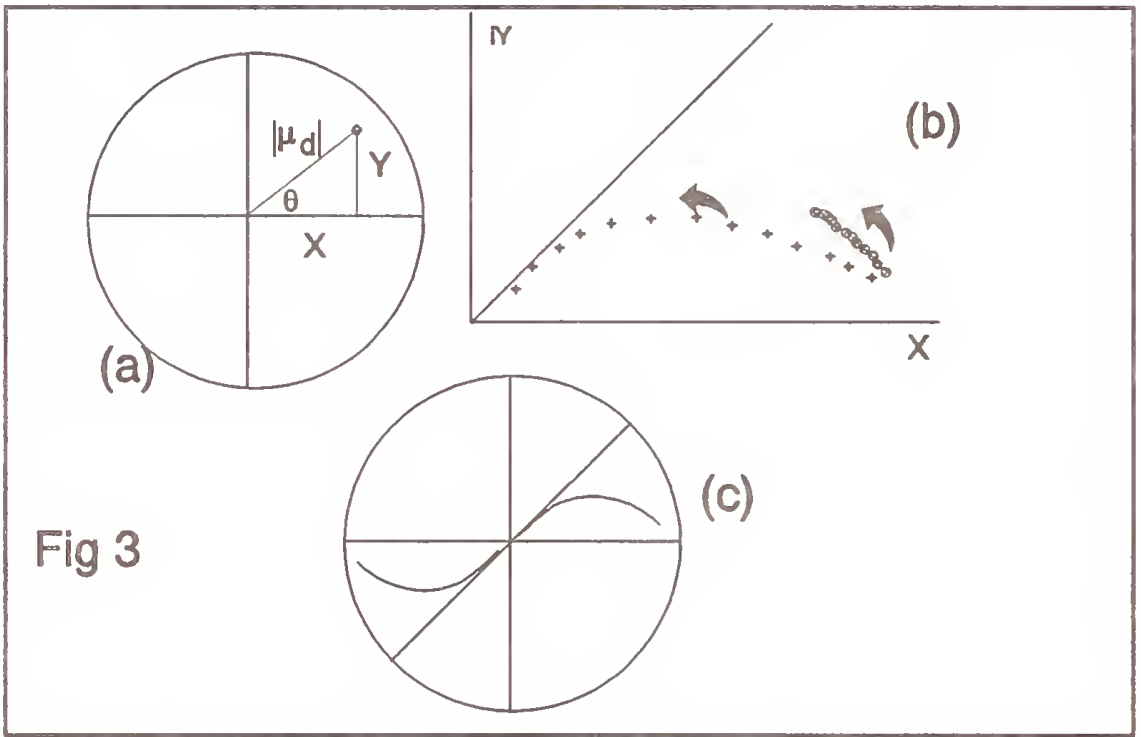

Figure 3: The mobility spectrum. (b) shows an enlarged picture of the first quadrant of Fig (a).

(increasing in the

direction of the arrow). For a given zeta, a small particle (circles) will show a larger magnitude than a larger particle $(+)$ but the difference is slight at the lowest frequency and increases as the frequency rises. The magnitudes always fall with increase in frequency whilst the phase angles always rise and tend to the limiting value of $45^{\circ}$, if the particles are large enough. Notice that for very small particles the magnitude does not change much, and neither does the phase angle, so the points tend to be bunched together more than for the larger particles. Changing the sign on the particles reflects the curve through the origin and changes the phase by $180^{\circ}$ (Figure $3(\mathrm{c})$ ) but for systems with $\lambda \approx 0$ the points are always confined to the two wedge-shaped sectors from $\theta=0$ to $45^{\circ}$ and $\theta=180^{\circ}$ to $225^{\circ}$. 
The measured mobility $\langle\mu\rangle$ for a dilute suspension is given by

$$
<\mu>=\int_{0}^{\infty} \mu(a) \cdot p(a) d a
$$

where $p(a) \mathrm{d} a$ is the (mass) fraction of particles with radius between $a+\mathrm{d} a / 2$ and $a-\mathrm{d} a / 2$. The measured spectrum is fitted by a least squares procedure to obtain the best parameters to describe the distribution function for the sizes. The particles are assumed to have a common zeta potential and to have a log-normal distribution of radii. The fitting procedure then determines the best fit values for zeta, the median radius, and a parameter which characterizes the spread of the radii; the algorithm fits both the magnitude and the phase angle spectrum over the entire frequency range. In practice the procedure is not simple but the complexities are not apparent to the user.

\section{RESULTS WITH THE ACOUSTOSIZER}

The performance of the AcoustoSizer on a set of nominally monodisperse samples can be judged from Fig. 4. The limits indicated by the solid horizontal lines cover the size from the 15 th percentile to the 85 th percentile as measured by sedimentation, using the Horiba Capa 700. The mark on the line indicates the position of the median size. The curves show the log-normal distribution estimated from the AcoustoSizer data. 


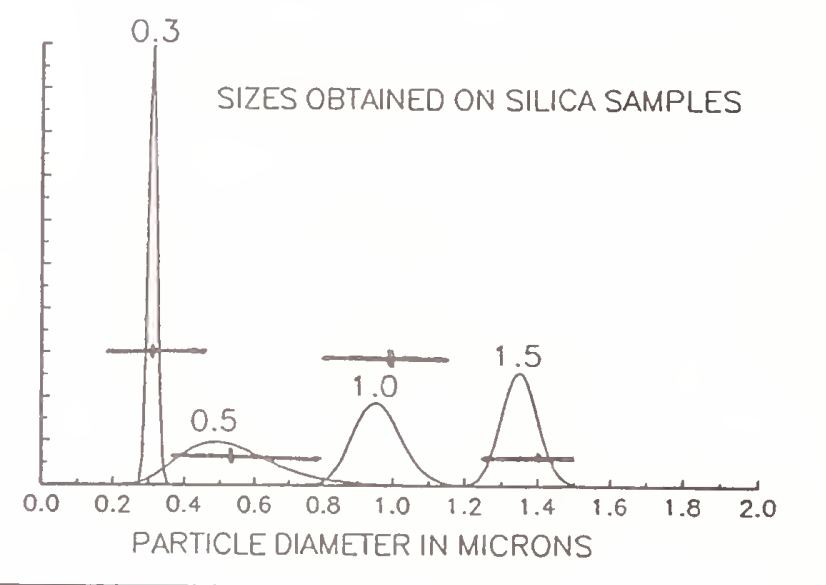

Figure 4: AcoustoSizer estimates of size are shown by the horizontal line. The mark indicates the mean of the assumed log normal distribution.

Fig. 5 shows the result of the same procedure applied to a sample of material for which the distribution is known with some confidence. These are crushed quartz samples prepared and sized by the Bureau of Common Reference of the European Community. The sizing was done by a settling procedure using direct measurements. As can be seen, the AcoustoSizer suggests a slightly broader distribution than the measured one but the median size is determined quite accurately and the result is certainly within the range of results obtained by other methods. 


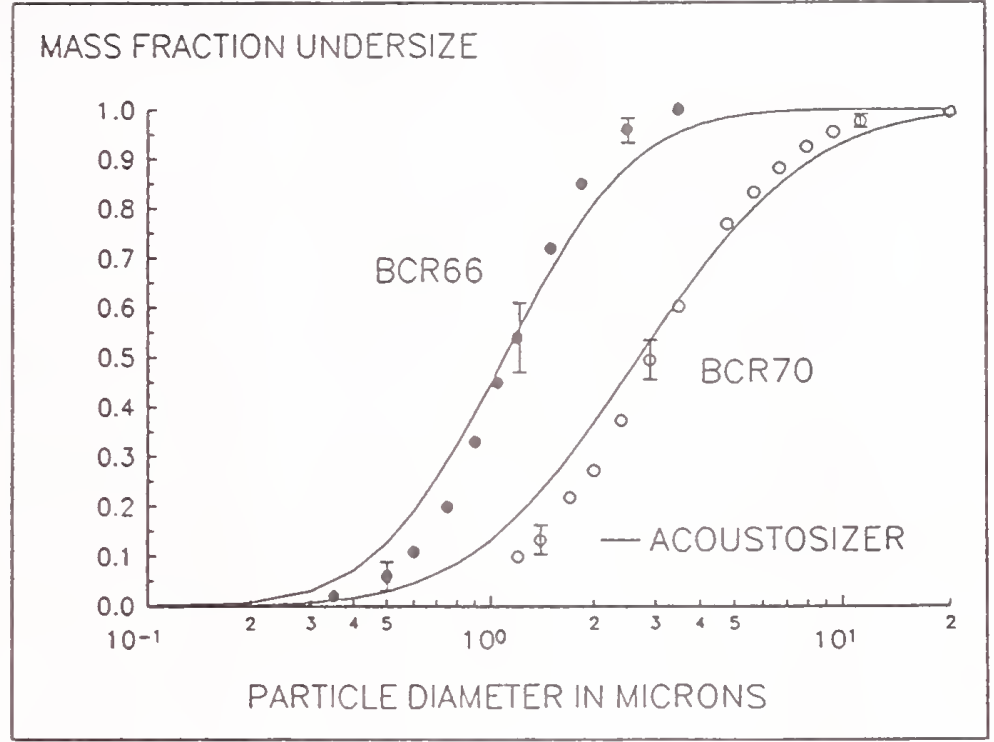

Figure 5: The full line represents the AcoustoSizer estimate. The points are the standard estimates for these materials.

Fig. 6 shows the results obtained for a variety of other materials using the AcoustoSizer and more conventional methods of sizing. It is clear that the new method gives results which are as satisfactory and consistent as any of the other methods to which it is compared.

The above measurements of size have all been conducted on samples which were well dispersed and at modest concentrations (up to about $5 \%$ ). The sols were well removed from their respective isoelectric points. The following table shows the results of a comparison of the estimated zeta potentials from the measured dynamic mobilities, using equation [3], compared with those calculated from the (d.c.) electrophoretic mobility.

Even when the zeta potential is brought close to the isoelectric point, $\left(\mathrm{TiO}_{2}\right.$ at $\mathrm{pH}$ 8.2) the agreement is excellent, provided one uses the correct apparent radius for the calculation. Unfortunately, we have found that in some systems, when the particles are unstable they can form a deposit on the electrodes and this gives rise to a spurious ESA signal which interferes with the measurement and leads to erroneous estimates of the phase angle. The size and charge estimates then become impossible. We are currently working on methods to obviate this difficulty. 
Comparison of Zeta potentials (in $\mathrm{mV}$ ) measured by the AcoustoSizer

with measurements using the Rank Bros Mark II apparatus and the Malvern ZetaSizer

\begin{tabular}{|c|c|c|c|c|}
\hline Colloid & pH & $\begin{array}{l}\text { Acousto- } \\
\text { Sizer }\end{array}$ & Rank & $\begin{array}{l}\text { Zeta } \\
\text { Sizer }\end{array}$ \\
\hline \multirow[t]{3}{*}{$\mathrm{TiO}_{2}$} & 4.0 & 37 & 37 & \\
\hline & 8.2 & -6 & -6 & \\
\hline & 4.0 & -20 & -22 & \\
\hline \multirow[t]{2}{*}{$\mathrm{Si}_{3} \mathrm{~N}_{4}$} & 3.5 & 26 & 24 & \\
\hline & 8.9 & -39 & -43 & \\
\hline \multirow[t]{2}{*}{ Kaolin } & 4.4 & -26 & -26 & \\
\hline & 4.8 & -37 & -43 & \\
\hline Alumina & 5.0 & 47 & 58 & 53 \\
\hline $\begin{array}{c}\mathrm{Si}_{3} \mathrm{~N}_{4} \\
\text { (Sample b) }\end{array}$ & 10.0 & -35 & -42 & -51 \\
\hline $\begin{array}{c}\mathrm{TiO}_{2} \\
\text { (Sample b) }\end{array}$ & 4.0 & 40 & 50 & 57 \\
\hline
\end{tabular}

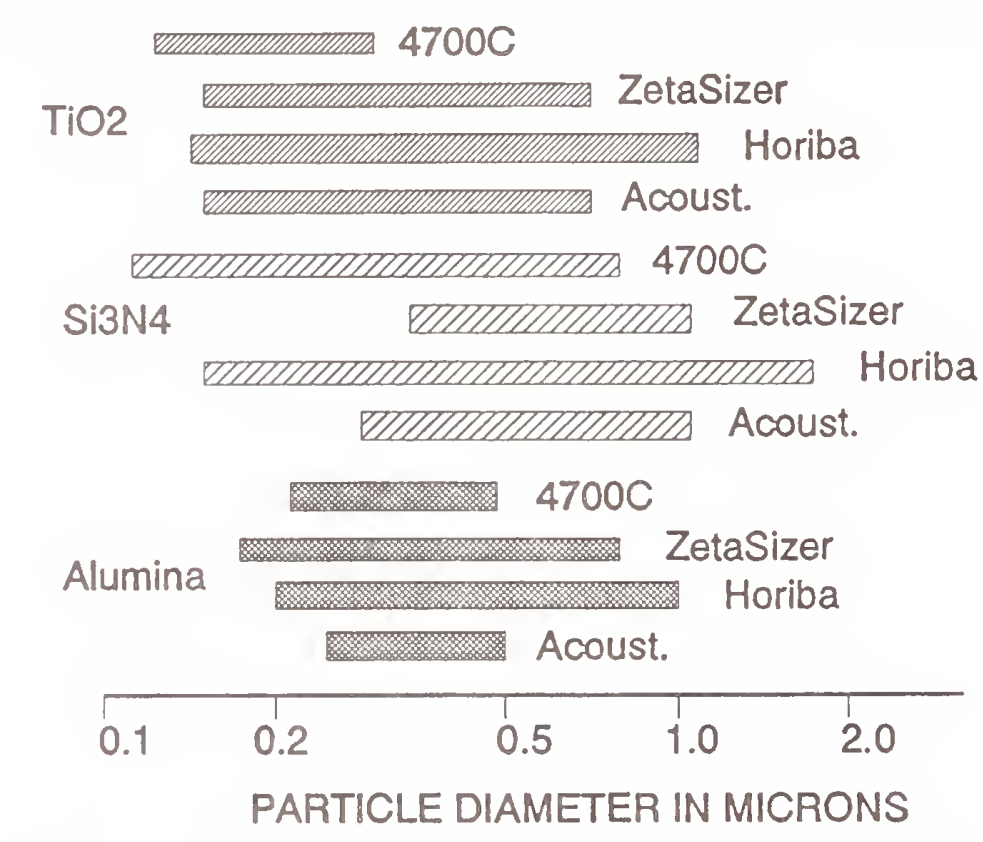

Figure 6: Comparison of various techniques for particle size analysis of different materials 


\section{MEASUREMENTS AT HIGHER PARTICLE CONCENTRATION}

At particle concentrations up to about $10 \%$ it is possible to assume that the acoustic impedance of the system is essentially the same as that of pure water. The zeta potential can also be calculated from the dilute solution theory (eqn [3]) with reasonable confidence. When the particle concentration is much higher it is necessary to take account of any changes in the acoustic impedance of the system in order to obtain reliable estimates of the dynamic mobility. There then remains the problem of determining the extent to which the theory can estimate charge and size from these dynamic mobility measurements.

The variation in impedance with volume fraction for a variety of samples is shown in Figure 7. Rather surprisingly, it turns out that the impedance varies linearly with volume fraction for these systems, and it is a real quantity which is independent of frequency. If that is found to be true for all systems, it will be a great deal easier to allow for it.

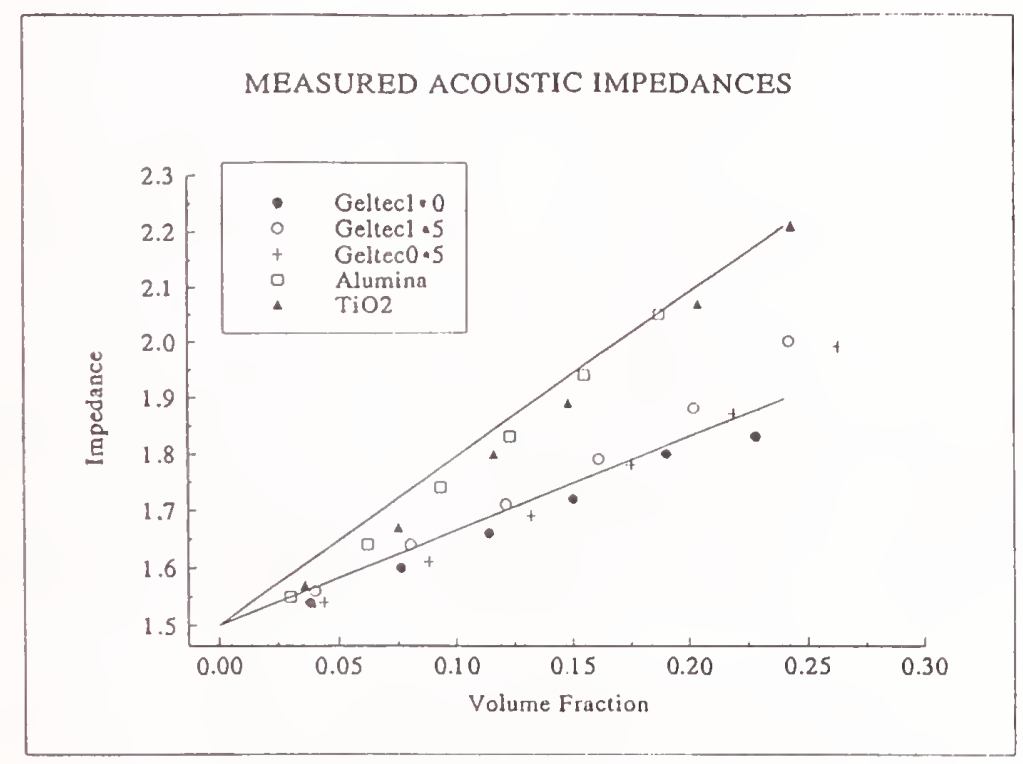

Figure 7: Measured acoustic impedance of different systems. The two lines are drawn using a simple relationship which assumes that the impedance is a function only of the volume fraction of the particles and the particle density. 
Even with this simplification, we still need a theoretical model to describe how the particle concentration is expected to influence the dynamic mobility. The most likely sort of model to use is a form of cell in which each particle is surrounded by an appropriate volume of liquid and the mathematical boundary conditions on the surface of the cell are arranged to produce a reasonable approximation to the conditions operating in the total volume of the suspension. We have set up such a cell model and from it have calculated the anticipated dependence of the magnitude and phase of the dynamic mobility as a function of frequency.

The result is shown in Fig. 8 and it is obvious that this particular cell model is not representing the behaviour as well as we would have hoped. That is rather surprising since the form of the boundary conditions used here has been used successfully in other applications of a very similar sort. The cell model does not suggest nearly enough influence of the concentration at the highest frequencies and it is difficult to see how to modify it. We have, as yet, no inkling of what could be causing the large effect at the uppermost frequency.

Fortunately it is possible to adopt an alternative, semi-empirical procedure. We can take a known system (like the Geltech silica) and determine the values of $\zeta, a_{\mathrm{m}}$ and $\sigma$ in dilute (less than $5 \%$ ) suspension. In practice we assume that the particles have a log-normal size distribution and determine the median size, $a_{\mathrm{n}}$ and the spread, $\sigma$ of that distribution where $a_{\mathrm{m}}$ $\pm \sigma$ covers the range from 15 to $85 \%$ of the sizes. We can then determine how $\zeta, a_{\mathrm{m}}$ and $\sigma$ appear to depend on the volume fraction, $\phi$ for the Geltech. (It turns out that $\zeta$ and $a_{\mathrm{m}}$ both decrease and $\sigma$ increases with volume fraction). We can then correct the estimated value for the unknown $\zeta$ at a given $\phi$ value by multiplying by the ratio of the true $\zeta$ to the apparent $\zeta$ for the standard Geltech system. The same procedure can be applied to the other variables. 
MOBILITY MAGNITUDES

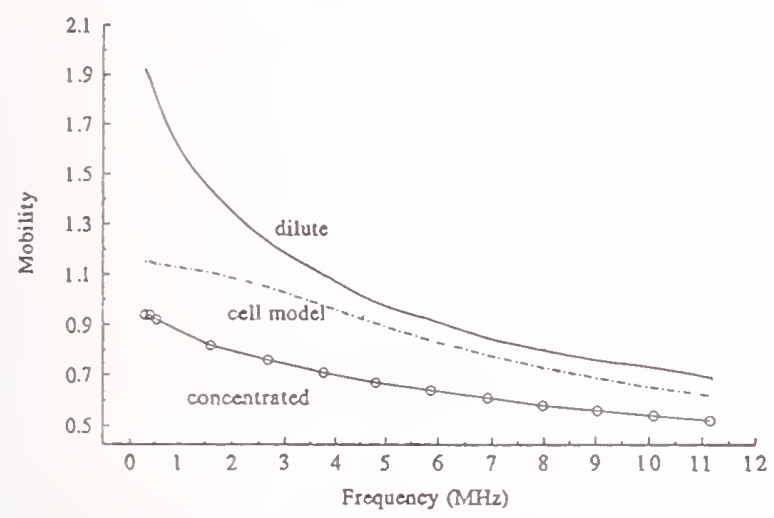

PHASE LAG

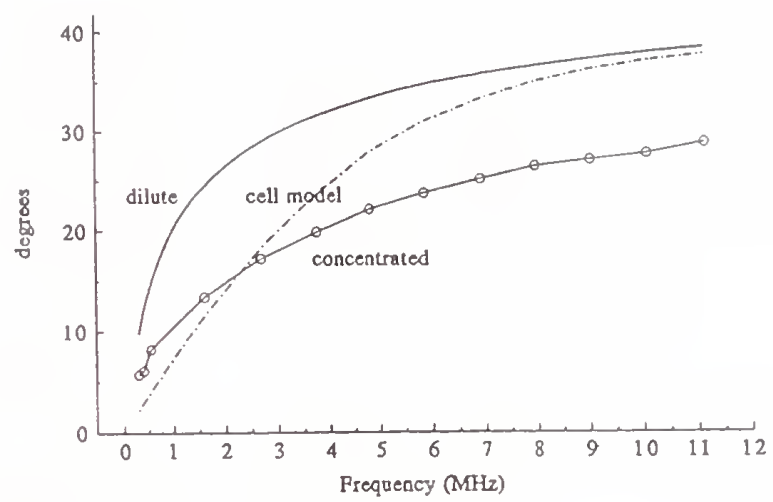

Figure 8. The magnitude and phase lag measured for a typical system and compared to the theoretical expectation for the current cell model.

The result of this procedure when applied to suspensions of $\mathrm{TiO}_{2}$ and alumina is shown in Figures 9 and 10. The estimated median size of the alumina is very nearly constant up to about $20 \%$ whilst the values for $\mathrm{TiO}_{2}$ show a smooth variation of around $50 \%$ over the range. The zeta values show a steady drop of around $10 \%$ for the alumina and perhaps $20 \%$ variation for the titania. The result is, however, extremely promising and is certainly greatly superior to any alternative procedure for examining these quantities in concentrated systems.

In many industrial situations the particle concentration is not varying greatly and one would expect that even if the measured values have no exact quantitative significance, the estimated values should be able to tell something about what is happening in such systems. We presume that a particular system will show a reproducible response in terms of the magnitude and phase of the dynamic mobility as a function of frequency, even if the behaviour is not well described by the theory for smooth spherical particles described above. Indeed, we have done a preliminary investigation on one such system - a suspension of the clay mineral kaolinite. 

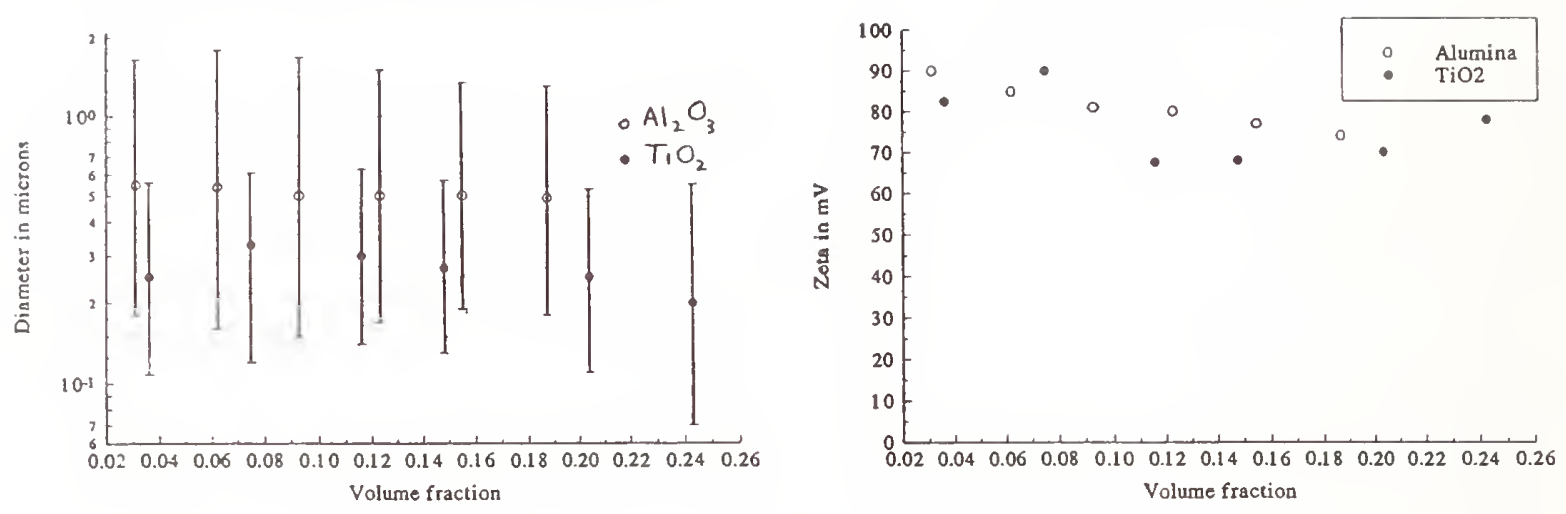

Figure 9. Calculated sizes for alumina and titania as a function of volume fraction using the quasi-empirical procedure described in the text. The bars in this case represent the range from the 15 th to the 85 th percentile.

Figure 10. Estimated zeta potentials for alumina and titania using the same procedure as for Figure 9.

Kaolinite particles show a number of anomalous electroacoustic properties. To begin with, both the magnitude and the phase of the dynamic mobility, as a function of frequency, are smaller than would be expected from other measurements. The fact that these particles behave as though they were smaller than their nominal size is not surprising. They are disc-shaped and our theoretical calculations ${ }^{10}$ suggest that such particles will give a phase angle signal corresponding to a particle with a radius reduced in the ratio $\sqrt{ } k$ where $k$ is the axial ratio of the particles (ratio of width of the disc to its thickness). More surprising at first sight is the fact that the magnitude of the signal actually increases with frequency. Once again this is understandable, at least qualitatively, by the fact that the parameter $\lambda$ in equation [3] is very large. Instead of the value of 0.02-0.03 expected from the estimated zeta potential, its measured value is close to unity. This large value for $\lambda$ is referred to as anomalous surface conductance and the fact that kaolinite exhibits it to such a high degree means that it will have to be treated using a special version of the sizing algorithm if we are to obtain the necessary accuracy for both size and charge. 


\section{CONCLUSIONS}

Electroacoustics provides a very convenient procedure for following the zeta potential or charge of a colloidal particle in suspension with the addition of some reagent. The most readily exploited is the effect of acid and base on the surface charge of oxide and similar materials. We have extended that to an investigation of the effect of added surface active ions and have shown that the electroacoustic method is rapid and convenient.

With the advent of the AcoustoSizer the range of application of the method is much wider. Particle size can be measured directly and this makes determination of the zeta potential more reliable as well. The new device can make adequate estimates of both size and charge in suspensions with particle concentrations up to almost $30 \%$ by volume (about $60 \%$ by weight). The mathematical analysis takes some 2 minutes using our 80286 PC but with the $80486 \mathrm{cpu}$ which will be used in the commercial instrument the analysis will be done in real time if the titration is conducted at the usual rate to ensure that the solution remains in equilibrium with the particle surface.

\section{ACKNOWLEDGMENTS}

We thank Matec Applied Sciences for the loan of the prototype version of the AcoustoSizer and the Australian Department of Industry Technology and Commerce and the Australian Research Council for grants to assist us in the development of the instrument and the basic science of electroacoustics respectively. 


\section{REFERENCES}

1. O'Brien, R. W., Midmore, B. R., Lamb, Alice, and Hunter, R. J. (1990) Faraday Disc. Chem. Soc., 90, 301-12.

2. Scales, P. and Jones, E. (1992) Langmuir 8, 385.

3. James, M., Hunter, R. J. and O’Brien, R. W. (1992) Langmuir 8, 420-3.

4. Rowlands, W. N. and Hunter, R. J. (1992) Clays and Clay Minerals 40, 287-91.

5. Hunter, R. J. and James, M. (1993) Clays and Clay Minerals (accepted).

6. Shubin, V., Hunter, R. J. and O'Brien, R. W. (1993) J. Colloid Interface Sci., (accepted).

7. O’Brien, R. W. (1988) J. Fluid. Mech. 190, 71-86.

8. Midmore, B. R., Hunter, R. J. and O'Brien, R. W. (1987) J. Colloid Interface Sci. 120, 210.

Midmore, B. R. and Hunter, R. J. (1988) J. Colloid Interface Sci, 122, 521.

9. Healy, T. W. and James, R. O. (1972) J. Colloid Interface Sci. 40, 42, 53 \& 65.

10. O’Brien, R. W. (1992) US Patent No 5,059,909, Oct 22, 1991.

11. Loewenberg, M. and O'Brien, R. W. (1992) J. Colloid Interface Sci. 150, 158-68. 


\title{
THEORETICAL CONSIDERATIONS IN ACOUSTOPHORETIC ANALYSIS OF CONCENTRATED COLLOIDS
}

\author{
Hemant P. Pendse, Terry A. Strout and Arvind Shanna \\ Industrial Process Control Sensor Systems Program \\ UNIVERSITY OF MAINE \\ ORONO, ME 04469
}

\begin{abstract}
In recent years several advances have been made in ultrasound based instruments and sensors for monitoring zeta potential and particle size distributions of colloidal suspensions. This paper deals with the ultrasonic response of colloidal slurries, over a wide frequency range, with particular attention to the effects of particle size distribution (PSD) and concentration. Measurements of ultrasonic attenuation spectra are used in a newly developed AcoustoPhor System to get PSD of colloidal dispersions.
\end{abstract}

\section{INTRODUCTION}

As sound travels through a suspension, the particles undergo oscillations relative to the suspending medium as consequence of difference in the densities of particle and medium phases. This relative motion is termed acoustophoresis.

Acoustophoresis induces a macroscopic colloid vibration potential (CVP) arising from the relaxation of ionic atmospheres of suspended particles. Measurements of CVP amplitude at a fixed operating frequency have been used to monitor zeta potential of concentrated aqueous pigment slurries in the Acoustophoretic Titrator (Pen Kem System 7000). In the Acoustophoretic Titrator the CVP phase is used to determine the zeta potential sign (Pendse, 1987; Marlow, Fairhurst and Pendse, 1988). In general, CVP amplitude and phase are found to depend on operating frequency, as well as particle size and concentration. Monitoring changes in the CVP phase during repeated acoustophoretic titrations has been suggested as a tool for detecting degree of flocculation (Pendse and Strout, 1990a). However, ability to use measurements of amplitude and phase of the CVP to determine the zeta potential and the PSD simultaneously, is inherently dependent on availability of 
appropriate predictive models that account for electric double layer relaxation associated with the acoustophoresis at moderate and high particle volume fractions. Preliminary analysis based on available dilute-limit models indicates that the frequency-dependent response of vibration potential amplitude and phase is complicated by (i) interacting roles of relative magnitudes of electric double layer thickness and oscillatory boundary layer thickness compared with particle size, and (ii) issues related to superposition of contributions of different sized particles in a polydisperse suspension, in addition to the contributions of dissolved ions and polyelectrolytes.

Acoustophoresis also causes attenuation of sound due to viscous dissipation associated with the particle motion relative to the suspending medium. Pen Kem has developed a new commercial laboratory instrument, called AcoustoPhor System for monitoring particle size distributions of concentrated colloidal slurries. The instrument is based on estimation of PSD functions using measurements of ultrasound attenuation coefficients at several frequencies over 1 to $100 \mathrm{MHz}$ by solving appropriate integral equations. The mathematical algorithm employed in the deconvolution or inversion of measured spectra utilizes the results of a recent theoretical model developed, by the University of Maine researchers, to predict the behavior of ultrasound as it passes through a slurry of polydisperse particles at a known concentration, as high as 50 volume percent. In a cooperative research project, Pen Kem developed and tested prototype sensor electronics and computer system with the measurement software capable of programmed ultrasonic spectroscopy. The slurry characterization chambers and the sample delivery system along with the sensor operating software were developed by P\&G Systems with design and fabrication assistance from Techna Machine. One on-line and two laboratory sensor prototypes have been developed and tested extensively in laboratory and production settings using several industrial mineral pigment slurries. The prototype sensor instrument system was tested successfully in the University of Maine and Du Pont research laboratories. Evaluation to date shows that the sensor is capable of providing particle size distributions, without any assumptions regarding their functional form, over diameters ranging from 0.1 to 100 micrometers in slurries with particle concentrations of 5 to 50 volume percents. 
An on-line prototype was evaluated at Du Pont's semiworks facility for pigment production during several months of field testing. Field evaluation has shown that the new ultrasound based PSD sensor system is capable of handling several types of mineral pigment slurries commonly encountered in industry under processing concentrations. During the field tests, the system has operated reliably as an on-line monitoring tool during protracted runs of continuous operation extending over several days, providing data sensitive to changes in the processing conditions. The main objectives in these tests were to study sensitivity of the analyzer to changes in the processing conditions, and to study robustness of the prototype design for extended operations in manufacturing plant environments. During several twentyfour hours per day and five days per week operations, the analyzer was found to be operating in a robust and reliable manner. The repeatability of data was also found to be excellent (Pendse, 1992).

In the following sections we present a brief theoretical background on this technique along with some illustrative results.

\section{BACKGROUND}

\section{Acoustophoresis and Associated Sound Attenuation}

The extent of attenuation depends on the length of the acoustic path, the concentration and sizes of the particles, the frequency of sound, and the physical properties of the particulate and fluid phases. In particular, the density contrast, $\sigma$ defined as the difference between the densities of the particulate and fluid phases divided by the fluid density is an important characteristic parameter in connection with the acoustophoresis and hence the associated viscous attenuation. The acoustophoretic velocity is zero when the density contrast is zero. The expression for the acoustophoretic velocity, $\nu_{\mathrm{A}}$ normalized by the undisturbed fluid velocity, $u$ takes the form:

$$
\frac{v_{A}}{u}=\frac{4}{9} s^{2} \sigma C_{v}(s, \phi)
$$


with

$$
s=\sqrt{\frac{D^{2} \omega \rho_{0}}{8 \eta_{0}}}
$$

where

$$
\begin{array}{lll}
\mathrm{D} & = & \text { particle diameter, } \\
\omega & = & \text { sound frequency in radians/second, }(2 \pi f), \\
f & = & \text { sound frequency in cycles/seconds, } \\
\rho 0 & = & \text { density of the suspending medium, } \\
\eta 0 & = & \text { viscosity of the suspending medium, } \\
\mathrm{s}^{2} & = & \text { vibrational Reynold's number, corresponds to } \\
\text { (particle radius/oscillatory boundary layer thickness) }
\end{array}
$$

Based on the considerations of the rate of viscous energy dissipation in a suspension of spherical solid particles subjected to a sound wave, one obtains the following expression for the attenuation coefficient contribution due to monodisperse particles, $\alpha_{\text {mono: }}$ :

$$
\frac{\alpha_{m o n o} C_{0}}{\omega \phi}=\frac{2}{9} s^{2} \sigma^{2} C_{A}(s, \phi)
$$

where

$$
\begin{array}{lll}
c_{0} & = & \text { sound speed in the suspending medium, and } \\
C_{\mathrm{A}}(s, \phi) & =\text { attenuation coefficient correction factor }
\end{array}
$$

For the case of polydisperse particles, the attenuation coefficient contribution as a function of operating sound frequency $f, \alpha(f)$ becomes: 


$$
\alpha(f)=\alpha_{\text {medium }}(f)+\int \alpha_{\text {mono }}(f, D) g(D) d D
$$

where

$$
\begin{aligned}
& g(D)=\quad \begin{array}{l}
\text { PSD by mass fraction with } g(D) d D \text { equal to the mass fraction } \\
\text { of particles having diameters in range of } D \text { to } D+d D, \text { and }
\end{array} \\
& \alpha_{\text {mono }}(f, D)=\quad \begin{array}{l}
\text { attenuation coefficient contribution at a frequency } f \text { due to a } \\
\text { monodisperse population with all particles having a diameter } \\
\text { equal to } D
\end{array}
\end{aligned}
$$

The particular expression for $\alpha_{\text {mono }}$ mentioned above takes into consideration the viscous dissipation associated with the acoustophoresis properly accounted for finite values of both the vibrational Reynold's number and the particle volume fraction. The non-linearity associated with the concentration dependence of attenuation coefficients is accounted for in the factor $C_{\mathrm{A}}(s, \phi)$. For very dilute suspensions $(\phi \rightarrow 0)$, and for small diameters and low frequencies $(s \rightarrow 0), C_{\mathrm{V}}(s, \phi)$ and $C_{\mathrm{A}}(s, \phi)$ approach 1 . For suspensions of submicron particles with considerable density contrast the viscous contribution to the attenuation often dominates over the thermal and scattering contributions in the frequency range of practical interest corresponding to the long-wavelength limit $(\lambda>>\pi D$ and $\sigma>>1)$. These conditions correspond to ultrafine inorganic pigment slurries commonly encountered in industry.

The correction factor for the attenuation coefficient, $C_{A}(s, \phi)$ is obtained using the coupledphase formulation for sound propagation through suspensions with the viscous dissipation term based on the oscillating sphere-in-cell model (Strout, 1991; Sharma, 1993). Use of the oscillatory sphere-in-cell model extends the coupled-phase approach to a broad range of concentrations while retaining appropriate size and frequency dependence in predictions of viscous attenuation coefficients. The frequency response of the attenuation coefficient is of interest because the frequency of the applied sound can be readily varied in practice to obtain a broad-band spectrum which uniquely characterizes a suspension. For frequencies and particle sizes corresponding to vibrational Reynold's numbers larger than unity, the drag force corrections become significantly different from those estimated on the basis of steady-state cell models. Ability of our present model to account for attenuation coefficient dependence of vibrational 
Reynold's number over a wide range at moderate and high particle volume fractions allows us to properly interpret measured attenuation spectra in terms of particle size distributions.

General Formulation of the Integral Problem for Estimating PSD Using Ultrasound Attenuation Spectra

Using measurements of the attenuation coefficient as a function of operating frequency, $\alpha(f)$ covering an appropriate frequency range and a known expression for $\alpha_{\text {mono }}(f, D)$, one can obtain the PSD function, $g(D)$ by inverting the above mentioned integral equation. The problem encountered in determining $g(D)$ from known $\alpha(f)$ is known by mathematicians as a Fredholm integral problem of the first kind. It is important to reformulate the governing integral equation in terms of transformed variables as follows:

$$
R(f)=\int K(f, X) p(X) d X
$$

where

$$
\begin{gathered}
R(f)=\frac{\alpha(f)-\alpha_{\text {medium }}(f)}{r(f)} \\
X=q(D) \\
p(X) d X=g(D) d(D)
\end{gathered}
$$

and

$$
K(f, X)=\frac{\alpha_{\text {mono }}(f, D(X))}{r(f)}
$$

The transformations defined by Equations (6) and (7) are selected to obtain appropriately normalized and non-dimensionalized variables $R$ and $X$. Proper selection of functions $r(f)$ 
and $q(D)$ can yield a well-behaved Fredholm integral problem. Note that $r(f)$ is independent of diameter $D$, and $q(D)$ is independent of frequency $f$. In this context the function $R(f)$ is referred to as the response function, and the function $K(f, X)$ as the kernel function.

In practice the measured response is not known accurately. Hence the above equation is only satisfied within measurement errors. This integral problem does not possess a solution if the kernel function $K(f, X)$ is separable into factors as $K(f) \cdot K^{n}(X)$. Hence, for a separable kernel function one can not determine $p(X)$ from known $R(f)$. Another peculiar nature of this integral problem can be appreciated by noting that for any $K(f, X)$ as long as it is reasonably smooth and not a sharply peaked kernel.

$$
R(f)=\int K(f, X)\{(X)+Q \sin (\chi X)\} d X
$$

as $\chi \rightarrow \infty$ for an arbitrary constant $Q$

Therefore, a true solution $p(X)$ and a test function obtained by an addition of a highly oscillatory error function $\{p(X)+Q \sin (\chi X)\}$ both will satisfy the governing integral equation. Thus a Fredholm integral problem of the first kind becomes a non-trivial problem. The success in inverting the Fredholm integral equation of the first kind depends on the measurement errors and the shape of the kernel function.

\section{Representation of the Integral Problem in Matrix Equation Form}

The integral equation can be transformed to a matrix equation by first considering discrete values of the operation variable (frequency $f$ ), and then replacing the integral by an appropriate quadrature formula using a weighted sum of the base-point functional values of 
the integrands as shown below:

$$
\begin{gathered}
R_{i}=\int K_{i}(X) p(X) d X \text { for } i=1,2, \ldots, M \\
K_{i}(X)=K\left(f_{i}, X\right) \text { for } i=1,2, \ldots, M
\end{gathered}
$$

$$
R_{i}=\sum_{j} K_{i, j} W P_{j} \text { with summation over } j=1,2, \ldots, M
$$

$$
\text { for } i=1,2, \ldots, M
$$

where

$$
\begin{aligned}
W j \quad & \quad \text { Quadrature weights for the numerical integration } \\
& \text { formulation, } \\
K_{\mathrm{ij}} & =\quad K\left(f_{i}, X_{j}\right) \\
R_{\mathrm{i}} & =\quad R(j), \text { and } \\
P_{\mathrm{j}} & =\quad p\left(X_{j}\right)
\end{aligned}
$$

Defining

$$
S_{j}=W_{j} p_{j}
$$

we get

$$
\mathbf{R}=\mathbf{K} \cdot \mathbf{S}
$$

where

$$
R_{i}=\sum_{j} K_{i} S_{j} \quad \text { with summation over } j=1,2, \ldots N \text {; }
$$




$$
\text { for } i=1,2, \ldots, M
$$

Here, $\mathbf{K}$ is an $M \times N$ matrix, and $\mathbf{R}$ and $\mathbf{S}$ are column vectors of dimension $M$ and $N$, respectively. One needs to pay attention to problems associated with near-singular coefficient matrix, $\mathbf{K}$ involved in the above equation. There are several algorithms reported in literature to solve Fredholm integral problems of the first kind. However, no single algorithm is found to be suitable for handling several widely different kernel functions when the response function is known with only modest accuracy as commonly encountered in practice. One needs to properly account for measurement as well as modeling errors. The solution depends on the choice of the kernel function and particular implementation of inversion algorithm.

We have extensively studied several deconvolution algorithms using simulated response functions appropriate for ultrasonic spectroscopy of industrial colloidal slurries (Pendse and Strout, 1990b). We have been quite successful in working with unimodal, as well as bimodal distributions. In terms of its ability to differentiate two distributions with relatively small differences in means and standard deviations our present deconvolution software is remarkably successful especially in the light of the fact that no assumptions regarding the form of the size distribution function are involved. 


\section{ATTENUATION SPECTRA AND PSD ESTIMATION}

A theoretical expression for $\alpha_{\text {mono }}(f, D)$, appropriate for finite particle concentrations (1 to 50 volume \%), forms the basis of our present deconvolution scheme. Focusing our attention on aqueous slurries of inorganic pigments with high density-contrast, we set out to test adequacy of the our model results using well-dispersed aqueous suspensions of titanium dioxide pigment. We obtained attenuation coefficient spectra over a wide frequency range on slurries of different particle concentrations. These slurries were prepared by careful dilutions of a pre-dispersed base slurry formulated at $75 \mathrm{wt} \%$ solids. Independent PSD analyses of these slurries using SediGraph 5100 confirmed that all of these slurries are characterized by a single particle distribution curve independent of their particle concentrations. The SediGraph PSD corresponds to median diameter of $0.35 \mu \mathrm{m}$, modal diameter of $0.31 \mu \mathrm{m}$, and geometric standard deviation ( $g s d$ ) of 1.6. Comparison of experimental measurements and corresponding predictions of attenuation coefficient spectra is shown in Figure 1. Experimental data is shown for attenuation coefficients covering ranges of 3.5 to $42.3 \mathrm{vol} \%$ (i.e., 13 to $75 \mathrm{wt} \%$ ) for particle concentration and 3 to $100 \mathrm{MHz}$ for sound frequencies. The seven concentration response curves correspond to seven operating frequencies of $3.00,5.57$, $10.34,19.20,35.65,66.20$ and $100.00 \mathrm{MHz}$. The curves shift up with increasing frequency. The coefficient of variation, i.e., ratio of standard deviation to mean for the measured attenuation coefficients is found to be less than $2 \%$. The attenuation coefficients range from 2 to $2000 \mathrm{~dB} / \mathrm{cm}$ increasing monotonically with increasing frequency.

The model predictions reported here were based on an assumed log-normal PSD characterized by geometric mean $(\mathrm{gm})$ of $0.33 \mu \mathrm{m}$ and $\mathrm{gsd}$ of 1.65 . The overall agreement 

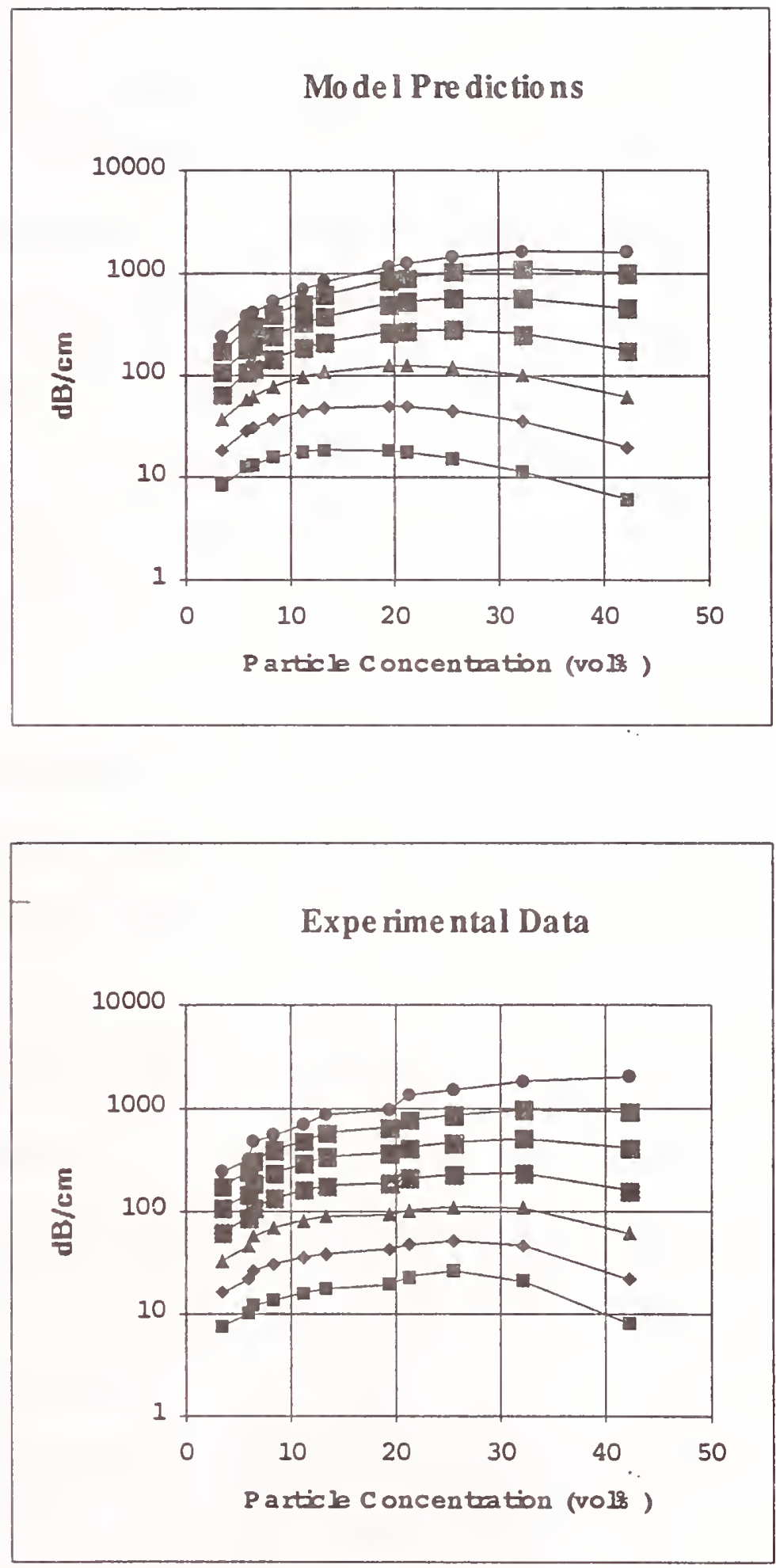

Figure 1. Variation of attenuation coefficient with particle concentration and frequency for well-dispersed titanium dioxide slurries 
between predictions and experimental measurements is remarkably well, with the overall root-mean-square relative deviation being $19 \%$ over such wide ranges of volume fraction (4 to 40 vol\%) and vibrational Reynold's number $(0.1$ to 10$)$. From Figure 1 it is clear that the attenuation coefficient versus volume fraction relationship is non-monotonic, with the peak location shifting to higher volume fractions with increasing frequency. This comparison of measurements done on a set of well characterized, well-dispersed slurries and corresponding predictions based on a single realistic PSD shows that the theory predicts appropriate dependence of attenuation coefficient on particle volume fraction and operating sound frequency over very wide ranges. The quantitative agreement of course will depend on adequacy of the assumed size distribution.

Figure 2 shows frequency response of the attenuation coefficients for two test slurries with 3.5 and $42.3 \mathrm{vol} \%$ solids concentration. These correspond to 13 and $75 \mathrm{wt} \%$ solids concentrations, respectively. The attenuation coefficients are normalized by dividing them by corresponding volume fractions and frequencies, for convenience. The peak in the normalized spectrum for $13 \mathrm{wt} \%$ slurry is apparent. The peak frequency for the spectrum for $75 \mathrm{wt} \%$ slurry is above $100 \mathrm{MHz}$. The two spectra corresponding to 13 and $75 \mathrm{wt} \%$ solids concentrations are quite different in magnitude and shape, even when normalized by the corresponding particle volume fractions. The need to properly account for dependence of ultrasonic attenuation spectra on particle volume fraction is obvious. Also shown in Figure 2 are model predictions based on three different assumed log-normal particle size distributions: (i) $g m=0.33 \mu \mathrm{m}$ and $g s d=1.65$, (ii) $g m=0.27 \mu \mathrm{m}$ and $g s d=1.65$, and (iii) $g m=0.33 \mu \mathrm{m}$ and $g s d=1.35$. None of the three test log-normal particle distributions appear to fit experimental spectra over the entire frequency range. The calculated attenuation coefficient 


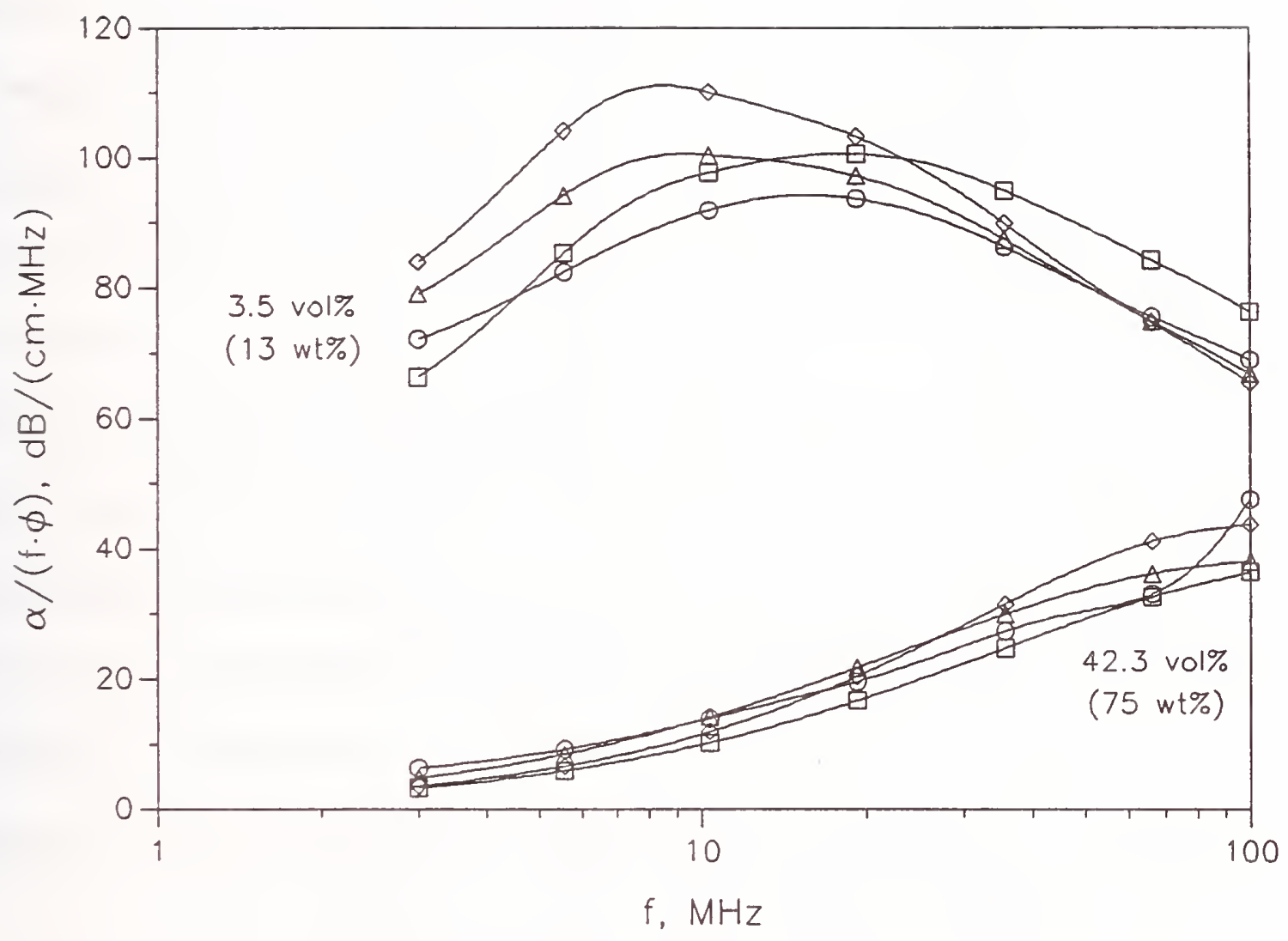

- Experimental data

$\rightarrow$ Model predictions with $g \mathrm{~m}=0.27 \mu \mathrm{m}$ and $\mathrm{gsd}=1.65$

- Model predictions with $\mathrm{gm}=0.33 \mu \mathrm{m}$ and $\mathrm{gsd}=1.35$

$\triangle$ Model predictions with $\mathrm{gm}=0.33 \mu \mathrm{m}$ and $\mathrm{gsd}=1.65$

Figure 2. Attenuation spectra for two test slurries 
spectra are seen to be sensitive to shifts in the $g m$, as well as shifts in the gsd. Upward shift in the peak frequency with the downward shift in the $g m$ diameter is especially clear from the model predictions for the $13 \mathrm{wt} \%$ slurry. Broadening of the PSD is reflected in the broadening of the attenuation spectra.

Using measured attenuation spectra one can estimate particle size distributions for the test slurries. Using our present deconvolution methodology we obtain particle size distributions without imposing any functional form. We impose the obvious nonnegativity constraint. Estimated particle size distributions obtained using the measured spectra are shown in Figure 3 for the two extreme cases of solids concentrations, 75 and 13 wt\%, respectively. It is interesting to compare the PSD estimates obtained by deconvolution of measured attenuation spectra for slurries of widely different solids concentrations with the results obtained from the SediGraph on samples further diluted prior to analysis. Ultrasonic PSD estimates appear to be a little broader and more skewed that the SediGraph PSD. The median diameters based on the ultrasonic PSD estimates are less than that reported by the SediGraph analysis, and are in fact closer to the modal diameter reported by the SediGraph analysis. Our experience with several pigment slurries shows this trend consistently. Similarities in the these PSD results are notable.

\section{CONCLUSIONS}

Ultrasonic spectroscopy of colloidal dispersions appears to be a powerful tool for their characterization. Recent instrument developments allow one to obtain attenuation coefficient spectra over 1 to $100 \mathrm{MHz}$ covering the range of 1 to $2000 \mathrm{~dB} / \mathrm{cm}$. For the case of high density contrast 


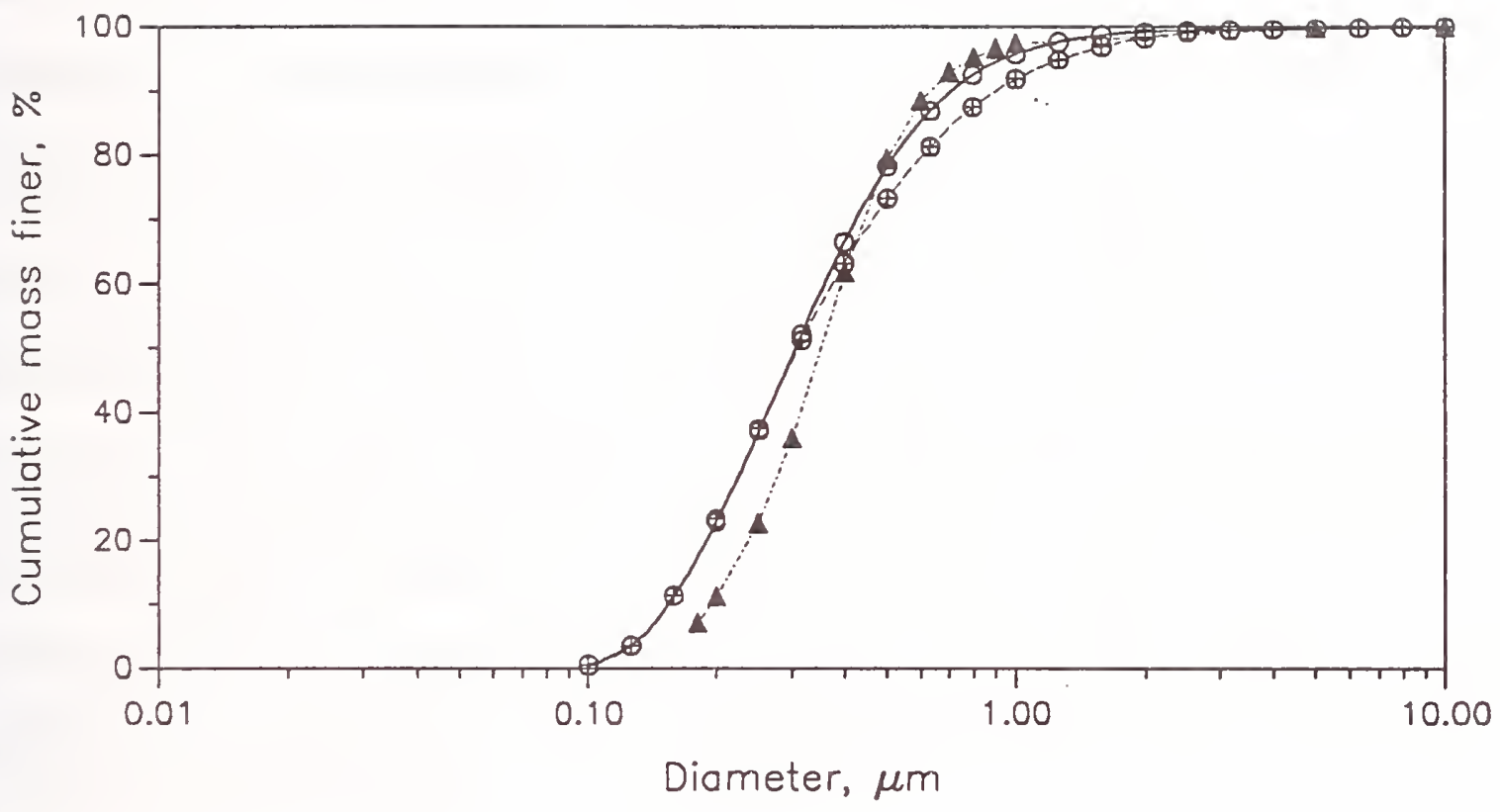

....... SediGraph 5100 onolysis

- Estimated PSD bosed on deconvolution for 42.3 vol\% (75 wt\%)

-- Estimated PSD based on deconvolution for 3.5 vol\% (13 wt\%) .

Figure 3. Particle size distributions for two test slurries 
suspensions, viscous loss associated with acoustophoresis is the dominant contribution to sound attenuation. Availability of a predictive, theoretical model that accounts for hydrodynamic interactions underlying acoustophoresis in multiparticle systems with moderate and high particle volume fractions, over a wide range of vibrational Reynolds numbers allows one to interpret measured attenuation spectra in terms of particle size distributions. This model is validated in the light of experimental data on attenuation spectra obtained using a set of well-dispersed suspensions of different solids concentrations. Attempts to use CVP phase measurements for monitoring particle size distributions can be expected to involve difficulties associated with proper accounting of interacting effects of variations in particle concentration, as well as dissolved ion and polyelectrolyte concentrations. The strategy (Pendse and Strout, 1990b) of using the two acoustophoresis related effects together in a complimentary fashion, electric double layer relaxation for zeta potential analysis combined with viscous attenuation effect for PSD characterization, may prove to be quite successful. Ultrasound spectroscopy may find interesting applications in characterization and quality control of a wide variety of industrial colloidal dispersions including suspensions and emulsions.

\section{ACKNOWLEDGMENT}

The authors are grateful for the support of Department of Energy, Office of Industrial Technologies under grant number DE-FC05-88CE40864, Du Pont Chemicals, and Pen Kem. This paper is based in-part on a presentation at the NIST Workshop entitled "Electroacoustics for Characterization of Particulates in Suspension," held in Gaithersburg, MD in February 1993. 


\section{REFERENCES}

1. Marlow B. J., D. Fairhurst, and H. P. Pendse, "Colloid Vibration Potential and the Electrokinetic Characterization of Concentrated Colloids, "Langinuir, 4, 611-626 (1988).

2. Pendse, H. P., "Electrokinetic Vibration Potential of Colloid Suspensions," NSF Project Report, Grant Number CBT-8410523 (1987).

3. Pendse, H. P., "Particle Size Distribution Sensor: Current Success and Future Work," DOE/Industry Advanced Sensors Technical Conference, Warwick, RI, (1992).

4. Pendse, H. P., and Strout, T. A., "On Effects of Particle Size in Acoustophoretic Analysis of Colloidal Zeta Potential," AlChE Annual Meeting, Chicago, IL (1990a).

5. Pendse, H. P., and Strout, T. A., "Ultrasound-Based Sensors for Characterization of Colloidal Slurries," Proceedings Sensors Expo West, 205B (1990b).

6. Sharma, A., "Experimental Aspects of Ultrasonic Spectroscopy," Ph.D. Dissertation, University of Maine (1993).

7. Strout, T. A., "Attenuation of Sound in High-Concentration Suspensions: Development and Application of an Oscillatory Cell Model," Ph.D. Dissertation, University of Maine (1991). 


\title{
NEW DEVELOPMENTS IN ELECTROACOUSTIC METHODS AND INSTRUMENTATION
}

David W. Cannon

Matec Applied Sciences

Hopkinton, MA

\begin{abstract}
Electroacoustic phenomena arise due to the interaction of electric fields and sound waves in a suspension of charged particles. The Electrokinetic Sonic Amplitude (ESA) is the sound wave generated by the particles when an alternating electric field is applied to the suspension. The Colloid Vibration Potential (CVP) is the alternating potential developed between two points when a sound wave is propagated through the suspension. Commercial instruments for measuring the ESA and CVP have been available since the middle 1980's and have been used to determine the particle charge or zeta potential. Recent developments in both theory and instrumentation have resulted in the Matec AcoustoSizer, a new instrument for measuring both the particle size and zeta potential in concentrated suspensions. The historical development of the technique and the basic measurement principles for determining particle size and charge in the AcoustoSizer are presented.
\end{abstract}

\section{INTRODUCTION}

Significant progress in the science of electroacoustic phenomena in colloids has been made over the past several years. The availability of commercial instruments coupled with important advances in theory have enabled the technology to move from a research curiosity to one of the most significant particle characterization techniques to emerge since light scattering. Electroacoustic instruments are being used for electrokinetic characterization in major industrial and academic research laboratories around the world for applications ranging from the characterization of ceramic powders to apolar liquid toners used in imaging technology. The popularity of electroacoustic methods has grown rapidly over the past several years because of the significant advantages offered over traditional methods for determining electrokinetic properties. 
These advantages include:

1. Measurements can be performed on concentrated suspensions that are inaccessible to optical techniques such as microelectrophoresis and electrophoretic light scattering.

2. The measurements can be made on stirred or flowing samples allowing rapidly sedimenting or viscous materials to be characterized. There is also promise for the technique to be applied to in-line monitoring of process streams.

3. Data acquisition is rapid and can be combined with automatic titrators to provide electrokinetic data over a wide range of solution conditions.

The promise of electroacoustics has been the ability to determine the electrokinetic charge or zeta potential in concentrated systems without the need for dilution. While progress has been made, the existing commercial instruments on the market have not been able to fully achieve this goal. Important issues like the dependence on particle size, instrument calibration, and the interpretation of measurements at high particle concentrations have been raised by several authors ${ }^{1-5}$. Through the theory developed by O'Brien6,7 we now have a much better understanding of electroacoustic effects in concentrated systems. In particular, the role of particle size has been studied in great detail and has enabled the development of new instrumental methods that allow zeta potential and particle size to be determined simultaneously. For the past 5 years, Matec Applied Sciences has been working with an Australian company, Colloidal Dynamics, and the Colloids Group at the University of Sydney to develop new electroacoustic measurement techniques and theory to measure particle size and zeta potential in concentrated suspensions. This effort has resulted in the Matec AcoustoSizer. The objective of this paper is to describe the design criteria for the instrument and the instrumental methods developed to achieve them. 


\section{ELECTROACOUSTIC EFFECTS AND THE DYNAMIC MOBILITY}

\section{Ultrasonic Vibration Potentials}

All of the early developments in electroacoustic instrumentation were concerned with the measurement of Ultrasonic Vibration Potentials (UVP). The ESA effect was not discovered until 1982. ${ }^{8}$ The UVP effect was first predicted by Debye9 in 1933 where he suggested it as a method for measuring the effective mass of ions in solution. Later, Rutgers ${ }^{10}$ and Hermans $s^{11}$ independently proposed UVP for measuring the particle charge in colloidal suspensions. Ultrasonic Vibration Potentials have been studied extensively by Yeager and coworkers who were the first to detect the "Debye effect" in electrolytes. A thorough review of research in the area prior to 1982 is given in reference (12). Marlow et al. ${ }^{13}$ describe a commercial instrument marketed by the Pen Kem company for applying the UVP technique to studying the electrokinetic properties of colloids.

Ultrasonic Vibration Potentials are created when sound waves are propagated through a suspension of charged particles if the material wave creates a periodic displacement (polarization current) between oppositely charged particles. In the case of electrolyte solutions the effect occurs if the cation and anion have different effective masses and frictional coefficients. The viscous and inertial forces acting on the ions due to the passage of the sound wave cause oppositely charged ions to take on different velocities creating periodically changing electric charge densities in the solution. In a colloidal suspension, the UVP effect arises from a periodic displacement of the particles relative to their surrounding electrical double layers. If there is density difference between the particles and the liquid, the particles will take on a different velocity in the sound wave than the surrounding liquid. Since the ions in the double layer will move with the liquid, there will be a relative motion between the double layer ions and the particle leading to a periodic polarization of the double layer. The dipole fields created by this polarization sum to produce a macroscopic alternating electric field at the frequency of the sound wave. According to the terminology established by Yeager $^{14}$, the UVP of an electrolyte is known as the Ion Vibration Potential (IVP) and the UVP of a colloid is termed the Colloidal Vibration Potential (CVP). The IVP and CVP are reported as the amplitude of the measured potential 
normalized by the velocity amplitude of the sound wave measured at the electrodes.

The UVP is measured by placing electrodes in the sound field with an electrode spacing other than an integer multiple of the sound wavelength and measuring the open circuit voltage. O'Brien ${ }^{6}$ has pointed out that the electrical response at the electrodes can also be measured as the short circuit current which has the advantage that the current is directly proportional to the charge on the particles while the UVP is proportional to the charge divided by the electrical conductivity of the suspension. Keeping with the foregoing terminology it seems appropriate to name the short circuit current as the Colloidal Vibration Current (CVC). The CVC is easily measured but has not been implemented in any of the available commercial instruments.

\section{Electrokinetic Sonic Amplitude}

A second type of electroacoustic effect occurs when an alternating electric field is applied to a suspension of charged particles. The particles move back and forth in the liquid due to the force of the electric field creating tiny pressure disturbances around the particles in the liquid. If there is a density difference between the particles and the liquid a macroscopic acoustic wave is developed at the boundaries of the suspension (the electrodes). This effect has been termed the Electrokinetic Sonic Amplitude (ESA) and was discovered at Matec ${ }^{8}$ in 1982. Matec Applied Sciences markets the ESA-8000 system which can be configured to measure either the ESA or CVP to characterize the electrokinetic properties of colloidal suspensions.

\section{The Dynamic Mobility}

O'Brien ${ }^{6}$ was the first to develop a theory for the ESA effect and showed that it is linked to the CVP through a reciprocal relation. More significantly he showed that the ESA and CVP effects are related to a fundamental property of the particles known as the dynamic mobility. The dynamic mobility, $\mu_{\mathrm{d}}$, is defined as the particle velocity, $\mathbf{V}$ divided by the electric field, $\mathrm{E}$ for a charged particle moving under the force of a sinusoidal electric field with angular frequency $\omega$. 


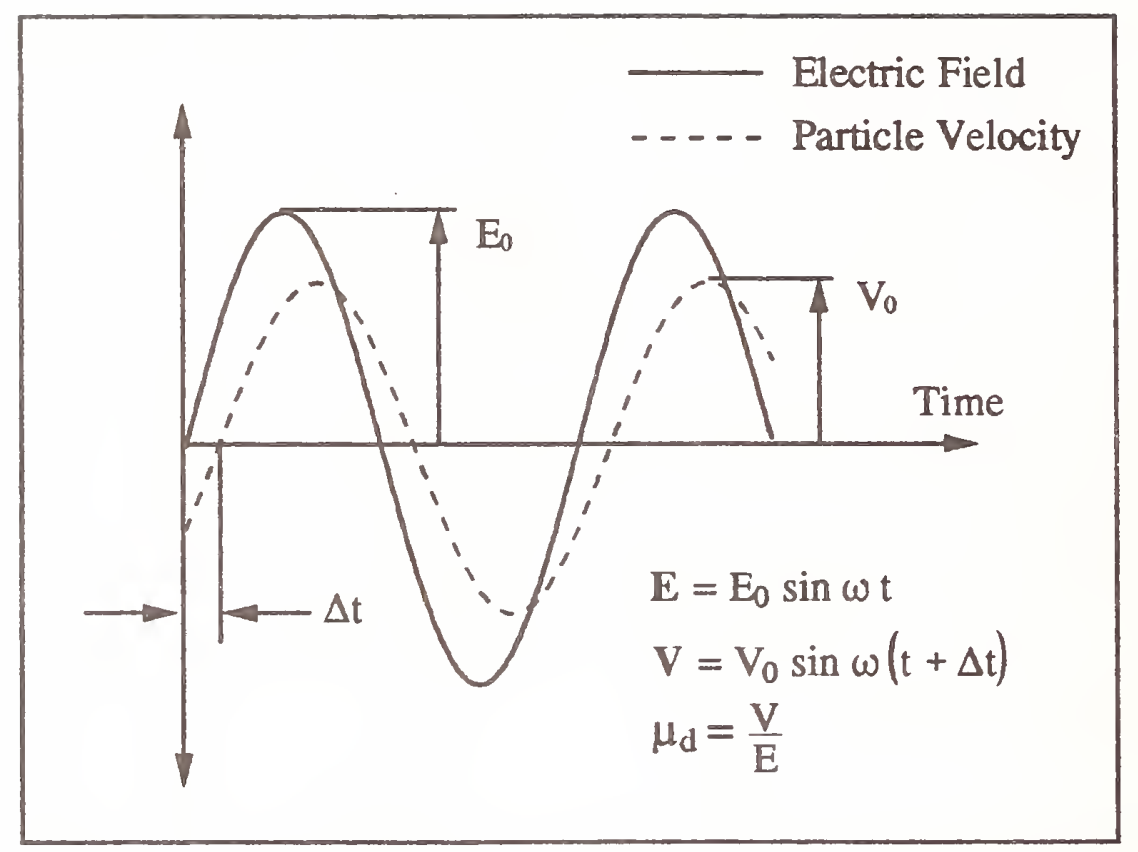

Figure 1. The definition of the dynamic mobility

The dynamic mobility is a complex quantity meaning that it has both a magnitude and argument (or phase angle). The magnitude of $\mu_{\mathrm{d}}$ is $\mathrm{V}_{0} / \mathrm{E}_{0}$, the amplitude of the particle velocity divided by the amplitude of the electric field. The argument of $\mu_{\mathrm{d}}$ relative to the electric field is $\omega(\Delta t)$. The argument of $\mu_{\delta}$ gives the time delay or phase lag between the particle motion and the applied field. The dynamic mobility of a colloidal particle is a function of the particle zeta potential, particle radius, double layer thickness, and the frequency of the applied field. As the frequency of the electric field approaches zero the dynamic mobility reduces to the familiar electrophoretic mobility defined for a DC electric field. When the frequency of the electric field is increased, the particle velocity will begin to lag the field and the velocity amplitude will drop because of inertial forces which are proportional to the particle size. The AcoustoSizer is a new instrument designed to make electroacoustic measurements over a range of frequencies to yield the dynamic mobility spectrum of a concentrated suspension. The particle size and zeta potential of the suspension can be calculated from the dynamic mobility spectrum. The first step in determining particle charge and size requires highly accurate and reproducible measurements of an electroacoustic effect. 
The existing commercial instruments for making electroacoustic measurements lack certain capabilities required in a device for determining particle size and charge. The AcoustoSizer does, however, draw several important design features from the Matec ESA-8000 system and an overview of that instrument serves as a good starting point for explaining the motivation behind the design principles of the AcoustoSizer. Figure 2 gives a schematic block diagram of the ESA-8000 system with the model PPL-80 flow through sensor.

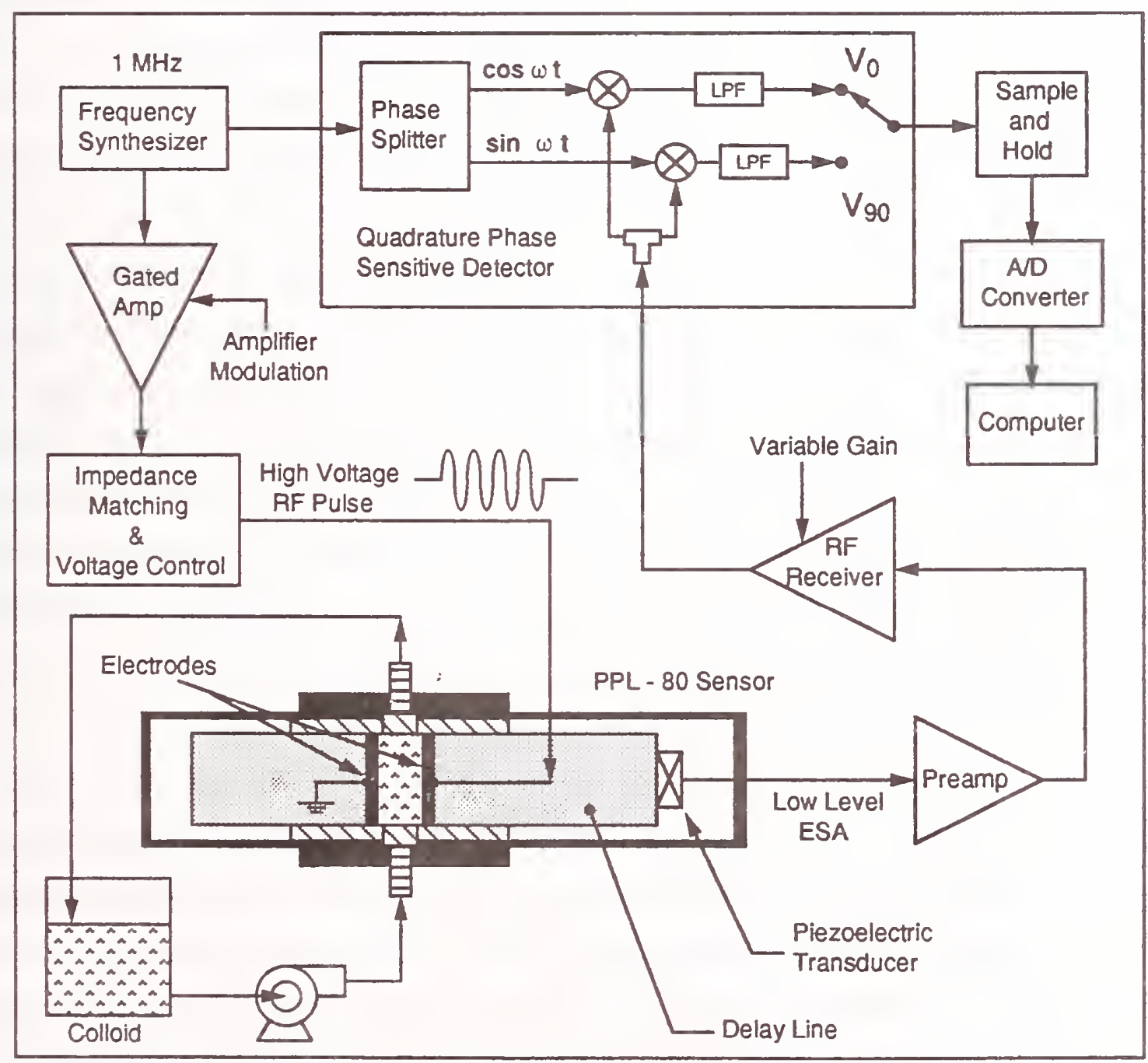

Figure 2. ESA-8000 System Block Diagram 
The instrument can be configured to measure either the ESA or CVP of a suspension depending upon how connections are made to the sensor. Figure 2 gives the arrangement for the ESA measurement mode. The PPL-80 measurement cell consists of two parallel gold electrodes laminated to solid rods sealed in a teflon housing inside the PPL-80. One of the rods has a piezoelectric transducer mounted on the end opposite the electrode. The colloid under test is pumped through the housing between the two gold electrodes. An ESA measurement begins by applying a $1 \mathrm{MHz}$ pulsed sinusoidal voltage to the electrodes in the sensor. When the electric field is applied to the electrodes, the charged particles in the suspension are accelerated back and forth generating tiny pressure waves in the liquid. The pressure fields from neighboring particles tend to cancel except at the boundaries of the suspension. The net effect is to generate a macroscopic acoustic wave at each electrode surface that behaves as if the electrodes were vibrating instead of the particles. A portion of the acoustic energy is transmitted into the solid rods supporting the electrodes and the rest travels through the suspension toward the opposite electrode. In the PPL- 80 design the electrode spacing is chosen such that the sound wave being generated at one electrode adds constructively to the sound wave arriving from the opposite electrode. This leads to acoustic resonance in the cavity between the electrodes. The required spacing must be approximately an odd multiple of one half the acoustic wavelength, $\lambda$, in the colloid. The PPL-80 design uses a spacing of $1.5 \lambda$ which is roughly $2.2 \mathrm{~mm}$ in water at $1 \mathrm{MHz}$. The acoustic resonance amplifies the ESA effect. Depending on the construction details of the sensor, the ESA signal is amplified by a factor of 3 to 5 times. The pressure wave travels down the solid rod and generates a voltage at the piezoelectric transducer.

In a CVP experiment, virtually the same arrangement is used except the voltage pulse from the gated amplifier is applied to the piezoelectric transducer. The voltage applied across the transducer causes it to expand and contract at the frequency of the applied voltage causing a sound pulse to travel down the rod. When the sound pulse reaches the electrode-colloid interface, some of the sound energy is transmitted into the cavity formed by the electrodes and the rest is reflected back towards the transducer. Acoustic resonance occurs if the electrode spacing is an integer multiple of one half the acoustic wavelength. If the electrodes are spaced an odd multiple of one half the acoustic wavelength, the CVP effect 
is maximized and can be measured by connecting the electrodes to a high input impedance amplifier. O'Brien ${ }^{6}$ derived a reciprocal relation linking the ESA and CVP effects and it can be written as:

$$
V_{E S A}=Z V_{C V P}
$$

$\mathrm{V}_{\mathrm{BSA}}$ is the voltage generated at the transducer divided by the voltage applied to the electrodes in an ESA experiment and $\mathrm{V}_{\mathrm{CVP}}$ is the the CVP voltage generated by the sound wave in the colloid divided by the voltage applied across the transducer to generate the sound wave. $\mathrm{Z}$ is the electrical impedance of the transducer divided by the cell impedance. From this relationship it is easy to see that the ESA effect will give a higher signal to noise ratio when the value of $\mathrm{Z}$ is greater than one. In the ESA-8000 system, this occurs in aqueous media at electrolyte concentrations of about $10^{-3} \mathrm{M}$ and greater. At lower suspension conductivities the CVP will give the best signal to noise but the conductivity of the suspension at the frequency of the electroacoustic effect must be measured to calculate the dynamic mobility from the CVP. The existing commercial instruments measure the conductivity at a lower frequency which gives only the real part of the conductivity at the frequency used in the electroacoustic measurements. This in turn leads to a measurement error when converting the CVP to a dynamic mobility. The ESA measurement has the advantage that it is independent of the suspension conductivity and can be used over a wide conductivity range. The ESA method is being successfully applied to electrokinetic measurements in non-aqueous low dielectric constant media ${ }^{15,16}$.

\section{Pulse Modulation vs Continuous Wave Methods}

Sinusoidal voltage pulses are used in the ESA system instead of continuous wave signals for a very important reason. At the frequencies used in the ESA system, it is very difficult to prevent the large voltages developed by the transmitter from interfering with the small signals generated by the ESA or CVP effects. The ESA and CVP signals are often on the order of microvolts while the transmitter voltages are typically hundreds of volts. By using voltage pulses and the solid rods in the sensor, the signal from the electroacoustic effect can 
be separated in time from the transmitter signal. This is illustrated in Figure 3 which shows a typical oscilloscope display of signals from various points in the ESA signal processing schematic (Figure 2). The top waveform is the sine wave output of the frequency synthesizer which operates at a frequency near $1 \mathrm{MHz}$. The low level synthesizer output is amplified into a high voltage pulse by a gated amplifier. The second waveform in Figure 3 is the modulation gate which turns the gated amplifier on and off. The gated amplifier output is typically 100 volts peak to peak for aqueous suspensions but voltages as high as 1000 volts peak to peak are used in low dielectric constant non-aqueous media. The bottom trace in Figure 3 is the amplified signal from the piezoelectric transducer in the sensor. The first waveform from the left hand side is the "cross talk" or electromagnetically induced interference from the transmitted voltage pulse. The second waveform in the bottom trace is the ESA signal which is delayed in time by the time it takes for sound waves to travel the length of the solid rod (acoustic delay line). The time duration of the voltage pulse and the length of the rod are selected so that the voltage pulse is long enough for the resonance in the cavity to reach steady state but short enough that the electrode voltage is turned off by the time the ESA signal arrives at the transducer. The pulse modulation technique allows very small signals to be measured with high accuracy and was employed by Yeager $^{17}$ in the first successful quantitative measurements of the IVP. 


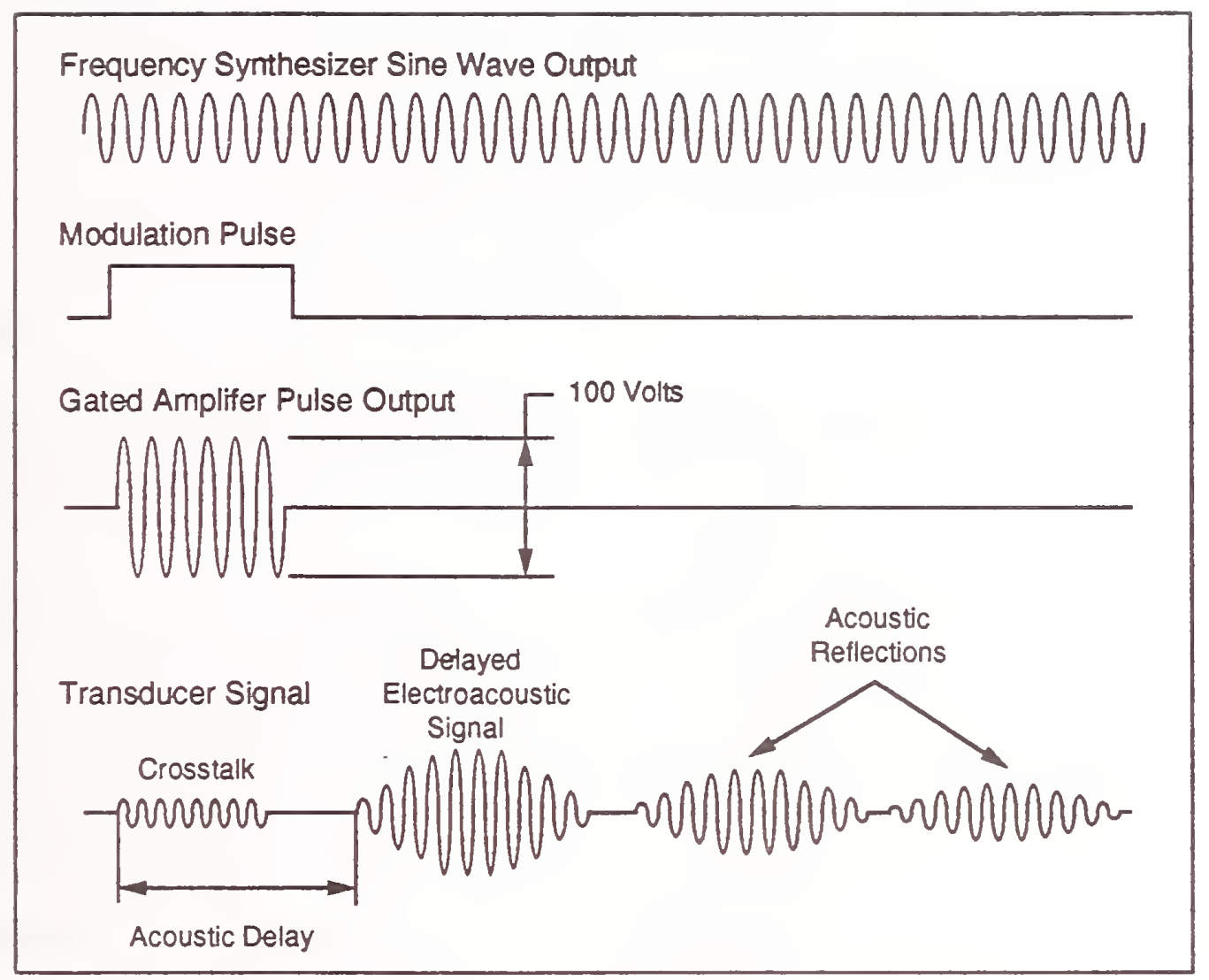

Figure 3. Pulse modulated acoustic measurement concepts.

\section{ESA-8000 Signal Processing and the Calculation of Zeta Potential}

The signal processing of the ESA and CVP is the same except for the connections to the sensor (Figure 2). The ESA or CVP signals from the measurement cell are amplified first by a high impedance narrow band preamplifier located near the measurement cell. The output of the preamplifier is further amplified by a variable gain amplifier before being mixed with the reference synthesized sine wave source in a quadrature phase sensitive detector. Quadrature detection allows both the amplitude and phase angle of the signal to be measured. The two outputs of the phase sensitive detector, $\mathrm{V}_{0}$ and $\mathrm{V}_{90}$ are proportional to the in phase and quadrature components of the measured signal. That is:

$$
V_{0}=A \cos \Phi \text { and } V_{90}=A \sin \Phi
$$


where $A$ is the amplitude of the signal being measured and $\Phi$ is the phase angle of the signal relative to the sine wave reference. The voltages $V_{0}$ and $V_{90}$ are measured by a sample and hold circuit and read by the computer through an A/D converter. The amplitude and phase angle of the signal at the input to the phase sensitive detector are given by:

$$
A=\left(V_{0}^{2}+V_{90}{ }^{2}\right)^{1 / 2} \text { and } \Phi=\operatorname{Tan}^{-1}\left(V_{90} / V_{0}\right)
$$

In practice, there are error terms which must be included in these equations that result from the phase splitter signals not being perfectly in quadrature. Reference (18) describes the procedure for eliminating the error terms.

In order to convert the measured electrical signal to the dynamic mobility and eventually to a zeta potential, a formula is required linking the electroacoustic effects to the dynamic mobility. This formula is obtained by solving the Electroacoustic Equations derived by $\mathrm{O}^{\prime} \mathrm{Brien}^{7}$ using boundary conditions appropriate for the PPL-80 measurement cell. Using a simple one dimensional parallel plate model of the sensor the following formula is obtained for the ESA pressure wave, $\mathbf{P}$, in the delay rods per unit electric field, $\mathbf{E}$, between the electrodes ${ }^{19}$.

$$
E S A=\frac{P}{E}=\Phi \frac{\Delta \rho}{\rho} \mu_{d} Z_{r}\left[1+\frac{\omega \chi}{k \rho}\left(\omega M-i Z_{r}\right) \cot k L\right]^{-1}
$$

where $\Phi$ is the particle volume fraction, $\Delta \rho$ is the difference between the particle density and the liquid density, $\rho . \mathrm{Z}_{\mathrm{r}}$ is the acoustic impedance of the solid delay rod. The acoustic impedance is defined as the ratio of pressure to particle velocity in an acoustic wave and is analogous to electrical impedance defined as the ratio of voltage to current. The term inside the brackets in equation [4] describes the acoustic resonance of the electrode cavity. Here $\omega$ is the angular frequency $(2 \pi f), M$ is the mass per unit area of the electrodes and $\mathrm{k}$ is the propagation constant for the acoustic wave in the suspension given by: 


$$
k^{2}=\frac{\omega^{2} \chi}{(1+i \omega \phi \Delta \rho \alpha)}
$$

where $\chi$ is the mass averaged bulk compressibility of the suspension and $\alpha$ is a term defined by O'Brien as a transport property of the suspension linking the particle velocity to the macroscopic pressure gradient in the suspension?

Equations [4] and [5] are valid for suspensions of arbitrary concentration. Unfortunately, they contain terms that depend on the properties of the suspension which vary with the particle volume fraction. Both $\alpha$ and $\chi$ are functions of particle concentration and must be determined experimentally in a concentrated suspension. In a dilute suspension, the term (1 $+i \omega \phi \Delta \rho \alpha)$ in equation [5] approaches 1 and $\mathrm{k}$ reduces to the frequency, $\omega$, divided by the speed of sound, c. Under this approximation, and with a fixed frequency and separation distance between the electrodes, the expression for the ESA pressure wave reduces to equation [6] where the term in the square brackets can be taken to be a constant under the restriction of dilute suspensions.

$$
E S A=\frac{P}{E}=\Phi \frac{\Delta \rho}{\rho} \mu_{d} Z_{x} Z_{s}\left[Z_{s}+\left(\omega M-i Z_{x}\right) \cot \left(\frac{\omega L}{c}\right)\right]^{-1}
$$

$\mathrm{Z}_{\mathbf{s}}$ is the acoustic impedance of the suspension which is the same as the pure solvent for a dilute suspension. For a fixed electrode spacing and frequency, the terms to right of $\mu_{d}$ in equation [6] can be taken as a constant and the ESA voltage measured by the ESA-8000 electronics given by:

$$
V_{E S A}=C(\Omega) \Phi \Delta \rho \mu_{d}
$$

The $C(\Omega)$ term in equation [7] lumps several instrument constants together with the constant terms in equation [6]. Instrument constants include the voltage - pressure calibration for the transducer, fixed acoustic propagation losses through the delay rod and fixed electronic gains in the instrument. The voltage measured by the instrument electronics can now be related to 
the $1 \mathrm{MHz}$ dynamic mobility of the suspension with an expression involving only instrument constants, the volume fraction of the particles and the density difference between the particles and the liquid. The ESA-8000 software computes the particle zeta potential using the following formulae 8 and 11 for the dynamic mobility of a dilute suspension of spheres ${ }^{6}$ :

(a) For aqueous media assuming thin double layers

$$
\begin{gathered}
\mu_{d}=\frac{\varepsilon \zeta}{\eta} G\left(\frac{\omega a^{2}}{\nu}\right) \\
G(\alpha)=\left[1-\frac{i \alpha(3+2 \Delta \rho / \rho)}{9[1+(1-i) \sqrt{\alpha / 2}]}\right]^{-1}
\end{gathered}
$$

where: $\alpha=\left(\frac{\omega a^{2}}{v}\right)$, a is the particle radius and $\nu$ is the kinematic viscosity of the liquid.

(b) For non-aqueous media assuming thick double layers

$$
\begin{gathered}
\mu_{d}=\frac{2 \epsilon \zeta}{3 \eta} G^{\prime}\left(\frac{\omega a^{2}}{v}\right) \\
G^{\prime}(\alpha)=\left[\left(1+\sqrt{\frac{\alpha}{2}}\right)-i\left[\sqrt{\frac{\alpha}{2}}+\frac{\alpha}{9}\left(1+\frac{2 \rho_{p}}{\rho}\right)\right]\right]^{-1}
\end{gathered}
$$

Equations [8] and [10] are identical to the well known Smoluchowski and Huckel equations ${ }^{20}$ for the static DC electrophoretic mobility except for the $\mathrm{G}(\alpha)$ and $\mathrm{G}^{\prime}(\alpha)$ terms. The $\mathrm{G}$ terms describe the effect of particle inertia on the dynamic mobility which causes it to decrease with both increasing frequency and increasing particle size. This dependency on particle size and frequency has been exploited in the AcoustoSizer for measuring both particle size and 
zeta potential. Because the ESA-8000 system operates at only one frequency, the particle size must be known to calculate zeta potential from the dynamic mobility.

\section{Standard Colloid Calibration}

In order to obtain a quantitative measurement of the dynamic mobility a calibration must be performed to determine the instrument constant in equation [7]. This is accomplished by placing a colloid with known particle size and zeta potential in the cell. The first step in calibrating involves sweeping the frequency of the applied field over a narrow frequency range to find the resonant frequency of the cell where the acoustic signal goes through a maximum. The resonant frequency can vary due to changes in the sound velocity of the medium which is a function of temperature, the particle volume fraction and electrolyte concentration. The magnitude and phase of the reference colloid signal is then measured at the resonant frequency of the cell. The instrument constant is determined by calculating the dynamic mobility of the standard using equation [8] or [10] and solving for $C(\Omega)$ using equation [7]. Reference (2) describes the calibration procedure using several different standards as a cross check.

\section{ESA Phase Angle Calibration}

The phase angle of the signal measured by the ESA-8000 electronics is not the phase angle of the ESA pressure wave relative to the electric field. Rather, it is the phase angle of the ESA signal relative to the sine wave reference and is arbitrary. The phase angle measurement includes phase shifts through the measurement electronics and also depends strongly on the time it takes the sound pulse to travel the length of the acoustic delay rod. From Figure 3 it should be apparent that a small change in the time delay of the ESA signal results in a large change in the phase relationship between the signal and the reference sine wave. The ESA-8000 system uses the phase of the ESA signal to determine the polarity of the particle charge. When the particle charge changes sign, the phase of the acoustic signal changes by 180 degrees. By following the change in the ESA phase angle from some known reference point the polarity of the particle charge can be determined. This process requires a reference measurement on a particle sample with a known charge polarity. The 
phase angle measured for the reference colloid is stored and used to calculate the relative phase angle of the sample to be analyzed. The phase angle reported by the ESA system is then the phase angle measured on the sample under test minus the phase angle of the reference colloid. If temperature is held constant, any phase difference between the sample and the reference is associated with the particle properties since the phase shifts through the electronics and acoustic delay line will cancel out. There are other factors which affect the measured phase angle in the ESA system besides the polarity of the particle charge. These include phase shifts due to changes in particle size and also changes in the acoustic resonance in the sample cell that arise from differences between the acoustic properties of the phase reference sample and the colloid under test. These effects are rarely large enough to cause problems with determining the charge polarity. A strong background electrolyte signal will also affect the ESA signal phase and magnitude and these signals will not, in general, be in phase with one another. The measured ESA signal is the vectorial sum of the ESA signal from the colloid and the electrolyte. Strong background electrolyte signals can result in an apparent shift in the colloid isoelectric point.

Once calibrated, the ESA-8000 system provides a robust and rapid way to determine the 1 $\mathrm{MHz}$ dynamic mobility and zeta potential of moderately concentrated colloidal suspensions. The measurement technique is accurate to within $+/-2 \%$ for suspension concentrations up to 5 volume percent for systems where the particle size is not too large $(<1$ micron) and well known. For higher particle concentrations the assumptions used to derive equation [7] are no longer valid and an error will be introduced that is proportional to $\phi \Delta \rho / \rho$. In practice the acoustic properties of a concentrated suspension cannot be calculated and must be measured directly. In spite of this limitation, the relative dynamic mobility or zeta potential measured in highly concentrated systems provides a powerful and rapid method for monitoring the change in electrokinetic charge as a function of $\mathrm{pH}$ or chemical additive. For samples with particle sizes significantly greater than one micron, the $1 \mathrm{MHz}$ dynamic mobility becomes a strong function of the particle size distribution. Under these conditions, the size distribution must be measured and the correct inertial term for the dynamic mobility must be calculated to accurately calculate the particle zeta potential. James et al. ${ }^{3}$ describe the procedure for calculating the correct $\mathrm{G}$ terms under these conditions. 


\section{ACOUSTOSIZER DESIGN PRINCIPLES}

The AcoustoSizer takes advantage of the dynamic mobility's sensitivity to particle size and the frequency of the applied field by measuring the frequency dependence of the dynamic mobility. As the frequency of the applied field is increased, the inertial forces acting on the particles increase causing both a decrease in particle mobility magnitude and an increase in phase lag. The magnitude spectrum combined with the phase lag spectrum provide enough information to calculate zeta potential and size. Figure 4 illustrates how the magnitude and phase lag of the dynamic mobility vary with frequency for two different particle sizes with the same zeta potential.
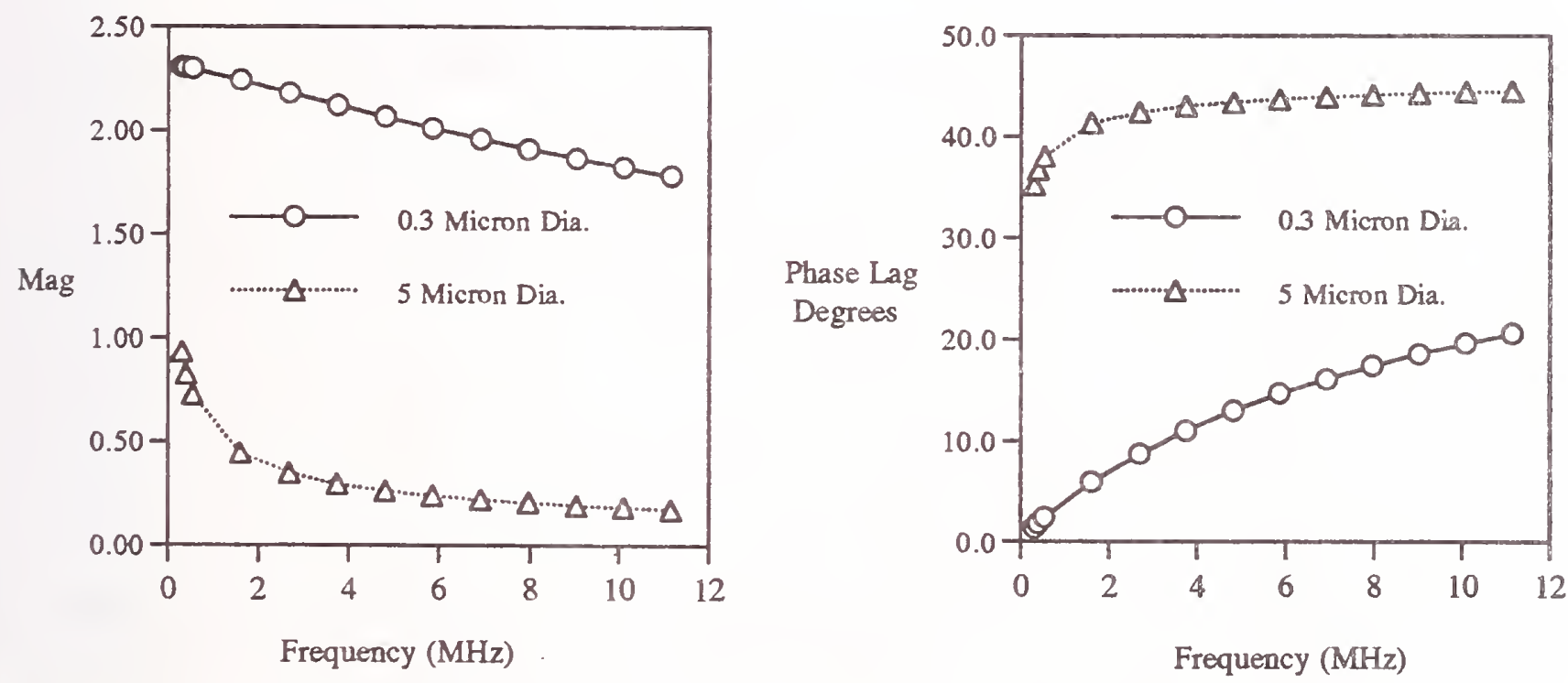

Figure 4. Dynamic mobility magnitude and phase lag vs frequency for 0.3 and 5.0 micron diameter spheres. $\Delta p / p=1.0 ; \zeta=30 \mathrm{mV}$

The mobility spectrum data is acquired by measuring the ESA of the suspension over a range of frequencies. The frequency range spanned by the instrument determines the range of particle sizes that can be measured. Particles at the lower sizing limit must have a measurable phase lag at the highest frequency in order to be sized. Particles at the upper sizing limit must move at a sufficient velocity at the lowest frequency to produce a measurable electroacoustic signal. The frequency must also be low enough so that the phase lag of the largest particle size is smaller than the asymptotic limit of 45 degrees for particles 
with thin double layers. These conditions are met when the quantity $\alpha=\left(\frac{\omega a^{2}}{v}\right)$ is near unity. The AcoustoSizer measures ESA over a frequency range of $300 \mathrm{kHz}$ to $12 \mathrm{MHz}$ to determine particle size in the range of 0.1 to 10 microns.

Several new instrumental methods had to be developed for the AcoustoSizer because the design of the ESA-8000 system was not suitable for frequency domain measurements. Factors limiting the ESA-8000 design for sizing purposes include:

1. Narrow band measurement cell design

The measurement cell is designed to operate over a narrow frequency range centered about 1 $\mathrm{MHz}$. The design has been tuned and optimized to provide high sensitivity at the resonant frequency of the cell. Matching the frequency response of the narrow band piezoelectric transducer to the frequency response of the electrode cavity is very difficult over the wide frequency range required for particle size measurements.

\section{Phase angle measurement accuracy}

The ESA-8000 system was not designed to measure the phase angle of the ESA signal with high accuracy since only changes of 180 degrees need to be detected to determine charge polarity. The phase of the ESA signal measured by the ESA-8000 system is very sensitive to changes in temperature, the acoustic properties of the suspension and phase shifts in the electronics. Electroacoustic particle sizing requires very accurate and reproducible phase angle measurements over the entire frequency range of the instrument.

3. Acoustic Properties of the suspension

The acoustic properties of the suspension must be measured in order to convert the measured ESA signal to the dynamic mobility in concentrated suspensions. There is no provision for this measurement in the ESA-8000 design. 


\section{Essential Features of the AcoustoSizer Design}

The AcoustoSizer has been designed to overcome the problems described above and can accurately measure the dynamic mobility spectrum of a suspension over a very wide particle concentration range. New designs have been developed for both the measurement cell and the instrument electronics. Figure 5 gives an overall schematic diagram of the measurement system.

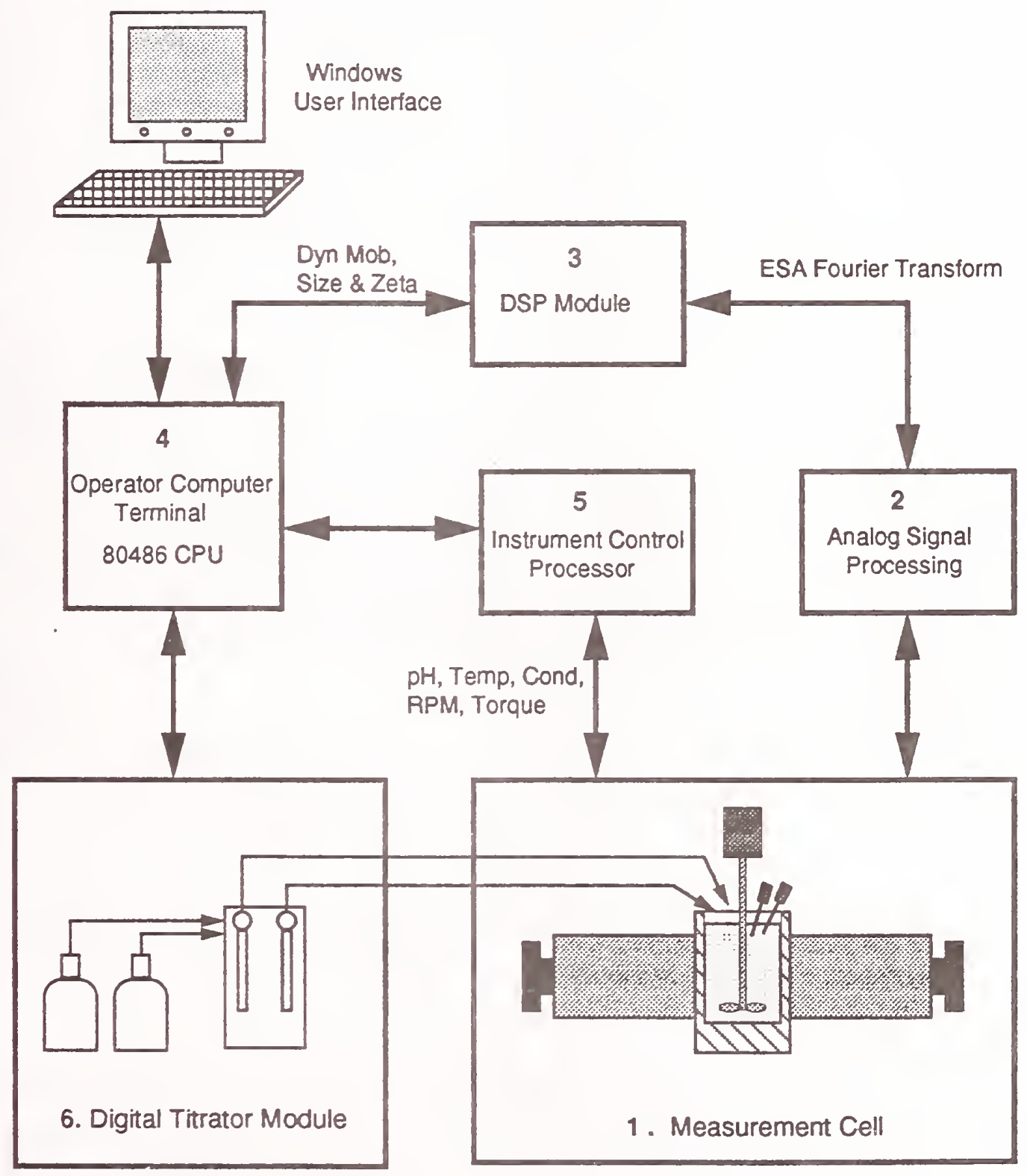

Figure 5. AcoustoSizer System Block Diagram. 
The instrument system is highly integrated combining measurement cell, signal processing electronics, computer and automatic titrator into a single instrument enclosure. The electroacoustic measurement sub-system consists of the measurement cell (1), analog signal processing electronics (2), and the DSP module (3). Each of the major instrument subsections is described below.

\section{The AcoustoSizer Measurement Cell}

A simplified schematic diagram of the AcoustoSizer measurement cell and front end signal processing is shown in Figure 6. The suspension under test is contained in an inert epoxy housing and kept under constant agitation by an overhead propeller type mixer. Probes for measuring $\mathrm{pH}$, conductivity and temperature are inserted into the suspension from above and are not shown in Figure 6. A heat exchanger for controlling the sample temperature is also inserted into the cell housing from above.

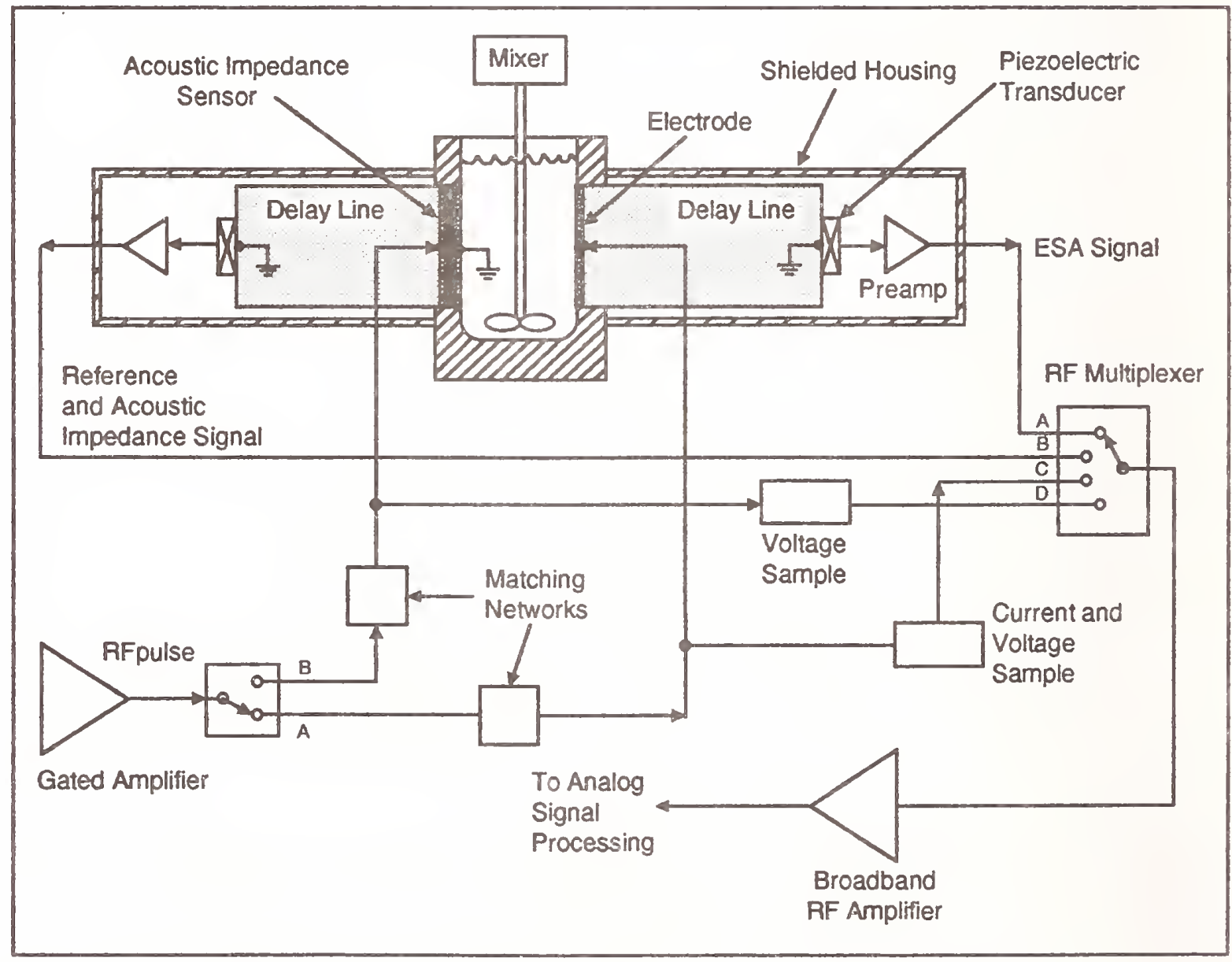

Figure 6. AcoustoSizer measurement cell and front end signal processing schematic. 
Two solid acoustic delay lines are mounted on opposite walls of the housing for carrying out ESA measurements on the suspension. Inert metal electrodes are mounted on the suspension side of each delay line and are sealed into the wall of the housing so that the electrodes are in contact with the suspension.

There are several important features of the AcoustoSizer cell design that distinguish it from the ESA-8000 sensors. The first is the use of two acoustic delay lines. Like the ESA system, the AcoustoSizer employs pulse modulation techniques to avoid problems with cross-talk between the transmitter and receiver electronics. The right hand delay line in Figure 6 is used to measure the ESA spectrum of the suspension. For each test frequency, a radio frequency ( $\mathrm{rf}$ ) pulse generated by a gated amplifier is applied to the electrodes in the measurement cell. The high frequency electric field applied across the suspension generates ESA pressure waves at each electrode which travel out into the suspension and down each delay line. The ESA pressure wave in the right hand delay line is detected by a piezoelectric transducer which produces an electrical signal that is amplified and directed to the signal processing electronics through a switch. The spacing between the electrodes in the AcoustoSizer measurement cell is large compared with the PPL-80 design. A wide spacing is used so that the ESA signals from each electrode do not interfere with one another. The ESA pulse width and electrode spacing is chosen so that the time duration of the ESA signal is shorter than the time required for the ESA signal from the opposite electrode to travel the distance between the electrodes. This eliminates the acoustic resonance between the electrodes that is employed in the PPL-80 design. Eliminating the resonance makes it much easier to carry out ESA measurements over a wide frequency range.

Solving the Electroacoustic Equations for the geometry used in the AcoustoSizer cell the following formula is obtained for the ESA pressure wave in the delay line.

$$
E S A=C(\Omega) \phi\left(\frac{\Delta \rho}{\rho}\right) \mu_{d} \frac{Z_{s} Z_{r}}{Z_{s}+Z_{r}}
$$

where $C(\Omega)$ is an instrument constant that does not depend on the properties of the particles, and the subsripts $s$ and $r$ refer to the suspension and delay rod respectively. Equation [12] is valid for both dilute and concentrated suspensions. In order to use this equation to compute 
the dynamic mobility, the acoustic impedance $\left(Z_{S}\right)$ of the suspension must be measured. This is the purpose of the second delay line in the measurement cell. Accurate measurement of the acoustic impedance is critical for converting the measured ESA signal in the delay line to the dynamic mobility in concentrated suspensions. The dependence of the ESA signal on important factors such as the presence of air bubbles and acoustic dissipation by the particles is governed by the $Z_{S}$ term in equation [12]. Measurement of the acoustic impedance is carried out by switching the output of the gated amplifier to a proprietary sensor mounted on the left hand delay line. The sensor generates an acoustic signal that varies with the acoustic impedance of the suspension and is detected by a piezoelectric transducer on the opposite end of the delay line. A very important feature of this arrangement is that the ESA and acoustic impedance signals travel through identical signal processing paths. This provides a means for ratioing out phase shifts in the signals that result from the physical measurement system. Examples include phase shifts due to thermal expansion of the delay lines and the effect of variable propagation delays in the electronics. The AcoustoSizer measurement cell also contains circuitry for measuring the current and voltage waveforms applied to the electrodes and the voltage applied to the acoustic impedance sensor. The unique design of the AcoustoSizer measurement cell and signal processing achieves absolute phase angle measurement accuracies better than one degree and magnitude measurement accuracies better than one percent over the entire operating frequency range.

\section{Analog Signal Processing Electronics}

The low-level signals from the measurement cell are switched to the input of the AcoustoSizer analog signal processing electronics where they undergo further treatment to produce the Fourier Transform of the signals. The Fourier Transform of the signals is required because the theory developed for relating the ESA spectrum to the dynamic mobility spectrum assumes that the applied fields are continuous sinusoids of a pure frequency, $\omega$. A pulsed sinusoid has its own characteristic frequency spectrum which varies with the width and shape of the pulse. The analog signal processing electronics measure the Fourier component of the ESA pulse at the frequency of the synthesized sine wave source supplied to the gated amplifier. The ESA spectrum is constructed by varying the frequency of the rf 
pulse applied to the electrodes in a series of steps over the frequency range of $300 \mathrm{kHz}$ to 12 $\mathrm{MHz}$. The process is repeated with the acoustic impedance sensor to produce the acoustic impedance spectrum of the suspension. Using equation [12] the dynamic mobility spectrum is calculated from the measured ESA and acoustic impedance spectra.

\section{DSP Processor}

The DSP module is a high speed "Digital Signal Processor" based microcomputer optimized for computationally intensive routines such as matrix operations. The DSP module controls the ESA data acquisition process and calculates the dynamic mobility, particle size distribution and zeta potential from the measured ESA and acoustic impedance spectra. The use of a high speed DSP co-processor in conjunction with the central operator computer terminal allows particle size and zeta potential data to be collected very rapidly achieving close to real time operation. This is an important feature for carrying out automated titrations.

For a polydisperse suspension, the dynamic mobility is given by the following integral equation ${ }^{3}$.

$$
\left\langle\mu_{d^{\prime}}\right\rangle=\int_{0}^{\infty} \mu_{d}(\tau) p(\tau) d \tau
$$

where $\left\langle\mu_{\mathrm{d}}\right\rangle$ is the mass averaged dynamic mobility of the suspension, $\mathrm{p}(\tau) \mathrm{d} \tau$ is the mass fraction of particles having radii between $\tau-\mathrm{d} \tau / 2$ and $\tau+\mathrm{d} \tau / 2$, and $\mu_{\mathrm{d}}(\tau)$ is the dynamic mobility for a particle of radius $\tau$. The calculation of $\left\langle\mu_{\mathrm{d}}\right\rangle$ is straight forward using equation [12] once the ESA and acoustic impedance spectra have been measured. The inversion of equation [13] to solve for the zeta potential and particle size distribution is not a trivial exercise however. Special numerical techniques are required to deconvolve the mobility spectrum. The software developed for the AcoustoSizer to date assumes the size distribution function $\mathrm{p}(\tau) \mathrm{d} \tau$ is a known function such as the log-normal distribution. The mass median size, 15 to 85 mass percentile size range and zeta potential of the suspension is adjusted using a least squares fitting routine to obtain the best fit to the measured dynamic mobility spectrum. The equation used for $\mu_{d}(\tau)$ in [13] depends on the nature of the sample 
under test. For thin double layer systems with concentrations below 10 volume percent, equation [8] can be used. At higher solids levels analytical formulae are not available for the dynamic mobility and a modified cell model approach is used. The details of the procedure along with AcoustoSizer results for a series of dilute and concentrated model colloidal systems are presented in a companion paper ${ }^{21}$.

\section{Operator Computer Terminal}

The AcoustoSizer Operator Computer Terminal consists of a fully imbedded 80486 computer including hard disk and floppy disk mass storage capabilities. The instrument control software utilizes the Microsoft Windows 3.1 user interface for intuitive and simple instrument operation. The central 80486 computer communicates with the DSP and Instrument Control Processor to acquire and process data. The distributed computing approach used in the instrument ensures that the data acquisition and analysis process proceeds smoothly and quickly while allowing the user to archive, analyze or print data simultaneously. All of the mobility, size, and zeta potential data are displayed on the high resolution color display. Temperature, $\mathrm{pH}$, conductivity, mixer speed, suspension relative viscosity data and temperature control functions are also provided on a front panel display located next to the measurement cell.

\section{Instrument Control Processor}

The AcoustoSizer instrument control processor is a microcontroller responsible for low level non-electroacoustic control and data acquisition functions in the AcoustoSizer. This module measures the $\mathrm{pH}$, conductivity, and temperature of the suspension under test. Additional functions include mixer speed control, torque and viscosity measurements, and temperature control utilizing a solid state Peltier temperature control system.

\section{Digital Titrator Module}

A dual syringe pump automatic titrator module is also incorporated in the AcoustoSizer instrument system. This module and accompanying control software provides for carrying out automated additions of reagents such as dispersants, flocculants, acids and bases for 
rapidly assessing their affect on the suspension zeta potential and particle size distribution.

\section{Calibration of the AcoustoSizer}

Similar to the ESA-8000 system, instrument constants must be determined for the AcoustoSizer in order to relate the voltage spectra measured at the sensing transducers to the actual ESA pressure wave in the delay line. The instrument constant is given as $C(\Omega)$ in equation [12] and is a function of frequency and instrument gain but not the properties of the particles. A standard colloid with a known zeta potential and particle size distribution is required for determining $C(\Omega)$. In practice it is very difficult to obtain suspensions with a well known size distribution and zeta potential. We have found that a series of spherical mono-disperse silica powders manufactured by GELTECH ${ }^{22}$ serve as excellent calibration standards for the AcoustoSizer. GELTECH's nominal 1.0 micron diameter material was chosen for calibrating the instrument. Typically the material is dispersed at high $\mathrm{pH}$ and at a sufficient background electrolyte concentration so that the assumption of a thin double layer is valid. The particle volume fraction is usually in the range of $2-3$ volume percent to provide good signal to noise while remaining in a concentration range where particle-particle interactions can be neglected. The zeta potential of the calibrant is determined by microelectrophoresis. The GELTECH 1.0 micron standard was found to be reasonably mono-disperse. The particle size of the calibration standard was verified by SEM and a centrifugal sedimentation based particle size analyzer, the Horiba Capa-700.

\section{SUMMARY AND CONCLUSIONS}

New developments in theory and electroacoustic measurement technology have resulted in the AcoustoSizer, an instrument for determining the particle size distribution and zeta potential of concentrated suspensions. It is the first commercial instrument capable of making accurate electroacoustic measurements in highly concentrated systems. The frequency dependent dynamic mobility can be measured in suspensions ranging from 1 to over 40 volume percent and particle size can be determined in the range of 0.1 to 10 microns diameter. The emergence of this technology should provide a rich set of new data on the 
microstructure of concentrated systems. The technology is well suited to a broad set of slurry processing applications in the minerals, ceramics, pigments and coatings industries.

\section{ACKNOWLEDGEMENTS}

I would particularly like to recognize my principal collaborator in the development of the AcoustoSizer, Richard O'Brien, who has played a pivotal role in the development of both the theory instrumentation for the technology. In preparing this overview I have drawn heavily from his work including many private communications. The contribution of Gary Petersen and Bruce Chick of Ritec, Inc. in the development of key aspects of the signal processing electronics is also gratefully acknowledged. 


\section{REFERENCES}

(1) O'Brien, R. W.; Midmore, B. R.; Lamb, Alice; Hunter, R. J., Faraday Disc. Chem. Soc. $1990,90,301$.

(2) Klingbiel, R. T.; Coll, H.; James, R. O.; Texter, J., Colloids and Surfaces, 1992, 68, 103.

(3) James, M.; Hunter, R. J.; O’Brien, R. W., Langmuir, 1992, 8, 420.

(4) Scales, P. J.; Jones, E., Langmuir, 1992, 8, 385.

(5) Isaacs, E. E.; Huang, H.; Babchin, A. J.; Chow, R. S., Colloids and Surfaces, 1990, 46, 177.

(6) O’Brien, R. W., J. Fluid Mech., 1988, 190, 71.

(7) O'Brien, R. W., J. Fluid Mech., 1990, 212, 81.

(8) Oja, T.; Petersen, G. L.; Cannon, D. W., US Patent \#4,497,208 (1985).

(9) Debye, P. J., Chem. Phys., 1933, 1, 13.

(10) Rutgers, A. Physica, 1938, 5, 674.

(11) Hermans, J., Phil. Mag., 1938, 25, 426.

(12) Zana, R.; Yeager, E. B., Mod. Aspects Electrochem., 1982, 14, 1.

(13) Marlow, B. J.; Fairhurst, D.; Pendse, H. P., Langmuir, 1988, 4, 611.

(14) Yeager, E.; Hovorka, F., J. Acoust. Soc. Am., 1953, $25,443$.

(15) Pearlstine, K.; Page, L.; El-Saued, L., Jour. Imaging Science, 1991, 35, 55.

(16) Pearlstine, K.; Swanson, J. R., J. Colloid Interface Sci., 1992, 151, 343.

(17) Yeager, E.; Dietrick, H.; Hovorka, F., J. Acoust. Soc. Am., 1953, 25, 456.

(18) Petersen, G. P., Matec Instruments' Application Note; "The Measurement of Acoustic Velocity and Attenuation Using Phase Detection and Matec Instruments' MBS-8000 Computer Controlled System" (1986), Matec Instruments, 75 South Street Hopkinton, MA 01748.

(19) O’Brien, R. W., Private communication, (1990).

(20) Hunter, R. J., In Zeta Potential in Colloid Science; Academic Press: New York, 1981. 
(21) O'Brien, R. W.; Rowlands, W. N.; Hunter, R. J., Proceedings of The NIST Workshop on Electroacoustics for Characterization of Particulates in Suspensions, 1993, National Institute of Standards and Technology, Gaithersburg, MD 20899.

(22) GELTECH Inc., 1 Progress Blvd., Box 8, Alachua, FL 32615. 


\title{
SESSION II
}

\section{APPLICATIONS AND PROCEDURES}

Session Chairman

\author{
H. P. Pendse \\ University of Maine
}





\title{
CALIBRATION OF ELECTROACOUSTIC (ESA) APPARATUS \\ USING LATTICES AND OXIDE COLLOIDS
}

\author{
R. O. James \\ Imaging Research Laboratories \\ Eastman Kodak Company \\ Rochester, NY 14650-2112
}

The electrokinetic sonic amplitude (ESA) of aqueous suspensions of polystyrene and poly(methyl methacrylate) lattices and of oxide colloids is reported as a function of volume fraction. The use of these readily available materials as convenient standards for the calibration of ESA apparatus is described. We review recent results obtained in our laboratories. The precision of these techniques appears competitive with other classical electrokinetic methods.

\section{INTRODUCTION}

In many industrial activities including mineral processing, water treatment and in particular, the specialist coatings industries, it is important to assess and control the colloid stability of suspended particles. A wide variety of methods have been used to monitor colloid stability or as sometimes termed "the dispersion quality" or "the emulsion quality", depending on the particular colloid system under scrutiny.

These methods have included electrokinetic techniques such as microelectrophoresis for measurement of the electrophoretic mobility and calculation of the "zeta potential" of the particles in dilute suspension. New methods to determine the mobility, $\mu$, or zeta potential, $\zeta$, in moderately concentrated suspensions have been developed in the last decade. The methods are based upon electroacoustic effects that are associated with charged colloids $(1,2)$. These effects involve either the generation of sound waves by colloidal particles driven by ac electric fields, or the generation of ac electric fields, or currents by the 
vibration of particles driven by a sound wave $(3,4)$. A specific example of a commercially available instrument is the electrokinetic sonic amplitude (ESA) apparatus produced by Matec Applied Sciences (3). In this paper, we will discuss methods and materials to calibrate the ESA apparatus to provide data that can be compared under appropriate conditions to data collected with classical methods, e.g., microelectrophoresis. Before proceeding with that discussion, a few comments about the need for new techniques will be offered.

In the area of coatings industries, quality assessment can be made on semi-finished or coated materials by observation of physical properties like surface roughness, gloss, reflectivity, transmission, haze, scattering, and absorption of light and by various microscopic techniques, e.g., optical microscopy, electron microscopy, and force microscopy methods. All these methods can be sensitive to defects and nonuniformities such as coating lines, variable roughness, and high or low optical density areas that may be caused or associated with over-sized or aggregated solid particles, e.g., dispersed dyes, pigments or latexes.

While some of these techniques can also be used directly on the coating fluid, few of them have a direct relation to the fundamental factors involved in the colloid stability of the fluid. In most coating applications, the stability of dispersions and the uniformity of the particle size distribution is controlled by at least two principal factors: (a) the mutual repulsion of charged particles and their electrical double layers. (b) The presence of adsorbed polymeric or condensed layers on the particle that reduce the attractive van der Waals or dispersion forces between the coated particles in the fluid.

Of course, in most coating applications, polymeric film-forming materials are present to ensure adhesion to the substrate and to modify other physico-chemical properties of the coating. Some of these polymers or binders function to reduce the van der Waals attraction between particles. For example, in the photographic industry, gelatin is famous for its role in the stabilization of silver halide particles in photographic emulsions. In addition, gelatin may show zwitterionic character due to ionizable anionic and cationic functional groups. Thus adsorbed polymers like gelatin can also dominate the interfacial charge and potential of the particles and may cause some gel-coated particles to become $\mathrm{pH}$ sensitive. 
Conventional microelectrophoresis has a drawback to direct application in many coating formulations owing to its optical detection requirement that light scattered by a particle then passes to the viewer without interaction with another particle. This often limits the dispersion concentration to about $0.01 \% \mathrm{w} / \mathrm{w}$ depending somewhat on the size and refractive index of the particles. In many practical systems the percent solids (of particles) in a coating fluid may be from about $1 \%$ to about $60 \% \mathrm{w} / \mathrm{w}$. Hence, the effect of $\mathrm{pH}$ adjustments with acids or bases or of added dispersants and polymeric stabilizers on the particle charge is often not directly observable at the coating solids level by microelectrophoresis. In addition, because of solution handling and dilutions it is not a very rapid characterization technique.

Thus the arrival in the 1980s of an electroacoustic technique (3), and a theoretical basis (4-6) for the evaluation of dynamic electrophoretic mobilities of more concentrated dispersions has been welcomed by a number of research and development groups in the formulation and coating community. It offers the possibility of making assessment of the parameters that relate more directly to ionic adsorption and to the repulsive interaction part of the total particle interaction energy. These parameters can be vital to colloid stability and to uniform particle distribution in a wide variety of dispersion and coating applications.

\section{ESA CALIBRATION STRATEGY AND PROCEDURES}

The ESA apparatus measures the ultrasonic (acoustic) response of a colloidal suspension of non-bouyant particles to a burst of applied radio-frequency (RF) field. The ESA is the ratio of the pressure wave amplitude to the RF field amplitude. The pressure wave arises from the combined vibrations of charged particles as they follow the driving electric field $(7,8)$.

Because the ESA apparatus involves various gain factors and instrumental constants there is a need for a calibration procedure so that ESA signal data can be transformed to dynamic electrophoretic mobility data for use in fundamental studies of colloid stability and for applied stability and rheological studies. In these areas the sign and value of the mobility and of the derived zeta potential may be crucial to interpretation of stability data. 
The calibration procedure described here rests upon a theoretical basis for the relationship between the dynamic electroacoustic mobility (at say $1 \mathrm{MHz}$ ) and the static or $\mathrm{dc}$ electrophoretic mobility of particles as observed by microelectrophoresis in conventional apparatus. The theoretical results described by O'Brien $(4-8)$ and by others $(9,10)$ that relate the frequency dependent ESA and dynamic mobility, $\mu(\omega)$, to the static mobility, $\mu(0)$, have allowed the development of methods of calibration by commercially available colloidal materials.

The empirical relationship of ESA to experimental signals $(\mathrm{S})$ and the theoretically derived dependence of ESA to the properties of the colloidal material in the limit of low volume fraction are expressed as (2):

$$
\mathrm{S} \gamma=\mathrm{ESA}=0.01 \mathrm{c} \phi \Delta \rho \mu(\omega) \mathrm{G}_{\mathrm{f}}
$$

where $S$ is the observed signal $(\mathrm{mPa} \mathrm{m} / \mathrm{V})$,

$\gamma$ is an instrumental constant (at low volume fraction),

ESA is the electrokinetic sonic amplitude $(\mathrm{mPa} \mathrm{m} / \mathrm{V})$,

$\mathrm{c}$ is the speed of sound of the continuous phase $\left(\mathrm{m} \mathrm{sec}^{-1}\right)$,

$\Delta \rho$ is the difference in density $\left(\mathrm{g} \mathrm{cm}^{-3}\right)$ between the particle and the continuous phase,

$\mu(\omega)$ is the dynamic mobility $\left(\mu \mathrm{m} \mathrm{cm} \mathrm{sec}{ }^{-1} \mathrm{~V}^{-1}\right)$ at field frequency $\omega\left(\mathrm{rad} \mathrm{sec}^{-1}\right)$ and 8,45 is the particle volume fraction. 
$\mathrm{G}_{\mathrm{f}}$ is a geometrical and acoustic gain factor that describes the coupling between the colloid suspension and the piezo-electric transducer and depends on the elastic properties on the buffer rod.

In practice it is convenient to lump $\mathrm{G}_{\mathrm{f}}$ and $\gamma$ together and determine an instrumental constant $\gamma / \mathrm{G}_{\mathrm{f}}$ such that:

$$
\mathrm{S}\left(\gamma / \mathrm{G}_{\mathrm{f}}\right)=\mathrm{ESA} / \mathrm{G}_{\mathrm{f}}=0.01 \mathrm{c} \Delta \rho \phi \mu(\omega)
$$

where the quantities on the RHS of Eq. 2 are only properties of the colloid dispersion. At higher volume fractions, outside the linear $\mathrm{S}-\phi$ range, $\gamma / \mathrm{G}_{\mathrm{f}}$ is dependent on electrode/probe geometry and on the volume fraction. This discussion of calibration procedures is limited to only the linear region which, in our experience, usually applies for volume fractions less than 0.05 .

The strategy for calibration of ESA apparatus involves use of appropriate materials to obtain data that may be evaluated using the theoretical models that are relevant to the particle or electrolyte data set. In the selection of materials for the calibration experiments, a number of the factors that should be considered are listed below.

1. Suitable colloid particles must be essentially insoluble in the solvent (water) with simple $\mathrm{pH}$ dependent surface charge reactions, preferably not redox reactions, e.g., oxides and lattices. Also, it is preferable that there is a $\mathrm{pH}$ region in which the electrophoretic mobility is not strongly dependent on $\mathrm{pH}$.

2. The particles must be non-bouyant. For aqueous systems near room temperature the particles should have a density greater than $1.05 \mathrm{~g} \mathrm{~cm}^{-3}$ or less than $0.95 \mathrm{~g} \mathrm{~cm}^{-3}$ and preferably have a density greater than about $2 \mathrm{~g} \mathrm{~cm}^{-3}$ in order that the $\Delta \rho$ term in Eq. 1 ensures a high signal-to-noise ratio.

3. The particles are preferably spherical or spheroidal and of narrow size distribution. In addition, they should be of such size and refractive index that they are easily observable 
in the microelectrophoresis cell. For example, the particle radius should be about or more than $100 \mathrm{~nm}$, but not so large as to sediment too quickly. Also the particle should be small enough that the acoustophoretic mobility is approximately the same as the electrophoretic mobility, $\mu(0)$. When these quantities are of very similar magnitude, errors and differences in sizing methods and in the details of model calculations are potentially less critical. For many particles, this requires that the radius be in the approximate range from 100 to $300 \mathrm{~nm}$ depending on the particle density $(1,2,11)$. Also, in order to use the theory developed by O'Brien (4), which is based on thin double layer approximations, i.e., ка $>>1$, it is desirable to adjust the ionic strength of the electrolyte to about $0.01 \mathrm{~mol} \mathrm{dm}^{-3}$ so that $к \mathrm{a}$ is greater than 30 and preferably greater than 50 , where $1 / \kappa$ is the Debye length or diffuse electrical double layer thickness.

For the relationship between the electroacoustic mobility $\mu(\omega)$ and the electrophoretic mobility, $\mu(0)$, we will use the theory developed by O'Brien (4). The important relations from that paper are given below.

$$
\mu(\omega)=(2 \epsilon \zeta / 3 \eta) \mathrm{G}(\alpha)(1+\mathrm{f})=\mu(0) \mathrm{G}(\alpha)
$$

where $\mathrm{f} \sim 0.5, \alpha=\omega^{2} / \nu, \mathrm{a}$ is the particle radius, $\nu=\eta / \rho$ is the kinematic viscosity, $\eta$ and $\rho$ are the viscosity and density of the continuous phase, and

$$
\mathrm{G}(\alpha)=\left\{1-\mathrm{i} \alpha(3+2 \Delta \rho / \rho) / 9\left(1+(1-\mathrm{i})(\alpha / 2)^{0.5}\right)\right\}^{-1}
$$

This leads to the modulus of the dynamic to dc mobility ratio

$$
|\mu(\omega) / \mu(0)|=\left|\left\{1-\mathrm{i} \alpha(3+2 \Delta \rho / \rho) / 9\left(1+(1-\mathrm{i})(\alpha / 2)^{0.5}\right)\right\}^{-1}\right|
$$


The modulus $|\mu(\omega) / \mu(0)|$ is plotted in Fig. 1 as a function of radius for several values of particle density in aqueous solutions and for ac field frequency at $\omega / 2 \pi=1 \mathrm{MHz}(1,11)$. It can be seen that for radius a $<0.2 \mu \mathrm{m},|\mu(\omega) / \mu(0)|>0.9$.

Thus particles that satisfy most of the above criteria should be quite useful as calibration materials for ESA using microelectrophoresis as the reference method.

Having calculated $\mu(\omega)$ for each particle, at say, fixed $\mathrm{pH}$, ionic strength, particle size and electrophoretic mobility, $\mu(0)$, the ESA signal amplitude, $\mathrm{S}$, is measured as a function of volume fraction, $\phi$, of particles in the suspension under the same solution conditions, e.g., $\mathrm{pH}$ and ionic strength. From the linear $\mathrm{S}-\phi$ regime at low volume fraction, the limiting slope, $\mathrm{d} S / \mathrm{d} \phi$, is determined and used to obtain the instrument calibration factor from:

$$
\gamma / \mathrm{G}_{\mathrm{f}}=0.01 \text { c } \Delta \rho \mu(\omega) /(\mathrm{dS} / \mathrm{d} \phi)
$$

Once $\gamma / G_{f}$ has been obtained and is self-consistent for several different colloidal materials it may be adopted as the calibration factor for the instrument.

At this point, one may then choose to establish other materials for evaluation as secondary standards that may offer materials advantages like availability or shelf-life and chemical stability. For example, there are a range of commercially available colloidal silica sols that could serve as secondary standards. An example is Du Pont Ludox TM, which is a stable silica sol and has a low concentration of $\mathrm{Na}_{2} \mathrm{O}$ so that sols of fairly high volume fraction can be prepared by dilution without greatly increasing the ionic strength above 0.01 mol $\mathrm{dm}^{-3}$. Ludox TM is a useful silica sol because it has particle density of $2.2 \mathrm{~g} \mathrm{~cm}^{-3}$ and a radius about $11 \mathrm{~nm}$, it is stable for at least a year and gives a good signal-to-noise ratio over a range of volume fractions. These silica sols are too small for conventional microelectrophoresis but have been investigated by moving boundary electrophoresis $(12,13)$ and also a large body of mobility or zeta potential data exists for the silica/water interface (14-24). Using ESA apparatus, one can determine the ESA signal, S, as a function of volume fraction of silica and use $\gamma / G_{f}, \Delta \rho$ and $c$ to obtain the dynamic mobility $\mu(\omega)$ from Eq. 2. Then it is possible to compute the static mobility from O'Brien's equations. In this case the particle size is small enough that these mobilities are the same, $\mu(0)=\mu(\omega)$. 
In addition, one referee reported that Ludox TM has been investigated using laser light scattering using a Coulter DELSA 440 apparatus (26). The result gave the electrophoretic mobility at $\mathrm{pH} 9.9$ of $-3.59(\mu \mathrm{m} / \mathrm{sec})(\mathrm{cm} / \mathrm{V})$ at $34.2^{\circ}$ scattering angle.

\section{EXPERIMENTAL}

Details of the experimental conditions and apparatus have been reported elsewhere $(1,2)$ so only a brief summary is given here. Electrophoretic mobilities were determined with a Rank Brothers Model Mk II microelectrophoresis apparatus at $25^{\circ} \mathrm{C}$. The microscope graticule was calibrated with a ruled scale on a microscope slide. The flat cell constant was $61.7 \pm 0.6 \mathrm{~cm}^{-1}$. Particles were timed in both directions by alternate reversal of the field of $7.28 \mathrm{~V} \mathrm{~cm}^{-1}$ and observed at the stationary fluid layer inside the front and back walls. The ESA signals were measured at $25^{\circ} \mathrm{C}$ with different Matec Applied Science MBS- 8000 electrokinetic sonic amplitude systems. A low volume parallel-plate flow cell (Matec PPL80) was used with electrode spacing of $3 / 2$ wavelengths. All measurements were done on samples adjusted to $0.01 \mathrm{~mol} \mathrm{dm}^{-3} \mathrm{KNO}_{3}$. The ESA apparatus field factor was first adjusted to give a signal $\mathrm{S}$ equal to $-3.67 \mathrm{mPa} \mathrm{m} / \mathrm{V}$ for a $10.0 \% \mathrm{v} / \mathrm{v}$ suspension of Ludox TM silica sol following the manufacturer's recommendation.

Poly(methyl methacrylate) latex (PMMA) (Soken MP-1201, Nachem, Braintree, MA) was obtained as a dry powder and dispersed by ultrasonic agitation in water at $10 \% \mathrm{v} / \mathrm{v}$. The particle density was $1.191 \mathrm{~g} \mathrm{~cm}^{-3}$.

Colloidal silica, Du Pont Ludox TM was obtained as a 51.2\% w/w sol. The particle density was taken as $2.2 \mathrm{~g} \mathrm{~cm}^{-3}$.

Fine alumina $\left(\alpha-\mathrm{Al}_{2} \mathrm{O}_{3}\right)$ abrasive powder, AKP-30, was obtained from Sumitomo Chemical Company, Ltd, Tokyo, was dispersed with a high speed Cowles mixer at $67.1 \%$ $\mathrm{w} / \mathrm{w}(33.8 \% \mathrm{v} / \mathrm{v})$ for $24 \mathrm{hr}$ at $\mathrm{pH} 4.0$. The particle density was taken as $3.98 \mathrm{~g} \mathrm{~cm}^{-3}$ and the size distribution was determined by centrifugal sedimentation.

Poly(styrene) latex (PS) with surface carboxyl groups was made by Dr. R. Sutton (Clinical Diagnostics Research Laboratories, Eastman Kodak Company). The particle 
density was $1.0541 \mathrm{~g} \mathrm{~cm}^{-3}$. Particle size for PS, PMMA and silica sols were determined from histograms obtained by electron-microscopy. For PS the average particle radius was $302 \pm 23 \mathrm{~nm}$.

\section{RESULTS AND DISCUSSION}

Results of the size analysis for the PMMA latex and silica sol are shown as normalized probability density distributions in Figs. 2 and 3. For PMMA the number average radius is $162 \pm 10 \mathrm{~nm}$, while for the Ludox TM the average radius is $11.5 \pm 2.3 \mathrm{~nm}$. This leads to $k \mathrm{a}$ values of 52.3 and 3.78 , respectively, for sols adjusted to $0.01 \mathrm{~mol} \mathrm{dm}^{-3} \mathrm{KNO}_{3}$. The size distribution for the AKP alumina sol is shown in Fig. 4 as a cumulative weight percent oversize plot for repeat determinations. The line is log normal distribution fit where the geometric mean radius by mass is $195 \mathrm{~nm}$ with a geometric standard deviation of 1.366. The arithmetic mean and volume mean radii and geometric mean radius by count were 153 , 169 , and $146 \mathrm{~nm}$, respectively (1). Using the volume mean radius (169 nm), the value of $\kappa \mathrm{a}$ in the electrolyte solution is 55; this is the value used to calculate $\mathrm{G}(\alpha)$ for AKP.

ESA and microelectrophoresis measurements were made in $0.01 \mathrm{~N} \mathrm{KNO}_{3}$ and at various constant $\mathrm{pH}$ values (6.8 for PS, 10 for PMMA, 4 for AKP, and 9 for Ludox TM). The static mobilities, $\mu(0)$, for PS, PMMA, and AKP are shown in Table I together with the zeta potential, $\zeta$, computed from the theoretical work of O'Brien and White (25).

The modulus of the ratio of dynamic mobility to static mobility $|\mu(\omega) / \mu(0)|$ calculated using Eq. 5 is shown in Fig. 1 at field frequency $\omega / 2 \pi=1 \mathrm{MHz}$ as a function of particle radius and for various values of particle density, e.g., $1.1,2.5,4$, and $6 \mathrm{~g} \mathrm{~cm}^{-3}$. It can be seen that for radius a less than $0.2 \mu \mathrm{m},|\mu(\omega) / \mu(0)|$ is greater than 0.9 for most kinds of particles. For larger particles, it becomes more important to know the density and particle size distribution accurately to get a good estimate of $|\mu(\omega) / \mu(0)|$ for subsequent evaluation of the instrument factor $\gamma / \mathrm{G}_{r}$.

Values of the mobility ratio were calculated for all of the sols (PS, PMMA, AKP and $\mathrm{TM})$ at $\omega / 2 \pi=1 \mathrm{MHz}$ and these appear in Table II. In all cases, owing to the combination 
of particle size, density and field frequency the mobility ratio is greater than 0.9.

To determine the instrument calibration factors it remains to evaluate the slope, $d S / d \phi$, from study of the volume fraction dependence of the ESA signal. Data for PS and AKP are shown in Fig. 5 where each sol exhibits a linear regime at low volume fraction $(\phi<0.05)$ with slopes of -2.76 and $139.5 \mathrm{mPa} \mathrm{m} / \mathrm{V}$, respectively. The low density of PS results in the low magnitude of the ESA signal and the slope and the negative sign reflects the charge of the dissociating carboxylate surface groups whose intrinsic dissociation constant is of the order $10^{-5}$. Likewise, the high density alumina AKP (which has an isoelectric point at $\mathrm{pH}=$ 9) yields a high positive slope due to the excess adsorption of protons at $\mathrm{pH} 4$. Values for $\mathrm{dS} / \mathrm{d} \phi$ and $\gamma / \mathrm{G}_{\mathrm{f}}$ derived from Eq. 6 are also listed in Table II. For the other sols, PMMA and TM, data for each sol was obtained on two different instruments and the ESA signal is plotted as a function of particle volume fraction in Figs. 6 and 7, respectively. Again, these sols demonstrate linear behavior at low volume fraction, giving slopes of $-6.89 \pm 0.24 \mathrm{mPa}$ $\mathrm{m} / \mathrm{V}$ and $-41.4 \pm 0.5 \mathrm{mPa} \mathrm{m} / \mathrm{V}$, respectively.

Using the slopes for PS, PMMA, and AKP and Eq. 6 the calibration factor was computed for each sol as 1.48 (1), 1.54 (2), and 1.23 (1), respectively, and listed in Table II. In an earlier report (1) we obtained a calibration factor of $1.4 \pm 0.15$ from an average of values for PS, PMMA, and AKP on a single instrument. However, since data presented here for PMMA was available from two different instruments and it had a narrower size distribution than the AKP or PS we will use the value 1.54 \pm 0.18 from the PMMA data for $\gamma / \mathrm{G}_{\mathrm{f}}$

This factor and the other sol properties now allow the estimation of the dynamic mobility of Ludox TM silica in $0.01 \mathrm{~mol} / \mathrm{dm}^{3} \mathrm{KNO}_{3}$. Using $\Delta \rho=1.20, \mathrm{dS} / \mathrm{d} \phi=-41.4$ and $\gamma / \mathrm{G}_{\mathrm{f}}=$ 1.54 gives a value $-3.59 \pm 0.41 \mu \mathrm{m} \mathrm{cm} \mathrm{s}^{-1} \mathrm{~V}^{-1}$ for $\mu(\omega)$. Since the silica particle radius is only $11.5 \mathrm{~nm}$, the $\mu(\omega) / \mu(0)$ ratio is unity and the implied static mobility is also $-3.59 \mu \mathrm{m} \mathrm{cm}$ $\mathrm{s}^{-1} \mathrm{~V}^{-1}$ at $\mathrm{pH}$. The result is consistent with a number of earlier studies using the moving boundary method on various Ludox silica samples $(12,13)$. The static mobility data may also be used to estimate the zeta potential of the sol particles using the theory of O'Brien and White $(25,26)$. We used their computer program to evaluate the relationship between zeta potential and mobility for ка values of 52.3 and 3.8, which apply to PMMA and Ludox TM 
in $0.01 \mathrm{~mol} \mathrm{dm}^{-3}$ univalent electrolyte. The results of calculations are shown in Fig. 8. From these plots, $\zeta_{\mathrm{PMMA}}$ is about $-52 \mathrm{mV}$ while and $\zeta_{\mathrm{TM}}$ is about $-78 \mathrm{mV}$ with a range from -64 to $-98 \mathrm{mV}$ for the $90 \%$ confidence level. Because the ka value for our Ludox TM experiments is near where a maximum exists in the O'Brien and White theory (25), larger uncertainty exists in the upper value of this range. This result, $\zeta_{\mathrm{TM}}=-78 \mathrm{mV}$, is consistent with other electrokinetic data for silica/electrolyte systems from a wide range of methods.

In conclusion, we have described a strategy and suitable materials that allow calibration of ESA apparatus by comparison with results obtained using the selected colloids with data obtained using standard microelectrophoresis methods. After calibration, dynamic mobilities can be converted to the steady state mobility and zeta potential, which may have useful application in the stability of dispersions and coating fluids.

\section{ACKNOWLEDGMENTS}

The collaboration and co-authorship of Hans Coll, Richard Klingbiel, Peter Scales, and John Texter is gratefully acknowledged. Discussions and correspondence with David Cannon, Robert Hunter, and Richard O'Brien over an extended time were very stimulating. I would also like to acknowledge private communications and pre-publication data or comments from Alex Babchin. In addition, one referee kindly supplied data on the mobility of Ludox TM in $0.01 \mathrm{M} \mathrm{KCl}$ at pH 9.9, which was obtained by Karin Evans formerly of Langley-Ford Instruments (Division of Coulter, Inc.). 


\section{REFERENCES}

(1) James, R. O.; Texter, J.; Scales, P. J. Langmuir 1991, 7, 1993.

(2) Klingbiel, R. T.; Coll, H.; James, R. O.; Texter, J. Colloids and Surfaces 1992, 68, 103.

(3) Oja, T.; Peterson, G. L.; Cannon, D. W. US Patent \#4,497,208 (1985).

(4) O'Brien, R. W. J. Fluid Mech. 1988, 190, 71.

(5) O'Brien, R. W. J. Fluid Mech. 1990, $\underline{212}, 81$.

(6) Loewenberg, M.; O’Brien, R. W. J. Colloid Interface Sci. 1992, 150, 158.

(7) O'Brien, R. W. Chemistry in Australia 1992, $\underline{59}, 158$.

(8) O'Brien, R. W. Australia Patent \#597,442 1988; US Patent 5,059,909 (1991).

(9) Babchin, A. J.; Chow, R. S.; Sawatzky, R. P. Adv. Colloid Interface Sci. 1989, $\underline{30}, 111$.

(10) Sawatzky, R. P.; Babchin A. J. J. Fluid Mech. 1993, 246, 321.

(11) Scales, P. J.; Jones, E. Langmuir 1992, ㅁ, 385.

(12) Long, R. P.; Ross, S. J. Colloid Sci. 1965, 20, 438.

(13) Allen, L. H.; Matejevic, E. J. Colloid Interface Sci. 1969, 31, 237; 1971, 35, 560.

(14) Depasse, J.; Watillon, A. J. Colloid Interface Sci. 1970, 33, 430.

(15) Goodwin, J. W.; Harbron, R. S.; Reynolds, P. A. Colloid Polym. Sci. 1990, 268, 766.

(16) Li, H. C.; de Bruyn, P. L. Surf. Sci. 1966, ㅁ, 203.

(17) Wiese, G. R.; James, R. O.; Healy, T. W. Discuss. Faraday Soc. 1971, 52, 302.

(18) Smit, W.; Holten, C. L. M.; Stein, H. N.; de Groeij J. J. N.; Theelen, H. M. J. J. Colloid Interface Sci. 1978, 63, 120.

(19) Prasad, N.; Pooley, F. D. J. Appl. Chem. Biotechnol. 1973, 23, 675.

(20) Bousse, L.; Mostarshed, S.; van der Shoot, b.; de Rooij, N. F.; Gimmel, P; Gopel, W. J. Colloid Interface Sci. 1991, 147, 22.

(21) Scales, P. J.; Grieser, F.; Healy, T. W.; White, L. R.; Chan, D. Y. C. Langmuir $1992, \underline{8}, 965$. 
(22) Churaev, N. V.; Nikologorskaja, E. A. Colloids Surf. 1991, 9ㅗ, 71.

(23) Kosmulski, M.; Matijevic, E. Langmuir 1992, ㅇ, 1060.

(24) Kosmulski. M.; Matijevic, E. Colloid Polym. Sci. 1992, 270, 1046.

(25) O'Brien R. W.; White, L. R. J. Chem. Soc. Faraday Trans. 2 1978, 74, 1607.

(26) Unpublished data: Karin Evans, Langley-Ford Instruments, Amherst, MA. 


\section{TABLE I}

Properties of Test Sols

\begin{tabular}{|c|c|c|c|c|c|c|}
\hline Sol & $\begin{array}{c}\rho \\
\mathrm{g} \mathrm{cm}^{-3}\end{array}$ & $\begin{array}{l}\text { Radius } \\
(\mathrm{a}, \mathrm{nm})\end{array}$ & ка & $\begin{array}{c}\mathrm{dS} / \mathrm{d} \phi \\
\mathrm{mPa} \mathrm{m} / \mathrm{V}\end{array}$ & $\begin{array}{c}\mu(0) \\
(\mu \mathrm{m} / \mathrm{S})(\mathrm{cm} / \mathrm{V})\end{array}$ & $\zeta \mathrm{mV}$ \\
\hline PS & 1.054 & 302 & 99 & -2.76 & -5.13 & -70 \\
\hline \multirow[t]{2}{*}{ PMMA } & 1.191 & 162 & 53 & $-6.89^{a}$ & -3.80 & -52 \\
\hline & & & & $-7.27^{b}$ & & \\
\hline $\mathrm{AKP}$ & 3.98 & $169^{\circ}$ & 55 & $139.5^{b}$ & +4.06 & +56 \\
\hline \multirow[t]{2}{*}{$\mathrm{TM}$} & 2.20 & 11.5 & 3.8 & $-41.5^{\mathrm{a}}$ & - & - \\
\hline & & & & $-43.4^{b}$ & & \\
\hline
\end{tabular}

${ }^{\mathrm{a}}$ Reference 2. ${ }^{\mathrm{b}}$ Reference $1 .{ }^{\mathrm{C}}$ Volume mean radius. 
TABLE II

Comparison of Calibration Constants for Test Sols

\begin{tabular}{|c|c|c|c|c|}
\hline Sol & $|\mu(\omega) / \mu(0)|$ & $y / G_{f}$ & $\begin{array}{c}\mathrm{m}(0) \\
\mu \mathrm{m} \mathrm{m} / \mathrm{s} \mathrm{V}\end{array}$ & $\zeta \mathrm{mV}$ \\
\hline PS & $0.950^{\circ}$ & $1.49^{\mathrm{b}}$ & -5.13 & -70 \\
\hline PMMA & $0.987^{\circ}$ & $\begin{array}{c}1.54^{\mathrm{a}} \\
\left(1.48^{b}\right)\end{array}$ & -3.80 & -52 \\
\hline $\mathrm{AKP}$ & $0.957^{\circ}$ & $1.23^{b}$ & +4.06 & +56 \\
\hline $\mathrm{TM}$ & $1.00^{\circ}$ & $\begin{array}{c}1.54^{\mathrm{a}} \\
\left(1.40^{\mathrm{b}}\right)\end{array}$ & $(-3.59)$ & $(-78)$ \\
\hline
\end{tabular}

aReference 2. ${ }^{\mathrm{b}}$ Reference 1. ${ }^{\mathrm{c}}$ Computed from Eq. 5. 


\section{FIGURE CAPTIONS}

Figure 1. Dependence of the modulus of the dynamic to static mobility ratio on particle size (a) and particle density $\rho$ at frequency $\omega / 2 \pi=1 \mathrm{MHz}$ from Eq. 5 for $\mathrm{p}=1.1(\mathbf{\square}), 2.5(\bullet), 4(\Delta)$, and $6(\diamond) \mathrm{g} \mathrm{cm}^{-3}$. The viscosity and density of the continuous phase were taken as 0.01 poise and $1 \mathrm{~g} \mathrm{~cm}^{-3}$, respectively.

Figure 2. Number density ( $p(a))$ normalized particle size distribution as a function of particle radius a for the PMMA latex, MP1201.

Figure 3. Number density ( $p(a))$ normalized particle size distribution as a function of radius a for the colloidal silica sol Ludox TM.

Figure 4. Cumulative percent oversize distribution for alumina AKP by centrifugal sedimentation analysis. The line is for a log normal distribution where the median radius by mass is $195 \mathrm{~nm}$ and the geometric standard deviation is 1.37 .

Figure 5. ESA signal, $S$, as a function of volume fraction $(\phi)$ for PS latex at pH 6.8 (ordinate values magnified 10X) and for alumina $\mathrm{AKP}$ at $\mathrm{pH}$ 4. The alumina particles have a positive charge while the PS latex is negative. The solution was $0.01 \mathrm{~mol} / \mathrm{dm}^{3} \mathrm{KNO}_{3}$ at $25^{\circ} \mathrm{C}$. 
Figure 6. ESA signal, $\mathrm{S}$, as a function of volume fraction (f) for PMMA latex MP-1201. The data points were obtained on two different MBS8000 instruments. The continuous line is a least-squares fit for $\phi<$ 0.03 and has a slope of $-6.89 \pm 0.24 \mathrm{mPa} \mathrm{m} / \mathrm{V}$.

Figure 7. ESA signal, $S$, as a function of volume fraction $(\phi)$ for the colloidal silica Ludox TM. The data points were obtained using two different MBS-8000 systems. The continuous line is a least-squares fit to data for $\phi<0.07$ and has a slope of $-41.4 \pm 0.5 \mathrm{mPa} \mathrm{m} / \mathrm{V}$.

Figure 8. Relationship between zeta potential and electrophoretic mobility for PMMA latex, $к \mathrm{a}=53$, and for Ludox TM, $к \mathrm{a}=3.8$ calculated using the O'Brien and White method (25) and computer program. 


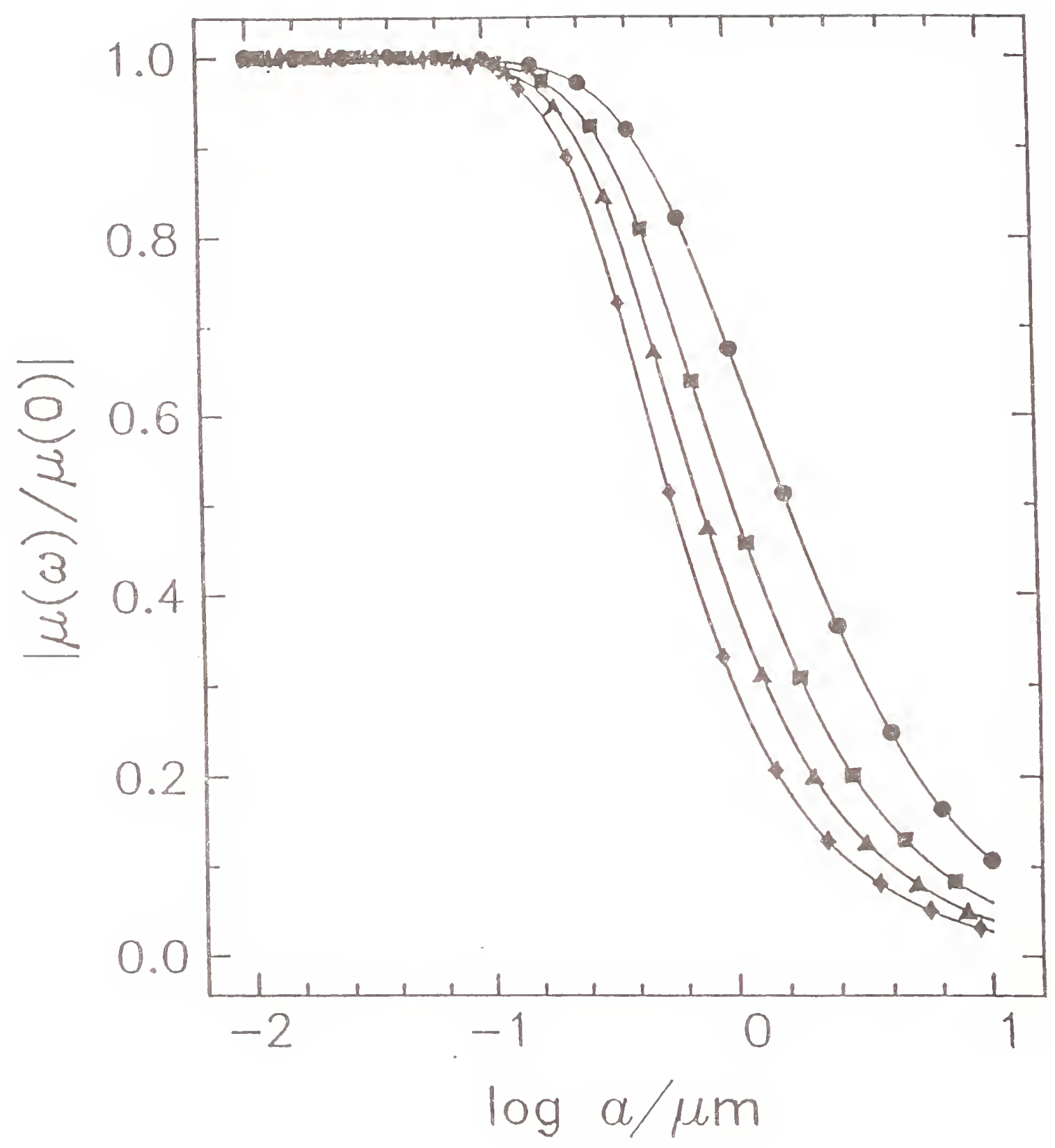

Figure 1 . 


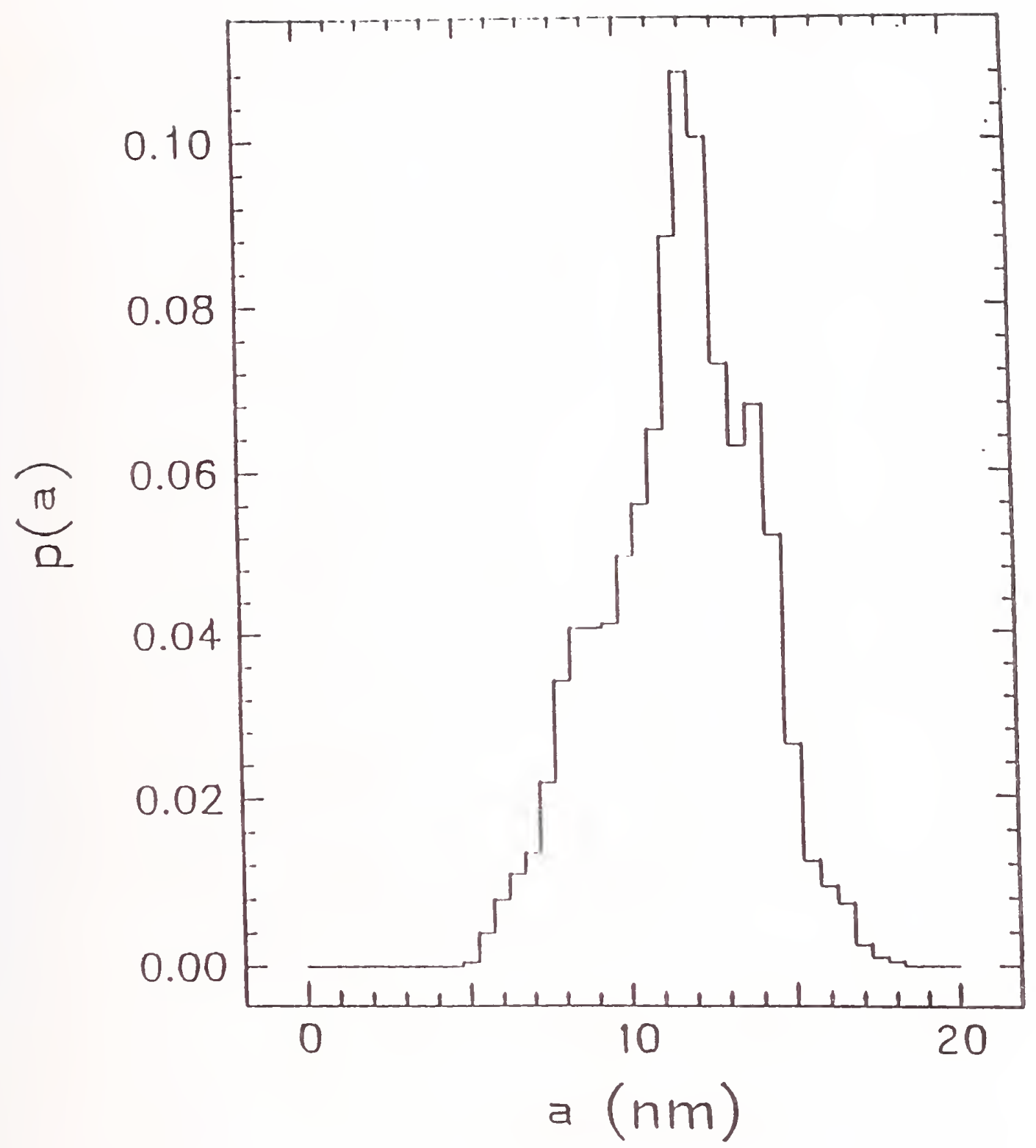

Figure 2 


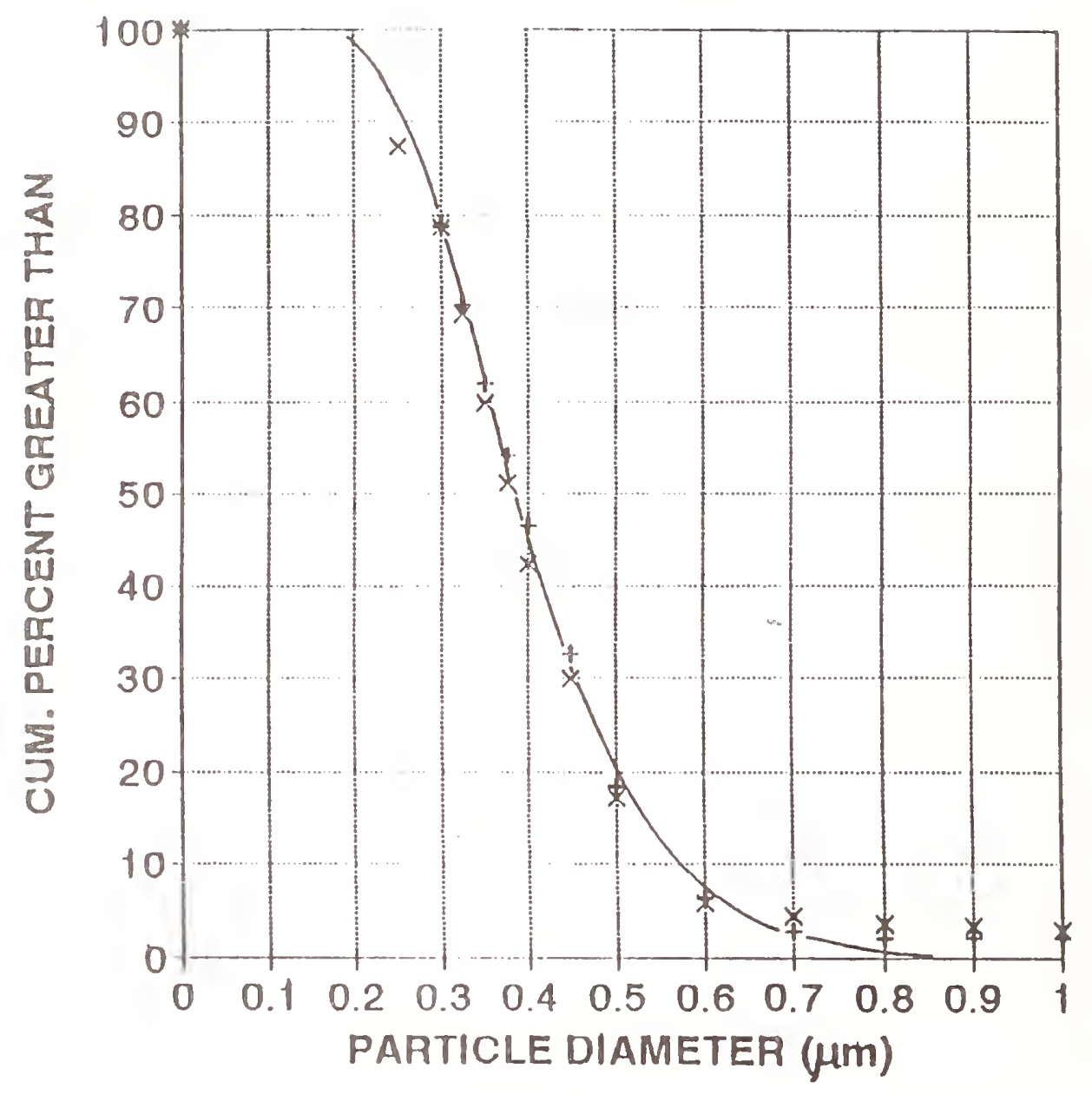

Figure 3 


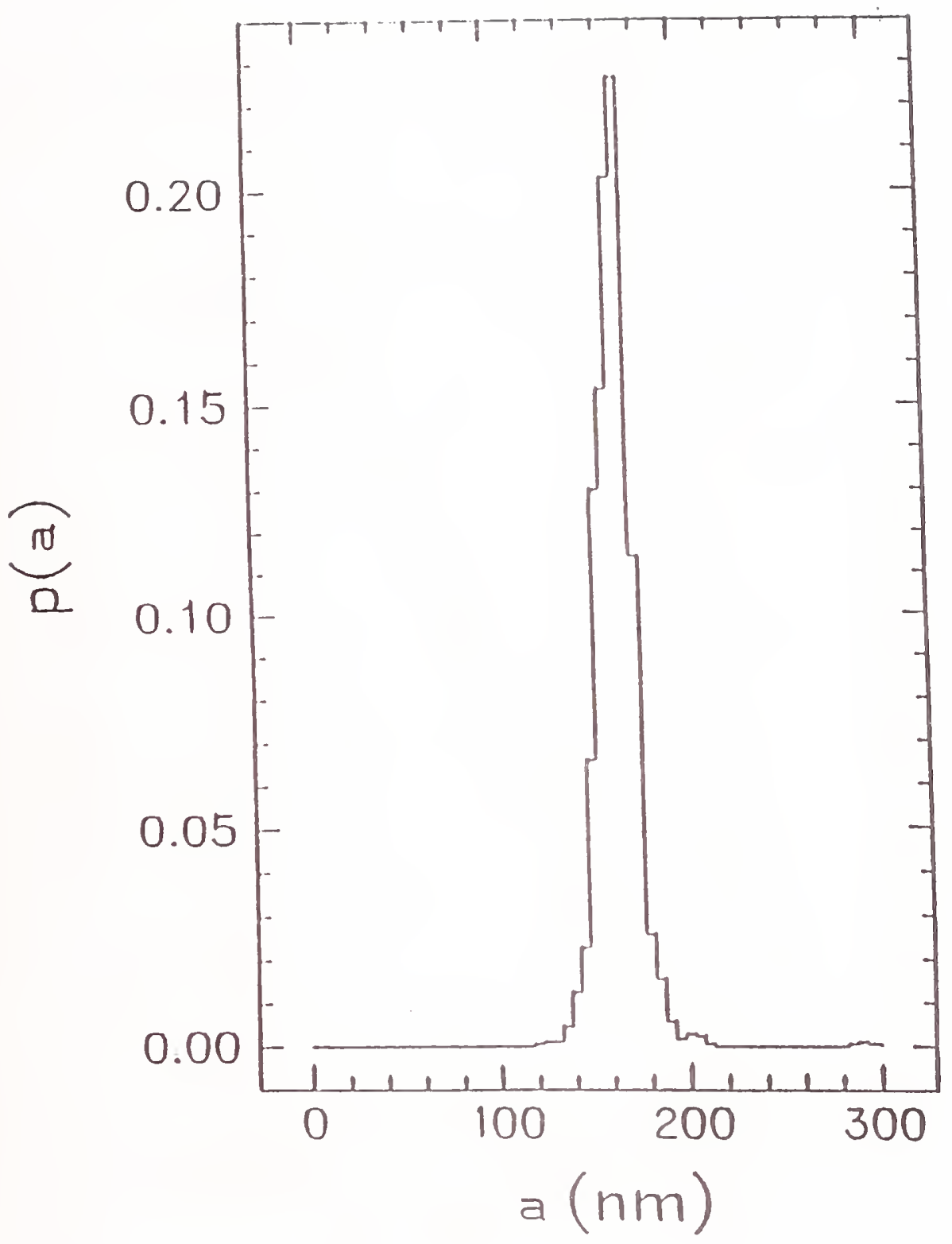

Figure 4 


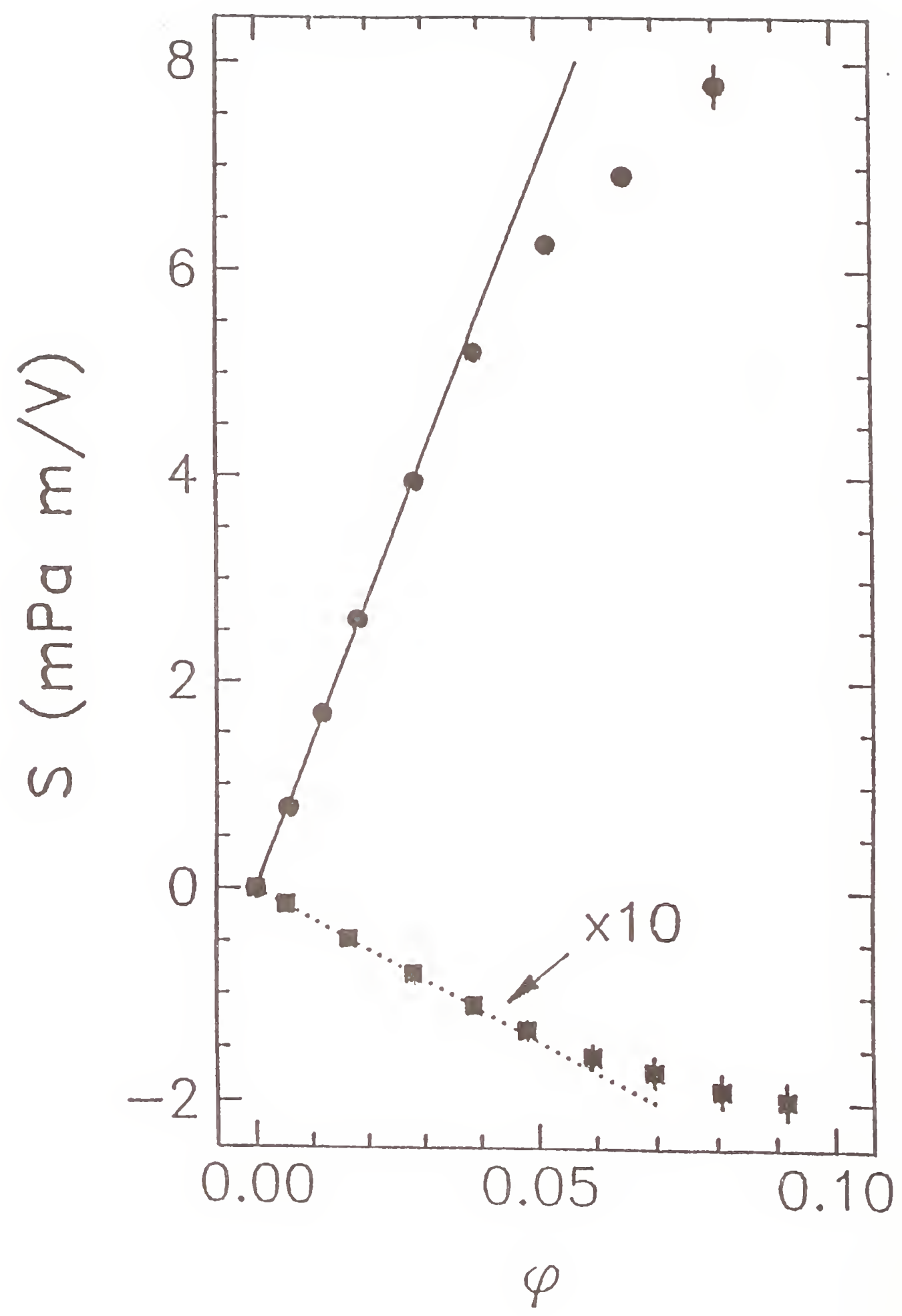

Figure 5 


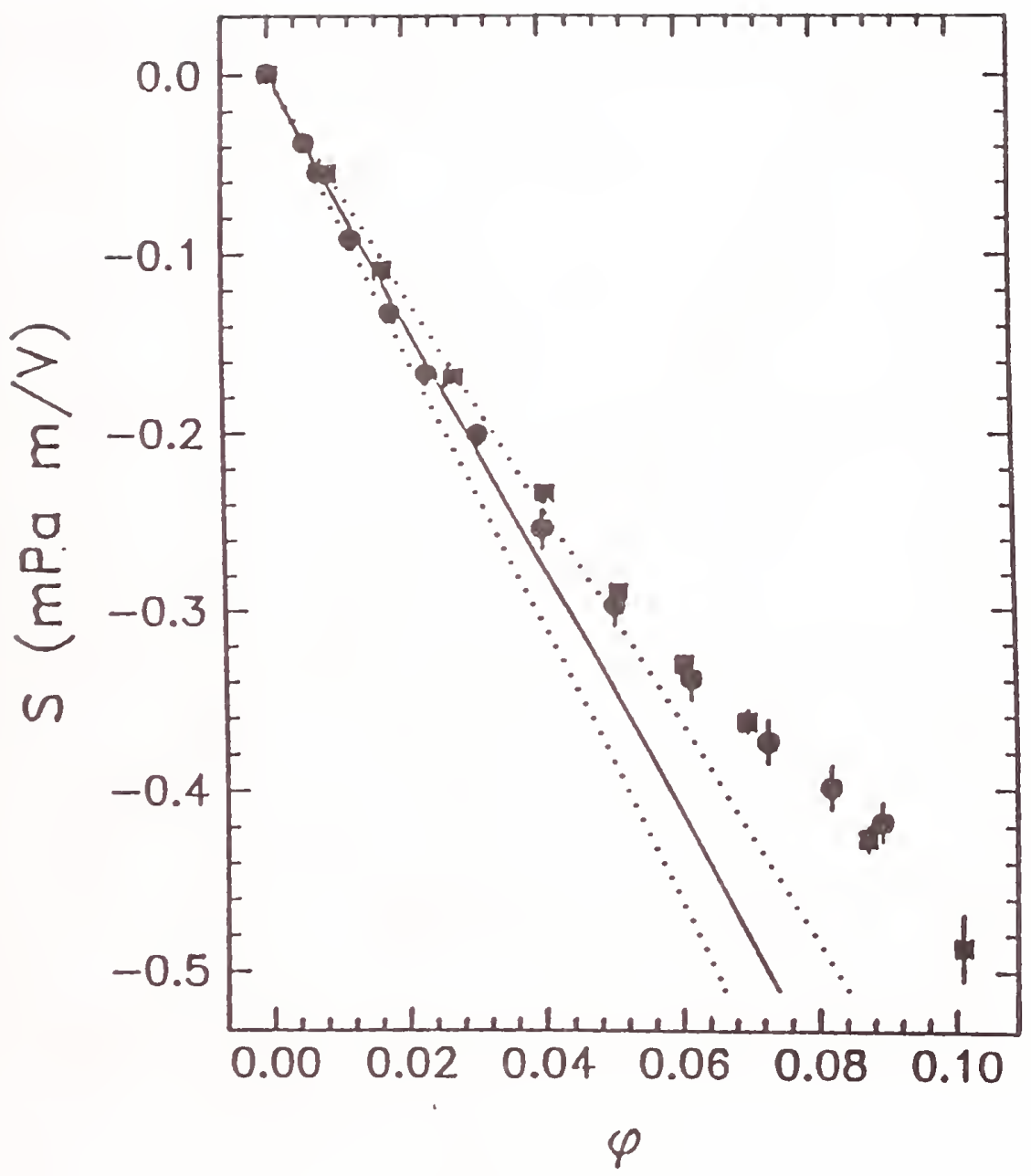

Figure 6 


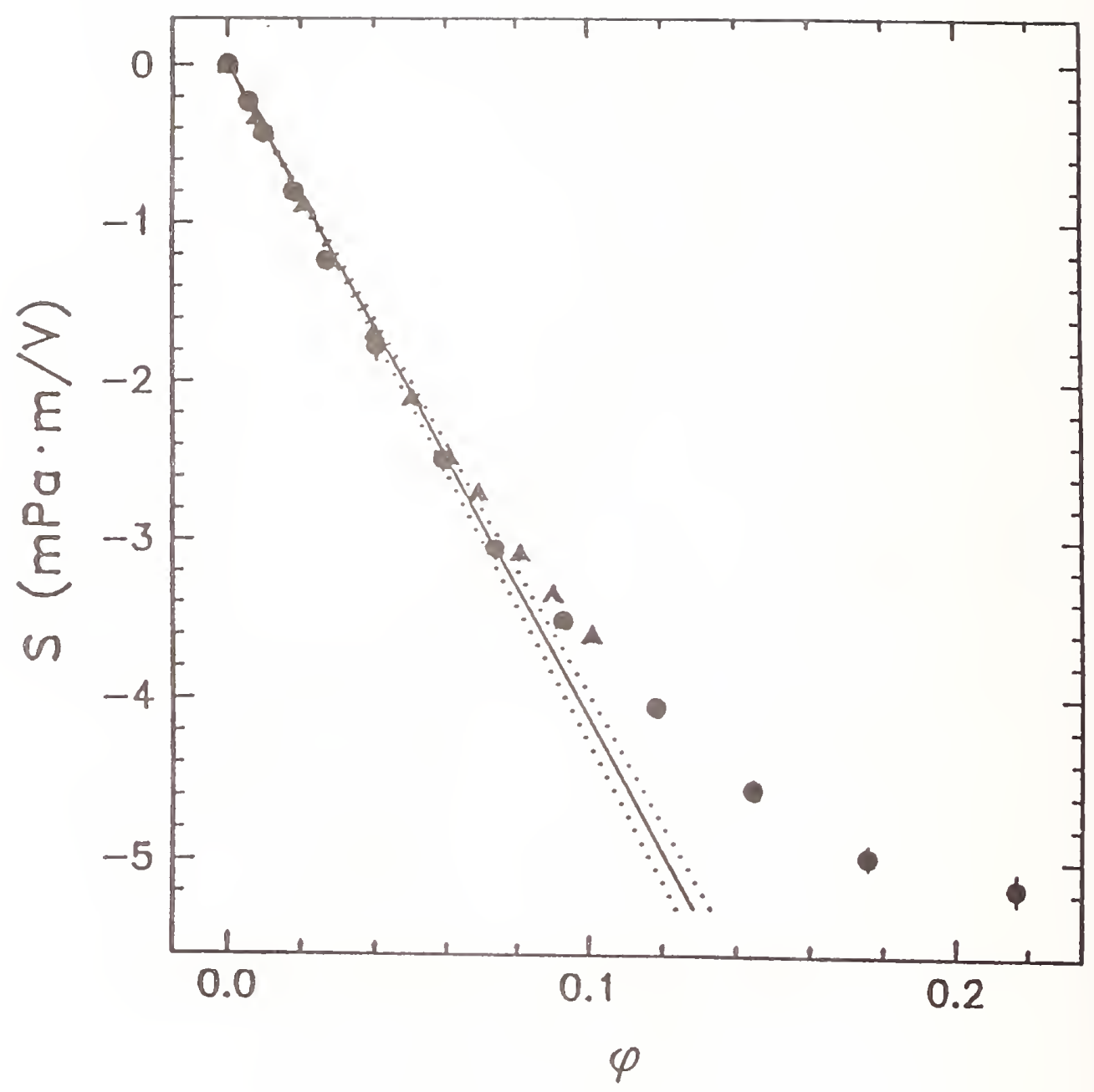

Figure 7 


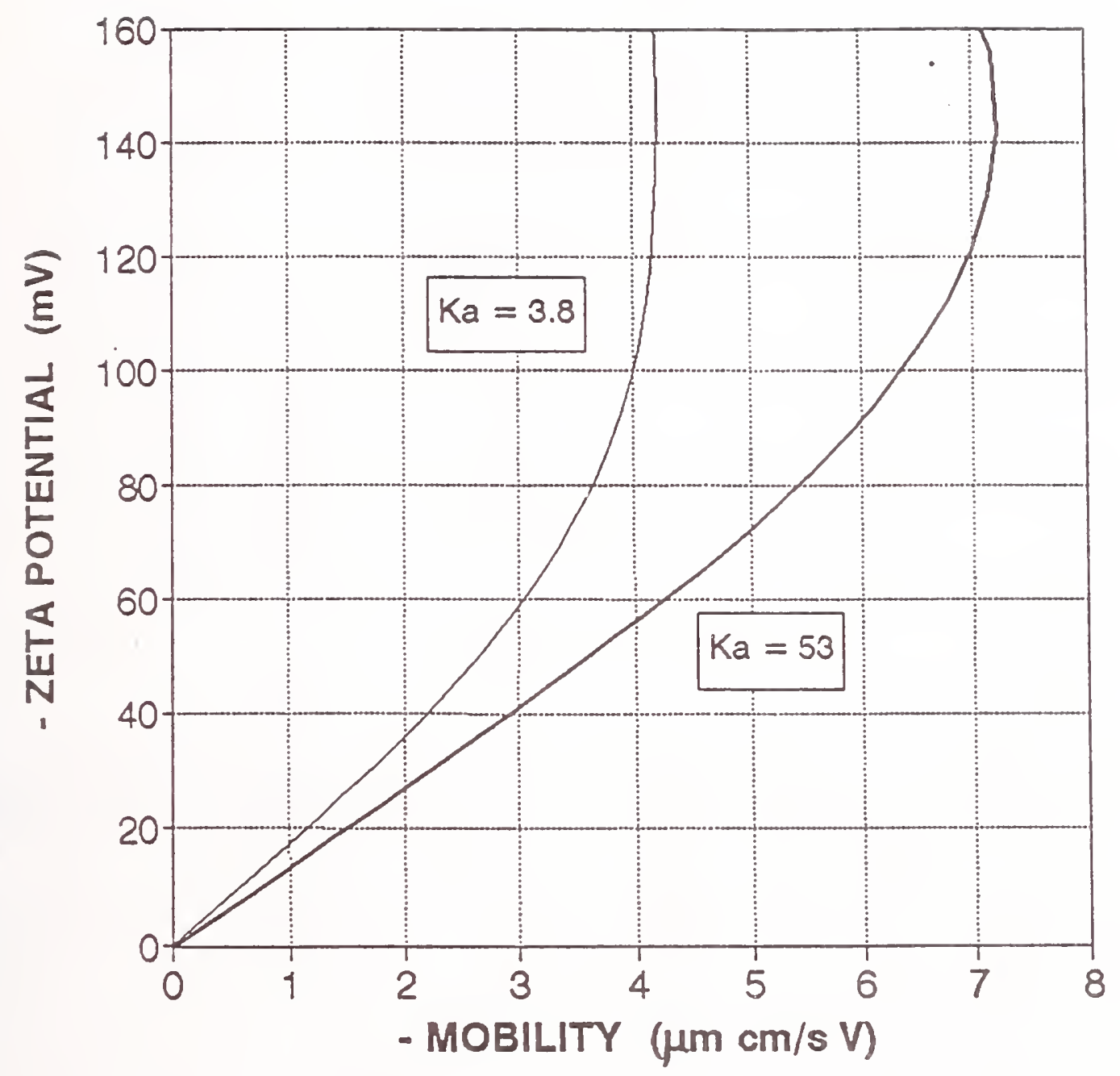

Figure 8 


\title{
ELECTROKINETIC MEASUREMENTS OF CONCENTRATED SUSPENSIONS IN
} APOLAR LIQUIDS

\author{
R. E. Kornbrekke \\ The Lubrizol Corporation \\ Wickliffe, $\mathrm{OH}$ \\ I. D. Morrison \\ Xerox Corporation \\ Webster, NY \\ and \\ T. Oja \\ Matec Applied Sciences \\ Hopkinton, MA
}

\begin{abstract}
We have used electroacoustic (Electrokinetic Sonic Amplitude, ESA) measurements to study the charging of carbon black suspended in alkanes containing dispersants. Systems with various types of dispersants and 0.5 to 15 volume $\%$ carbon black were used. The state of adsorption was determined from FTIR measurements, and the conductivity of the mixtures was also measured. These measurements have given insight into some of the mechanisms of particle charging in low dielectric liquids. The particles charge only after a sufficient amount of dispersant has adsorbed onto the solid. Charging is due to a combination of selective interaction (an acid-base type) coupled with ion pair dissociation which is probably facilitated by associative interactions. The degree of charging is affected by the particle concentration; the charging per particle decreases as the concentration increases. This is probably related to double layer overlap. We have found that particle charging in these systems correlates, to a large extent, with the suspension stability.
\end{abstract}

(Since the complete manuscript could not be released for publication, only the presentation slides are included for this paper). 


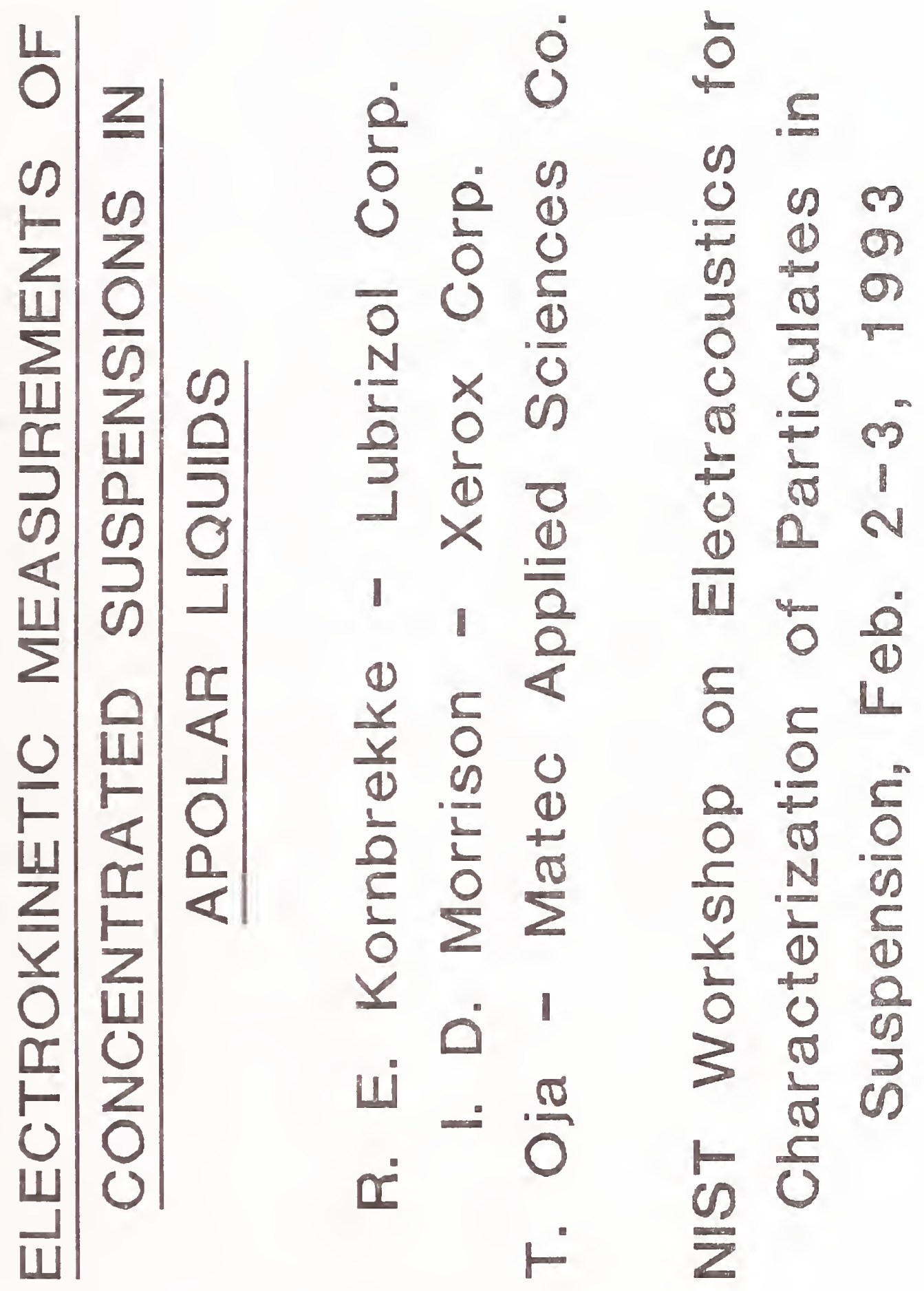




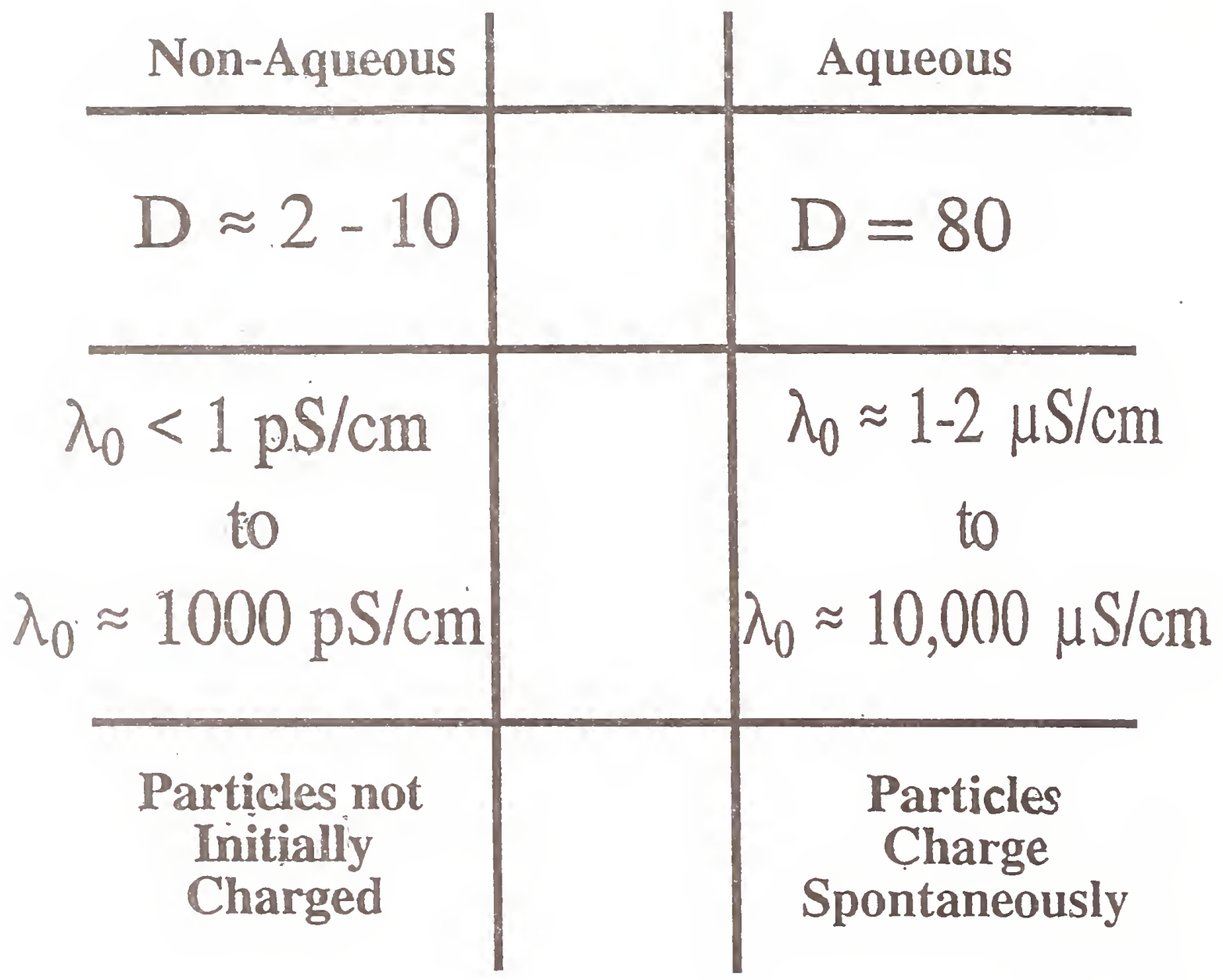




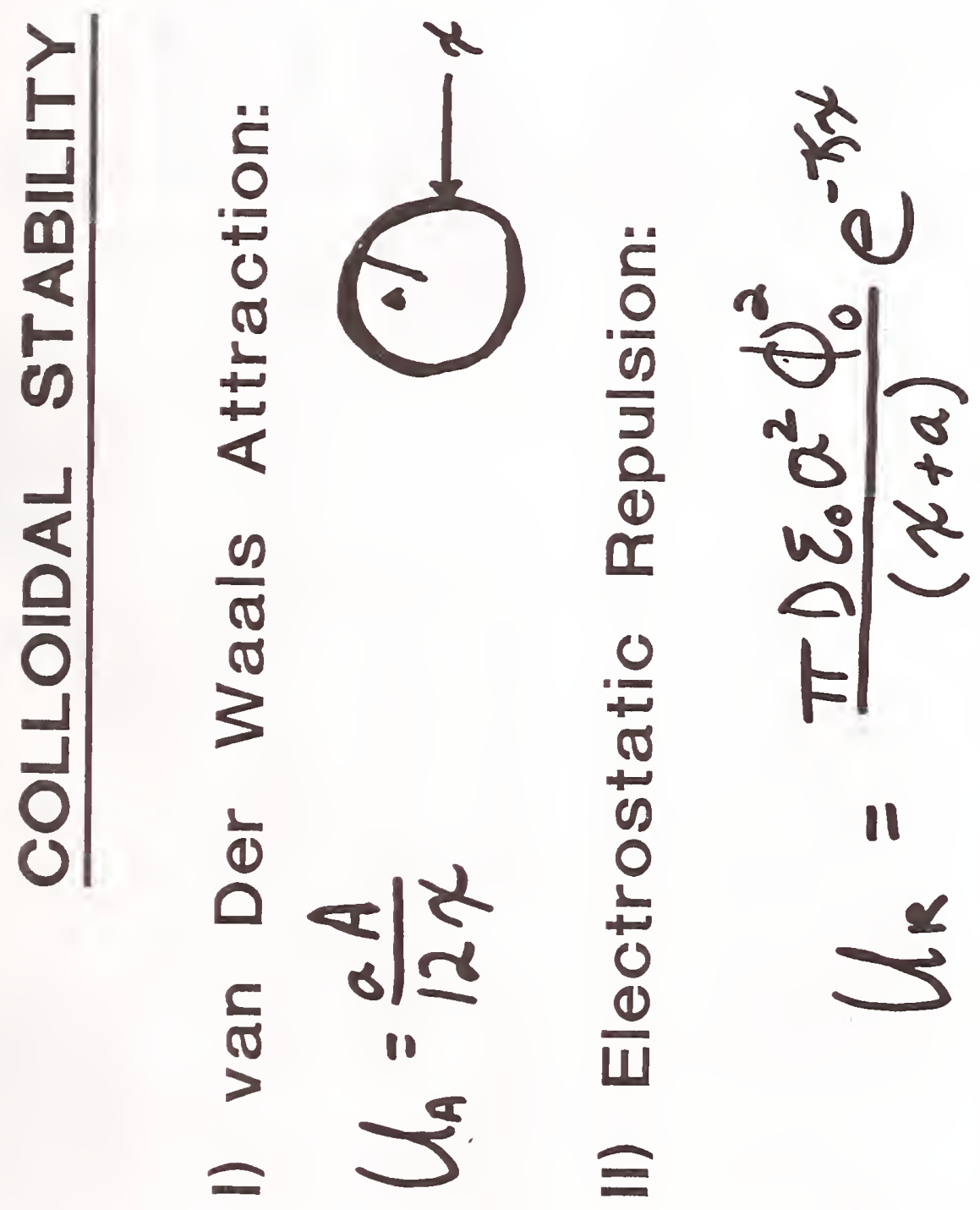




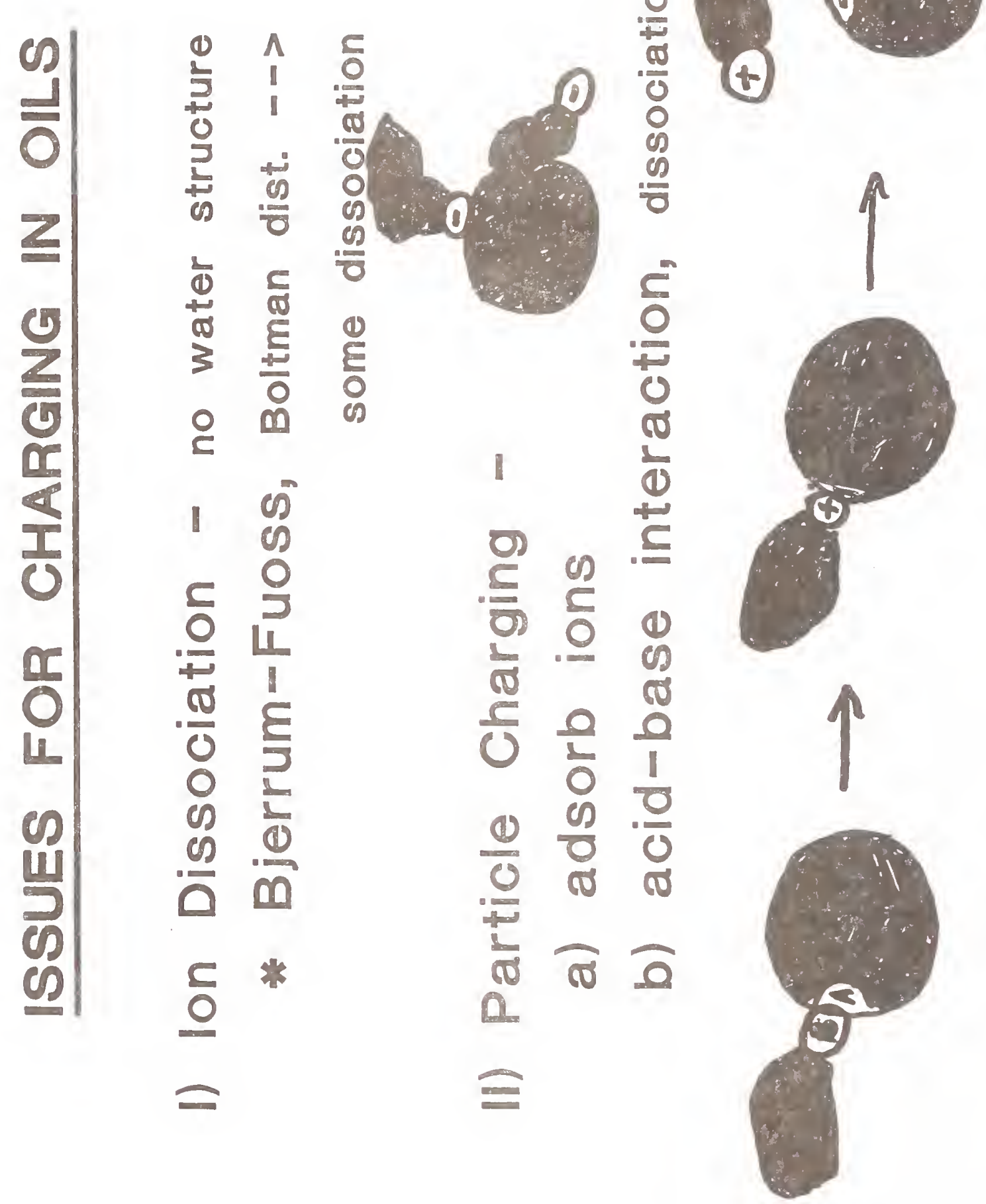




\section{EXPERIMENTAL PROCEDURE}

SYSTEM:

Sterling NS Carbon Black

Polyisobutylene Succinimide (PIBSI)

$(\mathrm{Mn}=1695, \mathrm{TBN}=46)$

Dodecane or Isopar G, L

Mix, equilibrate,

Measure:

* Conductivity - $18 \mathrm{~Hz}$.

* ESA - Zeta Potential

* Liquid (supernatant) Concentration FTIR Carbonyl $(1705 / \mathrm{cm}$.)

* Dispersion Stability 


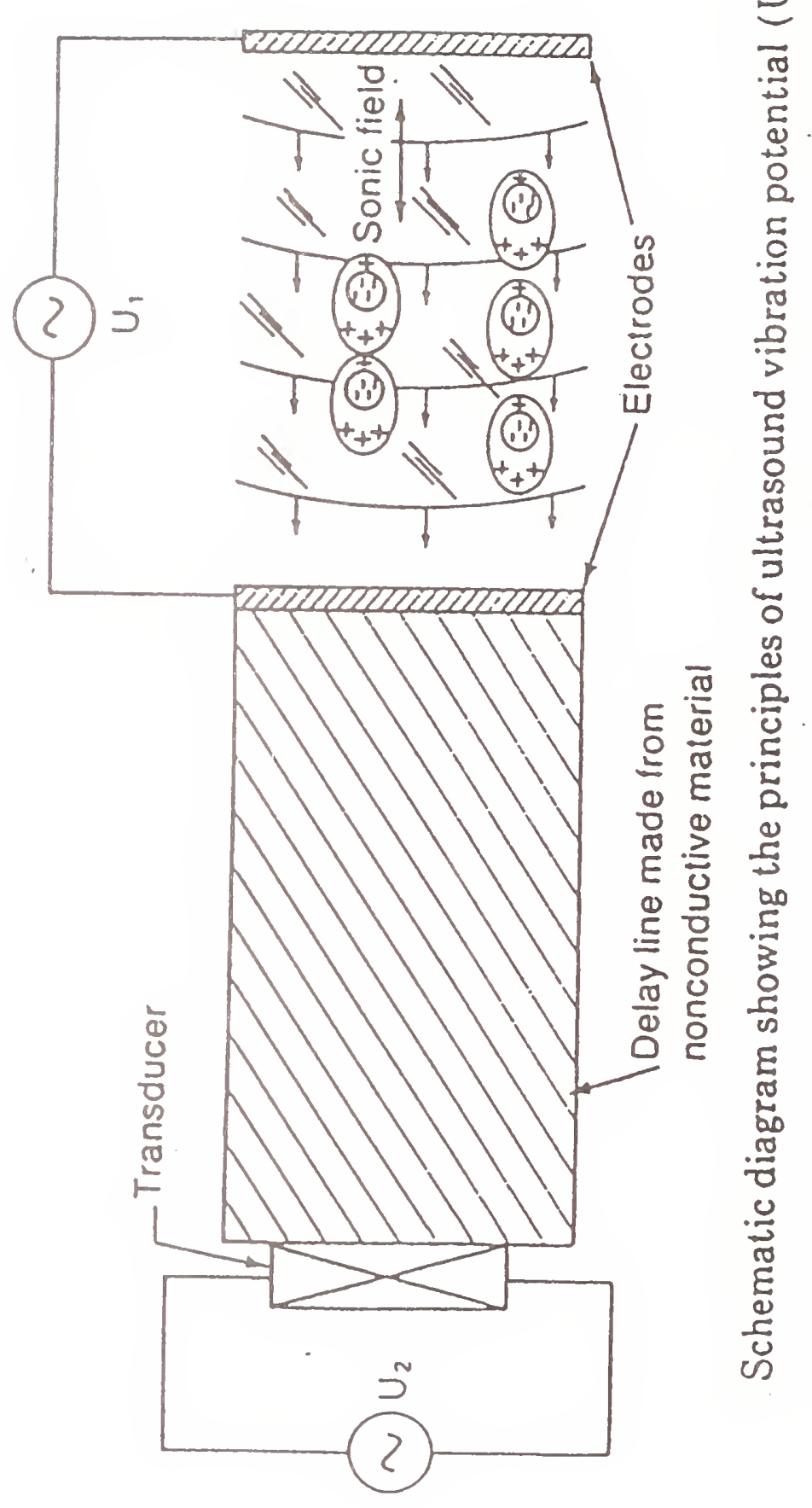




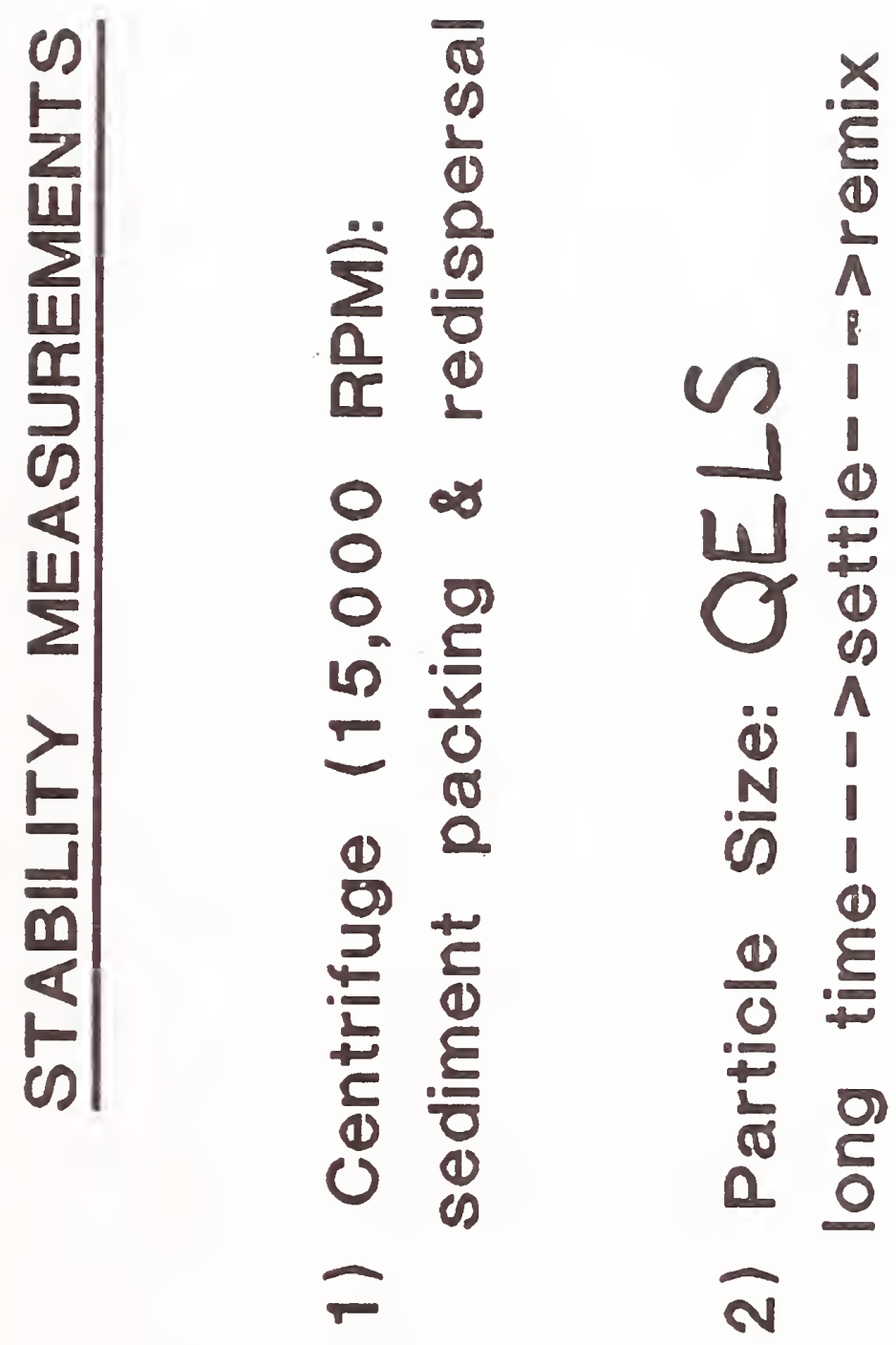




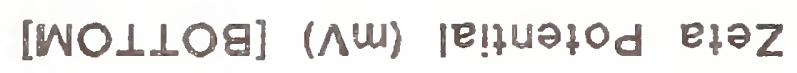

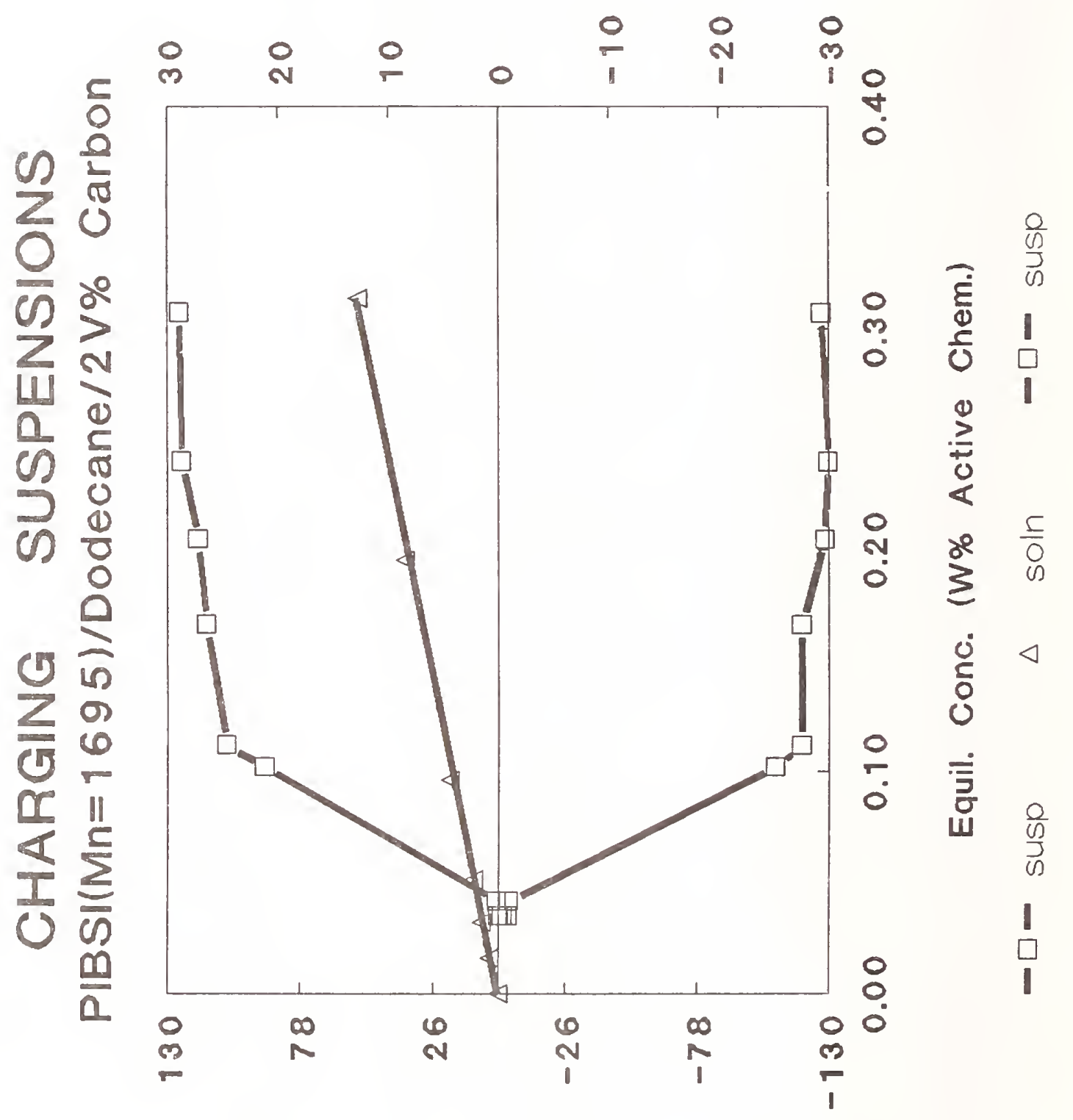

[dOL] (mo/sd) $\mathrm{A} !$ !n!lonpuoo 


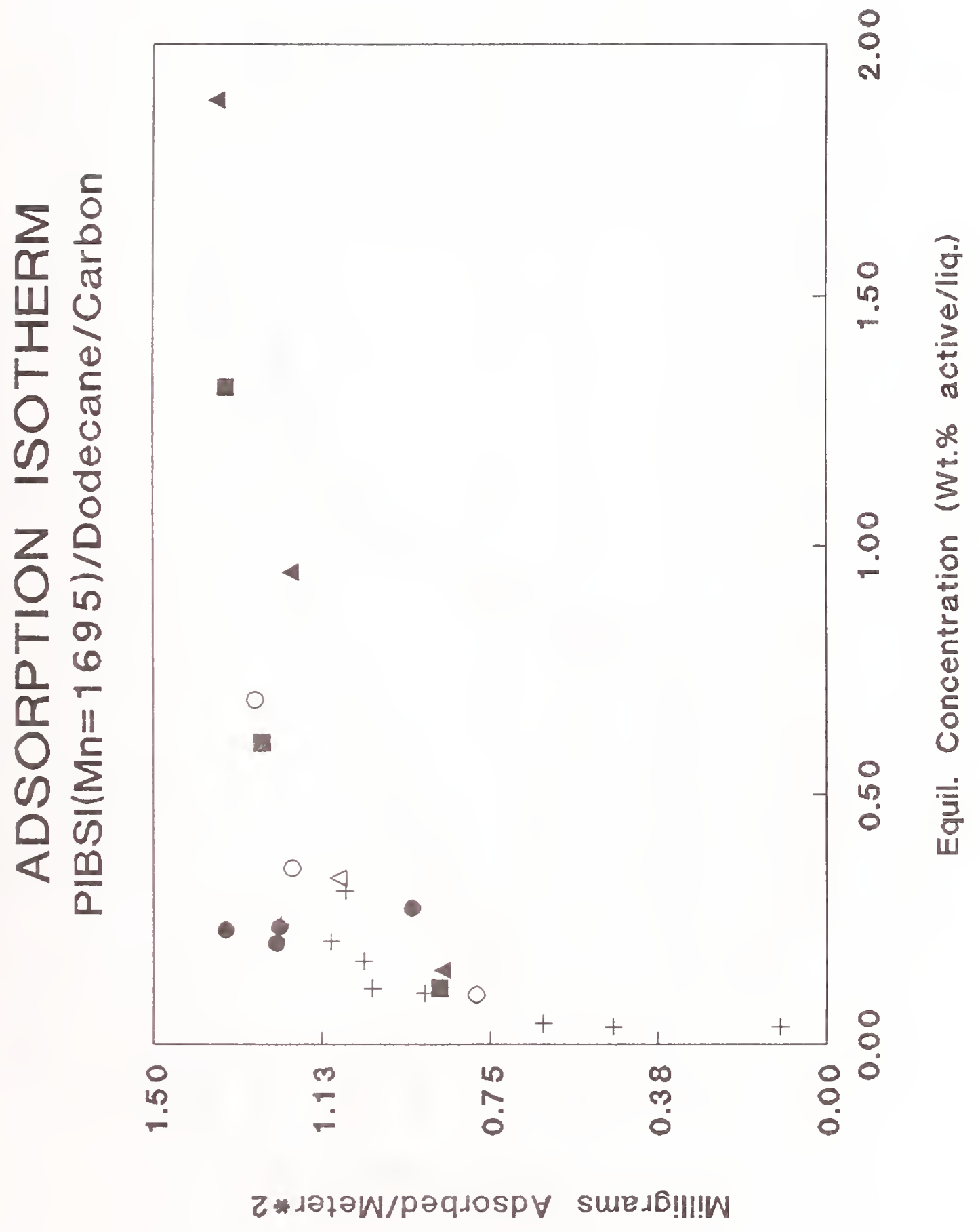




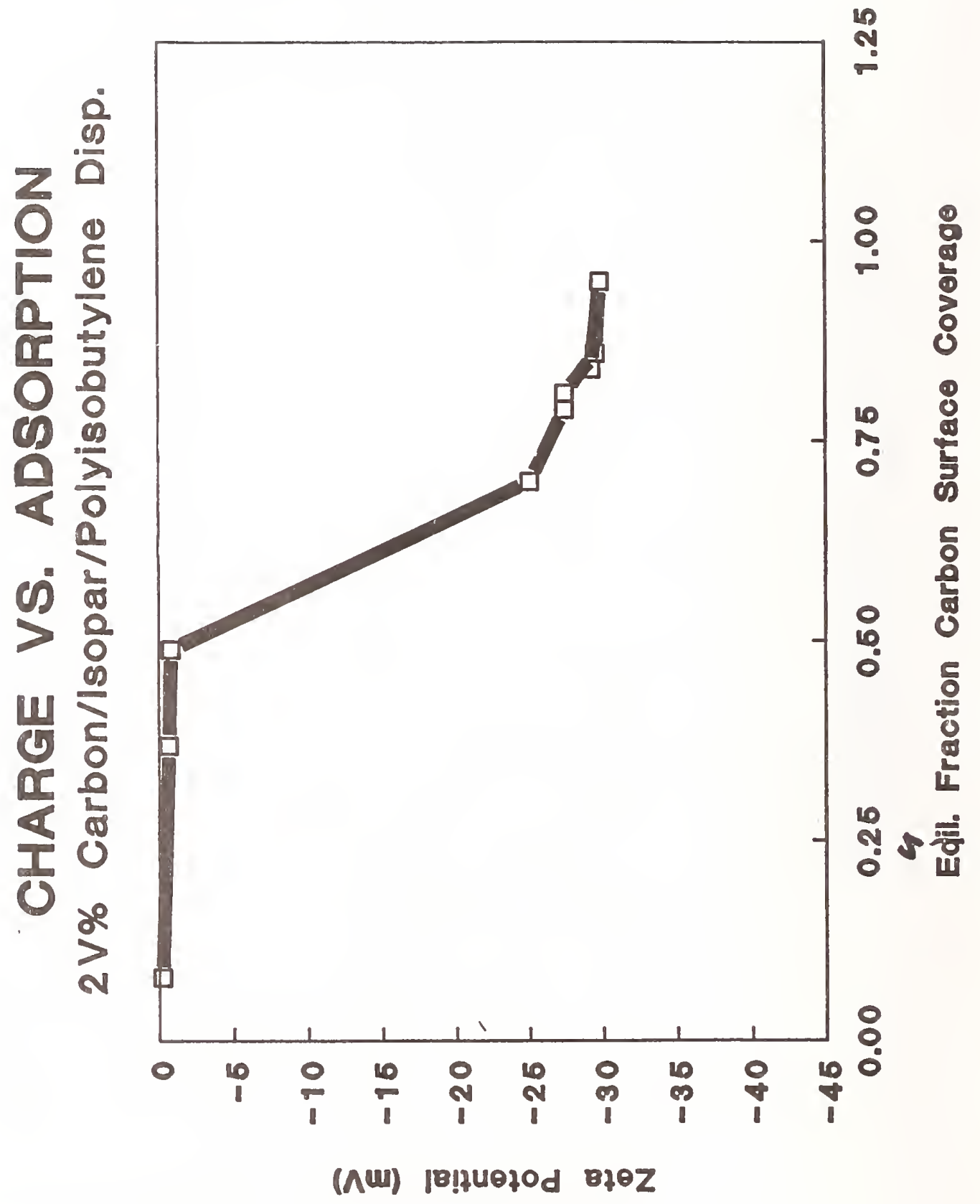




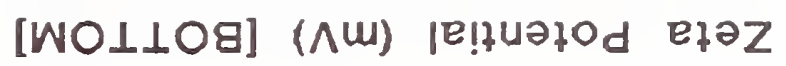

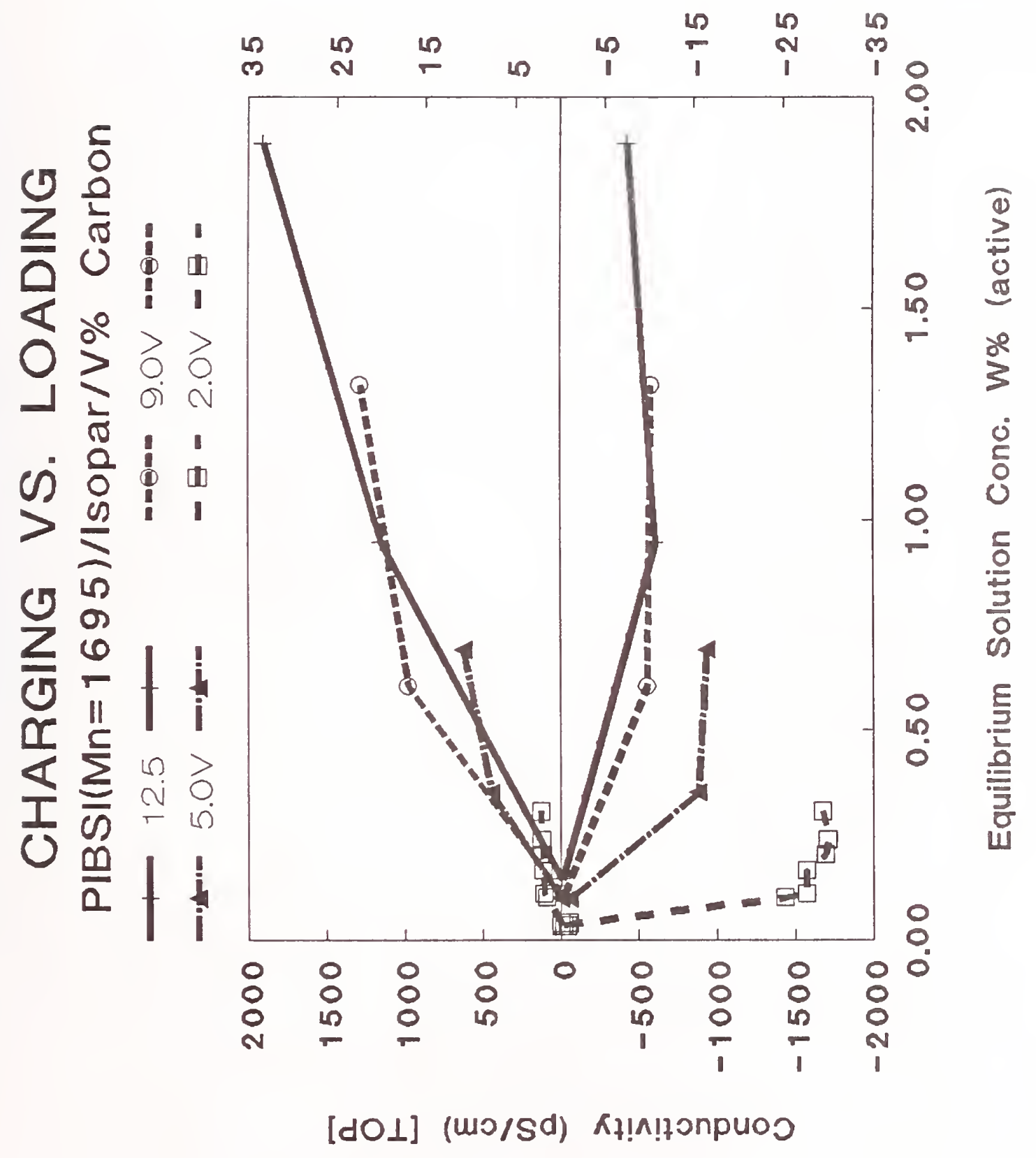




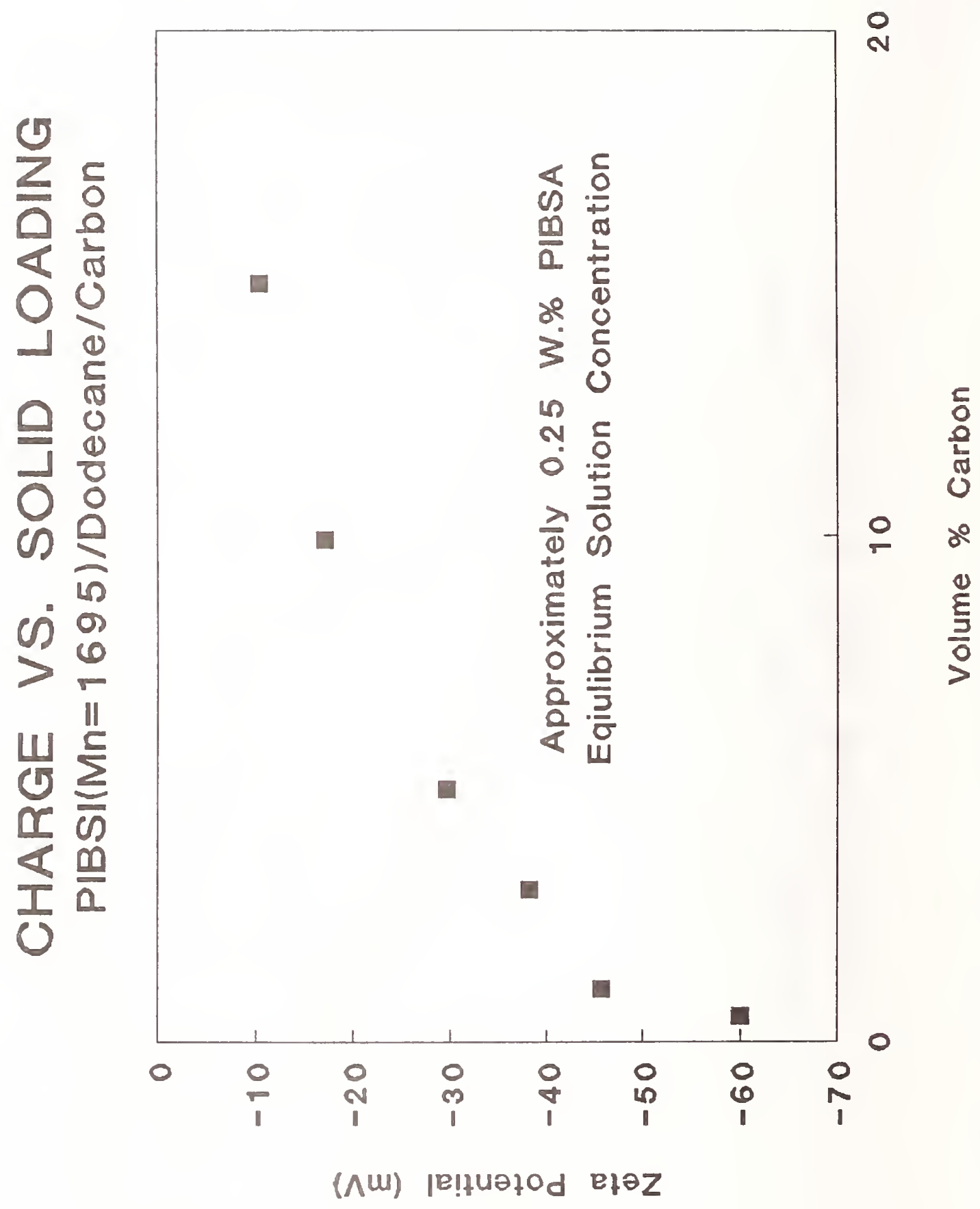




\section{SUSPENSION STABILITY}

$\frac{\text { System }}{5 \mathrm{~V} \% \mathrm{C}}$

Sediment

$\underline{Z e t a(m V)}$

$\% \mathrm{PIBS|}$

0.01

not resuspend

$-1.1$

0.35

resuspends

$-17.9$

0.69

resuspends easily

$-19.2$

$9 \mathrm{~V} \% \mathrm{C}$

$\% \mathrm{PIBS|}$

0.11

not resuspend

$-0.3$

0.60

resuspends easily

$-11.4$

1.31

resuspends easily

$-12.0$

$12.5 \% \mathrm{C}$

$\%$ PIBSI

0.14

resuspends easily

$-0.1$

0.95

resuspends easily

$-7.9$

1.89

resuspends easily

$-8.9$ 


\section{ELECTROSTATIC STABILIZATION}

1) Huckel regime:

\begin{tabular}{c|c} 
radius(um) & zeta(mV) \\
\hline 0.05 & -100 \\
0.1 & -71 \\
0.3 & -41 \\
1.0 & -22 \\
5.0 & -10
\end{tabular}

* Partial Electrostatic Stabilization 
Electrophoretic Mobility $=u \equiv \frac{\mathrm{V}}{\mathrm{E}} \quad \varepsilon \equiv \varepsilon_{0}, \mathrm{D}$

\begin{tabular}{c|c|c} 
Non-Aqueous & & Aqueous \\
\hline $\mathrm{u}=\frac{2 \varepsilon \zeta}{3 \eta}$ & & $\mathrm{u}=-\frac{}{\eta}$
\end{tabular}

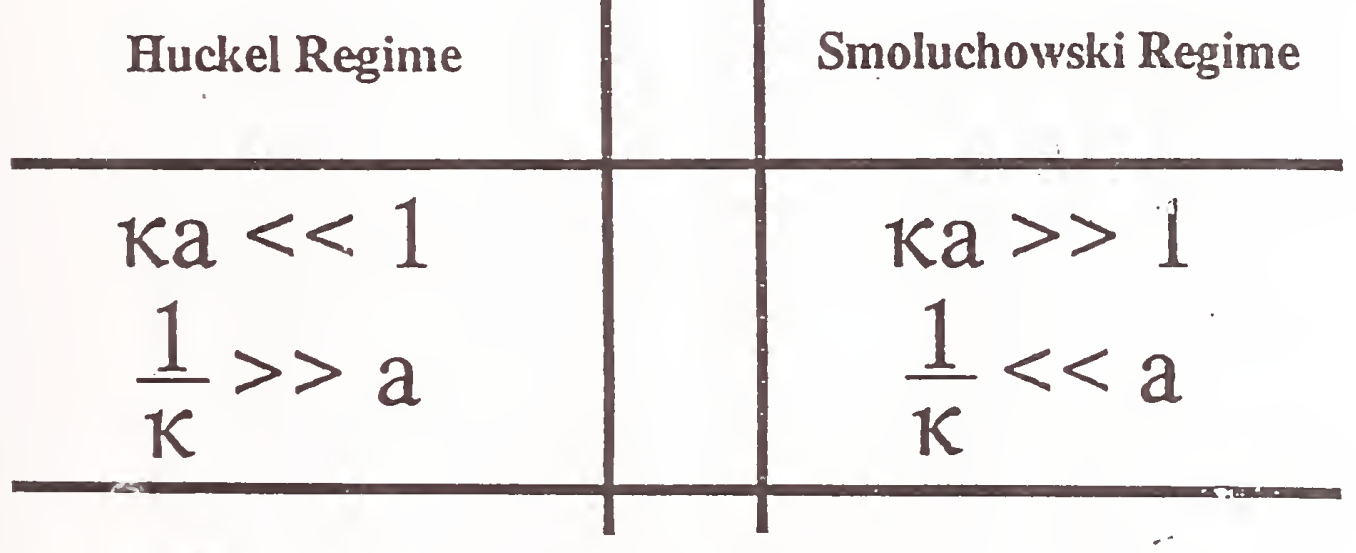

$$
\frac{1}{\kappa}=\left(\frac{\varepsilon k T}{e^{2} \sum \mathbf{n}_{\mathrm{i}}^{\mathrm{o}} \mathrm{Z}_{\mathrm{i}}^{2}}\right)^{\frac{1}{2}}
$$

$\frac{1}{\kappa} \equiv$ Thickness of the Diffuse Layer

Electrical Potential Decays to $2 \%$ of the Original Value at $\frac{3}{\kappa}$ 
At $0.5 \mathrm{~V} \%$ for Carbon Black

$\mathrm{a}=100 \mathrm{~nm}$

$\mathrm{R}_{1}=\frac{1.81 \times 100 \mathrm{~nm}}{(0.005)^{\frac{1}{3}}}=1060 \mathrm{~nm}$

But $\frac{1}{\kappa}=291 \mathrm{~nm}$

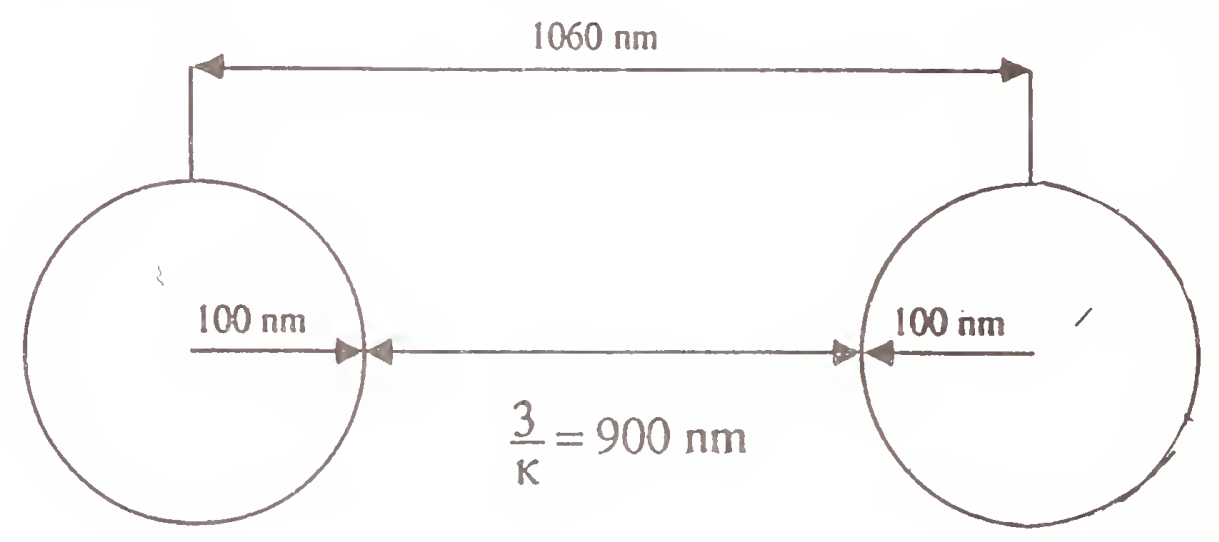

Electrical Double Layer Overlap occurs even at Relatively Low Particle Volume Fractions. 


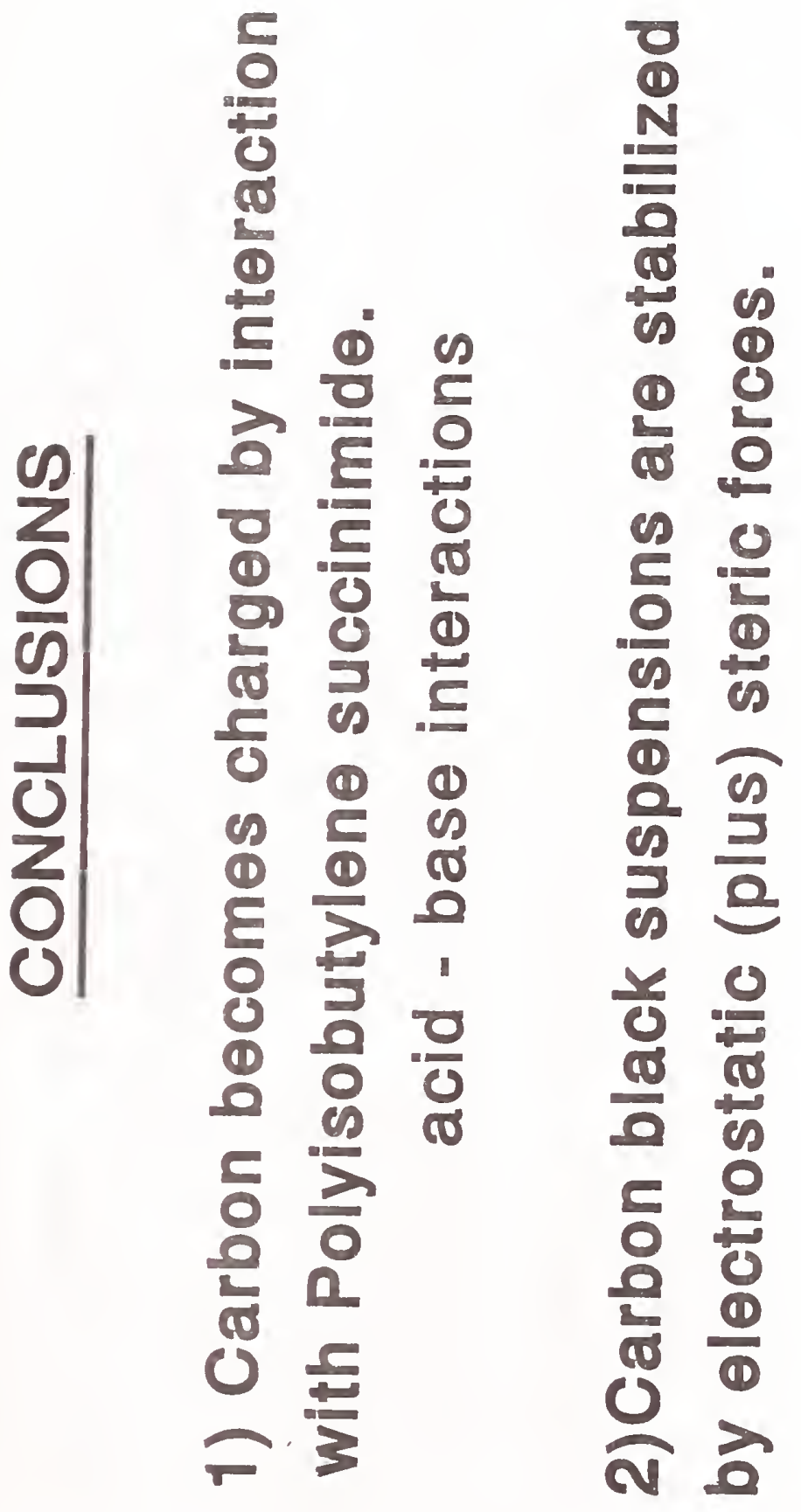




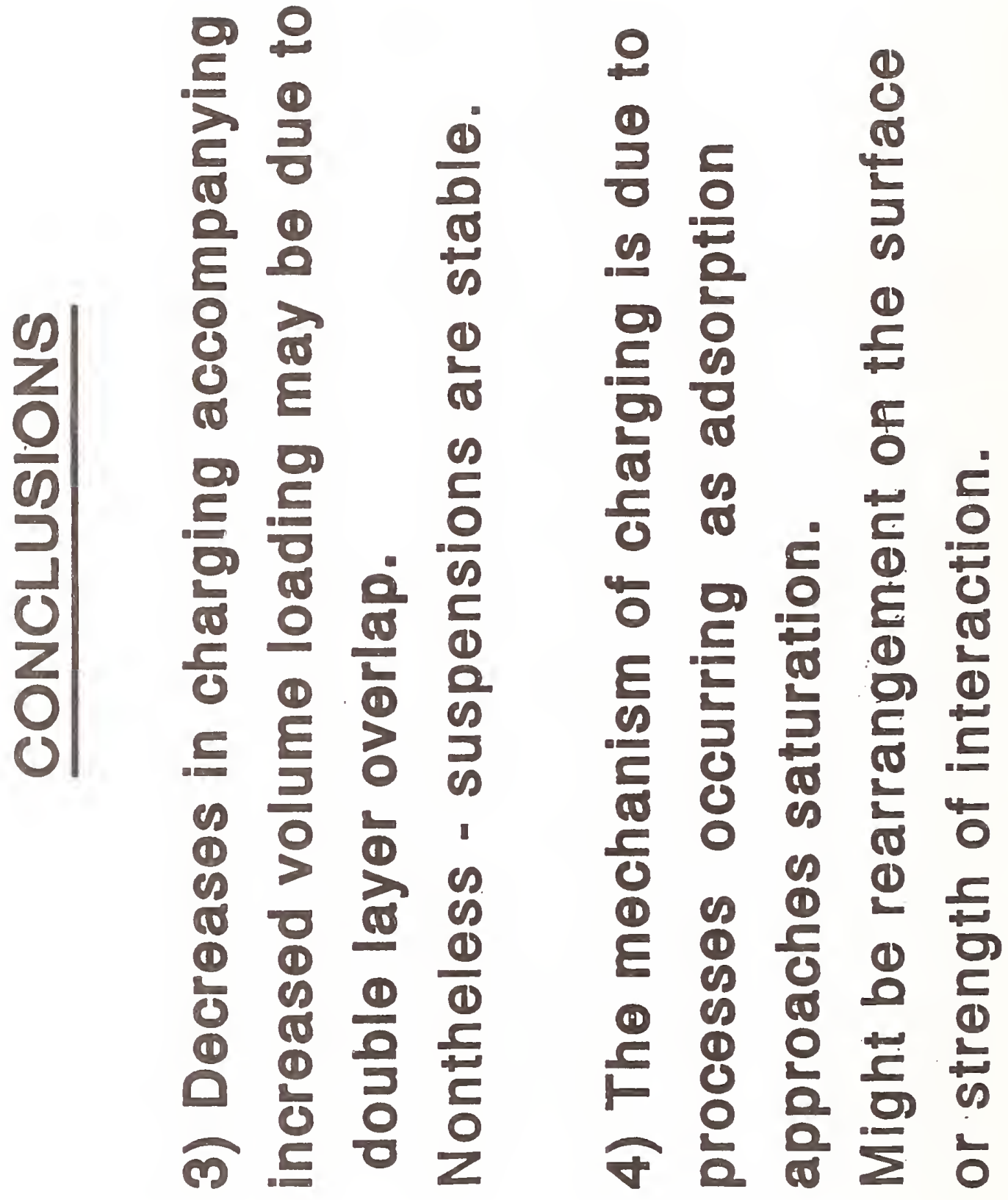




\title{
ACOUSTOPHORETIC CHARACTERIZATION OF COLLOIDAL DIAMOND PARTICLES
}

\author{
J. L. Valdes \\ AT\&T Bell Laboratories \\ Murray Hill, New Jersey 07974
}

\begin{abstract}
The interfacial chemistry of sub-micron diamond particles is of great practical importance in determining the stability and electrophoretic behavior of these colloidal dispersions. The electrokinetic sonic amplitude (ESA) technique is used to probe concentrated dispersions of natural and synthetic diamond particles in both aqueous and non-aqueous solutions. Acoustophoretic characterization of colloidal diamond dispersions have been studied over a broad $\mathrm{pH}$ range $(2<\mathrm{pH}<12)$. In aqueous dispersions, the zeta potential of both synthetic and natural diamond particles are dependent on the $\mathrm{pH}$ of the solution. Natural diamond was found to have an appreciably more negative zeta potential than synthetic dispersions of diamond under the same conditions. These results are consistent with the presence of very reactive, unsaturated covalent bonds that lead to the formation of oxygen-containing ionizable groups on the surface of natural diamond. The zeta potential of colloidal diamond dispersions was found to be considerably diminished in non-aqueous solvents (e.g. $\zeta=2-5$ $\mathrm{mV}$ in methanol). Acoustophoretic characterization of colloidal diamond dispersions is essential to the development of an electrophoretic process for the selected-area nucleation and patterning of diamond thin films.
\end{abstract}

\section{INTRODUCTION}

High thermal conductivity, excellent dielectric and optical properties, and superior mechanical strength make diamond thin films promising for a variety of important technological applications. In a recent paper, we demonstrated a new electrophoretic process for selected-area nucleation and patterning of silicon substrates for chemical vapor deposition (CVD) growth of polycrystalline diamond films (1-3). In this process, seeding of the substrate to be nucleated for diamond film growth is accomplished by electrophoretic deposition from a dispersion of colloidal diamond particles. Once deposited on a suitable substrate, the diamond crystallites serve as homoepitaxial centers for CVD growth of a contiguous film. 
The design of an optimized electrophoretic process for the deposition of colloidal diamond particles requires a consideration of numerous process parameters. One of the most important variables in this process is the electrophoretic mobility of diamond dispersions. The mobility controls not only the electromigrational transport of particles in the bulk and the degree of stability of the dispersion itself, but also has been found to impact on the density and selectivity of particle nucleation on the substrate.

Despite all of the unique physical and chemical properties ordinarily associated with diamond as a material, colloidal particles of diamond have a remarkably rich surface chemistry. The surface chemistry on diamond is believed to derive from the presence of very reactive, unsaturated covalent bonds. In the presence of water or oxygen, these covalent "dangling" bonds react to form a multitude of carbon-oxygen surface groups (4). Infrared spectroscopy of diamond powders has revealed the presence of various oxygen-containing functional groups on the surface, including: carbonyl, carboxylic, lactone, tertiary alcohol, cross-linked oxygen, and epoxide. When diamond particles are immersed in a polar liquid, the surface acquires a charge due to the ionization of these groups, and also from selective ion adsorption, dissolution or ion exchange. The electrophoretic mobility is determined largely by the degree of particle charging and will in general depend on the type of particles, the dispersion medium, ions present in the solution, and on the pretreatment of the diamond powders.

Electrokinetic measurements provide one of the most powerful tools for probing the interfacial chemistry and stability of colloidal diamond dispersions. Electrokinetic properties such as the particle mobility, zeta potential, surface charge density, and the iso-electric point are essential in developing an optimized electrophoretic nucleation process. In this work, we conducted an investigation using the electrokinetic sonic amplitude (ESA) method to characterize and explore the interfacial chemistry of colloidal diamond particles.

In the ESA method, an alternating electric field (E) is applied to a dispersion containing a known concentration (volume fraction, $\Phi$ ) of colloidal diamond particles (5). In response to their surface electrical charge, the particles will move in an oscillatory manner causing the transfer of momentum to the fluid phase with concomitant generation of an acoustical pressure sound wave. The magnitude of the electrokinetic sonic amplitude defined as the 
magnitude of the sound wave $(\mathrm{P})$ per unit electric field is shown to be directly proportional to the dynamic electrophoretic mobility $\mu_{d}^{*}$ by the following relationship $(6,7)$,

$$
E S A(\omega) \equiv \frac{P}{E}=c \Delta \rho \Phi \mu_{d}^{*}(\omega)
$$

where ( $\omega$ is the angular frequency, $\mathrm{c}$ is the velocity of sound in the suspension, and $\Delta \rho$ is the density difference between the particles and the medium. For most systems of practical interest, the double layer is usually thin compared to the characteristic size of the colloidal particle. For these systems, we will adopt the zero limit formula for the DC electrophoretic mobility as given by the Smoluchowski equation and represent the dynamic mobility as,

$$
\mu_{d}^{*}=\frac{\varepsilon \zeta}{\eta} G\left(\omega \frac{a^{2}}{v}\right)
$$

where $\zeta$ is the zeta potential, $\eta$ is the Newtonian shear viscosity, and $\nu$ is the kinematic viscosity of the bulk suspension. For sub-micrometer colloidal particles of diamond having an intrinsic density of $3.51 \mathrm{~g} / \mathrm{cc}$ in an aqueous dispersion and at operating frequencies of $1 \mathrm{MHz}$, the inertial correction term is relatively unimportant, $\mathrm{G} \sim$ unity.

\section{EXPERIMENTAL}

Electroacoustic experiments were conducted with the MATEC-ESA MBS-8000 electronics system. Electrokinetic measurements were performed in a continuously stirred Teflon ${ }^{\circledR}$ vessel containing approximately $200 \mathrm{~cm}^{3}$ suspension. Concurrent with the electroacoustic response the $\mathrm{pH}$, temperature, and electrical conductivity of the solution were measured. Characterization of colloidal diamond dispersions was studied by electroacoustic measurements of the zeta potential under controlled temperature $\left(25^{\circ} \mathrm{C}\right)$ and over a broad $\mathrm{pH}$ range $(2 \leq \mathrm{pH} \leq 12)$. 
Colloidal dispersions were made using either synthetic or natural diamond particles (Industrial Diamond Corp. (IDC), PA or Diamond Abrasives Corp. (DAC), NY) in high purity, organic-free, $18 \mathrm{M} \Omega-\mathrm{cm}$ resistivity water. The volume fraction of particles in all of the experiments reported here was maintained constant at $\Phi=0.5 \%$. Colloidal dispersions were prepared just prior to electroacoustic measurements by combining the requisite quantities of diamond powders in high purity water and ultrasonicating for approximately fifteen minutes. The natural $\mathrm{pH}$ of the resultant solution was approximately 5.6. The $\mathrm{pH}$ was adjusted from this initial value to around 12.2 by the addition of small aliquots from a $1 \mathrm{M}$ solution of electronic grade potassium hydroxide. Zeta potential-pH titrations were carried out using a $1 \mathrm{M}$ solution of hydrochloric acid. No other additional salts or indifferent electrolyte was used in these experiments. Electroacoustic experiments were also conducted with colloidal dispersions of diamond in low sodium MOS-grade methanol.

\section{RESULTS AND DISCUSSION}

In Figure 1 are shown experimental data derived from the electroacoustic characterization of colloidal diamond particles. The contribution of the acoustophoretic signal from the background electrolyte in these experiments is shown in Figure 2 in terms of the zeta potential. Over the range of $\mathrm{pH}$ values studied there is essentially no contribution from the electrolyte medium. In aqueous media, the zeta potential of both synthetic and natural diamond particles were found to be dependent on the $\mathrm{pH}$ of the solution. Natural diamond particles were found to exhibit considerably more negative zeta potentials than their synthetic counterparts. For both natural and synthetic diamond, the zeta potential is relatively insensitive to $\mathrm{pH}$ in the range 4.0 to 12.0 . For $\mathrm{pH}$ values below $\mathrm{pH}=4$, the zeta potential of natural diamond decreases rapidly with $\mathrm{pH}$ as it approaches the iso-electric point. The iso-electric point for natural diamond (Type IDC\#3, i.e., Batch \#3 from IDC) was found to be at a $\mathrm{pH} \sim 2$ whereas an iso-electric point at $\mathrm{pH} \sim 3.4$ was measured for the synthetic diamond. 
The hydrophilic nature of colloidal diamond particles is a property attributed in large part to the population density of oxygen-containing functional groups on the surface. In these systems, the principal particle charging mechanism results from the ionization of acidic-hydrogen associated with certain functional groups on the surface of diamond. The zeta potential measurements shown in Figure 1 suggest that synthetic diamond is considerably more hydrophobic in nature than natural diamond. Produced under high pressure and temperature conditions, synthetic diamond is likely to contain a dearth of oxygen surface groups which are responsible for the apparent higher degree of particle charging observed for natural diamond particles. Moreover, a reduction in the number of "dangling" bonds and the possible formation of a graphite-type layer on the surface may also explain the hydrophobicity of synthetic diamond.

The development of a practical electrophoretic process for the nucleation and patterning of silicon substrates requires a dispersion with consistent electrokinetic properties. We examined several samples of natural colloidal diamond to ascertain the degree of variability in these dispersions. In Figure 3 are shown experimental data for the acoustophoretic characterization of various natural diamond dispersions. The curves labeled IDC\#1, IDC 2 , and IDC\#3 correspond to three different batches of $0-1 / 2 \mu \mathrm{m}$ natural diamond particles obtained from IDC. Diamond powder obtained from DAC was specified to be of similar grade (with respect to particle size and its distribution) as the natural diamond obtained from IDC. Despite the apparent similarity in grade specifications, IDC and DAC powders exhibit a clearly visible difference in hue. Moreover, the zeta potential data shown in Figure 3 also suggests that a significant variation in the degree of particle charging exists among the various batches of the same grade natural diamond. The degree of particle charging in batch IDC\#2 was found to be considerably less $(\Delta \zeta \sim-20 \mathrm{mV}$ at $\mathrm{pH}=8)$ than that measured for either IDC\#l and IDC\#3. However, all of the diamond powders analyzed from IDC showed similar characteristics in the shape of the overall zeta potential-pH curves. In contrast, dispersions containing DAC particles exhibit a pronounced kink in the zeta potential curve around a $\mathrm{pH}$ of 6-7 which is not observed with any of the IDC material. This distinct feature suggests that the charging of DAC colloidal particles may derive from a wholly 
different surface functional group; one that has an appreciably less acidic hydrogen associated with it. The type of surface group on diamond may have an important role in determining the level of adhesion of colloidal particles on the nucleated substrate.

The electrophoretic nucleation process relies on the development of sufficient charge on the particle to allow for effective bulk transport of the colloids. To this end, we investigated the feasibility of improving the electrophoretic mobility of natural diamond particles by increasing the number of surface active sites. Strong oxidizing agents such as perchloric acid or nitric acid are effective in promoting the oxidation of surfaces for many materials. In Figure 4 are plotted experimental data on the zeta potential showing the effect of oxidizing the surface of natural diamond (IDC\#2) using concentrated nitric acid. We found that while the shape of the overall zeta potential-pH curve remains approximately the same, a substantial increase $(\Delta \zeta \sim-25 \mathrm{mV})$ in the zeta potential is afforded by treating the surface of natural diamond with a strong oxidizing agent. Only a minor shift of the iso-electric point $(\Delta$ i.e.p. $\sim 0.4)$, to lower $\mathrm{pH}$ values, was measured for the treated surface. These results suggest that oxidizing the surface of natural diamond increases the density of surface sites rather than alter the specific chemical moieties on the surface.

Chemical modification of the surface of natural diamond was examined using a hydrogen microwave plasma discharge. Natural diamond powder (IDC\#2) was treated in a hydrogen microwave plasma reactor at the following conditions: temperature, $800^{\circ} \mathrm{C}$; pressure, 10 torr; microwave power, 600 watts; for 5 hours. The zeta potential-pH curve of the treated sample is compared to the untreated IDC\#2 in Figure 5. Plasma treatment of the diamond material significantly alters both the magnitude $(\Delta \zeta \sim 22 \mathrm{mV})$ and shape of the overall zeta potential-pH curve. The iso-electric point of the treated diamond $(\mathrm{pH}=7.0)$ is also shifted by about 5 decades from the $\mathrm{pH}$ value corresponding to the isoelectric point of the untreated diamond powder $(\mathrm{pH}=2.0)$. These results strongly suggest that hydrogen plasma activation of the diamond surface leads to considerable modification in the surface chemistry of diamond. The electroacoustic characterization shows that plasma-treated diamond affords a material with functional groups that have less acidic type of protons. Further investigation of the surface by spectroscopic techniques is necessary to fully investigate the various functional derivatizations effected by the plasma treatment. 
In non-aqueous systems, the zeta potential of colloidal particles is generally not as pronounced as in protic solvents unless chemical agents and/or surfactants are added deliberatively in order to promote charging of particles. In Figure 6 are shown electroacoustic data on the zeta potential of various natural diamond particles in methanol. As expected, the zeta potential of natural diamond in methanol was found to be considerably diminished (e.g. $\zeta \sim-2$ to $-5 \mathrm{mV}$ ).

Electrophoretic deposition was conducted from dispersions containing approximately $0.2 \mathrm{~g} / \mathrm{l}$ of IDC natural diamond in high purity water and at the natural $\mathrm{pH}$ of the solution $(\mathrm{pH}=5.6)$. Typically, the deposition substrate consisted of a 4-inch p-type silicon wafer (10 ohm-cm conductivity) biased to positive potentials. A gold-plated metal disk of equivalent diameter served as the negative counter-electrode. Electric fields of $5-40 \mathrm{~V} / \mathrm{cm}$ and deposition times of 1-10 minutes were employed to provide effective nucleation of the substrate. A scanning electron micrograph (SEM) of diamond particles electrophoretically deposited onto a silicon substrate is shown in Figure 7. Diamond particles were found to be well-adherent to the silicon surface. The nucleation density afforded by this electrophoretic process $\left(10^{8}-10^{9}\right.$ nuclei $\left./ \mathrm{cm}^{2}\right)$ is many orders of magnitude higher than that can be achieved by any other substrate nucleation method.

Using conventional photolithographic techniques, the electrophoretic process can be applied to selected-area nucleation and patterning for the growth of CVD diamond films. As shown in Figure 8, a silicon wafer is first patterned with oxide $\left(\mathrm{SiO}_{2}\right)$. When immersed in an aqueous solution at the appropriate $\mathrm{pH}$, regions masked by the oxide develop an inherent negative surface charge that effectively precludes the deposition of diamond particles with a negative zeta potential. Deposition of diamond on silicon biased at a positive potential remains largely uninterrupted. Colloidal diamond particles with high zeta potentials are desirable for maximizing the coulombic repulsion forces in order to effect the selective deposition of diamond. 


\section{CONCLUSIONS}

The electrokinetic properties of sub-micron diamond particles are of great practical importance in determining the stability and chemical behavior of colloidal dispersions. We have used the electrokinetic sonic amplitude (ESA) technique to investigate the interfacial chemistry and particle charging mechanisms of colloidal diamond dispersions in aqueous and non-aqueous solutions. Such information is of paramount importance to the development of an optimal process for the nucleation and patterning of diamond thin films. 


\section{REFERENCES}

[1] Valdes, J. L., Mitchell, J. W., Mucha, J. A., Seibles, L., and Huggins, H., J. Electrochem. Soc., 138, 635 (1991).

[2] Valdes, J. L., Mitchell, J. W., Mucha, J. A., Seibles, L., and Huggins, H., Applications of Diamond Films and Related Materials, 73, 423 (1991).

[3] Valdes, J. L., Mitchell, I. W., US Patent \# 5,128,006, "Deposition of Diamond Films on Semiconductor Substrates."

[4] Shergold, H. L., and Hartley, C. J., Int. J. Mineral. Processing, 9, 219 (1982).

[5] O'Brien, R. W., Midmore, B. R., Lambs, A., Hunter, R. J., Faraday Discuss. Chem. Soc., 90, 301-312 (1990).

[6] O'Brien, R. W., J. Fluid Mech., 190, 71 (1988).

[7] O'Brien, R. W., J. Fluid Mech., 212, 81 (1990). 


\section{FIGURE CAPTIONS}

Figure 1. Acoustophoretic characterization of natural and synthetic colloidal diamond. Experiments were conducted with $0-1 / 2 \mu \mathrm{m}$-grade diamond powder at a volume fraction of $\Phi=0.5 \%$ and at room temperature. Isoelectric point for natural diamond was found to be at $\mathrm{pH} \sim 2$ and for synthetic diamond at $\mathrm{pH}$ $\sim 3.4$.

Figure 2. Acoustophoretic signal from the electrolyte medium.

Figure 3. Dependence of zeta potential on $\mathrm{pH}$ for several samples of sub-micron natural diamond powders.

Figure 4. The effect of surface oxidation by treatment with concentrated nitric acid on the zeta potential of natural diamond.

Figure 5. Chemical modification of the surface of natural diamond powder using a hydrogen microwave plasma.

Figure 6. The zeta potential of various colloidal dispersions of natural diamond in methanol.

Figure 7. Scanning electron micrograph (SEM) of natural diamond particles deposited electrophoretically onto a silicon $\langle 100\rangle$ wafer. Deposition was effected from a dispersion containing $0.2 \mathrm{~g} / \mathrm{L}$ of natural diamond (IDC\#2) at the natural $\mathrm{pH}$ of the solution $(\mathrm{pH}=5.6)$.

Figure 8. Electrophoretic nucleation process for selected-area nucleation and patterning of diamond thin films. 


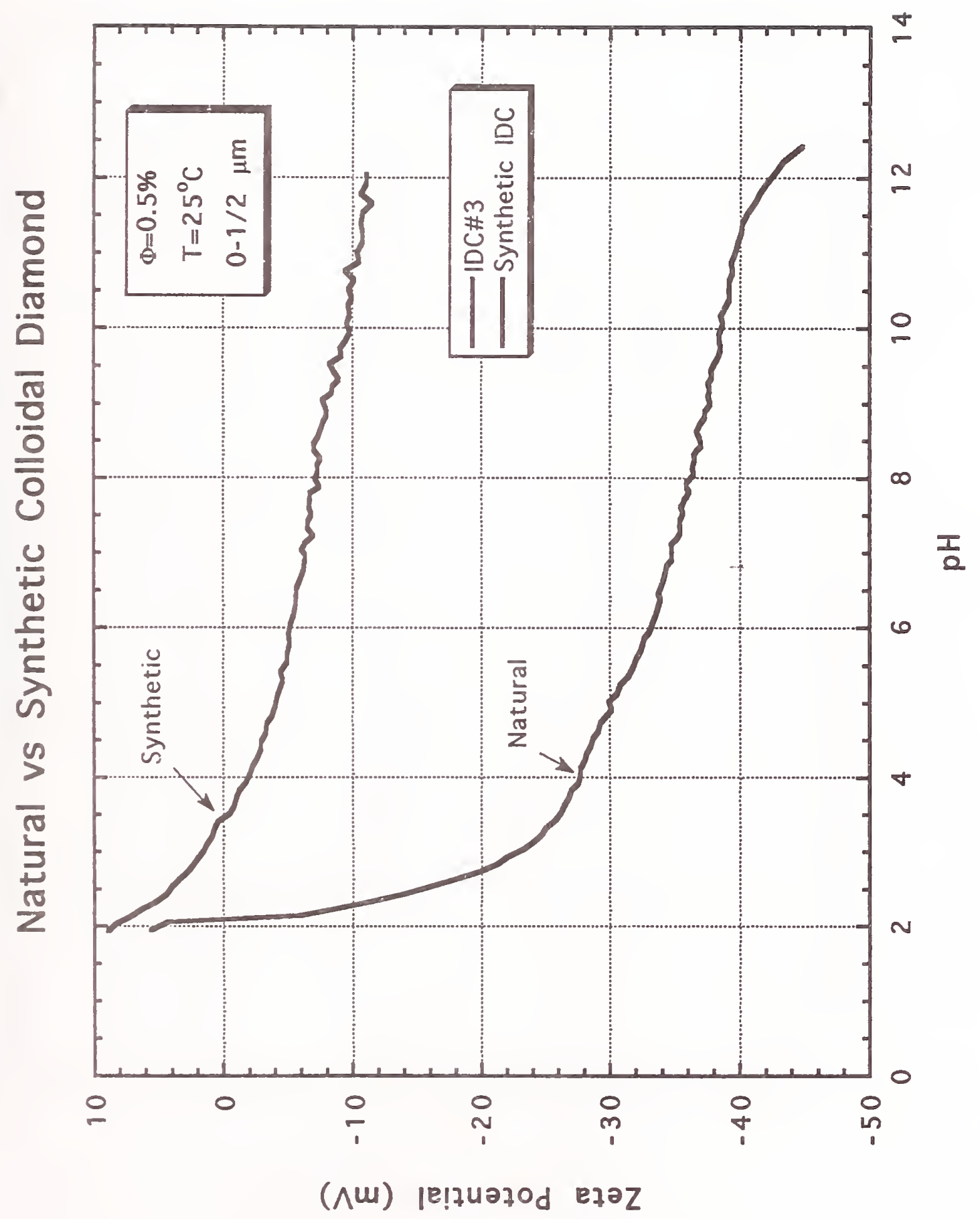




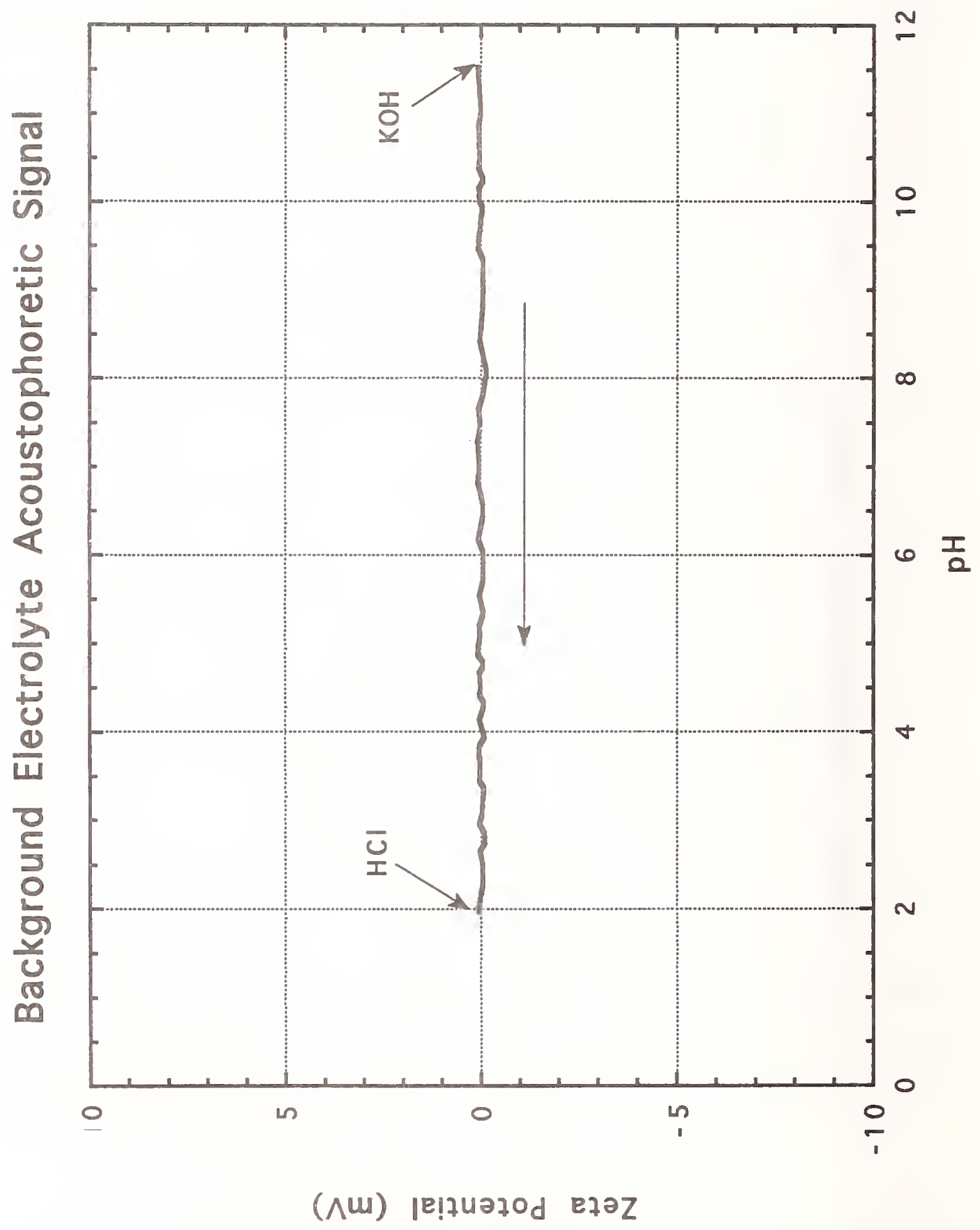




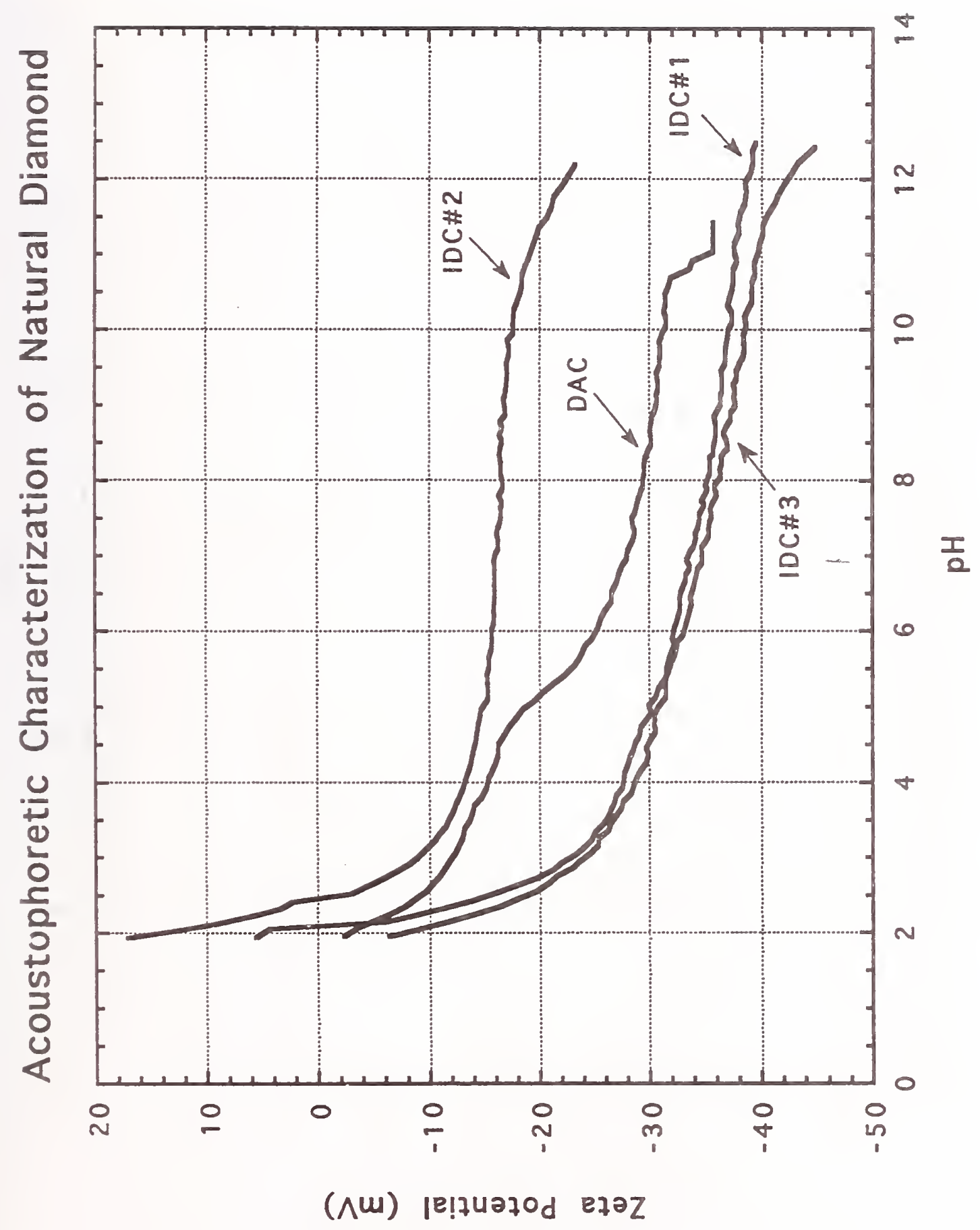




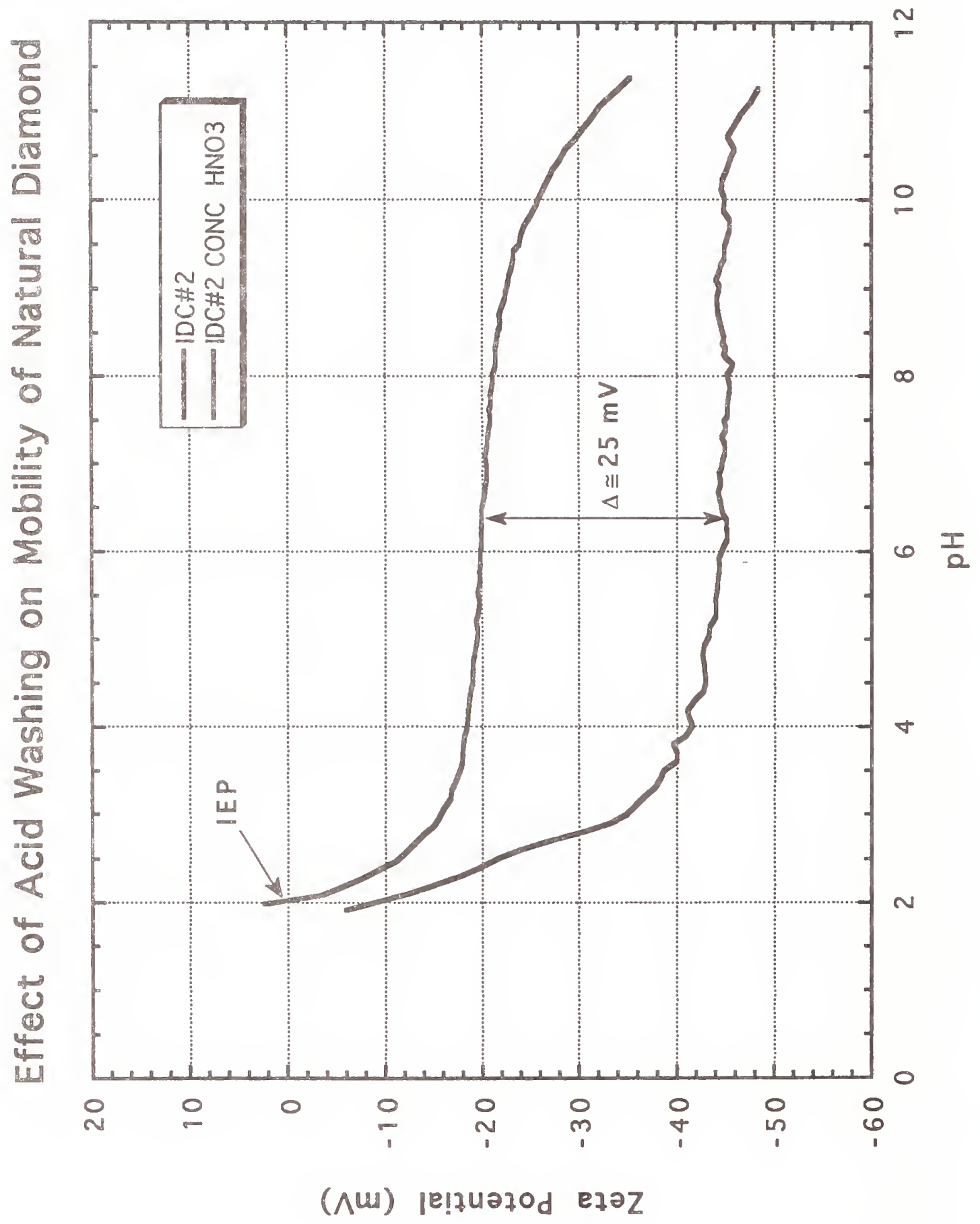




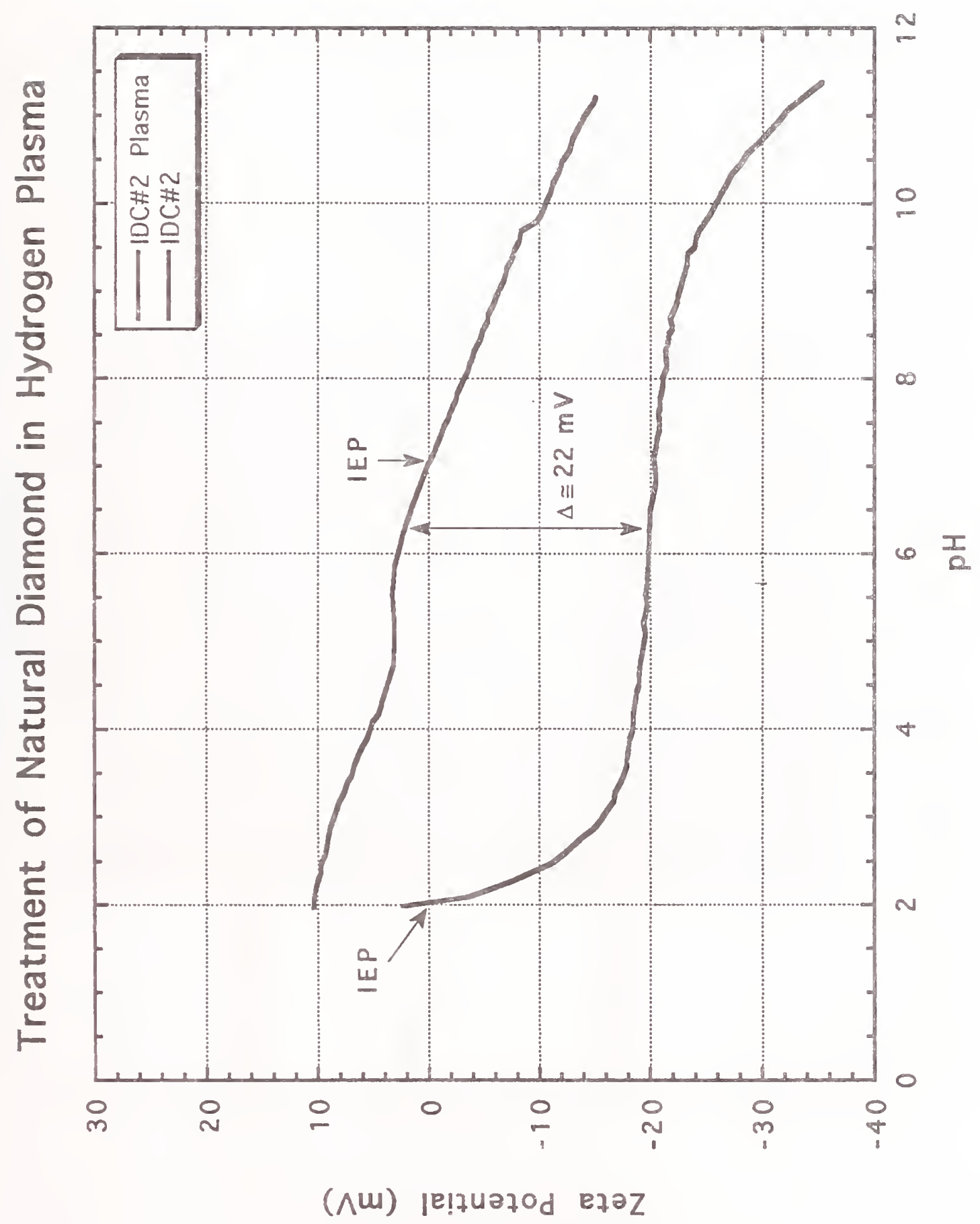




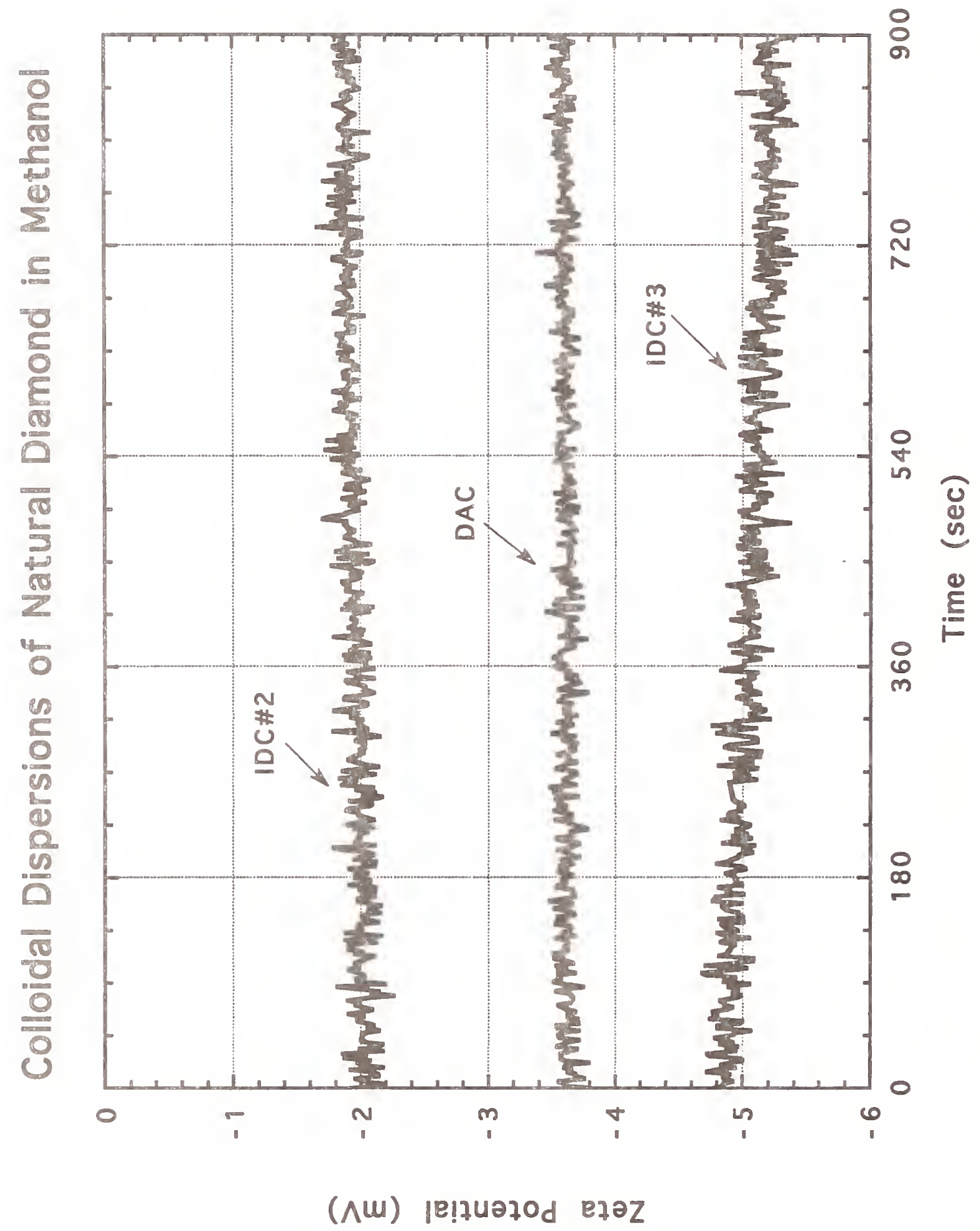




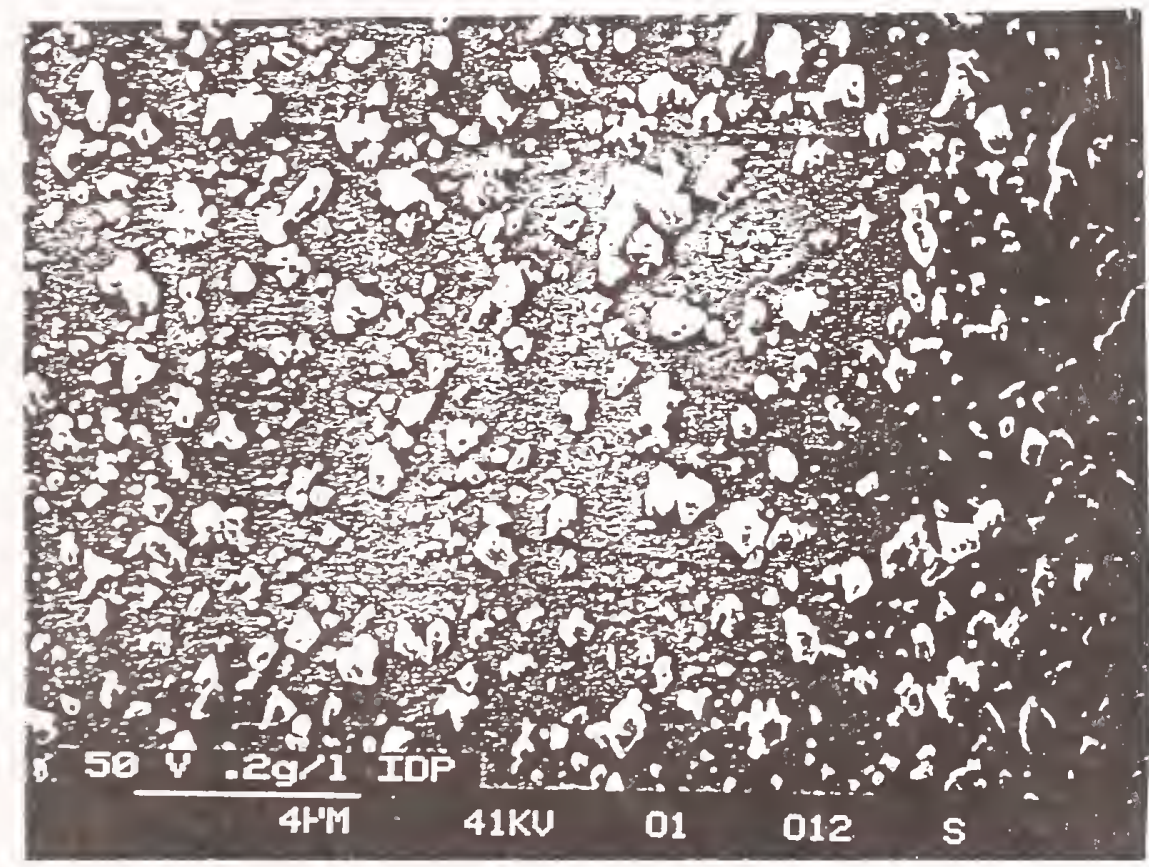

FIGURE 7 


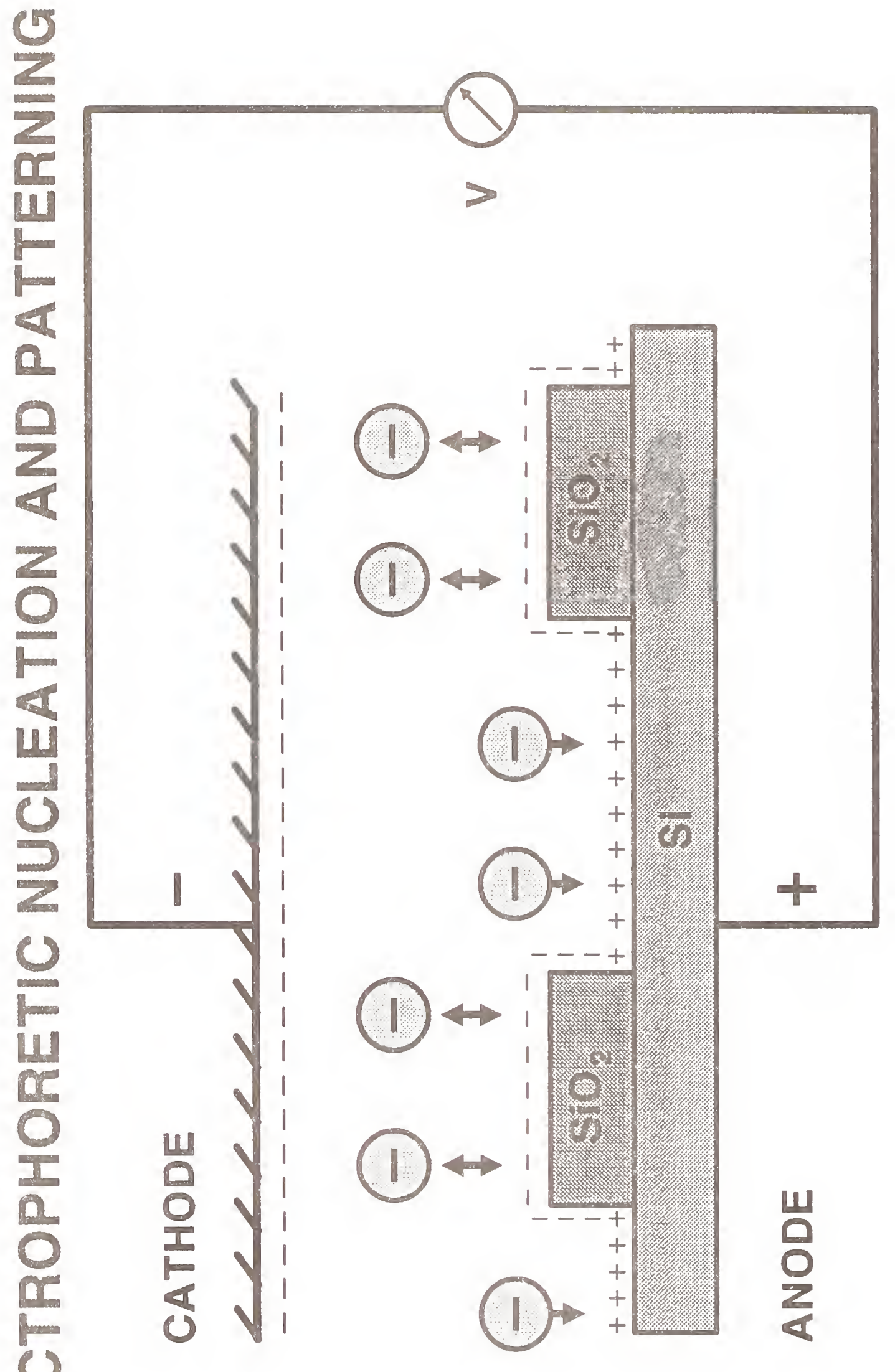

III

네

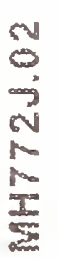




\section{SESSION III}

\section{ADVANCED CERAMICS APPLICATION}

Session Chairman

R. Mountain

National Institute of Standards and Technology 



\title{
ESA MEASUREMENTS FOR SILICA AND ALUMINA: BACKGROUND
} ELECTROLYTE CORRECTIONS

\author{
F. N. Desai' ${ }^{2}$ H. R. Hammad ${ }^{1}$, and K. F. Hayes ${ }^{* 1}$ \\ ${ }^{1}$ Department of Civil and Environmental Engineering \\ The University of Michigan \\ Ann Arbor, Michigan 48109-2125 \\ ${ }^{2}$ The Procter \& Gamble Company \\ Winton Hill Technical Center \\ 6300 Center Hill Road \\ Cincinnati, $\mathrm{OH} 45224$
}

•To whom all correspondence should be addressed

ABSTRACT

The ESA technique has been used to determine the electrophoretic mobility and zeta potential of colloidal systems. For a solid suspension in an electrolyte solution, the measured ESA signal is a vector sum of two signals, one for the solid and the other for the background electrolyte. Under certain conditions, for example when the ionic strength is high, the background contribution can become a significant fraction of the measured ESA signal and background subtraction has to be done. In this paper, the background correction technique has been applied to silica and alumina over a range of $\mathrm{pH}$ and ionic strengths.

\section{INTRODUCTION}

The Electrokinetic Sonic Amplitude (ESA) technique is an electroacoustic method that has recently been used to determine the electrophoretic mobility and zeta potential of colloidal system $\mathrm{s}^{1-7}$. The ESA technique has a wider range of applicability than conventional microelectrophoresis; for example, ESA measurements can be made on transparent or opaque particles that could be as large as $150 \mu \mathrm{m}$ with solid concentration as high as $50 \%$ by volume. However, for converting ESA to zeta potential, the upper limit of particle concentration is $5 \%$ by volume $e^{3 / 4}$. 
One limitation of the ESA technique is that a significant error is introduced in the measured ESA value when the ionic strength is high. In the presence of a background electrolyte, the measured ESA signal is a vector sum of two signals: one for the solid and the other for the background electrolyte. The background electrolyte signal can become important when i) the ionic strength is high, ii) the pH is close to the isoelectric point (iep), iii) the density difference between the solid and the liquid is small, iv) the solid concentration is relatively low, or v) the solid has a low charge density. For example, background corrections will be important for polymer solids for which the density difference between the solid and the liquid is small and hence the measured ESA is relatively low. Under these circumstances, the background electrolyte signal has to be subtracted from the measured ESA signal to give the true ESA.

Some researchers ${ }^{3,4}$ have avoided the problem of background correction by using a high enough solid concentration such that the background contribution was less than $5 \%$ of the measured ESA signal. This is not possible in all cases since the solid concentration is limited to $5 \%$ by volume if the ESA data has to be converted to zeta potential. Furthermore, if the solid is expensive or difficult to purify, it is advantageous to use as little solid as possible. Based on the background correction procedure for the colloid vibration potential (CVP) outlined by Mariow et al. ${ }^{2}$, Desai et al. ${ }^{8}$ have demonstrated how the background correction technique can be applied to ESA measurements on quartz. The objective of this paper is to apply this technique to silica and alumina suspensions over a wide range of $\mathrm{pH}$ and ionic strengths.

\section{BACKGROUND ELECTROLYTE CORRECTIONS}

The magnitude and the phase angle of the ESA signal can be measured with the Matec ESA-8000 (Matec Applied Sciences, Hopkinton, MA). The phase angle is measured with respect to a phase reference. The phase reference material is generally taken as the solid in water in the absence of any background electrolyte, assuming that the polarity of this solid is known a priori. 
Figure 1 gives the magnitude and the phase angles for the various ESA signals. The measured ESA $\left(\overline{E S A}_{\text {mens }}\right)$ is a vector sum of the true ESA for the solid $\left(\overline{E S A}_{\text {true }}\right)$ and the ESA for the background electrolyte $\left(\overline{E S A}_{\operatorname{bkg} \mathrm{d}}\right)$.

$\overline{E S A}_{\text {meas }}=\overline{E S A}_{\text {ure }}+\overline{E S A}_{\text {bkgd }}$

Equation (1) can be resolved into its two components:

$\mathrm{ESA}_{\text {meas }} \cos \theta=\mathrm{ESA}_{\text {true }} \cos \beta+\mathrm{ESA}_{\text {bkgd }} \cos \phi$

$\mathrm{ESA}_{\text {meas }} \sin \theta=$ ESAtrue $\sin \beta+\mathrm{ESA}_{\text {bkg }} \sin \phi$

Here $\theta, \phi$, and $\beta$ are the phase angles of the measured, background, and true ESA signals respectively. It should be noted that the absolute ESA values are used in equations (2) and (3) above. The actual sign of $\mathrm{ESA}_{\text {true }}$ can be determined from the phase angle, $\beta$. If $\beta<90^{\circ}$, the sign of $\mathrm{ESA}_{\text {true }}$ is the same as that of the phase reference; whereas, if $\beta>90^{\circ}$, the sign of $\mathrm{ESA}_{\text {true }}$ is opposite to that of the phase reference. If $\mathrm{ESA}_{\text {meas }}, \mathrm{ESA}_{\mathrm{bkgd}}, \theta$, and $\phi$ are measured experimentally, equations (2) and (3) can be solved simultaneously to give $\mathrm{ESA}_{\text {true }}$ and $\beta$.

\section{EXPERIMENTAL}

\section{Materials}

The silica used in this study was purchased from Geltech Corp. (Alachua, FL). The particles were spherical and monodispersed with particle density of $2 \mathrm{~g} / \mathrm{cm}^{3}$. The median particle diameter was about $1.6 \mu \mathrm{m}$ as measured with the Capa-500 Centrifugal Particle Size Distribution Analyzer (Horiba Instruments, Irvine, CA). This value is close to the diameter of $1.5 \mu \mathrm{m}$ specified by Geltech Corp. The Geltech silica had a purity of $99.9 \%$ and was used without any purification. 
The alpha alumina was purchased from Buehler (Lake Bluff, IL). The median particle diameter, measured with the Capa-500, was about $4 \mu \mathrm{m}$, which is larger than the $1 \mu \mathrm{m}$ size specified by the manufacturer. This was the case even when the sample was sonicated anywhere between 2 and 6 hours. The particle density was $3.97 \mathrm{~g} / \mathrm{cm}^{3}$. This alumina was used without any purification.

\section{Method}

Potentiometric titrations were performed on the ESA-8000 for silica and alumina at three ionic strengths. The concentration of solids was $5 \%$ by volume. The solids were hydrated for 24 hours, followed by sonication for about 2 hours. The ESA probe was calibrated with $10 \%$ by volume Ludox TM (Du Pont, Wilmington, DE). The ESA value for the Ludox should be $-5.32 \mathrm{mPa} \mathrm{m} / \mathrm{V}$ at $25^{\circ} \mathrm{C}$ or the zeta potential should be $-38 \mathrm{mV}$ as specified by Matec Applied Sciences. In all experiments, the temperature was kept constant at $25.0 \pm 0.2^{\circ} \mathrm{C}$ and a nitrogen blanket was maintained above the solution in the titration cell to prevent carbon dioxide contamination.

The following experimental procedure was followed for each solid at a particular ionic strength. After calibrating the ESA probe with Ludox, the solid in pure water was poured into the titration cell and used as the phase reference material. Next, the ionic strength was adjusted with the appropriate amount of $\mathrm{NaCl}$. Potentiometric titrations were performed by measuring the magnitude and phase angle of the ESA signal, temperature, electrical conductivity, and amount of acid or base added as a function of $\mathrm{pH}$. For silica, the $\mathrm{pH}$ was raised to 8 and the potentiometric titration conducted from $\mathrm{pH} 8$ to 2 . For alumina, the $\mathrm{pH}$ was raised to 10 and the titration was carried out from $\mathrm{pH} 10$ to 6 .

Blank titrations were also performed in the absence of the solid to give the background electrolyte signal as a function of $\mathrm{pH}$ and ionic strength. These titrations were performed using the Geltech and alpha alumina phase references. Keeping the phase reference the same, in effect, keeps the frequency of the ac signal constant. The precision of the ESA measurements was $\pm 0.01 \mathrm{mPa} \mathrm{m} / \mathrm{V}$ and that of the phase angle measurements was $\pm 5^{\circ}$. 


\section{RESULTS AND DISCUSSION}

\section{Geltech Silica}

Even though the ESA signal for the background electrolyte was measured as a function of $\mathrm{pH}$ and ionic strength, only the mean values of $\mathrm{ESA}_{\mathrm{bkgd}}$ and $\phi$ were used for each ionic strength since the ESA signal for the background electrolyte did not depend significantly on $\mathrm{pH}$ in the $\mathrm{pH}$ range 2 to 10 . Table 1 gives the background signal (ESA $\mathrm{bkgd}_{\mathrm{b}}$ and $\left.\phi\right)$ as a function of ionic strength with Geltech silica as the phase reference.

Figure 2 shows the measured ESA and the phase angle for the Geltech silica as a function of $\mathrm{pH}$ and ionic strength. For 0.001 and $0.01 \mathrm{M}$ ionic strengths, the measured phase angle, $\theta$, is close to zero indicating that the ESA does not change sign in the $\mathrm{pH}$ range of 8 to 2 . However, for $0.1 \mathrm{M}$ ionic strength, the uncorrected ESA becomes positive at a $\mathrm{pH}$ of about 4.7. This change in sign of the ESA signal is also reflected in the measured phase angle, $\phi$, which goes from about 0 to $180^{\circ}$ at a $\mathrm{pH}$ of about 4.7 .

The data for $0.1 \mathrm{M}$ ionic strength suggests that the iep is at a $\mathrm{pH}$ of 4.7. However, the background correction shows that for $0.1 \mathrm{M}$ ionic strength, the background corrected ESA value is negative at least down to $\mathrm{pH} 2$ (Figure 3) as expected ${ }^{9,10}$. The background correction has little effect at low ionic strength (at least less than $0.01 \mathrm{M}$ ), but a substantial effect at $0.1 \mathrm{M}$ ionic strength. As the ionic strength increases, two factors tend to increase the relative importance of the background signal: $\mathrm{ESA}_{\mathrm{bkgd}}$ increases and $\mathrm{ESA}_{\text {true }}$ decreases.

\section{Alpha alumina}

Table 2 gives the background electrolyte signal as a function of ionic strength with alpha alumina as the phase reference. Comparison of Tables 1 and 2 shows that ESA $A_{\text {bkgd }}$ is approximately independent of the phase reference material within experimental error, whereas $\phi$ is a strong function of the phase reference. This underscores the need for measuring the background electrolyte signal whenever a new phase reference material is used.

Figure 4 shows the measured ESA and phase angle as a function of $\mathrm{pH}$ and ionic strength. The common intersection point (cip) occurs at an ESA value greater than zero with an iep of about 8.7 suggesting that there may be contamination in the sample. However, the 
electrolyte background correction shifts the cip to ESA value of zero (Figure 5) indicating that there is no impurity, with the iep at $\mathrm{pH} 9$, the same as the value that has been previously reported for alpha alumina ${ }^{11}$. As for silica, the background electrolyte has a significant effect at $0.1 \mathrm{M}$ ionic strength and a negligible effect at the lower ionic strengths.

Several factors, including type and concentration of solid, electrolyte concentration, and $\mathrm{pH}$, determine the ionic strength at which background corrections become important. As shown by Hackley and Malghan ${ }^{12}$ in a companion paper, the background electrolyte signal also depends on the ions that make up the electrolyte; for example, the ESA signal for $\mathrm{Ba}\left(\mathrm{NO}_{3}\right)_{2}$ is about twice that for $\mathrm{NaCl}$. The relative importance of each of the factors must be considered to insure that interpretation of the ESA signal is made correctly.

\section{CONCLUSIONS}

The background electrolyte correction method extends the range of experimental conditions (like $\mathrm{pH}$, ionic strength, and solid concentration) over which ESA data can be converted to electrophoretic mobility and zeta potential. The background electrolyte signal is only a weak function of $\mathrm{pH}$ in the $\mathrm{pH}$ range 2 to 10 , but a strong function of ionic strength. The magnitude of the ESA signal for the background electrolyte, $\mathrm{ESA}_{\mathrm{bkgd}}$, is independent of the phase reference material, whereas the phase angle, $\phi$, is a strong function of the phase reference.

For both silica and alumina, the background electrolyte has a negligible effect below $0.01 \mathrm{M}$ ionic strength, but a significant effect at $0.1 \mathrm{M}$ ionic strength. For silica, at $0.1 \mathrm{M}$ ionic strength, the measured ESA changes sign around a $\mathrm{pH}$ of 4.7 suggesting that silica is positively charged below that $\mathrm{pH}$. However, background correction rightly shows that the true ESA does not change sign at least down to a pH of 2. For alumina, ESA measurements without background correction suggest possible contamination in the sample. However, after the background correction is made, the iep and cip are coincident indicating no contamination. 


\section{ACKNOWLEDGMENT}

We gratefully acknowledge the support of Dr. Frank J. Wobber, Program Manager, Subsurface Science Program, Office of Health and Environmental Research, U.S.

Department of Energy (Grant DOE-FG02-89-ER60820) for funding this research.

\section{NOMENCLATURE}

CVP colloid vibration potential

ESA $_{\text {bkgd }} \quad$ magnitude of the measured ESA signal for the background electrolyte

ESA $_{\text {meas }} \quad$ magnitude of the measured ESA signal for the solid

ESA $_{\text {true }} \quad$ magnitude of the background-corrected ESA signal for the solid

Greek symbols

$\beta$

$\phi$

$\theta$ phase angle of the background-corrected (true) ESA signal for the solid phase angle of the measured ESA signal for the background electrolyte phase angle of the measured ESA signal for the solid

\section{BIBLIOGRAPHY}

(1) O'Brien, R. W., J. Fluid Mech., 1988, 190, 71.

(2) Marlow, B. J.; Fairhurst, D.; Pendse, H. P., Langmuir 1988, 4, 611.

(3) James, R. O.; Texter, J.; and Scales, P. J., Langmuir 1991, 7, 1993.

(4) Scales, P. J.; Jones, E., Langmuir 1992, 8, 385.

(5) James, M.; Hunter, R. J.; O’Brien, R. W., Langmuir 1992, 8, 420.

(6) Babchin, A. J.; Chow, R. S.; Sawatzky, R. P., Adv. Colloid Interface Sci. 1989, 30,111 .

(7) Sawatzky, R. P.; Babchin, A. J., J. Fluid Mech., 1992.

(8) Desai, F. N.; Hammad, H. R.; Hayes, K. F., submitted to Langmuir April 1993. 
(9) Allen, L. H.; Matijevic, E., J. Colloid Interface Sci. 1969, 31(3), 287.

(10) Li, H. C.; de Bruijn, P. L., Suff Sci. 1966, 5, 203.

(11) Hunter, R. J., Zeta Potential in Colloidal Science. Principles and Applications, Academic Press, London, 1981, 229.

(12) Hackley, V.; Malghan, S. Workshop on Electroacoustics for Characterization of Particulates, 1993, NIST, Gaithersburg, MD. 
Table 1: Background Electrolyte Signals with Geltech Silica as Phase Reference

\begin{tabular}{|c|c|c|}
\hline$[\mathrm{NaCl}](\mathrm{M})$ & $\mathrm{ESA}_{\text {bkgd }}(\mathrm{mPa} \mathrm{M} / \mathrm{V})$ & Phase angle, $\phi,(\mathrm{deg})$. \\
\hline 0.001 & 0.026 & 165 \\
\hline 0.01 & 0.050 & 150 \\
\hline 0.1 & 0.125 & 175 \\
\hline
\end{tabular}

Table 2: Background Electrolyte Signals with Alpha Alumina as Phase Reference

\begin{tabular}{|c|c|c|}
\hline$[\mathrm{NaCl}](\mathrm{M})$ & $\mathrm{ESA}_{\mathrm{bkgd}}(\mathrm{mPa} \mathrm{M} / \mathrm{V})$ & Phase angle, $\phi,(\mathrm{deg})$. \\
\hline 0.001 & 0.026 & -8 \\
\hline 0.01 & 0.045 & -20 \\
\hline 0.1 & 0.130 & 0 \\
\hline
\end{tabular}


Figure 1: Magnitude and phase angle for the ESA signals

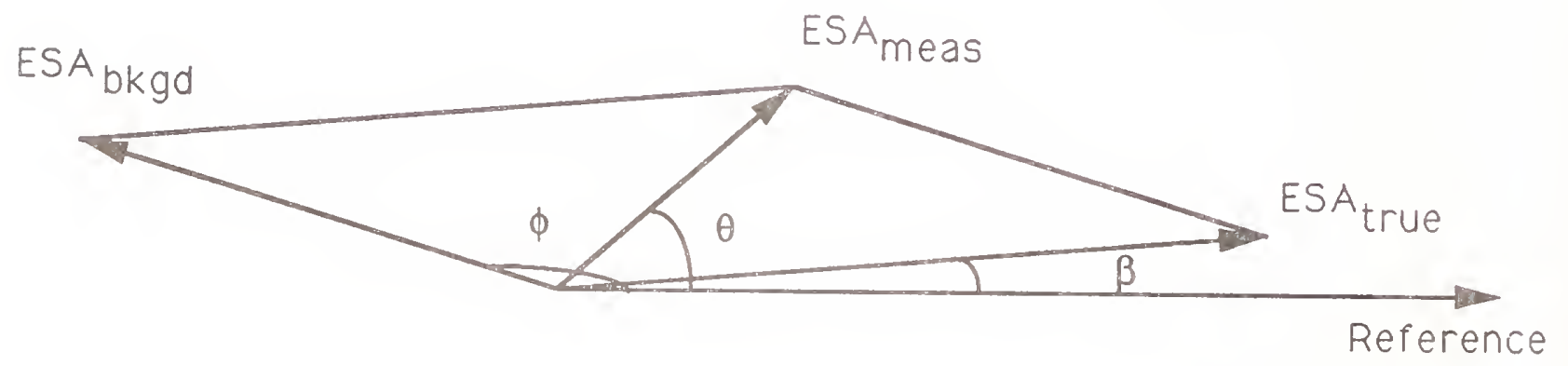


Figure 2: Measured ESA and phase angle for Geltech silica
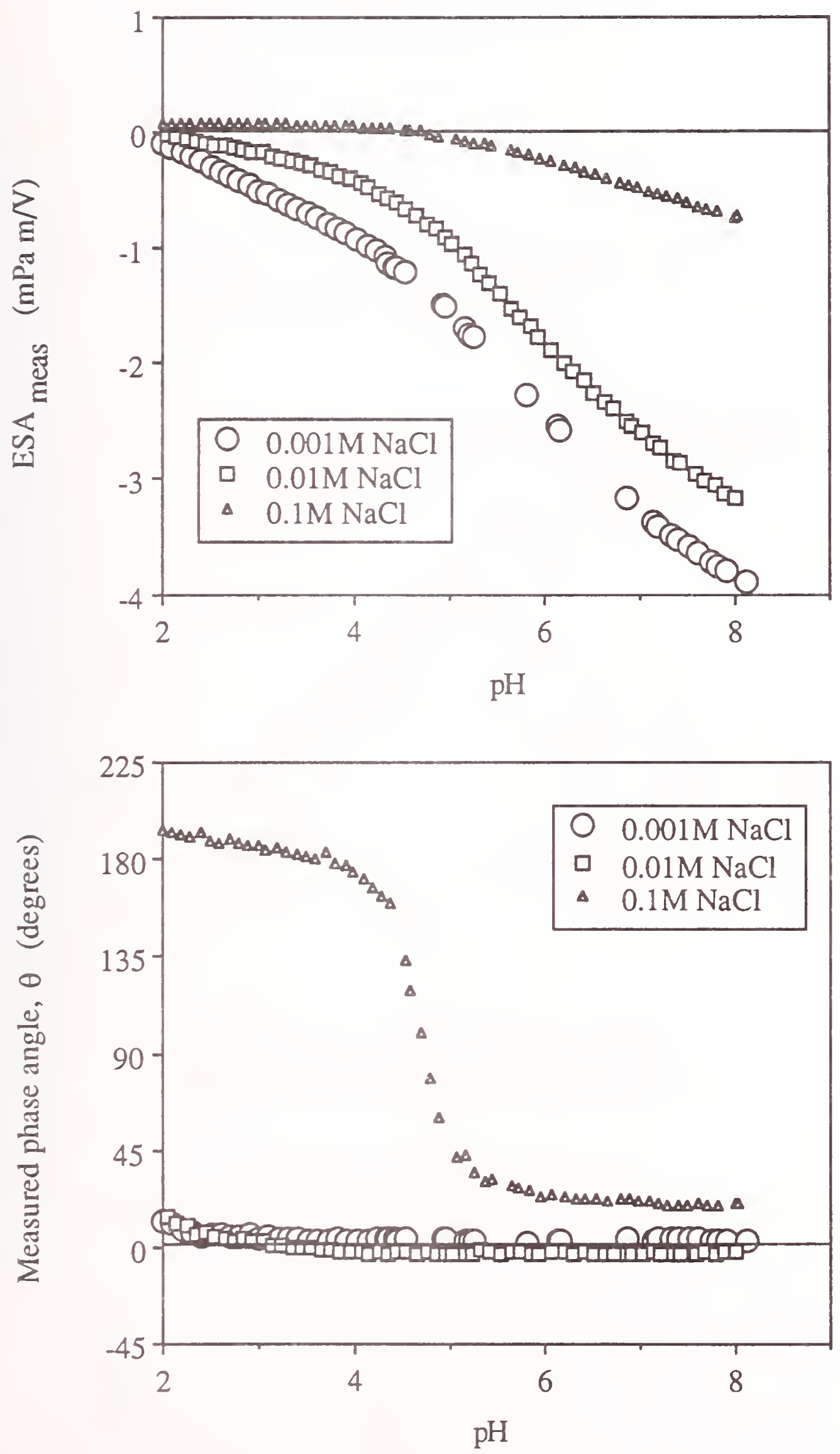
Figure 3: Measured and background-corrected ESA for Geltech silica

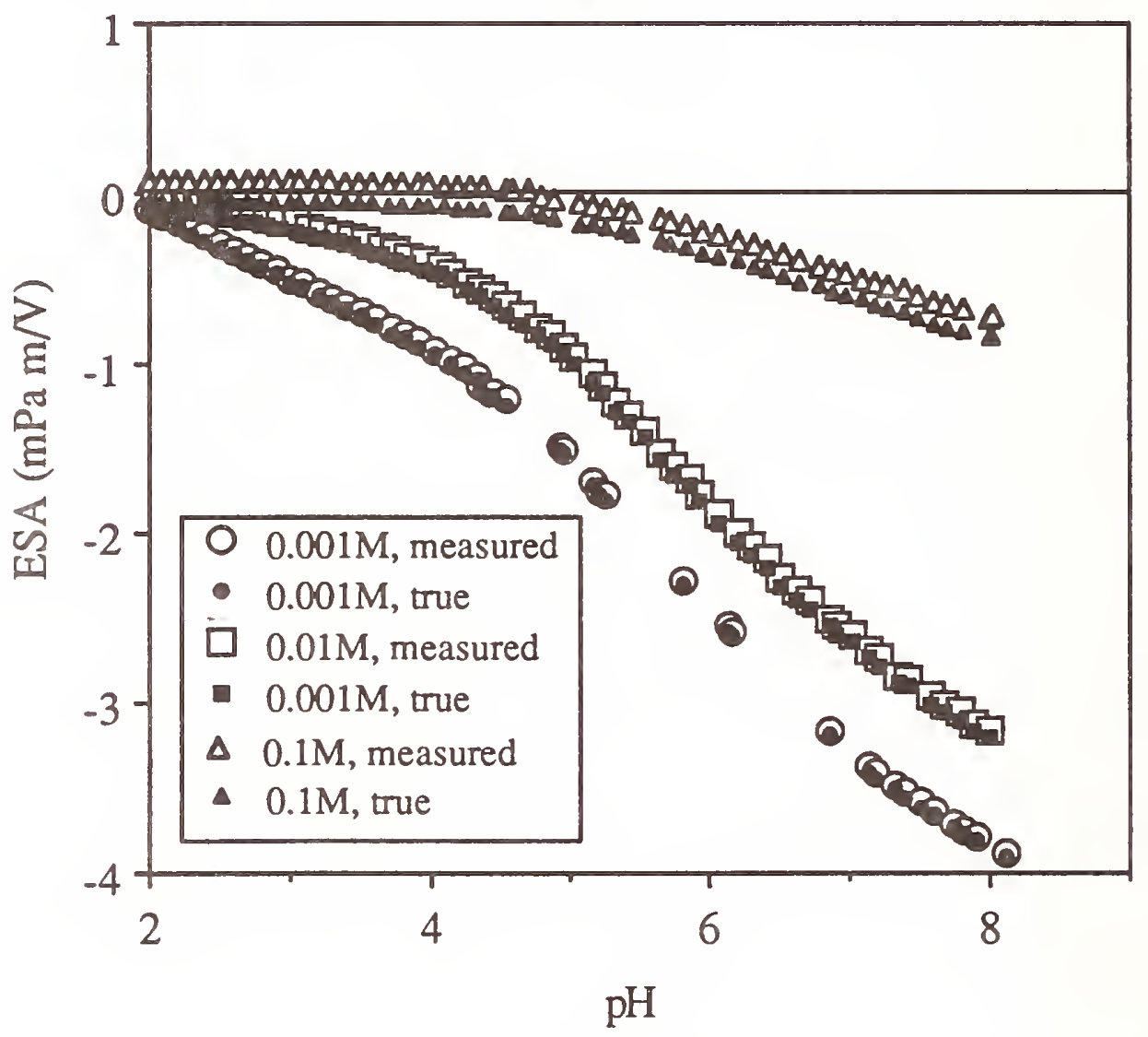


Figure 4: Measured ESA and phase angle for alpha alumina
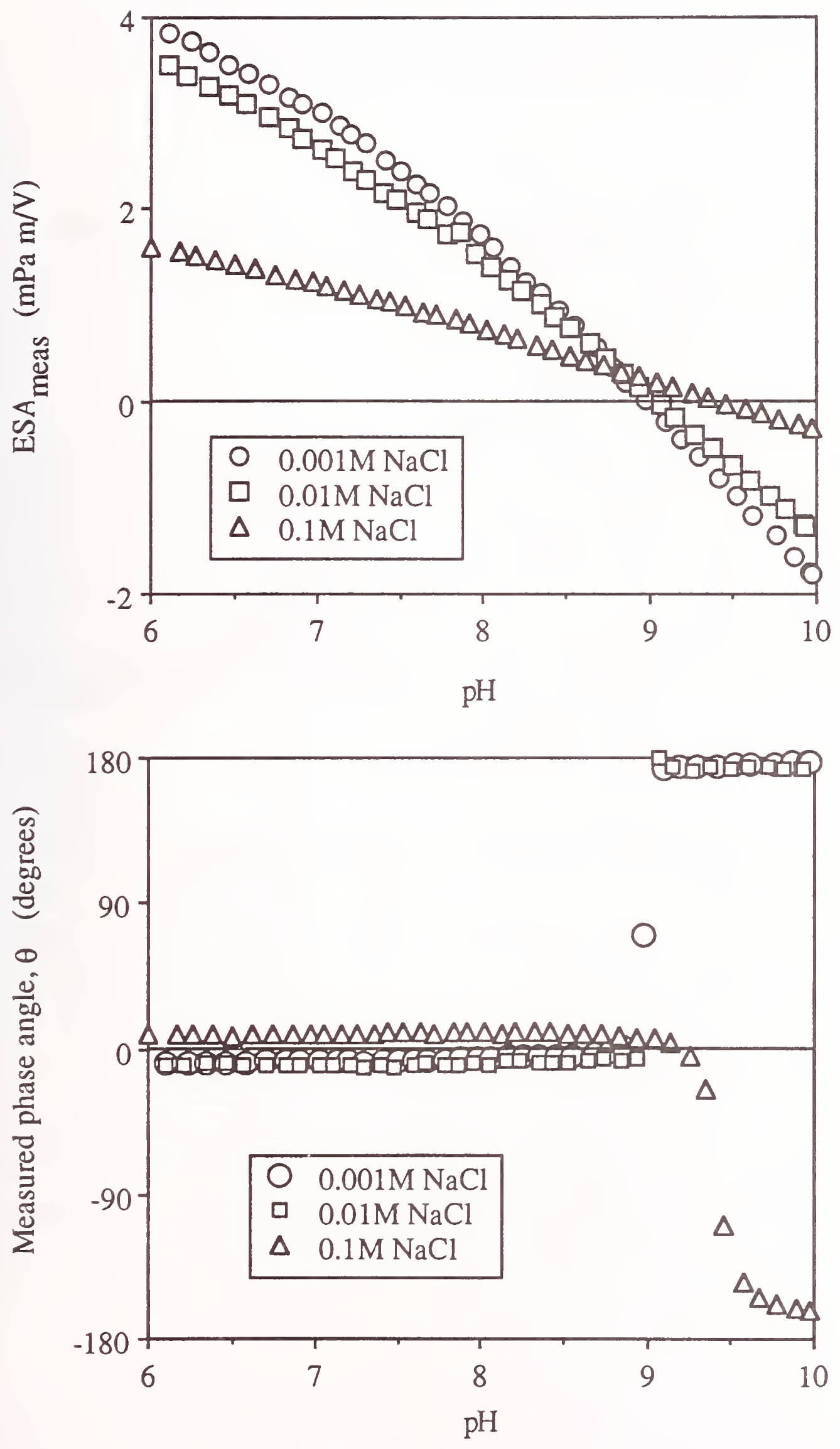
Figure 5: Background-corrected ESA for alpha alumina

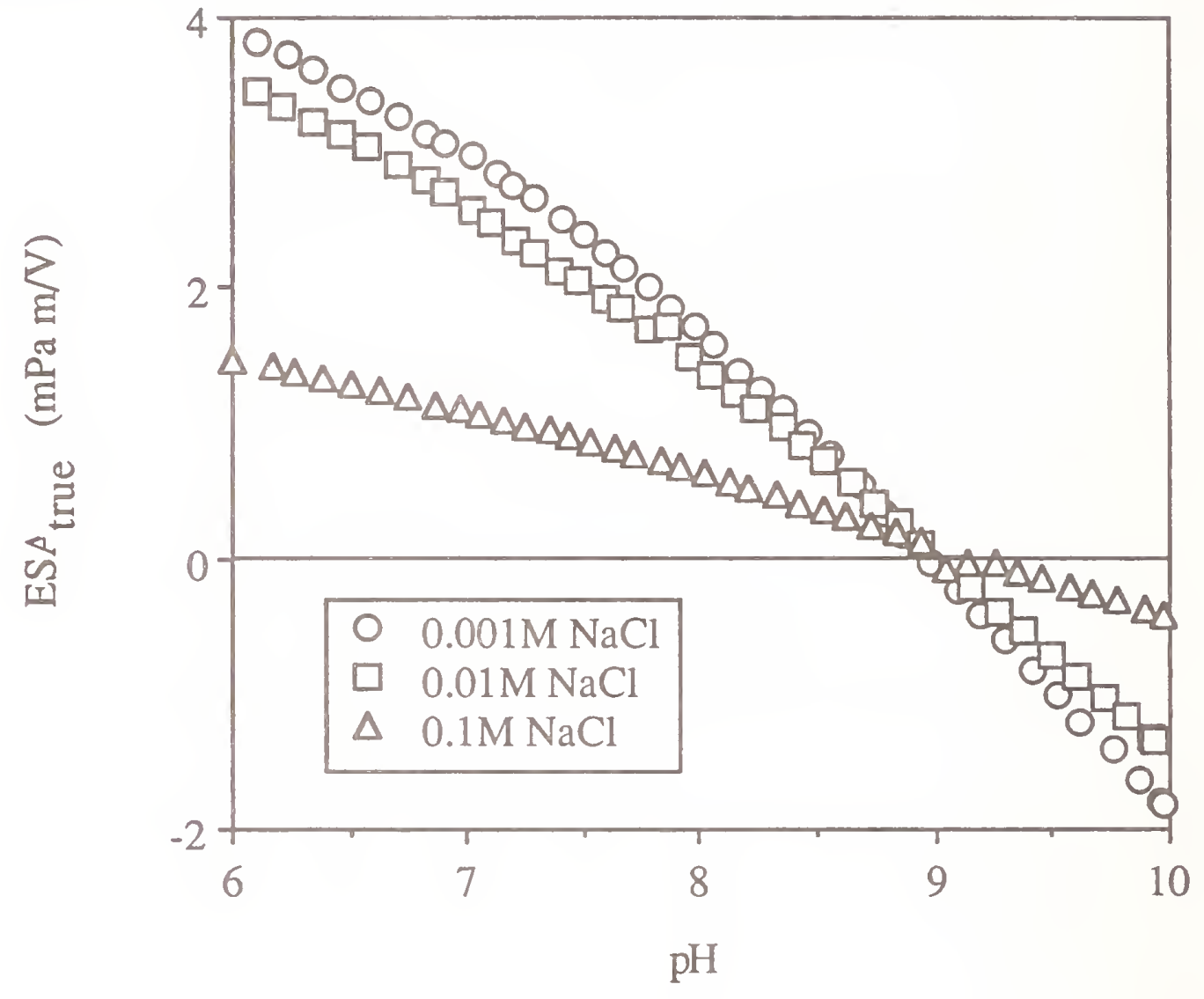




\title{
OXIDE AND NON-OXIDE POWDER PROCESSING APPLICATIONS USING ELECTROACOUSTIC CHARACTERIZATION
}

\author{
J. P. Pollinger \\ AlliedSignal Ceramic Components \\ Torrance, California
}

\begin{abstract}
Aqueous suspensions of ceramic powders are used in a variety of powder compact forming techniques for structural ceramics. The development and implementation of practical electroacoustic techniques for characterization and development of aqueous powder suspensions is presented. Topics to be presented include evaluation of powder aging in suspension, characterization of dispersion of powders by $\mathrm{pH}$ and dispersant additions, development of compatible multiple powder suspensions, intrepretation of high solids content suspension information, and correlation of acoustophoresis measurements with other suspension characterization techniques. The example powder system discussed is silicon nitride and yttrium oxide, typically used in fabricating structural silicon nitride material.
\end{abstract}

\section{INTRODUCTION}

Most structural ceramic components are fabricated by densification (sintering) of powder compacts composed of a mixture of powders. Generation of ceramic components with high strengths, reliability (Weibull modulus), and dimensional control places many requirements on the as-formed powder compact, such as a homogeneous distribution of the powder constituents with no agglomerates and uniform high powder packing density. If colloidal filtration is the powder compact forming route, then the suspension properties will control the resulting powder compact properties. If it is assumed that the powder composition and particle sizes/distributions are constrained by the final desired microstructure and properties, then the colloidal suspension properties that can be manipulated are the degree of dispersion of the powders and the suspension solids content. 
The dispersion of colloidal particles in an aqueous medium by the generation of electrostatic or steric repulsion has been extensively discussed in the literature [1-5]. In the case of electrostatic forces, the generation of high zeta potentials is generally observed to result in stable dispersions through the generation of large mutually repulsive forces between particles. The suspension will pack into high density compacts during filtration due to the mutual repulsion between particles. Low zeta potentials result in the domination of short distance attractive forces such as Van der Waals forces, which result in the generation of poorly packed floc structures (agglomerates) which rapidly settle out and generate increased suspension viscosities and instability. The poor packing, results in inhomogeneous, low packing density compacts.

For multicomponent powder suspensions, powders with same-sign and large magnitude of zeta potentials exhibit well dispersed stable suspensions, while powders with opposite sign of zeta potentials exhibit hetero-coagulation, instability, and rapid settling $[6,7]$. Heterocoagulation can be a benefit and has been utilized in suspensions where the powder constituents have greatly different densities and differential segregation can occur in a highly dispersed suspension [8-10]. Electrostatic forces are typically manipulated by changing $\mathrm{pH}$ of the suspension through the addition of acids, bases, and polyelectrolytes.

Steric repulsion can be generated simultaneously with electrostatic repulsion by the addition of long chain polyion dispersants to the suspension. One end of the polymer attaches to the particle surface, while the other end extends out and repels like-polymer chain ends attached to other particles. If the polymer is added to particles with low surface charge, the charged polymers also generate a large charge and create an appreciable electrostatic repulsion[11]. In practice, $\mathrm{pH}$ manipulation and dispersant additions are used in combination to stabilize suspensions.

Solids content of suspension also influences a number of suspension and cast part properties. Besides exhibiting higher viscosities, higher solids content suspensions may exhibit greater tendencies towards shear thinning and instability (manifested as gelation) because of decreased particle repulsive forces due to double layer overlap and compression when the particle separation distances in the suspension approach the particle double layer thicknesses [12,13]. As a result, pourability and conformability of suspensions in rnolds will 
be reduced, as well as the time over which the suspensions can be used. Higher solids content do result in shorter casting times and higher powder compact packing densities due to the smaller amount of liquid to be removed during filtration and the smaller amount of rearrangement needed for particles transitioning from the suspension to cast compact[14].

Thus, the characterization of colloidal suspensions is critical to allowing insight into suspension properties and how they can be manipulated into generating the desired suspension characteristics and resulting powder compact properties. Many techniques are used to characterize colloidal suspensions. Techniques that measure macroscopic suspension properties include rheological measurements, settling tests, and measurement of packing densities of cast powder compacts. But insight into the fundamental nature of particle interactions allows the highest degree of understanding of colloidal suspension stability, and subsequently its manipulation. The most widely used technique is electrophoresis which allows determination of electophoretic mobility as a function of suspension properties, such as $\mathrm{pH}$ and electrolyte concentration. A major limitation of electrophoresis is that it is restricted to very dilute suspensions.

Recently acoustophoresis equipment has been developed that utilizes high frequency electric fields to generate relative motion in charged colloidal particles with surface charge in suspension, called the electrokinetic sonic amplitude (ESA) effect. The movement of the particles generates a high frequency sound wave which is related to the charge on the particle surfaces and from which the electrophoretic mobility and zeta potential of the particles can be calculated. The mobility of particles in suspension can be monitored as the $\mathrm{pH}$ of the suspension is varied, and as dispersant is added. The acoustophoresis equipment is capable of measuring highly loaded colloidal suspensions, up to $50 \mathrm{vol} \%$.

At AlliedSignal Ceramic Components, the acoustophoresis technique has been evaluated, developed, and implemented for the characterization of oxide and non-oxide powder based aqueous suspensions, to be used in the fabrication of structural ceramics by colloidal filtration, or by compaction of spray dried granules generated from suspensions. The following sections will discuss the development and implementation of acoustophoresis characterization, comparison and correlation with other colloidal suspension characterization techniques, examination of $\mathrm{pH}$ and surfactant titration techniques, effect of suspension solids 
content, and applications correlating colloidal properties with other particle characterisitics. The example ceramic system discussed is silicon nitride and yttrium oxide in aqueous suspensions (a typical silicon nitride structural ceramic composition would have 4 to $8 \mathrm{wt} \%$ yttrium oxide added as a sintering aid).

\section{ACOUSTOPHORESIS TECHNIQUE DEVELOPMENT}

Acoustophoresis techniques have been developed and refined at AlliedSignal Ceramic Components over the last five years, for application with silicon nitride based aqueous suspensions. The first step in the characterization process is to prepare the colloidal suspension. For characterization of a single powder suspension, a 1 vol \% solids suspension is prepared in a teflon container (nominal suspension volume is $250 \mathrm{ml}$ ). The powder is added to deionized water and the suspension is mixed for 15 minutes using a teflon coated magnetic stir bar. The stir bar speed is adjusted to ensure that no powder settles to the bottom of the container. The suspension is then ultrasonicated using an ultrasonic horn for 1 minute at 100 watts power, to break up any agglomerates and assure that maximum colloid surface area is available for evaluation. The suspension is then placed into the acoustophoresis measurement equipment $t^{\mathrm{a}}$ and constantly stirred. The suspension properties are then monitored until they have equilibrated. Properties monitored are zeta potential, suspension $\mathrm{pH}$, and suspension conductivity. Through experience and knowledge of the planned suspension applications, the suspension is considered to be equilibrated when none of the measured properties has varied more than $5 \%$ in 15 minutes. It has been demonstrated that silicon nitride powders react in water, due to the reiative instability of surface amine groups and their replacement on the surface over time by silanol groups[7]. But it has been observed in this example powder system that the yttium oxide powder is much more reactive in water (as demonstrated by the aging results in Figure 1) than representative silicon nitride powders. Yttrium oxide powder hydrolyzes readily in water and is generally observed to become unstable in aqueous silicon nitride-yttrium oxide suspensions long before the silicon

${ }^{\text {aMatec Model }} 8000$ 
nitride powders. After the powder suspension is determined to be equilibrated, the $\mathrm{pH}$ of the suspension is noted, as it is a characteristic of the powder as a result of its desorbed and dissolved surface species.

The properties of the suspension are then characterized. Acoustophoresis measurements are made using the ESA method. The Ultrasonic Vibration Potential method is not used, as it requires knowing the suspension high-frequency conductivity, which is not measured. The zeta potential is typically determined by titrating the $\mathrm{pH}$, from the equilibrated suspension $\mathrm{pH}$, to either the desired low or high $\mathrm{pH}$ endpoints. Thus two experiments are required to generate a $\mathrm{pH}$ vs. zeta potential curve. Titrations are only performed in one direction for each experiment, since this approximates suspension preparation for actual components, i.e. the suspension is only adjusted to its desired final $\mathrm{pH}$ and there is no buildup of undesirable counterions. The titrants used are $2.0 \mathrm{~N} \mathrm{HNO}_{3}$ and $2.0 \mathrm{~N} \mathrm{NH}_{4} \mathrm{OH}$, since this acid and base are used in production suspension $\mathrm{pH}$ manipulation, as they contain no detrimental cations or anions. The relative strength of the titrants limits the achievable $\mathrm{pH}$ range to $2.0-10.5$. A 25 second hold is utilized between titrant additions to allow the suspension time to equilibrate. Figure 2 shows a typical zeta potential vs. pH curve for a silicon nitride powder. It is important to note that no electrolyte is added to these suspensions. Electrolytes such as $\mathrm{KCl}$ and $\mathrm{NaCl}$ are typically added to colloidal suspensions for electrophoresis experiments to generate a constant ionic environment for suspension property measurements. But alkali and alkaline earth cations and halide anions are detrimental to silicon nitride (and many other structural ceramics) high temperature properties and are not added to colloidal suspensions used in component fabrication. Experiments have been performed to evaluate the influence of electrolyte additions on measured zeta potentials in $\mathrm{pH}$ titrations of silicon nitride powders (see Figure 3). The only influence of the electrolyte was to decrease the relative zeta potential, with the greatest effect at low and high $\mathrm{pH}$. Thus, no detriment is observed by eliminating electrolyte additions for acoustophoresis measurements.

The effect of dispersant additions on zeta potential is determined by titrating the dispersants into suspensions. In some cases, the $\mathrm{pH}$ is manually adjusted to be maintained at a desired level during the titration, so that the effect of the dispersant can be determined at a constant $\mathrm{pH}$. 


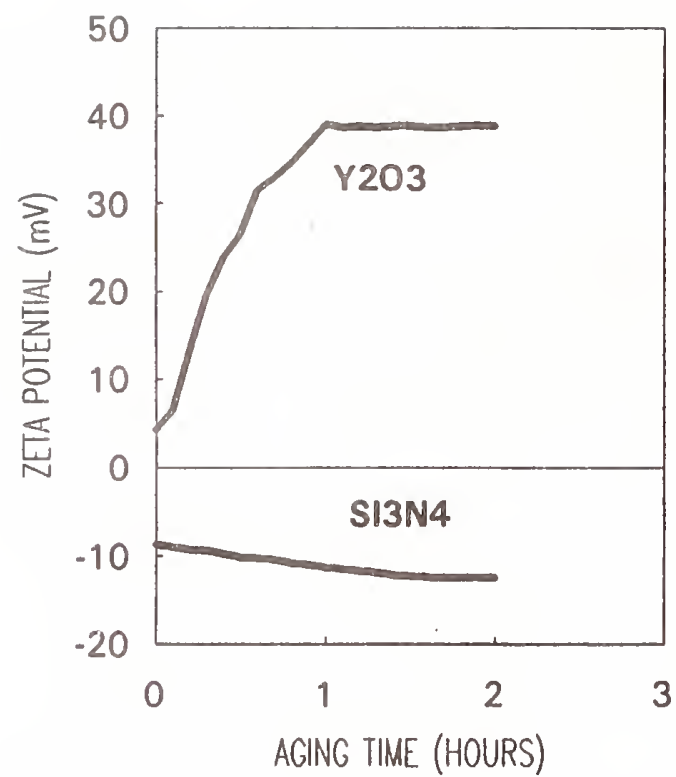

Figure 1. Aging Comparison of $\mathrm{Si}_{3} \mathrm{~N}_{4}$ and $\mathrm{Y}_{2} \mathrm{O}_{3}$ $\mathrm{Si}_{3} \mathrm{~N}_{4}$ and $\mathrm{Y}_{2} \mathrm{O}_{3}$ Powders in Water.

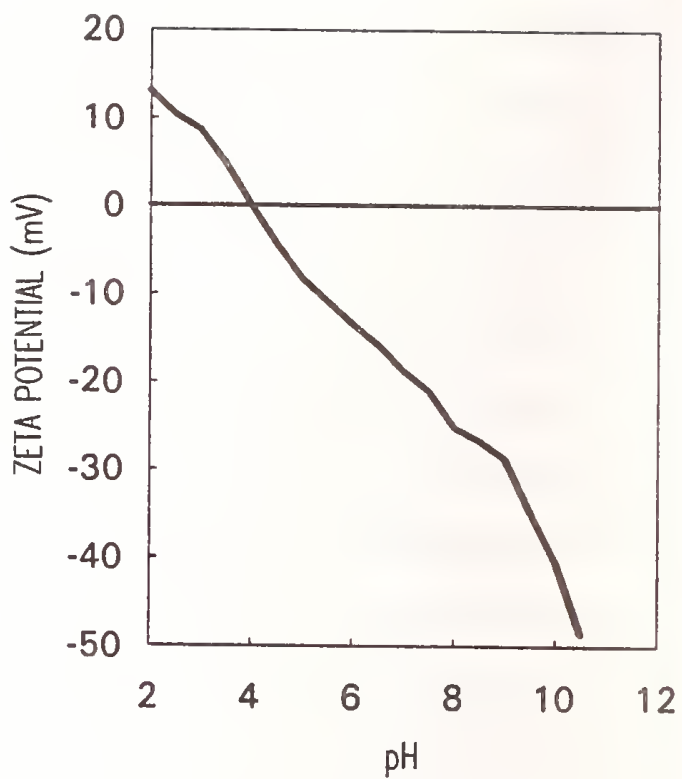

Figure 2. Typical Zeta Potential vs. $\mathrm{pH}$ Curve for $\mathrm{Si}_{3} \mathrm{~N}_{4}$ Powder.

The time between dispersant additions is typically 25 seconds, to allow for suspension equilibration after the previous dispersant addition. A typical dispersant titration curve for silicon nitride is presented in Figure 4.

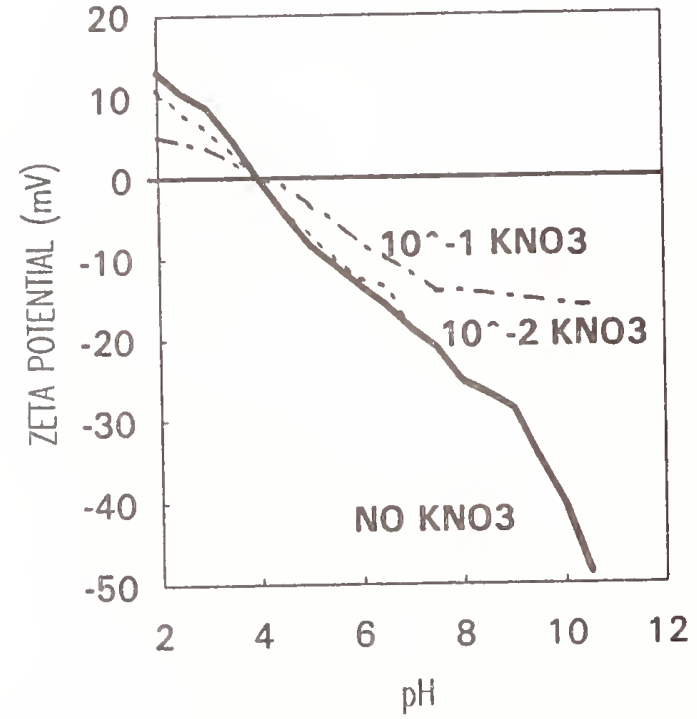

Figure 3. Effect of Electrolyte Additions on Zeta Potential of $\mathrm{Si}_{3} \mathrm{~N}_{4}$ Powder inWater.

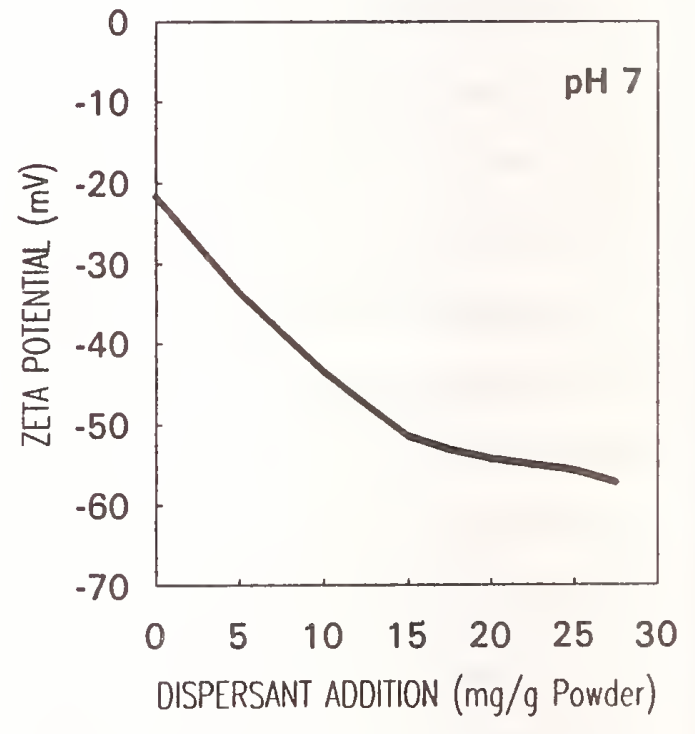

Figure 4. Example of Surfactant Titration Curve For $\mathrm{Si}_{3} \mathrm{~N}_{4}$ Powder. 


\section{APPLICATION TO SILICON NITRIDE - YTTRIA SUSPENSIONS}

\section{Single Component Suspension Characterization}

The first step taken in the development of silicon nitride based aqueous suspensions is to characterize the individual powder zeta potentials as a function of $\mathrm{pH}$. In order to correlate zeta potential with other suspension characterization techniques that require higher solids content suspensions for measurement sensitivity, 5 vol\% solids suspensions were utilized. Figure 5 shows the zeta potential vs. pH curves of silicon nitride (Denka SN-9S) and yttria (Shin-Etsu SU) powders. The $\mathrm{Y}_{2} \mathrm{O}_{3}$ was only evaluated above $\mathrm{pH} 7$, since it dissolves under acidic conditions. The isoelectric point (iep) of the $\mathrm{Si}_{3} \mathrm{~N}_{4}$ is 4.0 and the iep of the $\mathrm{Y}_{2} \mathrm{O}_{3}$ is 9.25. The powders exhibit opposite sign zeta potentials at $\mathrm{pH} 7$. As the $\mathrm{pH}$ is increased, the $\mathrm{Y}_{2} \mathrm{O}_{3}$ moves through its iep and becomes negatively charged, the same sign charge as the $\mathrm{Si}_{3} \mathrm{~N}_{4}$ powder. As pH 10.5 is reached, zeta potential values of the two powders increase to large values. The zeta potential values measured were correlated with three other suspension characterization methods - suspension viscosity, suspension sediment height, and suspension supernatent turbidity.

The suspensions used for these methods were prepared at specific $\mathrm{pH}$ values, not titrated. The viscosity of the suspensions, measured using a high sensitivity couvette viscometer ${ }^{\mathrm{b}}$ is shown in Figure 6. Viscosity measurements correlate well with zeta potential values, i.e. viscosities are lowest where zeta potential values are highest, and the $\mathrm{Y}_{2} \mathrm{O}_{3}$ viscosity is highest at its iep, where there are no repulsive forces and floccing is allowed to occur.

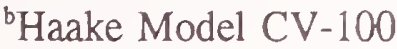




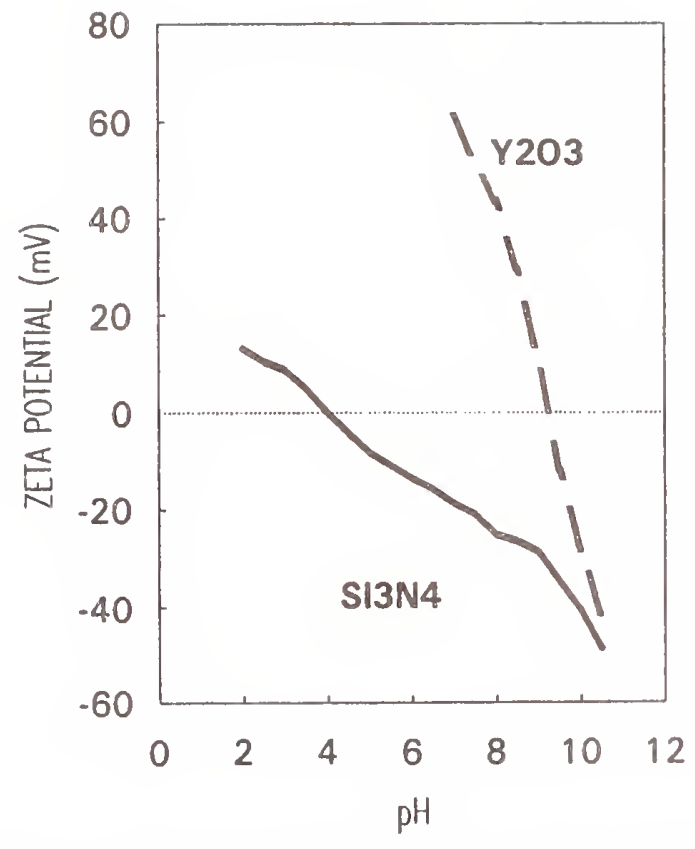

Figure 5. Zeta Potential vs. pH Curves for $\mathrm{Si}_{3} \mathrm{~N}_{4}$ and $\mathrm{Y}_{2} \mathrm{O}_{3}$ Powders.

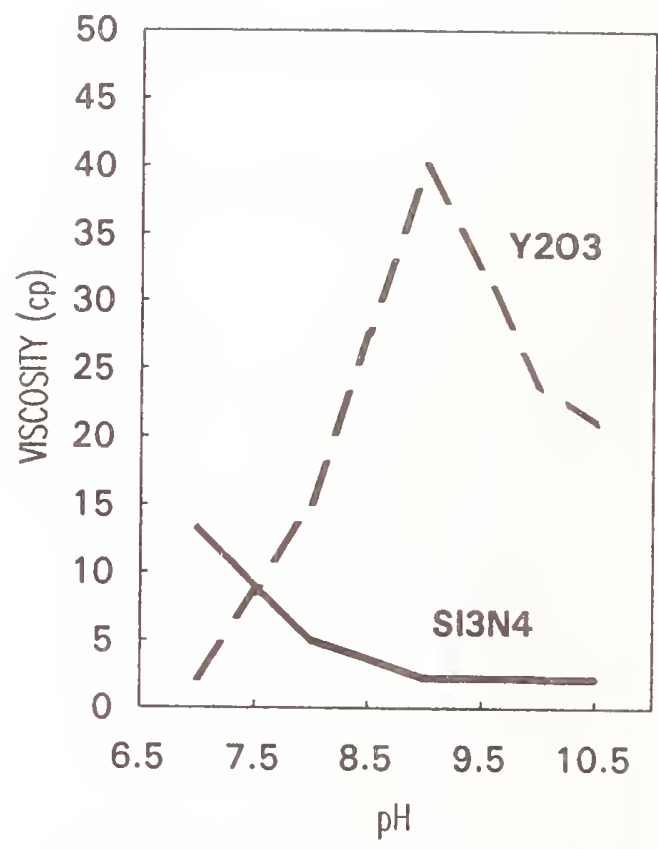

Figure 6. Viscosity of $\mathrm{Si}_{3} \mathrm{~N}_{4}$ and $\mathrm{Y}_{2} \mathrm{O}_{3}$ Powders as a Function of $\mathrm{pH}$.

Sediment height tests were conducted with equal amounts of suspension placed into test tubes for 48 hours. The relative sediment heights were then examined. The intrepretation of sediment height tests is that if a suspension is allowed to stand for an extended time, the particles will settle due to gravity. Powders that are well dispersed will pack into high density structures, forming a short sediment height. Poorly dispersed (low zeta potential) powders will pack poorly into low density structures, resulting in high sediment heights. Figure 7 shows relative sediment height results as a function of $\mathrm{pH}$. The results correlate with zeta potential measurements in that highest sediment heights are achieved at the lowest zeta potential values. Supernatant turbidity tests were conducted by examining the settling test suspensions placed in test tubes after 48 hours. The suspension supernatant turbidity was rated by visual inspection from 1 to 5 , with 1 being clear and 5 being opaque. Interpretation of turbidity tests is that well dispersed particles will mutually repel one another and that some will stay suspended for long times, clouding the supernatant. Poorly dispersed particles will floc and settle rapidly, leaving a clear supernatant. The results again correlate with zeta potential measurements in that opaque or nearly opaque supernatants are seen for high zeta potential suspensions, while a clear supernatant is observed for $\mathrm{Y}_{2} \mathrm{O}_{3}$ at its iep (see Figure 8). 


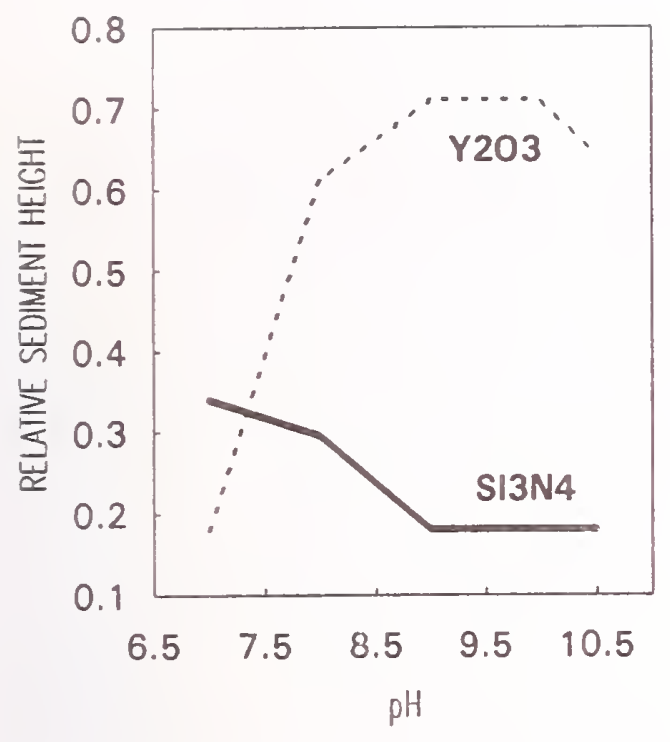

Figure 7. Sediment Height of $\mathrm{Si}_{3} \mathrm{~N}_{4}$ and $\mathrm{Y}_{2} \mathrm{O}_{3}$ Suspensions as a Function of $\mathrm{pH}$.

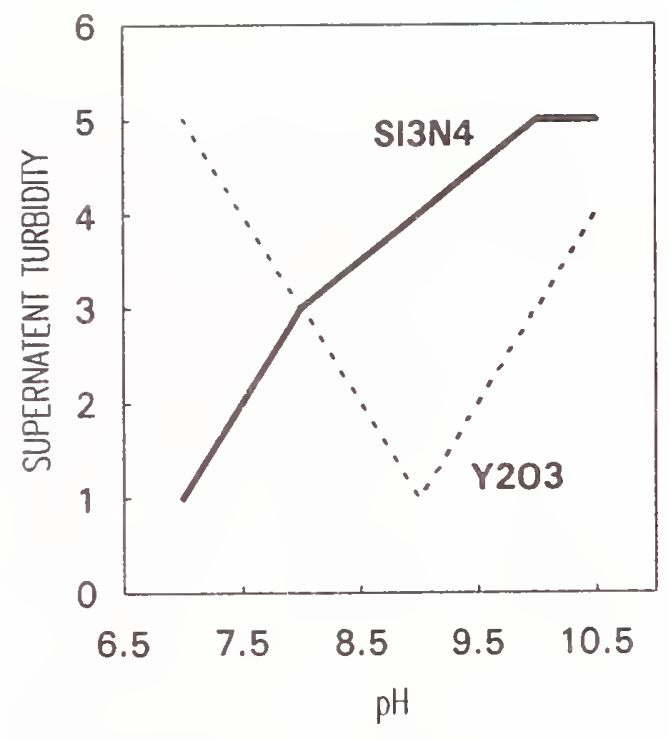

Figure 8. Supernatent Turbidity of $\mathrm{Si}_{3} \mathrm{~N}_{4}$ $\mathrm{Y}_{2} \mathrm{O}_{3}$ Suspensions as a Function of $\mathrm{pH}$.

The correlation is excellent for all three techniques, when correlated with the zeta potential measurement results, but the advantages of acoustophoretic measurement of the zeta potential is clear. It is both more rapid (two $\mathrm{pH}$ titrations vs. preparation of a number of different $\mathrm{pH}$ suspensions, and waiting for sedimentation to take place in the case of sediment height and supernatent turbidity tests), and it provides information and insight on the fundamental forces controlling suspension properties.

\section{Multicomponent Suspension Characterization}

The second step taken in the development of $\mathrm{Si}_{3} \mathrm{~N}_{4}-\mathrm{Y}_{2} \mathrm{O}_{3}$ suspensions was to examine the effect of combining the two powders in suspension. Examination of the individual powder zeta potential vs. pH curves in Figure 5 indicates that powders will have opposite sign zeta potentials at $\mathrm{pH} 7$ and hetero-coagulation will occur. As the $\mathrm{pH}$ is increased, the $\mathrm{Y}_{2} \mathrm{O}_{3}$ will pass through its iep and become the same sign charge. Mutual repulsion will then occur with dispersion being maximized at $\mathrm{pH} 10.5$, where both powders exhibit large magnitude zeta potentials. To determine if the individual zeta potential values of the two powders could be used to predict multicomponent suspension properties, two suspension compositions were prepared - $50 \mathrm{wt} \% \mathrm{Si}_{3} \mathrm{~N}_{4} / 50 \mathrm{wt} \% \quad \mathrm{Y}_{2} \mathrm{O}_{3}$, and $95 \mathrm{wt} \% \mathrm{Si}_{3} \mathrm{~N}_{4} / 5 \mathrm{wt} \% \quad \mathrm{Y}_{2} \mathrm{O}_{3}$. 
Earlier experiments indicated that titrating the suspensions through regions of opposite sign zeta potentials induces hetero-coagulation that could not be reversed as the titration is continued. Thus, individual suspensions were prepared at specific $\mathrm{pH}$ values of $7,8,9,10$, and 10.5. Since the acoustophoresis equipment measures the sum signal of the suspension, the zeta potential measured for multicomponent powder suspensions is a relative value. It is useful to calculate the relative zeta potential value for qualitative use in evaluating multicomponent suspensions, so the particle density and particle size inputs to the zeta potential calculation software are weighted using weight percent. The results are presented in Figure 9. For the $95 \mathrm{wt} \% \mathrm{Si}_{3} \mathrm{~N}_{4}$ suspension, the zeta potential measured is essentially that of the $\mathrm{Si}_{3} \mathrm{~N}_{4}$ powder by itself in suspension. This can be expected due to the fact that the acoustophoresis equipment is measuring the resulting zeta potential of the entire suspension and that $5 \% \mathrm{Y}_{2} \mathrm{O}_{3}$ will have a minimal effect on the suspension properties, even if it has an opposite sign zeta potential below $\mathrm{pH} 9$, compared to the $\mathrm{Si}_{3} \mathrm{~N}_{4}$. On the other hand, the 50 $\mathrm{wt} \% \mathrm{Si}_{3} \mathrm{~N}_{4}$ suspension shows a dramatic effect as a function of $\mathrm{pH}$.

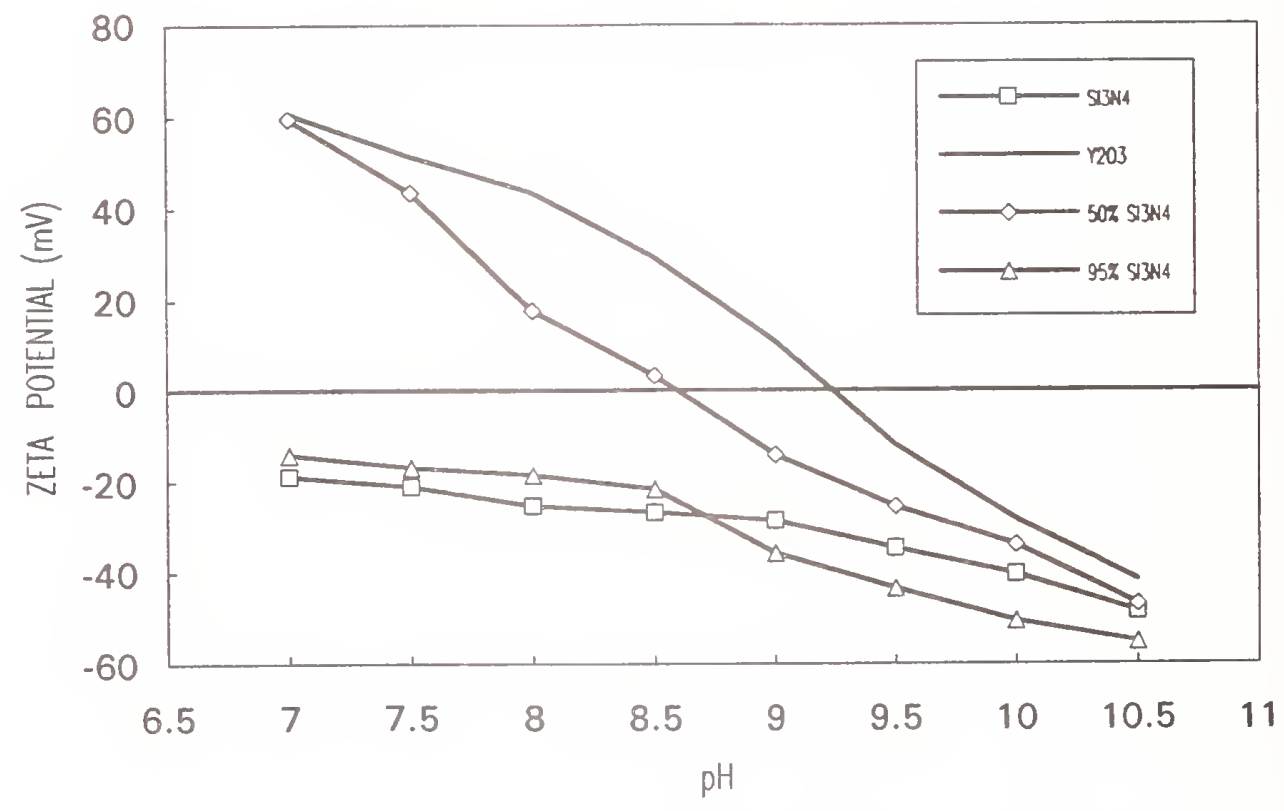

Figure 9. Comparison of Multicomponent $\mathrm{Si}_{3} \mathrm{~N}_{4} / \mathrm{Y}_{2} \mathrm{O}_{3}$ Suspension Zeta Potential as a Function of $\mathrm{pH}$, with the Separate Components. 
Its measured zeta potential is approximately halfway between that of the $\mathrm{Si}_{3} \mathrm{~N}_{4}$ and $\mathrm{Y}_{2} \mathrm{O}_{3}$ powders between $\mathrm{pH}$ values 8.8 and 10.5. This can be expected since the powders are both negatively charged and the suspension zeta potential should be approximately an average between the two powder zeta potentials. Below $\mathrm{pH} 8$, the zeta potential tracks with the $\mathrm{Y}_{2} \mathrm{O}_{3}$ zeta potential, indicating that either the $\mathrm{Y}_{2} \mathrm{O}_{3}$ particles have heterocoagulated onto the larger $\mathrm{Si}_{3} \mathrm{~N}_{4}$ particles, thereby shielding the $\mathrm{Si}_{3} \mathrm{~N}_{4}$ surface charge, or possibly that Yttrium cations have dissolved from the $\mathrm{Y}_{2} \mathrm{O}_{3}$ particle surfaces and adsorbed onto the $\mathrm{Si}_{3} \mathrm{~N}_{4}$ surfaces, a process observed in other oxide materials by Wiese and Healy[15-16].

The viscosity of the two multicomponent suspensions was also measured and the results are presented in Figure 10. The $95 \mathrm{wt} \% \mathrm{Si}_{3} \mathrm{~N}_{4}$ suspension tracks with the viscosity of the $\mathrm{Si}_{3} \mathrm{~N}_{4}$ suspension, while the $50 \mathrm{wt} \% \mathrm{Si}_{3} \mathrm{~N}_{4}$ suspension viscosity tracks with its measured zeta potential. At $\mathrm{pH} \mathrm{7,} \mathrm{although} \mathrm{strong} \mathrm{hetero-coagulation} \mathrm{is} \mathrm{predicted} \mathrm{from} \mathrm{the} \mathrm{individual}$ powder suspension zeta potentials, the result is a low viscosity suspension with high zeta

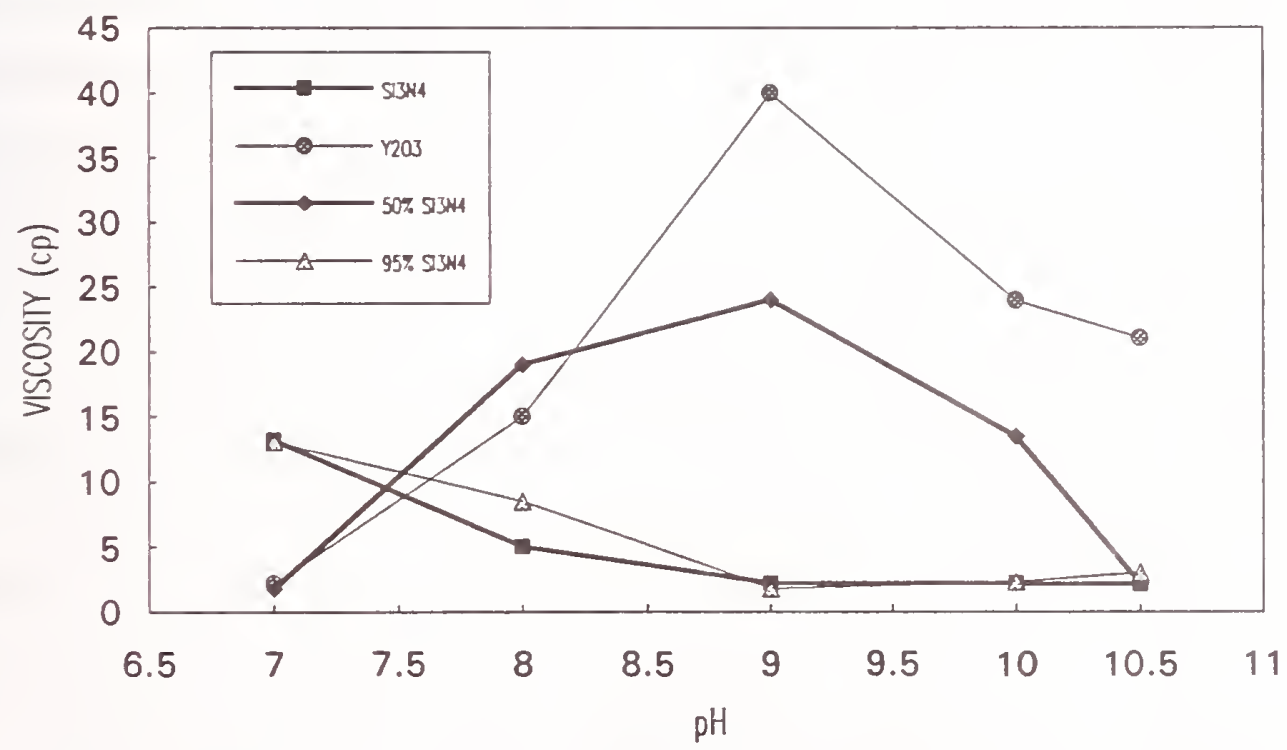

Figure 10. Viscosity of Multicomponent Suspensions as a Function of $\mathrm{pH}$, Compared with the Separate Components.

potential. In addition, a sediment height evaluation was performed at $\mathrm{pH} 7$ on the 50 wt $\%$ $\mathrm{Si}_{3} \mathrm{~N}_{4}$ suspension and it showed a higher sediment height than either of the individual powder suspensions at $\mathrm{pH} 7$. This indicates that there is some type of hetero-coagulation occurring, but that it is not strongly influencing rheology. 
In summary, evaluation of multicomponent suspensions by acoustophoresis (even though a qualitative measurement) is valuable in determining how the individual constituents will react when placed together, and how they can be manipulated, even though the acoustophoresis technique only measures the overall suspension zeta potential. But in order to effectively interpret results, the zeta potential of the individual powder must be known as a function of $\mathrm{pH}$ before trying to interpret the multicomponent suspension results, and suspension characterization techniques such as viscosity measurements, can provide additional useful information and insight.

\section{Manipulation of Zeta Potential Sign by Dispersants}

Multicomponent suspensions have a desired $\mathrm{pH}$ level at which they must be prepared, in order to maximize the zeta potential and degree of dispersion by the major powder component. Many times the minor powder additions have the opposite sign zeta potential of the major powder constituent at that $\mathrm{pH}$ and if added directly to the suspension will result in hetero-coagulation. The zeta potential sign can potentially be manipulated by addition of a dispersant and the result can be measured using acoustophoresis. A specific example is the following $\mathrm{Si}_{3} \mathrm{~N}_{4}$-based suspension. The $\mathrm{Si}_{3} \mathrm{~N}_{4}$ powder shown in Figure 2 can be most effectively dispersed at a $\mathrm{pH}$ range from 9 to 10.5 . But a selected $\mathrm{Y}_{2} \mathrm{O}_{3}$ powder ${ }^{c}$ has the opposite sign zeta potential (positive) at that $\mathrm{pH}$ range. In order to prevent heterocoagulation, the zeta potential of the $\mathrm{Y}_{2} \mathrm{O}_{3}$ was changed to negative by the addition of a sodium polyacrylate dispersant. ${ }^{d}$ The dispersant titration curve is shown in Figure 11. Obviously, the $\mathrm{Y}_{2} \mathrm{O}_{3}$ must be predispersed with the dispersant before addition to the $\mathrm{Si}_{3} \mathrm{~N}_{4}$, and the $\mathrm{Si}_{3} \mathrm{~N}_{4}$ powder must also be compatible with the dispersant, because some of the dispersant adsorbed on the $\mathrm{Y}_{2} \mathrm{O}_{3}$ particles may desorb and interact with the $\mathrm{Si}_{3} \mathrm{~N}_{4}$.

${ }^{\mathrm{c} M o l y c o r p ~ \# 5600 ~}$

${ }^{\mathrm{d} W . R . ~ G r a c e ~ D a x a d ~} 30$ 


\section{Effect of Solids Content}

Acoustophoresis has the ability to measure zeta potential in high solids content suspensions. But the equations relating the electroacoustic effects to mobility and zeta potential are based on dilute suspensions of particles. As the solids content is increased to where electrical double-layer interactions and hydrodynamic interactions occur, the relationships become nonlinear. At even higher solids contents, particle-particle interactions occur and reduce dynamic mobility. For most aqueous suspensions, the non-linear behavior occurs above approximately $10 \mathrm{vol} \%$. $\mathrm{Si}_{3} \mathrm{~N}_{4}$ aqueous suspensions were prepared at a range of solids contents to evaluate acoustophoretic zeta potential measurements. The results are presented in Figure 12. The calculated zeta potential is in fact the same for $10 \mathrm{vol} \%$ solids content and less. The calculated zeta potential does decrease above $10 \mathrm{vol} \%$ solids. Viscosity was also measured for each suspension prepared and the results are shown in Figure 12. The viscosity does not start to increase appreciably until 20 vol\% solids.

In order to determine the sensitivity of acoustophoresis at high solids loadings, $\mathrm{Si}_{3} \mathrm{~N}_{4}$ suspensions and $95 \% \mathrm{Si}_{3} \mathrm{~N}_{4} / 5 \% \mathrm{Y}_{2} \mathrm{O}_{3}$ suspensions were prepared at 37.7 vol\% solids at $\mathrm{pH} 9$ and 10.5 .

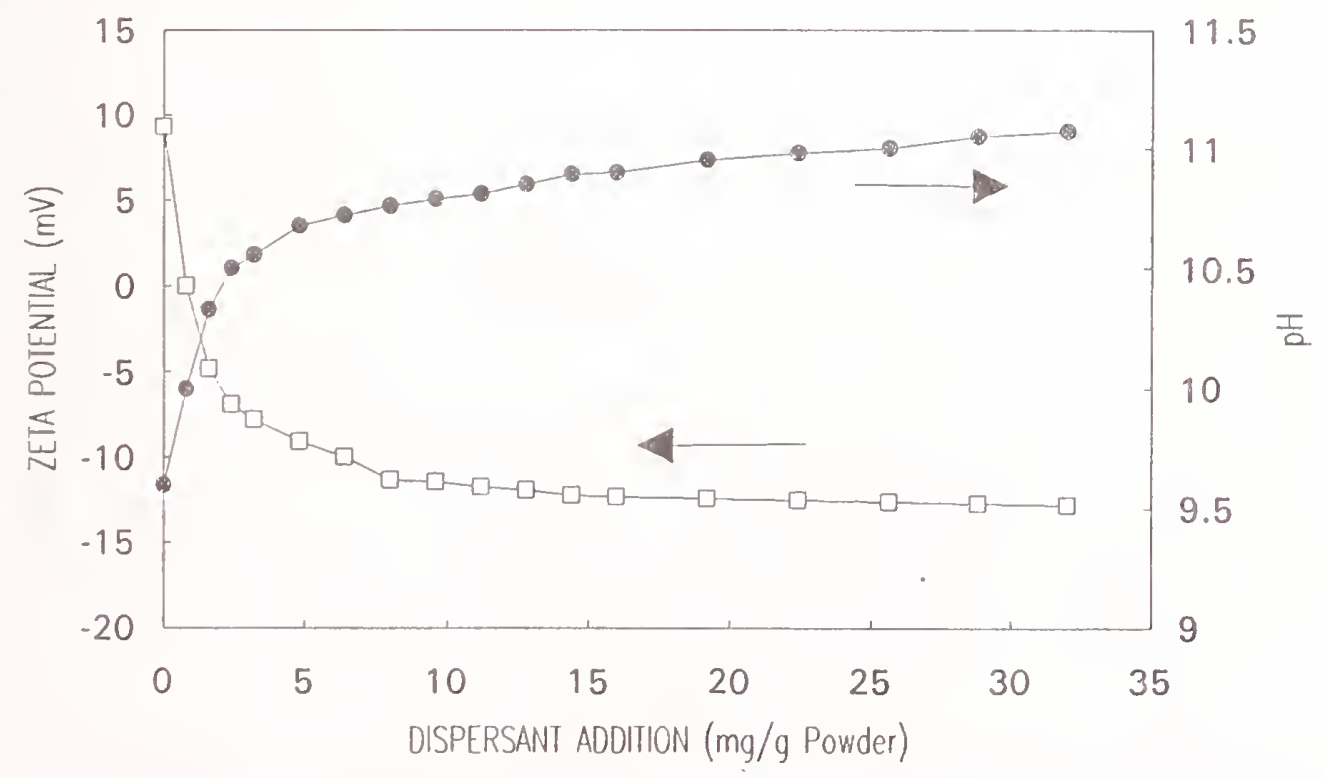

Figure 11. Reversal of Zeta Potential Sign of $\mathrm{Y}_{2} \mathrm{O}_{3}$ Powder in Aqueous Suspension by Dispersant Additions. 


\section{Correlation of Zeta Potential with $\mathrm{Si}_{3} \underline{\mathrm{N}}_{1}$ Oxygen Content}

Zeta potential measurements can also be used to evaluate other properties that affect zeta potential of powders. An example is the correlation that can be made between $\mathrm{Si}_{3} \mathrm{~N}_{4}$ isoelectric point (iep) and surface oxygen content. In an experiment to evaluate this correlation, $\mathrm{Si}_{3} \mathrm{~N}_{4}$ aqueous suspensions were milled in deionized water for a range of times, up to 168 hours. For each milling time, the suspensions were titrated using acoustophoresis to determine the iep, then the suspension was quickly dried and the oxygen content of the powders determined using a fusion technique. ${ }^{e}$ The results are presented in Figure 14. The $\mathrm{Si}_{3} \mathrm{~N}_{4}$ powder iep decreases in $\mathrm{pH}$ with increasing oxygen content. This is a result of the substitution of surface amine groups by silanol groups, which result in the surface becoming more $\mathrm{SiO}_{2}$-like. The $\mathrm{Si}_{3} \mathrm{~N}_{4}$ iep varies nearly linearly with oxygen content up to approximately $2.5 \mathrm{wt} \%$ oxygen, then the oxygen content increases rapidly with little change in iep. The transition at $2.5 \mathrm{wt} \%$ oxygen potentially indicates the complete coverage of the $\mathrm{Si}_{3} \mathrm{~N}_{4}$ particle surface by silanol groups. Further increases in oxygen content would not be expected to affect the $\mathrm{Si}_{3} \mathrm{~N}_{4}$ iep, since the surface will continue to be fully covered with silanol groups.

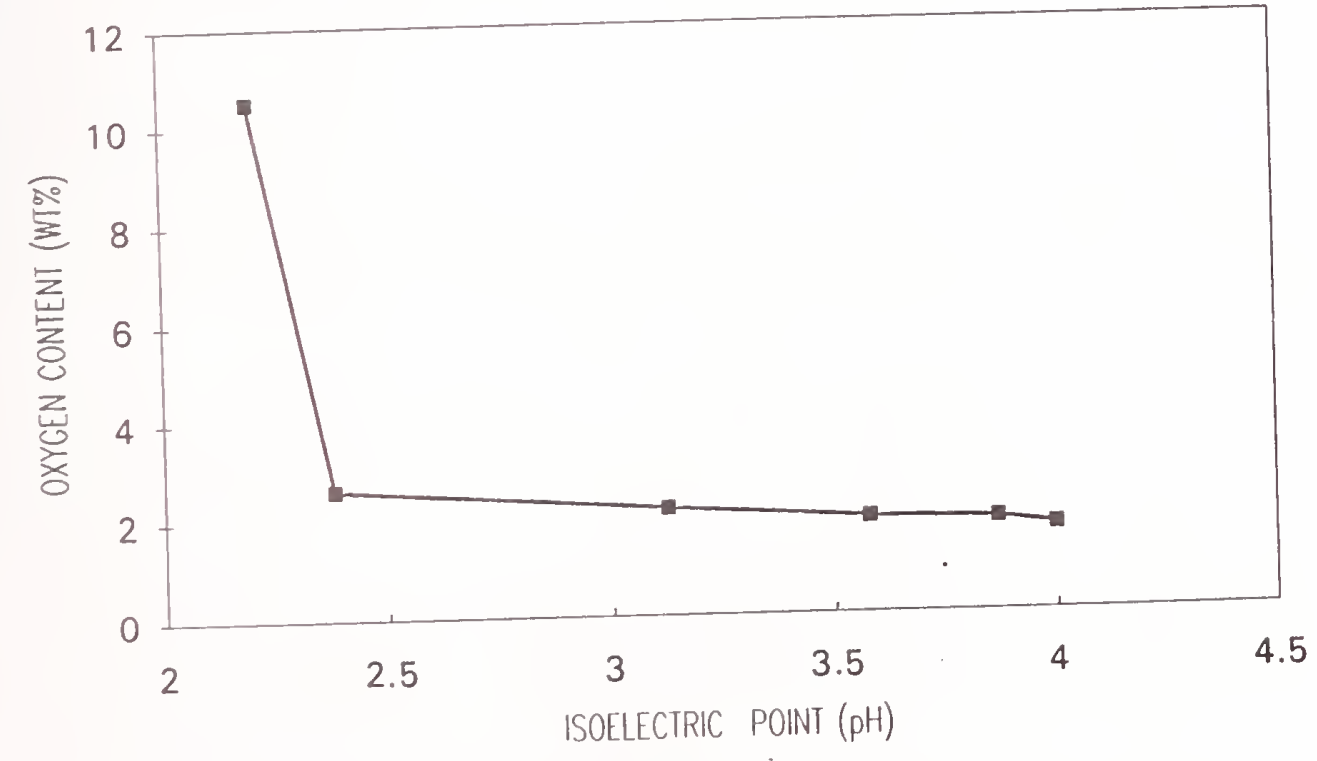

Figure 14. Correlation of the Isoelectric Point of $\mathrm{Si}_{3} \mathrm{~N}_{4}$ in Aqueous Suspension (Milled at Various Times) with Oxygen Content. 


\section{SUMMARY}

In conclusion, the development and implementation of acoustophoresis techniques for the determination of zeta potential in aqueous ceramic powder suspensions has been described. The acoustophoretic measurements correlate well with other suspension characterization methods for low solids content suspensions and are more rapid than conventional methods. At high solids contents, viscosity is a more sensitive indicator of suspension properties. Acoustophoresis techniques can also be used to characterize multicomponent powder suspensions, if single component suspension properties of the constituent powders are known. Powder properties which affect zeta potential, such as $\mathrm{Si}_{3} \mathrm{~N}_{4}$ powder surface oxygen content, can also be correlated with zeta potential measured by acoustophoresis. 


\section{REFERENCES}

1. J. T. G. Overbeek, "Recent Developments in the Understanding of Colloid Stability," J. Colloid Interface Sci., 58 [2] 408- 22 (1977).

2. J. T. G. Overbeek, "How Colloid Stability Affects the Behavior of Suspensions," in Emergent Process Methods for High Technology Ceramics, edited by R. F. Davis, H. Palmour III, and R. L. Porter, Plenum Press, NY, 25-44 (1982).

3. J. Lyklema, "Interfacial Electrochemistry of Disperse Systems," ibid 1-24.

4. M. D. Sacks et al., "Dispersion and Rheology in Ceramic Processing," in Ceramic Powder Science, edited by G. L. Messing, K. S. Mazdiyasni, J. W. McCauley, and R. A. Haber, Am. Ceram. Soc., Ohio, 495-516 (1987).

5. R. O. James, "Characterization of Colloids in Aqueous Systems," in Ceramic Powder Science, edited by G. L. Messing, K. S. Mazdiyasni, J. W. McCauley, and R. A. Haber, Am. Ceram. Soc., Ohio, 350-410 (1987).

6. T. W. Healy, G. R. Wiese, D. E. Yates, and B. V. Kavanaugh, "Hetero-coagulation in Mixed Oxide Colloidal Dispersions," J. Colloid Interface Sci., 42 [3] 647-9 (1973).

7. J. P. Pollinger, D. D. Newson, and J. J. Nick, "The Implementation of Acoustophoresis for the Characterization of Silicon Nitride Based Aqueous Suspensions," to be Published in Characterization Techniques for Solid Solution Interfaces in Ceramic Systems, edited by J. H. Adair and J. A. Casey, Am. Ceram. Soc.

8. E. Carlstrom and F. F. Lange, "Mixing of Flocced Suspensions," J. Am. Ceram. Soc., 67 [8] C169-70 (1984).

9. F. F. Lange, B. I. Davis, and E. Wright, "Processing-Related Fracture Origins: IV, Elimination of Voids Produced by Organic Inclusions," J. Am. Ceram. Soc., 69 [1] 6669 (1986).

10. T. M. Shaw and B. A. Pethica, "Preparation and Sintering of Homogeneous Silicon Nitride Green Compacts," J. Am. Ceram. Soc., 69 [2] 88-93 (1986). 
11. R. G. Horn, "Surface Forces and Their Action in Ceramic Materials," J. Am. Ceram. Soc., 73 [5] 1117-35 (1990).

12. W. Albers and J. T. G. Overbeek, "Stability of Emulsions of Water in Oil: II. Charge as a Factor of Stabilization Against Flocculation," J. Colloid Sci., 14 [5] 510-18 (1959).

13. E. M. Deliso, A. S. Rao, and W. R. Cannon, "Electrokinetic Behavior of $\mathrm{Al}_{2} \mathrm{O}_{3}$ and $\mathrm{ZrO}_{2}$ Powders in Dilute and Concentrated Aqueous Dispersions," in Ceramic Powder Science, edited by G. L. Messing, K. S. Mazdiyasni, J. W. McCauley, and R. A. Haber, Am. Ceram. Soc., Ohio, 525-536 (1987).

14. N. Ise, et. al., "Ordering of Charged Particles in Solution," Naturwissenschaften, 69, S.544 (1982).

15. G. R. Wiese and T. W. Healy, "Coagulation and Electrokinetic Behavior of $\mathrm{TiO}_{2}$ and $\mathrm{Al}_{2} \mathrm{O}_{3}$ Colloidal Dispersions," J. Colloid Interface Sci., 51 [3] 427-33 (1975).

16. G. R. Wiese and T. W. Healy, "Adsorption of $\mathrm{Al}(\mathrm{III})$ at the $\mathrm{TiO}_{2}-\mathrm{H}_{2} \mathrm{O}$ Interface," J. Colloid Interface Sci., 51 [3] 434-42 (1975). 

SILICON NITRIDE POWDERS

\author{
Vincent A. Hackley and Subhas G. Malghan \\ National Institute of Standards \& Technology \\ Ceramics Division \\ Gaithersburg, Maryland 20899
}

\begin{abstract}
The electrokinetic sonic amplitude (esa) measurement may be used to characterize silicon nitride powders in aqueous suspension at solids concentrations approaching those encountered in real processing environments. The effects of powder and measurement parameters on esa analysis has been investigated. The role played by solution ionic species in modifying surface charge behavior and the ionic contribution to the esa signal of the solid phase was examined in detail.
\end{abstract}

\title{
INTRODUCTION
}

Colloidal filtration (slip casting) and spray drying methods for processing of silicon nitride powders into structural ceramics require the application of aqueous colloidal dispersion techniques. The preparation and property control of dense powder slurries containing multiple components (e.g., sintering aids, dispersants and binders) which interact in complex ways is a difficult problem [1]. In addition, the silicon nitride surface may, depending on powder history, contain varying amounts of oxide or inorganic contaminants. These components can alter the interfacial chemistry during processing. Wide variations in physical and surface chemical properties of powders often occur between manufacturers and even between batches from the same source. It is desirable to understand how these variations will affect suspension behavior, then apply this information to compensate for property fluctuations during or prior to processing.

The colloid stability and rheology of aqueous suspensions derive primarily from the interfacial electrical properties of the dispersed particles. For $\mathrm{Si}_{3} \mathrm{~N}_{4}$, the surface is 
zwitterionic consisting primarily of silanol $(\mathrm{Si}-\mathrm{OH})$ and amine $(=\mathrm{N}-\mathrm{H})$ functional groups, which can dissociate to produce a negative or positive charge that is $\mathrm{pH}$ dependent [2]. The isoelectric point (iep) defines the $\mathrm{pH}$ at which positive and negative species are balanced, yielding a net potential of zero on the particles. This condition is usually determined by electrokinetic measurements of the shear-plane or zeta potential [3]. The iep for $\mathrm{Si}_{3} \mathrm{~N}_{4}$ can vary from about $\mathrm{pH} 2$ to 9 , and is greatly affected by the adsorption of ionized solution species and the degree of surface oxidation [4-7]. Electroacoustic techniques, based on the response of charged particles to an alternating electric field or sound wave, have recently been developed for the analysis of interfacial electrochemistry in dense suspensions. Detailed descriptions of measurement systems based on the electroacoustic effect are to be found elsewhere within this volume.

We have measured the electrokinetic sonic amplitude (esa) which is related to the colloid dynamic mobility, $\mu(\omega)$, and zeta potential through the following relationship [8]:

$$
\text { esa }=\frac{P}{E}=c \Delta \rho \phi f_{g} \mu(\omega)
$$

where $P / E$ is the pressure amplitude over the applied ac electric field, $c$ is the speed of sound, $\Delta \rho$ is the difference in density between the particle and suspending medium, $\phi$ is the solid volume fraction and $\mathrm{f}_{\mathrm{g}}$ is a geometric factor. The dynamic and static (electrophoretic) mobility differ by an inertial factor which is close to 1 for the conditions of most of the experiments reported in this paper. The polarity of the particle charge is calibrated against a known material, after which any change in polarity is characterized by a rapid shift (through $180^{\circ}$ ) in the measured phase angle. 


\section{EXPERIMENTAL}

The $\mathrm{Si}_{3} \mathrm{~N}_{4}$ powder used in these studies has a mean particle size of $0.4 \mu \mathrm{m}$ (determined by light diffraction) and a BET surface area of $10.1 \mathrm{~m}^{2} / \mathrm{g}$. This material, type SNE- $10^{\dagger}$, is produced by Ube Industries (Tokyo, Japan). In general, sample suspensions were prepared in the following manner. A weighed amount of powder was mixed into the appropriate amount of solution containing the desired salt and deionized water (pre-acidified with nitric acid in most cases) and allowed to equilibrate for $10 \mathrm{~min}$. The suspension was then dispersed using ultrasonication. If multivalent ions were used in an experiment, they were added in solution form following sonication and then equilibrated for $10 \mathrm{~min}$. The esa measurements and acid-base titrations were performed using an ESA-8000 system from Matec Applied Sciences (Hopkinton, MA). Head space in the sample cell was blanketed with nitrogen gas and maintained at positive pressure to exclude atmospheric $\mathrm{CO}_{2}$ during an experiment. Samples were titrated at $60 \mathrm{~s}$ intervals unless otherwise noted. Nitric acid and sodium hydroxide titrants were used. All chemicals were analytical grade or higher.

All particle concentrations are reported as \% volume fraction of solids. Ion concentrations are expressed in either molar or equivalent weight units $(\mathrm{eq} / \mathrm{L})$. We define an equivalent weight as that fraction of the molecular weight corresponding to the reciprocal valance of an ion as given in the text or graphs. For example, $\mathrm{Mg}^{2+}$ and $\mathrm{HPO}_{4}{ }^{2-}$ have an equivalent weight equal to $1 / 2$ of the molecular weight, and therefore a $1 \mathrm{~mol} / \mathrm{L}$ solution of these ions contains $2 \mathrm{eq} / \mathrm{L}$. For all 1:1 electrolytes, the molecular and equivalent weights are identical.

$\uparrow$ Certain trade names and company products are mentioned in the text or identified in illustrations in order to adequately specify the experimental procedure and equipment used. In no case does such identification imply recommendation or endorsement by National Institute of Standards and Technology, nor does it imply that the products are necessarily the best available for the purpose. 


\section{RESULTS AND DISCUSSION}

\section{Measurement and Powder Parameters}

Relative to more established methods, such as microelectrophoresis, the esa technique has not been tested over a sufficiently broad range of experimental conditions and sample diversity to warrant a priori acceptance of results. Our first goal is to examine the dependence of esa measurements on various physicochemical properties of aqueous ceramic powder suspensions, and establish procedures and conditions for obtaining reproducible results that can be interpreted in a manner consistent with known properties. Our second goal is to perform fundamental studies of non-oxide surface chemistry which may relate directly to processing concerns.

Figure 1 illustrates the high degree of reproducibility obtained from base titrations performed on typical $1 \%$ suspensions over several days and under controlled experimental conditions. In particular the iep, found to be 6.4 in this case, is determined with an error not exceeding $\pm 0.05 \mathrm{pH}$ units. At higher solids concentrations, errors associated with the measurement of $\mathrm{pH}$ using a glass-membrane electrode may play a significant role in determining the overall error in the iep value. In Figure 2 we examine the effect of titration speed on esa as a function of $\mathrm{pH}$. The adjustable parameter is the delay time between each incremental titrant addition and the next measurement. Although significant variations in esa are observed with titration speed in the alkaline $\mathrm{pH}$ range, the iep (6.15 in this case), exhibits only a small spread. There is, however, indication of a trend toward increasing esa with longer delay times. The different iep in Figs. 1 and 2 is partly a consequence of using titrants which differed in dissolved $\mathrm{CO}_{2}$. This effect will be discussed at greater length in the section on Effects of Surface Contamination.

Three sample-related parameters will now be addressed, namely sample stability, volume fraction and extent of surface oxidation. The notion of sample stability is a complex problem in which several factors may play a role simultaneously. Evidence has indicated that for many non-oxide powders, the surface is unstable in aqueous solution [4], possibly undergoing hydrolysis, dissolution, or other yet undefined reactions. Other factors that may affect 
stability are changes in the state of dispersion and slow surface reactions in which equilibrium is not reached during the duration of an experiment. In most experimental scenarios, stability becomes important over a period of one to several hours, whereas in a processing application this period may be extended. The results of three contiguous cyclic acid-base titrations of $\mathrm{Si}_{3} \mathrm{~N}_{4}$ in $0.1 \mathrm{eq} / \mathrm{L} \mathrm{NaNO}{ }_{3}$ solution are plotted in Figure 3. Each cycle took about $1.6 \mathrm{~h}$ to complete, and no significant hysteresis or iep shift was observed. These results indicate that this suspension is electrochemically stable over at least a $5 \mathrm{~h}$ period.

In Equation (1) the esa signal is linearly dependent on the particle volume fraction. Typically, this relationship is observed up to roughly $10 \%$ solids $[5,9]$. However, significant deviations from dilute behavior often occur above $5 \%$ solids, and changes in the effective particle size due to aggregation may lead to further deviations from linearity. Figure 4 shows the esa signal as a function of solids concentration and suspension $\mathrm{pH}$ for a series of base titrations ( $\mathrm{pH} 4$ to 8 ). The iep is independent of volume fraction up to $6 \%$ solids, at which point a small shift is observed. Examination of the iso-pH curves in the lower part of Figure 4 indicates some deviation from linearity at $6 \%$, particularly for negative polarity values. Furthermore, the curves are not symmetric about the iep. These effects may be partially explained by irreversible changes in particle aggregate mass that occur upon passing through the iep. In any event, this data clearly illustrates a demarcation between $4 \%$ and $6 \%$, which may be interpreted as a transition from dilute to moderately concentrated regimes.

Depending on the synthesis route and treatment history, commercial $\mathrm{Si}_{3} \mathrm{~N}_{4}$ powders may contain varying amounts of oxygen, both in the bulk phase and as a surface oxide phase [6]. The presence of oxygen greatly effects the distribution and dissociation behavior of surface charge groups, which in turn modifies the iep and dispersion properties. The surface oxide exists essentially as a silicate layer encapsulating the bulk phase. Assuming a silica structure, the surface oxide thickness can be estimated from electron spectroscopy methods. In previous work we showed that the iep, determined from esa measurements on several commercial powders, decreased linearly with increasing oxide thickness [4]. Presumably, the iep decrease is a consequence of acidic silanol groups derived from surface hydrolysis. 
Linear regression analysis predicts an iep of about 9.7 for pure $\mathrm{Si}_{3} \mathrm{~N}_{4}$ and estimates a surface oxide thickness of $1.5 \mathrm{~nm}$ is necessary to obtain a silica-like surface. Additionally, experiments indicate that surface oxide can be removed and the iep increased using mild surface treatment such as aqueous soxhlet extraction.

\section{The Role of Ions in Solution}

There are generally four mechanisms by which ions dissolved in solution can affect the electroacoustic measurement of particulates in suspension: the ion vibration potential, electrostatic screening, specific adsorption and heterogeneous precipitation. In the first mechanism, or more accurately its reciprocal effect in an applied ac potential, the ions make an independent contribution to the esa signal and phase angle. This occurs as a result of, and the amplitude is proportional to, the difference in relative effective mass between cations and anions during field induced charge separation. The ion vibration potential was predicted by Debye in 1933 [10], and has been used to obtain information about ion size in solution. For colloid systems this effect represents a background signal that may require a correction. The ion signal is relatively small in most cases, but can become significant when the particle concentration is low, the ionic strength is high or the measurement is made close to the iep of the particles. Additionally, this signal can cause the apparent iep to shift, and lead to an incorrect interpretation of results [11]. Figure 5 shows the measured ion contribution to the esa signal for a series of ion pairs at $0.1 \mathrm{eq} / \mathrm{L}$ concentration. This data may be used to predict potential background problems with an electrolyte. A blank measurement of the ion signal from an equivalent solution or supernatant, followed by a simple subtraction procedure will generally suffice as background correction. Since the ion signal is nearly pHindependent from $\mathrm{pH} 5$ to 9 , a single background measurement is usually sufficient in this range. Of the four mechanisms listed above, only the ion vibration potential is unrelated to the solid phase.

The second mechanism, screening, is generally indifferent toward the nature of the particle surface, and depends only on ionic strength $I$, through it's affect on the electrical double-layer parameter $\kappa[3]$ : 


$$
\kappa=3.288 \sqrt{I}\left(\mathrm{~nm}^{-1}\right)
$$

in water at $25^{\circ} \mathrm{C}$. As $I$ increases, the effective thickness of the counter-ion envelope $1 / \kappa$ around each particle is compressed (potential falls off more rapidly), which reduces the zeta potential and mobility accordingly. This effect is illustrated in Fig. 6 for $\mathrm{Si}_{3} \mathrm{~N}_{4}$ in $\mathrm{NaNO}_{3}$ solutions of increasing $I$. The data are presented in the form of dynamic mobility calculated directly from esa. The well-defined common intersection point (cip) in Fig. 6 is indicative of an "indifferent" electrolyte. The cip defines the "natural" iep which characterizes a powder surface, while the absence of a cip implies that specific interactions are involved. The latter situation occurs when an ion interacts chemically with the particle surface, penetrating the inner or compact part of the double-layer. This type of interaction may consist of varying degrees of covalent or coordinate (complexation) bonding, and constitutes the third mechanism by which ions affect the esa measurement.

Generally, specific adsorption of anions and cations will cause the iep to shift toward the acidic and alkaline directions, respectively (see Fig. 7). This affect is easily explained. In the case of specific anion adsorption, more protons are necessary to compensate for the additional negative charge brought to the surface by the anion. The $\mathrm{pH}$ at which this new charge equilibrium is balanced (the iep) must therefore decrease. Specific adsorption of cations is explained in an analogous manner.

In Fig. 8, mobility curves calculated from esa measurements of $\mathrm{Si}_{3} \mathrm{~N}_{4}$ suspensions are compared for the (potassium) halide series relative to the curve for $\mathrm{NaNO}_{3}$. It is seen that only fluoride, which is known to interact strongly with silicon, gives any indication of specificity for the $\mathrm{Si}_{3} \mathrm{~N}_{4}$ surface, and then only a small interaction is apparent. The other electrolytes are indifferent. Figure 9 shows the mobility curves for $\mathrm{Si}_{3} \mathrm{~N}_{4}$ in the presence of several (potassium and sodium) oxyanions. The interaction with this class of ions is observably stronger. This is particularly true for sodium hexametaphosphate $\left(\mathrm{NaPO}_{3}\right)_{6}$, which does not have a measurable iep. Figure 10 shows mobility curves for divalent cations including the alkaline-earth (nitrate) series and the transition metal ion copper. The alkaline- 
earths behave similarly, exhibiting weak specificity with no clear dependence upon ion size. In contrast, the interaction of $\mathrm{Cu}^{2+}$ with the $\mathrm{Si}_{3} \mathrm{~N}_{4}$-solution interface is clearly more complex, and belongs to a separate class. This type of mobility curve is typical for hydrolyzable metal cations on hydrous oxide surfaces [12], and is an example of the fourth mechanism, heterogeneous precipitation.

The interaction of hydrolyzable cations with $\mathrm{Si}_{3} \mathrm{~N}_{4}$ is illustrated in Fig. 11, which shows a series of dynamic mobility curves for suspensions containing increasing yttrium nitrate concentrations in $0.01 \mathrm{eq} / \mathrm{L} \mathrm{NaNO}_{3}$ as supporting electrolyte. At acidic $\mathrm{pH}$ values (below 6) the hydrated $\mathrm{Y}^{3+}$ ion adsorbs weakly to the positively charged $\mathrm{Si}_{3} \mathrm{~N}_{4}$ surface. With increasing concentration of $\mathrm{Y}\left(\mathrm{NO}_{3}\right)_{3}$, a maximum in the mobility curve develops between $\mathrm{pH}$ 6 and 8 and the iep is shifted toward increasingly alkaline $\mathrm{pH}$ values. The sharp increase in mobility which occurs near $\mathrm{pH} 8$ is due to strong adsorption of hydrolyzed $\mathrm{Y}(\mathrm{OH})_{\mathrm{X}}{ }^{(3-\mathrm{X})+}$ and cationic polynuclear species which predominate in solution in this $\mathrm{pH}$ and concentration range [13]. As the $\mathrm{pH}$ increases, further hydrolysis leads to precipitation of a new metalhydroxide surface phase. This new phase controls the electrokinetic behavior of the dispersed particles, conferring on them the iep of the freshly precipitated hydroxide. This process has been investigated as a method for adding sintering aids based on oxides of yttrium, aluminum and the rare-earths [1].

\section{Effects of Surface Contamination}

Commercial $\mathrm{Si}_{3} \mathrm{~N}_{4}$ powders often contain minor and trace constituents that may affect surface properties. Oxygen and carbon are ubiquitous, being found to some degree on all non-oxide powder surfaces exposed to air. The effect of oxygen on the electrochemical behavior was discussed previously. Some other common elemental contaminants are $\mathrm{Fe}, \mathrm{Al}$, $\mathrm{Ca}, \mathrm{Mg}, \mathrm{Co}, \mathrm{Ni}$ and $\mathrm{F}$. The effect these contaminants may have on the surface properties are contingent upon their form and their concentrations near the surface. Still, based on data presented above we can predict that metallic impurities such as Fe, if they exist in oxidized forms, will generally produce a more basic $\mathrm{Si}_{3} \mathrm{~N}_{4}$ surface (higher iep). Synthetic routes based on nitridation of $\mathrm{Si}$ sometimes employ hydrofluoric acid leaching to remove residual $\mathrm{Si}$, resulting in fluorine contamination [4]. This impurity will most likely exist as $\overrightarrow{\mathrm{F}}$ or 
bound to silicon near the surface. Our results indicate that $\mathrm{F}^{-}$interacts weakly with $\mathrm{Si}_{3} \mathrm{~N}_{4}$, causing a small increase in surface acidity (lower iep). Contamination may also occur during processing if the powders come in contact with metallic surfaces, for instance in the milling operation.

Another potential source of contamination is atmospheric $\mathrm{CO}_{2}$, a component that is not normally controlled in the processing environment, but may affect interpretation of titration experiments. It has previously been demonstrated for hydrous iron oxides [14] that $\mathrm{CO}_{2}$ dissolved in solution can react chemically with surface hydroxyls to form an adsorbed carbonate anion. Evidence for this type of interaction has recently been observed in our laboratory for $\mathrm{Si}_{3} \mathrm{~N}_{4}$ using esa measurements. In this case, contamination is thought to have originated in the base titrant, where $\mathrm{CO}_{2}$ has a substantial solubility. During a series of titration experiments, a sudden and consistent shift in the measured iep was observed. All samples were purged with nitrogen, had come from the same powder batch and were analyzed over a relatively short period of time under identical conditions. The difference had occurred when the base titrant being used was replaced with a new low-carbonate standard solution. Figure 12 illustrates this effect for $2 \%$ volume fraction $\mathrm{Si}_{3} \mathrm{~N}_{4}$ in $0.01 \mathrm{eq} / \mathrm{L} \mathrm{NaNO}$. These titrations were performed using a low-carbonate $0.1 \mathrm{~N} \mathrm{NaOH}$ standard and a prepared $\mathrm{NaOH}$ solution in which $\mathrm{CO}_{2}$ contamination was not controlled. Further investigation of the $\mathrm{CO}_{2}$ effect is on-going, but caution should be exercised when preparing or storing alkaline titrant solutions if $\mathrm{CO}_{2}$ is a suspect reactant.

\section{SUMMARY}

We have employed electroacoustic methods to characterize and study the electrokinetic behavior of $\mathrm{Si}_{3} \mathrm{~N}_{4}$ precursor powders in moderately concentrated aqueous suspension. The electrokinetic properties of a suspension are closely connected to its dispersion properties, and often reflect changes which occur as a result of reactions at the particle-solution interface. Various parameters that affect the measurement of $\mathrm{Si}_{3} \mathrm{~N}_{4}$ suspensions have been analyzed. The effects of dissolved inorganic ionic species on the electrokinetic sonic 
amplitude (esa) has been examined in terms of four general mechanisms. These mechanisisms are the result of ion electroacoustic effects or ion interactions (screening, adsorption and precipitation) at the solid-solution interface. The implication of these investigations regarding the effects of contamination and the use of supporting electrolytes is discussed in detail.

\section{REFERENCES}

[1]. P. Greil, "Processing of Silicon Nitride Ceramics," Mat. Sci. Engineer., A109, 27-35 (1989).

[2]. G. Busca, V. Lorenzelli, G. Porcile, M. Baraton, P. Quintard and R. Marchand, "FT -IR Study of the Surface Properties of Silicon Nitride," Mat. Chem. Phys., 14, $123-140$ (1986).

[3]. R. J. Hunter, "Zeta Potemial in Colloid Science," Academic Press, London (1988).

[4]. V. A. Hackley, P. S. Wang and S. G. Malghan, "Effects of Soxhlet Extraction on the Surface Oxide Layer of Silicon Nitride Powders," to be published in Mat. Chem. Phys. (1993).

[5]. J. P. Pollinger, D. D. Newson and J. J. Nick, "The Implementation of Acoustophoresis for the Characterization of Silicon Nitride Based Aqueous Suspensions, Multicomponent Silicon Nitride Based Aqueous Suspensions," to be published in Ceramic Transactions (1993).

[6]. P. Greil, "Evaluation of Oxygen Content on Silicon Nitride Powder Surface from the Measurement of the Isoelectric Point," J. Europ. Ceram. Soc., 7, 353-59 (1991).

[7]. L. Bergström, "Surface Chemistry of Silicon Nitride Powders: Electrokinetic Behavior and ESCA Studies," Colloids Surf., 49, 183-197 (1990).

[8]. R. W. O’Brien, "The Electroacoustic Equations for a Colloidal Suspension," J. Fluid Mech., 212, 81-93 (1990).

[9]. V. A. Hackley, NIST, unpublished work.

[10]. P. Debye, "A Method for the Determination of the Mass of Electrolytic Ions," J. Chem Phys., 1, 13-16 (1933).

[11]. V. A. Hackley, R. S. Premachandran and S. G. Malghan, "Electrokinetic Sonic Analysis of Silicon Nitride Suspensions," to be published in Ceramic Transactions (1993). 
[12]. R. O. James and T. W. Healy, "Adsorption of Hydrolyzable Metal Ions at the Oxide Water Interface," J. Colloid Interface Sci., 40, 53-64 (1972).

[13]. C. F. Baes, Jr. and R. E. Mesmer, "The Hydrolysis of Cations," John Wiley \& Sons, New York (1976).

[14]. W. A. Zeltner and M. A. Anderson, "Surface Charge Development at the Goethite/Aqueous Solution Interface: Effects of $\mathrm{CO}_{2}$ Adsorption, "Langmuir, 4, 469-74 (1988); V. A. Hackley, University of Wisconsin, unpublished data. 



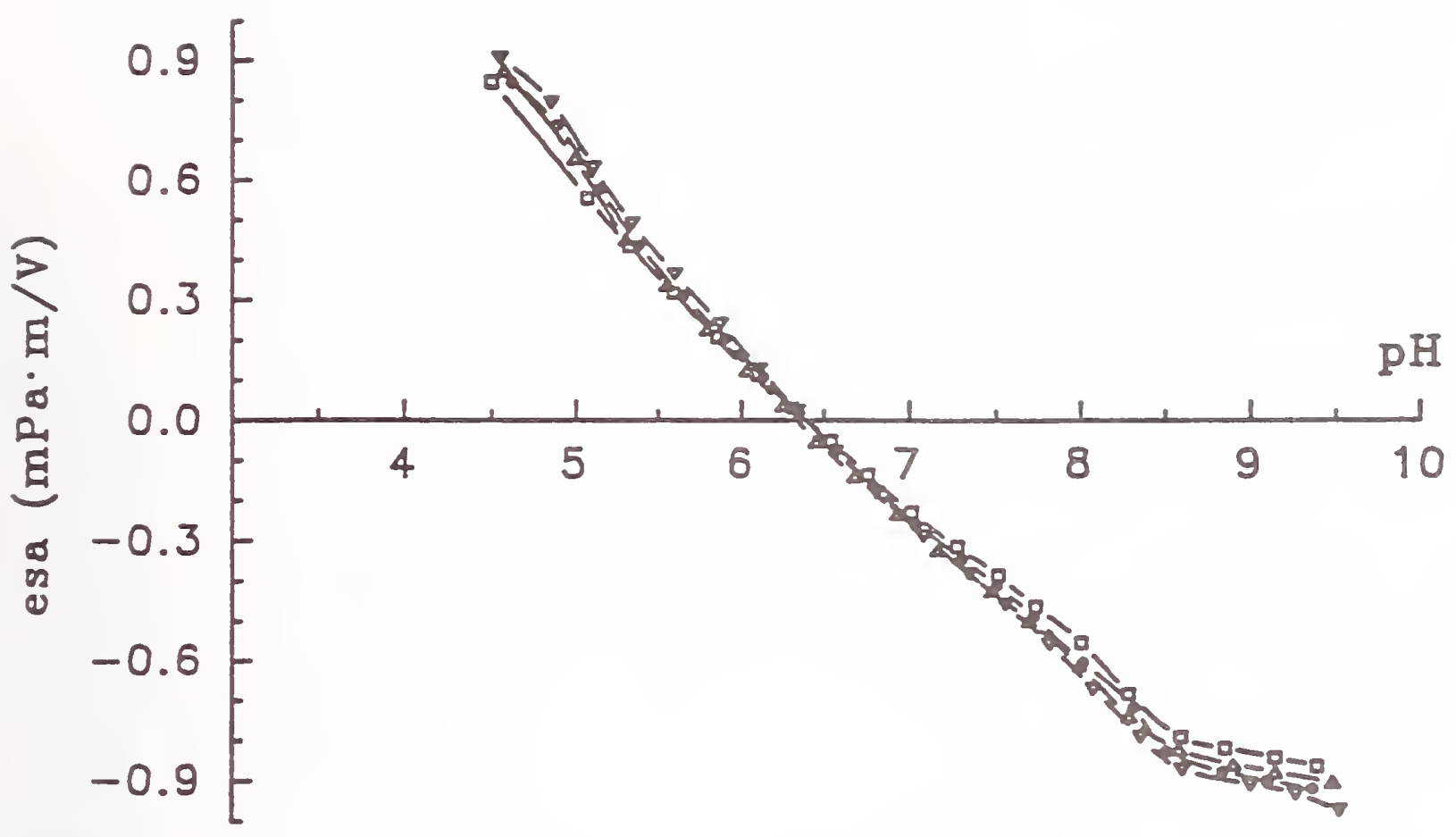

Fig. 1 Reproducibility of electroacoustic measurements and isoelectric point determination during $\mathrm{NH}_{4} \mathrm{OH}$ titrations of $1 \%$ volume fraction $\mathrm{Si}_{3} \mathrm{~N}_{4}$ aqueous suspensions.

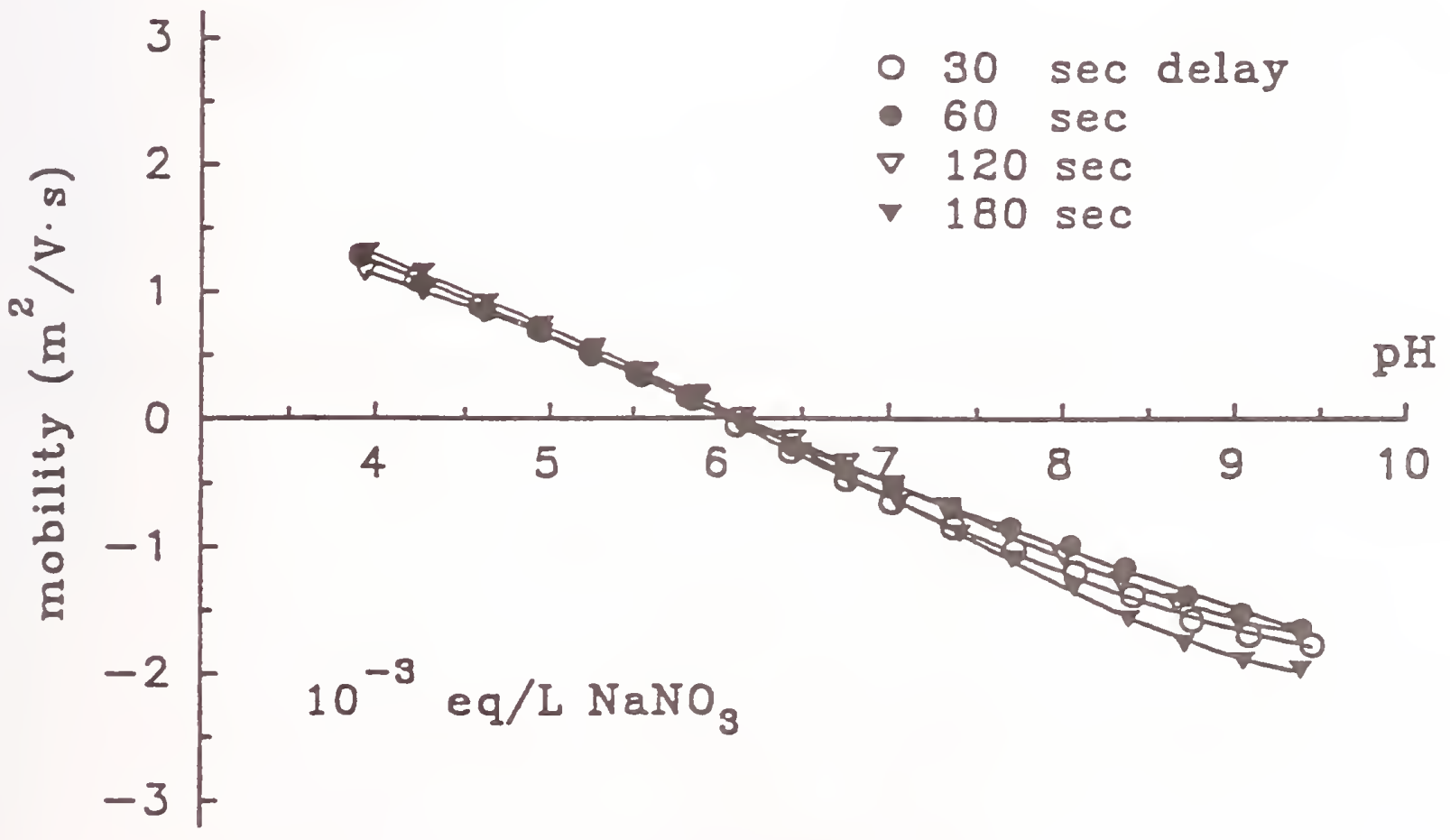

Fig. 2 The effect of the delay time between incremental titrant additions on the electroacoustic measurement of dynamic mobility for a $2 \%$ volume fraction $\mathrm{Si}_{3} \mathrm{~N}_{4}$ suspension. 


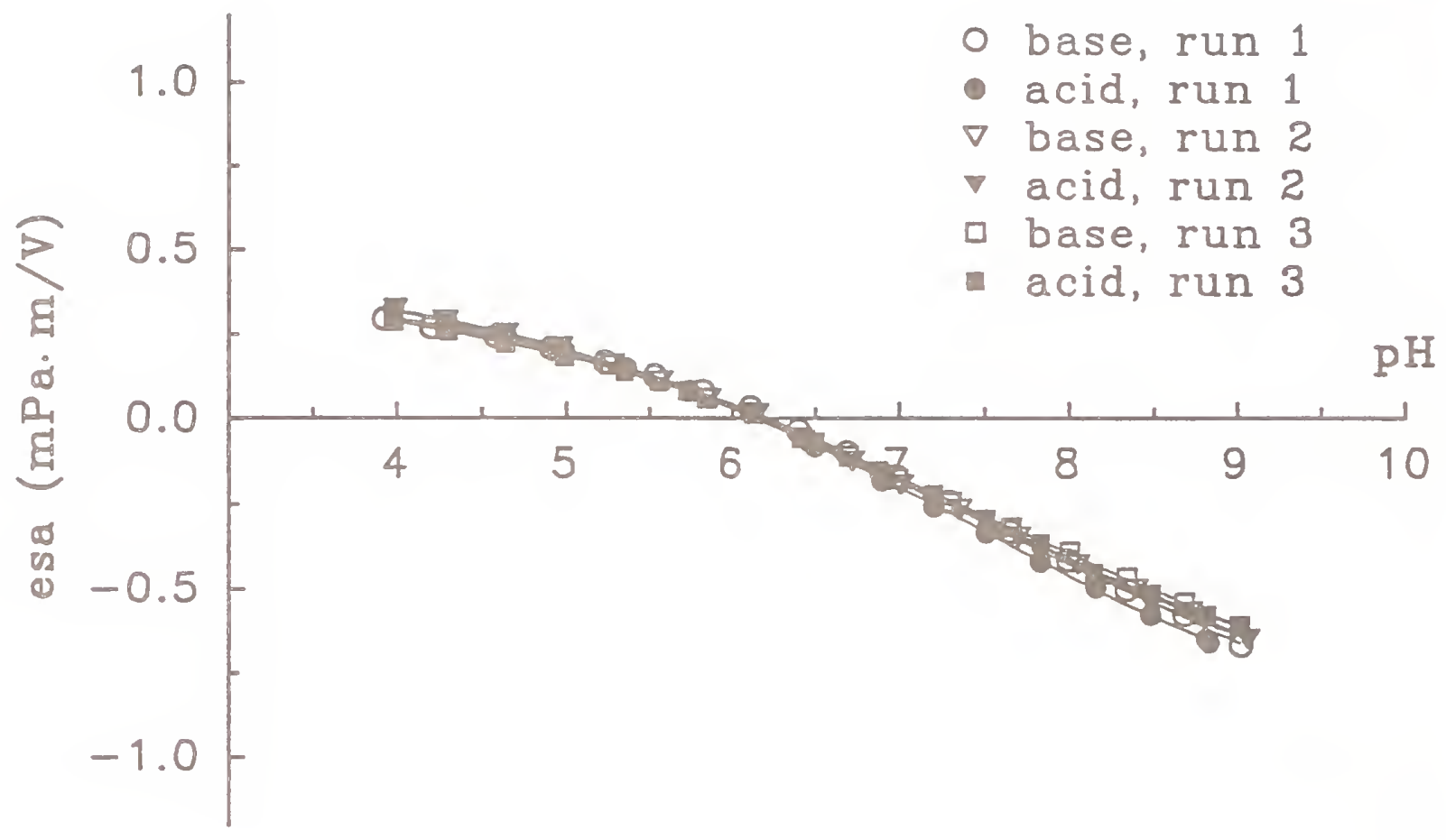

Fig. 3 The stability of $\mathrm{Si}_{3} \mathrm{~N}_{4}$ during three contiguous acid-base titration cycles in $0.1 \mathrm{eq} / \mathrm{L} \mathrm{NaNO}_{3}$. 

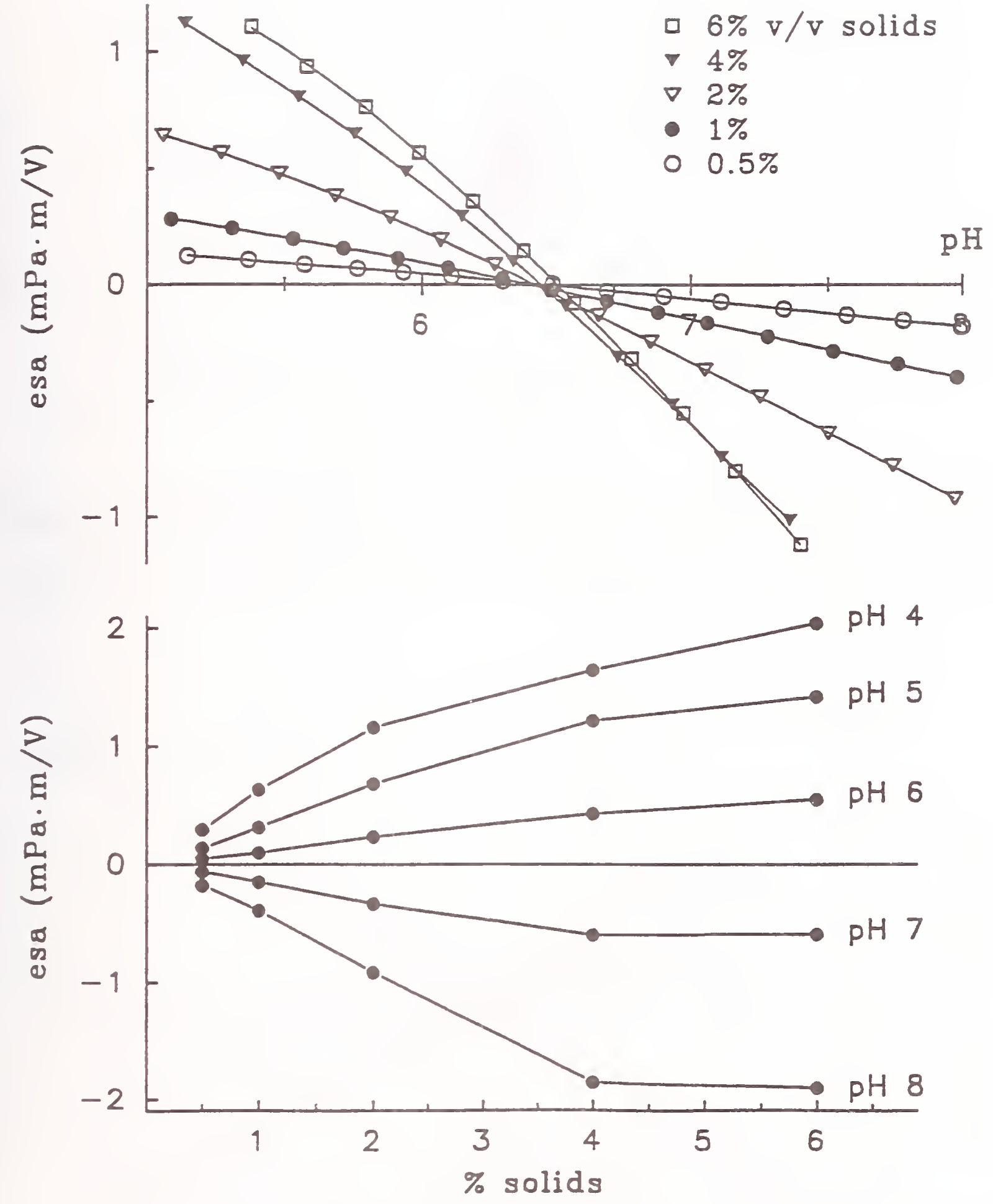

Fig. 4 The dependence of the electrokinetic sonic amplitude and isoelectric point on the volume fraction of $\mathrm{Si}_{3} \mathrm{~N}_{4}$ and suspension $\mathrm{pH}$. 


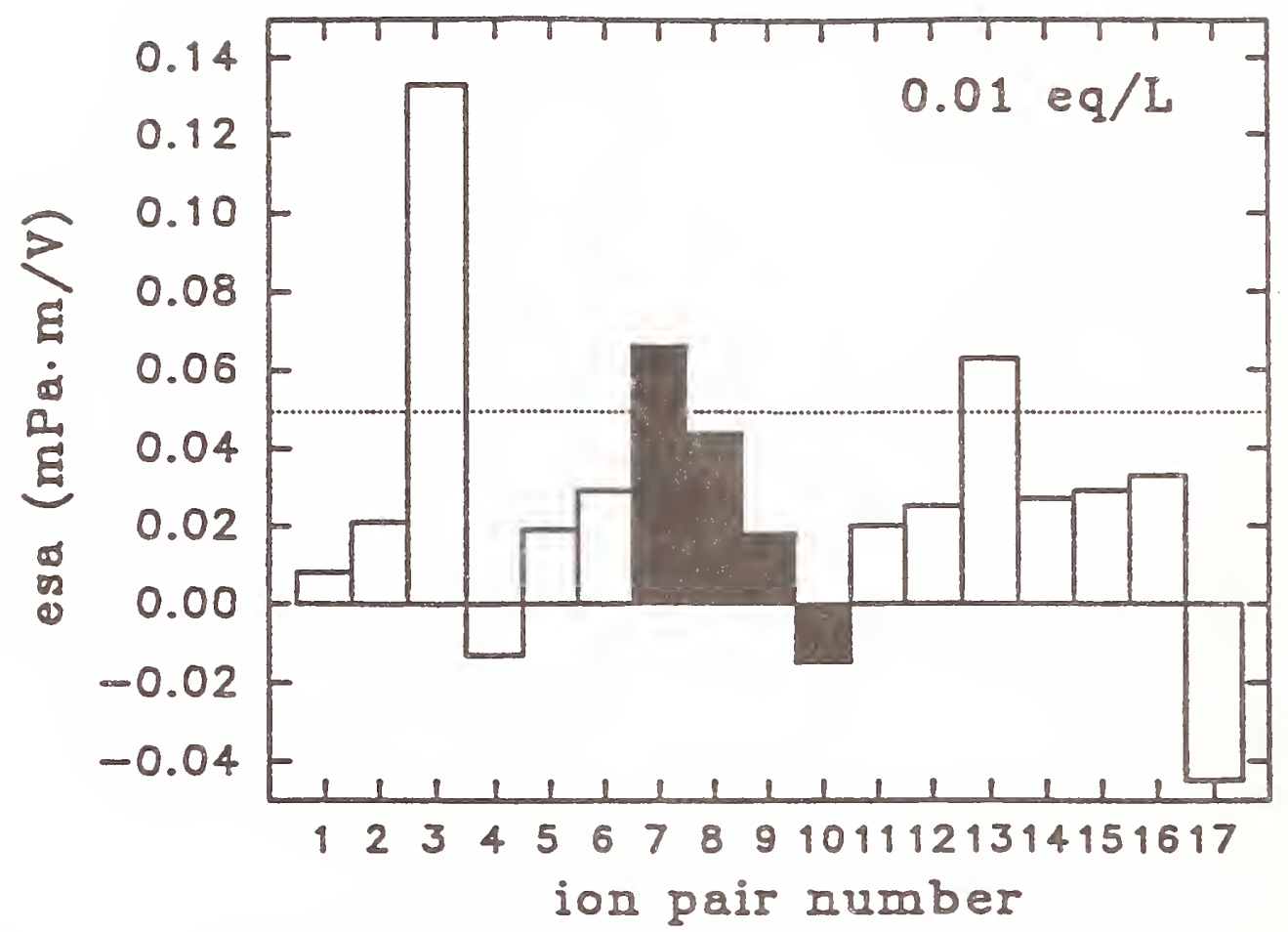

Fig. 5 The background esa signal for a series of ion pairs in solution at $0.01 \mathrm{eq} / \mathrm{L}$. Ion pair number corresponds to following species: (1) $\mathrm{NaNO}_{3}$, (2) $\mathrm{KNO}_{3}$, (3) $\mathrm{CsNO}_{3}$, (4) $\mathrm{NH}_{4} \mathrm{NO}_{3}$, (5) LiCl, (6) $\mathrm{NaCl}$, (7) $\mathrm{KF}$, (8) $\mathrm{KCl}$, (9) $\mathrm{KBr}$, (10) $\mathrm{KI}$, (11) $\mathrm{Mg}\left(\mathrm{NO}_{3}\right)_{2}$, (12) $\mathrm{Ca}\left(\mathrm{NO}_{3}\right)_{2}$, (13) $\mathrm{Ba}\left(\mathrm{NO}_{3}\right)_{2}$, (14) $\mathrm{K}_{2} \mathrm{CrO}_{4}$, (15) $\mathrm{Na}_{2} \mathrm{SiO}_{3}$, (16) $\mathrm{K}_{2} \mathrm{HPO}_{4}$, (17) $\left(\mathrm{NaPO}_{3}\right)_{6}$.

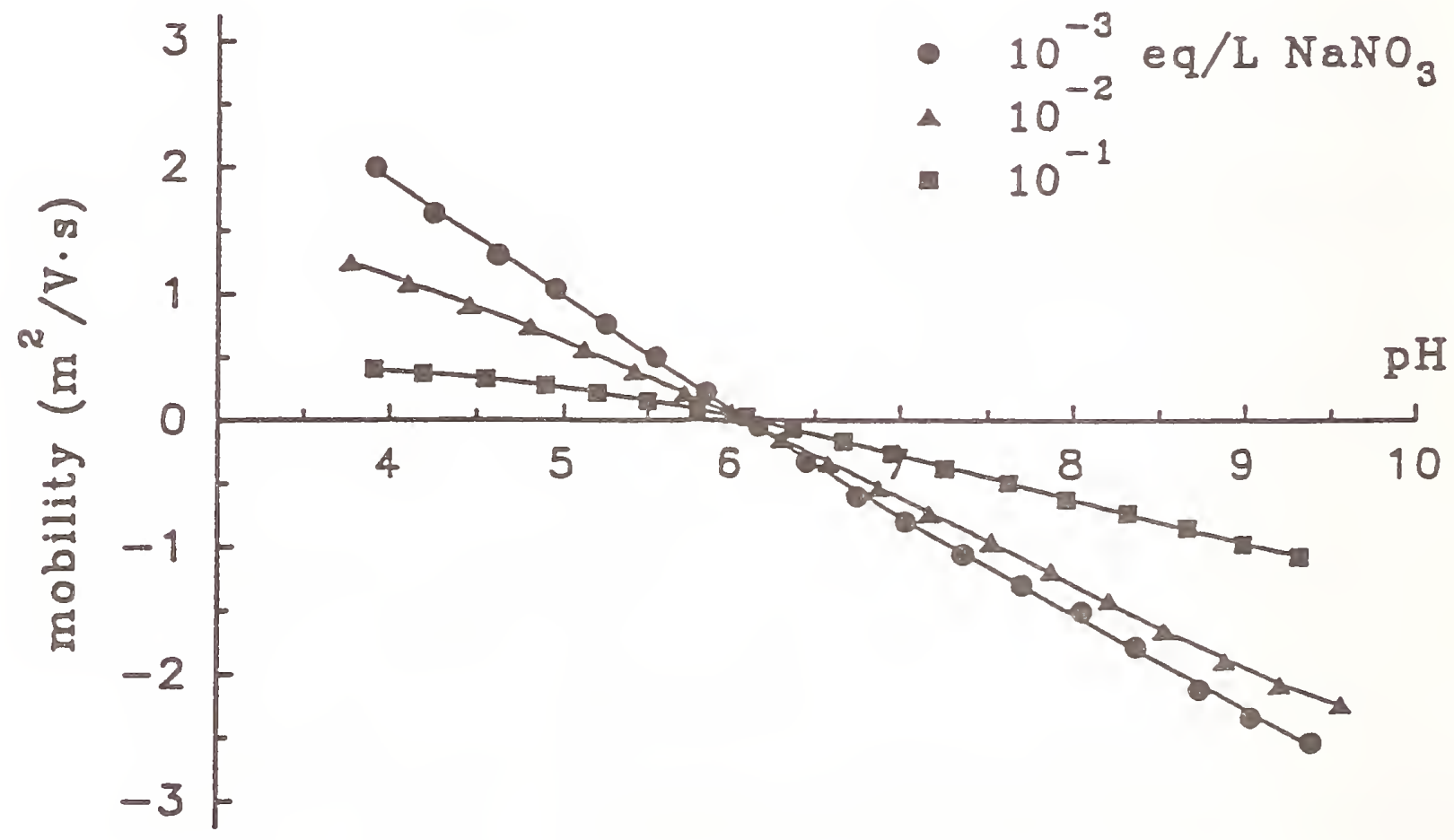

Fig. 6 Dynamic mobility as a function of $\mathrm{pH}$ for $2 \%$ volume fraction $\mathrm{Si}_{3} \mathrm{~N}_{4}$ in $\mathrm{NaNO}_{3}$ solution at three ionic strengths. 


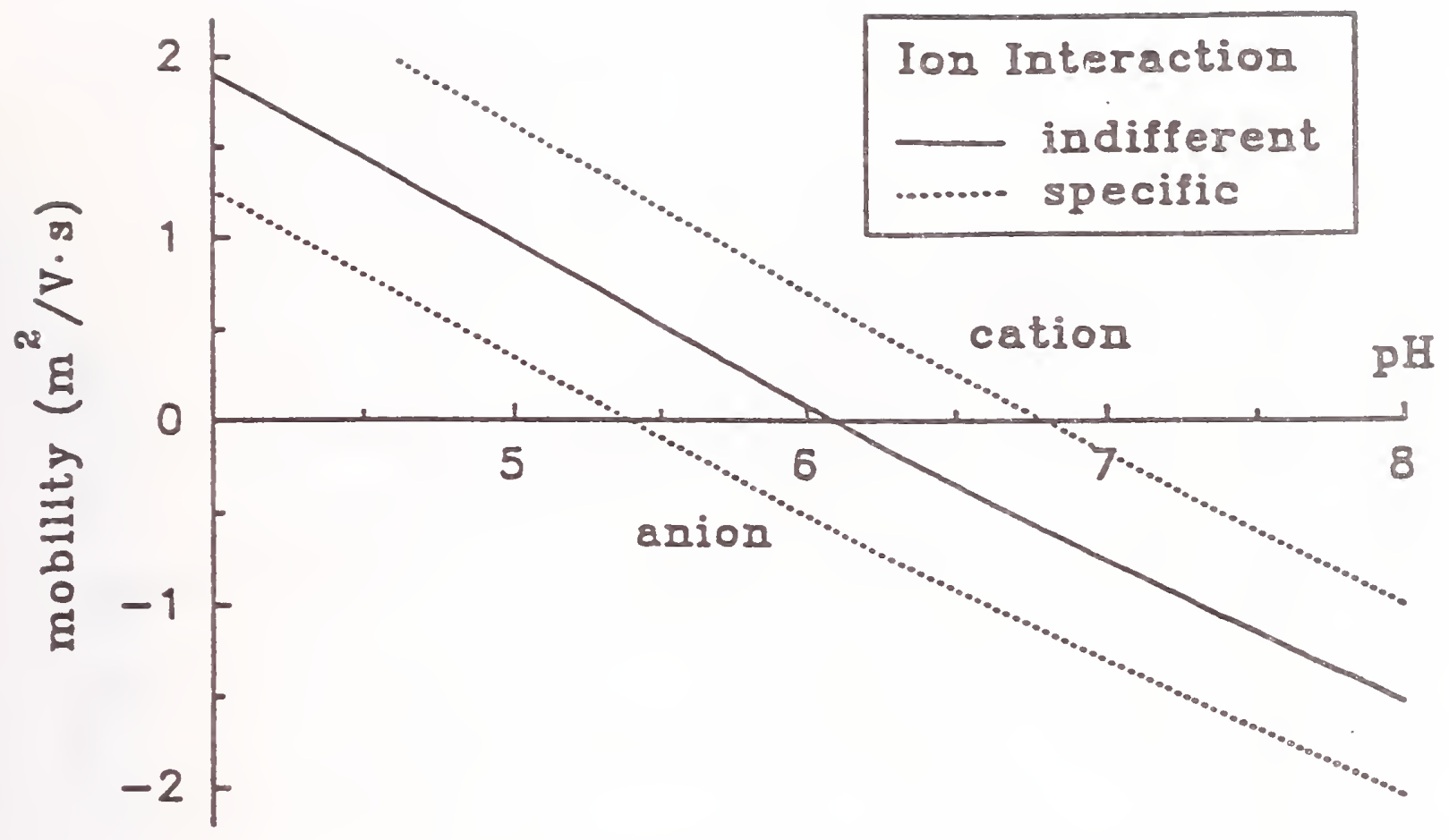

Fig. 7 Schematic illustration of the effect of specific adsorption on the mobility and isoelectric point of a colloid suspension.

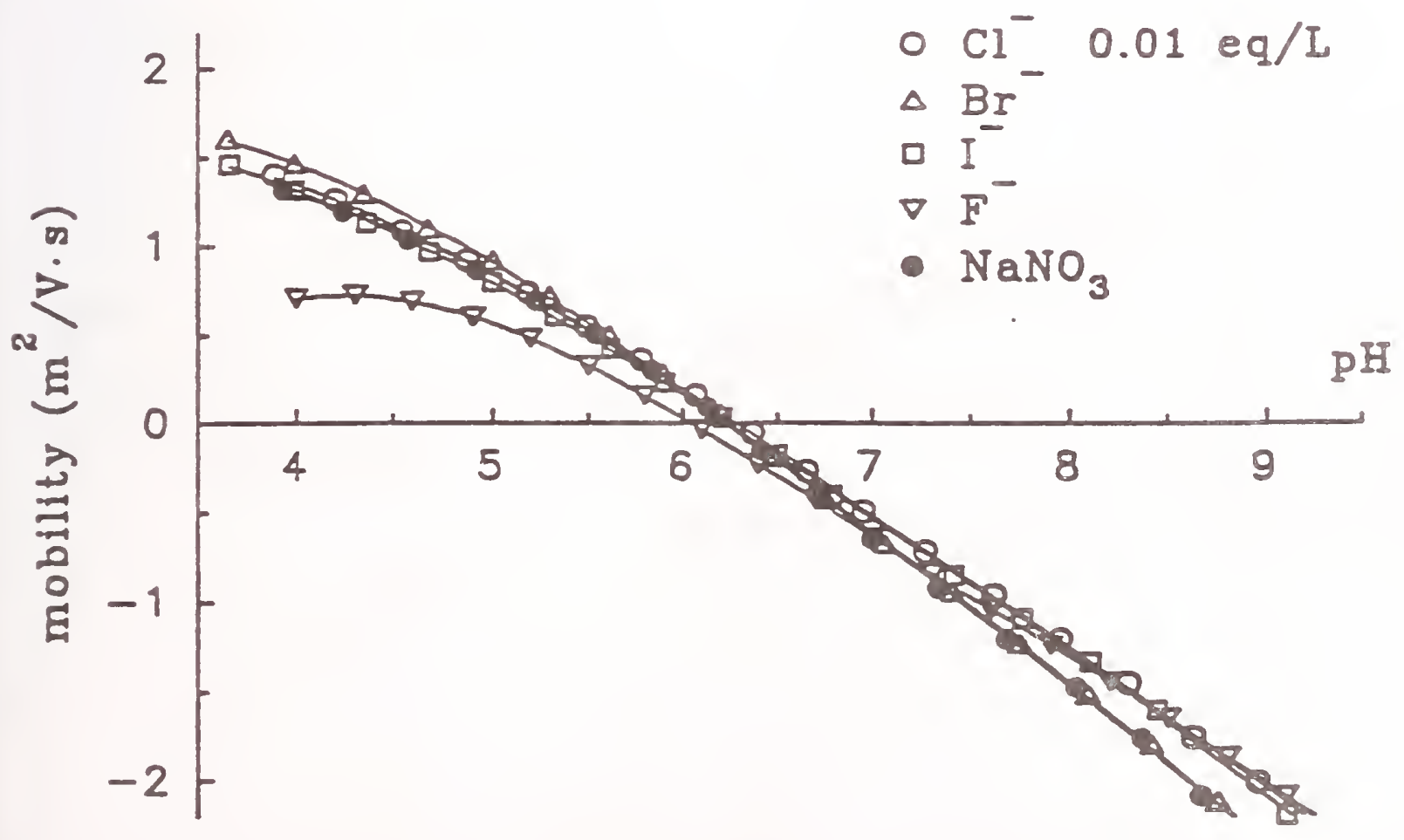

Fig. 8 Dynamic mobility as a function of $\mathrm{pH}$ for $2 \%$ volume fraction $\mathrm{Si}_{3} \mathrm{~N}_{4}$ in a series of (potassium) halide solutions at $0.01 \mathrm{eq} / \mathrm{L}$, relative to the curve for $\mathrm{NaNO}_{3}$. Fluoride shows a small degree of specificity for the surface. 


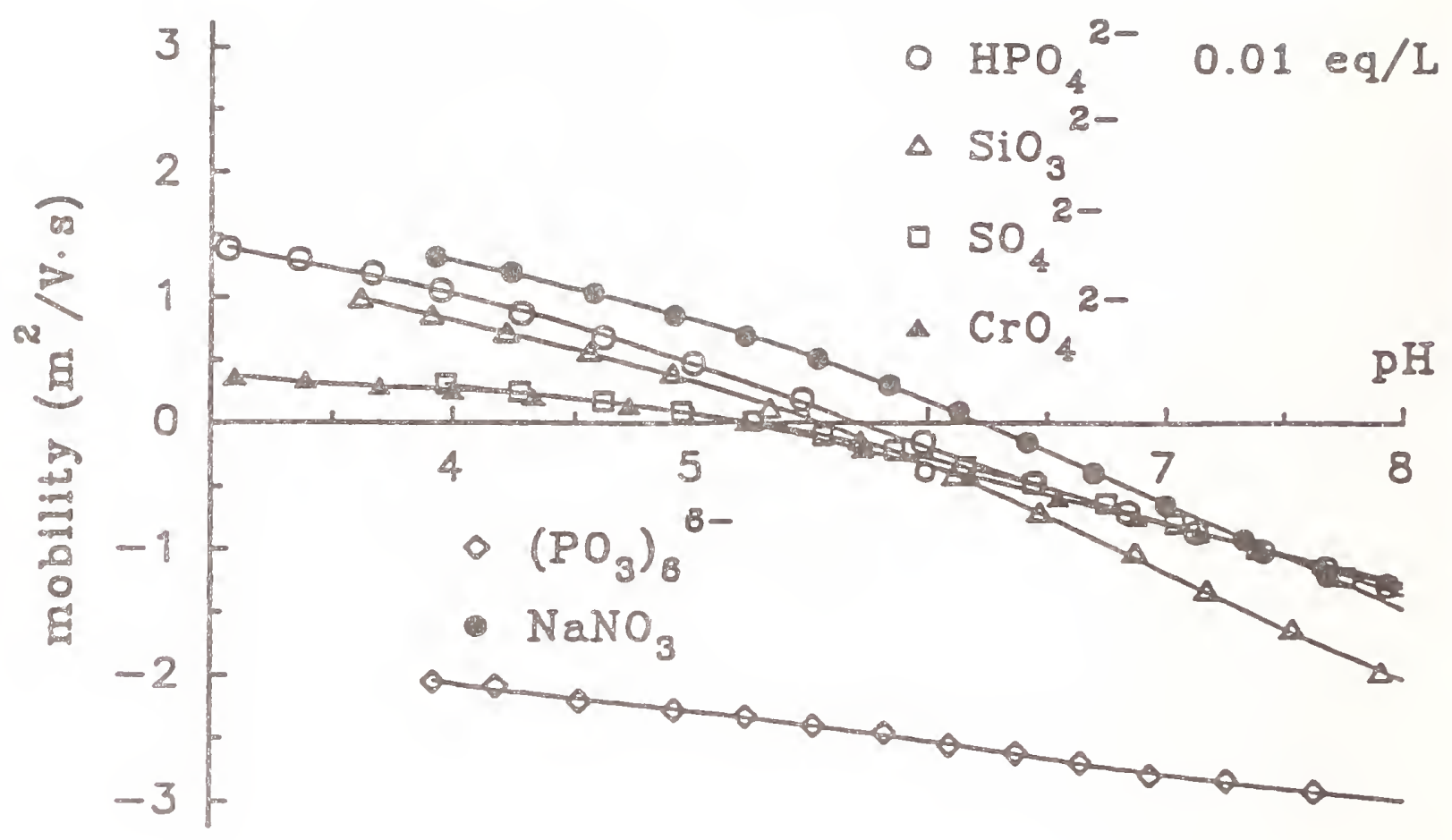

Fig. 9 Dynamic mobility as a function of $\mathrm{pH}$ for $2 \%$ volume fraction $\mathrm{Si}_{3} \mathrm{~N}_{4}$ in a series of oxyanion solutions at $0.01 \mathrm{eq} / \mathrm{L}$, relative to the curve for $\mathrm{NaNO}_{3}$.

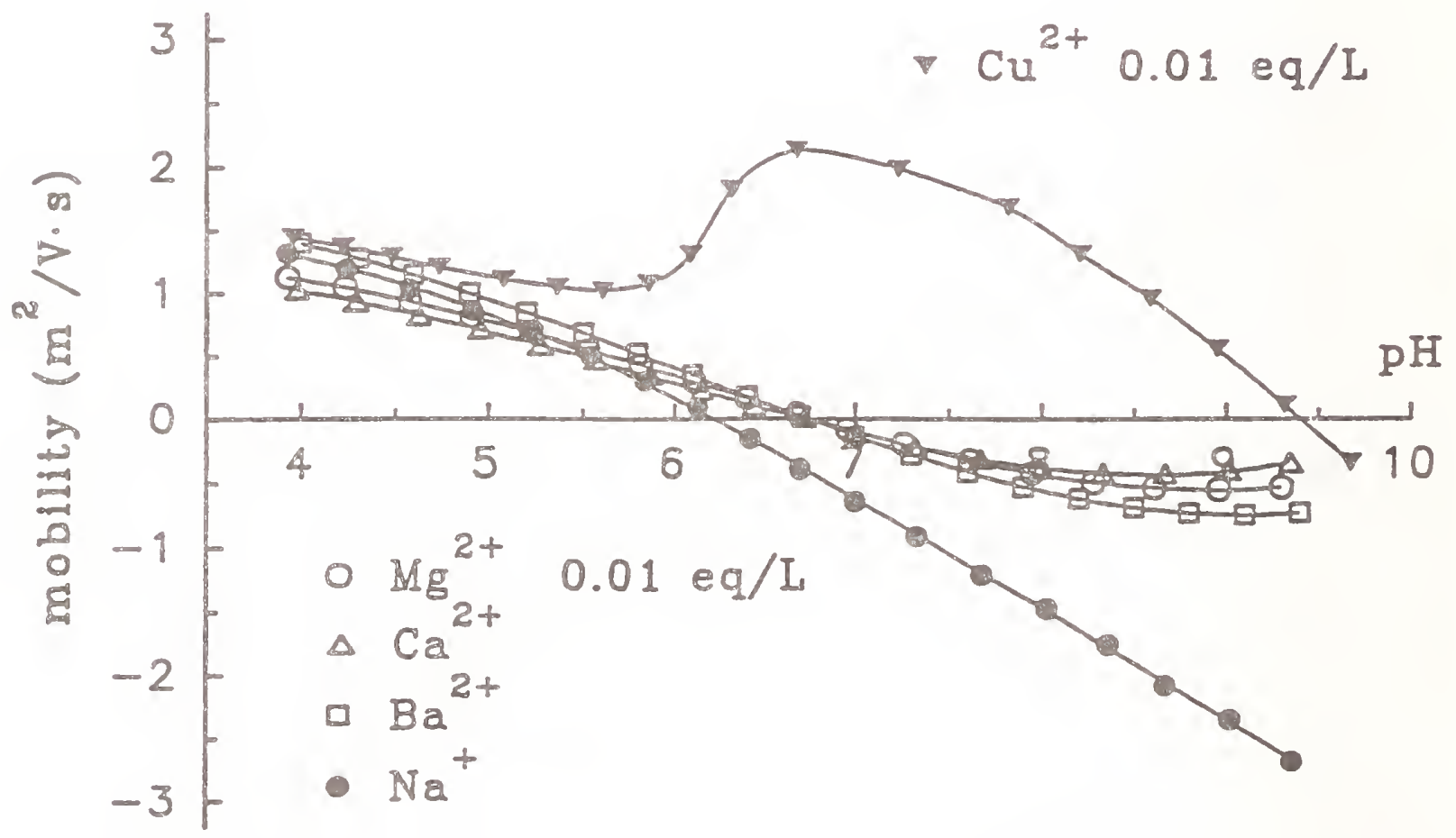

Fig. 10 Dynamic mobility as a function of $\mathrm{pH}$ for $2 \%$ volume fraction $\mathrm{Si}_{3} \mathrm{~N}_{4}$ in a series of divalent cation (nitrate salt) solutions at $0.01 \mathrm{eq} / \mathrm{L}$, relative to the curve for $\mathrm{NaNO}_{3}$. Note the distinct difference in behavior between the hydrolyzable $\mathrm{Cu}^{2+}$ ion and the alkaline-earth ions. 


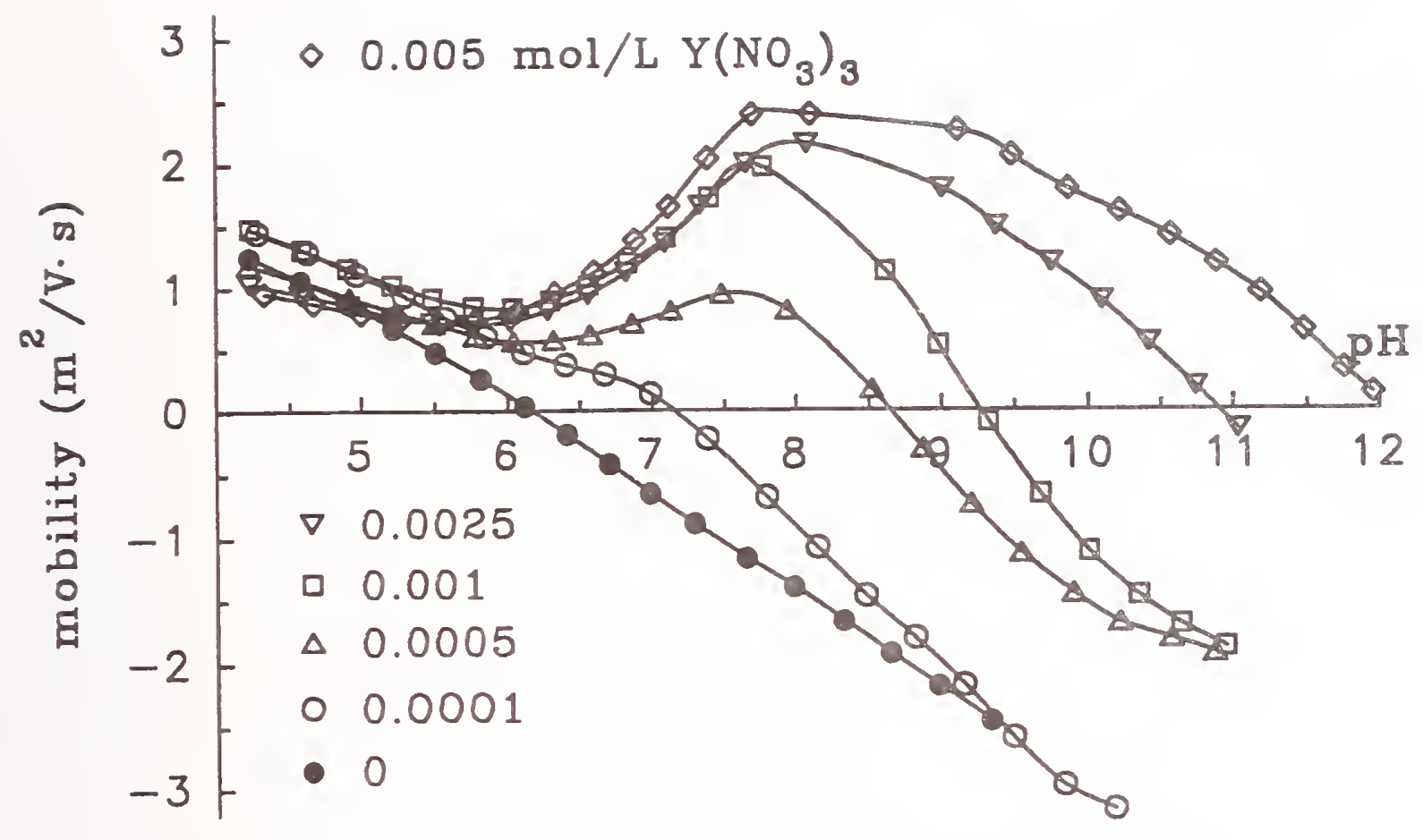

Fig. 11 Dynamic mobility as a function of $\mathrm{pH}$ and $\mathrm{Y}\left(\mathrm{NO}_{3}\right)_{3}$ concentration for $2 \%$ volume fraction $\mathrm{Si}_{3} \mathrm{~N}_{4}$ in $0.01 \mathrm{eq} / \mathrm{L} \mathrm{NaNO}_{3}$. The formation of a maximum in the mobility curve is due to the heterogeneous precipitation of $\mathrm{Y}(\mathrm{OH})_{3}$ at the $\mathrm{Si}_{3} \mathrm{~N}_{4}$ surface.

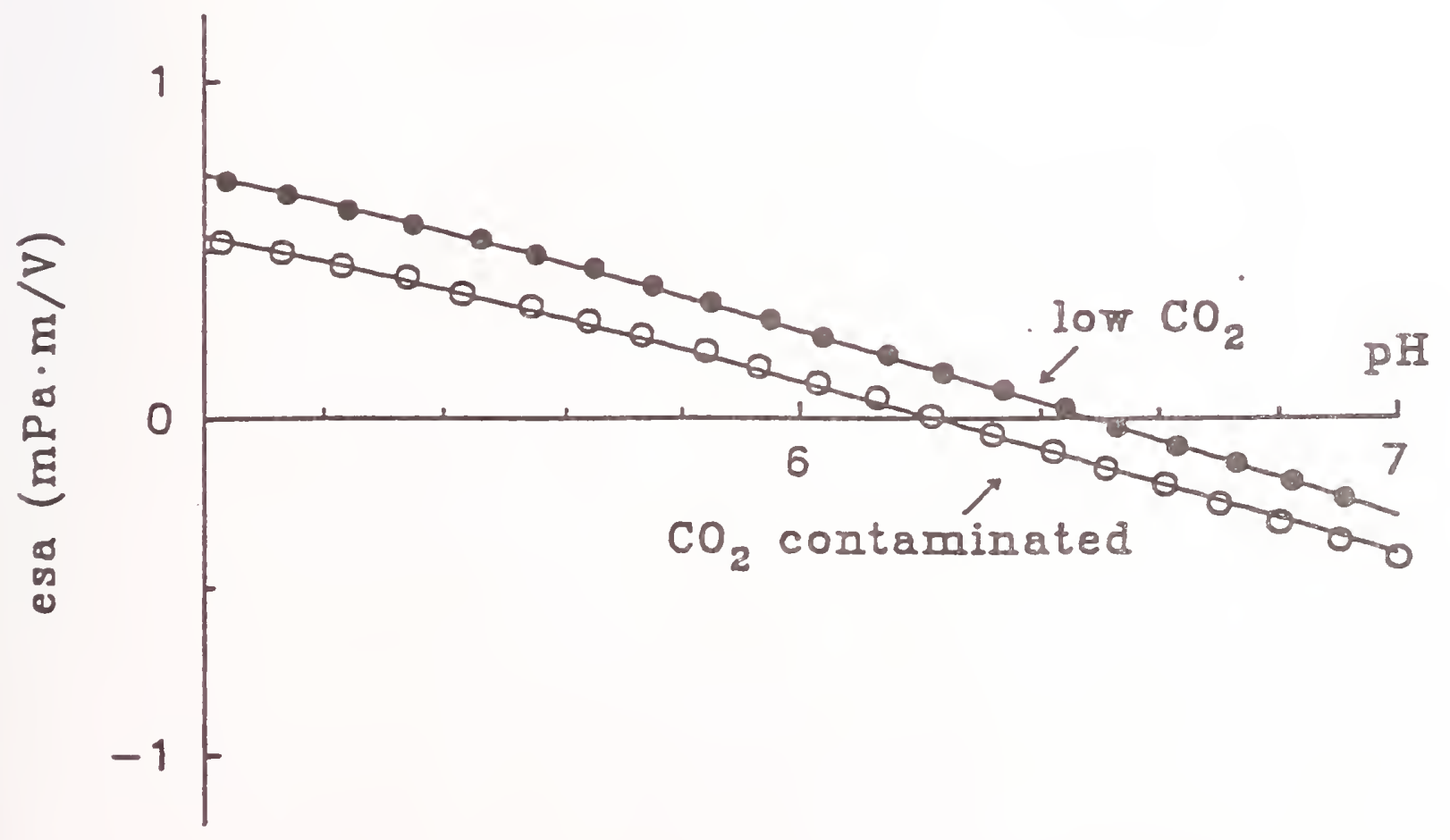

Fig. 12 The effect of $\mathrm{CO}_{2}$ contamination of a base titrant on the isoelectric point of a $2 \%$ volume fraction $\mathrm{Si}_{3} \mathrm{~N}_{4}$ suspension in $0.01 \mathrm{eq} / \mathrm{L} \mathrm{NaNO}_{3}$. 



\title{
SESSION IV
}

\section{CLAYS, CEMENTS, AND COAL}

\section{Session Chairman}

\author{
B. M. Moudgil \\ University of Florida
}


Yan C. Huang ${ }^{2}$

Research Associate

Department of Chemistry

Lehigh University

Bethlehem, PA

USA

Frederick M. Fowkes*

Professor of Chemistry

Department of Chemistry

Lehigh University

Bethlehem, PA

USA

*Deceased.
Nigel D. Sanders

Project Manager

Surface \& Colloid Science

Specialty Minerals Inc.

Bethlehem, PA

USA

Thomas B. Lloyd

Research Scientist

Department of Chemistry

Lehigh University

Bethlehem, PA

USA

\section{ABSTRACT}

The rheology of coating colors is now well recognized to be a function of both particle packing and surface chemical effects. In this paper we explore the complex rheology of precipitated calcium carbonate (PCC) slurries where surface chemistry has been carefully controlled. Surface properties of PCC were characterized with a combination of surface charge measurements (via a Matec electrokinetic sonic amplitude analyzer) and adsorption calorimetry. Viscoelasticity was assessed with a Bohlin rheometer. We have found that the unique surface chemical features of PCC (namely, the presence of strongly adsorbed calcium ions as interactive sites) determine the nature of the binding configuration of conventional anionic polyelectrolytes to the PCC surface and hence the rheology of the slurry. We further suggest ways in which these unique features may be used to improve the rheology of a PCCbased coating color where particle size distribution (packing) has been chosen to optimize coating structure.

${ }^{1}$ This paper is an updated version from the original which was presented at the 1991 TAPPI Coating Conference, Montreal, Quebec, May 1991, Copyright TAPPI 1991.

${ }^{2}$ Current Address: International Paper, Corporate Research Center, Long Meadow Road, Tuxedo Park, New York, USA 


\section{INTRODUCTION}

Precipitated calcium carbonate (PCC) is a bright, white pigment which is being used extensively for high and low gloss paper coatings. Generally a sodium polyacrylate dispersant is added to stabilize calcium carbonate slurries at high solids content. The calcium carbonate particles are generally regarded to be negatively charged, i.e. the dispersion has an anionic character. [1] The precise relationship between the charging effect of the polyelectrolyte and pigment dispersion stability in terms of rheological properties of slurries is not well known though many theories have been presented.

A recent study by the authors of this paper shows that the surface of undispersed calcium carbonate has a finite number of reactive sites $\left(1.58 \times 10^{-6}\right.$ moles $\left./ \mathrm{m}^{2}\right)$ which spontaneously and uniformly interact with calcium ions in solutions $(\Delta \mathrm{H}=-6.8 \mathrm{kcal} / \mathrm{mole}$ and $\Delta G^{\circ}=-6.9 \mathrm{kcal} / \mathrm{mole}$ at $23^{\circ} \mathrm{C}$ ). [2] Slurries of undispersed calcium carbonate in solutions of ca. $4 \mathrm{ppm}$ or above $\mathrm{Ca}^{2+}$ have a cationic character because the $\mathrm{Ca}^{2+}$ adsorption favors a positive $\zeta$-potential on the $\mathrm{CaCO}_{3}$ particles.

It has been found that determining electrostatic charging via $\zeta$-potential measurements is a useful analytical tool to monitor interactions between dispersion components and hence to access dispersion stability. [3] The purpose of the present study is to relate various rheological properties of calcium carbonate dispersions to the mechanism of electrostatic charging on the calcium carbonate particles, which is in turn related to the surface chemistry at the solid/liquid interfaces.

\section{EXPERIMENTAL}

\section{Materials}

The PCC used in the studies is the prismatic form of calcite. This material consists of $0.7 \mu \mathrm{m}$ particles with a specific surface area of $8.63 \mathrm{~m}^{2} / \mathrm{g}$ and a true density of $2.442 \mathrm{~g} / \mathrm{ml}$. Doppler electrophoretic light-scattering measurement at low solids $(0.1 \%$; Coulter DELSA 440) yield an average $\zeta$-potential of $+18.3 \pm 0.8 \mathrm{mV}$ at $\mathrm{pH}=8$ and $\mathrm{pCa}=3.1$ in $0.01 \mathrm{M}$ 
$\mathrm{KCl}$, which is in good agreement with the results of Foxall, et al. (+21 mV). [4] Ion scattering spectroscopy (ISS) and X-Ray Photoelectron Spectroscopy (ESCA) surface elemental composition data confirm that the material has a clean $\mathrm{CaCO}_{3}$ surface.

The sodium polyacrylate dispersant (NaPA, 43.7 wt \% in water) is a commercial product and is a $90 \%$ neutralized polyacrylic acid $\left(\mathrm{M}_{\mathrm{w}}=3400\right)$. Calcium chloride $\left(\mathrm{CaCl}_{2}\right.$ $2 \mathrm{H}_{2} \mathrm{O}$ ) is of reagent grade.

\section{Methods}

Potentiometric titrations were performed at room temperature with a Matec electrokinetic sonic amplitude analyzer (ESA; Model 8050), interfaced with an AT personal computer. The ESA, which can be related to particle mobility and therefore $\zeta$-potential, is the acoustic response of a pigment slurry under an external, alternating electric field (ca. 1 $\mathrm{MHz}$ ). An aliquot quantity of a titrant solution was injected stepwise by a Hamilton motorized microsyringe (Microlab 900) into the reaction vessel where ESA, conductivity and $\mathrm{pH}$ (all pre-calibrated) were simultaneously determined for each step of the addition of the titrant solution. A complete titration curve was obtained by dividing the whole titration process into several segments to ensure that the frequency of the electrical field was always optimized.

Calcium ion concentrations were determined at room temperature with a $\mathrm{Ca}^{2+}$ ion selective electrode (ISE; Orion, Model 93-20) connected to an pH/ISE analyzer (Orion, Model 940). The ion analyzer was precalibrated to the $\mathrm{Ca}^{2+}$ concentration range of interest.

Dynamic light scattering was performed at $23^{\circ} \mathrm{C}$ with a Coulter N4 MD multi-angle, photon correlation spectrometer (PCS). An instrumental capability (via a package of hardwired computer firmware) to determine the pre-scale factor and the delay time increment of the autocorrelation function was always used in the PCS experiments.

Electrophoretic light scattering was performed at $25^{\circ} \mathrm{C}$ with a Coulter Doppler-shift electrophoretic light-scattering apparatus (DELSA, Model 440) which is a PC computer software-driven instrument. The electrical field across the cell was programmed at a constant current of $30 \mathrm{~mA}$ and a frequency shift of $500 \mathrm{~Hz}$. 
Flow and viscosity curves were determined at $25^{\circ} \mathrm{C}$ with a Bohlin VOR rheometer. A coaxial cylinder (C25) with a moving cup of radius $27.5 \mathrm{~mm}$ and a fixed bob of radius 25.0 $\mathrm{mm}$ and a torsion bar of $3.76 \mathrm{gcm}$ were used.

Solution titration microcalorimetry to determine heats of interaction of the sodium polyacrylate with $\mathrm{PCC}$ and calcium ions, respectively, was performed with a Tronac 1250 batch-type microcalorimeter running in an isoperibol mode.

\section{RESULTS AND DISCUSSION}

\section{Electrostatic Charging of $\mathrm{CaCO}_{3}$ Particles in Sodium Polyacrylate Solutions}

PCC slurries, $11.54 \%$ and $20.69 \%$ by weight, were titrated with a sodium polyacrylate dispersant solution, $\zeta$-potentials were determined during the titration process by the Matec ESA-8050 system, and $20 \mathrm{~cm}^{3}$ of the NaPA titrant solution $\left(0.3 \mathrm{~g} / \mathrm{cm}^{3}\right)$ were added to each of the slurries by the end of the titration. The PCC slurries were prepared by dispersing 30 and $60 \mathrm{~g} \mathrm{CaCO}_{3}$, respectively, into $230 \mathrm{~cm}^{3} \mathrm{DDI}$ water. The $\mathrm{pH}$ of these dispersions was always about $9.5 \pm 0.2$ and rose only slightly upon addition of sodium polyacrylate in accord with a slight decrease in calcium ion concentration and the $\mathrm{CaCO}_{3}$ solubility equilibria. [2] Figures 1 and 2 depict the $\zeta$-potential and conductivity versus the NaPA addition, respectively. In Figure 2, the conductivity of the $\mathrm{CaCO}_{3}$ slurries was plotted against the NaPA concentration shown as the cumulative grams of the NaPA added per liter of the continuous phase. The conductivity curve of the $11.54 \mathrm{wt} \%$ slurry is drawn with a dotted line and a dashed line is drawn for the 20.69 wt \% slurry. Since the two slurries were prepared with the same volume of DDI water, there was no significant difference in the conductivity of the two slurries. The conductivity of the NaPA solution in particle-free, $\mathrm{CaCO}_{3}$-saturated DDI water was found to bear a linear relationship with the NaPA concentration (the solid line). The particle-free, $\mathrm{CaCO}_{3}$-saturated DDI water was prepared by storing DDI water over $\mathrm{CaCO}_{3}$ and then filtering the $\mathrm{CaCO}_{3}$ suspension through $0.2 \mu \mathrm{m}$ pore-size syringe filters (Acrodisc Chemical Resistant, Gelman Sciences).

Comparing the conductivity in the solutions with and without the $\mathrm{CaCO}_{3}$ particles, both of the two slurries had slightly higher conductivity than the solution without any $\mathrm{CaCO}_{3}$ 
particles at low levels of the NaPA addition, which suggests that the negatively charged $\mathrm{CaCO}_{3}$ particles were slightly conductive. As the NaPA concentration became higher, the conductivity of the two slurries approached that of the particle-free NaPA solution. The conductivity of the 11.54 wt\% slurry became equal to that of the particle-free NaPA solution at a lower level of the NaPA addition than the $20.69 \mathrm{wt} \%$ slurry. This effect was very likely an indication that the negatively charged $\mathrm{CaCO}_{3}$ particles were experiencing a viscous drag, which lowered their drift velocity and therefore the conductivity. When the NaPA addition was increased, polyacrylate concentrations both on the $\mathrm{CaCO}_{3}$ surface and in the bulk phase, also increased and the viscous drag was enhanced, thus decreasing the conductivity of the particles. For the less concentrated slurry $(11.54 \mathrm{wt} \%)$, the build-up of the surface polyacrylate-concentration occurred sooner than the $20.69 \mathrm{wt} \%$ slurry; hence, the viscous drag was seen at an earlier stage of the titration.

In Figure 1, the $\zeta$-potentials were plotted as a function of the NaPA addition, expressed in the NaPA-to- $\mathrm{CaCO}_{3} \mathrm{w} / \mathrm{w}$ percentage (i.e. the dispersant level). Before the addition of the NaPA dispersant, the $\mathrm{CaCO}_{3}$ slurries were positively charged (about $3 \mathrm{mV}$ ); with the addition, the polarity of the $\zeta$-potentials altered immediately. The positive $\zeta$-potentials were provided by adsorbed $\mathrm{Ca}^{2+}$ ions while the negative $\zeta$-potentials were due to the anionic polyacrylate chains. Microcalorimetric determination shows the adsorption of NaPA to be endothermic (i.e. heat was absorbed). The magnitude of the heat is high $(+4.5 \mathrm{kcal} / \mathrm{mole}$ of monomer), demonstrating a strong interaction. [10]

As can be seen, the negative $\zeta$-potentials increased with the dispersant level until a peak in the electrostatic charging was reached. The peak electrostatic charging corresponds to an optimum dispersant level at $1-2 \mathrm{w} / \mathrm{w} \%$ sodium polyacrylate. The optimum dispersant level was independent of the $\mathrm{CaCO}_{3}$ concentration, but the peak electrostatic charging was lower for the 20.69 wt $\%$ slurry. The humps on the $\zeta$-potentiometric titration diagrams, occurring not far beyond the optimum NaPA level, are probably an indication of multilayer adsorption and/or changing orientation of the adsorbed polyacrylate chains. [1] The multilayer/orientation change effect was also seen in the microcalorimetric titration curves which show a heat "tail" after the initial heat pulse due to NaPA adsorption. 
When the dispersant concentration went beyond the optimum level, the $\zeta$-potentials started to decrease from their peak values. The decrease in $\zeta$-potential with increasing electrolyte concentration is due to an effect known as electrical double layer compression. Charged particles have an electrical double layer around them. The electrical double layer can be regarded as consisting of two regions: an inner region which included adsorbed $\zeta$ potential-determining ions, and a diffuse region in which ions are distributed according to the influence of electrical forces and random thermal motions. The extent of the diffuse double layer around a particle is, in general, on the order of some tens of nanometers, but the exact magnitude depends on the electrolyte concentration. Increasing the electrolyte concentration causes the diffuse double layer to shrink closer to the particle, so that the electrostatic $\zeta$ potential falls more quickly with distance [5]. Since $\zeta$-potential is the electrostatic $\zeta$ potential measured at the shear plane, which is located at some fixed distance from the particle surface, it decreases as the bulk electrolyte concentration increases.

Also shown in Figure 1, the negative $\zeta$-potential was higher in the less concentrated slurry at any dispersant level around and beyond the peak electrostatic charging. The same trend of the $\zeta$-potential dependence was reported in cationic $\mathrm{CaCO}_{3}$ suspensions where $\mathrm{Ca}^{2+}$ was the $\zeta$-potential determining ion [2]. The effect of particle concentration on $\zeta$-potential, especially in concentrated dispersions, has not been studied extensively so far; however, this effect is genuine and has been observed also in oil-in-water emulsions, [6] clay-particle dispersions, [7] and silica and PMMA latex suspensions [8]. As detailed in the following section, studies of the bulk electrolyte-concentration effect and the particle-concentration effect on $\zeta$-potential were conducted in our laboratory using the adsorption of $\mathrm{Ca}^{2+}$ ions from $\mathrm{CaCl}_{2}$ solutions on $\mathrm{CaCO}_{3}$ particles [2] as a model system.

\section{Electrical Double Layer Compression due to Bulk Electrolyte Concentration and Charged}

\section{Particle Concentration}

$\zeta$-potential and conductivity plots, similar to those in Figures 1 and 2, for six $\mathrm{CaCO}_{3}$ slurries are shown in Figures 3 and 4 . These slurries, ranging from 8 to 21 wt $\%$, were prepared by dispersing proper amounts of $\mathrm{CaCO}_{3}$ into $230 \mathrm{ml}$ DDI water, respectively, each followed by a $\zeta$-potentiometric titration with $20 \mathrm{ml}$ of a 1.02 molar $\mathrm{CaCl}_{2}$ solution. As can 
be seen, the adsorbed $\mathrm{Ca}^{2+}$ ions were the $\zeta$-potential-determining ions, giving the slurries a cationic nature. The peak electrostatic charging was about $+18.5 \mathrm{mV}$ at the addition of 0.1 millimoles of $\mathrm{Ca}^{2+}$ ions per gram $\mathrm{CaCO}_{3}$, which is in excellent agreement with our previous studies on the adsorption of $\mathrm{Ca}^{2+}$ ions from $\mathrm{CaCl}_{2}$ solutions [2]. In this case, the addition of $\mathrm{Ca}^{2+}$ causes a drop in $\mathrm{pH}$ from the initial $9.5 \pm 0.2$ to $8.1 \pm 0.1$ as a direct consequence of $\mathrm{CaCO}_{3}$ solubility equilibria [2]. The $\zeta$-potentials in the six slurries rose uniformly with the addition of the $\mathrm{Ca}^{2+}$ ions before reaching the peak electrostatic charging; no $\mathrm{CaCO}_{3}$ concentration-dependence was observed at this stage of the titration. As soon as the addition of $\mathrm{Ca}^{2+}$ ions exceeded this level, the $\zeta$-potential began to decrease. The more concentrated the slurry, the more rapidly the $\xi$-potentials decreased.

In Figure 4 the conductivity of the slurries, for the data points beyond the peak electrostatic charging, was plotted against the bulk $\mathrm{Ca}^{2+}$ equilibrium concentration, which was evaluated by subtracting the monolayer adsorption coverage $\left(13.6 \mu\right.$ moles $/ \mathrm{gm} \mathrm{CaCO}_{3}$ [2] from the total amount of the $\mathrm{Ca}^{2+}$ ion addition. In the particle free, $\mathrm{CaCO}_{3}$-saturated DDI water, the conductivity was found to increase linearly with the $\mathrm{Ca}^{2+}$ equilibrium concentration (the solid line). At any bulk $\mathrm{Ca}^{2+}$ concentrations, there was little difference in the conductivity of the six slurries of the various particle concentrations, indicating saturation of the $\mathrm{Ca}^{2+}$ adsorption. Again, the positively charged $\mathrm{CaCO}_{3}$ particles were seen to be slightly conductive; in other words, the $\mathrm{CaCO}_{3}$ particles with $\mathrm{Ca}^{2+}$ adsorbed are actually conducting species somewhat like ions. Being huge in size and therefore very slow in drift velocity as compared to the $\mathrm{Ca}^{2+}\left(\mathrm{CO}_{3}\right)^{2-}, \mathrm{Cl}^{-}, \mathrm{H}^{+}$, and $\mathrm{OH}^{-}$ions in the solutions, the positively charged $\mathrm{CaCO}_{3}$ particles contributed to the total conductivity far less than the ions. The viscous drag effect observed in the $\mathrm{CaCO}_{3} / \mathrm{NaPA}$ system was virtually insignificant in the $\mathrm{CaCO}_{3} / \mathrm{CaCl}_{2}$ system, largely because $\mathrm{Ca}^{2+},\left(\mathrm{CO}_{3}\right)^{2-}, \mathrm{Cl}^{-}, \mathrm{H}^{+}$, and $\mathrm{OH}^{-}$ions are by orders of magnitude smaller than that of the anionic polyacrylate chains.

Further studies on the dependance of $\zeta$-potential upon bulk $\mathrm{Ca}^{2+}$ concentration and $\mathrm{CaCO}_{3}$ particle concentration were performed in the $\mathrm{CaCO}_{3}$ slurries of $11.54,13.53$ and $16.67 \mathrm{wt} \%$, respectively, by determining the bulk $\mathrm{Ca}^{2+}$ equilibrium concentrations at various stages of the titration using $\mathrm{a} \mathrm{Ca}^{2+}$ ion selective electrode. In Figure 5 the $\zeta$-potentials and conductivity (the left and right ordinates, respectively) of the slurries were both plotted 
against the bulk $\mathrm{Ca}^{2+}$ ion equilibrium millimolarity. Data points for the conductivity measurements are all overprinted with a small square-shaped dot, and the average conductivity of the three slurries is drawn with a dashed line. The $\zeta$-potentials were found to decrease with the increasing bulk $\mathrm{Ca}^{2+}$ concentration and with the increasing $\mathrm{CaCO}_{3}$ particle concentration. The $\zeta$-potential-dependence upon the $\mathrm{CaCO}_{3}$ particle concentration is not a conductivity effect. As can be seen, the conductivity of the three slurries all increased linearly with the bulk $\mathrm{Ca}^{2+}$ equilibrium concentration; at any bulk $\mathrm{Ca}^{2+}$ concentration, the conductivity values of the three slurries are nearly identical, but this is obviously not the case for the $\zeta$-potentials.

For the three $\mathrm{CaCO}_{3}$ particle concentrations, the $\zeta$-potential versus $\mathrm{Ca}^{2+}$ equilibrium concentration plots appear to be three straight lines almost parallel to one another with the least concentrated slurry (11.54 wt\%) on the top and the most concentrated (16.67 wt\%) at the bottom. The fact that the rate of $\xi$-potential decrease with respect to the bulk $\mathrm{Ca}^{2+}$ concentration $(-0.0962 \mathrm{mV} / \mathrm{mM})$ is independent of particle concentration suggests that in the $\mathrm{CaCO}_{3} / \mathrm{CaCl}_{2}$ system the $\mathrm{CaCO}_{3}$ particle concentration is an additional factor to be considered in the analysis. One possibility is that the electrical double layer compression due to increasing bulk electrolyte concentration and due to increasing charged particle concentration work on the same principle, because the positively charged $\mathrm{CaCO}_{3}$ particles are of ion-like behavior to some extent. When the particle concentration is increased, the diffuse double layer is compressed towards the particle surface as if the electrolyte concentration were increased, and the electrostatic $\zeta$-potential decays more quickly with distance.

In Figure 6 the $\xi$-potential were plotted against interparticle separation distance for the bulk $\mathrm{Ca}^{2+}$ concentrations at $25,40,60$ and $80 \mathrm{mM}$, respectively. Based on the geometry of a cube with a $\mathrm{CaCO}_{3}$ particle at the center of the cube, the calculated interparticle separation distances are 616,718 and $801 \mathrm{~nm}$ for the $16.67 ; 13.53$ and $11.54 \mathrm{wt} \%$ slurries, respectively. The higher the solids concentration, the shorter the interparticle separation distance. As can be seen, at the four bulk $\mathrm{Ca}^{2+}$ concentrations, the $\zeta$-potential increased with the interparticle separation distance at a same rate $(0.00663 \mathrm{mV} / \mathrm{nm})$.

By combining the two factors, bulk electrolyte concentration and charged particle concentration, into one equation, the change in $\zeta$-potential after the peak electrostatic 
charging can be written as $\mathrm{d} \zeta=-0.0962 \mathrm{dC}_{\mathrm{aq}}+0.00663 \mathrm{dH}$ where the $\zeta$ stands for $\zeta$ potential in $\mathrm{mV}$, the $\mathrm{C}_{\mathrm{aq}}$ is the bulk $\mathrm{Ca}^{2+}$ equilibrium concentration in millimolarity, and the $\mathrm{H}$ is the interparticle separation distance in $\mathrm{nm}$. In the foregoing analysis we have, however, assumed a constant dispersed particle radius. If flocculation affects the effective radius for ESA conversion to $\zeta$-potential slightly, this could be an additional $\mathrm{CaCO}_{3}$ solids-dependent factor.

\section{Mesophase Structural Elements of Polyacrylate Aggregates in Solutions}

To ascertain the self-association between polyacrylate chains, two NaPA solutions were prepared: one of $4.3 \mathrm{wt} \% \mathrm{NaPA}$ in DDI water at $\mathrm{pH} 7$ and the other of $5.0 \mathrm{wt} \% \mathrm{NaPA}$ in particle-free, $\mathrm{CaCO}_{3}$-saturated DDI Water at $\mathrm{pH}$ 9.5. This concentration range of $4.3-5.0$ wt $\%$ NaPA in solutions corresponds to an NaPA level of $1.8-2.1 \mathrm{w} / \mathrm{w} \%$ in a $70 \mathrm{wt} \%$ $\mathrm{CaCO}_{3}$ slurry. The solutions were repeatedly filtered through the Acrodisc $0.2 \mu \mathrm{m}$ pore-size syringe filters and then stored in glass vials for two days before dynamic light scattering experiments via the Coulter N4 MD photon correlation spectrometer.

As shown in Figures 7 and 8, mesophase structural elements with well defined dynamic correlation lengths were observed in both the solutions. The smoothly decaying and well-converged autocorrelation functions at the lower right-hand corner of Figures 7 and 8 ascertains the detection of the structural elements in solutions. The fact that the solutions have been repeatedly filtered excluded possibilities of the presence of adventitious dust, so the structural elements must be aggregates of polyacrylate chains. The light scattering angle, $15.5^{\circ}$, was chosen for illustration because it is more sensitive to weak scatterers than a larger angle. In the solution that contains about $7.04 \times 10^{-5} \mathrm{M} \mathrm{Ca}^{2+}$ ions (Figure 8) the scattered light intensity (1.95 X $10^{4}$ photon counts per second) was about 2.3 times higher than the one without $\mathrm{Ca}^{2+}\left(6.94 \times 10^{3}\right.$ counts/sec. in Figure 7), indicating a higher degree of aggregation. As can be seen through a comparison between Figures 7 and 8, the dynamic correlation lengths of the mesophase structural elements were also increased due to the higher degree of aggregation. Results of mean aggregate sizes from the unimodal analysis increased from 1830 to $3150 \mathrm{~nm}$ (almost double). The size distribution processor (SDP) gave both the systems bimodal diagnosis and suggested that a small aggregate size fraction at $326 \mathrm{~nm}$ was 
present in the solution in DDI water only but not in the $\mathrm{Ca}^{2+}$-containing solution which instead had a large size fraction at $5600 \mathrm{~nm}$. Both solutions, however, contain aggregates of 1800 to $2310 \mathrm{~nm}$ in size.

The self-association (or aggregation) between the polyacrylate chains is, most likely, brought about by the bridging of the carboxylate groups which are surrounded with the $\mathrm{Na}^{+}$ counter-ions. It is conceivable that the divalent $\mathrm{Ca}^{2+}$ causes an even greater degree of bridging (specific interactions) than is seen for $\mathrm{Na}^{+}$. Thus, the mesophase structural elements with $\mathrm{Ca}^{2+}$ are larger in dimension.

\section{Specific Interactions Between Calcium Ions and Polyacrylate Chains}

The electrophoretic mobilities of the same two NaPA solutions $\left(\mathrm{CaCO}_{3}\right.$ particle-free) were measured in the Coulter DELSA and $\xi$-potentials calculated. Here the velocity of the charged mesophase structural elements in an electrical field is measured by determining the Doppler shift of light scattered at four angles, $8.6^{\circ}, 17.1^{\circ}, 25.6^{\circ}$ and $34.2^{\circ}$. Figures 9 and 10 show that both the systems have negative $\xi$-potentials with the $\mathrm{Ca}^{2+}$-containing system being less negative, approximately $-30 \mathrm{mV}$ versus $-40 \mathrm{mV}$. Also the latter (Figure 10) has a much narrower distribution of the $\xi$-potential than the DDI water-only system (Figure 9). It appears that under the electrical field the mesophase structural elements are of net negative charge, most likely due to carboxlyate end groups with $\mathrm{Na}^{+}$and $\mathrm{H}^{+}$as counter-ions. The weakly held aggregates may be in a flux of changing size and charge. When $\mathrm{Ca}^{2+}$ was added, the attractive forces were greater and the aggregates more stable as to size and charge. Some $\mathrm{Ca}^{2+}$ ions must have been trapped inside the polyacrylate mesophase structural elements, thus neutralizing part of the negative electrostatic charging. This is the evidence that among the three kinds of counter-ions in the system, namely, $\mathrm{Ca}^{2+}, \mathrm{Na}^{+}$and $\mathrm{H}^{+}$, the $\mathrm{Ca}^{2+}$ ions have the strongest specific interactions with the polyacrylate chains.

The specific interactions observed in the solution phase between $\mathrm{Ca}^{2+}$ and polyacrylate chains may well be the analogy of the interactions occurring at a $\mathrm{CaCO}_{3} /$ polyacrylate interface; thus, further investigation was made by studying flow and viscosity curves of $\mathrm{CaCO}_{3}$ slurries (Figure 11). Two $50 \mathrm{wt} \% \mathrm{CaCO}_{3}$ slurries were prepared each with the addition of $2.9 \mathrm{w} / \mathrm{w} \% \mathrm{NaPA}$ based on the weight of the $\mathrm{CaCO}_{3}$. Then $1.6 \mathrm{w} / \mathrm{w} \% \mathrm{CaCl}_{3}$ 
was added to one of the slurries, also based on the weight of the $\mathrm{CaCO}_{3}$. After mixing thoroughly in a blender, the two slurries were tumbled on a laboratory rolling mill for 10 days to let the dispersion contents equilibrate. Twenty minutes before the viscometry experiments, the two slurries were removed from the rolling mill and sonicated vigorously for ten minutes each using an ultrasonic probe.

In Figure 11 the viscosity and shear stress (the left and right ordinates, respectively) were plotted against the shear rate, which was programmed to sweep downwards from 1500 to $1.2 \mathrm{~s}^{-1}$ in 32 steps (each step at a fixed shear rate). At each shear rate, a ten-second shear was applied prior to the measurements: then, the viscosity and shear stress were measured over a twenty-second period.

The two slurries showed shear-thinning behavior. For the shear rates lower than 10 $\mathrm{s}^{-1}$, the NaPA-only system had a more rapidly decreasing viscosity profile which started to level off as the shear rate further increased, but the viscosity of the $\mathrm{Ca}^{2+}$-containing system continued to decrease and became lower than the NaPA-only system when the shear rate exceeded $300 \mathrm{~s}^{-1}$. The flow curves (the correlation between shear stress and shear rate) indicate that the NaPA-only system had some dilatancy, while the $\mathrm{Ca}^{2+}$ containing system had a higher yield-point value (i.e., more elastic), which suggests that due to the addition of $\mathrm{Ca}^{2+}$ the polyacrylate chains are bonded more strongly to the $\mathrm{CaCO}_{3}$ surface as compared to the NaPA-only system. [9]

In the adsorption of spin-labelled polyacrylic acid onto calcium phosphate surface, it was demonstrated by electron spin resonance (ESR) spectroscopy that the specific interactions between $\mathrm{Ca}^{2+}$ and polyacrylate chains are more intense than the interactions between $\mathrm{Na}^{+}$ and polyacrylate chains [1]. The polymer was found to be adsorbed in a flat conformation with more than $90 \%$ of its segment adsorbed (immobilized) on the surface. The adsorption was favored by the $\mathrm{Ca}^{2+}$ because considerably more polymer segments were found in the conformation of loops when a significant part of the surface $\mathrm{Ca}^{2+}$ was replaced by $\mathrm{Na}^{+}$. 


\section{CONCLUSIONS}

In this paper we have demonstrated that the detailed chemical nature of the surfaces of a coating pigment can strongly impact the way in which the pigment particles interact with commercial dispersants. The conformation of a polyacrylate dispersant on the surface of PCC, for example, is highly dependent upon the concentration of $\mathrm{Ca}^{2+}$ surface sites. By regulating the surface $\mathrm{Ca}^{2+}$ site concentration to achieve an optimum conformation, the rheology of PCC-based coating colors may be improved.

\section{ACKNOWLEDGEMENTS}

We would like to thank John H. Schaefer of Specialty Minerals for performing the particle size and surface area measurements and Ali R. Bashey of Specialty Minerals for the ISS, ESCA and microcalorimetric data. 


\section{REFERENCES}

1. F. Th. Hesselink, "Adsorption of Polyelectrolytes from Dilute Solution" in Adsorption from Solution at the Solid/Liquid Interface, G. D. Parfitt and C. H. Rochester, Eds., Academic Press, Orlando, 1983. p. 377.

2. Y. C. Huang, F. M. Fowkes, T. B. Lloyd, and N. D. Sanders "The Adsorption of Calcium Ions from Calcium Chloride Solutions onto Calcium Carbonate Particles" Langmuir, 7(8): 1742 - 1748 (1991).

3. N. D. Sanders and J. H. Schaefer, TAPPI Proceedings, "Zeta Potential Distributions in Pulp/PCC Mixtures" 1989 Papermakers Conference: 69 (1989).

4. T. Foxall, G. C. Peterson, H. M. Rendall, and A. L. Smith, J. Chem. Soc., Faraday Trans. I, "Charge Determination at Calcium Salt/Aqueous Solution Interface" 75: 1034 (1979).

5. R. J. Hunter, Foundations of Colloid Science, Oxford University Press, New York, 1987, p. 91.

6. R. J. Goetz, dissertation study, "Formation and Stabilization of Oil in Water Miniemulsions", in progress, Lehigh University.

7. A. Tuncan, dissertation study, "The Influence of Ocean Disposal of Wastes on Depositional and Post Depositional Mechanics of Marine Clays", in progress, Lehigh University.

8. R. T. Klingbiel, H. Coll, R. O. James, and J. Texter, "Colloidal Standards for the Measurement of Electrokinetic Sonic Amplitude", presented at the 21st Annual Meeting of the Fine Particle Society, San Diego, California, August, 1990. 
9. S. Ross and I. D. Morrison, Colloidal Systems and Interfaces, Wiley, New York, 1988, p. 17.

10. N. D. Sanders and A. R. Bashey, Proceedings 1991 International Paper Physics Conference, TAPPI Press, Atlanta, pp. 473-482. 


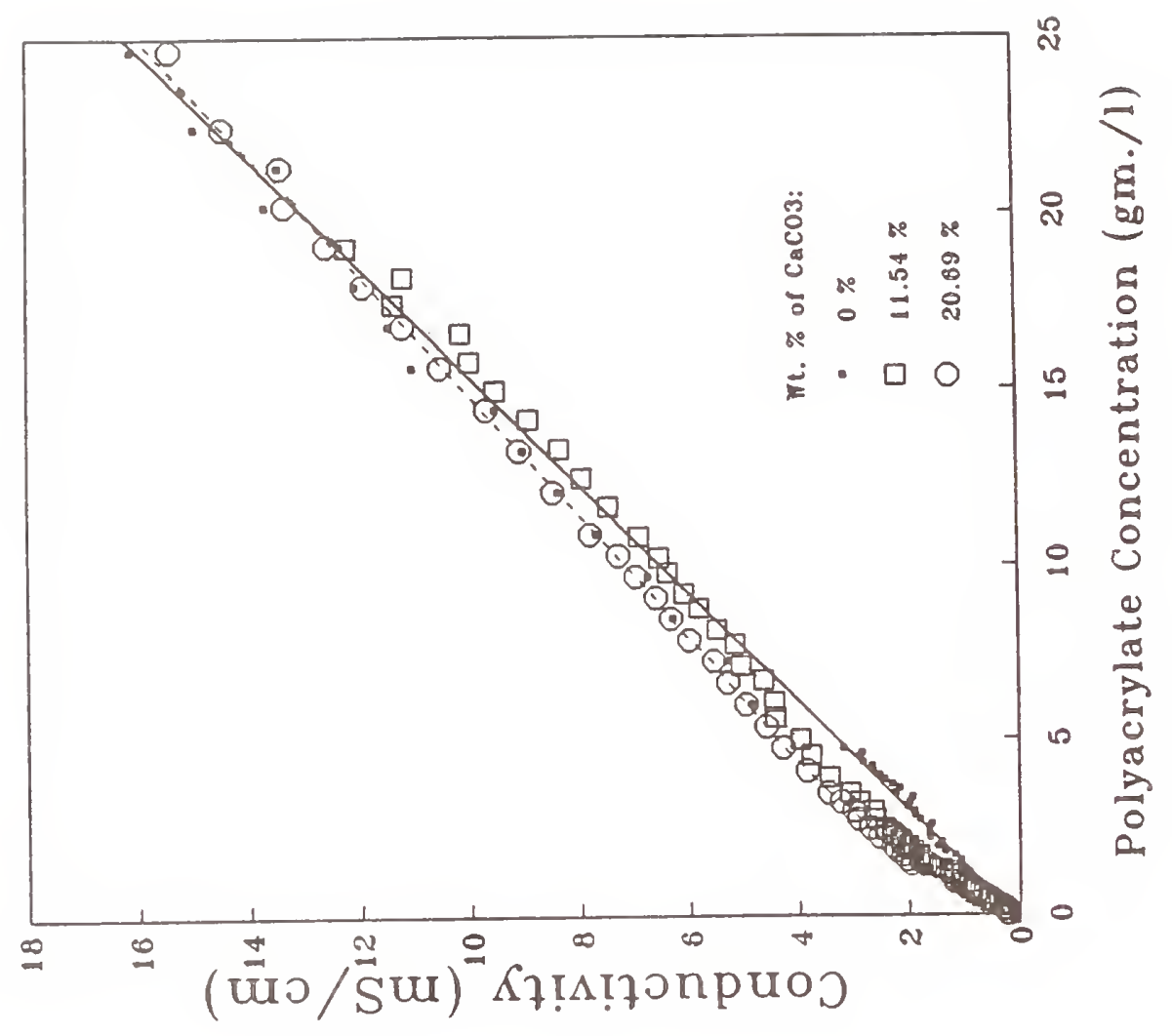

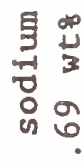

ㅇํํ

แ 뜸

范

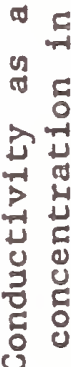

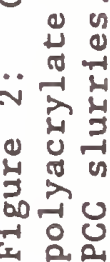

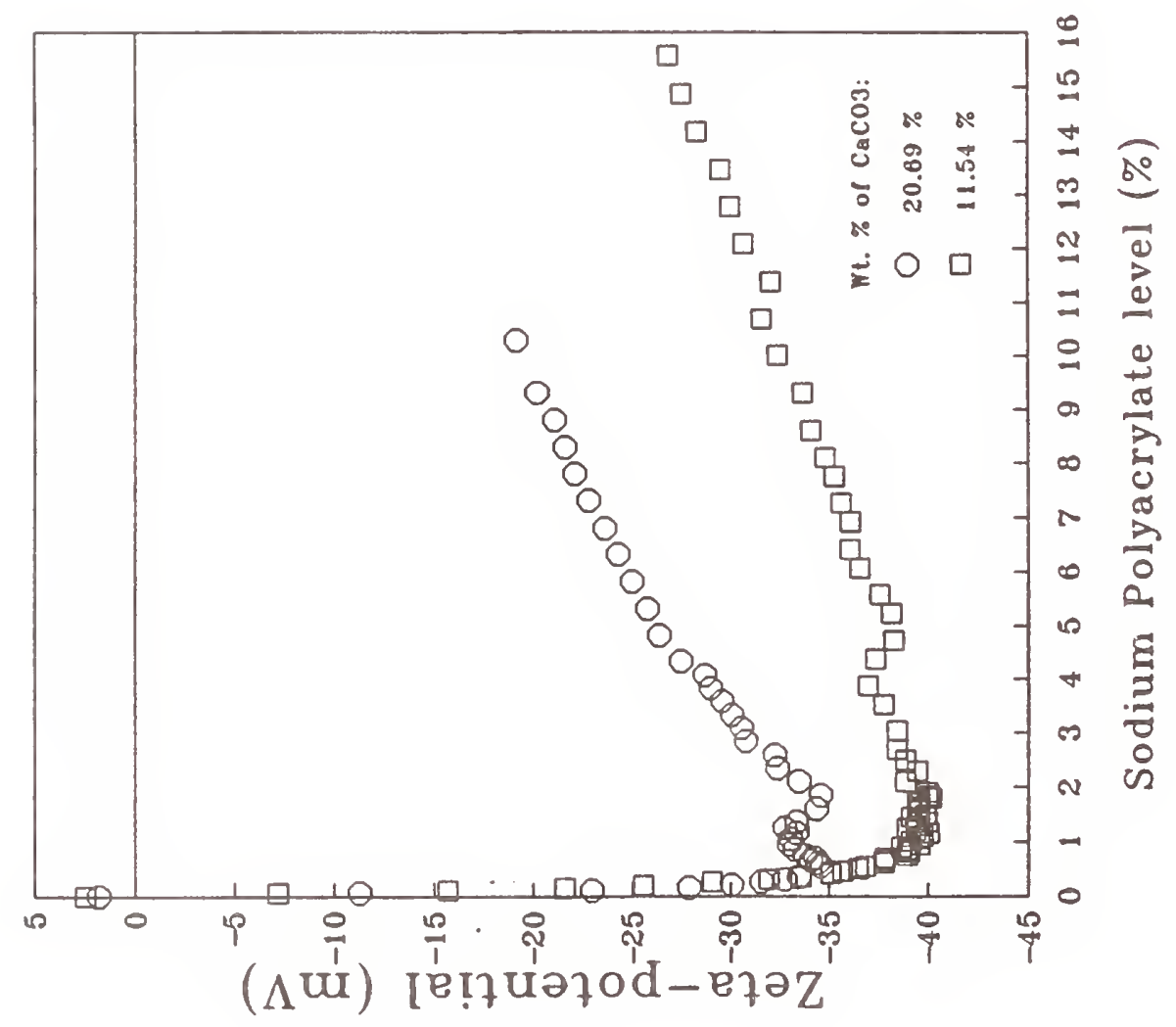

葛呟

ज峲

$\exists$ 동

岂量

동

론

出

击

대

c)

나 $\infty$

的号

펀

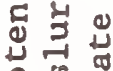

ด

ค

is

中

분

낭

วิ당

용 


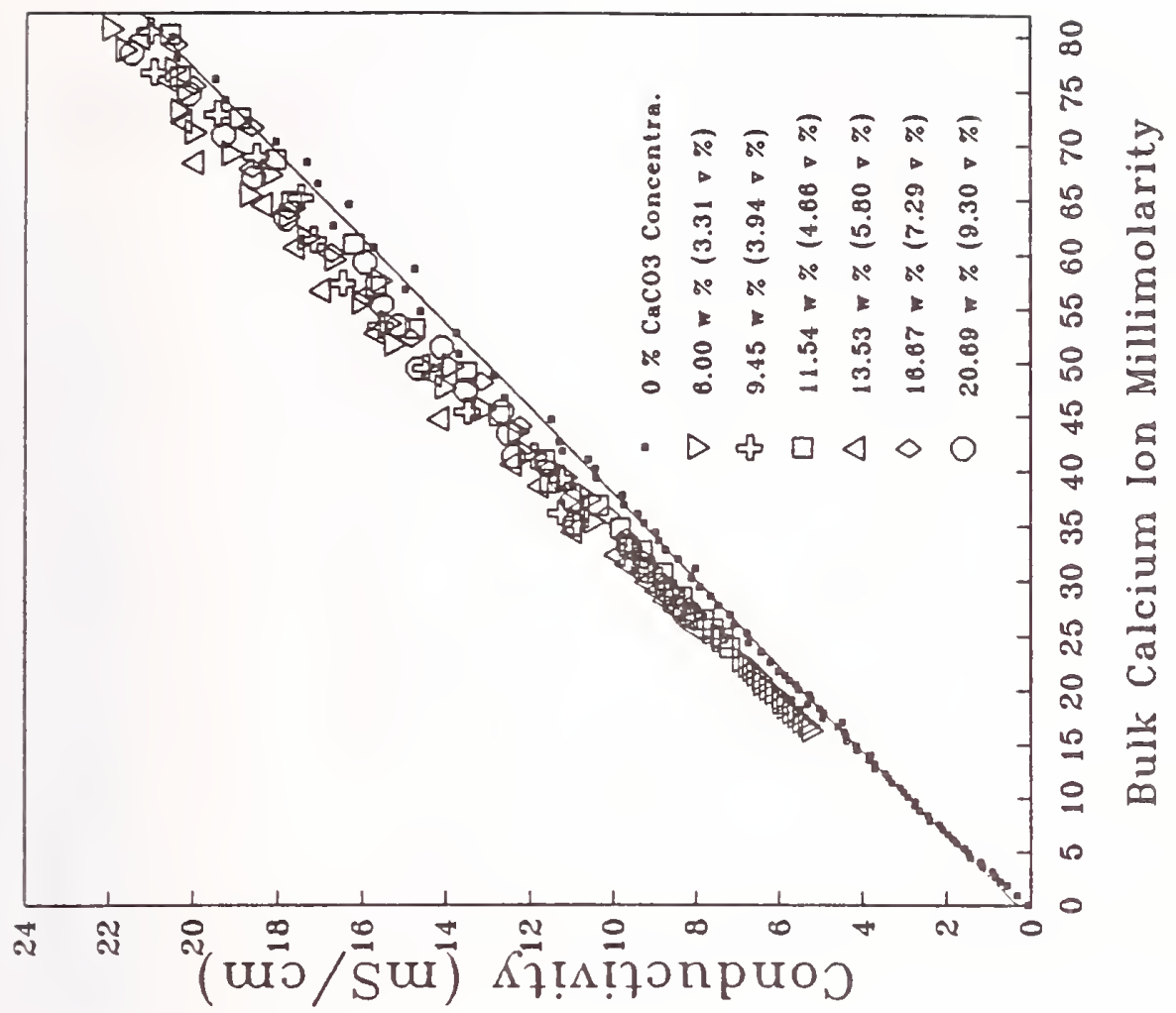

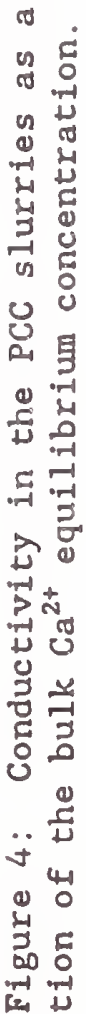

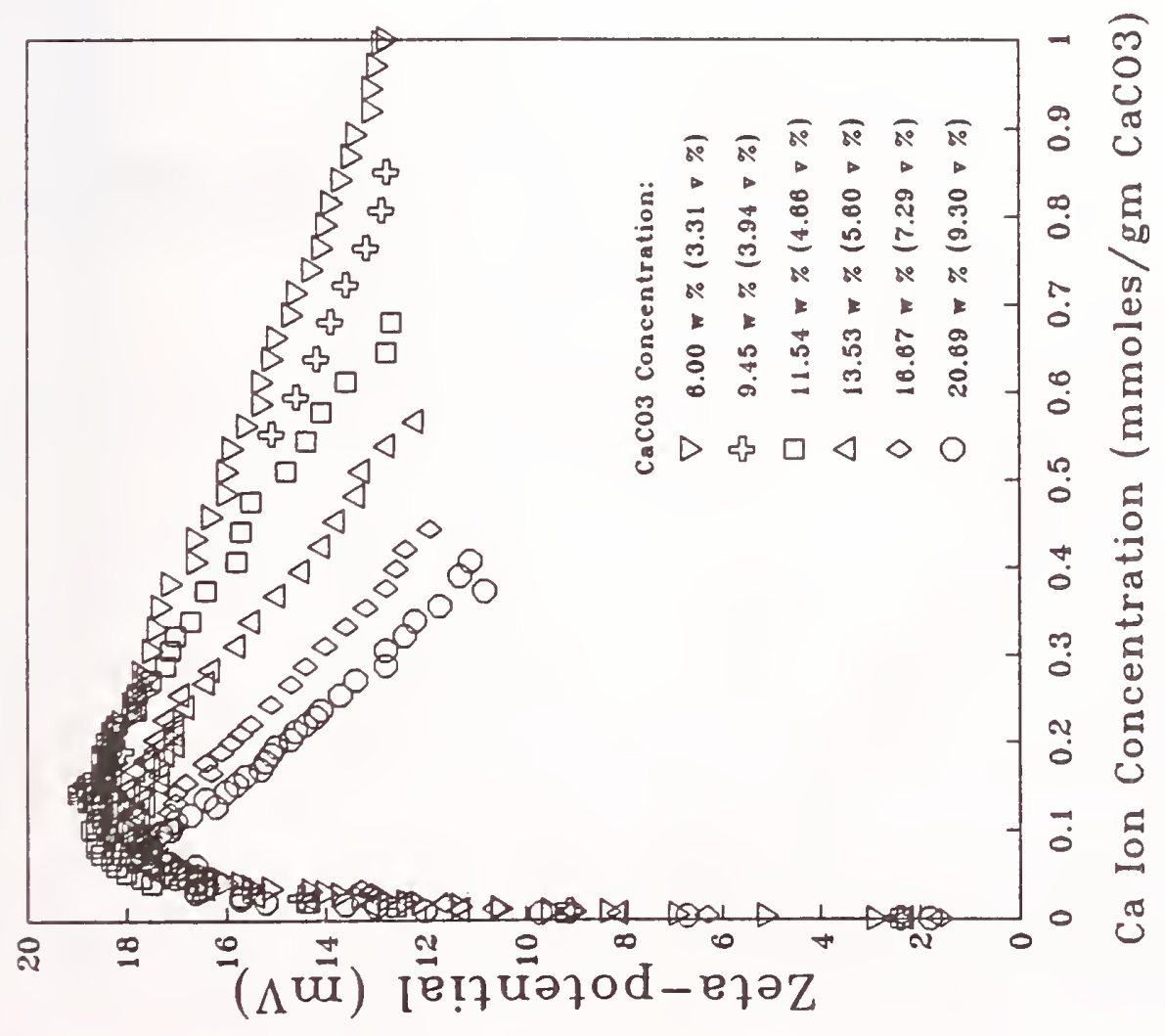

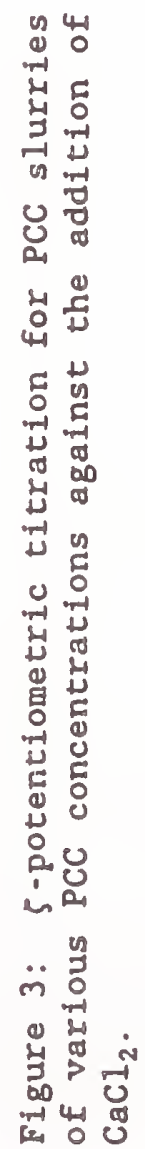




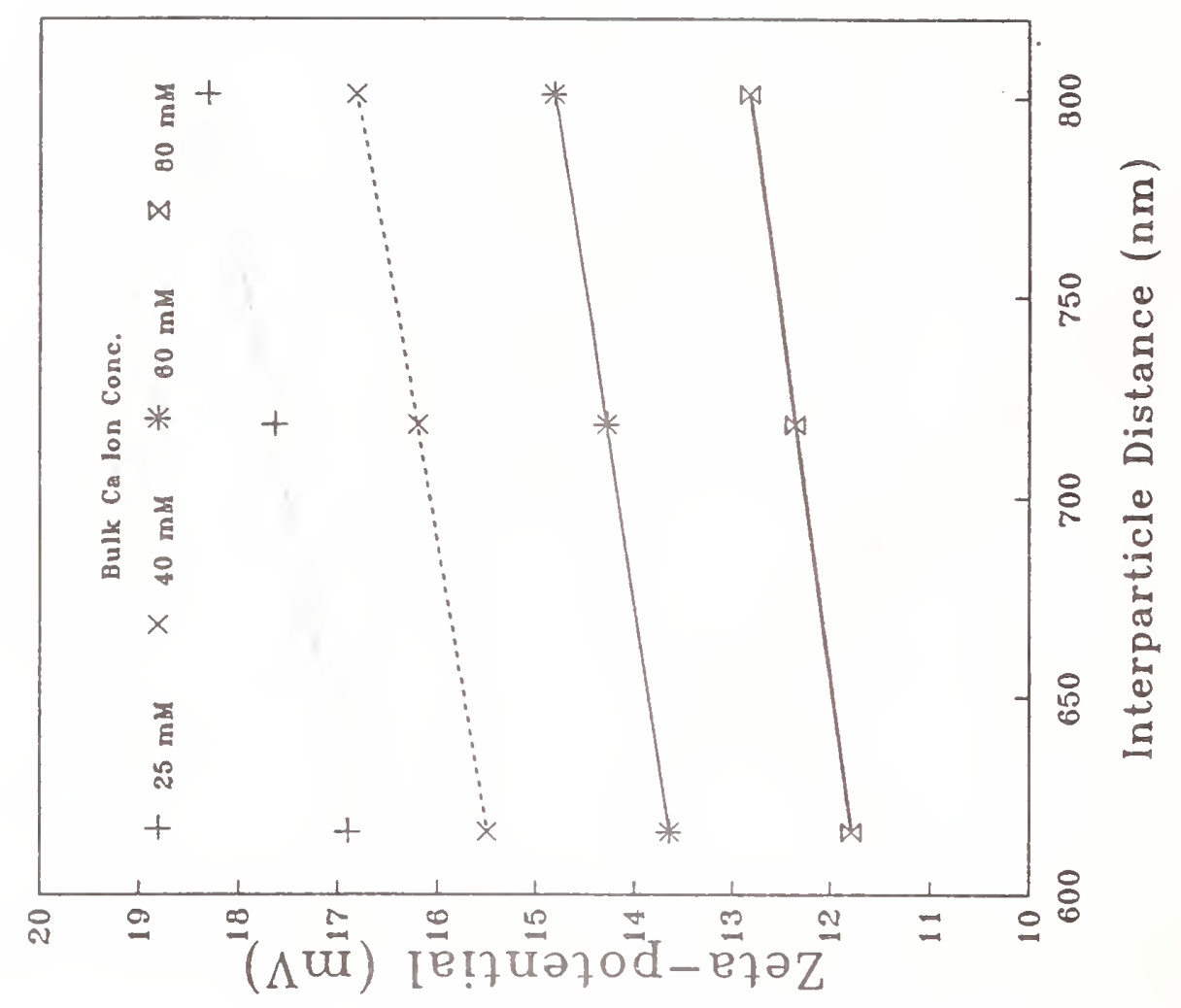

嵌

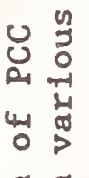

5.

पै

造

व

a

*

न

क्ष

ए

ป็

的㟧

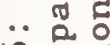

6 过

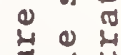

$3-1$

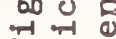

is is

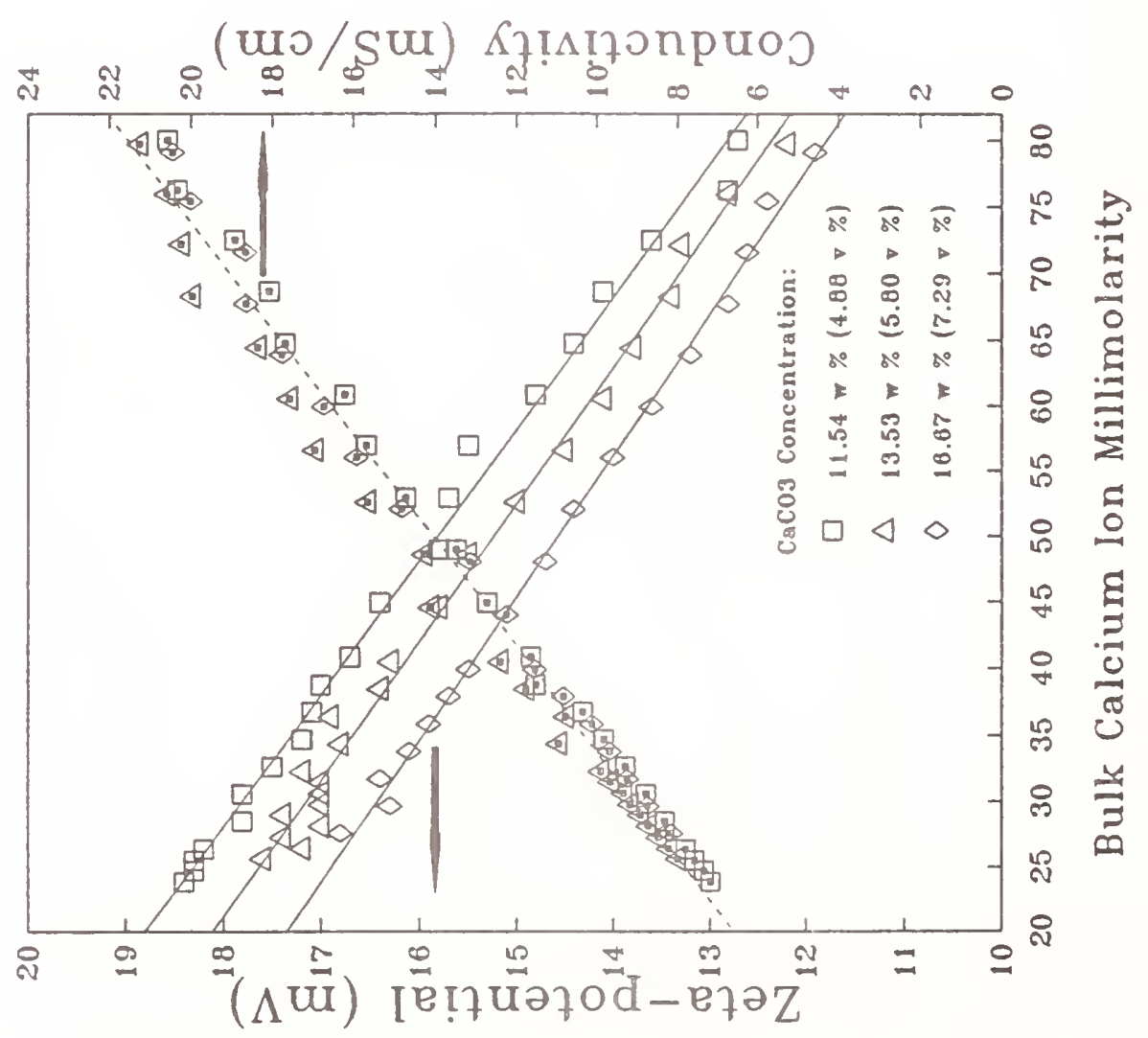

듕

등

in I

蛋

岂

윰

E i

0

듕

$+5$

离

곡워

4.

○

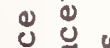

है ป

30

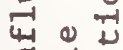

ज्ञ

H

in

म

베 

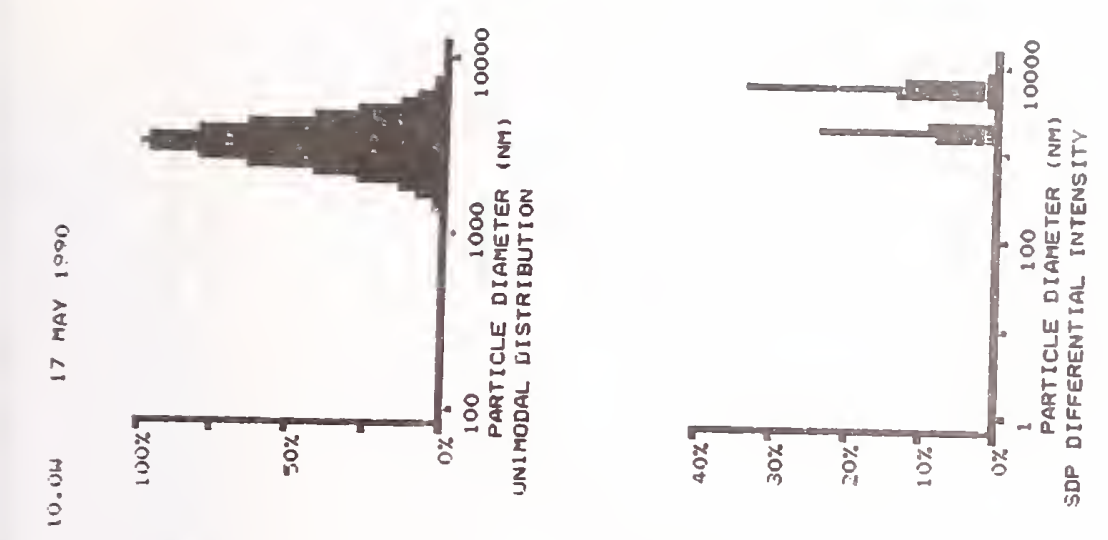

으늠
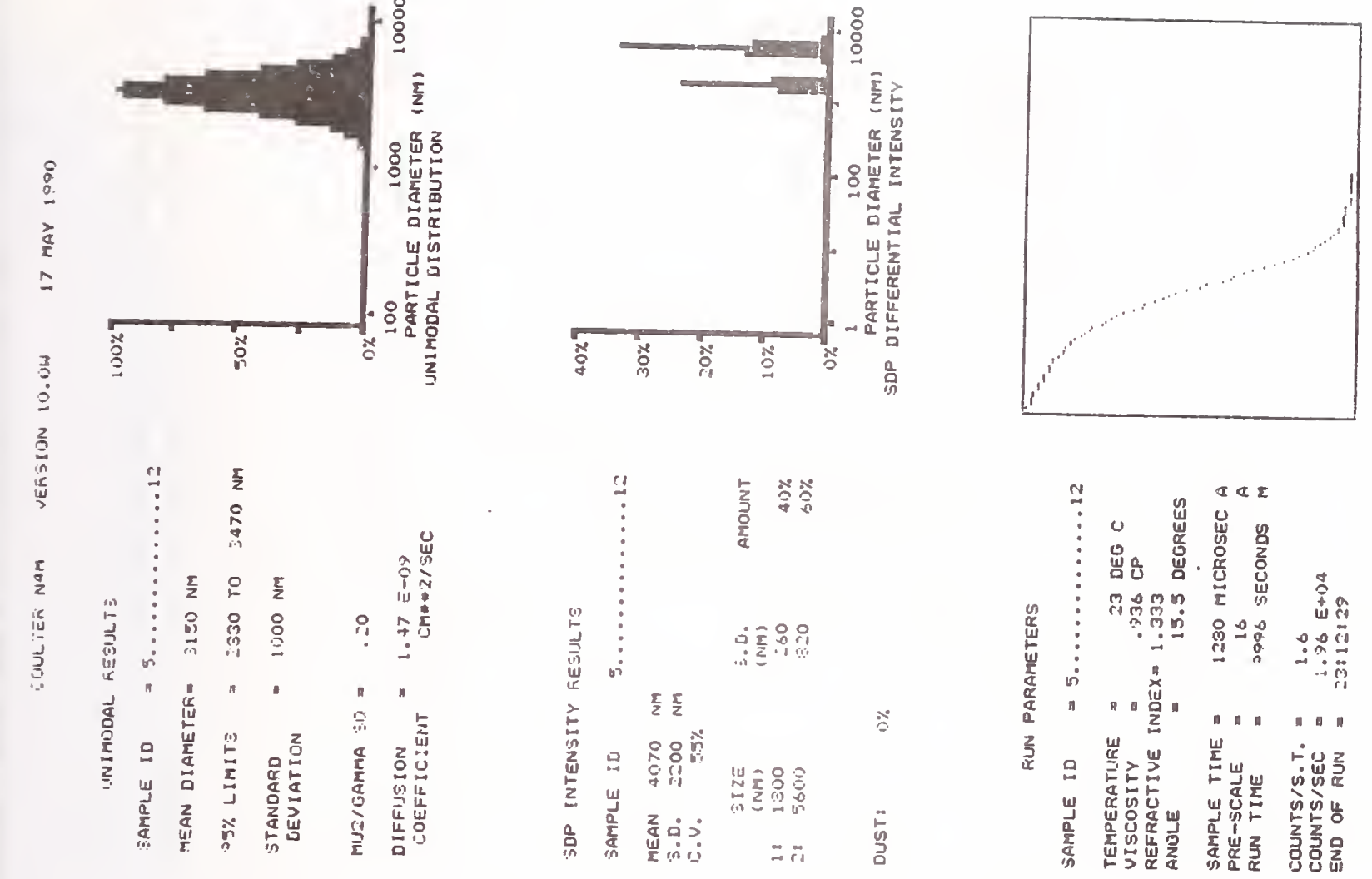

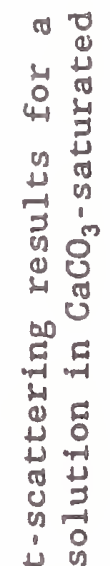
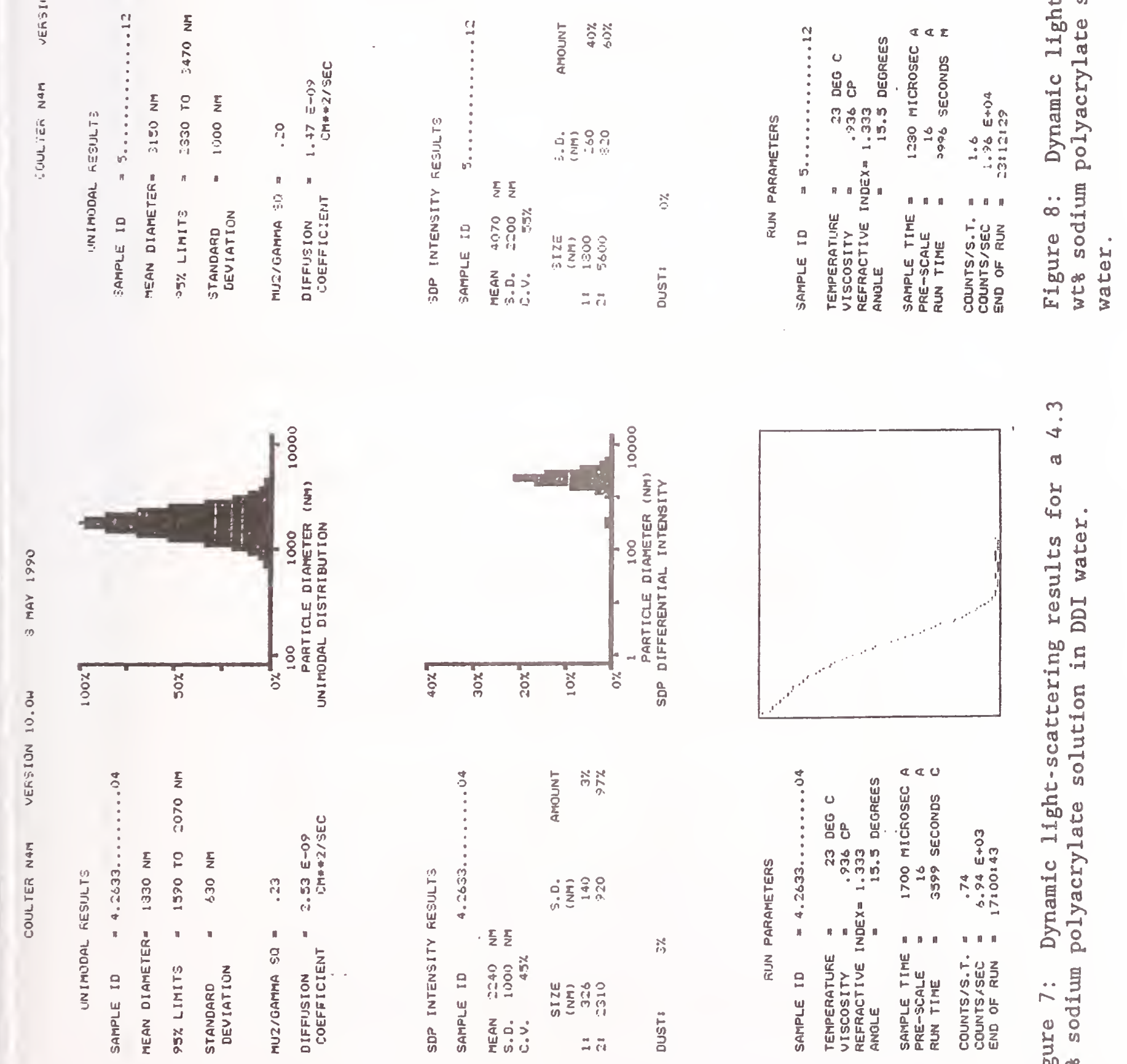

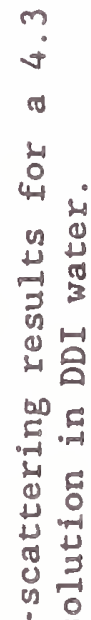

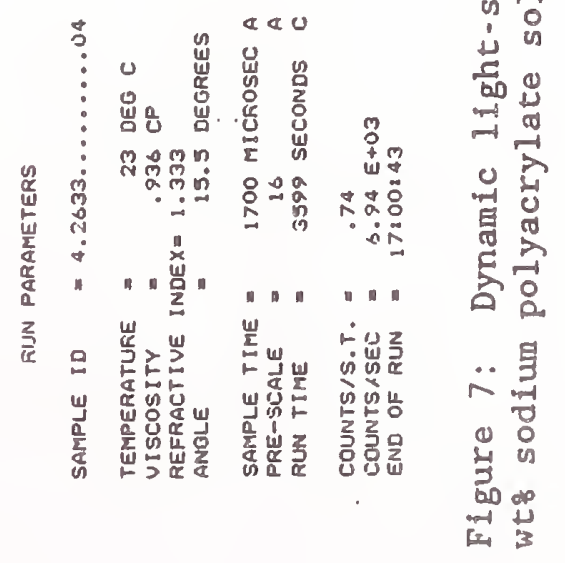



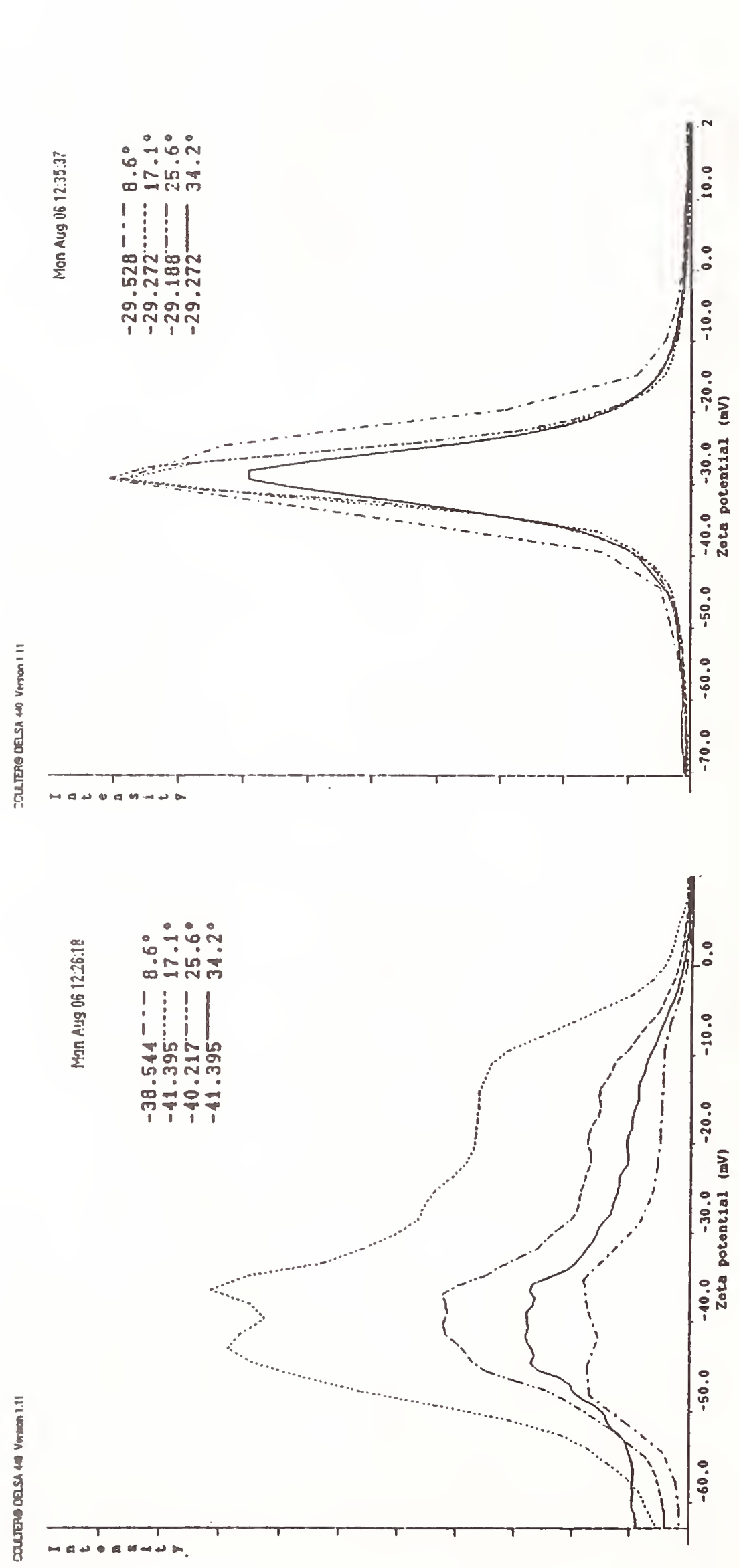

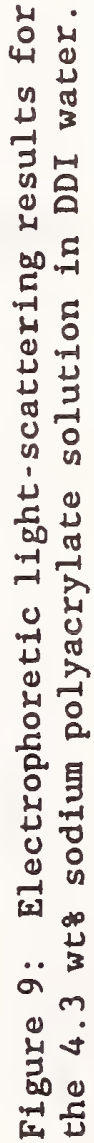




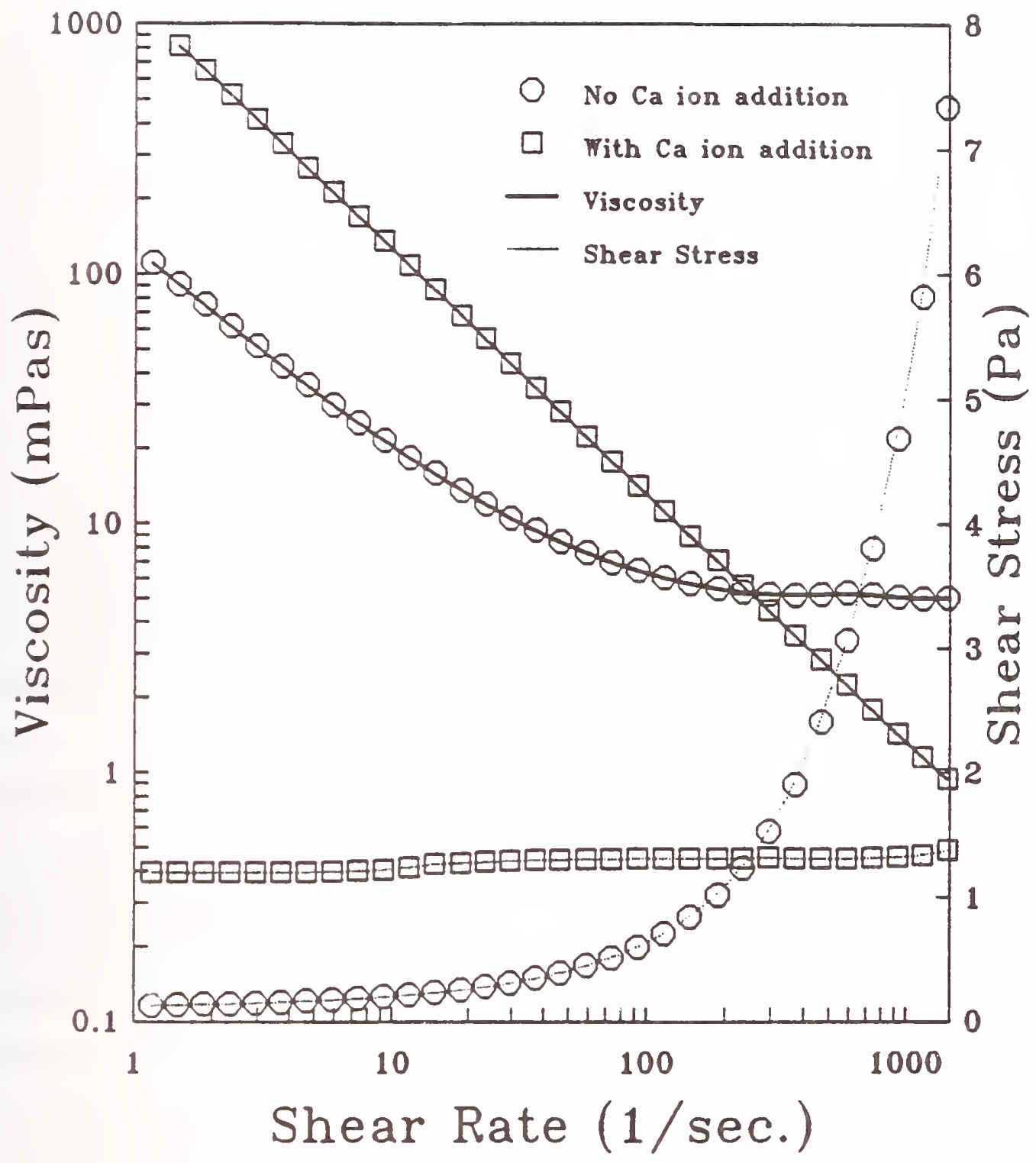

Figure 11: Influence of the addition of $\mathrm{Ca}^{2+}$ to the PCC/NaPA slurry on flow and viscosity curves. 
H. Polat, M. Polat and S. Chander

Mineral Processing Section

Penn State University

University Park, PA 16802

\begin{abstract}
The feasibility of electrokinetic sonic amplitude (ESA) technique to characterize coal slurries was investigated in this study. The ESA of coal samples of different rank was measured as a function of particle size and electrolyte concentrations. For comparison, measurements were also made with alumina suspensions. Substantial differences were observed in the electro-kinetic behavior between coal and alumina samples. The reasons for the unusual behavior of coal were discussed.
\end{abstract}

\title{
INTRODUCTION
}

Electrokinetic characterization of concentrated slurries is important in process control. Concentrated slurries are processed in the ceramics, paint, pharmaceutical and coal industries daily. Traditional methods of electrokinetic characterization require samples of low solids concentration which necessitates dilution of the original slurry. In addition, many characterization methods are constrained to very narrow particle sizes, usually very fine, which may not be the actual state of the sample to be examined. Although the phenomenon of acoustophoresis, which is a type of electrokinetic phenomena, has been known for several decades (Debye, 1933), only recently was it exploited in the study of concentrated slurries (O'Brien, 1986). Depending on the signal being measured, acoustophoretic measurements can be divided into two distinct categories: ultrasonic vibration potential (UVP) and electrokinetic sonic amplitude (ESA). In the former case, an alternating pressure wave, or sound wave, is applied to a slurry which causes the displacement of the particles suspended in liquid. A measurable alternating electric field will be developed due to this displacement 
whose magnitude is a function of inertia of the particles. In the latter case, an alternating electric field is applied to a suspension of particles. The resulting motion is a vibration of particles between the electrodes which creates an alternating pressure wave that can be measured quantitatively and is related to the zeta potential (O'Brien, 1986; James et al., 1992).

The electrokinetic behavior of coal particles in concentrated slurries may provide information on coal surface properties and on the interactions among coal particles suspended in liquid. Such information may be extremely important in explaining coal-water slurry properties. Coal is predominantly composed of organic material with imbedded mineral matter and shows a different structure depending on its rank, degree of oxidation and mineral matter content. Hence, its electrokinetic behavior is difficult to predict. There are numerous studies in the literature on the electrokinetic behavior of coals (Sun, 1954; Fuerstaneau, 1983; Guiterrez-Rodriguez and Aplan, 1984). Nevertheless, they all involve measurements of the electrophoretic mobility in dilute suspensions. Introduction of the ESA and UVP techniques provides a good opportunity toward carrying out an investigation with more realistic slurries. In this study, the feasibility of the ESA technique to characterize coal slurries was investigated.

\section{EXPERIMENTAL PROCEDURE}

The instrument used in the experimental work was a Model 8000 Electrokinetic Sonic Amplitude Measurement system manufactured by Matec Applied Sciences. It consists of a main unit which controls four separate probes to measure ESA, $\mathrm{pH}$, conductivity, and temperature. The system also includes a frequency synthesizer to regulate the alternating electrical field, an automatic titrator for continuous $\mathrm{pH}$ adjustment and a computer to acquire and process data. All the measurement probes are inserted from the top in a teflon cell of a volume of about $300 \mathrm{ml}$.

The relationship between the electrokinetic sonic amplitude, ESA, and the zeta potential was developed by O'Brien (1986) and may be written as: 


$$
\zeta G(\alpha)=\frac{(E S A) \eta_{l i q}}{\varepsilon \phi \Delta \rho v_{l i q}}
$$

where

$$
\frac{1}{G(\alpha)}=1-\frac{i \alpha\left(3+\frac{2 \Delta \rho}{\rho_{\text {liq }}}\right)}{9\left[1+(1-i) \sqrt{\frac{\alpha}{2}}\right]}
$$

and

$$
\alpha=\frac{\omega a^{2} \rho_{1 i q}}{\eta_{l i q}}
$$

ESA is the electrokinetic sonic amplitude which is the pressure sensed by the probe due to oscillating particles per unit length of electrical field in units of $\mathrm{mPa} / \mathrm{V} . \mathrm{m}, \zeta$ is the zeta potential of the particle in $\mathrm{V}, \eta$ is the viscosity of the liquid in $\mathrm{kg} / \mathrm{m} . \mathrm{sec}, \epsilon$ is the dielectric permittivity of the suspension, $\Phi$ is the volume fraction of the particles, $\Delta \rho$ is the density difference between the particles and the liquid in $\mathrm{kg} / \mathrm{m}^{3}, \rho_{\text {liq }}$ is the density of the liquid in $\mathrm{kg} / \mathrm{m}^{3}, \nu_{\text {liq }}$ is the velocity of sound in the suspension in $\mathrm{m} / \mathrm{sec}, \mathrm{G}(\alpha)^{-1}$ is the inertial term, a is the particle radius in $\mathrm{m}$ and $\omega$ is the angular frequency in $\mathrm{sec}^{-1}$.

The complex quantity, $\mathrm{G}(\alpha)$, takes into account the inertial effects of particles. This term is equal to unity and drops out for particles less than about $0.3 \mu \mathrm{m}$ for alumina and about $1.0 \mu \mathrm{m}$ for coal. For particles larger than these sizes, the zeta potential has to be corrected for the inertia effect using $\mathrm{G}(\alpha)$. Following appropriate mathematical manipulations, $\mathrm{G}(\alpha)$ can be expresses as a real quantity as: 


$$
\frac{1}{G(\alpha)}=i \sqrt{\left(1+\frac{\left(3 A^{3}+2 A^{3} \frac{\Delta \rho}{\rho}\right)}{9\left(A^{2}+A+0.5\right)}\right)^{2}+\left(\frac{(1+A)\left(3 A^{2}+2 A^{2} \frac{\Delta \rho}{\rho}\right)}{9\left(A^{2}+A+0.5\right)}\right)^{2}}
$$

where

$$
A=\sqrt{\frac{\alpha}{2}}
$$

It has to be noted that the calculations given above are valid for relatively dilute slurries, less than $10 \%$ by volume, and for $\kappa$ a values greater than 50 where $\kappa$ is the reciprocal thickness of the double layer. Calculations using the ionic strength of the double distilled water used in our studies resulted in a $к$ a value of 48 which is well within the limitations of the formulae above.

Ions in solution generate electroacoustic signals as was predicted by Debye in 1933 and lead to an appreciable background signal at high ionic strengths. This phenomenon might become especially important in cases where a weak ESA signal should be expected, such as at low solids concentration and where there are small density differences between the solution and particles. In addition, small ESA readings near the point of zero charge is another factor for amplifying the significance of the background signal. Hence, a background correction which would eliminate the contributions from the ion in solution may be necessary under certain circumstances.

\section{MATERIALS}

A $\gamma$-alumina and two coal samples, namely anthracite and a sub bituminous A, were used in this study. The $\gamma$-alumina was used to establish the measurement procedure since its electrokinetic behavior was well known. Alumina samples of three nominal sizes, namely 0.05, 0.3 and $1.0 \mu \mathrm{m}$, were obtained from the Buehler Company, Illinois. 
The sub bituminous A coal and anthracite samples were obtained from Penn State Coal Data Bank. The samples were from Colorado Q Seam in Moffat County-Craig Township, Colorado and the Primrose Seam in Schuylkill County-Coaldale Township, Pennsylvania, respectively. Approximate analysis of the coal samples are given in Table 1.

Before the electrokinetic analysis the coal samples were ground below nominal $-37 \mu \mathrm{m}$ and observed to have similar size distributions. The mean size of the samples were about $12 \mu \mathrm{m}$ and well within the range of the practical size requirements of the ESA device. According to the manufacturer the device should be able to measure electrostatic signal for particles ranging in size from $150 \mu \mathrm{m}$ down to the molecular sizes (Matec manual, 1989).

A suspension of the sample was prepared using double distilled water. It was placed in an ultrasonic bath for 30 minutes to disperse the particles and the slurry temperature was adjusted to $23.0^{\circ}$ in a temperature bath. The sample was placed in the cell of the ESA device. A moderate stirring was applied in the cell to prevent settling of the particles.

Table 1. Characteristics of coal samples used in this investigation.

\begin{tabular}{|l|l|l|l|l|}
\hline & \multicolumn{3}{|l|}{ Sub-bituminous A coal } & \multicolumn{2}{l|}{ Anthracite } \\
\hline \hline Component, \% & As Received & Dry & As Received & Dry \\
\hline Moisture & 13.03 & - & 3.15 & - \\
\hline Ash & 5.65 & 6.50 & 11.00 & 11.36 \\
\hline Carbon & 61.14 & 70.30 & 81.09 & 83.73 \\
\hline Hydrogen & 4.10 & 4.71 & 1.50 & 1.55 \\
\hline Nitrogen & 1.64 & 1.88 & 0.67 & 0.69 \\
\hline Total Sulfur & 0.32 & 0.37 & 0.46 & 0.48 \\
\hline Oxygen & 14.12 & 16.24 & 2.12 & 2.19 \\
\hline
\end{tabular}




\section{RESULTS AND DISCUSSIONS}

\section{Effect of Solids Concentration}

To determine the change in ESA with varying amounts of solids and to choose an appropriate solids concentration for the subsequent tests, experiments were carried out with $\gamma$-alumina, at different slurry concentrations. Three slurry concentration of $0.1 \%, 1.0 \%$, and $5.0 \%$ by weight were investigated. The results are given in Figure 1. As expected, the point of zero charge did not change when the amount of alumina in the slurry was increased. However, the strength of the ESA signal decreased drastically with decreasing alumina concentration as predicted by Equation 1. It was observed that a solids concentration of at least $5.0 \%$ was desirable to obtain a strong signal, especially in the case of coal samples whose densities were small compared to the alumina sample.

\section{Effect of Particle Size}

A set of experiments were conducted with alumina using three different samples with mean particle sizes of $0.05 \mu \mathrm{m}, 0.3 \mu \mathrm{m}$ and $1.0 \mu \mathrm{m}$. The results are given in Figure 2 . Particle size was seen to have an effect on the magnitude of the ESA signal. The point of zero charge also displayed a slight shift to the acidic side with increase in particle size. Increase in the ESA signal strength with increasing particle size is contrary to Equations 1 through 4 which predict the signal strength should decrease with increasing particle size above $0.3 \mu \mathrm{m}$ and no change in the point of zero charge should take place. To explain this behavior, zeta potential was measured using conventional electrophoresis technique with the $0.05 \mu \mathrm{m}$ and $1.0 \mu \mathrm{m}$ alumina samples. The sample displayed the same amount of shift in the point of zero charge as in the ESA experiments, suggesting that this behavior was due to some chemical differences in the sample characteristics. Further experimentation with this sample was discontinued.

To determine the effect of particle size on ESA measurements with coal, sub bituminous A coal sample was used. Samples with different size distributions were obtained after grinding for 1, 2, 4, 8 and 16 minutes in a laboratory Bleuler mill. Size distributions of the samples were determined with a light scattering device, Malvern $2600 \mathrm{c}$ and are given in 
Figure 3. The measured ESA are given for various grinding times in Figure 4. In order to correct for the inertial effects, an effective inertial term, $\overline{\mathrm{G}}(\alpha)_{\text {eff }}$, was computed with the assumption that a given sample consisted of $\mathrm{n}$ discrete size intervals. The following relationship was used:

$$
\bar{G}(\alpha)_{e f f}=\sum_{i=1}^{n} f_{i} G(\alpha)_{i}
$$

where $f_{i}$ is the volume fraction of the $i$ th size interval and $\mathrm{G}(\alpha)_{i}$ is the interval term for the $i$ th size interval. The term, $\overline{\mathrm{G}}(\alpha)_{\text {eff }}$ was inserted in Equation 1 to calculate the corrected zeta potentials for each grinding time and the results are given in Figure 5. The figure shows a good agreement for the zeta potentials between the samples towards high $\mathrm{pH}$ values. The shift in the point of zero charge with increase in the grinding time at lower $\mathrm{pH}$ values might be due to creation of new unoxidized surface by grinding. It is well known that the point of zero charge moves to acidic side as the oxidation of the coal sample increases (Wen and Sun, 1977). The higher than expected zeta potential for the 1 minute ground sample was ascribed to the partial settling of this sample in the ESA cell which would have reduced the average

size of the particles in the suspension. Since the $\overline{\mathrm{G}}(\alpha)_{\text {eff }}$ term in Figure 5 was estimated using the actual size distribution, it resulted in overestimation of the zeta potentials (closed circles in Figure 5). If the $\mathrm{G}(\alpha)_{\text {eff }}$ term computed for the sample ground for 2 minutes was used to correct for the inertial effects, the 1 minute-curve approached the rest of the curves (open circles in Figure 5).

\section{Effect of Electrolyte Concentration}

\section{Background Correction}

Presence of ions in solution generate an electroacoustic signal. Addition of ions during the titration or adjustment of the ionic strength of the solution by electrolyte addition may increase the magnitude of this secondary signal to such an extent that it may be necessary to correct for it. In such a situation, the background signal should be subtracted from the actual ESA readings. The subtraction process has to be done vectorially since the ESA is a vector 
quantity. In a recent study to determine the effect of electrolyte concentration on the background signal on the ESA, Desai et al. (1993) measured ESA of different electrolyte solutions as a function of $\mathrm{pH}$, which were subtracted from the ESA of the slurries. The procedure required the selection of a common reference for both electrolyte solutions and the slurries. A dispersion of sample solids in distilled water was chosen as the reference material against which both the actual and the background signal were measured as a function of $\mathrm{pH}$ for different electrolyte concentrations. The "true" ESA signal was obtained using the equations:

$\mathrm{ESA}_{\text {true }} \operatorname{Cos} \beta=\mathrm{ESA}_{\text {measured }} \operatorname{Cos} \theta-\mathrm{ESA}_{\text {background }} \operatorname{Cos} \Phi$

and

$\mathrm{ESA}_{\text {true }} \operatorname{Sin} \beta=\mathrm{ESA}_{\text {measured }} \operatorname{Sin} \Theta-\mathrm{ESA}_{\text {background }} \operatorname{Sin} \Phi$

The various angles in Equations 7 and 8 are defined in Figure 6. The authors found that the electrolyte concentrations above $0.01 \mathrm{M}$ required background correction for the samples they utilized. They also stated that the background signal due to addition of acid or base during the titration cycle was negligible in the $\mathrm{pH}$ range of 4 to 10 .

A second procedure was followed in our experiments in which the reference selection was different. Instead of choosing a dispersion of solids in pure water as the reference, the dispersion of solids containing the appropriate amount of electrolyte was employed. Measurements on both the actual suspension and the blank electrolyte solution were carried out as a function of $\mathrm{pH}$ with respect to this reference. Equations, 7 and 8 were used to calculate the true magnitude of the ESA signal after background electrolyte correction. A comparison of the background correction using both the procedure of Desai et al., (1993) and that used in this study is given in Figure 7 for an the alumina sample in $0.1 \mathrm{M} \mathrm{KNO}_{3}$ solution. The figure shows that the true ESA signal after correction is significantly different than the measured ESA signal at this high electrolyte concentration, including a notable shift in the point of zero charge. The true ESA signal estimated by both the methods was quite similar. It was also observed in our study that the background electrolyte correction was not necessary for a pH range of 2 to 12 and when the electrolyte concentration was less than or 
equal to $0.01 \mathrm{M}$. For a concentration of $0.1 \mathrm{M}$ the background electrolyte correction was necessary. Therefore, the background correction in the absence of electrolytes was ignored in the other sections of this investigation. The ability to measure zeta potentials at higher electrolyte concentrations is quite useful since difficulties are encountered with conventional electrophoresis methods.

\section{ESA Corrected for Background Electrolyte for Alumina and Coal Slurries}

A set of experiments with alumina were carried out at different ionic strengths of $0.1 \mathrm{M}$, $0.01 \mathrm{M}$, and $0.001 \mathrm{M}$ obtained by adding an indifferent electrolyte, $\mathrm{KNO}_{3}$. The ESA results corrected for background electrolyte are given in Figure 8. It was found that the background electrolyte correction was necessary only for the electrolyte concentration of $0.1 \mathrm{M}$. There was no significant changes in the ESA at lower electrolyte concentrations. However, the ESA signal decreased appreciably at $0.1 \mathrm{M}$ electrolyte concentration, most probably due to the depression of the double layer and, therefore, small zeta potential values at the slipping plane. This data is in agreement with the data reported by Malghan and Lum (1990) for $\mathrm{Si}_{3} \mathrm{~N}_{4}$ powders in the presence of electrolytes. It may be noted that a shift in the point of zero charge to the basic side at high ionic strength prevailed even after the background correction, suggesting a different phenomenon to be responsible for such a behavior.

Another set of experiments at the same electrolyte concentrations were carried with the anthracite and sub-bituminous A coal samples and the results are given in Figures 9 and 10, respectively. A decrease in the ESA signal was observed with increasing electrolyte concentrations as expecied with no noticeable change in the point of zero charge at low concentrations of $\mathrm{KNO}_{3}$. At an electrolyte concentration of $0.1 \mathrm{M}$, the ESA signal remained in the negative side for both coal samples and did not change its polarity in the $\mathrm{pH}$ range studied. Yoon and Sabey (1988) observed a decrease in the electrophoretic mobility of coal and a shift in the point of zero charge to the acidic side for all the electrolyte concentrations. The observed behavior suggests that the ESA measurements may be affected by some secondary factors at very high ionic strengths such as strong adsorption of the anions to the coal surface. Nevertheless, further studies are needed to explain the reasons behind such a behavior. Therefore, meaningful interpretation of the electrophoretic data should be done 
with caution under these circumstances.

\section{Reversibility of Titration}

To determine the reversibility of titration the suspension was subjected to a titration with an alkali after completing the acid titration. For the alumina sample, it was observed that neither the magnitude of the measured signal nor the point of zero charge changed significantly. However, both coal samples studied showed a different behavior when the same suspension was subjected to a second titration with an alkali. The results are given in Figures 11 and 12 for anthracite and sub-bituminous A coal, respectively. For both coals, the ESA signal remained in the positive side throughout the $\mathrm{pH}$ range covered during the second titration. A change in the sign of the ESA was not observed. To understand this behavior, an additional experiment was conducted in which second titration was carried out after filtering the solids and adding fresh water following the first titration. In the case of anthracite, the signal obtained was identical to the one obtained in the first titration process and crossed the point of zero charge value at the expected $\mathrm{pH}$ ("Second titration after filtering" curve in Figure 11). For the sub-bituminous coal, on the other hand, filtration alone was not enough to restore the original surface characteristics since the ESA signal still remained positive as can be seen from the data marked as "Second titration after filtering" in Figure 12. Filtration followed by a thorough washing of the solids was necessary to restore the electrokinetic characteristics of the sub-bituminous coal. For the filtered and washed sample the point of zero charge was observed at the expected $\mathrm{pH}$ as can be seen from the data marked as "Second titration after filtering and washing" in Figure 12. These results show that the surface characteristics of the coal particles were restored after filtration and thorough washing. The change in the ESA is probably due to some surface active species which weakly adsorb on the surface. 


\section{SUMMARY AND CONCLUSIONS}

The feasibility of electrokinetic sonic amplitude (ESA) technique to characterize coal slurries was determined in this investigation and the following conclusions were made:

1. Zeta potential could be estimated using correction factors for inertial effects. To determine the correction factor a good estimate of particle size distribution was necessary. In addition, some secondary effects such as settling in the cell or changes in the surface with change in particle size might also have to be taken into account.

2. Two procedures were used to make corrections for background electrolyte. Both the procedures gave comparable results. The background ESA correction was negligible for electrolyte concentrations less than or equal to $0.01 \mathrm{M}$ for $\mathrm{KNO}_{3}$. Hence, for most purposes, this correction might not be necessary.

3. Reversibility of titration was observed for alumina but not for the coal samples. For both the coal samples, the values of ESA in the second titration with alkali were markedly different than those obtained in the first titration with acid. In the reverse titration, the ESA did not change sign at the point of zero charge. For anthracite, filtration of the suspension after the first titration and addition of fresh water restored the original behavior, suggesting some form of weak specific adsorption onto the anthracite surface. For the sub-bituminous A coal, the adsorption was reversed only after filtration and thorough washing of the coal surface. These results imply that the strength of adsorption was a function of coal type. Further studies are needed to establish the mechanisms.

\section{Acknowledgements}

The authors acknowledge the financial assistance from the U.S. Department of Energy through its cooperative agreement No. DE-FC22-92PC92162 in support of this work. 


\section{REFERENCES}

1. Debye, P. W., J. Chem. Phys., Vol. 1, pp. 13, 1933.

2. Desai, F. N., Hammand, H. R., and Hayes, K. F., Background Electrolyte Correction for Electrokinetic Sonic Amplitude (ESA) Measurements, This publication, 1993.

3. Fuerstenau, D. W., Rosenbaum, J. M., and Laskowski, J., Effect of Surface Functional Groups in the Floatability of Coal, Colloids and Surfaces, Vol. 8, pp. 153174, 1983.

4. Gutierrez-Rodriguez, J. A. and Aplan, F. F., The Effect of Oxygen on the Hydrophobicity and Floatability of Coal, Colloids and Surfaces, Vol. 12, pp. 27-51, 1984.

5. James, M., Hunter, R. J., and O'Brien, R. W., Langmuir, Vol. 8, pp. 420, 1992.

6. Malghan, S. G. and Lum, L., Factors Effecting Interface Properties of Silicon Nitride Powders in Aqueous Environment, Am. Cer. Soc., Ed. G. L. Messing et al., Vol. 12, pp. 403-411, 1990.

7. MATEC ESA-8000 System Manual, MATEC Applied Sciences, 75 South Street, Hopkinton, Ma, 01748, 1989.

8. O'Brien, R. W., Electro-Acoustic Effects in a Dilute Suspension of Spherical Particles, J. of Fluid Mechanics, Vol. 190, pp. 71-86, 1986.

9. Sun, S. C., Hypothesis for Different Floatabilities of Coals, Carbons and Hydrocarbon Minerals, Transactions of AIME, Vol. 199, pp. 67-75, 1954.

10. Wen, W. W. and Sun, S. C., Transactions of AIME, Vol. 262, pp. 174-180, 1977

11. Yoon, R. H. and Sabey, J. B., Coal Flotation in Organic Salt Solutions, Interfacial Phenomena in Coal Technology, Edited by Gregory D. Botsaris and Yuli M. Glazman, Vol. 32, pp. 87, 1988. 


\section{FIGURE NAMES}

Figure 1. Effect of solids concentration on ESA for $\gamma$-alumina.

Figure 2. Effect of particle size on ESA for $\gamma$-alumina.

Figure 3. Size distributions of the sub-bituminous A coal samples for various grinding times.

Figure 4. Measured ESA values for the sub-bituminous A coal samples for various grinding times.

Figure 5. Zeta potential of sub-bituminous A coal samples calculated from ESA values.

Figure 6. Background correction for electrolyte signal.

Figure 7. ESA values measured and corrected for background electrolyte for $\gamma$-alumina sample in $0.1 \mathrm{M} \mathrm{KNO}_{3}$ solution.

Figure 8. Effect of electrolyte concentration on ESA for $\gamma$-alumina.

Figure 9. Effect of electrolyte concentration on ESA for anthracite.

Figure 10. Effect of electrolyte concentration on ESA for sub-bituminous A coal.

Figure 11. Effect of acid-base titrations on ESA for anthracite.

Figure 12. Effect of acid-base titrations on ESA for sub-bituminous A coal. 


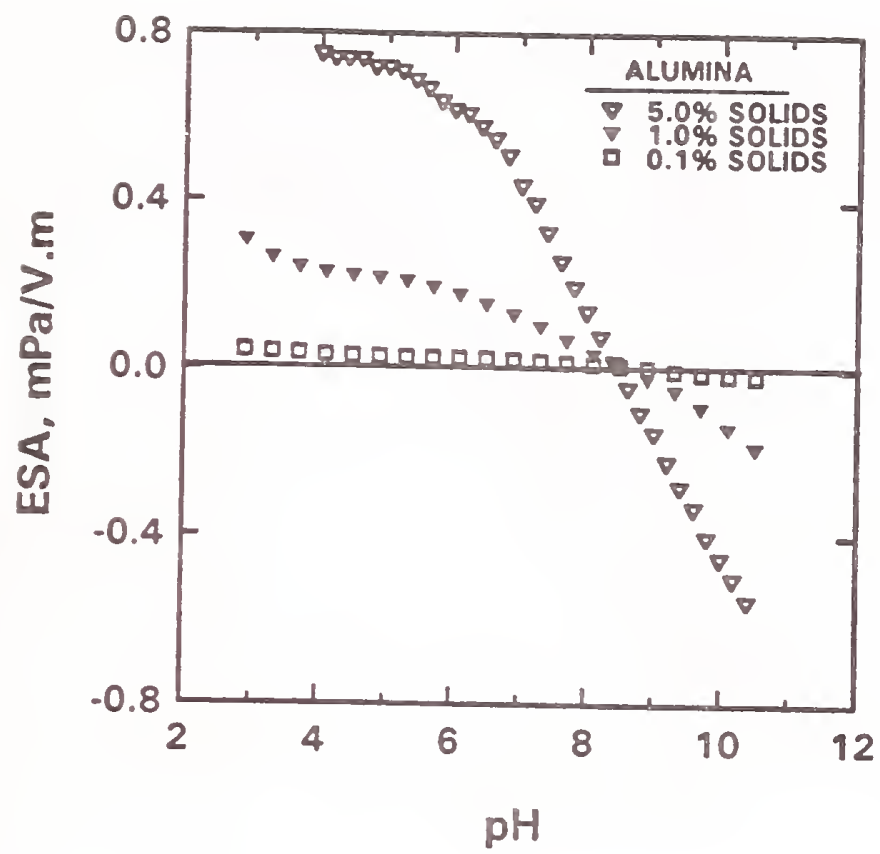

Figure 1. Effect of solids concentration on the ESA for $\gamma$-alumina.

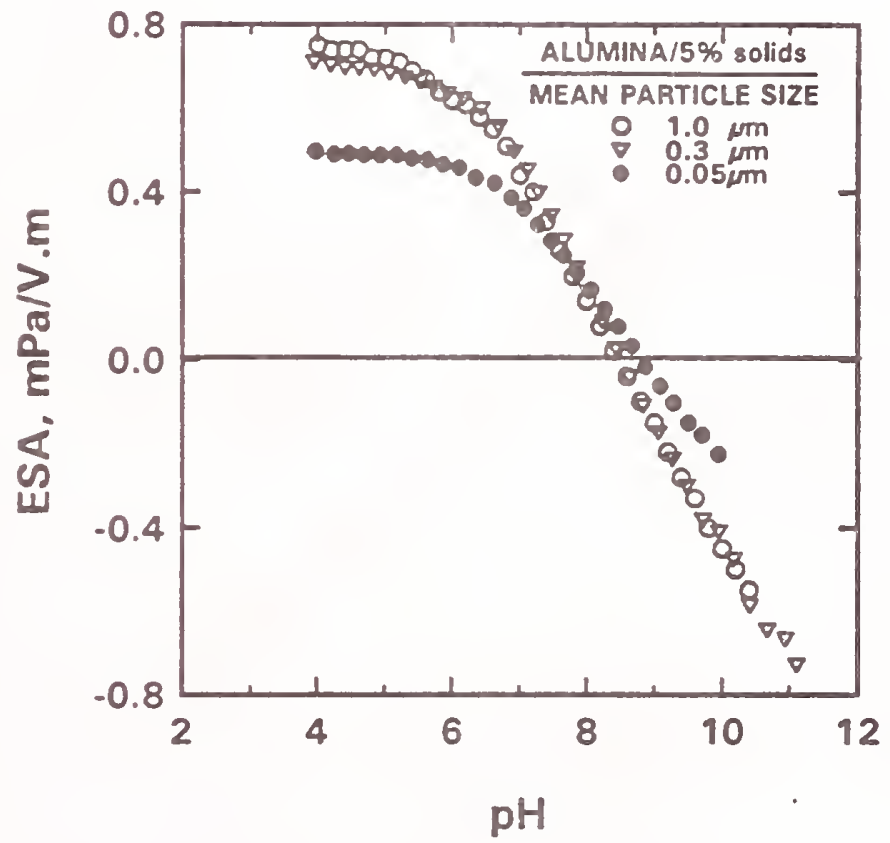

Figure 2. Effect of particle size on the ESA for $\gamma$-alumina. 


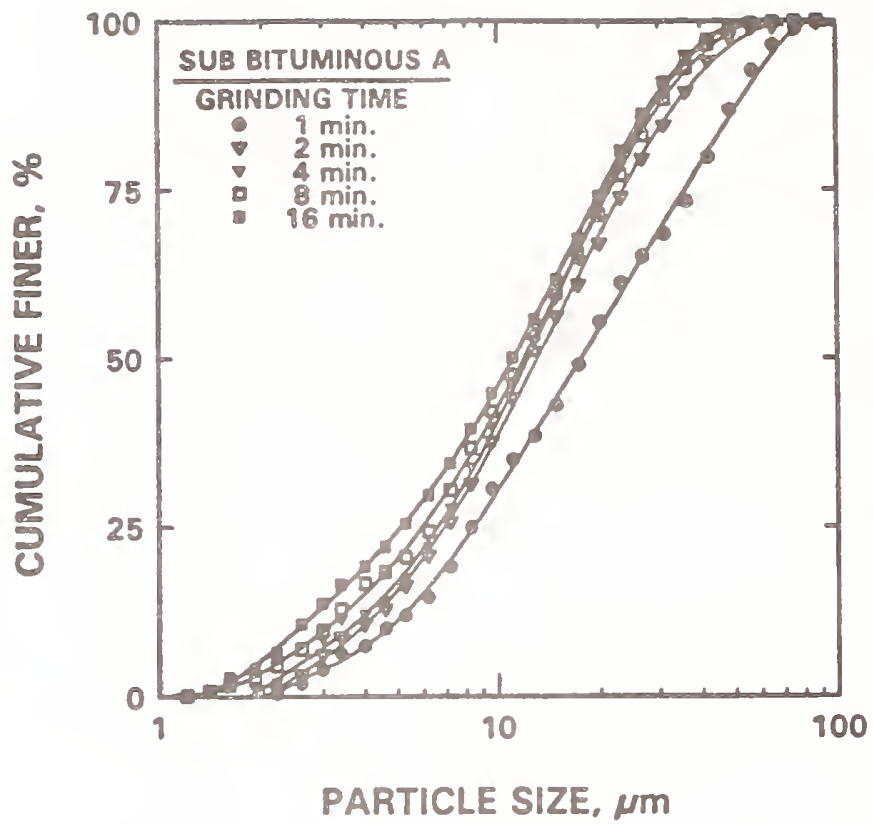

Figure 3. Size distributions of the sub-bituminous A coal samples ground for various times.

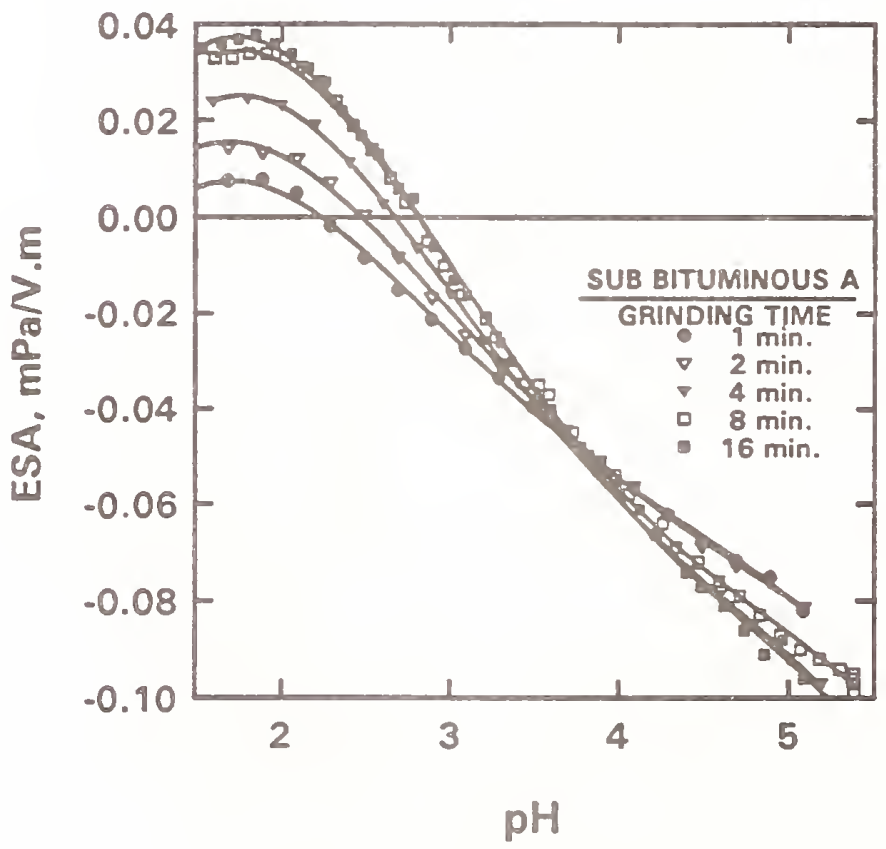

Figure 4. Measured ESA values for the sub-bituminous A coal samples. 


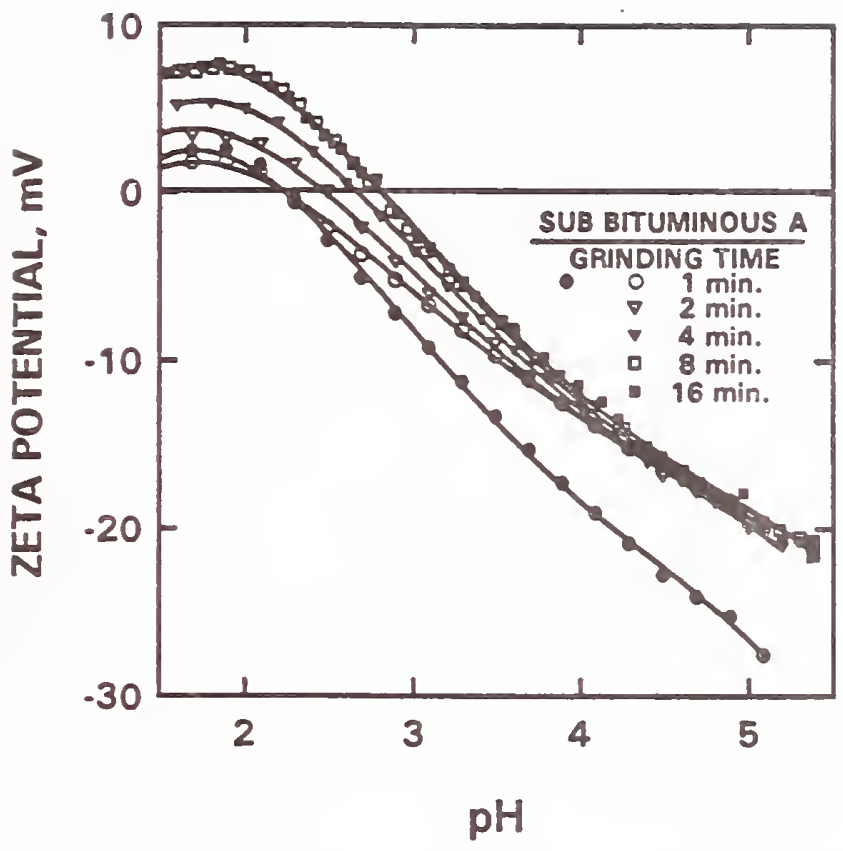

Figure 5. Zeta potential of sub-bituminous A coal samples calculated from ESA values.

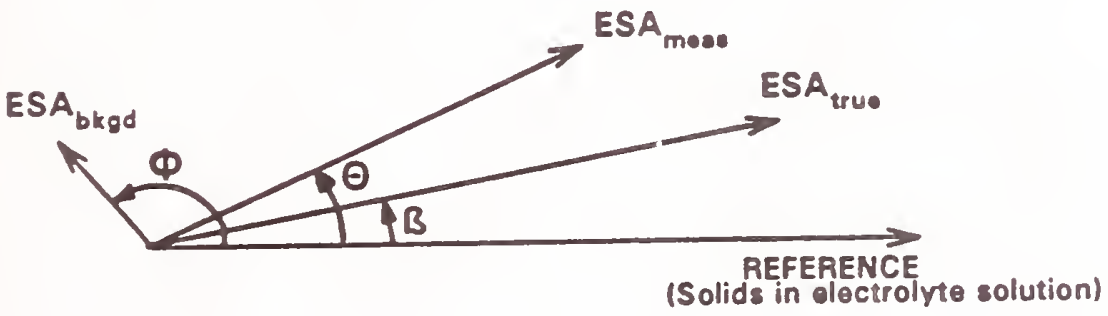

Figure 6. Background correction for electrolyte (Desai et al., 1993) 


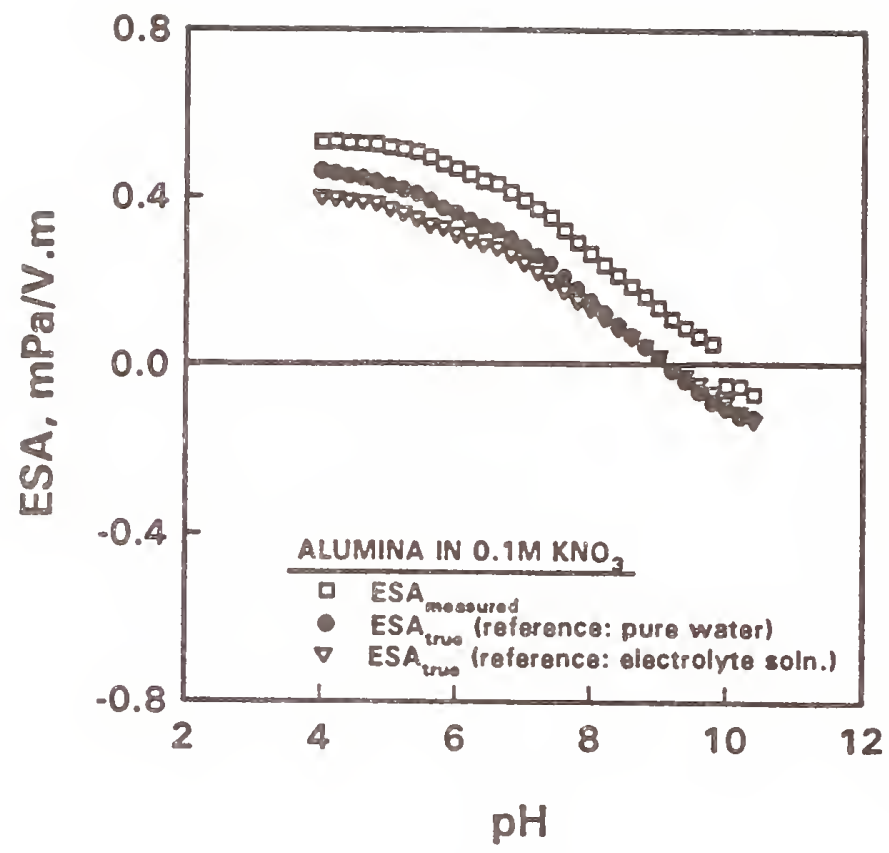

Figure 7. Measured and corrected ESA's for $\gamma$-alumina sample at $0.1 \mathrm{M} \mathrm{KNO}_{3}$.

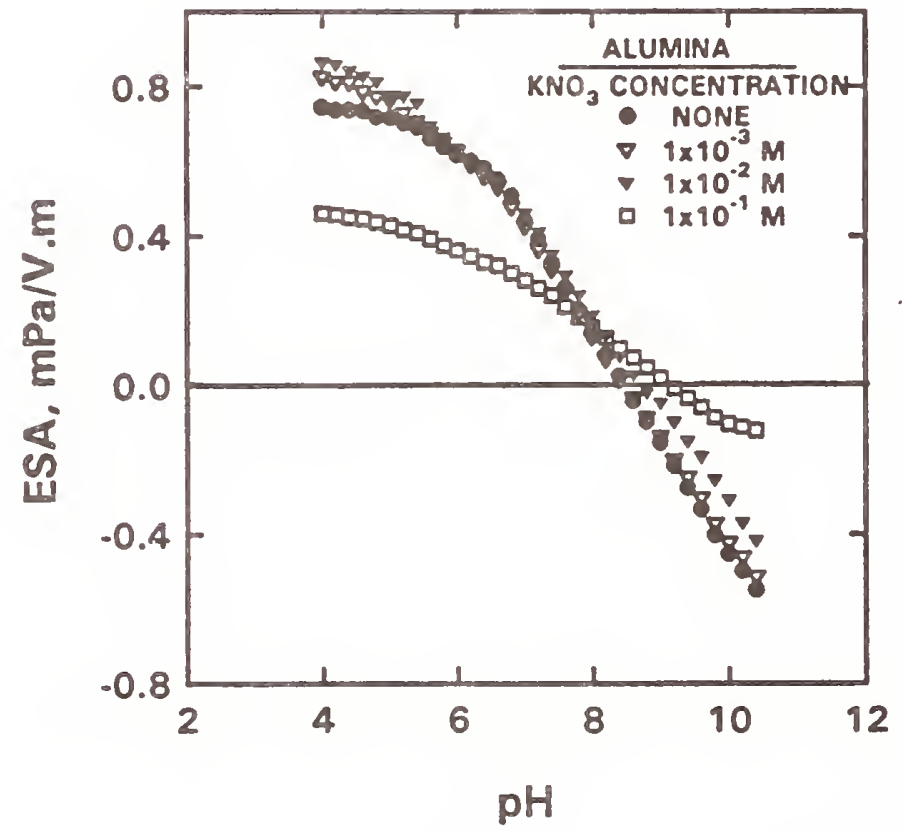

Figure 8. Effect of electrolyte concentration on the ESA for $\gamma$-alumina. 

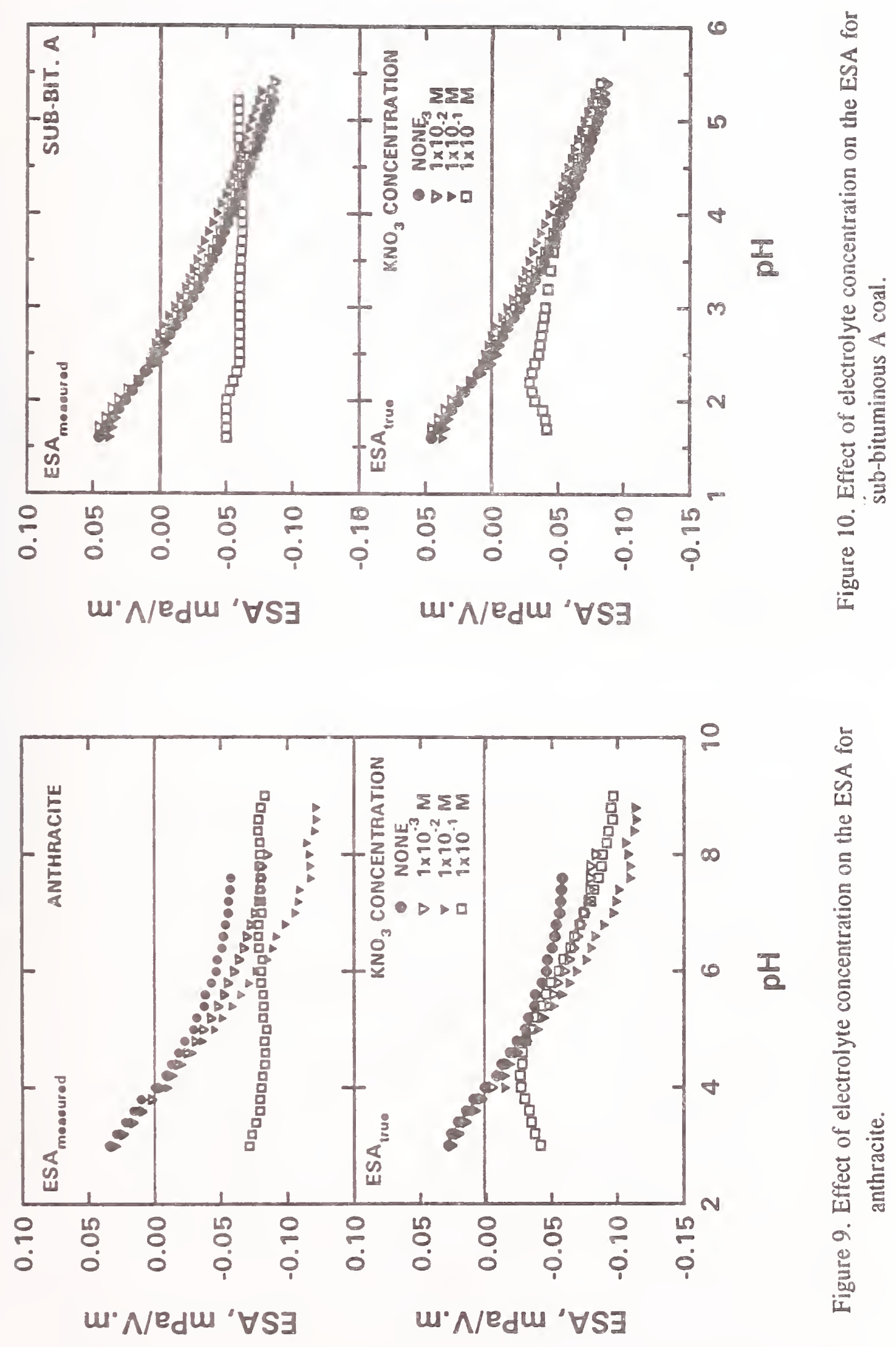


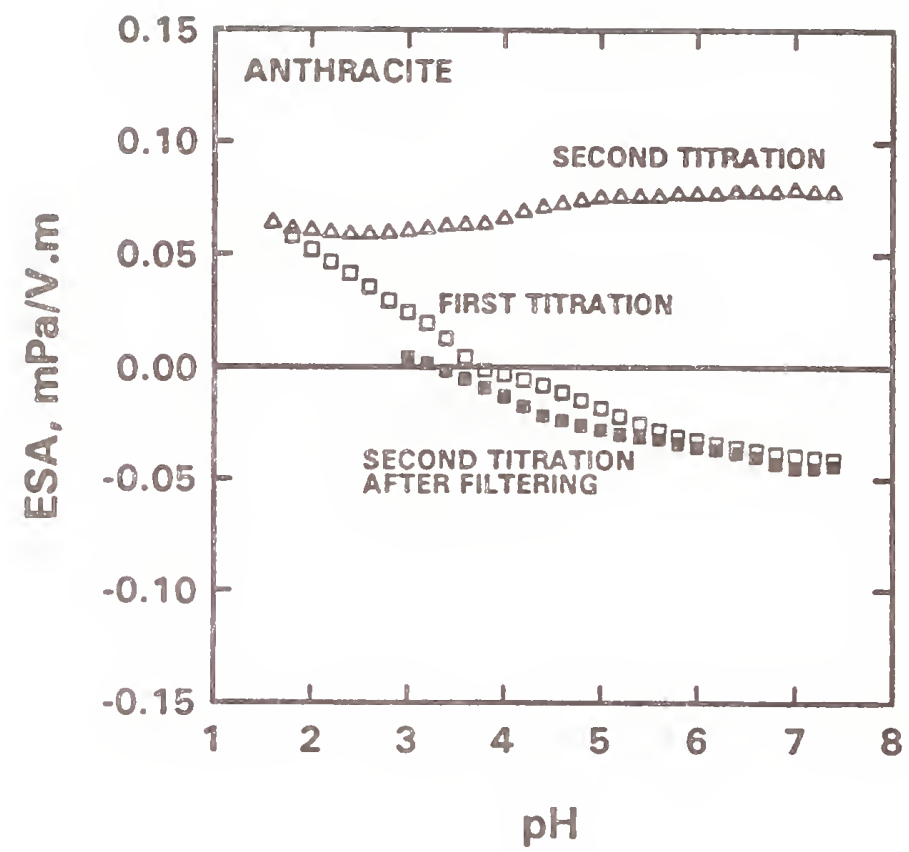

Figure 11. Effect of acid-base titrations on the ESA for anthracite.

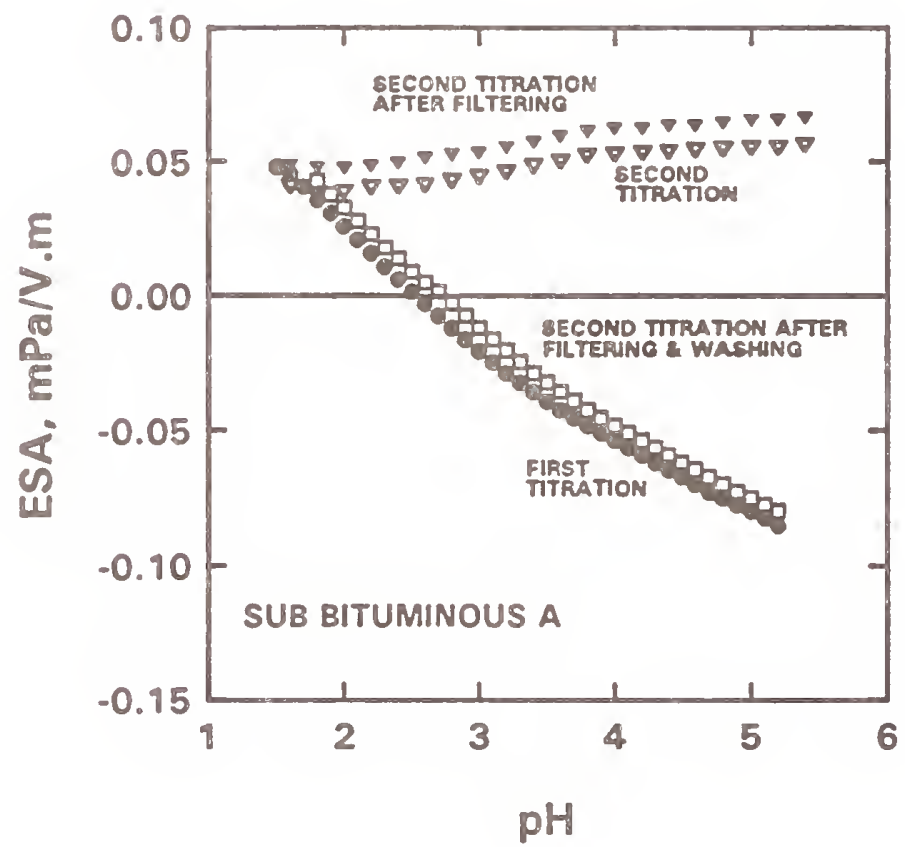

Figure 12. Effect of acid-base titrations on the ESA for sub-bituminous A coal. 


\author{
B. M. Moudgil and R. Damodaran \\ Department of Materials Science and Engineering \\ University of Florida \\ Gainesville, FL 32611
}

\begin{abstract}
Surface charge characterization of minerals is important in developing suitable solid-solid and solid-liquid separation techniques. Adsorption of surfactants or polymers on solids, which is dependent on the surface charge characteristics, govern the efficiency of physicochemical processes such as flotation and flocculation. Generally, relative surface charge measurements are adequate for process development purposes. Electrokinetics measurements were employed until a few years ago to evaluate the surface charge behavior of dilute as well as concentrated suspensions of minerals. Presently, electroacoustic techniques are being evaluated for surface charge characterization. In this paper, the opportunities and limitations of electroacoustic measurements, as applied to mineral processing operations are discussed.
\end{abstract}

\title{
INTRODUCTION
}

The study of colloidal phenomena is important in a vast array of materials, processes and physical properties. Colloids are typically liquids containing very small particles ranging in size from 1 to 1000 nanometers, common examples of which are milk, blood, pigments, pharmaceuticals, inks, dyes, ceramics and clays. In mineral processing, the theories of colloidal phenomena have been used for surface charge characterization of suspensions in which the particle sizes are in the range 0.2 to 10 micrometers. Many properties of colloidal suspensions, such as dispersion/aggregation, polymer and surfactant adsorption on solid particles, and aging phenomena are determined, directly or indirectly, by the presence of electrostatic charge on the surface of the particles in suspensions. The charge on the particles can develop from a variety of mechanisms, such as ionization of surface chemical groups, adsorption of ions, or unequal dissolution of ions from an ionic crystal lattice. The surface charge of the particle is balanced by an equal and opposite charge of "counter-ions" 
distributed as a diffuse cloud around the particle. The combined region of surface and diffuse charge is known as the "electrical double layer". The electrical potential at the plane of shear is termed the zeta potential which determines the electrostatic interaction forces between the particles. Important colloid properties, such as suspension rheology and suspension stability towards aggregation, are strongly influenced by the zeta potential.

Surface charge characterization of particles in suspensions find wide applications in important mineral processing operations such as flotation, filtration, sedimentation and selective flocculation. The desired state of dispersion or aggregation of the particles in suspension, required in such operations are achieved by control of the surface properties including its charge characteristics. Modification of the surface properties may be brought about by adsorption of surfactants or polymers. Polymer and surfactant interactions with the mineral particles are governed by hydrogen bonding, covalent bonding, electrostatic or hydrophobic interactions. Electrostatic interactions of polymer and surfactants are determined by the charge of the polymer or the surfactant as well as that of the solid substrate. Surface reactions in particles such as aging phenomena, which play an important role in determining surface properties as a function of time, can be monitored by surface charge characterization of the particles. Both dilute and concentrated slurries are encountered in mineral processing, which emphasizes the use of techniques to characterize surface charge both under dilute and concentrated conditions.

\section{ELECTROKINETIC MEASUREMENTS}

Until recently, the measurement and control of zeta potential in industrial processes has been done by electrokinetic measurement techniques which involve one of the four classical electrokinetic phenomena; electrophoresis, electro-osmosis, streaming potential and sedimentation potential. Of these four electrokinetic effects, electrophoresis and the streaming potential are more frequently employed in studies related to minerals. In microelectrophoresis, the velocity of individual particles under the influence of a known dc or low frequency electric field, is measured in extremely dilute suspensions (less than 100 ppm). Such low solids concentrations do not truly represent the practical systems, hence, the 
data obtained from such measurements has to be extrapolated to the conditions of the real system, which may not always be correct. Nevertheless, microelectrophoresis has been used extensively to characterize surface charge behavior of solid-liquid and liquid-liquid interfaces. It should be noted that more often it is the value of the iso-electric point and the trends in the behavior of the disperse systems that have significance, compared to the specific calculated values of the zeta potentials.

\section{Applications in Mineral Processing}

Electrokinetic measurements of zeta potential have been used to correlate bulk properties such as viscosity of suspensions with the surface chemical behavior of particles. A typical correlation between viscosity of concentrated alumina slurries ( $40 \mathrm{vol} \%)$ with the zeta potential of alumina measured using dilute suspensions $(100 \mathrm{ppm})$ is shown in Figure $1 .^{[1]}$ A sharp increase in viscosity (at $\mathrm{pH} 4.5-5.0$ and again at $\mathrm{pH} 10.8-11.2$ ) is observed with a slight increase in the zeta potential. These observations suggest that, zeta potentials measured using microelectrophoresis may not be directly correlated with the viscosity of a concentrated suspension. Electrokinetic measurements have also been used to study polymer adsorption onto mineral particles and polymer-surfactant interactions. Zeta potential measurements were conducted to investigate the nature of interactions between polyacrylamide type polymers, and anionic and cationic surfactants, in a study on the effects of such interactions on adsorption of various species on hematite and silica (see Figure 2). ${ }^{[2]}$ The effect of higher pulp densities encountered in mineral processing operations, however, can only be speculated because under such conditions the suspensions could aggregate, making it difficult to keep the particles suspended during the electrophoretic measurements. The electrokinetic behavior of the individual minerals and the changes taking place at the solid/liquid interface, when other minerals are present, are important considerations in flotation. Changes in the zeta potential of apatite and dolomite as a function of $\mathrm{pH}$ were used to study dissolution behavior, which affected the flotation results during separation of phosphate rock from dolomite. ${ }^{[3]}$ A correlation between mobility, settling, and surfactant adsorption density, shown in Figure $3,{ }^{[4]}$ emphasizes the use of electrokinetic measurements to monitor bulk properties. Again, the effect of solids loading can only be speculated. An 


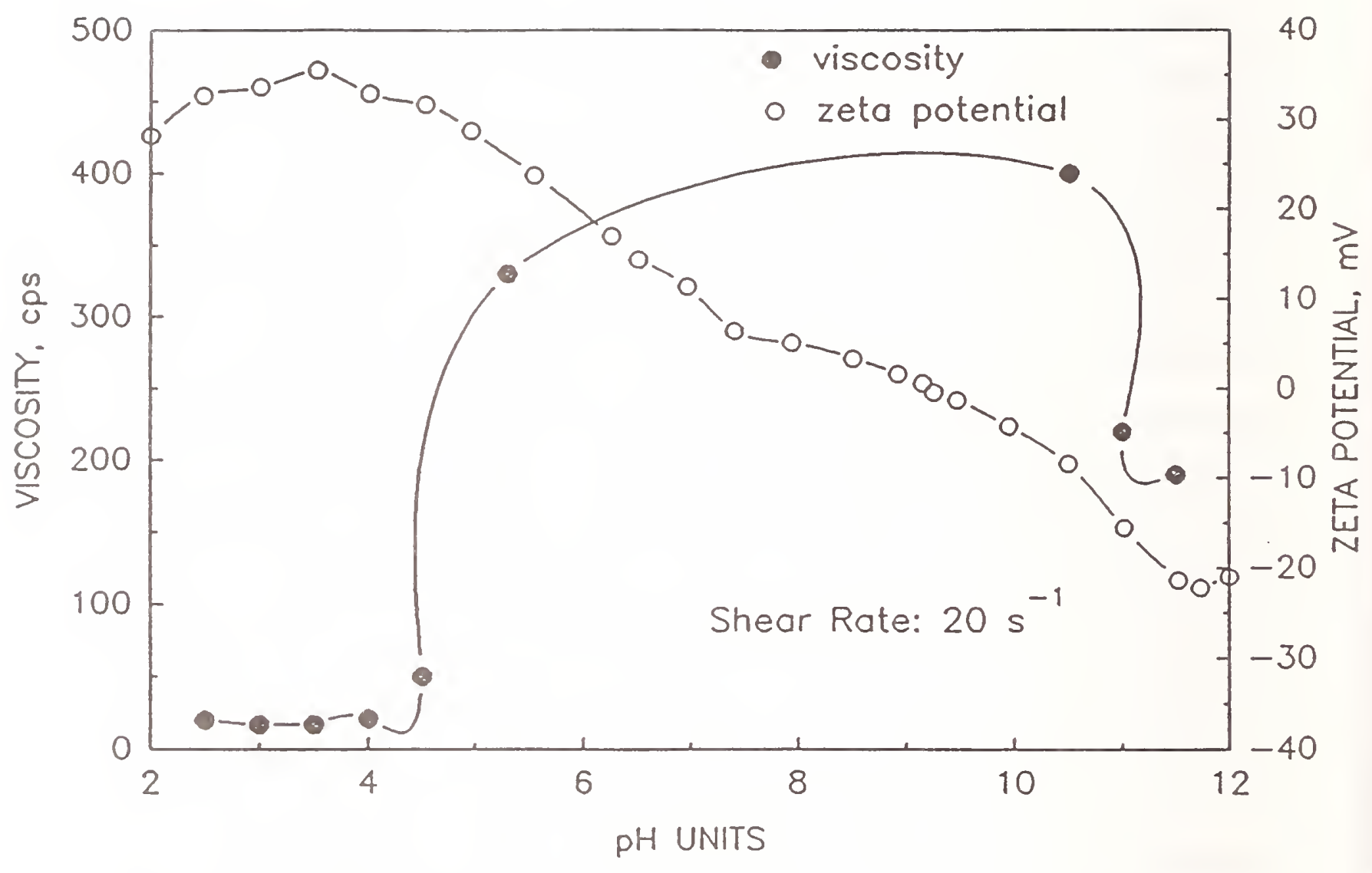

Figure 1. Correlation between zeta poteritial and viscosity (40 vol\% alumina) ${ }^{[1]}$ 


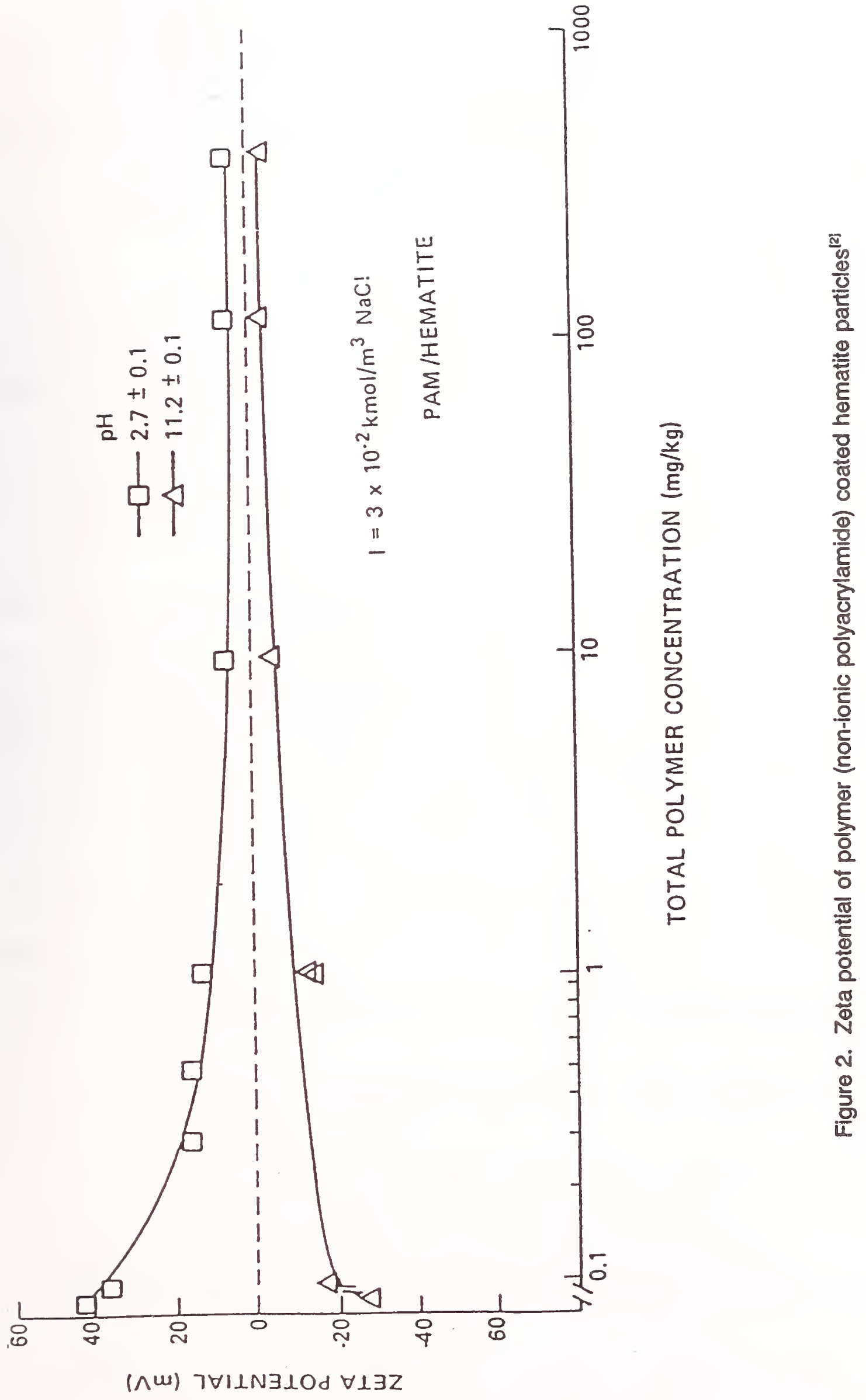




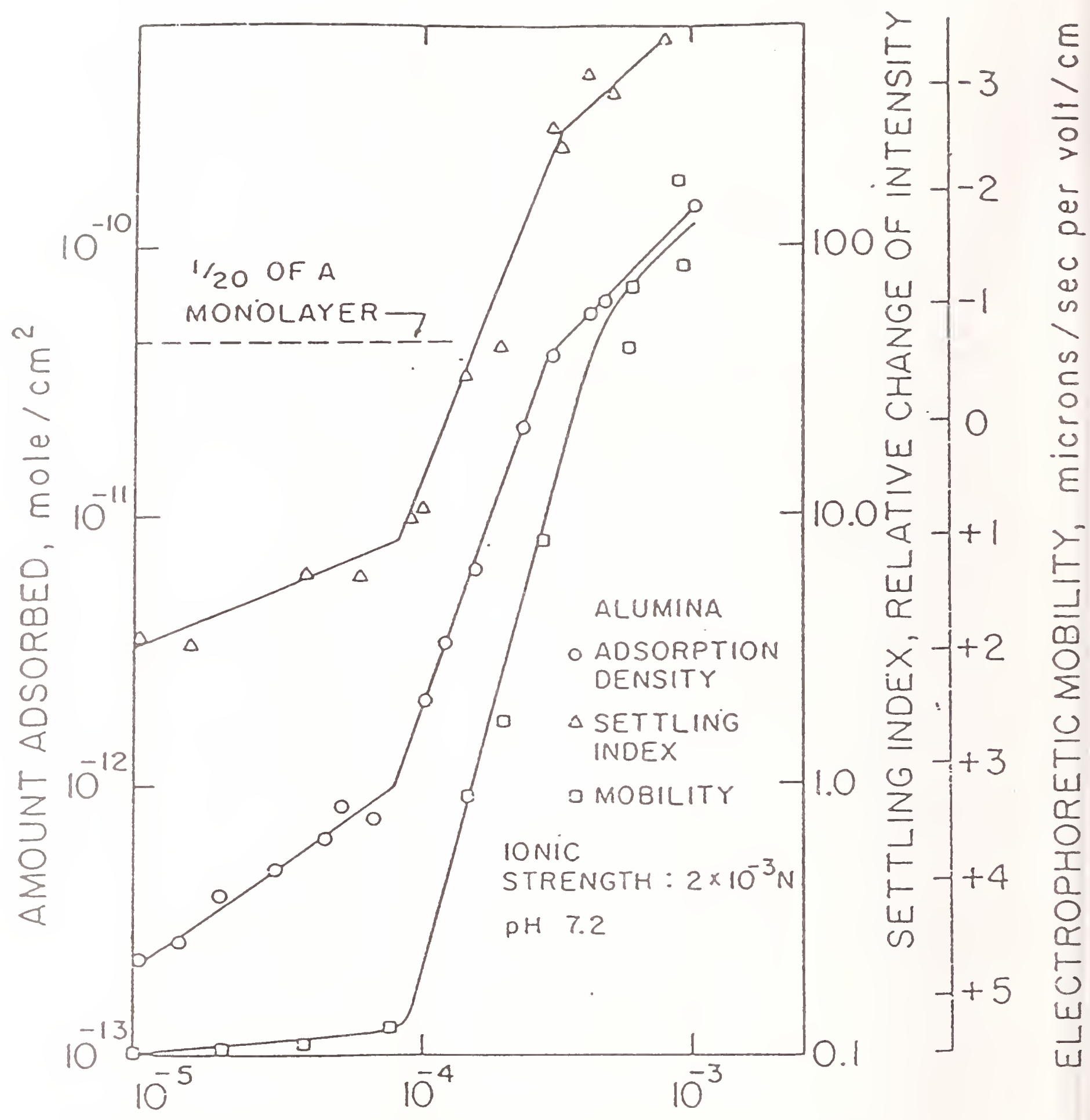

EQUILIBRIUM CONCENTRATION, mole/ liter

Figure 3. Correlation between mobility, settling and surfactant adsorption density ${ }^{[4]}$ 
useful review of zeta potential studies in mineral processing has been published by Mackenzie. ${ }^{[5]}$ The following technique for surface charge characterization of relatively concentrated suspensions became available in the last decade.

\section{ELECTROACOUSTIC MEASUREMENTS}

Acoustophoretic technique can be applied to characterize surface charge of colloidal particles (less than 1.0 micrometers). A wide range of sample concentrations, ranging from $100 \mathrm{ppm}$ to $50 \mathrm{vol} \%$ can be measured using this technique. Absence of optical imaging permits characterization of opaque systems. The most important advantage is the potential use of acoustophoresis in on-line analysis of real systems as in flocculation processes, quality control, dispersion and emulsion formulation, raw materials screening and process monitoring.

The electroacoustic instrument measures the response of charged particles to alternating electric and pressure fields instead of using static electric or pressure fields such as in electrophoresis or streaming potential methods. An applied alternating field induces a tangential motion between the particles and the liquid, resulting in a periodic distortion of the particle's electrical double later. If there is a density difference between the suspended particles and the liquid, the following electroacoustic phenomena occur;

1. When an alternating electric field is applied, the relative motion between the particles and liquid generates a sound wave at the frequency of the applied field. The particles vibrating in the electric field radiates sound which sums up to a coherent sound wave. This effect has been termed the Electrokinetic Sonic Amplitude or ESA of the colloid and is defined as the pressure amplitude per unit electric field generated by the colloid, and has units of pascals per volt per meter.

2. In the case of an applied alternating pressure field (sound wave), the inverse of the ESA effect occurs. The relative motion between the particles and liquid, due to their density difference, creates a periodic polarization of the electrical double layers and an alternating dipole moment at the frequency of the applied field, which sum to a potential 
that can be detected by placing a pair of electrodes in the sound field. This effect is called the Ultrasonic Vibration Potential or UVP and was first predicted for electrolyte solutions by Debye in 1933. ${ }^{[6]}$ UVP has units of volts per meter per second. The UVP in colloidal systems was first studied theoretically by Enderby ${ }^{[7]}$ in 1951 and more recently by $\mathrm{O}^{\prime} \mathrm{Brien}^{[8]}$ and White. ${ }^{[9]}$

The electroacoustic measurement system as illustrated in Figure 4, generally consists of a signal generator with a frequency synthesizer, a titrator assembly and a computer system controller. The titrator assembly consists of a sample cell and the sensors for detecting $\mathrm{pH}$, temperature, conductivity, electroacoustic signals, and a high torque stirrer. The probe assembly can be placed into a cell of any geometry and hence is ideal for on-line analysis.

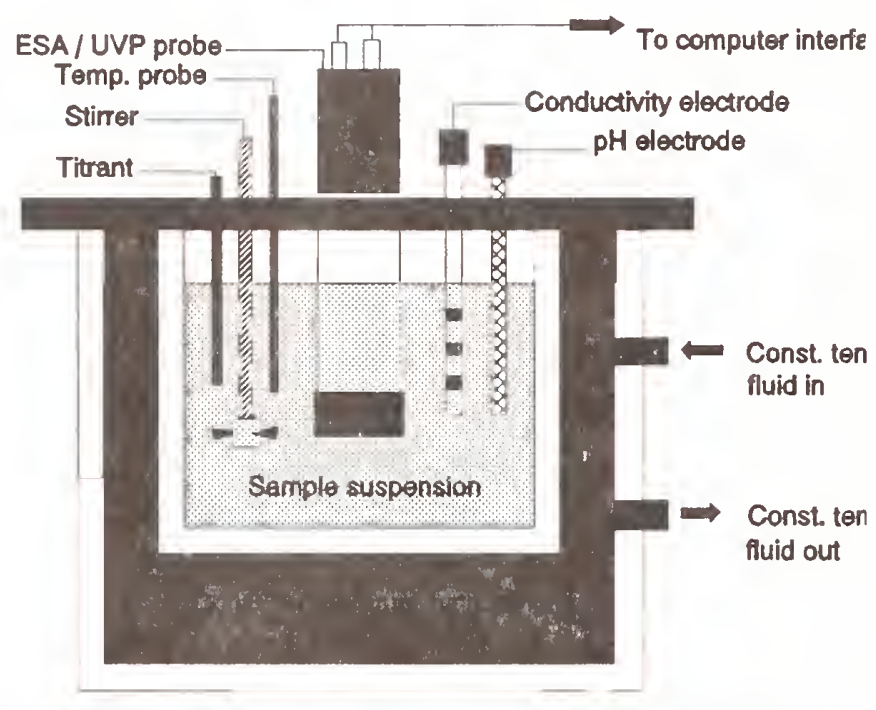

Figure 4. Schematic diagram of an ESA sample cell

\section{Relation Between ESA, UVP and Zeta Potential}

Rigorous theoretical treatments have been developed by O'Brien, ${ }^{[8]}$ Marlow et al., ${ }^{[10]}$ and Babchin et al. ${ }^{[1]}$, to arrive at relationships between UVP, ESA, the zeta potential and the electrophoretic mobility. A relationship between ESA, UVP and a high frequency or dynamic mobility $\left(\mu_{\omega}\right)$ of the form 


$$
\operatorname{UVP}\left(\mathrm{K}^{*}\right)=\mathrm{ESA}=\mathrm{c} \Delta \rho \phi \mu_{\omega} \gamma
$$

has been predicted, where $\mathrm{K}^{*}$ is the complex conductivity of the dispersion, $\mathrm{c}$ is the speed of sound in the fluid, $\Delta \rho$ is the density difference between the particle and the fluid, $\phi$ is the volume fraction of the particles in the fluid, and $\gamma$ is a geometrical and acoustic coupling factor. O'Brien gives an approximate relationship between $\mu_{\omega}$ and $\zeta$ for the case where $к$ a is less than 10:

$$
\mu_{\omega}=(2 \epsilon \zeta / 3 \eta)(1+f) \mathrm{G}(\alpha)
$$

where $\epsilon$ is the dielectric permittivity of the fluid, $\eta$ is the fluid viscosity, $f$ is a complex function of the frequency of the applied field and the diffusivity of the electrolyte ions in solution; $G(\alpha)$, a damping term, is a complex function of the frequency of the applied field $(\omega)$, the average radius of the particles in solution (a), and the kinematic viscosity $(\nu)$, such that

$$
\alpha=\omega \mathrm{a}^{2} / \nu
$$

and

$$
G(a)=\left[1-\frac{\frac{1}{9} i a\left(3+\frac{2 \Delta \rho}{\rho}\right)}{1+(1-i) \sqrt{\frac{a}{2}}}\right]^{-1}
$$

where $\rho$ is the fluid density. At high $\kappa$, the ratio of $\mu_{\omega}$ to $\mu_{\mathrm{e}}$ may be approximated to

$$
\mu_{\omega} / \mu_{\mathrm{e}}=2 / 3(1+f) \mathrm{G}(\alpha)
$$

In general, the magnitude of the ESA depends on the solids concentration in the suspension, the zeta potential of the particles and the density difference between the particles and the suspension medium. 


\section{Experimental Verifications}

A number of researchers have demonstrated excellent agreement between the calculated zeta potentials from electroacoustics and microelectrophoresis for a range of solids using the above mentioned theoretical treatments. However, it has been found that frequently the magnitude of the zeta potential calculated from dynamic mobilities are different from those calculated from other electrokinetic techniques. It was demonstrated by Scales and Jones ${ }^{[12]}$ that this discrepancy was due to an error in the input of the particle size. These investigators have arrived at correlations between the dynamic mobility and the electrophoretic mobility for different particle sizes and frequencies (see Figure 5). The required volume fraction to ensure an error of less than $5 \%$ in mobility, as a function of the density difference, plotted in Figure 6, for two different values of ionic strengths, arrived at, highlights the importance of electrolyte signal in such measurements. ${ }^{[12]}$ The density difference between most minerals and water is normally high enough to use a suspension concentration of 1 to 2 vol\%. The use of electroacoustic techniques for the accurate measurement of zeta potential is straightforward for monosized particles and for polydisperse dispersions where the particle size is distributed in a normal fashion about the mean.

Marlow et al. ${ }^{[10]}$ have reported good agreement between measured values of electrophoretic and electroacoustic mobilities for a $700 \mathrm{ppm}$ sample of $\mathrm{TiO}_{2}$ in $0.001 \mathrm{M} \mathrm{KCl}$ as a function of $\mathrm{pH}$ (see Figure 7). The electroacoustic mobilities agreed to within $10 \%$ of the electrophoretic mobilities, thus independently verifying the UVP theory for dilute suspensions. For higher suspension concentrations, a knowledge of the continuous phase conductivity is required to compare experimental and theoretical values. Electroacoustic mobilities for colloidal silica (3.5 nm particles) at $20 \% \mathrm{w} / \mathrm{w}$ as a function of $\mathrm{pH}$, measured by Marlow et al. ${ }^{[10]}$ highlights the adivantage over electrokinetic techniques in characterizing colloids which are optically opaque.

For polydisperse samples, O'Brien et al. ${ }^{[13]}$ have shown that the effective mass average particle size has to be taken into consideration for accurate measurements of zeta potential using the electroacoustic techniques. A comparison of zeta potential, obtained from microelectrophoresis and electroacoustics, as a function of $\mathrm{pH}$ is provided in Figure 8. 


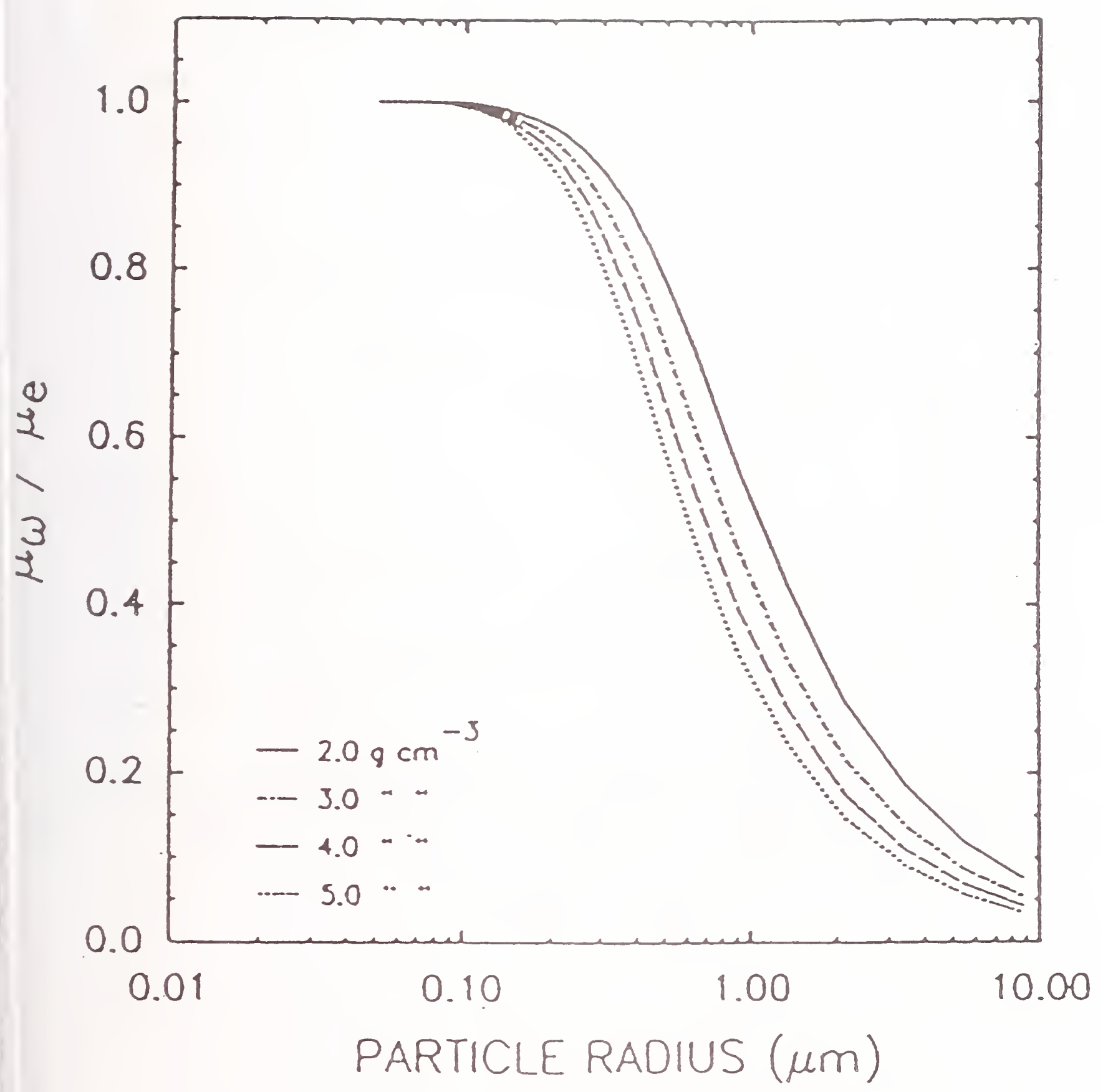

Figure 5. Calculated ratio of dynamic to electrophoretic mobilities (for $\mathrm{KCl}$ at $0.1 \mathrm{~mol} \mathrm{dm}^{-3}$, particle relative permittivity of 5 and a zeta potential value of $30 \mathrm{mV})^{[12]}$ 


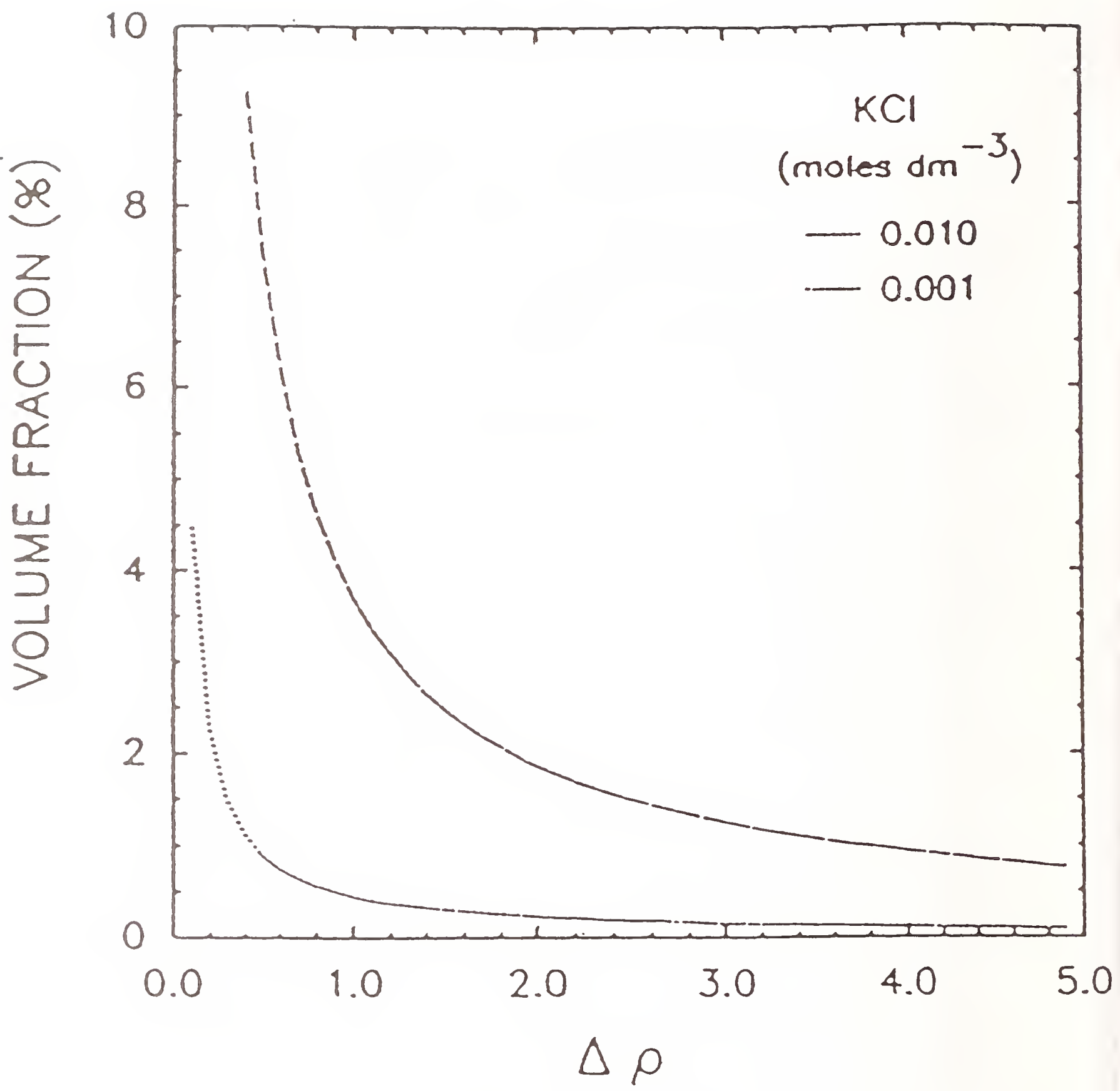

Figure 6. Volume fraction versus density difference for a $5 \%$ error cut (for two $\mathrm{KCl}$ concentrations) ${ }^{[12]}$ 


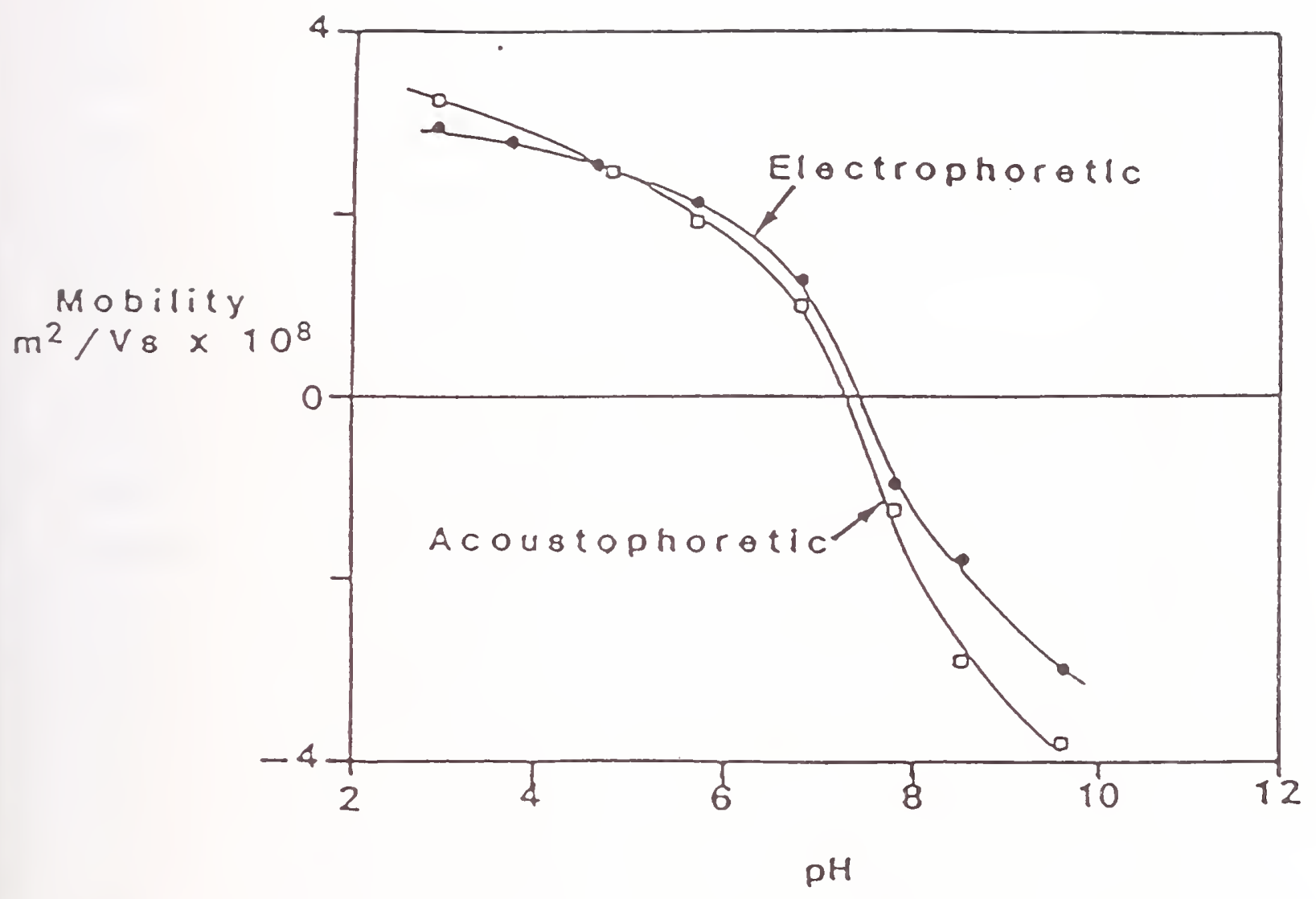

Figure 7. Comparison of electrophoretic and acoustophoretic mobilities (data is for a $700 \mathrm{ppm} \mathrm{TiO}_{2}$ sample in $\left.0.001 \mathrm{M} \mathrm{KCl}\right)^{[10]}$ 


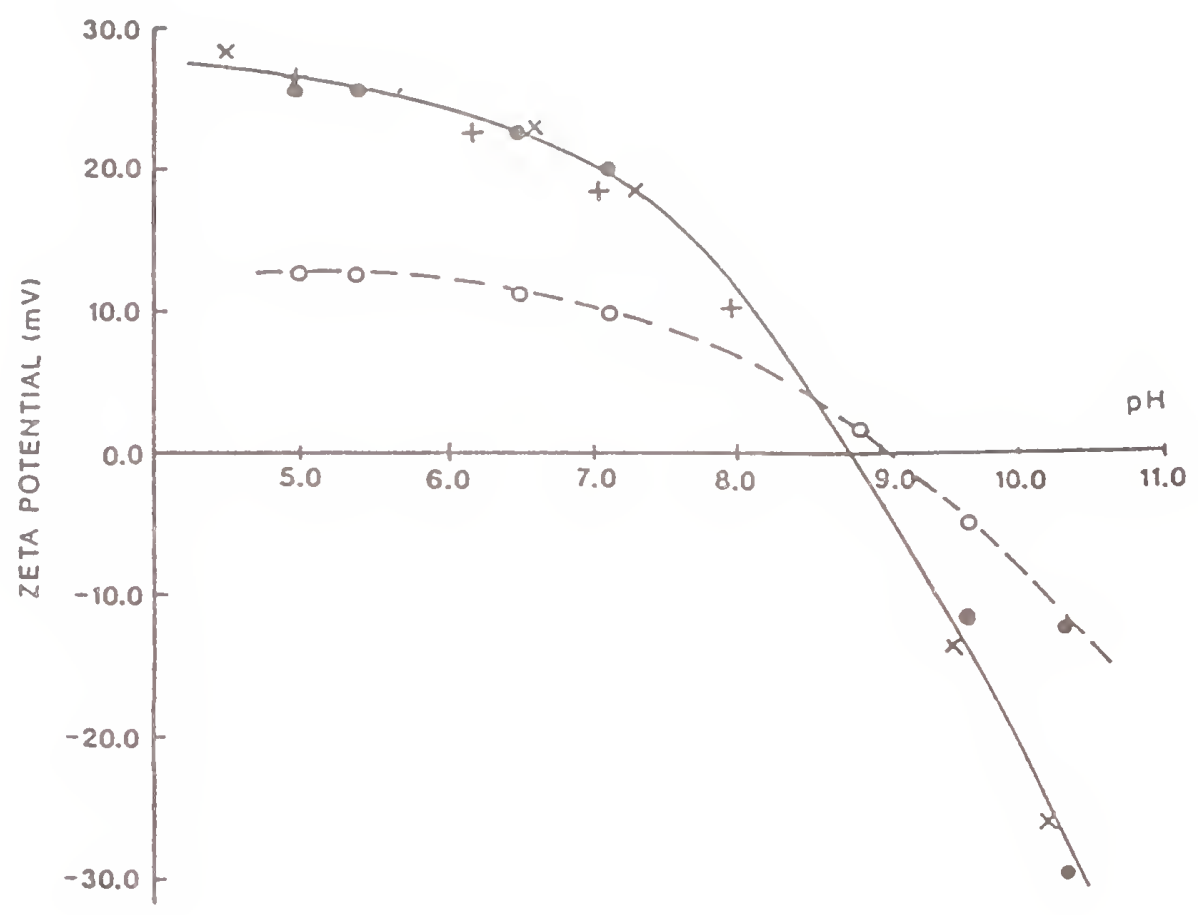

Figure 8. Effect of effective mass average radius correction on zeta potentials (alumina in $10^{-3} \mathrm{M} \mathrm{KCl}$ solution) ${ }^{[13]}$ 
The values of zeta potential, obtained using initial estimates of the mass average particle size on a $0.5 \mathrm{vol} \%$ suspension, are low compared to the data obtained using microelectrophoresis. However, electroacoustic results of a well dispersed sample show excellent agreement with the microelectrophoresis results. There is an excellent agreement of the corrected electroacoustic results, using light scattering estimates for the effective mass average radius at each $\mathrm{pH}$, with the microelectrophoresis values.

Miller and Berg ${ }^{[14]}$ found good agreement of microelectrophoresis mobility data for titania, to inertially-corrected mobility data calculated from ESA measurements upto the isoelectric point of titania, beyond which particle aggregation was reported to result in deviation between the two curves. Figure 9 compares the two techniques for titania/PVA adlayer system. Electroacoustic techniques have been demonstrated to be useful in measuring the effect of polymer molecular weight on zeta potential. It has been suggested that electroacoustic studies on titania dispersions with an adsorbed layer of PVA can be used to calculate an effective adsorbed layer thickness on the particles.

All the above mentioned studies have been conducted in suspensions of not more than 5 vol\% solids loading, which again, is not representative of the slurries encountered in mineral processing applications. Such operations involve solids loading of $20 \mathrm{vol} \%$ or more. Additionally, typical ceramic powders processed are at solids loading around $40 \mathrm{vol} \%$ or more. At such high concentrations, where particle-particle interactions cannot be ignored, existing theories of particle behavior cannot predict the bulk properties satisfactorily. Direct measurements of mobility at such a high solids loading have been reported by Springgate ${ }^{[15]}$ using electroacoustics. The effect of pH on the ESA values in a 40 vol\% alumina suspension were shown to follow the same general trend as in dilute suspensions (see Figure 10). However, the $\mathrm{pH}$ range in which ESA values are high is broader than that for a dilute suspension, indicating that concentrated alumina slurries can be stabilized at higher $\mathrm{pH}$ values than the dilute suspensions. 


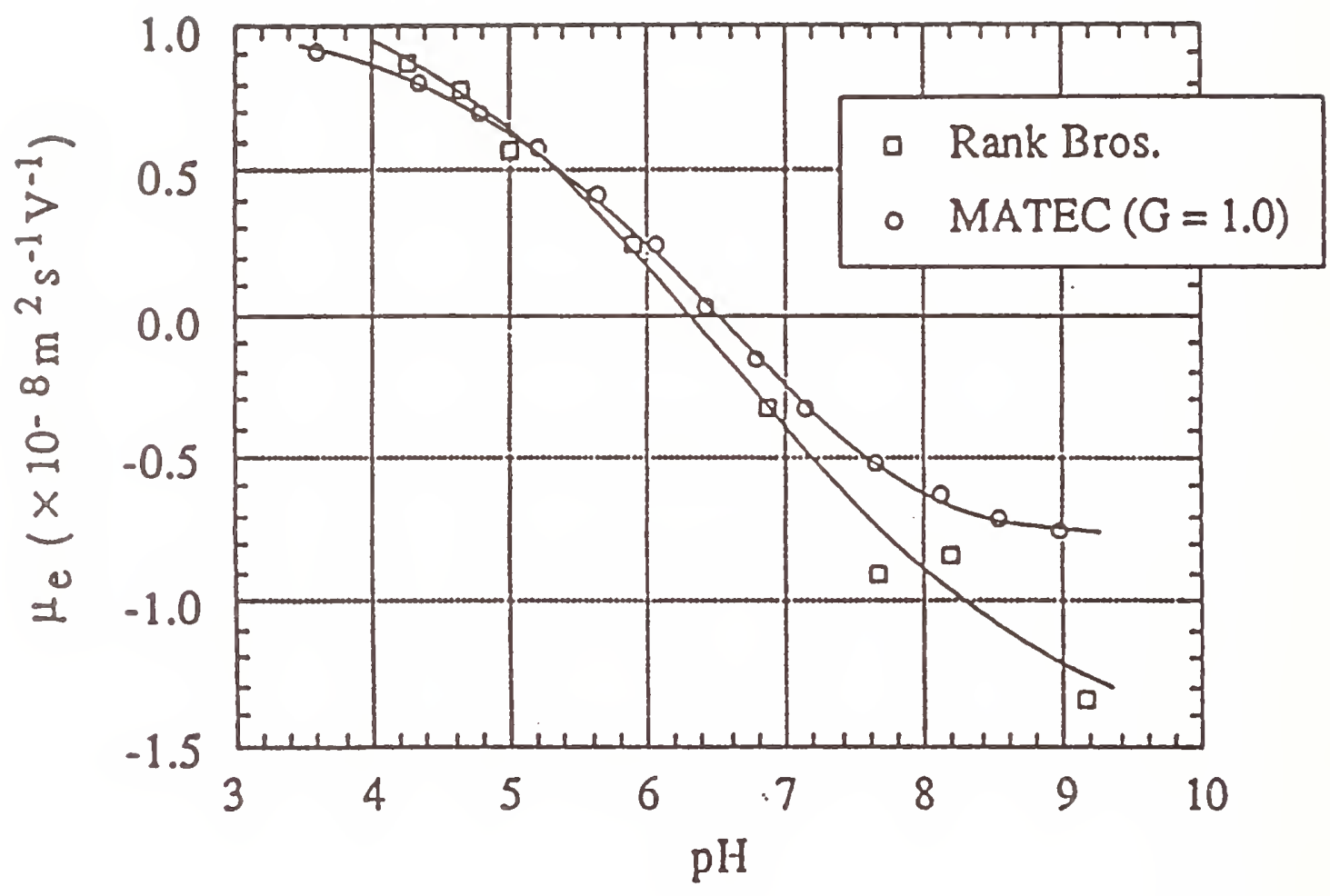

Figure 9. Comparison of electroacoustic and electrophoretic mobilities for a polymer coated titania dispersion ${ }^{[14]}$ 


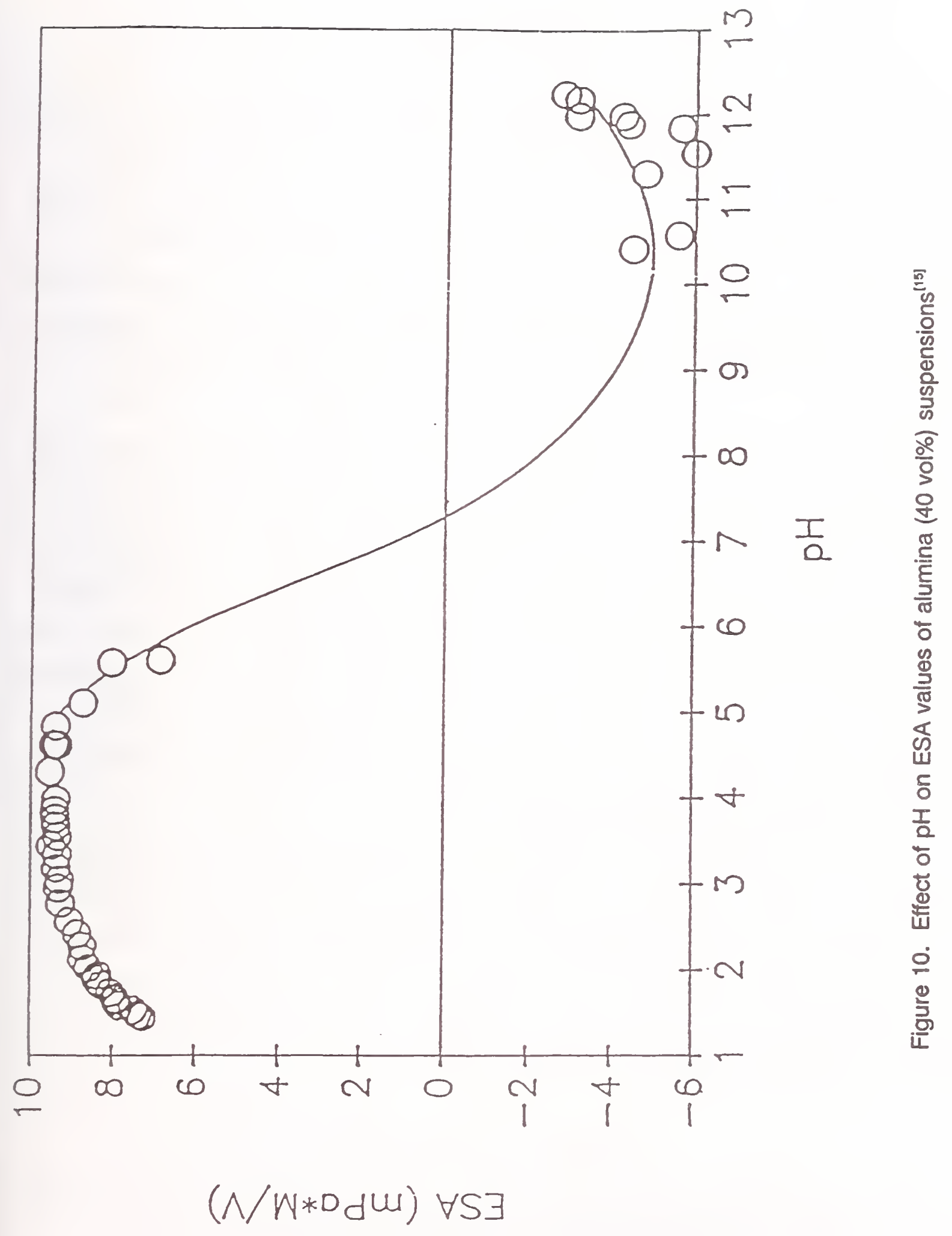




\section{CONCLUSIONS}

Electrokinetic techniques for surface charge characterization have traditionally been used to study surface chemical phenomena and arrive at correlations with bulk properties in mineral processing applications. Recently developed electroacoustic technique have made it possible to determine surface charge behavior of relatively concentrated suspensions. Dynamic mobility is related to electrophoretic mobility and an excellent correlation has been observed in zeta potential trends obtained by electroacoustic and electrophoretic techniques. Electroacoustic methods have a distinct advantage over electrokinetic measurement techniques.

Mineral suspensions are heterogeneous in nature and a range of particle size distribution is encountered. Electrokinetic measurements, which require small sample volumes and can be conducted only in dilute suspensions, have been adequate for most mineral processing applications. However, such measurements are limited to suspensions which are optically transparent and cannot be used in high viscosity media. Electroacoustic measurements, on the other hand, require larger samples, although smaller volumes can be used with a parallel plate sensor, and can be done in concentrated or flocculated suspensions. There are no optical or viscosity limitations to the type of suspension used and measurements in stirred and flowing systems are possible with a probe sensor.

Electroacoustic measurements for surface characterization are used in areas such as basic characterization of the solid-liquid or liquid-liquid interface of disperse systems, screening raw materials for differences in surface properties, and monitoring processes in real-time. Industrial applications of electroacoustic measurements include scale-up of flocculation processes, preparation of coal-water slurries, dispersion of clays and other minerals, evaluation of dispersing agents, and dewatering of clays.

\section{ACKNOWLEDGEMENTS}

The authors acknowledge the Minerals Resources Research Center, University of Florida for their financial support towards this work. 


\section{REFERENCES}

1. Kulkarni, N., M.S. Thesis, University of Florida, 1993.

2. Moudgil, B. M., Engg. Sci. D. Thesis, Columbia University, New York, 1981.

3. Moudgil, B. M. and Ince, D., Surfactants in solution, 10, 1989, Ed.K.L. Mittal, Plenum Press, New York.

4. Somasundaran, P., Ph D. Thesis, University of California, Berkeley, California, 1964.

5. Mackenzie, J. M. W., Mineral Sci. Engg., 3(3), 25, 1971.

6. Debye, P., J.Chem. Phys., 1, 13, 1933.

7. Enderby, J., Proc. Phys. Soc., 207, 321, 1951.

8. O’Brien, R. W., J. Fluid Mech., 190, 71, 1988.

9. White, L. R., Trans. Faraday Soc., 1987.

10. Marlow, B. J., Fairhurst, D., Pendse, H. P., Langmuir, 4,611, 1988.

11. Babchin, A. J., Chow, R. S., Sawatzky, R. P., Adv. Colloid Interface Sci., 30, 11, 1989.

12. Scales, P. J. and Jones, E., Langmuir, 8, 385, 1992.

13. James, M., Hunter, R. J., O’Brien, R. W., Langmuir, 8, 420, 1992.

14. Miller, N. P. and Berg, J. C., Colloids and Surfaces, 59, 119, 1991.

15. Springgate, M. E., M.S.Thesis, University of Florida, 1990. 


\title{
SURFACE CHARGE CHARACTERIZATION OF PORTLAND CEMENT IN THE PRESENCE OF SUPERPLASTICIZERS
}

\author{
David C. Darwin" Roger Y. Leung, and R. Trevor Taylor \\ W.R. Grace \& Co. - Conn. \\ Research Division \\ Washington Research Center \\ 7379 Route 32 \\ Columbia, MD 21044
}

"corresponding author

\begin{abstract}
The surface charge characterization techniques of electroacoustics, microelectrophoresis, and streaming potential are compared for the system of superplasticizer and portland cement. All techniques were found to have limitations with respect to the absolute zeta potential determination of realistic systems. Electroacoustic measurements were used to evaluate interparticle effects in portland cement slurries. The electroacoustic measurements on portland cement showed important differences from results obtained on other colloidal systems.
\end{abstract}

\section{INTRODUCTION}

Over one billion tons per year of Portland cement is manufactured in all parts of the world, and used as the primary binder in concrete for construction. Essentially, the production process involves mining minerals containing mainly calcium carbonate and silicon oxides. These are kiln fired, forming hard pellets called clinker. The clinker is then finely ground with a few percent calcium sulfate, usually in a ball mill. The resulting Portland cement is then a powder with a broad distribution of particle sizes, with mean radii typically from 3 to 10 microns.

In its end use application, portland cements are combined with water, sand, and stones to form concretes. Typical mass based water to cement ratios range from 0.3 to 0.8 . The 
cement particles and water form a paste or slurry. However, the mixture is not a typical colloid as most of the particles are quite large and show effects of settling. Analysis of the cement - water systəm is further hindered by the fact that the cement particles begin to react with the water from the moment of first contact. This results in surface and solution compositions that change with time.

Nevertheless, the cement - water system has received extensive study with respect to its electrokinetic properties [1-10]. Other researchers have gone a step further and examined the electrokinetic behavior of individual phases within the cement $[11,12]$. In the concrete industry it is common to admix various chemicals to control the reaction chemistry and rheology of hydrating cement suspensions. Many of these systems have been subject to electrokinetic analysis [13-24]. Among the most interesting of these admixtures are anionic polymers called superplasticizers. Superplasticizers are dispersants which reduce the yield stress of concretes and allow increased flow of fresh concrete without addition of water, which would serve to reduce the compressive strength of the hardened material if added. It is widely believed that superplasticizers adsorb onto cement particles and induce negative charge.

Past works generally used microelectrophoresis techniques to determine the zeta potential of cement particles in dilute solutions. The advent of electroacoustic analysis which can measure electrokinetic properties under concentrated slurry conditions has led us to investigate the properties of concentrated portland cement slurries. In this study, we compare the surface charging behavior of superplasticizers on portland cements using electroacoustic techniques, microelectrophoresis, and streaming potential. We then use electroacoustic methods to investigate the interparticle effects of electrokinetic measurements at higher cement volume fractions.

\section{EXPERIMENTAL}

Two portland cements were used, referred to as "New York" and "Texas". The mean particle radii were determined by laser light diffraction to be 5.5 and 4.9 microns, respectively. A particle size distribution for the Texas cement is shown in Figure 1. A 
compositional analysis of both cements is given in Table 1. The superplasticizer used was a commercial naphthalene sulfonate formaldehyde condensate (designated NSFC; Grace, WRDA-19). The superplasticizer was supplied as a $40 \%$ solution in water and was diluted to appropriate concentrations for use.

Electroacoustic measurements were made using a Matec Applied Sciences ESA-8000 System in ESA mode. The electroacoustic probe was calibrated to a LudoxTM silica standard, as described in the Matec manual. Conductivity and $\mathrm{pH}$ electrodes were calibrated using standard $\mathrm{KCI}$ solutions and standard $\mathrm{pH}$ buffers. For an electroacoustic measurement, cement was weighed out corresponding to the desired volume fraction, and the proper amount of deionized water was added. The resulting slurry was magnetically stirred for 4 minutes, and then put onto the ESA 8000 . Magnetic stirring was used in the ESA-8000 during the measurements to avoid particle settling. Superplasticizer was either added after 3 minutes of stirring in water with an additional 1 minute stir (for single point measurements), or auto-titrated into the cement slurry in small increments using a 30 second equilibration time.

Microelectrophoresis measurements were made on a Pen-Kem System 3000 instrument. For these measurements, $0.05 \mathrm{~g}$ of cement was dispersed in $500 \mathrm{~g}$ deionized water. The particles were stirred for 3 minutes. Superplasticizer was then added and allowed to stir for 1 minute. The suspension was then loaded into the Pen Kem sample cell for zeta potential determination.

Streaming potentials were measured in a Mutek PCD 02 particle charge detector. The experimental setup consists of a cylindrical vessel and a displacement piston both constructed of teflon. The reciprocal pision movement at $4 \mathrm{~Hz}$ forces the colloid to stream in a slit between the cylindrical vessel and the piston, inducing a streaming potential. For streaming potential measurements, $0.5 \mathrm{~g}$ of cement was dispersed in $500 \mathrm{~g}$ deionized water, and $10 \mathrm{~cm}^{3}$ solution was used in the experimental vessel. Superplasticizer was added to the vessel in increments by an autotitrator. The value of the streaming potential was then recorded as a function of the amount of superplasticizer added. 


\section{RESULTS AND DISCUSSION}

\section{Technique Comparison}

The basic experiment used in this work to compare the surface charge characterization techniques of electroacoustic analysis, microelectrophoresis, and streaming potential is the titration of superplasticizer onto the New York cement. Past results of this type of experiment using microelectrophoresis show that as higher dosages of superplasticizer are added the particles acquire negative charge with an eventual plateau value of the zeta potential $[14,17,19,20]$. This can be understood as the polyanionic polymer adsorbing onto the cement surface until it has reached a limiting value.

\section{A. Electroacoustic Analysis}

Zeta potential measurements using electroacoustic measurements were performed at various cement concentrations. Results shown in Figure 2 are for $200 \mathrm{~g}$ cement in $200 \mathrm{~g}$ water, corresponding to a volume fraction of 0.24 . These results were generated using incremental titration of NSFC. Qualitatively, the expected negative charging of the particles by superplasticizer is found. However, experiments done at lower volume fraction show positive changes in zeta potential. These experiments are described in detail later.

The y-axis of the plot is labelled "apparent zeta potential" because the true values of the zeta potential in these experiments are uncertain. Two effects contribute to this uncertainty. First, the broad particle size distribution causes an uncertainty in the calculation of zeta potential from ESA signal. This is due to the Matec software's calculation assuming monodisperse particle size distribution, where O'Brien's mathematical formulation holds [25]. The effects of particle size distribution on electroacoustic measurements were subsequently evaluated and discussed by Scales [26] and James [27]. The proper zeta potential can be calculated in principle by weighted integration of the ESA signal over the particle size distribution [28]. For the measurements presented here, the mass average particle size was used as suggested by James [27]. Specifically for this study, particle radii of 3.5 microns and 5.5 microns were used for the Texas and New York cements, respectively. The densities of the cements were taken to be $3.15 \mathrm{~g} / \mathrm{cm}^{3}$. Secondly, there is a 
limitation on the volume fraction range of validity for the ESA to zeta potential conversion using these assumptions. An upper bound of volume fraction no greater than 0.05 has been proposed [27], and in Figure 2 we have shown data acquired at 0.24 , for reasons that will be evident later.

\section{B. Microelectrophoresis}

Microelectrophoresis measurements were performed at various single concentrations of NSFC. A particle concentration of $50 \mathrm{mg}$ cement per $500 \mathrm{~g}$ deionized water was found to be optimum. Lower particle concentrations gave insufficient signal and higher concentrations of particles resulted in no variation of zeta potential as a function of NSFC concentration, presumably due to interparticle interactions. Results shown in Figure 3 indicate a negative charging trend qualitatively similar to that found by numerous investigators $[14,17,19,20]$. Our results are in good quantitative agreement with those presented in the work by Singh [20].

Limitations of the microelectrophoresis technique used here for cement included: rapid particle settling inside the microelectrophoresis sample cell, which limits signal levels and the ability to signal average over time, and also results in gathering signal from mainly the smaller particles; fresh solutions of cement and superplasticizer had to be prepared for each value of superplasticizer as titration of the reservoir cement suspension was found to be irreproducible; poor reproducibility of single point results, necessitating experiments to be performed on each solution 4 to 6 times to provide adequate statistics.

\section{Streaming Potential}

Streaming potentials as a function of superplasticizer concentration were measured in an autotitration mode. $0.5 \mathrm{~g}$ cement particles were dispersed in $500 \mathrm{~g}$ deionized water, and NSFC was incrementally titrated. Results of the experiments are shown in Figure 4. Changes in streaming potential are reported on the figure because it was found that the absolute value of the streaming potential was dependent on the initial cleanliness of the cell. Furthermore, we found that in doing control experiments the ionic composition of the solution must be equivalent to that of the cement which it serves as a reference for. This is expected because 
the streaming potential depends on the conductivity. In these experiments we used a sodiumlime-gypsum solution [NaLG; $21.3 \mathrm{~g} \mathrm{Na}_{2} \mathrm{SO}_{4}, 30 \mathrm{~g}$ calcium hydroxide, $5 \mathrm{~g}$ reagent grade gypsum, total volume of 1 liter, with particulate allowed to settle out], which simulates the ionic environment generated by portland cement. The NaLG solution was used at a concentration which matched the conductivity of the $0.01 \%$ cement suspension used for streaming potential. The results show negative charging similar to results observed by microelectrophoresis techniques. The two control experiments shown demonstrate that neither dilution effects from adding water to cement nor effects on the streaming potential from adding NSFC can be invoked to explain the negative streaming potential of cement particles in the presence of NSFC.

\section{Evaluation of Interparticle Effects by Electroacoustic Techniques}

The advent of electroacoustic techniques for surface charge characterization now allows one to investigate surface charge characteristics in concentrated slurries where interparticle effects can become important. This is especially important for portland cement applications, as typical use concentrations are $w / c=0.3$ to 0.5 . These concentrations are orders of magnitude above the regimes where microelectrophoresis and streaming potential measurements are applicable. Furthermore, ionic effects can be important in cements as under typical use conditions, the concentration of ions will be near $0.1 \mathrm{M}$, mostly in $\mathrm{Na}, \mathrm{K}$, $\mathrm{Ca}$ cations, and 504 and $\mathrm{OH}$ anions. Although the particle concentration in microelectrophoresis and streaming potential measurements cannot be increased to the realistic regime, it may be advisable to adjust the ionic strength to match the realistic regime.

Nagele et. al. have performed many studies on the zeta potential of portland cement as determined by microelectrophoresis [1-10], including the variation of cement zeta potential over time $[3-6,8,9]$. Nagele's zeta potential measurements in deionized water starts initially at $-13 \mathrm{mV}$ and rises to $-10 \mathrm{mV}$ after 100 minutes [Figure 3, Reference 8].

ESA time series data on the Texas cement is shown for various water to cement ratios in Figure 5. The absolute values of the zeta potentials are uncertain as discussed above, however, any integration scheme on a single cement will correct all of the numbers by the same function. Thus, either interparticle effects or ionic effects are responsible for the 
differences between the different concentrations. Clearly, the zeta potential of hydrating cement particles decreases with concentration. Our data also differ from that of Nagele [8] in that our zeta potentials decrease (became more negative) over the 30 minute measurement period, while Nagele's increase. This discrepancy could be due to the variations in ionic content of water, especially in the microelectrophoresis experiment as observed by Nagele [8]. Subsequently, Nagele used cement extract to simulate highly concentrated cement dispersions [9]. This changed the values of the zeta potential found, but not the initial positive trend with time. ESA is much less sensitive to the ionic strength of the water used as the large amounts of cement used dominate the ionic strength.

The charging action of sulfonated superplasticizers (and other admixtures) on portland cement has been extensively studied by microelectrophoresis [13-24]. The generally observed behavior is as the superplasticizer concentration is increased, the zeta potential of the cement particles becomes more negative until a plateau is reached. Our electroacoustic measurements presented in an earlier section verified this behavior for cement slurries of $\mathrm{w} / \mathrm{c}=1$.

In order to investigate interparticle effects on superplasticized cements, we performed measurements at volume fractions of 0.01 and 0.05 , regimes where the zeta potential conversion from ESA signals should be valid. Data from these experiments is shown in Figure 6, where once again the y-axis label of "apparent zeta potential should be noted when one evaluates the magnitude of these effects. Much to our surprise, the zeta potential of these titrations showed an increase in cement zeta potential with increasing superplasticizer concentration. These experiments were reproducible.

A number of effects might be expected to influence these results, including variations in the zeta potential over the time of the titration, ESA phase errors, and ionic strength effects. From the data in Figure 5 it is seen that the time variations should affect the 0.01 and 0.05 volume fraction experiments in the opposite direction to that observed, while the 0.24 volume fraction experiment would be relatively unaffected. To address phase and time effects, each experiment was individually phased to 0 to \pm 5 degrees phase angle before measurement and single point measurements were made with mixing cycle identical to that used in the microelectrophoresis experiments described above, yielding constant mixing time 
for all measurements. The observed trends are plotted in Figures $7 \mathrm{a}$ and $7 \mathrm{~b}$, and the trends are maintained as seen in the autotitration experiments. To investigate the effects of ionic strength, we adjusted the solution of some 0.01 volume fraction experiments to the conductivity observed at 0.05 volume fraction with the sodium-lime-gypsum solution described in the section on streaming potential. The additional ions shifted the apparent zeta potential from $-53.7 \mathrm{mV}$ to the unrealistic value of $-173 \mathrm{mV}$. Thus, the effect of additional ions is opposite to that we observed with cement, where higher concentrations of cement (and thus higher concentration of ions) produces zeta potentials nearer to zero.

The ESA signal is known to have non-linearities at volume fractions above 0.05 for silica [27]. In Figures $8 \mathrm{a}$ and $8 \mathrm{~b}$ we show the relationship for the Texas cement. As before, we used the mean mass averaged particle size in the Matec software which performs the ESA-zeta conversion. The data are not in line with that for silica, as a constant zeta potential is not observed with volume fraction nor is there a linearly decreasing ESA signal with increasing volume fraction. Figures $9 \mathrm{a}$ and $9 \mathrm{~b}$ show the volume fraction dependencies of the Texas cement dosed with $0.4 \%$ NSFC. Dependencies in this case are as expected with a flat zeta potential and a linearly decreasing ESA signal from volume fraction 0.01 to 0.15 . Experiments were also conducted with the samples diluted in cement extract to simulate ionic effects, and this was found to affect only the lowest volume fractions.

The unconventional dependencies of electroacoustically derived zeta potentials and ESA signals for portland cement explain why anomalous charging effects are observed upon the addition of superplasticizer, because the zeta potentials at the start of the experiment are artificially more negative than expected. When NSFC is added, the zeta potentials tend towards the same plateau for the 0.05 and 0.24 volume fractions. It is known that NSFC is a dispersant for cement particles, so one explanation may be flocculated particles when little superplasticizer is present. When observations are made at a volume fraction of 0.24 , these effects may be abated due to the sample being more like a slurry than a dispersion. Ionic effects cannot be totally ruled out, but they require additional investigation. Also, it should be noted that experiments such as these can be cement specific, thus the anomalies may not occur in all portland cements. 


\section{CONCLUSIONS}

In this work we have studied three methods of zeta potential determination, electroacoustics, microelectrophoresis, and streaming potential as applied to portland cement. The three techniques are in qualitative agreement regarding the generation of negative charge on cement particles caused by superplasticizer. Each of the techniques has limitations in terms of absolute zeta potential determination.

Electroacoustic techniques provide a method for evaluation of electrokinetic properties of portland cement at near realistic conditions. Empirically, electroacoustic techniques provide an ease of use advantage over the other techniques studied. For superplasticized cement slurries, puzzling electroacoustic data on the charging of cement particles by superplasticizer as a function of volume fraction remain unexplained. 


\section{REFERENCES}

1. Nagele, E. and Ney, P., Cem. Concr. Res., 12, 535-536 (1982).

2. Nagele, E., Chem. Eng. Sci., 40, 2141-2148 (1985).

3. Nagele, E., Cem. Concr. Res., 15, 453-462 (1985).

4. Nagele, E., Cem. Concr. Res., 16, 853-863 (1986).

5. Nagele, E., Cem. Concr. Res., 17, 573-580 (1987).

6. Nagele, E. and Schneider, U., Cem. Concr. Res. 17, 977-982 (1987).

7. Nagele, E. and Schneider, U., Cem. Concr. Res., 18, 257-264 (1988).

8. Nagele, E., Chem. Eng. Sci., 44, 1637-1645 (1989).

9. Nagele, E. and Schneider, U., Cem. Concr. Res., 19, 978-986 (1989).

10. Nagele, E., Cem. Concr. Res., 21, 478-483 (1991).

11. Suzuki, K., Nichikawa, T., Kato, K., Hayashi, H., and Ito, S., Cem. Concr. Res., 11 , 759-764 (1981).

12. Singh, N.B., Singh, A. K., and Singh, S.P., J. Amer. Ceram. Soc., $\underline{73}$, 3063-3068 (1990).

13. Daimon, M. and Roy, D.M., Cem. Concr. Res., $\underline{8}, 753-764$ (1978).

14. Daimon, M. and Roy, D.M., Cem. Concr. Res., $\underline{9}, 103-110 \quad$ (1979).

15. Collepardi, M., Monosi, S., Moriconi, G, and Corrardi, M., Cem. Concr. Res., 10, 455-462 (1980).

16. Massazza, F., Costa, U., and Barrila, A., J. Amer. Ceram. Soc., 65, 203-207 (1982).

17. Miyake, N., Ando, T., and Sakai, E., Cem. Concr. Res., 15, 295-302 (1985).

18. Andersen, P. J., Kumar, A., Roy, D. M., and Wolfe-Confer, D., Cem. Concr. Res., 16, 255-259 (1986).

19. Andersen, P. J., Cem. Concr. Res., 16, 931-940 (1986).

20. Singh, N. B. and Singh, S. P., J. Mat. Sci., 22, 2751-2758 (1987).

21. Andersen, P. J., Roy, D. M., and Gaidis, J. M., Cem. Concr. Res., 17, 805-813 (1987).

22. Mangialardi, T. and Paolini, A. E., Cem. Concr. Res., 18, 351-362 (1988). 
23. Andersen, P. J., Roy, D. M., and Gaidis, J. M., Cem. Concr. Res., 18, 980-986 (1988).

24. Nawa, T. Eguchi, H., and Fukaya, Y., Third Int. Conf. on Superplasticizers, 405-424 (1989).

25. O’Brien, R. W., J. Fluid Mech., 190, 71-86 (1988).

26. Scales, P. J. and Jones, E., Langmuir, $\underline{8}, 385-389$ (1992).

27. James, M., Hunter, R. J., and O'Brien, R. W., Langmuir, $\underline{8}, 420-423$ (1992).

28. Mann, R., Matec Applied Sciences, Private Communication. 
Table 1

Composition of Portland Cements Expressed as Oxides (\% by mass)

Component

$\mathrm{Na}_{2} \mathrm{O}$

$\mathrm{K}_{2} \mathrm{O}$

$\mathrm{MgO}$

$\mathrm{CaO}$

$\mathrm{A}_{2} \mathrm{O}_{3}$

$\mathrm{Fe}_{2} \mathrm{O}_{3}$

$\mathrm{SiO}_{2}$

$\mathrm{TiO}_{2}$

$\mathrm{SO}_{3}$

L.O.I.
New York

0.20

0.50

3.91

65.95

4.16

2.88

20.61

0.44

1.19

0.67
Texas

0.01

0.42

1.30

67.60

4.71

4.20

21.60

0.24

1.99

0.87 


\section{FIGURE CAPTIONS}

Figure 1. Particle size distribution for Texas cement as measured by laser light diffraction.

Figure 2. Change in apparent zeta potential of the New York cement as a function of superplasticizer concentration at volume fraction of $0.24(w / c=1)$, as measured by ElectroSonic Analysis.

Figure 3. Change in zeta potential of the New York cement as a function of superplasticizer concentration as measured by microelectrophoresis.

Figure 4. Change in streaming potential of New York cement as a function of superplasticizer concentration as measured by streaming potential. Effects of dilution by water and addition of superplasticizer to water are included for reference.

Figure 5. Time variation of zeta potential of Texas cement caused by cement hydration at three different volume fractions.

Figure 6. Change in apparent zeta potential of the Texas cement as a function of superplasticizer concentration for different volume fractions. The total time for this titration is close to the time scale of Figure 5.

Figure 7. Single point measurements of apparent zeta potential change of Texas cement as a function of superplasticizer concentration for a) high volume fractions, b) low volume fractions.

Figure 8. Dependence of electroacoustically determined quantities as a function of volume fraction for Texas cement; a) apparent zeta potential, b) ESA signal.

Figure 9. Dependence of electroacoustically determined quantities as a function of volume fraction for Texas cement with $0.4 \%$ NSFC added; a) apparent zeta potential, b) ESA signal. 


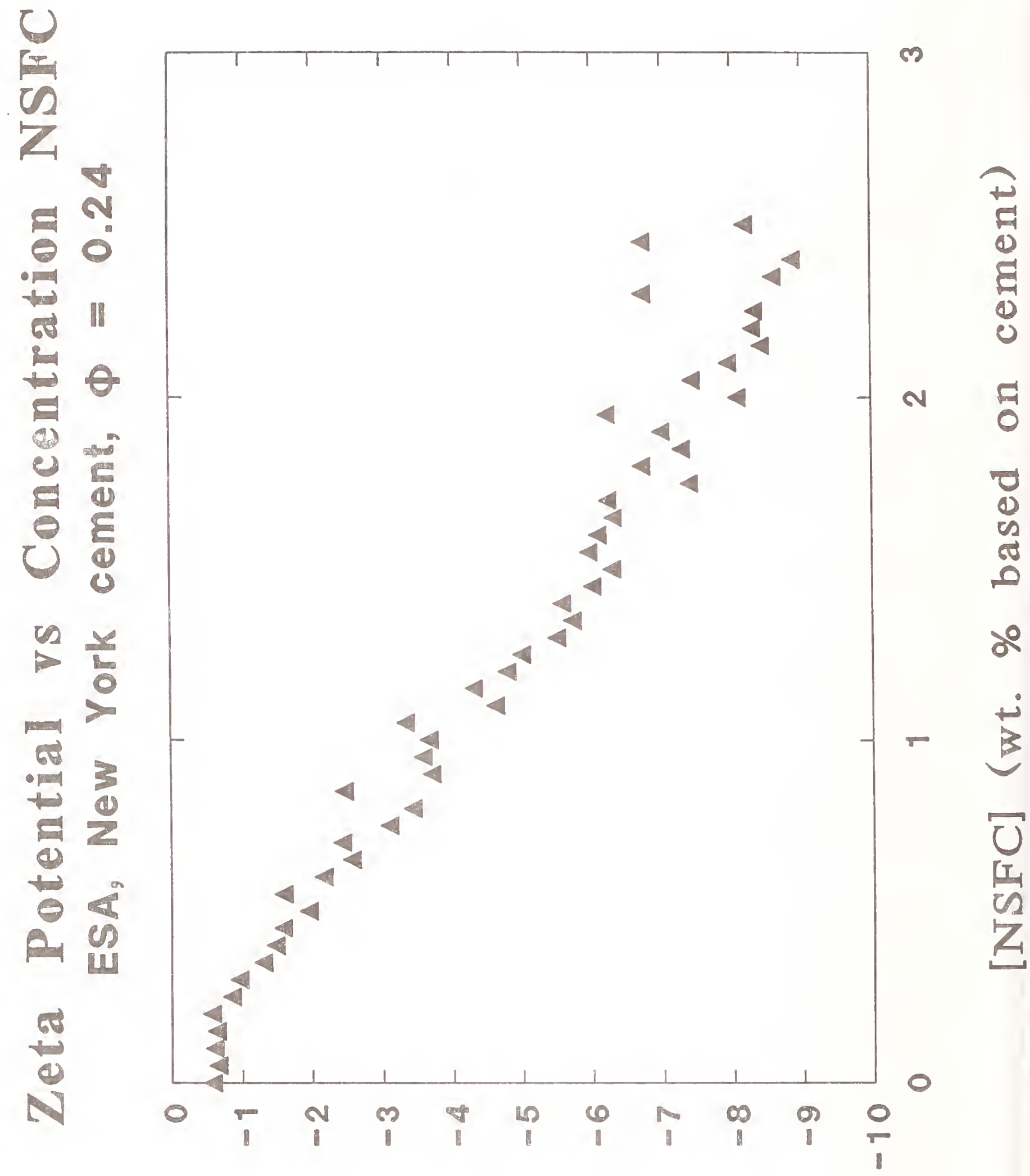

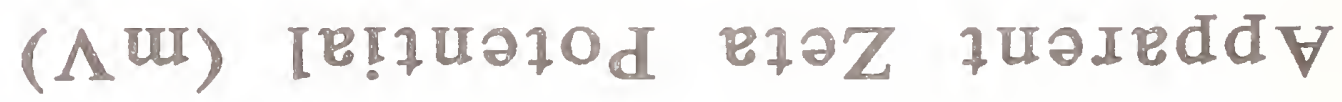


$\tau$

Z

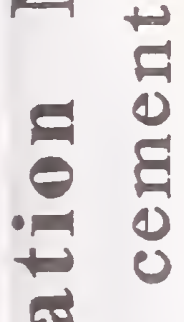

$\stackrel{0}{ \pm}$

0

H

U

$\ddot{\infty}$

$\dot{s} 0$

$\rightarrow$ 왕

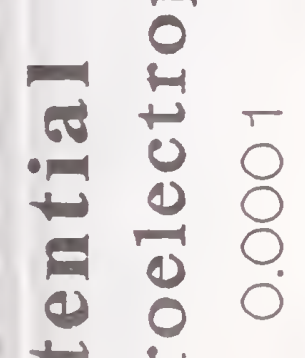

๖

- $\sum_{\Sigma} Q$

$\stackrel{5}{*}$
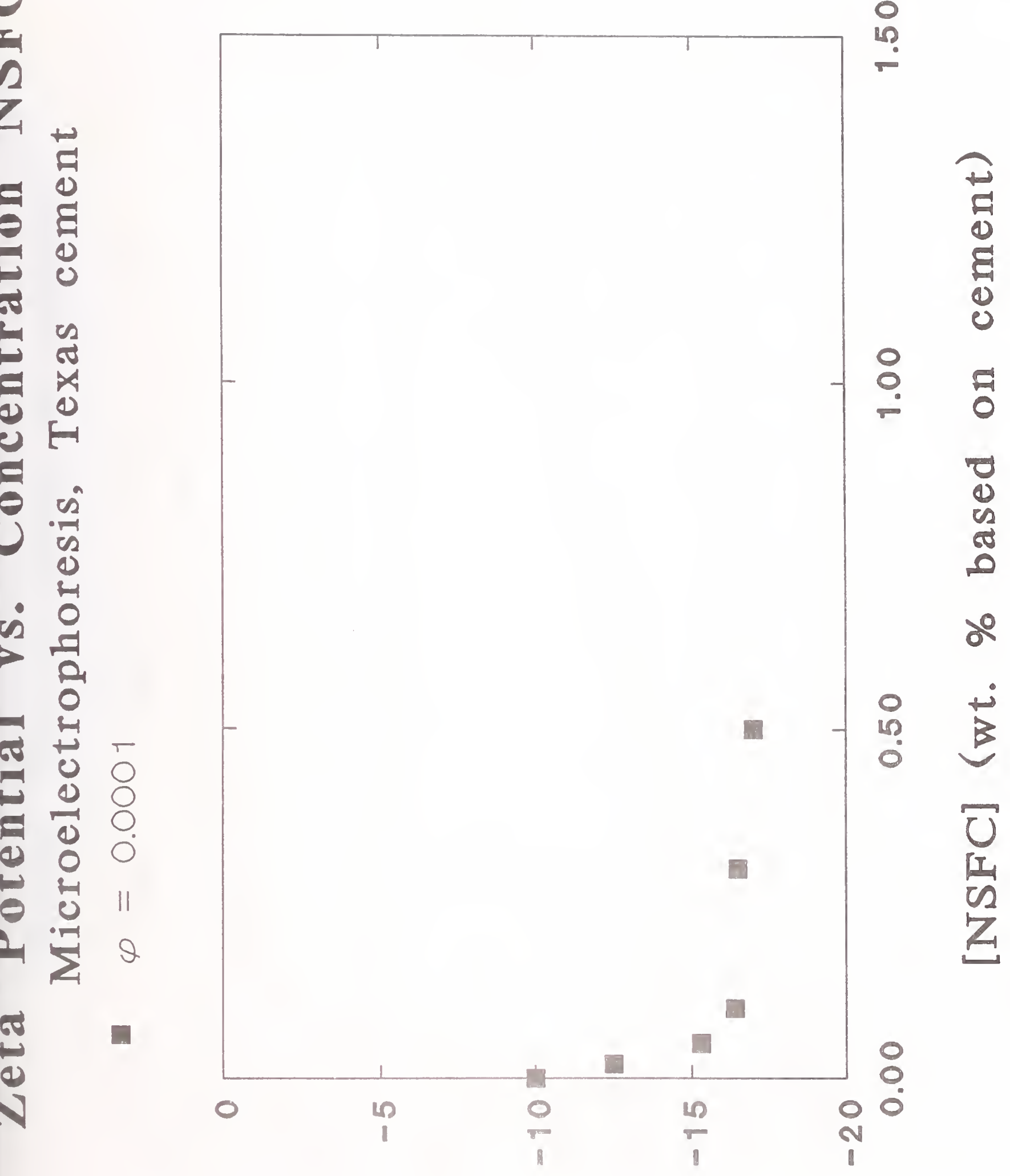

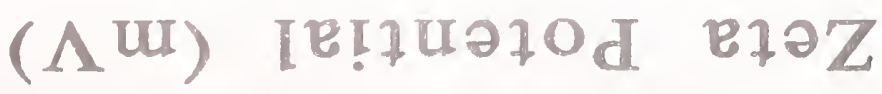




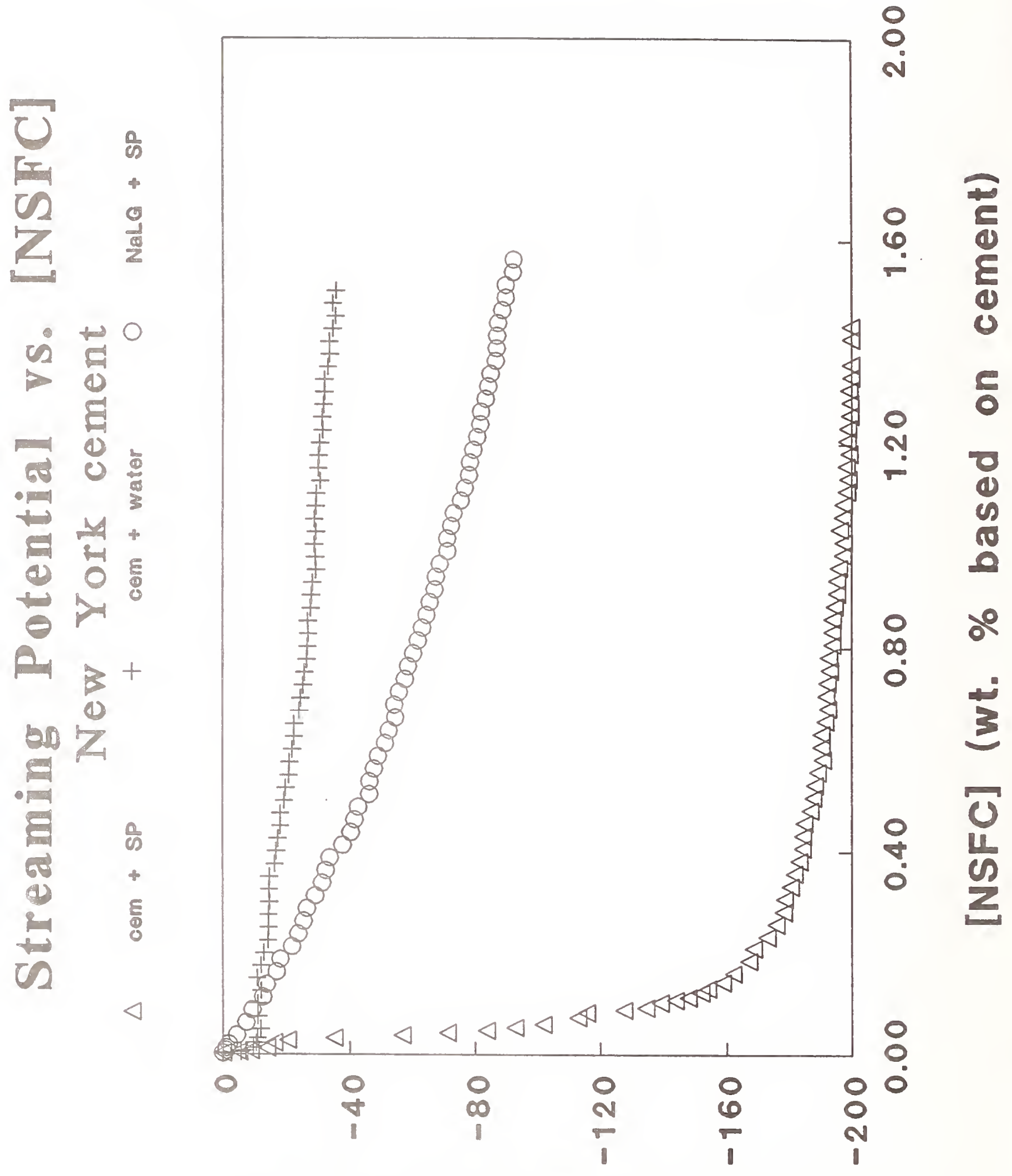

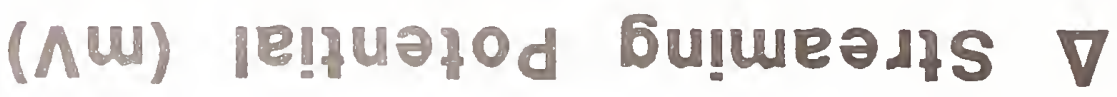




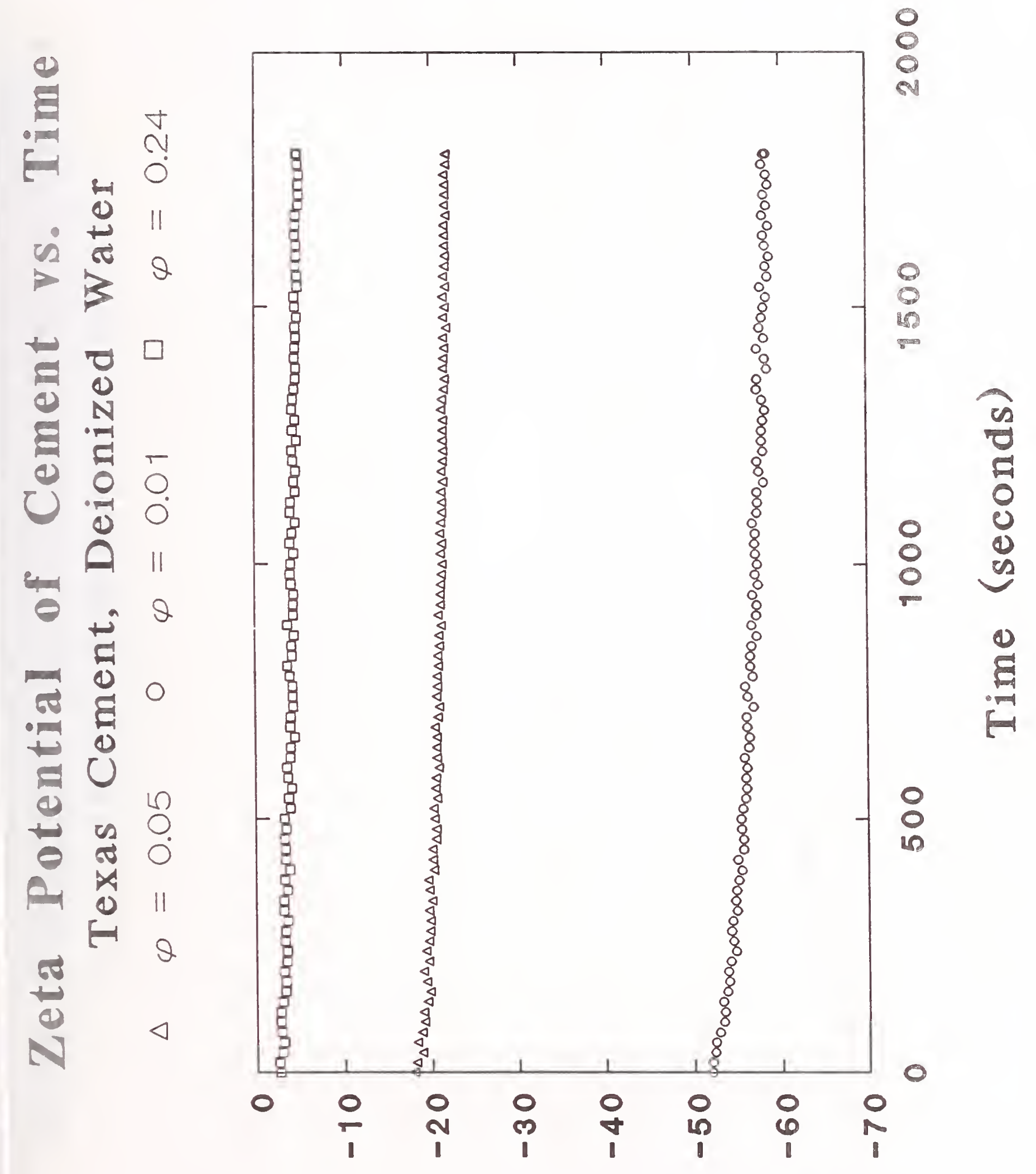

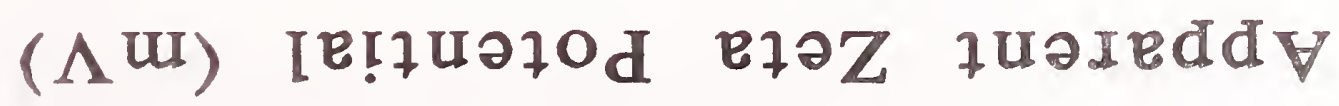




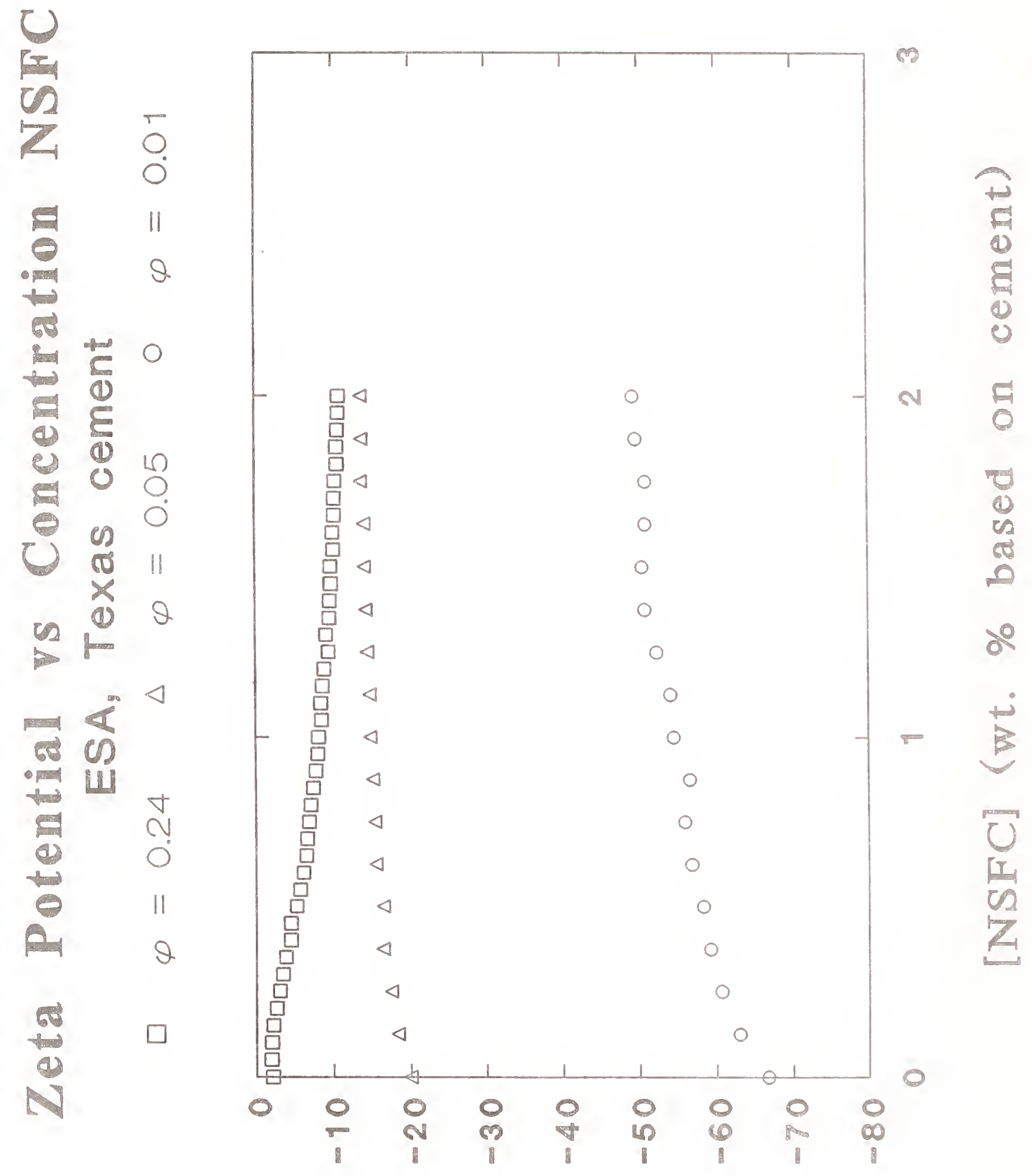

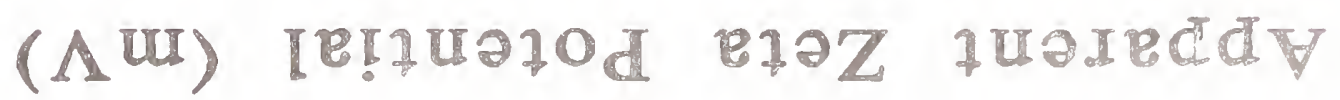




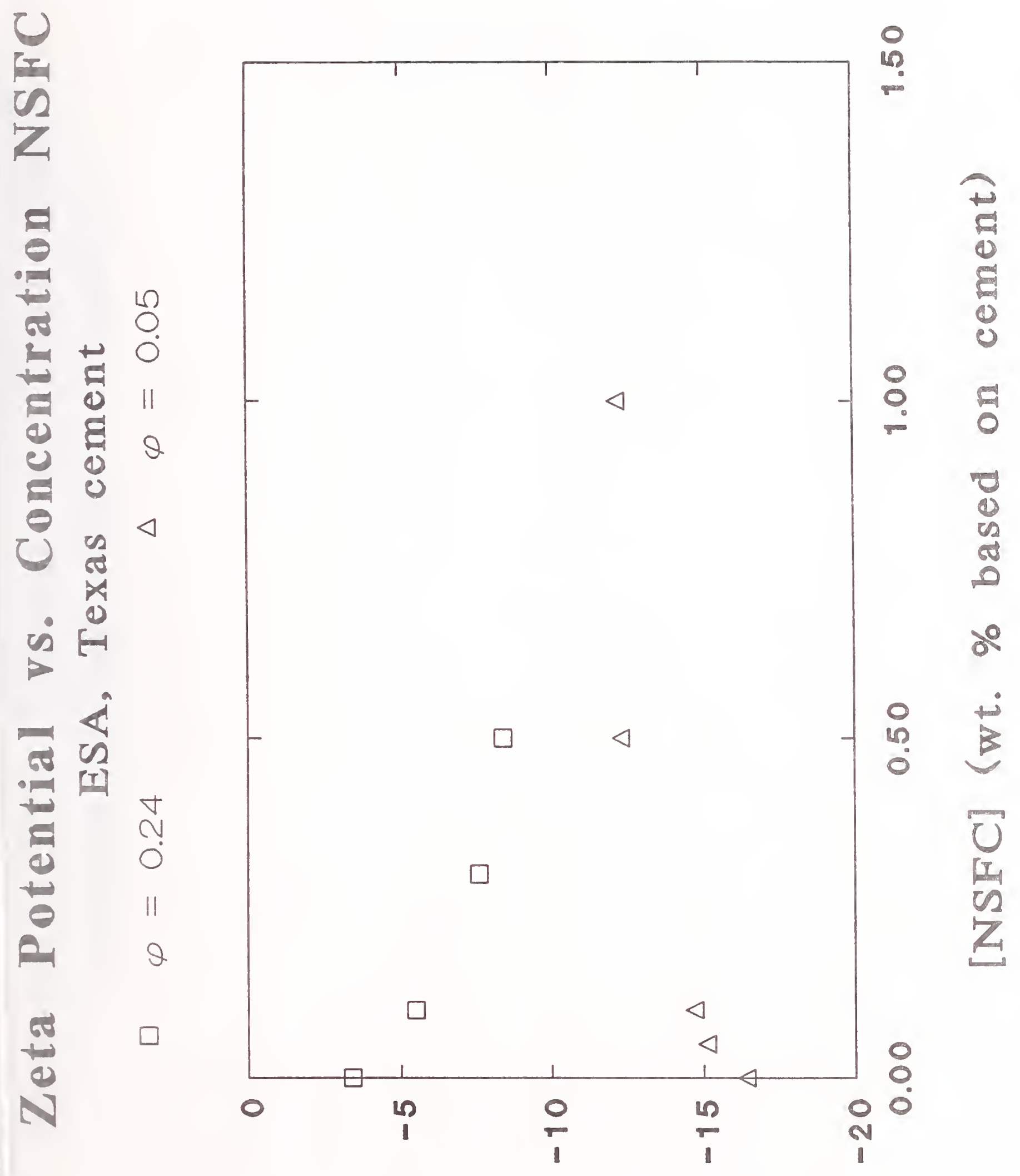

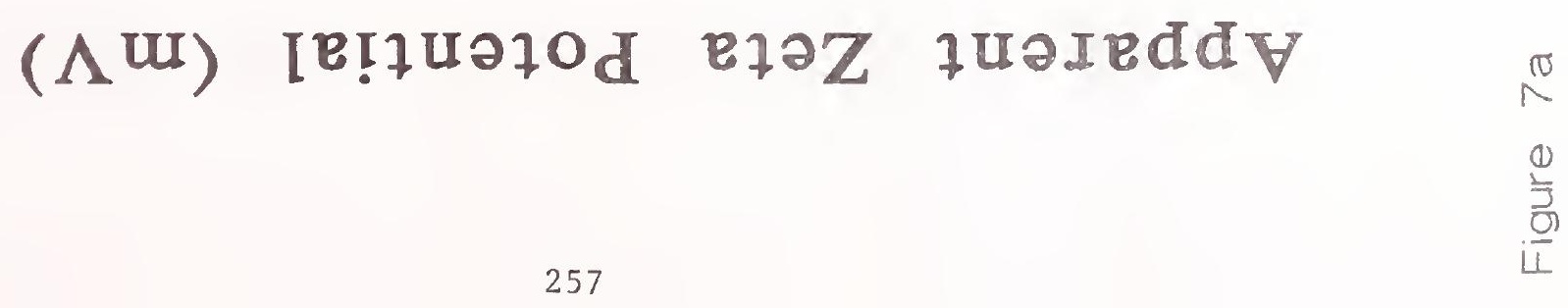


$\circlearrowright$

$\sqrt{2}$

C

$Z_{1}$

응

then

68

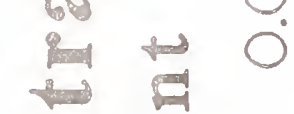

(료 (1) 11

(2)

(c) (2)

e 0

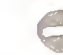

乙

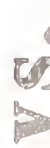

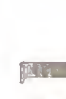

क्ष

(a)

4

(2)

$+$

(6)

2

ह5

(4)

N

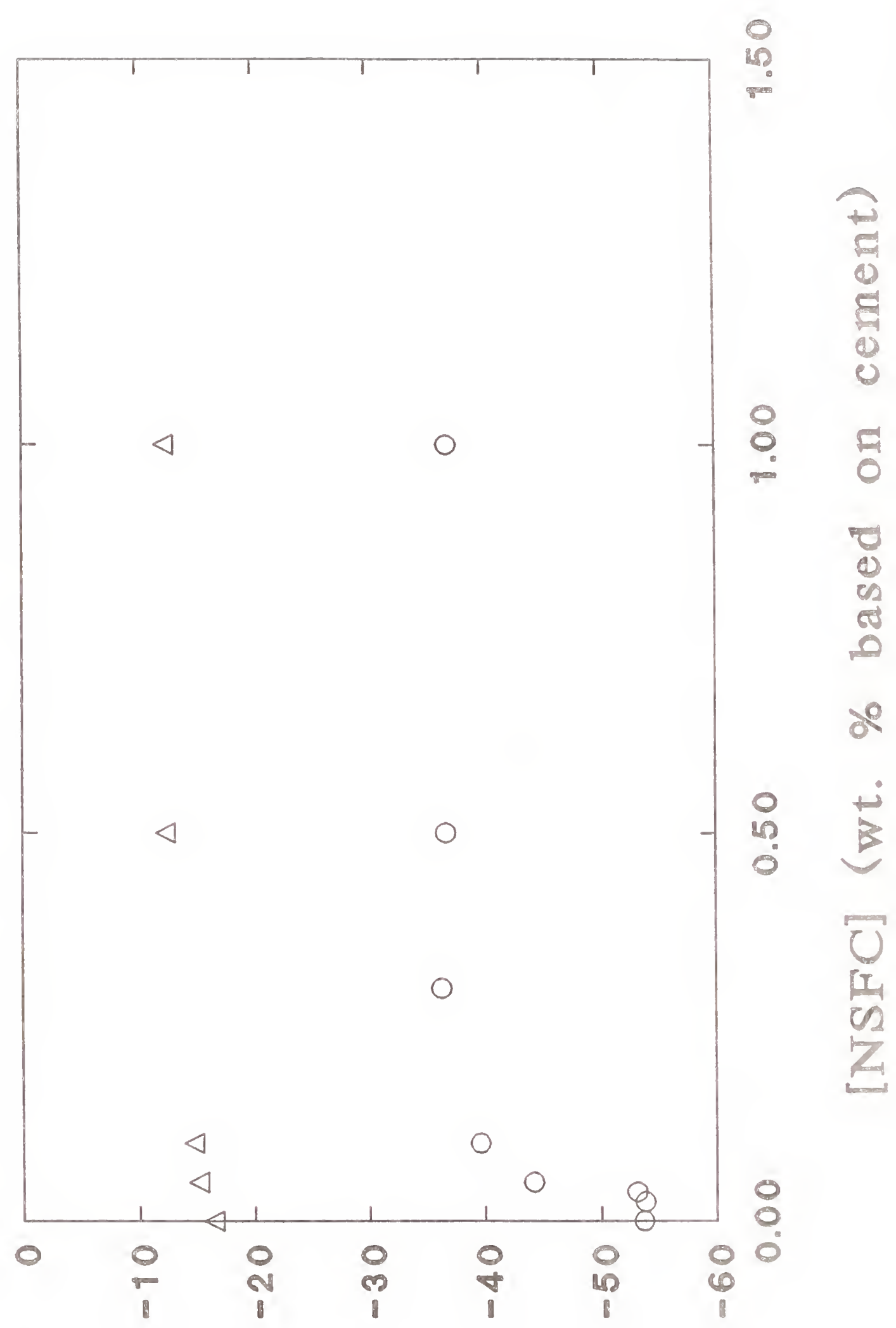

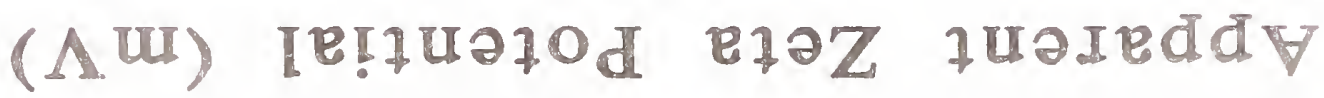




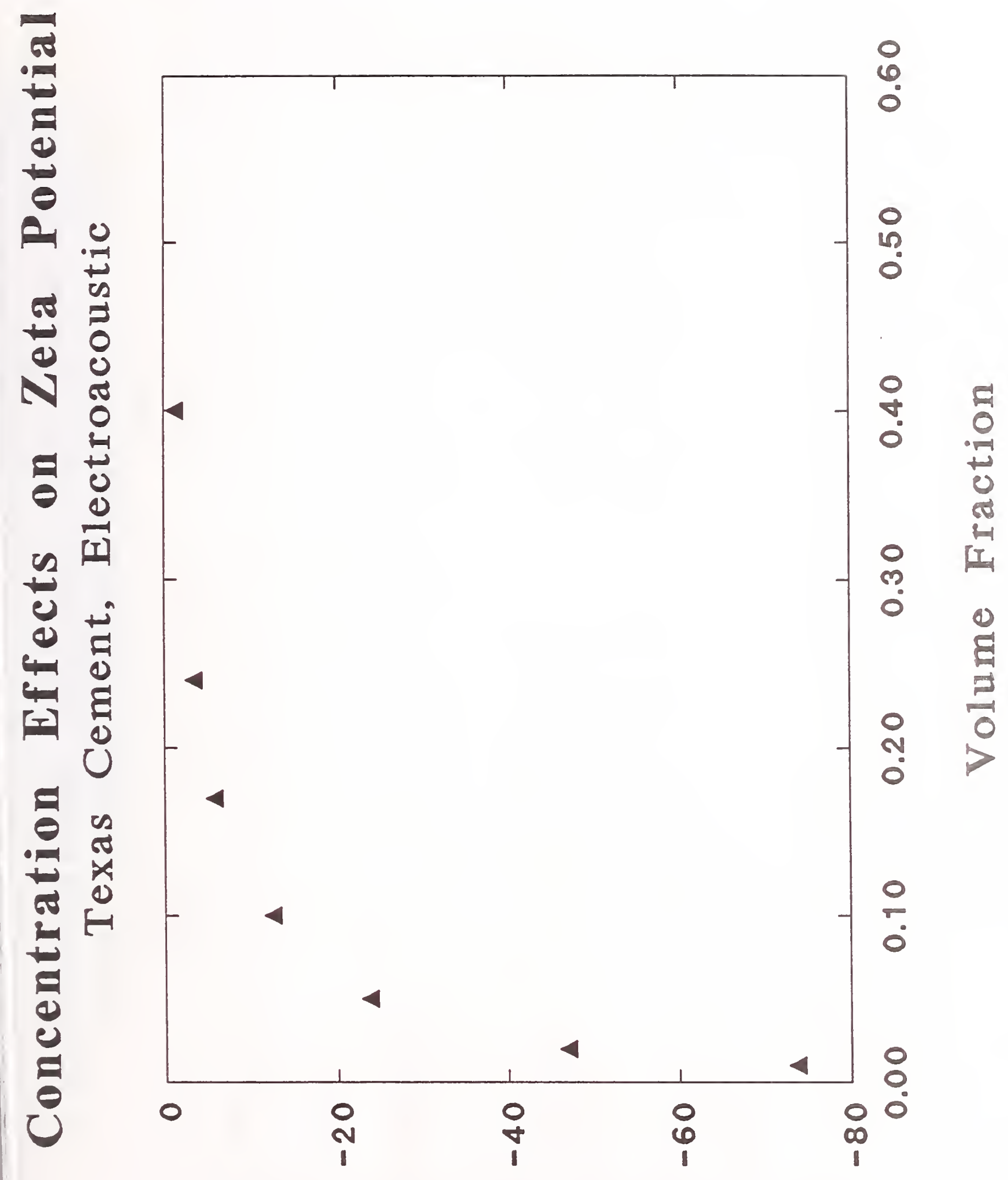

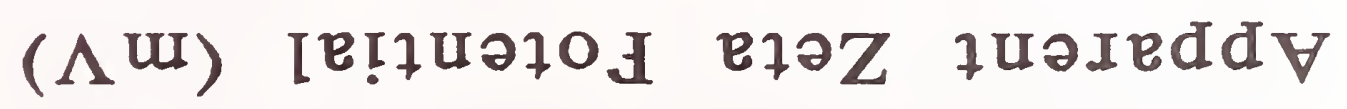




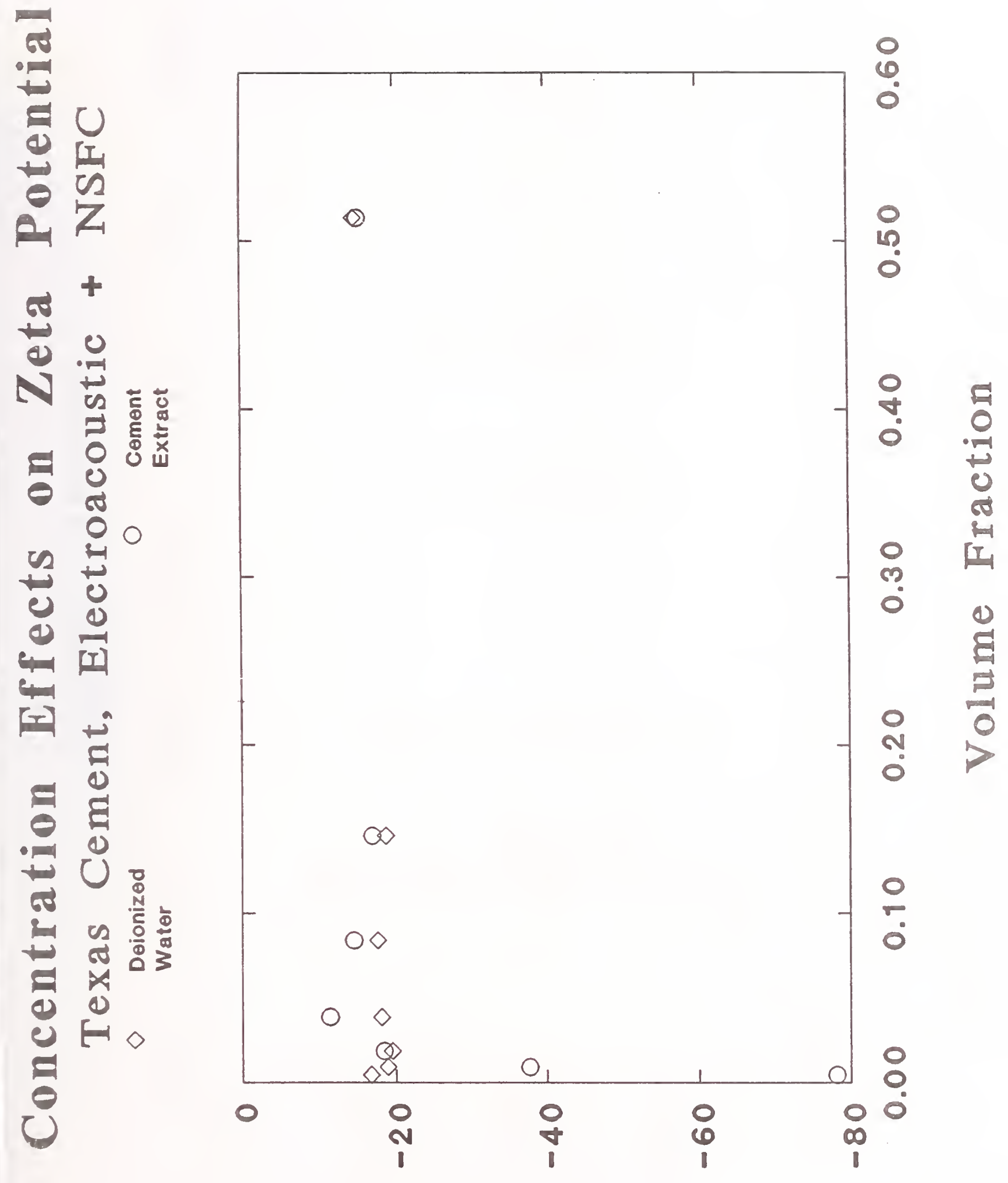

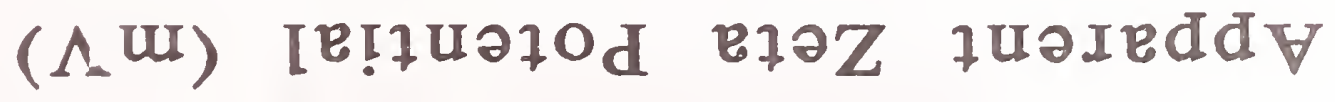




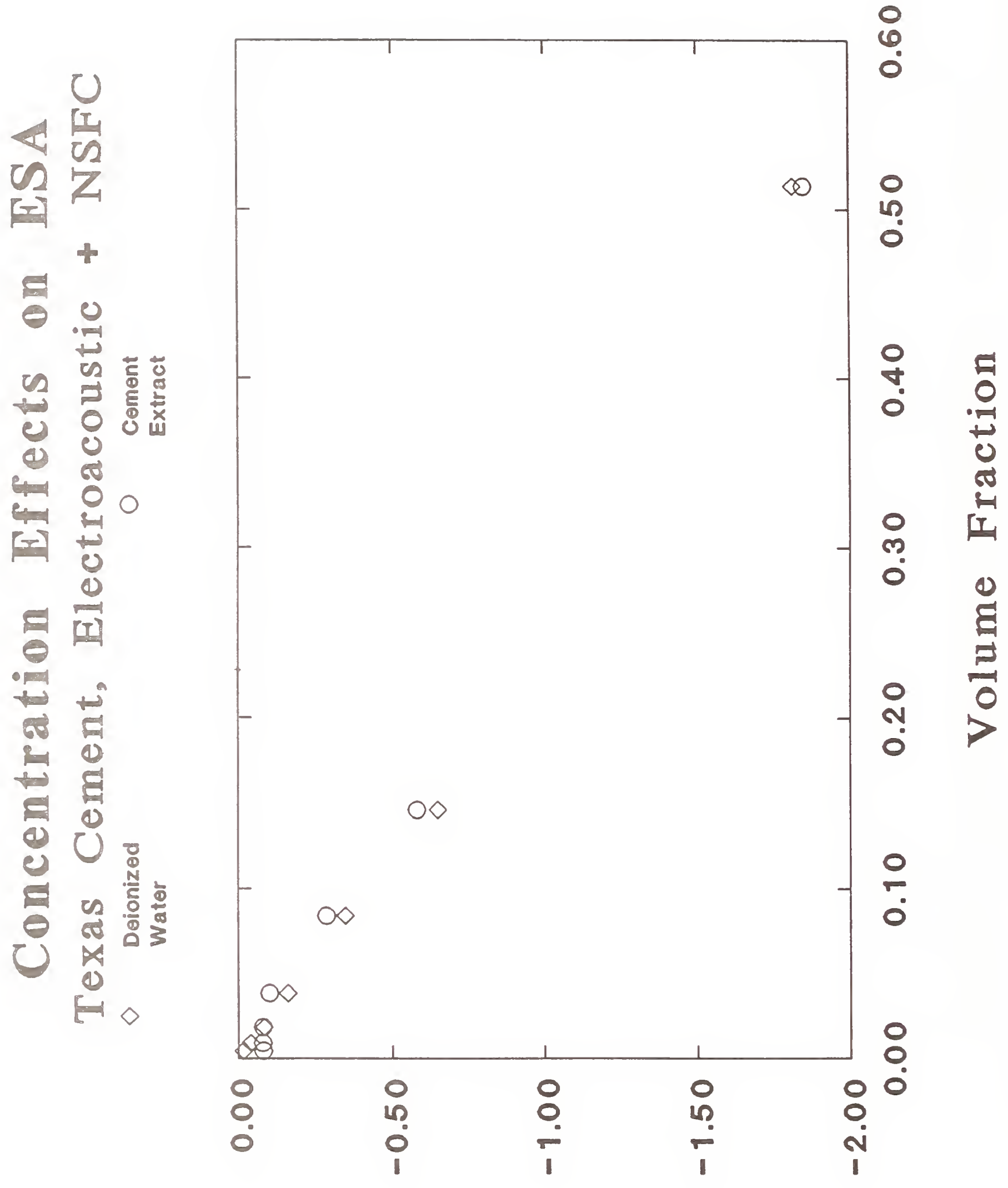

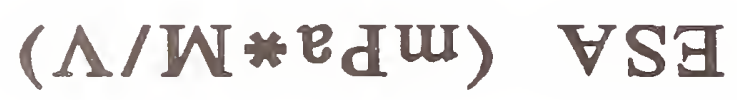

के 


\title{
SESSION V
}

\section{COATINGS}

\section{Session Chairman}

\author{
N. Sanders \\ Specialty Minerals Inc.
}





\title{
ROLE OF PARTICLE SIZE AND ELECTROKINETIC SONIC AMPLITUDE IN PERFORMANCE OF SOLVENT BASED GRAVURE INKS
}

\author{
R. Krishnan \\ Sun Chemical Corporation \\ 631 Central Avenue \\ Carlstadt, New Jersey 07072
}

\begin{abstract}
In Gravure printing, the destruction of chrome plating in the form of banding crops up time and time again. While several factors are involved in this phenomena, from the physico-chemical properties of ink, the particle size of pigments, and the Electrokinetic Sonic Amplitude (ESA) seems to play a major role.

Maximizing ESA offers a solution to this problem. This is probably due to the reduction in abrasiveness of the carbon black pigment as particle size decreases. Although these particles are sterically stabilized, maximum in ESA seems to correspond to a minimum in particle size in most cases.
\end{abstract}

\section{INTRODUCTION}

Of the three major printing processes, Lithographic, Gravure, and Letterpress, the Gravure printing is the preferred type for long run publications because of its high quality reproduction at high speeds. Gravure printing is also used in packaging materials such as paper and board since line cutting and creasing operations are easier because of the drying characteristics of inks. In addition, for printing on non-porous substrates such as aluminum foils it is ideal, because lithographic printing is easier only on water absorbing substrates. The printing image is engraved into a gravure cylinder in the form of cells which are filled with ink. A gravure cylinder consists of copper plated steel tube. A gelatine coating is exposed under a positive of the image and it hardens to a depth which depends on the light received. After etching with ferric chloride solution, a cylinder which consist of square cells of varying depth results. Darker tones correspond to deeper cells which carry more ink. Finally, the finished cylinder is chrome coated. 
A schematic of the gravure printing unit is shown in Figure 1. The printing unit is an ink duct in which the etched cylinder rotates in a low viscosity ink. Excess ink is scraped by means of a metal doctor blade. Printing occurs at the nip between the etched cylinder and a rubber covered impression roller. The printed web is then heated in a dryer and succeeding colors are printed ${ }^{[1]}$.

Most publication gravure inks are toluene based in which the polymeric binder is usually a calcium or zinc resinate. The pigments are Carbon Black, phthalo cyanine blue, diarylide yellow, and Lithol Rubine, corresponding to the four process colors.

The problem discussed in this paper with respect to the ink's performance is described as "banding". Banding is a scoring of the surface of the gravure cylinders which starts mainly

near the edges and progresses into printing areas. It occurs erratically, and predominantly on the cylinders running with black ink; although some cases of blue ink banding have been reported, very few of the other colors are involved. As the length of the run increases, banding progresses into the printing areas of the cylinders causing spoilage of the printed materials. Dechroming and rechroming of a banded cylinder sometime help to prolong the life of the cylinder, but it usually reoccurs. The erratic nature of the occurrence of this problem and the possible involvement of over twenty operating parameters make the analysis of the situation extremely complex. :

\section{Major Factors:}

Major factors which lead to the destruction of chrome plating in the form of scaling:

a) Chromium:

Different chrome electrolytes, which have an effect on wear behavior with regard to the respective chromium structure.

b) Wiper:

Wiper speed and wiper pressure are also factors which may have a considerable effect on chromium wear.

c) Printing Ink:

Physico-chemical properties of ink, on the basis of their composition and method of manufacture have wear a characteristic behavior. 


\section{RESULTS AND DISCUSSIONS}

Optical photomicrographs (Figures 2 and 3) of two typical banded cylinder shells reveal a network of surface cracking or reticulation in the chrome plated surface of the banded areas on both specimens.

This network of defects extends ahead of the actual banded area for a small distance and appears to be the initiating stage of the failure of the chrome plating.

A possible mechanism for the destruction which is very apparent in the center of each band might be the flaking off of the small chips of chrome loosened by this cracking or crazing phenomenon and getting trapped beneath the doctor blade. The hardness of the chrome flakes would then cause a rapid erosion and scoring of the cylinder's surface.

The photomicrographs (Figures 2 and 3 ) of a cylinder showing the leading edge of the band indicates an area extending beyond the heart of the band where the copper underlayer is exposed, which is covered with the crazed or cracked chromium surface which has not yet deteriorated to the point of flaking off and exposing the copper sub-surface. It also shows the beginning of the non banded area which is essentially free of these hairline surface defects in the chromed surface.

A doctor blade section taken from the press was also examined. Photomicrographs (Figures 4 and 5) of the upper edge of this blade, in the vicinity of the band show an increased amount of wear as evidenced by a low spot in the blade edge as well as a number of areas where metal wear is shown. They show an area where a leaf of blade metal has been pealed backward and is on the verge of breaking off and in addition, a wire edge on the verge of separating from the blade. The excessive amount of wear in this area would be expected if an unusual concentration of hard or abrasive materials, such as chrome flakes which may have separated in the banded areas to expose copper, collected under the blade while running. 


\section{Lubricity of Gravure Inks}

It has been proposed that banding may be correlated with the lubrication ability of gravure inks. Black ink (carbon black) was indeed found to be more abrasive than colored inks (organic pigments). However, a black ink with increased aromaticity (higher ratio of Toluene to Mineral Spirit) gave poor lubrication as compared to low aromatic ink. But in actual printing the high aromatic ink reduced the banding problem significantly.

\section{Particle Size of Pigments in the Ink}

A particle size analysis of the various inks involved were carried out using an Ultrafine Particle Size Analyzer (UPA) from Microtrac. It monitors Brownian motion of particles smaller than 5 microns as a means of measurement. Particles vibrating at various velocities by means of collisions with surrounding solvent molecules cause a shift in incident light frequency. Measurement of resulting frequency shifts (Doppler effect) allows a calculation of particle size and particle size distribution. The results are shown in Table I. A sample of the output corresponding to ink $\mathrm{C}$ is also attached (Figure 6). It shows a trend of lower particle size leading to less incidence of banding.

\section{Electrokinetic Sonic Amplitude of Inks}

In order to understand the reason for variations in particle size distribution, electroacoustic characterization of these inks was carried out. ${ }^{[3]}$ The formulations of these inks were essentially similar except for small variations in the amount of zwitter ionic surfactant used. The ratio of pigment to grinding (stability) resin was kept constant. The Electrokinetic Sonic Amplitude was measured using a MATEC system. The results are also shown in Table I.

Whenever the ESA is less that $0.5 \mu \mathrm{Pa} / \mathrm{Volt}$ Meter the incidence of banding was very high.

These experiments help us to offer a hypothesis for the problem of cylinder wear with solvent based inks. Although the solvent based inks are stabilized by steric stabilization the charge seems to play a major role in obtaining smaller particle size. For the systems studied, lower the ESA, higher is the particle size. In addition, under high shear conditions 
such as between a doctor blade and a gravure cylinder, with inks having lower ESA the particles may agglomerate which then become very abrasive creating cylinder banding.

\section{REFERENCES}

1. The Printing Ink Manual, Ed R.H. Lench, VanNostrand Reinhold (1988).

2. Microtrac Ultrafine Particle Analyzer Manual, Leeds \& Northrup (1990).

3. Matec Applied Science ESA 8000 Systems Manual. 


\section{TABLE I}

Mean Particle Size, ESA and Banding Tendency of Differenct Ink Samples

\begin{tabular}{lccl}
\multicolumn{1}{c}{ Sample } & $\begin{array}{c}\text { Mean Particle Size } \\
(\mu \mathrm{m})\end{array}$ & $\begin{array}{c}\text { Electrokinetic } \\
\text { Sonic Amplitude } \\
\mu \mathrm{Pa}^{*} \mathrm{~m} / \mathrm{V}\end{array}$ & \multicolumn{1}{c}{$\begin{array}{c}\text { Banding } \\
\text { Tendency }\end{array}$} \\
\cline { 2 - 3 } & 0.65 & 0.27 & High \\
$\mathrm{B}$ & 0.5 & 0.62 & Medium \\
$\mathrm{C}$ & 0.41 & 1.24 & None \\
$\mathrm{D}(\mathrm{A}+$ Zwitterionic & 0.46 & 0.75 & Very Low \\
$\quad$ Surfactant) & & &
\end{tabular}




\section{Figure Captions}

Figure 1. A schematic of gravure printing system.

Figure 2 and 3. Photomicrographs of typical banded cylinder shells showing a network of sruface crakcing.

Figure 4 and 5. Photomicrographs of a cylinder showing the leading edge of the band in which a copper underlayer is exposed.

Figure 6. Particle size distribution of ink $\mathrm{C}$ by using an instrument based Doppler effect. 


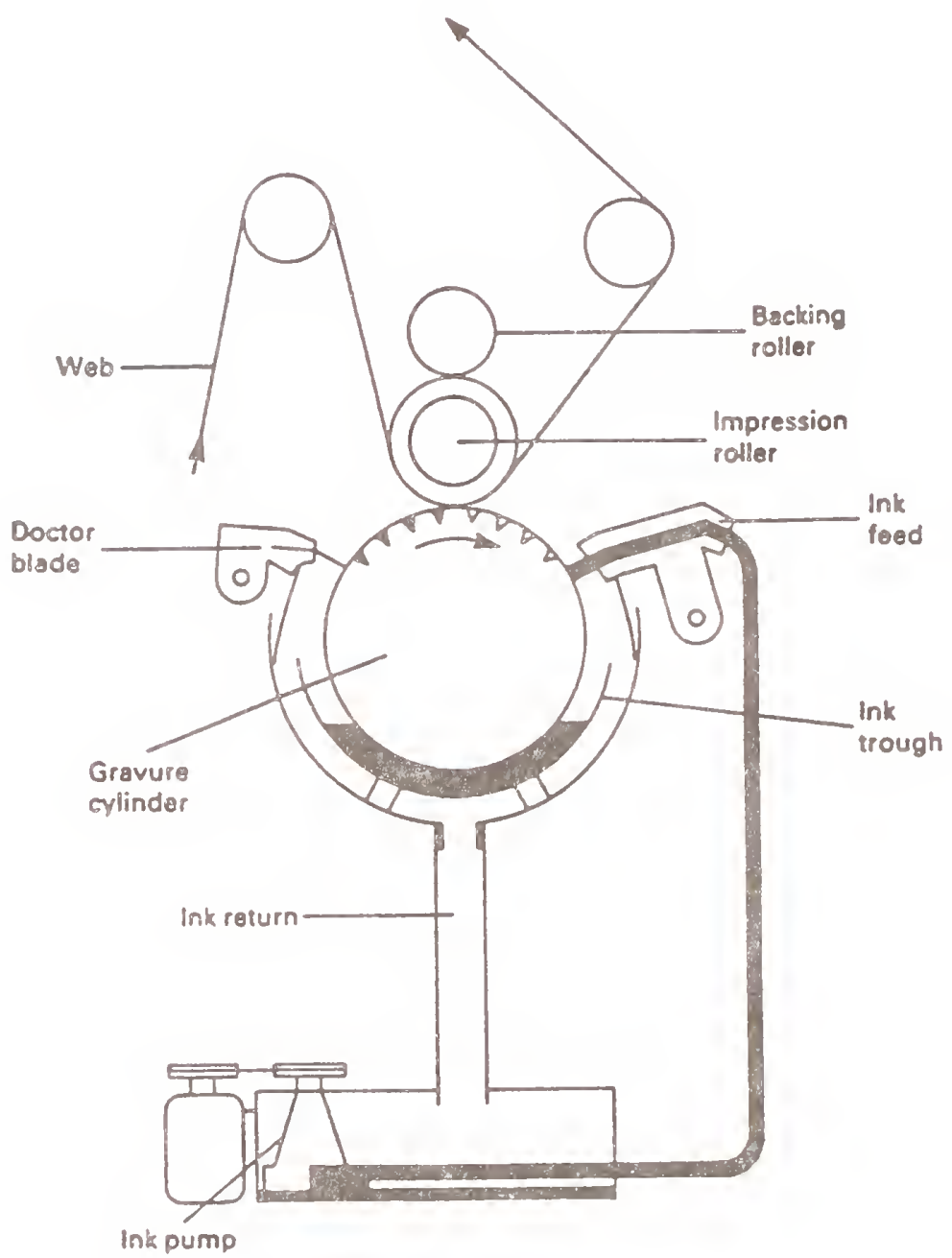




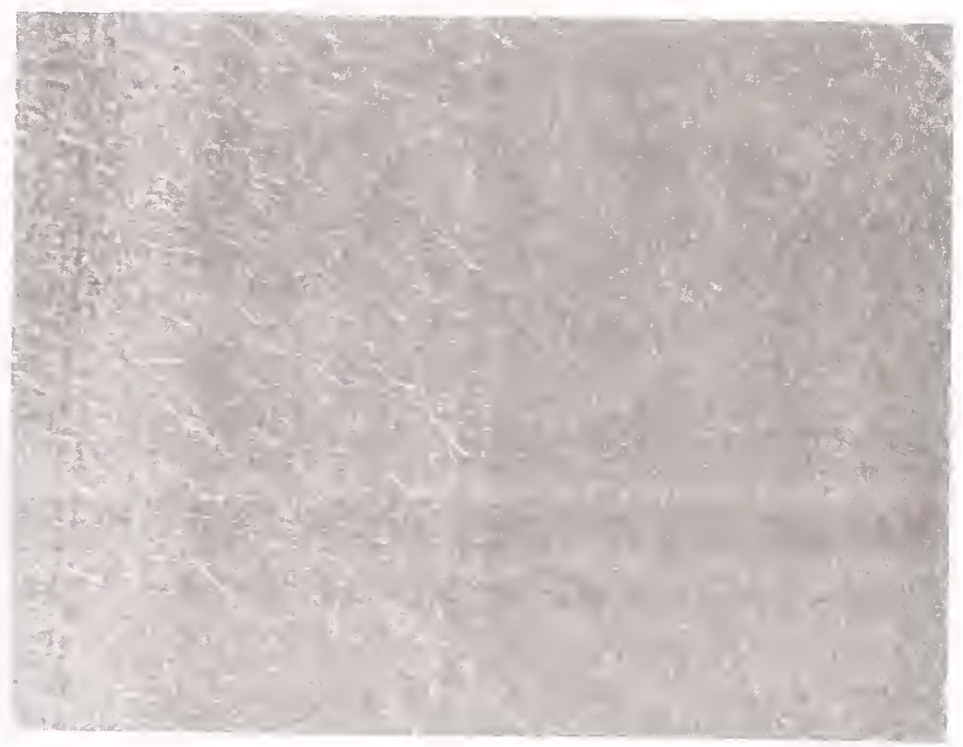

FIGURE 2

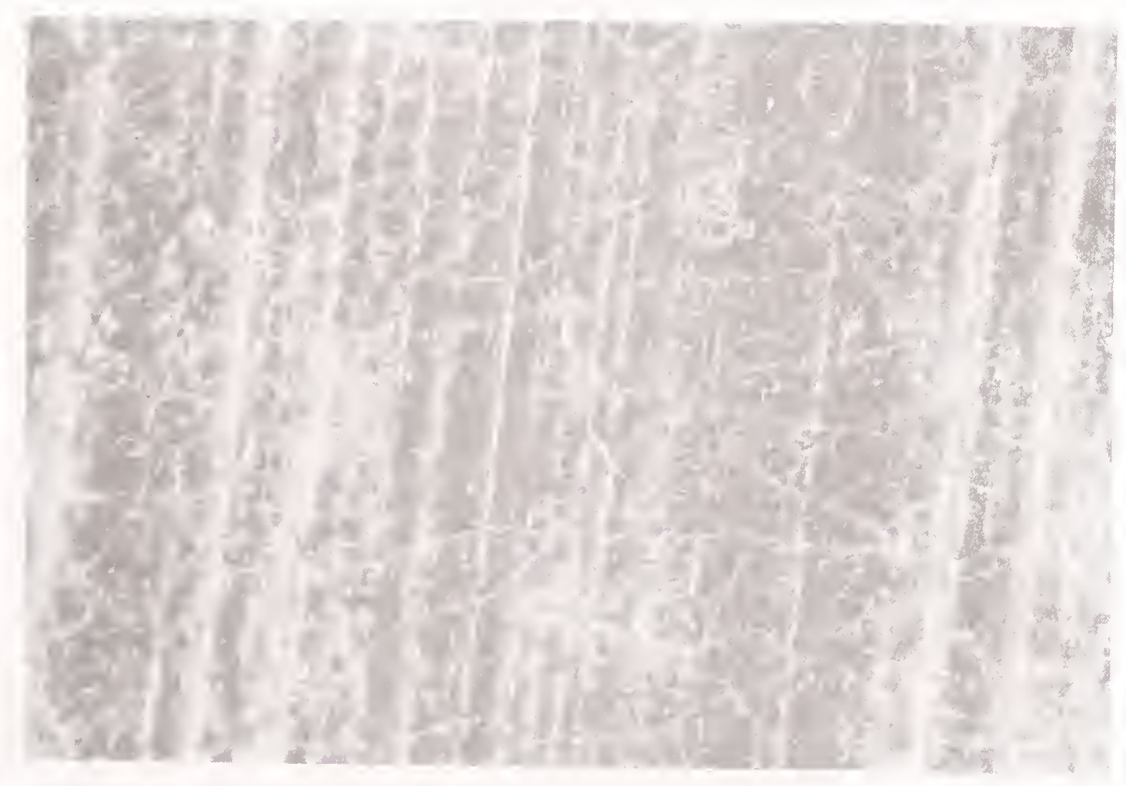

FIGURE 3 


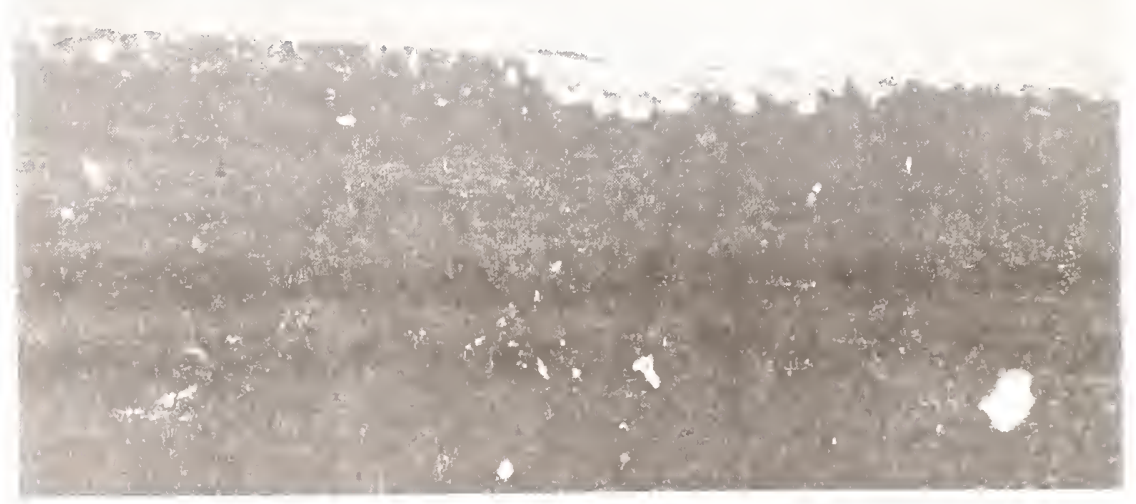

FIGURE 4

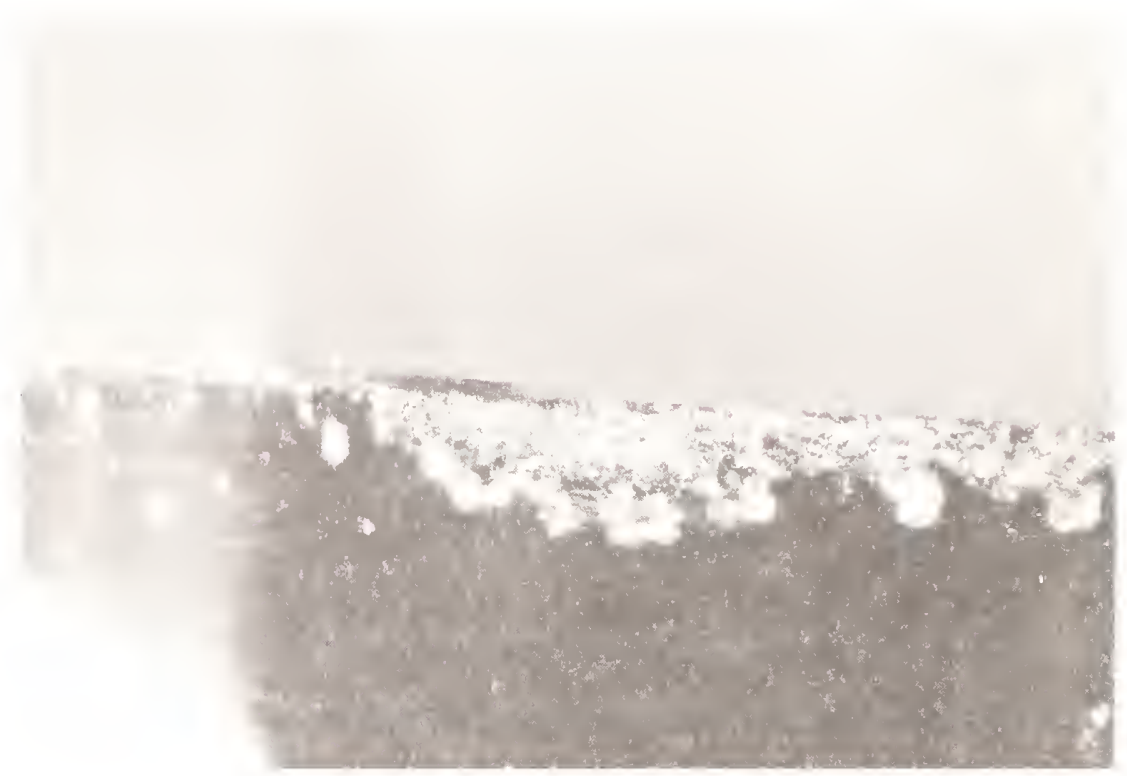

FIGURE 5 
Percent in Channel Graph Ultrafine Particle Analyzer Persion 2.00 , SUNSP

Sample ID 1: SIDGriRg

Mode: Rall Range

Distribution Rornat: Intensity

Bon five: 300 seconds

Bun 1: 1 of 2 runs

Loading Index: 0.903

Beflected Poner: 199 in

Abore Besidual: $0.00 \%$

Ploid Desc: SPABE 3

Cell Perperature: $25.1 \mathrm{C}$

Transparent Particles: Mo

Particle refractive Index: 1.50

Suroary Data

$c i=7.003$

$10 \%=0.0587$

$50 \%=0.1254$

$90 \%=0.5053$
Sample ID 2: toluene

Date: $9 / 28 / 92$ Tine: $10: 54$

Upper Channel Idge: 2.7500

Lower Channel idge: 0.0054

Chan. Progression: standard

Nuber of Channels: 36

Residuals: Rnabled

Belon Besidual: $0.00 \%$

Rluid Piscosity: $1.000 \mathrm{cp}$

$\mathrm{Cs}=\mathrm{n} / \mathrm{a}$

sd $=0.1409$

Particle refractive ludex: 1.50

Spherical Particles: No

Pluid Refractive !ndex: 1.33

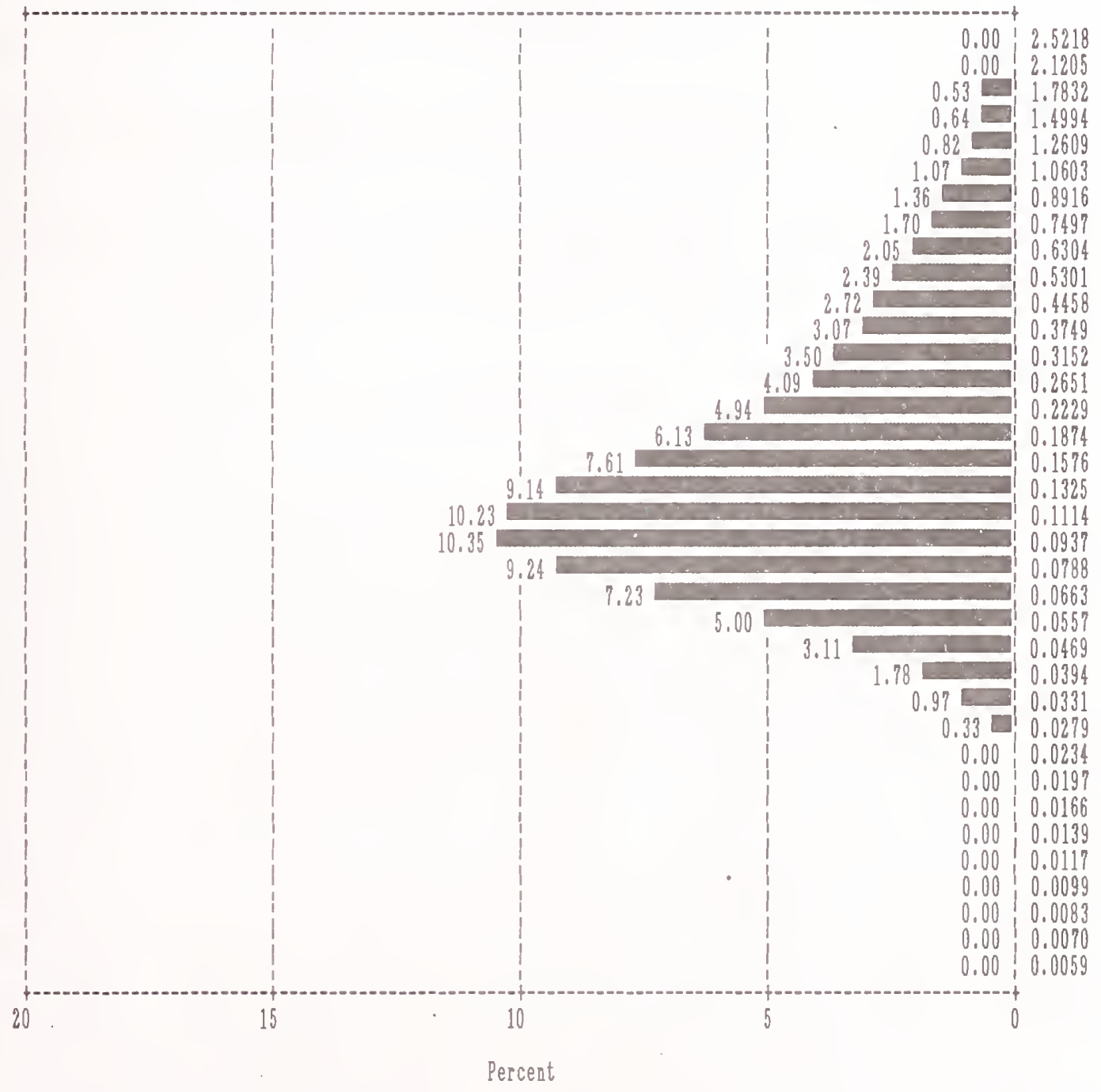




\title{
ELECTROKINETICS OF PHOSPHORS
}

\author{
Arunava Dutta \\ Osram Sylvania Inc \\ Research and Development \\ 100 Endicott Street \\ Danvers, MA 01923
}

\begin{abstract}
Electrokinetic studies have been conducted on a variety of fluorescent lamp phosphors using the ESA technique. In particular, the ESA vs pH and IEP of phosphors emitting in the UV, red, green, blue and white regions of the spectrum are investigated. The effects of nonluminescent additives, multicomponent phosphor blends, surface coatings, wash treatments and ionic polymers on the electrokinetic behavior of phosphors are studied.
\end{abstract}

\section{INTRODUCTION}

Phosphors are inorganic materials which produce luminescence when excited by high energy radiation. The incident radiation can be in the form of UV/visible photons or electrons and ions. Phosphors are used in a broad variety of applications which include general purpose fluorescent lamps, photocopying lamps, suntanning and medical lamps, high pressure mercury lamps, sign tubes, tagging and identification, cathode ray tubes, electroluminescent displays and X-ray intensifying screens. Every phosphor consists of a host crystal and an activator center which plays a key role in the luminescence mechanism.

This paper will focus on lamp phosphors in general and on fluorescent lamp phosphors in particular. A new generation highly energy efficient compact fluorescent lamp may have as many as four to five phosphors deposited on the inner curved glass surface of the lamp. Each phosphor has a particular emission spectrum in the visible range when excited by 254 $\mathrm{nm}$ UV photons which constitute the bulk of the radiation generated in a low pressure mercury discharge. The overall spectrum of visible light generated by such a lamp is an 
appropriate summation of the individual contributions from the various phosphors.

The phosphor coating on the interior surface of a fluorescent lamp is achieved by contacting the glass with an aqueous suspension of phosphors in a manner so as to cover the entire surface of the glass. Excess suspension is drained under gravity, following which the wet coating is dried by passing hot air over it, after which the dried coating is baked at an elevated temperature in air. In addition to the phosphors, the aqueous coating suspension may contain one or more of the following ingredients: very fine particle size alumina, metal salts, polymeric binders, surfactants, defoamers, cross-linking agents, plasticizers and dyes. The organic components of the suspension are removed from the coating film during the baking step, leaving behind primarily the phosphors and small amounts of non-luminescent metal oxides.

The texture of the phosphor coating in a finished lamp is very sensitive to the degree of dispersion of the phosphors in the coating suspension. Flocculation of the phosphor particles leads to unacceptable lamp texture and the conditions of the coating suspension have to be optimized to prevent this undesirable phenomenon. Flocculation may occur between particles of the same phosphor or between particles of different phosphors.

The stability of a phosphor coating suspension depends on a balance between electrostatic repulsive forces and the van der Waals attractive forces. The former are a result of the electrical double layer around the phosphor particles. The iso-electric points (IEP) of the various solids in the suspension and the dependence of the ESA (electrokinetic sonic amplitude) on $\mathrm{pH}$ constitute a key information that is necessary for an understanding of the stability of phosphor coating suspensions.

\section{ESA OR ZETA POTENTIAL?}

Electrokinetic studies were conducted using a Matec ESA-8000 system. In this apparatus [1] an alternating high frequency electric field is applied to a dispersion between two parallel electrodes, and the particles vibrate in this field producing a coherent sound wave. ESA, or electrokinetic sonic amplitude, is the pressure amplitude of this sound wave per unit of applied electric field. The units of ESA are $\mathrm{mPa} \cdot \mathrm{m} / \mathrm{V}$. The 
relevant equations [2] describing the operation of the apparatus are listed below:

$$
\begin{gathered}
E S A=\phi(\Delta \rho) C \mu_{d} \\
\mu_{d}=(\epsilon \zeta / \eta) G(\alpha) \\
\alpha=\omega r^{2} / v
\end{gathered}
$$

In the equations listed above, $\mu_{\mathrm{d}}$ is the dynamic mobility of the phosphor particles, $\phi$ is the volume fraction of the solids in the dispersion, $\Delta \rho$ is the difference in density between the phosphor and water, $\mathrm{c}$ is the speed of sound in the suspension, $\epsilon$ is the dielectric permittivity of the suspension, $\zeta$ is the zeta potential of the phosphor and $\eta$ is the dynamic viscosity of water. It is seen from Equation (1) that the magnitude of the ESA is directly proportional to the dynamic mobility of the phosphor. Equation (2) shows that the dynamic mobility is proportional to the zeta potential of the material. It follows that ESA is proportional to the zeta potential of the phosphor.

In this paper changes in $\zeta$ of the phosphor particles in the dispersion are followed by tracking the magnitude and polarity of the ESA signal. The reason that this investigation focuses on ESA which is a direct instrument output rather than $\zeta$ which is a computationally derived parameter is the uncertainty surrounding the applicability of the expression for $\mathrm{G}(\alpha)$, which is used to compute $\zeta$ from ESA, to the phosphor samples at hand. This makes the calculated value of $\zeta$ questionable.

$\mathrm{G}(\alpha)$ is an inertial correction which accounts for the decrease in dynamic mobility with particle size. The maximum value of $\mathrm{G}(\alpha)$ is unity which is attained by very fine sized particles. In Equation (3) $\omega$ is the angular frequency of the input electrical signal, $\nu$ is the kinematic viscosity of water and $\alpha$ is dimensionless. The assumptions [2] which are used in the derivation of $\mathrm{G}(\alpha)$ include a dilute suspension ( $<10 \mathrm{vol} \%$ solids), large particle radius 
(r) relative to the thickness of the double layer $(\kappa \mathrm{r}>50)$ and a narrow particle size distribution ( $\alpha<20 \%$ of mean size).

The double layer thickness $(1 / \kappa)$ varies from about $3 \mathrm{~nm}$ to $96 \mathrm{~nm}$ [3] for a change in ionic strength from $10^{-2} \mathrm{~mol} / 1$ to $10^{-5} \mathrm{~mol} / \mathrm{l}$. While electrolyte is not deliberately added in this work, the specific conductivity of the phosphor dispersion prior to potentiometric titration is influenced by a small degree of dissolution of ions from the phosphor surface. The anions and cations which leach out of the surface of the phosphors are polyvalent entities which significantly increase the ionic strength beyond what is expected for a 1:1 electrolyte of the same concentration. The specific conductivity of the phosphor dispersions is in the range of 30 to $50 \mu \mathrm{S} / \mathrm{cm}$. For the sake of comparison, a $10^{-3} \mathrm{M} \mathrm{KCl}$ solution has a conductivity of about $145 \mu \mathrm{S} / \mathrm{cm}$. Based on this comparison, it is estimated that the ionic strength is at least $10^{-4} \mathrm{~mol} / 1$ in this work which leads to a maximum value of about $30 \mathrm{~nm}$ for $1 / \kappa$. It follows that the phosphor particles must be larger than about $3 \mu \mathrm{m}$ in diameter for the criterion $\kappa \mathrm{r}>50$ to be satisfied. This is usually the case.

The solids loading assumption used in the derivation of $G(\alpha)$ is also satisfied since the maximum concentration of phosphors used in this study is about 2 vol\%. It is the third assumption which is violated by commercial fluorescent lamp phosphors. The particle size distribution is very broad: there are particles from about $2 \mu \mathrm{m}$ to about $20 \mu \mathrm{m}$ in diameter. No single particle size can uniquely describe the population for the purposes of calculation of $\zeta$. Any computed value of $\zeta$ using the expression for $\mathrm{G}(\alpha)$ is, therefore, questionable. It is for this reason that ESA rather than $\zeta$ is tracked in this research.

It follows from the nature of $\mathrm{G}(\alpha)$ that a larger particle size phosphor would produce a smaller ESA signal than a smaller size phosphor with the same surface chemistry. This is caused by the larger inertia of the bigger particles which cannot track the rapidly alternating electric field as fast as the smaller particles. The magnitude of the ESA signal is also sensitive to the phosphor derisity and concentration: the signal increases if one changes to a more dense phosphor or uses a more concentrated dispersion of the same phosphor. 


\section{EXPERIMENTAL TECHNIQUE AND SCOPE}

Table 1 lists the various phosphors which have been studied and their densities. The effect of a non-luminescent material like alumina on the electrokinetics of phosphor suspensions is also investigated. It is easier to refer to most phosphors by their acronyms and this practice is adopted in this paper. The BAM, CAT and YOE are rare earth (RE) activated phosphors which luminesce in the blue, green and red portions, respectively, of the visible spectrum when the phosphors are excited by $254 \mathrm{sm}$ photons in a fluorescent lamp. Good color rendition lamps may have this blend of $R E$ activated phosphors in certain proportions by weight. The $\mathbb{C W}$ phosphor has a white light emission spectrum and may be used in conjunction with RE phosphors or by itself. The remaining two phosphors in Table 1 do not have common acronyms and are categorized by their dominant emission wavelength. Thus, the strontium borate phosphor emits in the UVA region (320 to $400 \mathrm{~nm}$ ) of the spectrum while the cerium aluminate phosphor emits in the UVB region (260 to $320 \mathrm{~nm}$ ). These UV phosphors, which are referred to as UVA and UVB phosphors from now on, are used in sun tanning lamp applications.

The pH and conductivity probes of the Matec ESA-8000 system are calibrated prior to the measurement of every new phosphor dispersion. The ESA probe is calibrated with a Ludox TM colloidal silica dispersion, as recommended by Matec [1]. A specified mass of phosphor ranging from 10 to $15 \mathrm{~g}$ is added to $200 \mathrm{~cm}^{3}$ distilled water in a Teflon sample cell which contains a cross shaped Teflon magnetic stirrer. The cell is then inserted in the Matec apparatus. Water is circulated in a plexiglass jacket positioned around the cell to maintain the temperature of the contents of the sample cell at $25^{\circ} \mathrm{C} \pm 0.3^{\circ}$. In addition to the magnetic stirrer, the contents are also agitated by a motor driven propeller. The $\mathrm{pH}$, conductivity and ESA are monitored every minute for about $72 \mathrm{~h}$ using the Time Series mode of data acquisition. This length of time is sufficient to stabilize a phosphor dispersion in terms of ESA and pH.

Arrangements are made for potentiometric titration after the dispersion has stabilized. The automated titration system is first washed with acid/base as appropriate. Phase calibration is then conducted with a knowledge of the polarity of the sample at the end of the 
time series study. This information is derived from chemical considerations of the surface or by observing the changes in ESA vs pH during the time series. Titration is either done with $0.1 \mathrm{M} \mathrm{HNO}_{3}$ or with $0.1 \mathrm{M} \mathrm{NaOH}$. Specific adsorption of ions is not a problem with either of these titrants. IEP measurements are reproducible to within $0.15 \mathrm{pH}$ units.

TABLE 1

PHOSPHOR TYPES

\begin{tabular}{|c|c|}
\hline PHOSPHOR (ACRONYM) & DENSITY (g/cc) \\
\hline $\mathrm{BaMg}_{2} \mathrm{Al}_{16} \mathrm{O}_{27}: \mathrm{Eu}(\mathrm{BAM})$ & 3.70 \\
\hline$(\mathrm{Ce}, \mathrm{Tb}) \mathrm{MgAl}_{11} \mathrm{O}_{19}: \mathrm{Ce}, \mathrm{Tb}(\mathrm{CAT})$ & 4.15 \\
\hline $\mathrm{Y}_{2} \mathrm{O}_{3}: \mathrm{Eu}(\mathrm{YOE})$ & 5.15 \\
\hline $\mathrm{LaPO}_{4}: \mathrm{Ce}, \mathrm{Tb}(\mathrm{LAP})$ & 5.20 \\
\hline $\mathrm{Ca}_{5}(\mathrm{~F}, \mathrm{Cl})\left(\mathrm{PO}_{4}\right)_{3}: \mathrm{Sb}: \mathrm{Mn}(\mathrm{CW})$ & 3.10 \\
\hline $\mathrm{SrB}_{4} \mathrm{O}_{7}: \mathrm{Eu}(\mathrm{UVA})$ & 3.40 \\
\hline $\mathrm{Ce}\left(\mathrm{Sr}, \mathrm{Mg} \mathrm{Al}_{11} \mathrm{O}_{17}: \mathrm{Ce}(\mathrm{UVB})\right.$ & 4.30 \\
\hline $\mathrm{A} \ell_{2} \mathrm{O}_{3}(\mathrm{AOC})$ & 3.75 \\
\hline
\end{tabular}

RESULTS

\section{CW Phosphor and Effect of AOC}

Figure 1 shows the electrokinetic results obtained for a dispersion of $12.65 \mathrm{~g}$ of $\mathrm{CW}$ in $200 \mathrm{cc}$ of water (a $2 \mathrm{vol} \%$ solids dispersion). The stable $\mathrm{pH}$ of this dispersion prior to titration is about 6.4. The IEP of this phosphor is about 2.3 which reflects the presence of strongly anionic sites on the surface of this material. XPS (X-ray photoelectron spectroscopy) of the surface of this phosphor reveals the following atomic composition: $\mathrm{Ca}$ $17 \%, \mathrm{P} 17 \%$, O 62\%, F $3.8 \%$ and the balance is $\mathrm{Sb}$. 
Figures 2 through 5 are data for dispersions containing mixtures of $\mathrm{CW}$ and alumina in various proportions. The alumina is Aluminum Oxide C (AOC) from Degussa. AOC has a primary particle size of about $20 \mathrm{~nm}$ and a specific surface area of about $90 \mathrm{~m}^{2} / \mathrm{g}$ as compared to about $0.55 \mathrm{~m}^{2} / \mathrm{g}$ for the $\mathrm{CW}$ phosphor. Four distinct $\mathrm{CW} / \mathrm{AOC}$ blends are tested ranging from an AOC loading of $1.25 \%$ to $10 \%$ by mass of the phosphor. The total surface area presented by unaggregated $\mathrm{AOC}$ in water is about twice that of the $\mathrm{CW}$ phosphor when the AOC loading is $1.25 \%$. The stable $\mathrm{pH}$ of these $\mathrm{CW} / \mathrm{AOC}$ suspensions is only weakly sensitive to the concentration of AOC and is about 7.2. The total mass of the mixture is maintained at $12.65 \mathrm{~g}$ in $200 \mathrm{~cm}^{3}$ of water for all four CW/AOC blends.

Prior to potentiometric titration, every CW/AOC dispersion is referenced to itself for the purposes of phase calibration. There is a dramatic change in IEP from 2.3 to about 7.1 with the addition of only $1.25 \%$ AOC, as concluded from Figures 1 and 2. The measurements shown in Figures 3 through 5 indicate that the IEP of the $\mathrm{CW} / \mathrm{AOC}$ blend progressively increases from 7. 1 to about 8.5 as the concentration of AOC is raised from $1.25 \%$ to $10 \%$. There is a discontinuity in the ESA curve around the IEP in Figure 4. Such discontinuities have been discussed in the literature [4] and are usually attributed to ionic background interference. No electrolyte is, however, used in this work. The discontinuity in the ESA curve in Figure 4 is, therefore, not well understood and warrants further study. In Figure 5, where the AOC concentration is $10 \%$ compared to the $5 \%$ in Figure 4, there is also a discontinuity in the ESA curve but this is less prominent than that in Figure 4.

The IEP of AOC, as shown in Figure 6, is about 9.15. The AOC dispersion contains $6 \mathrm{~g}$ alumina in $200 \mathrm{~cm}^{3}$ water. The very small size of the AOC particles is primarily responsible for the large ESA signals associated with this material. The IEP of AOC and CW being 9.15 and 2.3 respectively, these materials will not remain individually dispersed at intermediate $\mathrm{pH}$ values. In particular, electrokinetic considerations would dictate that the $\mathrm{AOC}$ be positively charged and the $\mathrm{CW}$ be negatively charged at these $\mathrm{pH}$ values, leading to inter-particle flocculation. Given the much larger surface area of the $\mathrm{AOC}$ relative to $\mathrm{CW}$, this would translate to a portion of the surface of the $\mathrm{CW}$ phosphor being covered by the AOC. This reasoning is upheld by the following analysis of the electrokinetic data. 
The IEP of the CW/1.25\% AOC system is 7.1 which means that the net ESA is zero at this $\mathrm{pH}$. At this $\mathrm{pH}, \mathrm{AOC}$ and $\mathrm{CW}$ would individually have positive and negative surface charges respectively. Let us assume that at this $\mathrm{pH}$ the components are individually dispersed with no inter-particle flocculation due to electrostatic attraction. In other words, we are assuming that the $\mathrm{CW}$ surface is not covered by the $\mathrm{AOC}$ to any degree at this $\mathrm{pH}$. The ESA then can only be zero at this $\mathrm{pH}$ if the positive ESA contribution from the AOC is matched by an equal negative ESA contribution from the $\mathrm{CW}$ phosphor. These contributions are calculated using the information in Figures 1 and 6 which has to be adjusted for the appropriate concentration of the solids in the $\mathrm{CW} / 1.25 \%$ AOC blend. Under these conditions it can be shown that the net ESA would not be zero but rather a sizable negative quantity due to the strong negative ESA of the $\mathrm{CW}$ phosphor at this $\mathrm{pH}$. It follows that the net ESA can only be zero at $\mathrm{pH} 7.1$ if the effect of the AOC is to coat a portion of the surface of the CW phosphor thereby masking some of the negative contribution to the ESA. The extent of coverage of the $\mathrm{CW}$ surface by the $\mathrm{AOC}$ is a function of the $\mathrm{pH}$ and the relative total surface areas of the two solids exposed to the liquid.

\section{Effect of a Thin Film Coating on the cw Phosphor}

Figure 7 shows the results of an electrokinetic study for a dispersion of $\mathrm{CW}$ phosphor, $12.65 \mathrm{~g}$ in $200 \mathrm{~cm}^{3}$ water, which has a thin film $\left(\sim 400 \mathrm{~A}^{\circ}\right)$ coating of alumina on its surface. XPS shows a 95\% attenuation of the Ca signal from the phosphor substrate indicating a fair degree of surface coverage by the alumina coating. This coating may be applied by any one of a variety of known methods. A comparison of Figures 1 and 7 proves that the IEP of the strongly acidic CW may be changed to a basic value by depositing an alumina film on its surface. This has implications as regards the degree of stability of the overall coating suspension.

\section{Effect of YOE, CAT and BAM Phosphors}

Figure 8 shows the electrokinetic behavior of a red YOE phosphor dispersion which has $15 \mathrm{~g}$ of the powder in $200 \mathrm{~cm}^{3}$ water. The basic nature of the surface is reflected in the 
IEP of 8.75. XPS of the surface of the YOE phosphor reveals the following atomic composition: Y $24 \%, \mathrm{O} 74 \%$ and the balance is Europium. The $\mathrm{O} / \mathrm{Y}$ ratio is almost three, like in yttrium hydroxide, which may indicate a highly hydroxylated surface. The green CAT phosphor, $10 \mathrm{~g}$ in $200 \mathrm{~cm}^{3}$ water, has an even more basic surface as indicated by an IEP of 9.3 in Figure 9. XPS of the CAT phosphor yields the following surface atomic composition: $\mathrm{Al} 35 \%, \mathrm{O} 61 \%, \mathrm{Mg} 2.1 \%, \mathrm{~Tb} 1.3 \%$ and $\mathrm{Ce} 0.94 \%$. The blue BAM phosphor, $10 \mathrm{~g}$ in $200 \mathrm{~cm}^{3}$ water, is the most basic of the three RE phosphors as observed in Figure 10 which displays an IEP of 9.85 for this material. XPS of the blue BAM phosphor shows that the surface atomic composition is as follows: $\mathrm{Al} 37 \%, \mathrm{O} 60 \%, \mathrm{Mg} 1.5 \%, \mathrm{Ba}$ $1.1 \%$ and the balance is Europium.

The effect of YOE and CAT on the IEP of a phosphor blend with CW is also investigated. A three component blend of YQE, CAT and CW phosphors is prepared in 200 $\mathrm{cm}^{3}$ distilled water with $3.75 \mathrm{~g}$ each of the first two components and $7.5 \mathrm{~g}$ of the $\mathrm{CW}$ phosphor. This composition results in about a 2 vol\% total solids dispersion. In addition, the total surface area exposed to the water by the $\mathrm{CW}$ in this blend is comparable to the sum of the surface areas of the YOE and CAT phosphors. The electrokinetic characteristics of the blend is shown in Figure 11. The blend IEP is 7.65 which is much greater than the IEP of CW (2.3) and somewhat smaller than the IEP of either YQE (8.75) or CAT (9.3).

\section{Effect of Wash Treatment}

Various wash treatments are given to different phosphors at some final stage of the manufacturing process. While these treatments are meant to improve the brightness and/or lumen maintenance of these materials, these washes also influence the electrokinetic behavior of the phosphors which has to be accounted for while considering the stability of the coating suspension. Figure 12 shows the electrokinetic behavior of a green LAP phosphor, $10.5 \mathrm{~g}$ of powder in $200 \mathrm{~cm}^{3}$ water or a $1 \mathrm{vol} \%$ solids dispersion, which has not undergone any surface wash treatment. The IEP of this phosphor is 6.6. XPS of this phosphor provides the following surface atomic composition: La 5.8\%, P 14\%, O 74\%, Ce 4.6\% and Tb $2.3 \%$. There is more phosphorus on the surface than is required for the amount of lanthanum present. The same LAP phosphor after a KOH base wash is shown in Figure 13. XPS of 
the surface of this washed phosphor shows the following atomic composition: $\mathrm{La} 6.3 \%, \mathrm{P}$ $13 \%, \mathrm{O} 73 \%, \mathrm{Ce} 4.8 \%$ and $\mathrm{Tb} 2.2 \%$. The IEP of the washed phosphor has increased to 8.9. The increased IEP of the base washed phosphor will in turn influence the stability of any multicomponent phosphor blend in which the washed LAP is introduced.

\section{Effect of Ionic Polymers}

A dispersion of CW, $12.65 \mathrm{~g}$ in $200 \mathrm{~cm}^{3}$ water, is prepared in the Matec sample cell. At the end of $2.5 \mathrm{~h}$ of aging, the ESA is $-0.171 \mathrm{mPa} \mathrm{mN}$ while the $\mathrm{pH}$ is 6.25 . The polarity of the ESA indicates that the surface is negatively charged at this $\mathrm{pH}$, which should be the case given that the IEP for $\mathrm{CW}$ is about 2.3 . At this point, $1 \mathrm{~cm}^{3}$ of a strongly cationic, low molecular weight polymer (Betz 1190) is rapidly introduced into the dispersion. The addition of this polymer causes the ESA to change very quickly to +0.415 . This change in the polarity and magnitude of the ESA is not accompanied by any significant change in $\mathrm{pH}$.

The positive ESA and its large magnitude proves that the cationic polymer adsorbs strongly on the initially negative CW surface, transforming it to a positively charged surface. In order to have changed polarity, the surface must have gone through a zero ESA. Flocculation at this juncture is, however, avoided by the rapid increase in the magnitude of ESA. This rapid increase may be attributed to two critical parameters: the amount of polymer which is introduced and the short time scale over which the introduction is made.

Changes in the polarity and magnitude of ESA can have significant impact on the degree of dispersion of a coating suspension containing one or more phosphors. In particular, a cationic polymer treated surface will have a more basic IEP than a non-treated surface. In addition, the quality of thin film coatings deposited from a solution onto the phosphor surface can be greatly influenced by such changes in phosphor surface charge characteristics.

Having changed the initially negatively charged $\mathrm{CW}$ surface to a positively charged surface, $1 \mathrm{~cm}^{3}$ of a highly anionic polymer (BASF Sokalan PA30) is slowly introduced into the dispersion. The polarity of the ESA is now reversed going from +0.415 to a final value of -0.385 . The $\mathrm{pH}$ increases by about 2 units. The extent of change in the ESA cannot be explained by this amount of movement in $\mathrm{pH}$. Flocculation is visible when ESA decreases to 
about zero. The suspension disperses again as the ESA, now negative, increases in magnitude with gradual addition of the anionic polymer. Adsorption of an anionic polymer onto the surface of a cationic polymer treated $\mathrm{CW}$ phosphor can, therefore, change the surface polarity from positive to negative.

\section{UV Phosphors}

The electrokinetic behavior of an UVA strontium borate phosphor, $15 \mathrm{~g}$ in $200 \mathrm{~cm}^{3}$ water, is shown in Figure 14. The extremely basic nature of the surface of this phosphor is reflected in an IEP of about 11.2. This is one of the highest measured IEPs of any fluorescent lamp phosphor. XPS of the surface of this UVA phosphor gives an atomic composition as follows: $\mathrm{Sr} 6.8 \%, \mathrm{~B} 36 \%$, O 56\%, Eu $0.37 \%$ and $\mathrm{Ba} 0.78 \%$. A suntanning lamp is required to have the proper relative amounts of UVA and UVB emission. The electrokinetics of a strontium magnesium aluminate UVB phosphor, $15 \mathrm{~g}$ in $200 \mathrm{~cm}^{3}$ water, is shown in Figure 15. This phosphor has an IEP of 9.9. XPS of this phosphor provides surface composition as follows: $\mathrm{Al} 36 \%, \mathrm{O} 60 \%, \mathrm{Sr} 2.5 \%, \mathrm{Mg} 0.34 \%$ the balance being other atoms. The surface composition of a phosphor does not necessarily reflect its bulk composition. Cerium, for example, is not detectable on the surface of this phosphor. Rheological testing of an aqueous suspension of a blend of the UVA and UVB phosphors shows strong pseudoplasticity at $\mathrm{pH}$ between 10 and 11.5 . This is a result of interparticle flocculation.

\section{CONCLUSIONS}

The electrokinetic behavior of a large variety of fluorescent lamp phosphors has been investigated. These luminescent materials have a broad range of iso-electric points: about 2.3 for $\mathrm{CW}, 6.6$ for LAP, about 8.75 to 10 for the YOE, CAT and BAM rare earth phosphor and about 10 to 11 for the UV emitting phosphors. Addition of a very small amount of very fine size alumina causes a significant increase in the effective IEP of $\mathrm{CW} / \mathrm{AOC}$ blends relative to $\mathrm{CW}$. A thin film coating of alumina on $\mathrm{CW}$ increases its IEP by about $6 \mathrm{pH}$ units. A tricomponent blend of YEO, CAT and $\mathrm{CW}$ phosphors compared to 
$\mathrm{CW}$, has an effective IEP of 7.65. Potassium hydroxide base wash of the LAP green phosphor increases its IEP from almost neutral $\mathrm{pH}$ to about 9. Adsorption of cationic and anionic polymers onto the surface of the $\mathrm{CW}$ phosphor leads to charge reversal.

\section{REFERENCES}

1. Matec Applied Sciences, ESA-8000 System Operating Manual, 1992.

2. O’Brien, R. W., J. Fluid Mech., 190, 71 (1988).

3. Hunter, R. J., Zeta Potential in Colloid Science, Academic Press, 1981.

4. Hackley, V. A., Premachandran, R. S. and Malghan, S. G., Ceramic Transactions "Characterization Techniques for Solid-Solution Interfaces in Ceramic Systems," Ed. J. Adair, and J. Casey, American Ceramic Society: In Press, 1993. 


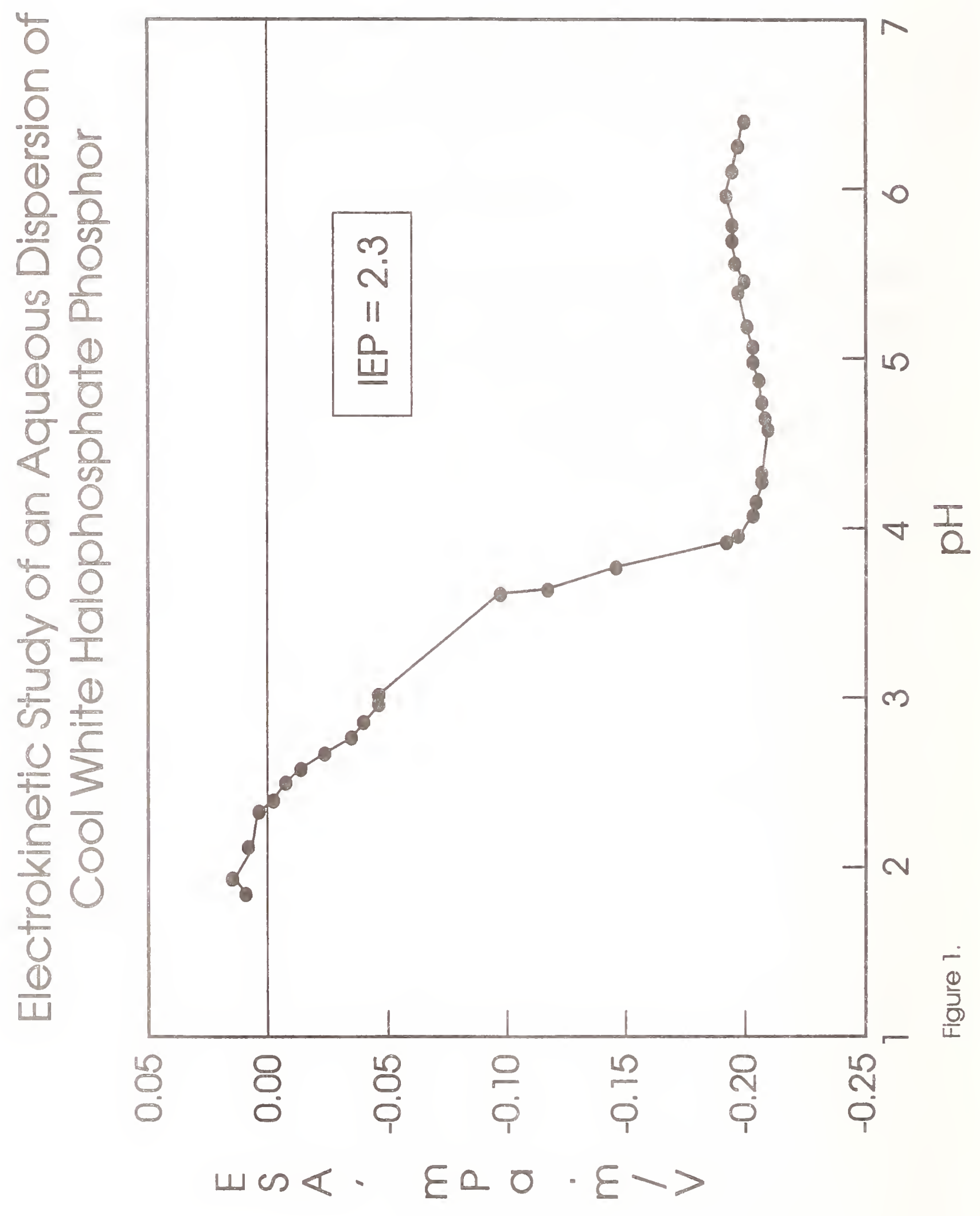




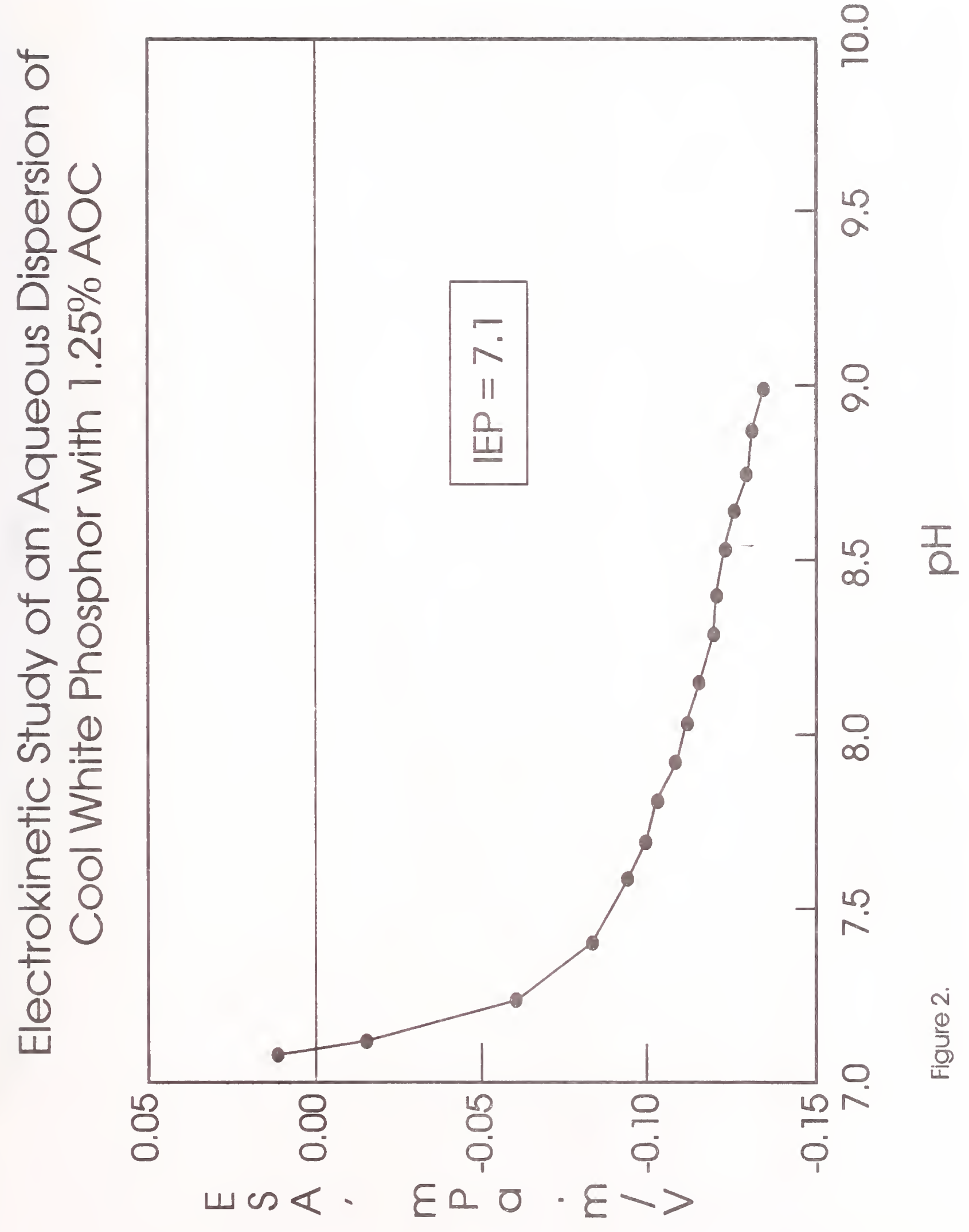




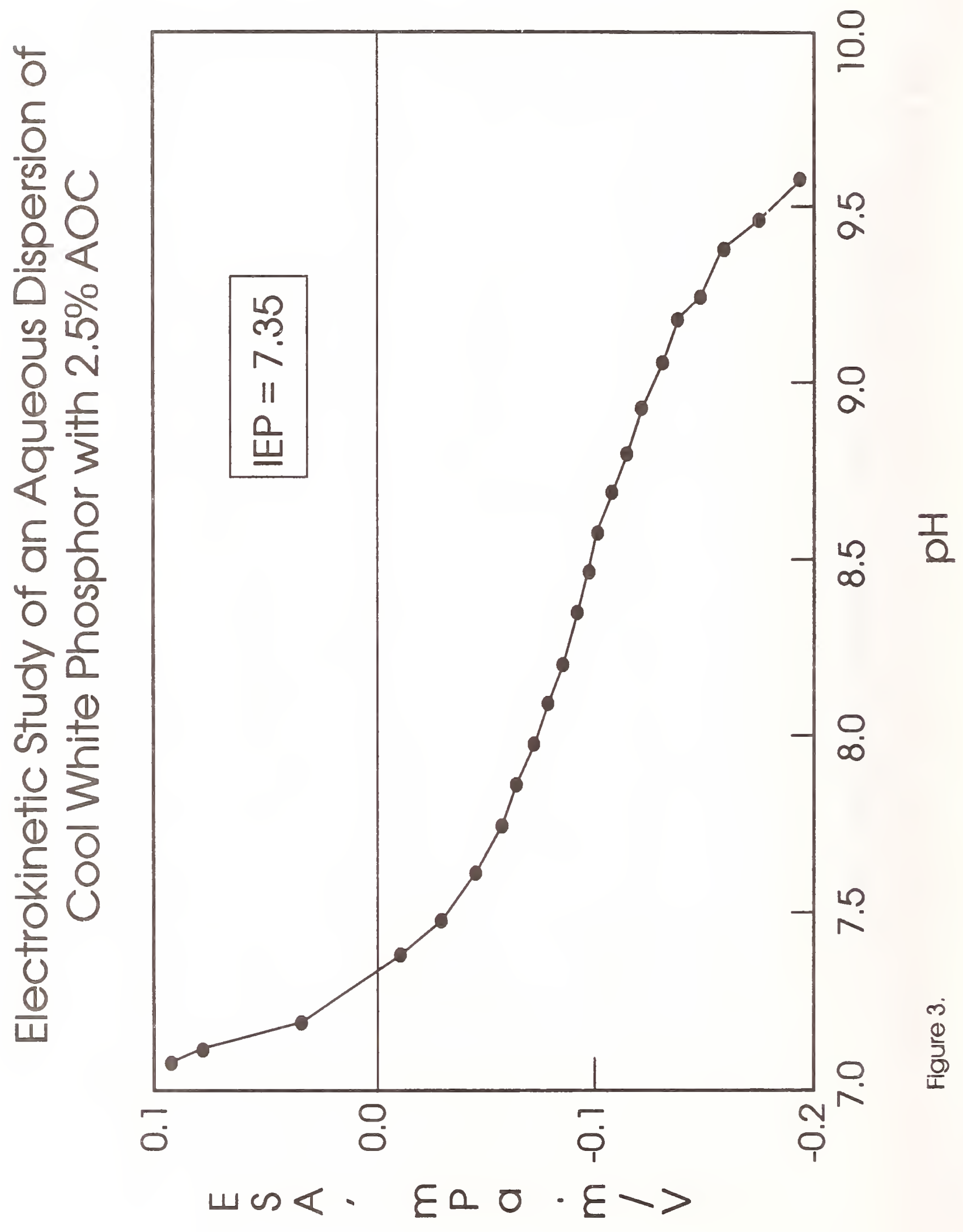




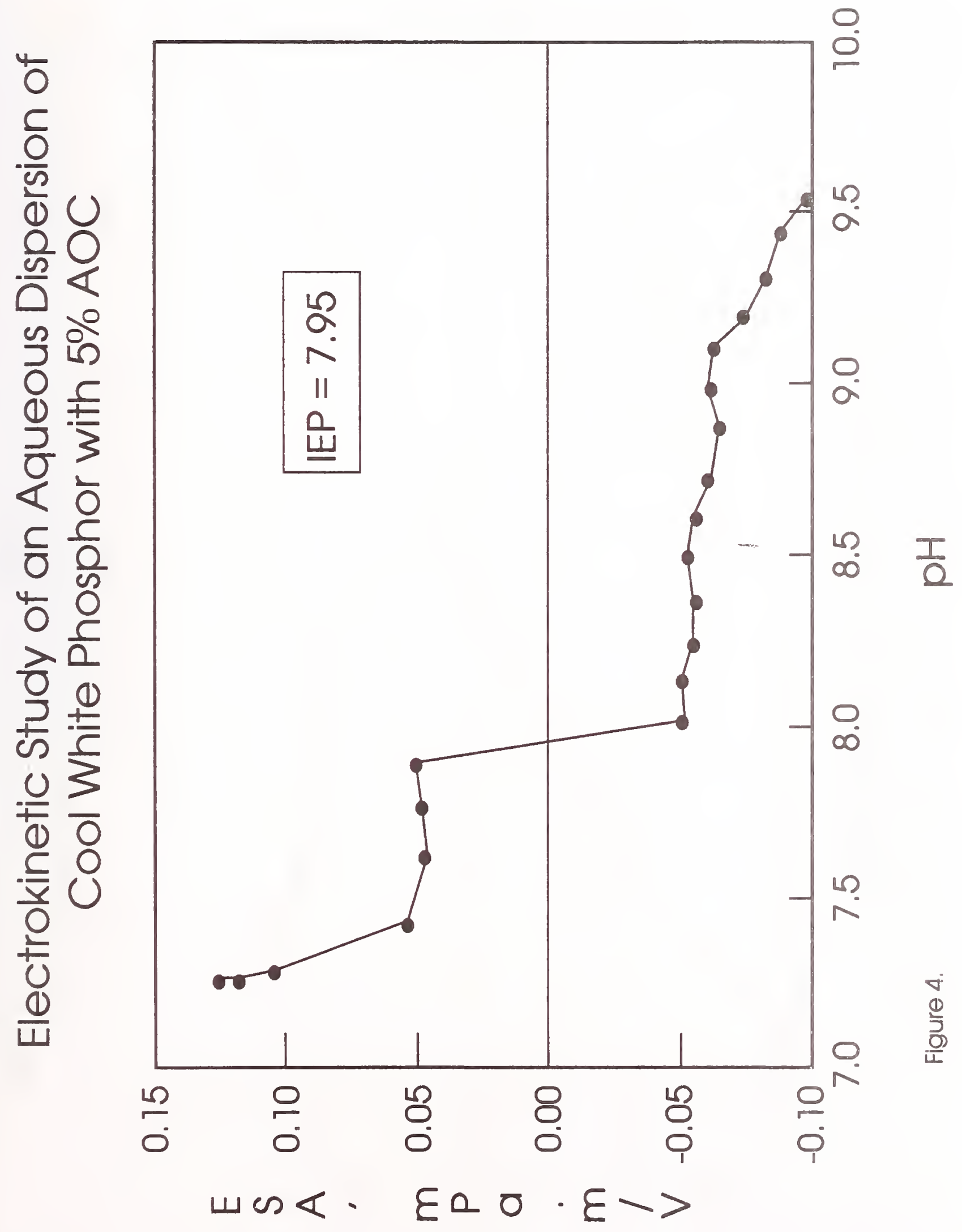




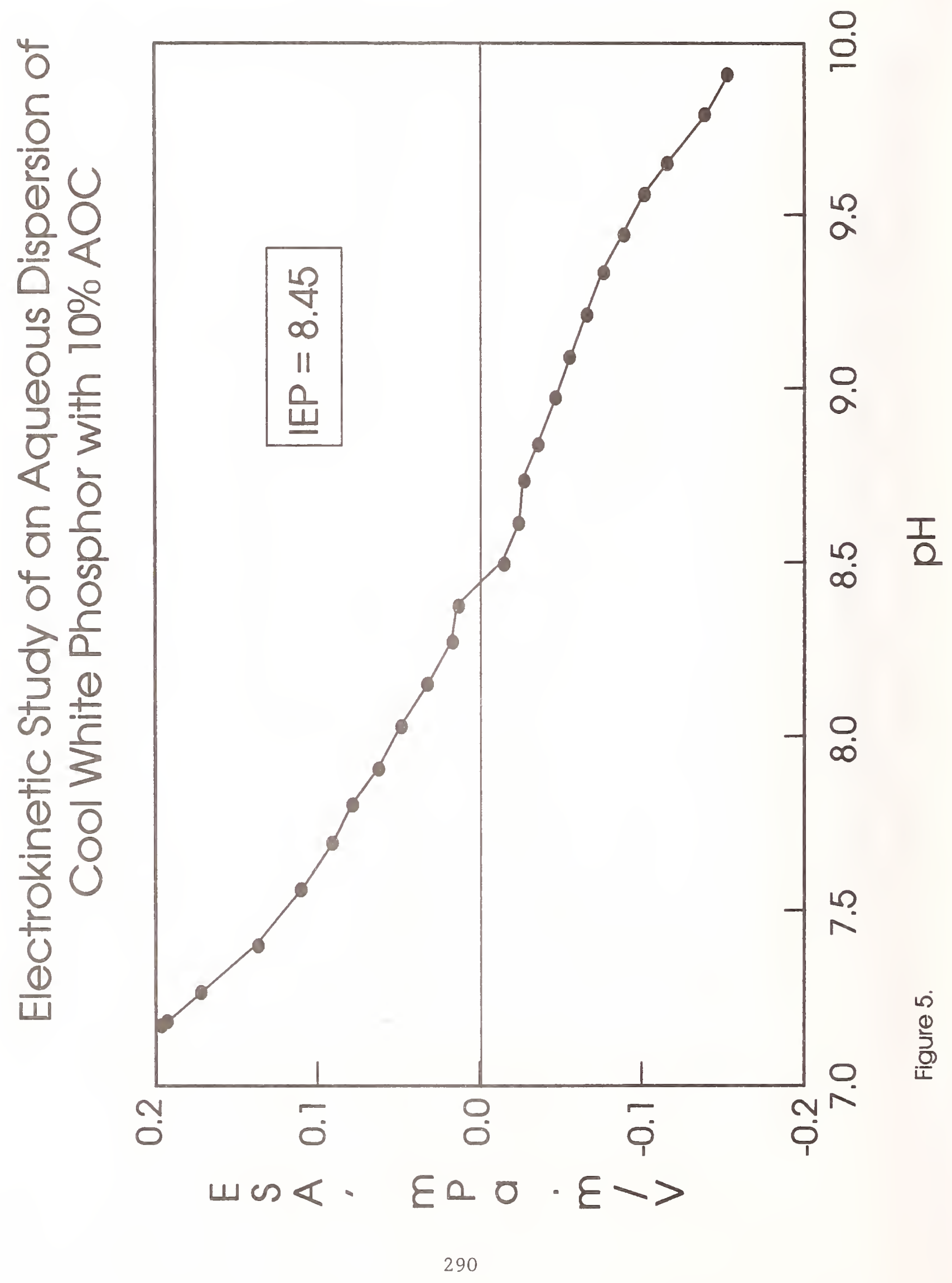




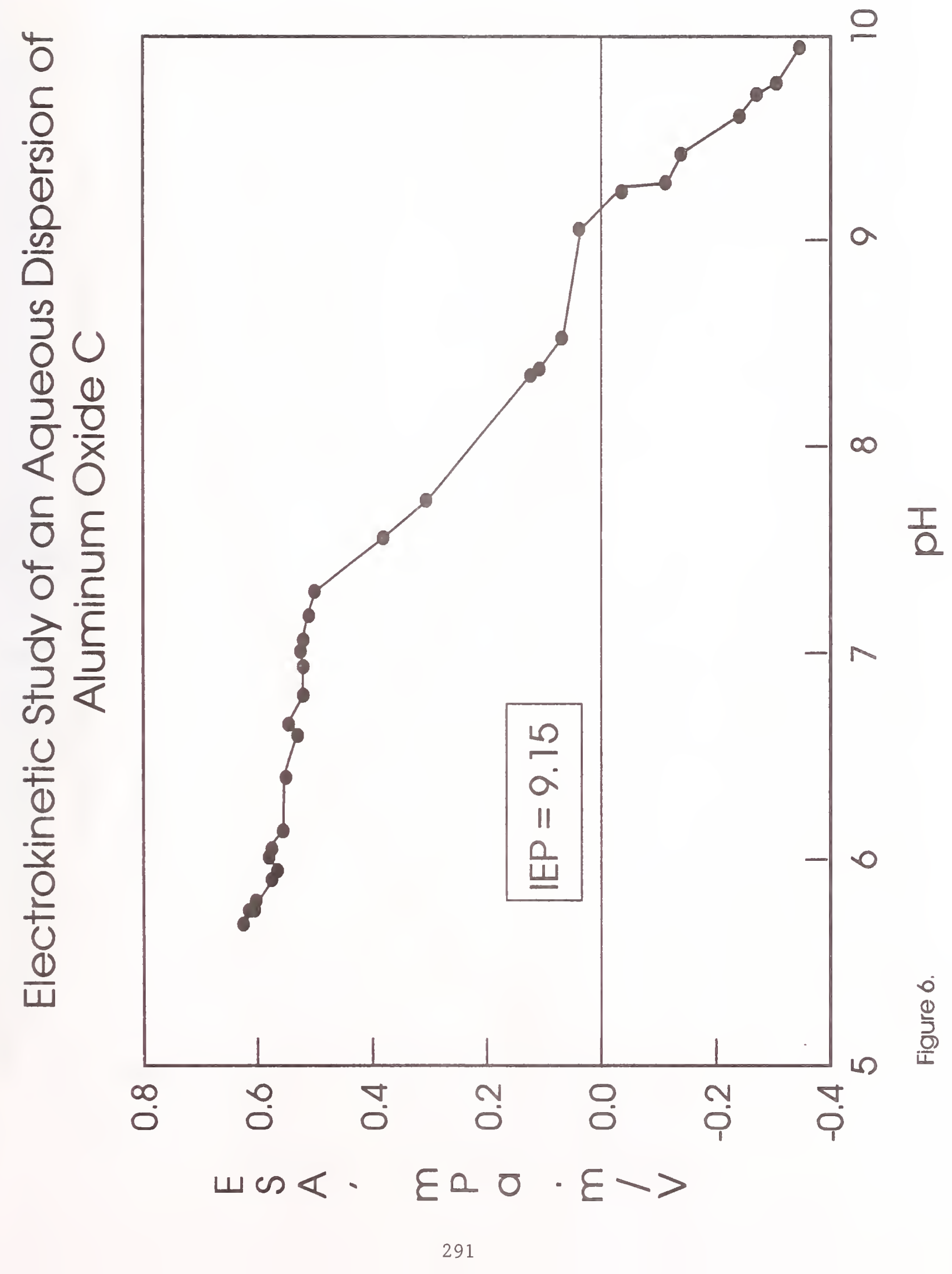




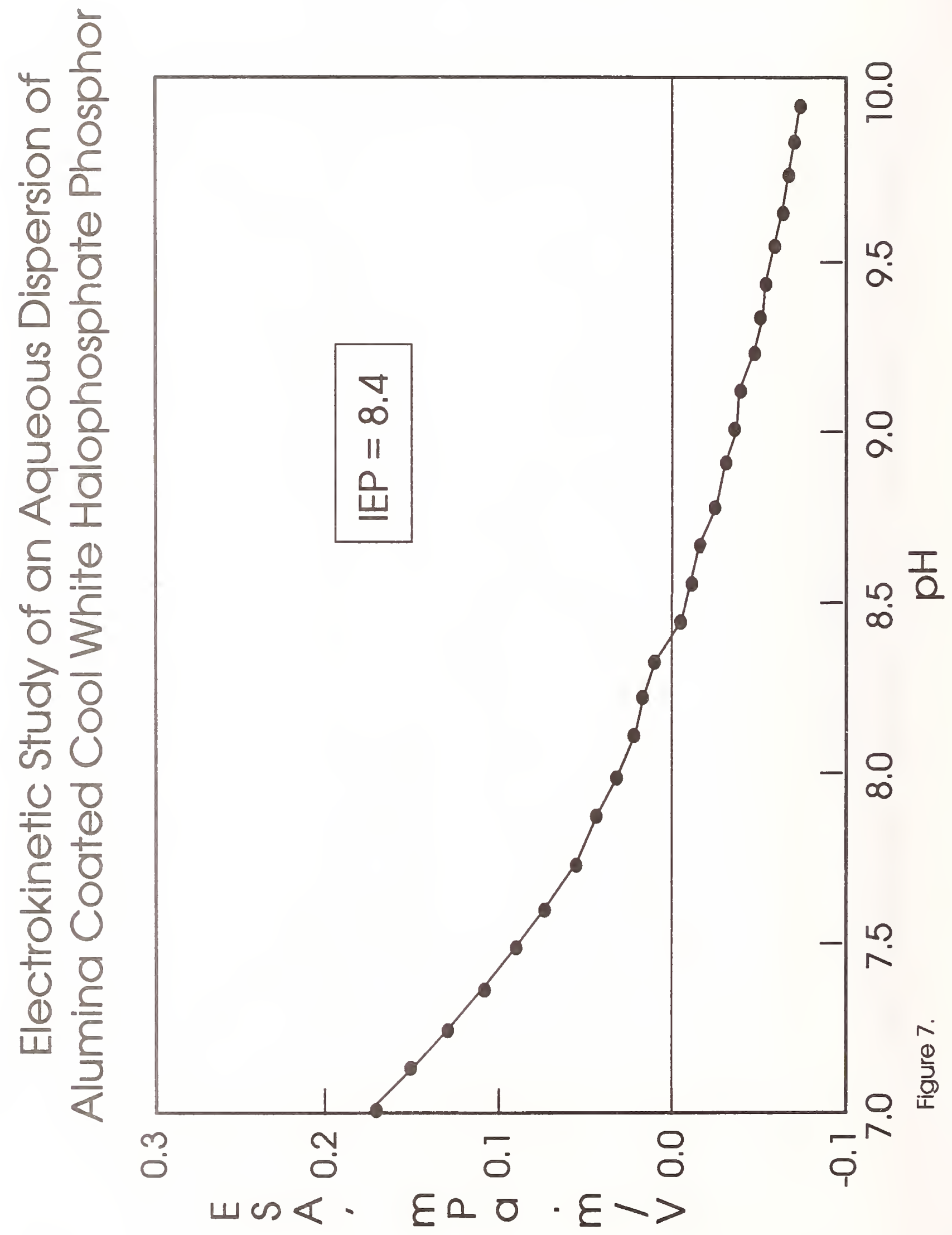




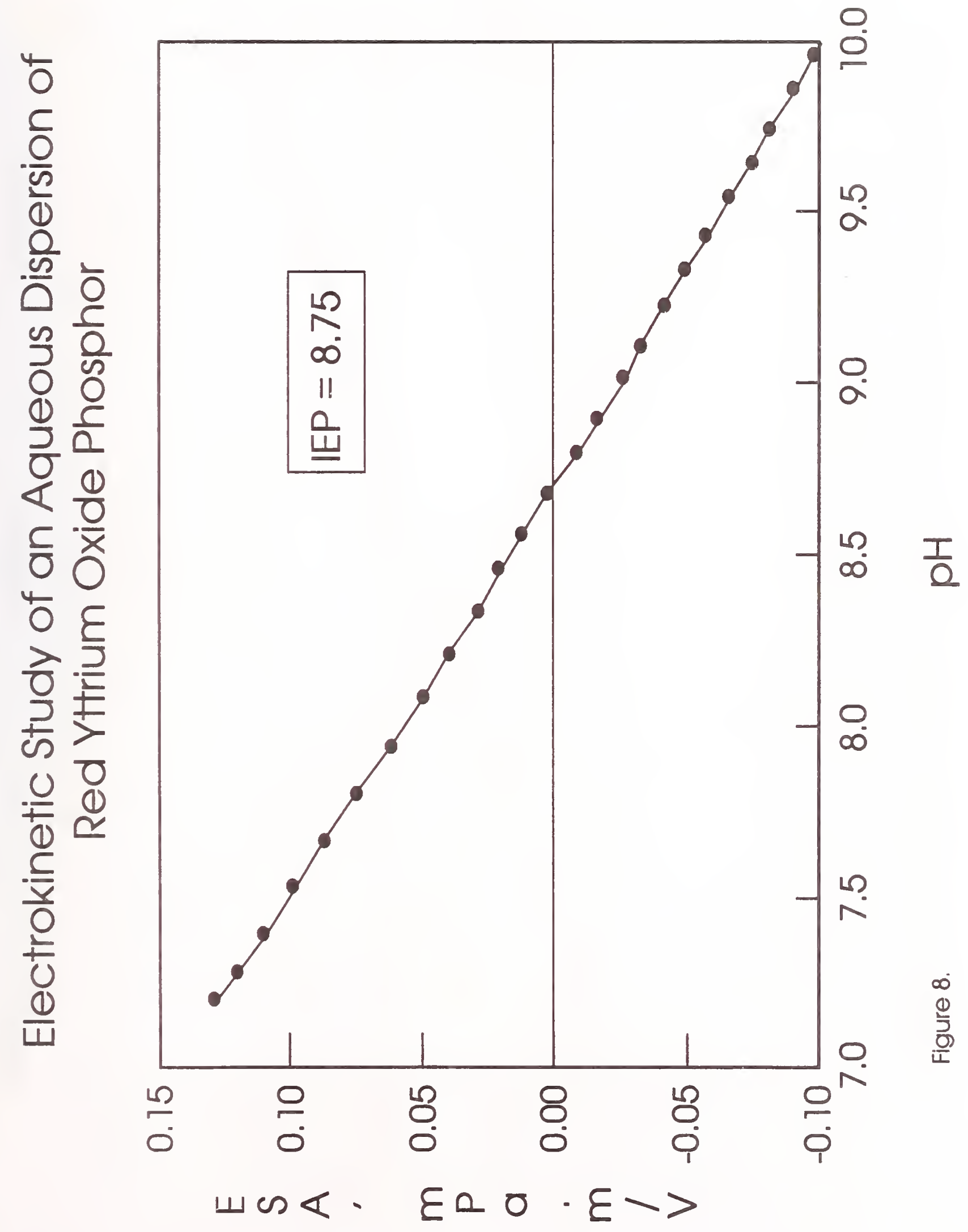




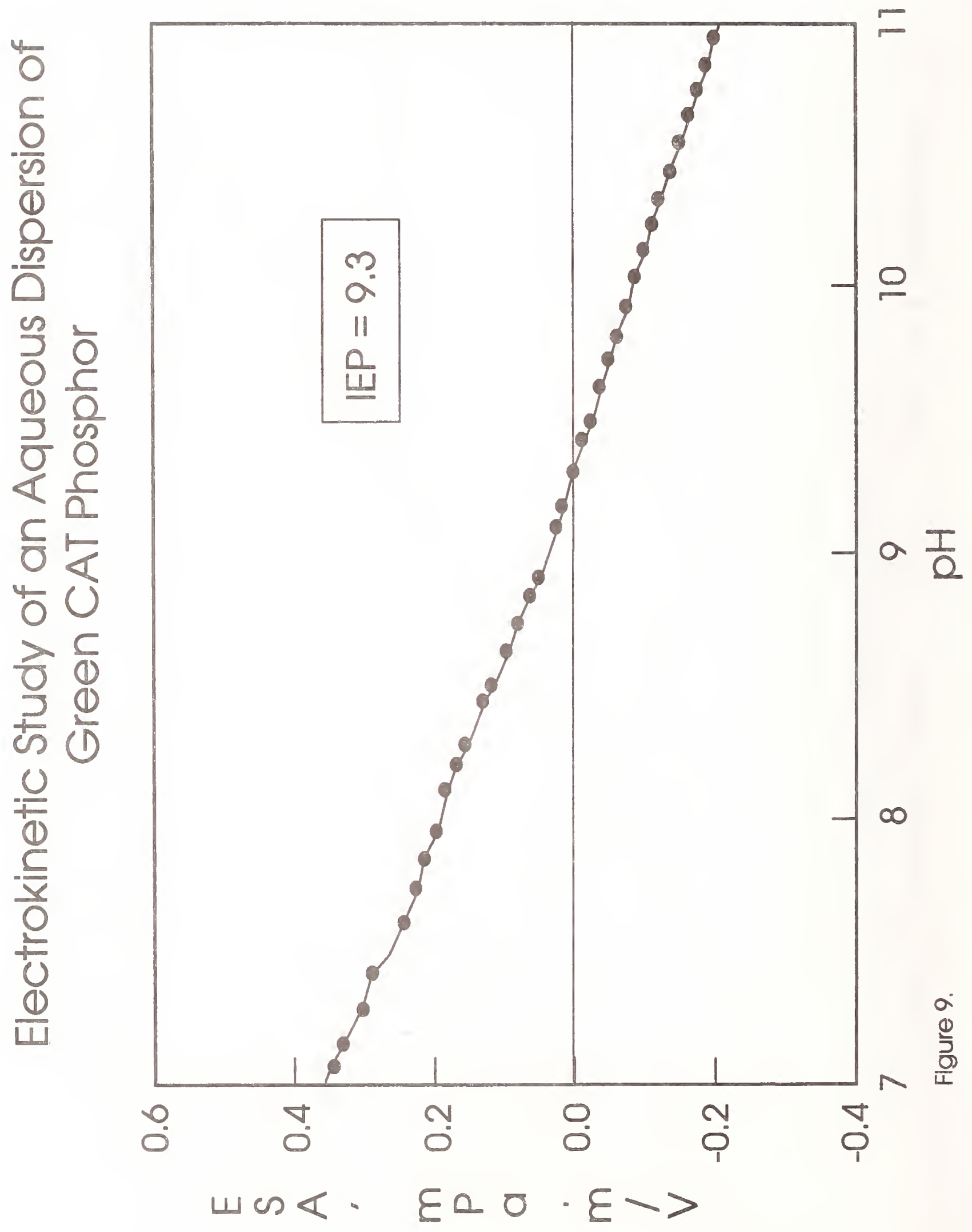




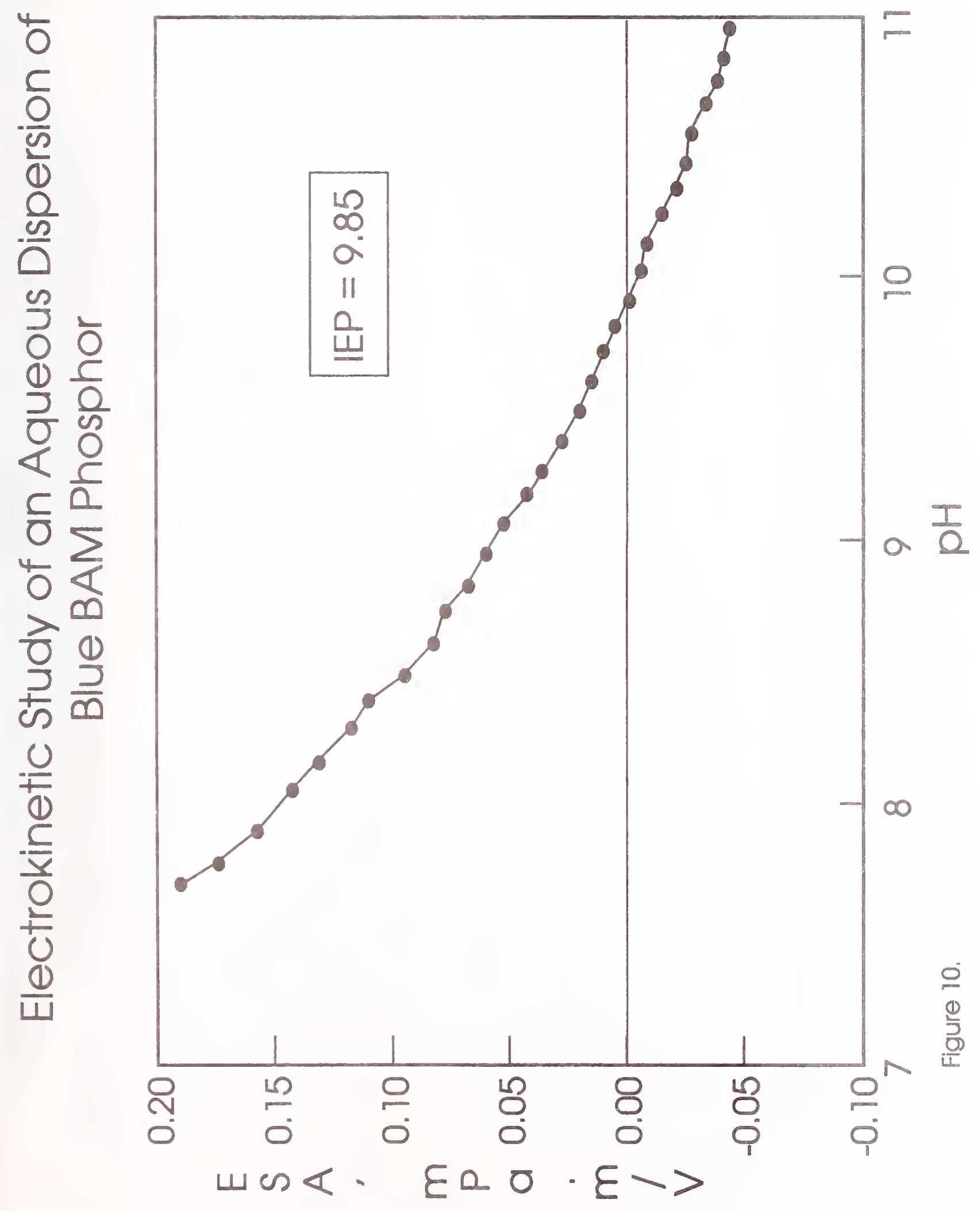




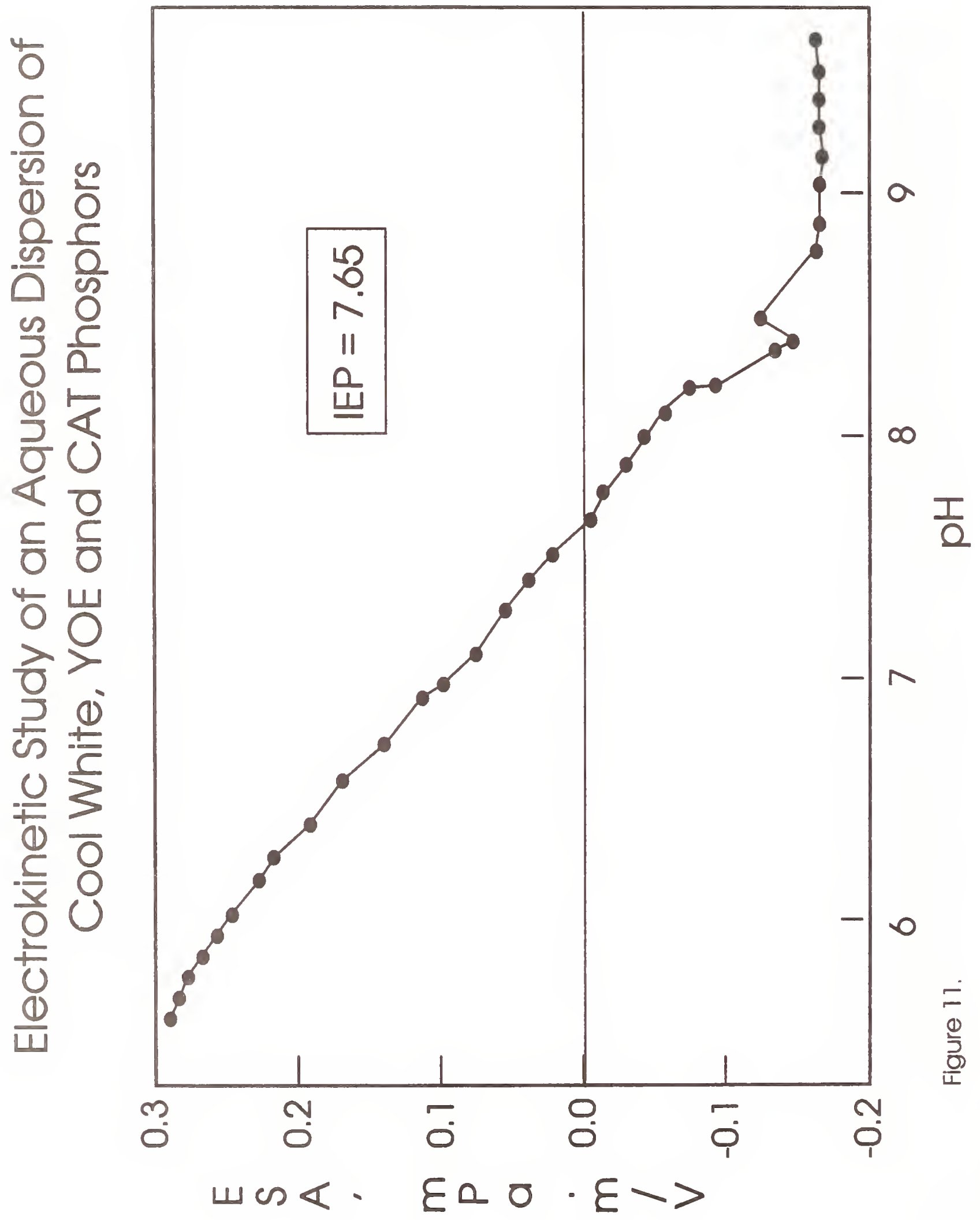




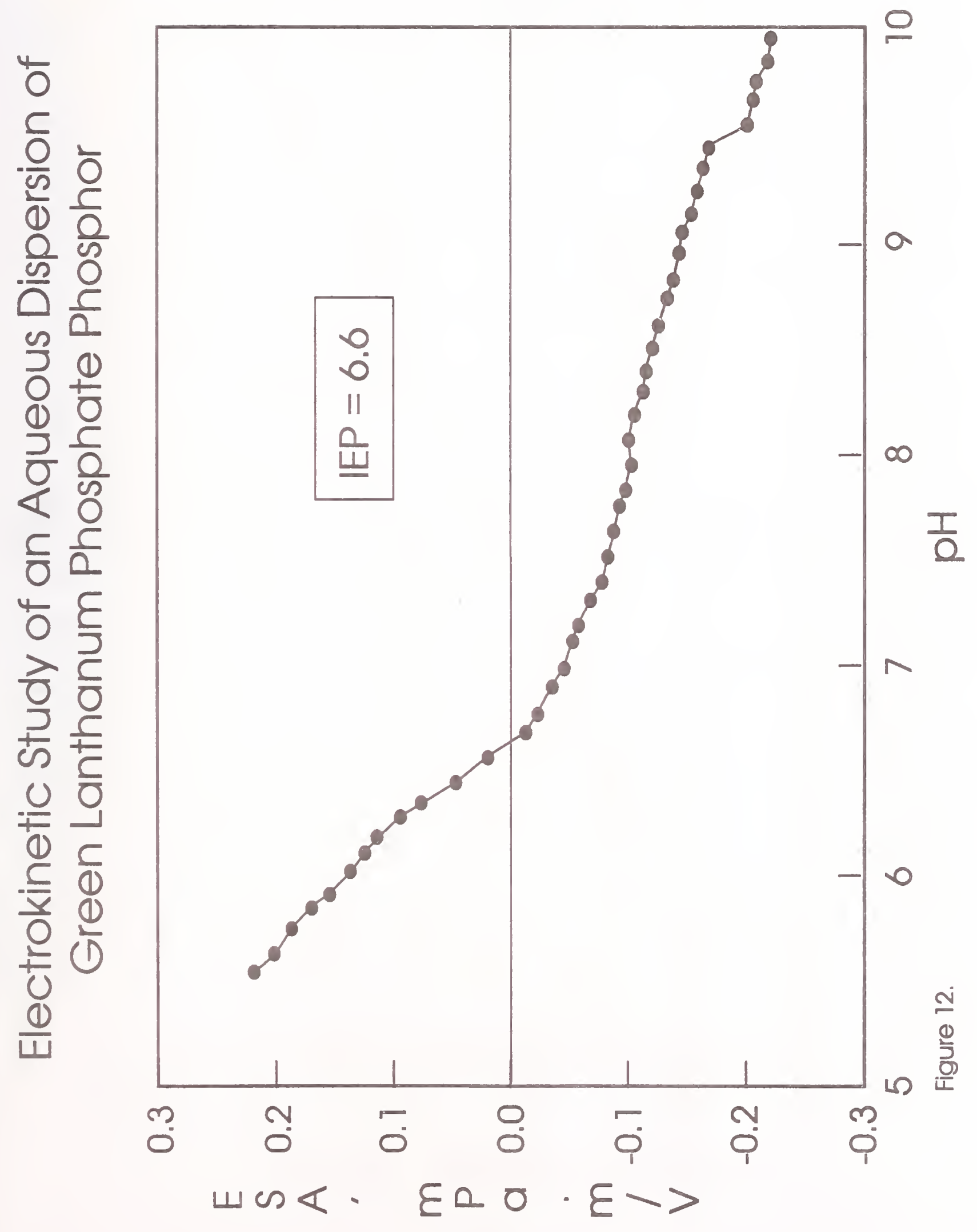




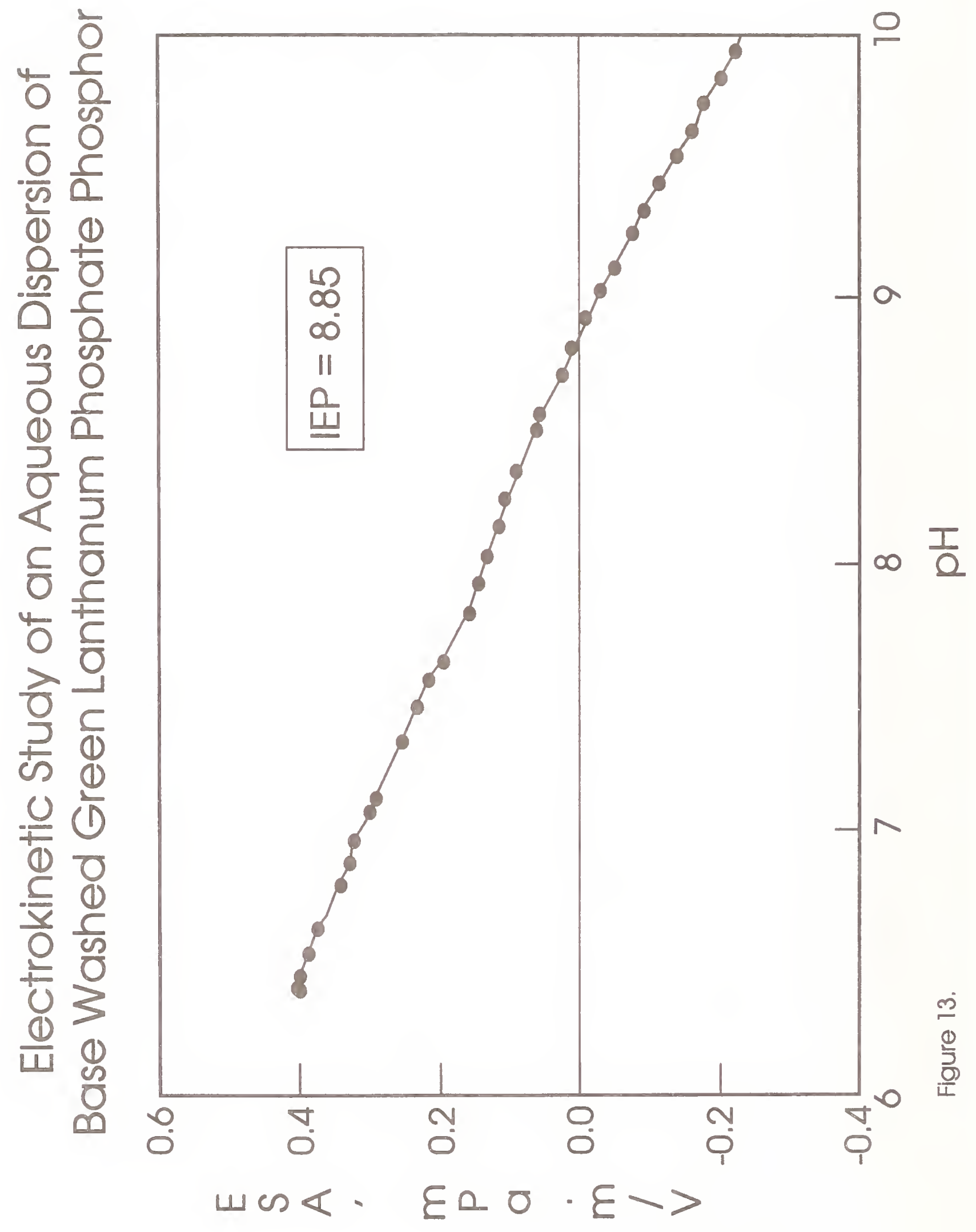




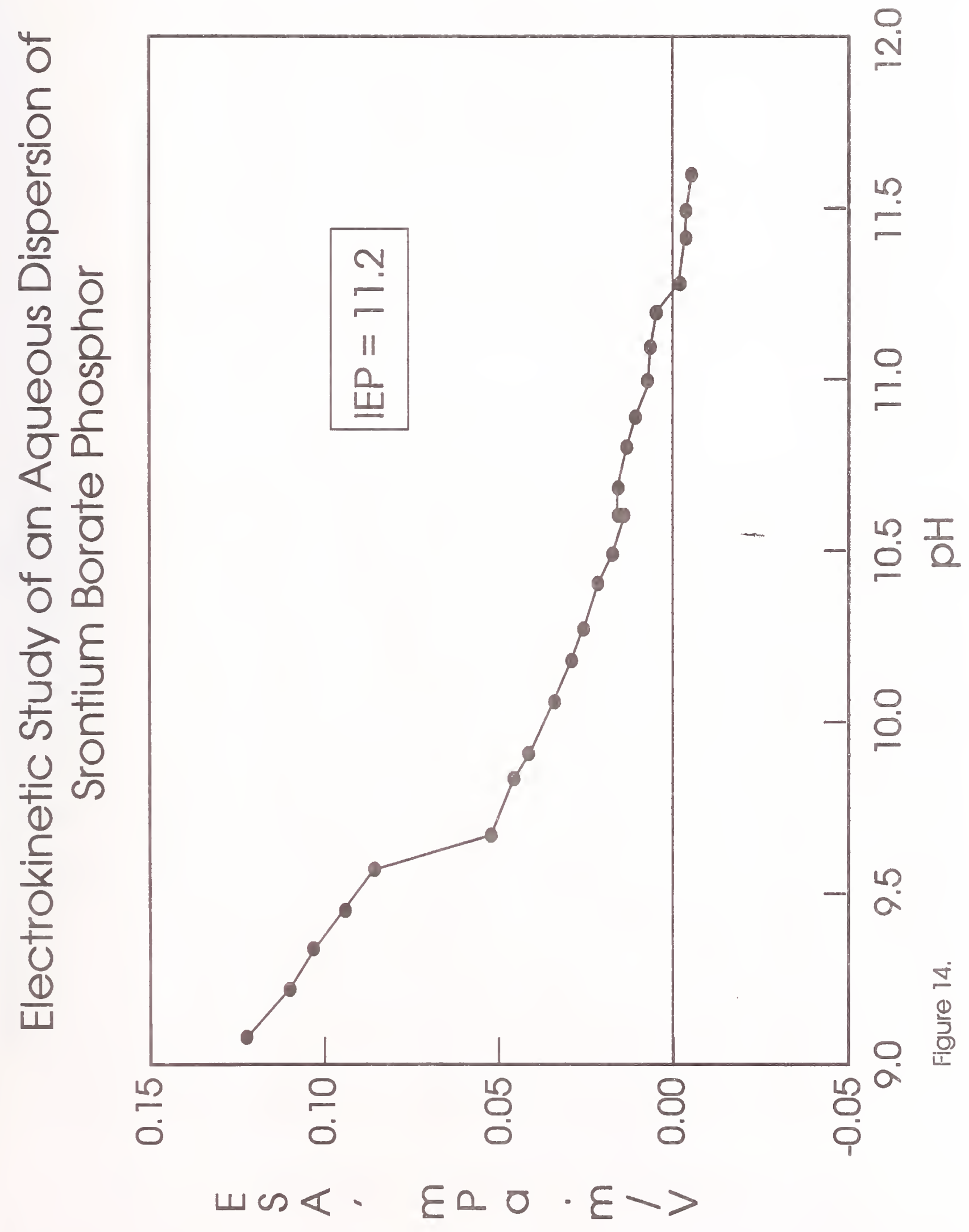




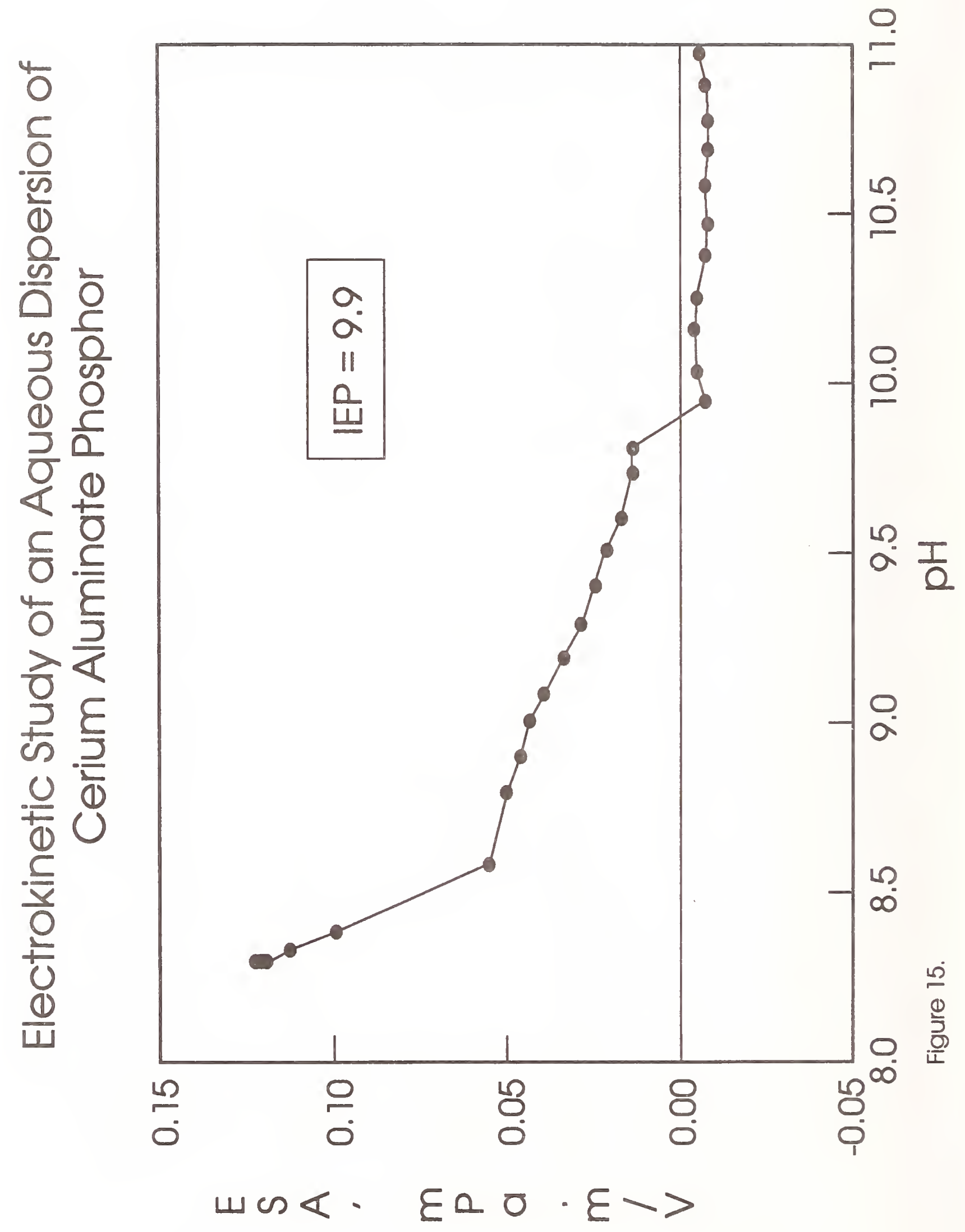




\title{
ADVANCES IN LIOUID TONER CHARGING MECHANISM ELECTROACOUSTIC MEASUREMENT OF LIOUID TONER CHARGE
}

\author{
James R. Larson \\ Webster Research Center \\ Xerox Corporation \\ Webster, NY 14580
}

\begin{abstract}
Methods for determining the electrical properties of liquid toners are critiqued with an emphasis on electroacoustic measurements. Advances in understanding the mechanism are discussed.
\end{abstract}

\section{INTRODUCTION}

Particles dispersed in a hydrocarbon liquid become charged by the addition of a charge director, a hydrocarbon soluble ionic surfactant, which aggregates into inverse micelles in non-polar media. This effect is utilized in liquid electrostatic developers for imagewise particle deposition which enables electrostatic printing. ${ }^{1}$ The charge director micelles ionize to form both positive and negative micelles which can compete with particles of the same sign for image charge during the image development process. Several techniques are available for the measurement of particle charges. These include methods by which particles are electrophoretically developed and the transient current monitored ${ }^{2,3}$, one method where micelle counter ions are stripped from the particles by fluid flow ${ }^{4}$, and particle charge can be related to particle mobility which can be measured by a number of techniques. ${ }^{5-7}$ All of these methods have some utility but their effectiveness will depend on how well they account for all of the charged species in the particle dispersion and other factors which effect charge on the particle. In this report a discussion of the influence of micelle ionization and applied electric fields on the particle charge and its measurement are presented. Electroacoustic methods ${ }^{7}$ are identified as being very effective in assessing particle charges because they operate at relatively large fields and are independent of the charged micelle activity. 


\section{EXPERIMENTAL}

The liquid toner dispersion was prepared by a hot melt blend-cold particle size reduction process which resulted in 1-2 micron polymer particles. The hydrocarbon dispersant used was Isopar L (Exxon Chemical Co., Houston, TX). The charge directors studied were Basic Barium Petronate ${ }^{\circledR}$ and Neutron Barium Petronate ${ }^{\circledR}$ (Witco Corporation, New York, NY). Viscosities were determined with the use of Cannon-Fenske Viscosimeter Tubes (Cannon Instrument Co., State College, PA). The conductivity of the liquid toner dispersions and charge director solutions were determined with a Scientifica 627 Conductivity Meter (Scientifica, Princeton, NJ). The measurement signal for this meter is a low distortion $18 \mathrm{hz}$ sine wave with an amplitude of 5.4 to 5.8 volts rms. Toner particle mobilities were determined with a MBS-8000 electrokinetic sonic analysis (ESA) system (Matec Applied Science, Hopkinton, MA). The system was calibrated in the aqueous mode per manufacturers recommendation to give an ESA signal corresponding to a zeta potential of -26 mv for a $10 \%(\mathrm{v} / \mathrm{v})$ suspension of Ludox-TM (DuPont). The system was then set up for non-aqueous measurements. The electrophoretic mobilities obtained are converted to zeta potentials using O'Brien's method. ${ }^{7}$

\section{PARTICLE CHARGING MECHANISM}

Acid-base interactions between the particles and the charge director micelles, equations (1) and (2), are believed to be an important mechanism for particle charging. A large body of evidence has been published supporting the acid-base mechanism. ${ }^{8-13}$ In the present discussion it is assumed that charging occurs from the transfer of an ion between a site on the particle surface and a micelle. The charging site on the particle may be a strongly acidic or basic site inherent to the particle composition or may be a site where ionic material is adsorbed onto the particle surface. 


\section{Negative Charging Particle}

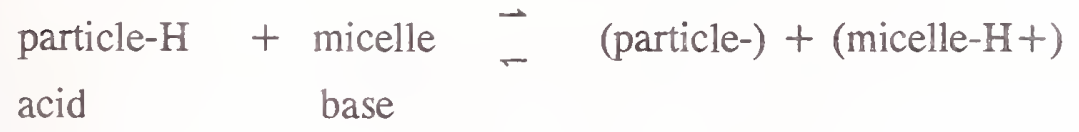

\section{Positive Charging Particle}

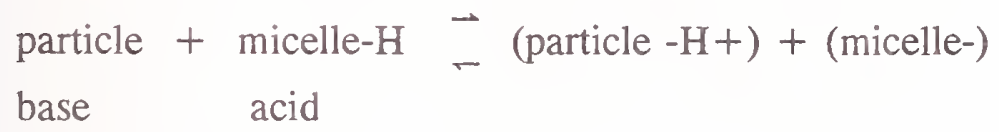

\section{PARTICLE CHARGE AND MICELLE IONIZATION}

Particle charging in a liquid toner system is assumed here to occur by ion exchange between the particle and a charge director micelle. The charge director micelles $\left(\mathrm{M}^{\circ}\right)$ can self ionize to form both positive and negative micelles $(\mathrm{M}+$ and $\mathrm{M}-)$. The micelle charges have long been recognized to compete with particles for image charge in electrostatic printing systems.

The linear relationship between the conductivity of charge director solutions and concentration, as seen in Figure 1, combined with the fact that only a small fraction of the micelles are ionized ${ }^{14}$ suggests that charge director ionization is a second order reversible reaction. This is consistent with the proposed mechanism for the ionization of Aerosol-OT in non-aqueous media ${ }^{15,16}$ and with the increase in conductivity of charge director solutions with increasing temperature that cannot be accounted for by viscosity changes alone. ${ }^{14}$ Toner particle charging has also been proposed to be a reversible second order process involving a charging site on the particle $S^{\circ}$ and a neutral micelle producing a charged site on a particle $(\mathrm{S}-) .{ }^{17}$ These processes are depicted in equations (3) - (6) for negatively charged particles.

In equations (3) - (6): $\left[\mathrm{M}^{0}\right]=$ number of neutral micelles/dispersion volume; $[\mathrm{M}+]=$ number of positively charged micelles/dispersion volume; $[\mathrm{M}-]=$ number of negatively charged micelles/dispersion volume; $\left[\mathrm{S}^{\circ}\right]=$ number of uncharged sites/particle volume and 
Figure1.

Conducsivity of Newtral Barium Potronate in Isopar $L$

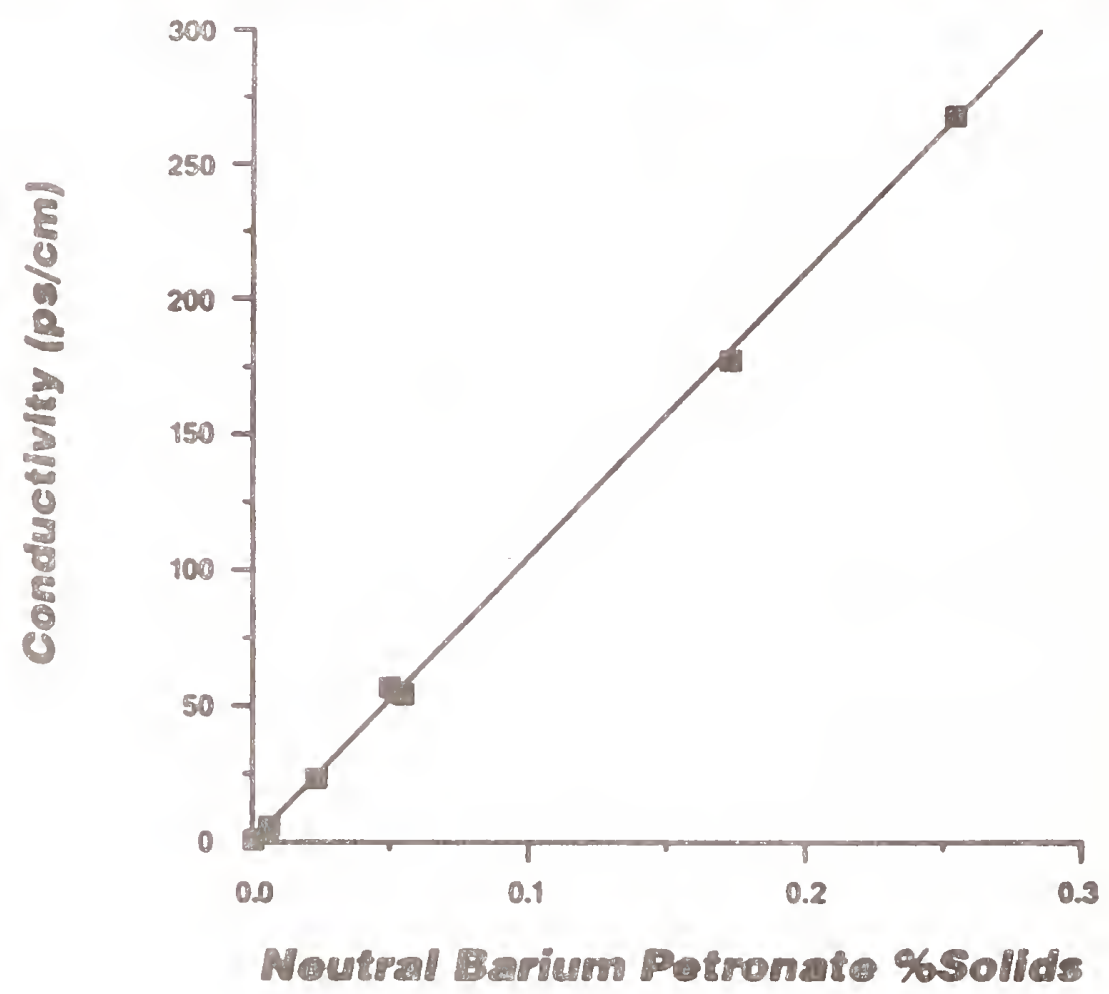

$[\mathrm{S}-]=$ number of negatively charged sites/particle volume. Examination of equations (4) and (6) leads to the conclusion that the level of micelle ionization will be influenced by the presence of the particles and vise versa. The interaction of charging sites on the particles $\left(\mathrm{S}^{\circ}\right)$ and micelles $\left(\mathrm{M}^{\circ}\right)$ forming positive micelles $(\mathrm{M}+)$ will force the micelle ionization equilibrium, equation (4), to the left, i.e., causing a reduction of micelle ionization. This is a significant point as attempts to account for the level of micelle ionization in a particle dispersion often require the measurement of the electrical properties of charge director solutions in the absence of particles and assume that the values obtained will be representative of the micelles in the particle dispersion. 
Micelle Ionization

$$
\begin{aligned}
& \mathrm{M}^{\circ}+\mathrm{M}^{\circ}-\mathrm{M}^{+}+\mathrm{M}^{-} \\
& \mathrm{K}_{\mathrm{i}}=\left[\mathrm{M}^{+}\right]\left[\mathrm{M}^{-}\right] /\left[\mathrm{M}^{\circ}\right]\left[\mathrm{M}^{\circ}\right]
\end{aligned}
$$

Particle Charging

$$
\begin{aligned}
& \mathrm{S}^{\circ}+\mathrm{M}^{\circ} \rightarrow \mathrm{M}^{+}+\mathrm{S}^{-} \\
& \mathrm{K}_{\mathrm{q}}=\left[\mathrm{M}^{+}\right]\left[\mathrm{S}^{-}\right] /\left[\mathrm{S}^{\circ}\right]\left[\mathrm{M}^{\circ}\right]
\end{aligned}
$$

\section{TEMPERATURE DEPENDENCE OF PARTICLE CHARGING}

\section{AND MICELLE IONIZATION}

Support for the conclusion that micelle ionization and toner charging cannot be dealt with separately is obtained by examining the effect of temperature on the two processes. The ionization of micelles in charge director solutions is governed by equation (4) and is expected to increase as $\mathrm{K}_{\mathrm{i}}$ increases. The effect of temperature on micelle ionization has been studied and $\mathrm{K}_{\mathrm{i}}$ has been found to increase with temperature as expected for an endothermic processes. ${ }^{17}$ An example of this is found in Figure 2 where it is shown that the temperature dependence of conductivity of charge director solutions is greater than the temperature dependence of the viscosity.

The effect of temperature on particle charging has also been studied. ${ }^{17}$ If particle charging and micelle ionization are independent then the effect of temperature on particle charging could be predicted by examination of equation (6) alone. In this case it would be reasonable to expect that $\mathrm{K}_{\mathrm{q}}$ would behave similarly to $\mathrm{K}_{\mathrm{i}}$ and that particle charging would increase with increasing temperature. However, if micelle ionization and particle charging are coupled then the expected effect of temperature on particle charging will be more complex.

The examination of the effect of temperature on particle charging is simplified by assuming that there is sufficient charge director concentration so that $\left[\mathrm{M}^{+}\right] \simeq\left[\mathrm{M}^{-}\right]$. Without particles $\left[\mathrm{M}^{+}\right]=\left[\mathrm{M}^{-}\right]$, however, with particles $\left[\mathrm{M}^{+}\right]>\left[\mathrm{M}^{-}\right]$. Under conditions of high charge director concentration $\left[\mathrm{M}^{+}\right] \simeq\left[\mathrm{M}^{-}\right]$:

$$
\text { then }\left[\mathrm{S}^{-}\right] /\left[\mathrm{S}^{0}\right] \simeq \mathrm{K}_{\mathrm{q}} / \mathrm{K}_{\mathrm{i}}
$$


Figure 2.

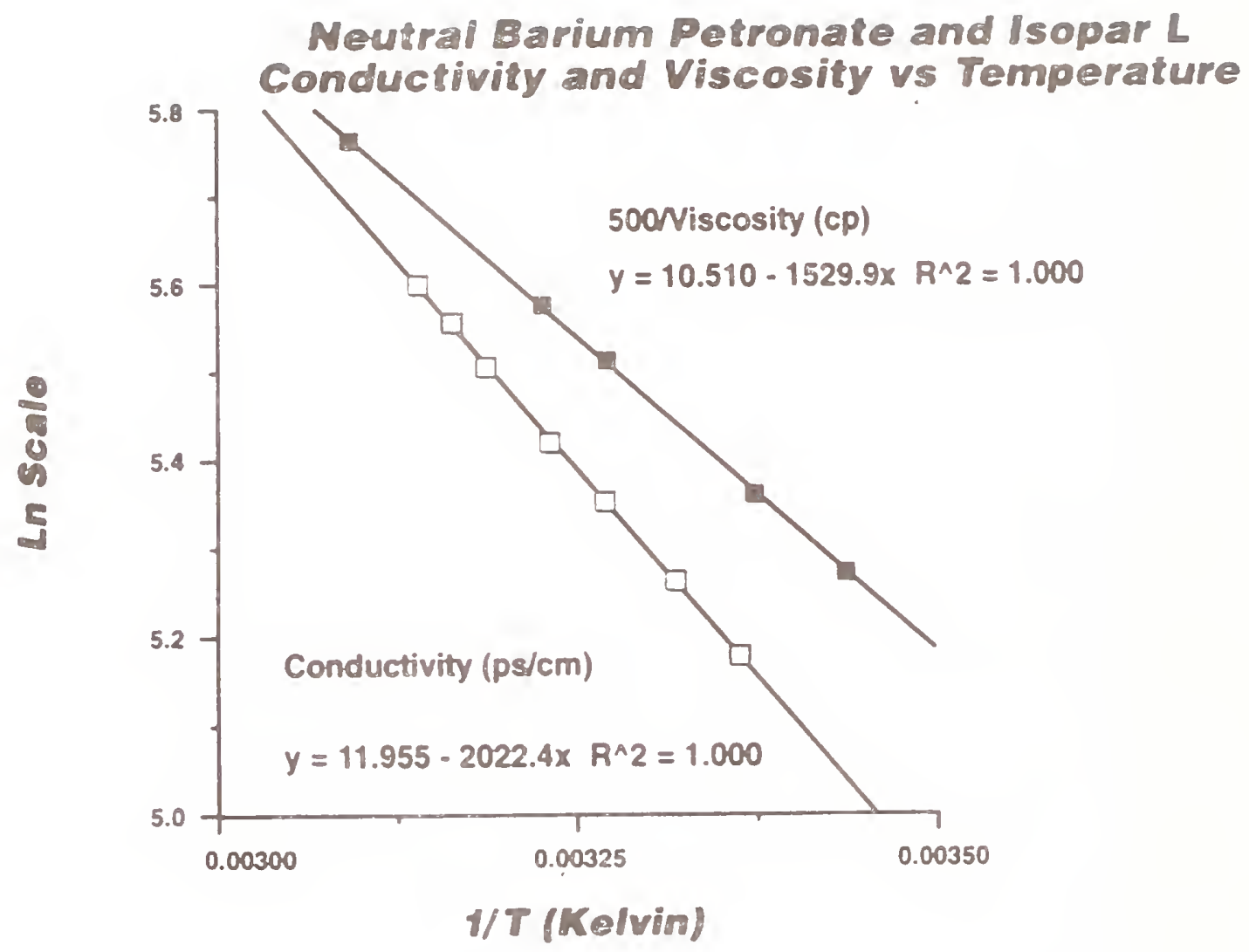

Taking $[\mathrm{S}]$ to be the concentration of surface sites, both charged and uncharged, and $\theta$ to be the fraction of surface sites that are charged.

$$
\left[\mathrm{S}^{-}\right] /\left[\mathrm{S}^{\circ}\right]=\left[\mathrm{S}^{-}\right] /\left(\left[\mathrm{S}^{-}-\left[\mathrm{S}^{-}\right]\right)=\theta /(1-\theta)\right.
$$

Combining [7] and [8] and rearranging gives

$$
\theta=\mathrm{K}_{\mathrm{q}} /\left(\mathrm{K}_{\mathrm{q}}+\sqrt{ } \mathrm{K}_{\mathrm{i}}\right)
$$

Examination of equation (9) leads to the conclusion that regardless of the relative magnitudes of $\mathrm{K}_{\mathrm{q}}$ and $\mathrm{K}_{\mathrm{i}}$ that the effect of temperature on particle charging will be moderated by the fact that the rate of increase of $\mathrm{K}_{\mathrm{q}}$ with temperature will likely be similar to that of $\mathrm{K}_{\mathrm{q}}+\sqrt{ } \mathrm{K}_{\mathrm{i}}$. 
Particle charging could increase or decrease with temperature depending on the relative temperature effects on $\mathrm{K}_{\mathrm{q}}$ and $\sqrt{ } \mathrm{K}_{\mathrm{i}}$.

This expectation is consistent with the experimental evidence available. Kohler, et.al., ${ }^{18}$ reported that the temperature dependence of particle surface charge for a liquid toner dispersion to be relatively invariant with temperature between $10^{\circ} \mathrm{C}$ and $50^{\circ} \mathrm{C}$. The zeta potential and charge/mass ratio of liquid toners using two different barium petroleum sulfonates as charged directors were measured by electroacoustic methods (ESA) and found to be relatively independent of temperature compared to the temperature dependence of the conductivity and micelle ion concentration found for the charge director solutions alone. ${ }^{17}$ This data is presented in Figures 3-4.

Figure 3.

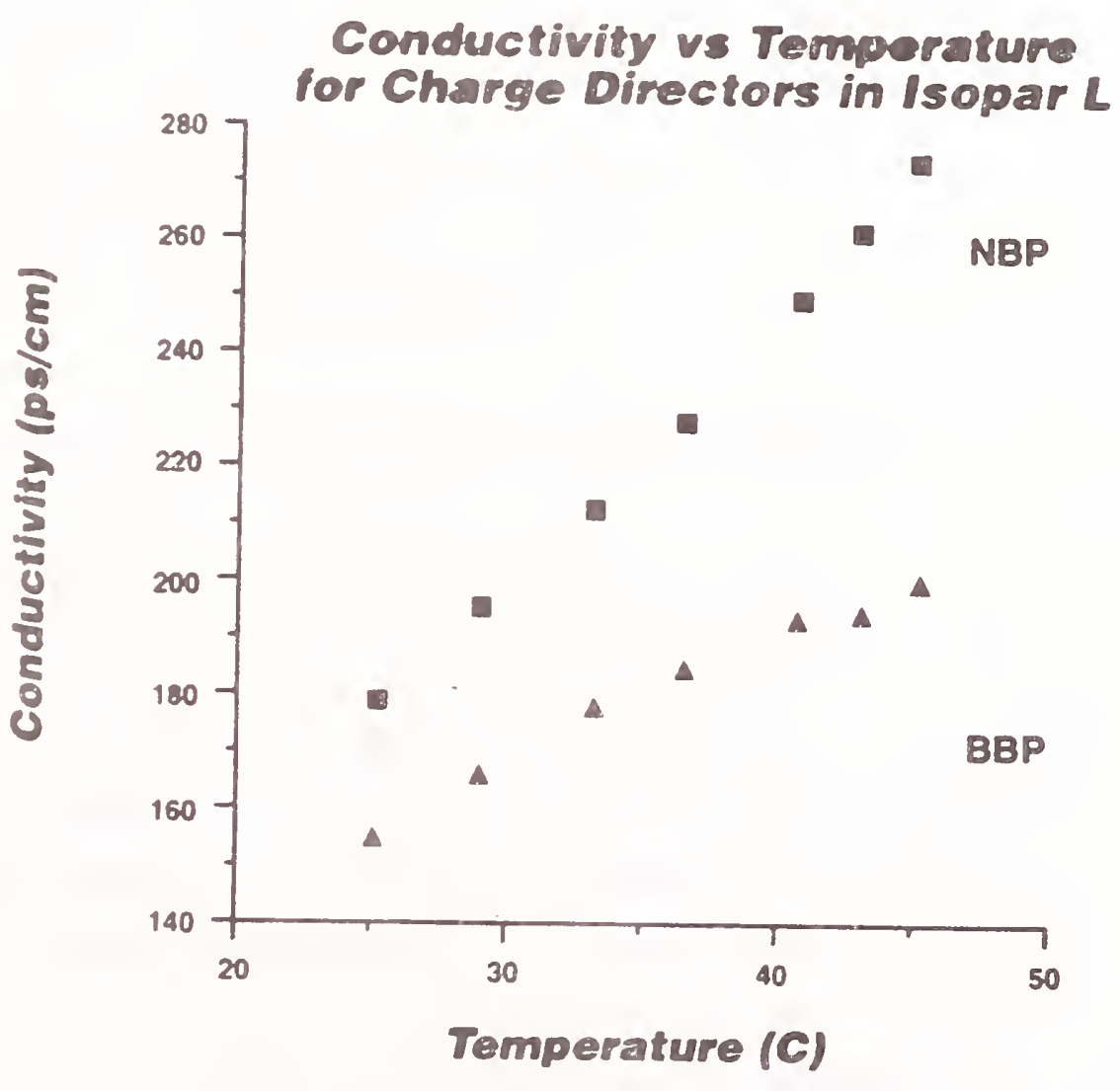


Figure 4.

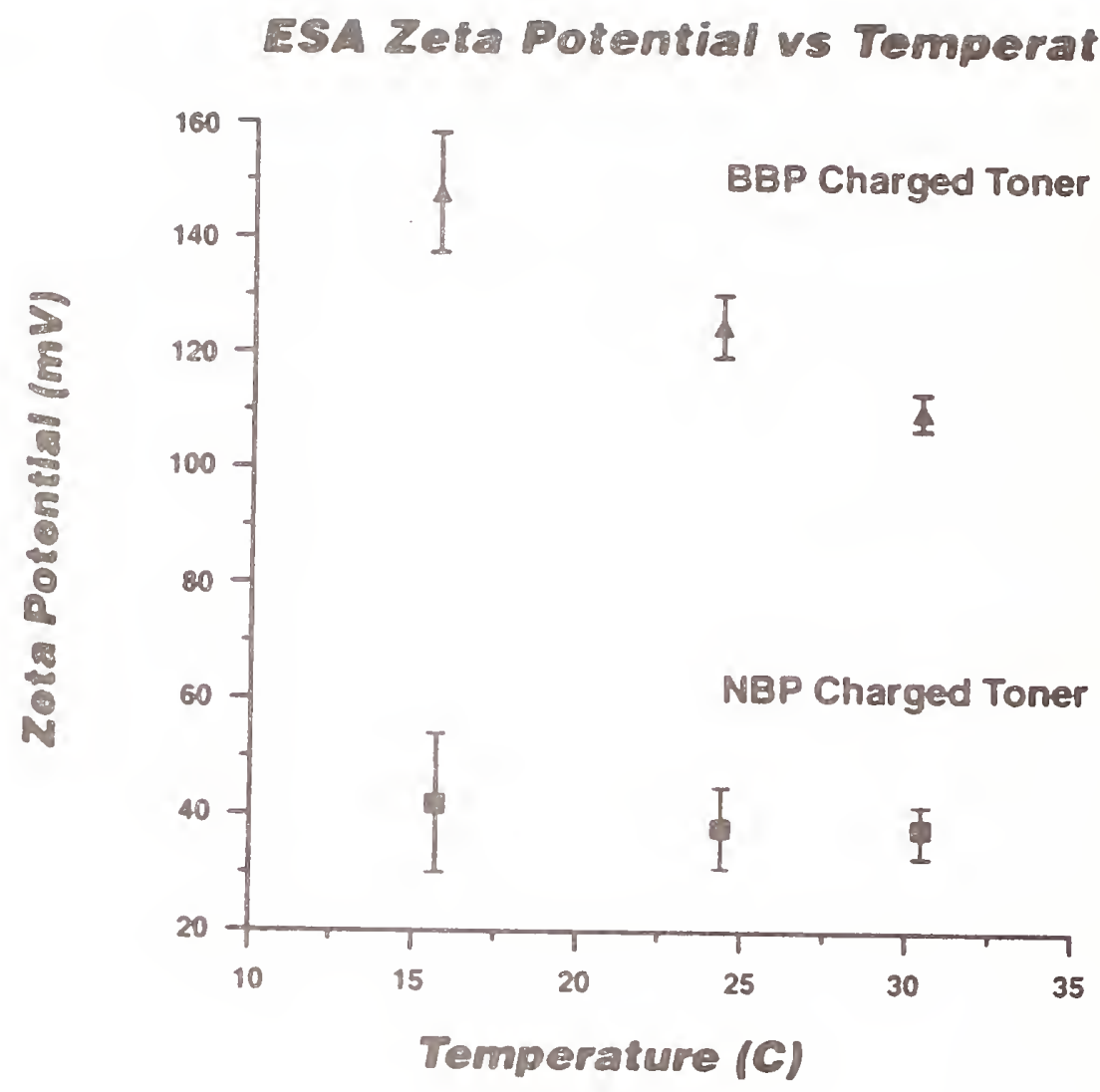

FIELD DEPENDENCE OF PARTICLE CHARGING

Particle charges can be calculated from the electrophoretic mobility of the particles with a knowledge of the particle size and the viscosity of the fluid medium. However, it has been observed that the particle electrophoretic mobilities are not constant with applied electric field as would be expected for particles of constant charge. The field dependence of mobility was first reported by Stotz ${ }^{19}$ for polystyrene spheres dispersed in an aliphatic hydrocarbon. Mobility increases as great as 10 fold were reported for field variations in the range of 10 to $1000 \mathrm{~V} / \mathrm{mm}$. Similar phenomena were observed by Novotny $\mathrm{y}^{20,21}$ for titanium dioxide dispersed in p-xylene where a four fold increase in the mobility was observed when the field was increased from $100 \mathrm{~V} / \mathrm{mm}$ to $1000 \mathrm{~V} / \mathrm{mm}$. This effect has also been reported for liquid toner dispersions by Kohler ${ }^{18}$, Niv ${ }^{6}$, and Larson. ${ }^{22}$ 
The increase in particle charge is explained by the stripping of counter charges from the double layer. As the double layer is removed the field dependence is expected to decrease and eventually at very high electric fields the mobility is expected to be field independent. ${ }^{19}$ Field independent mobility has been observed at high fields in a number of cases..$^{6,18,19}$

\section{MICELLE IONIZATION AND FIELD DEPENDENT CHARGING: CHARGE MEASUREMENT METHODS}

A common approach to measure the charge on particle dispersions of the type being discussed here is to electrophoretically develop the particles in a parallel cell arrangement and measure the mass developed and calculate the charge deposited by monitoring the transient electrical current. ${ }^{2,3}$ This method is effective in determining the total number of charges in the system, charges obtained from both particle charging and micelle ionization, relative to the mass of the particles.

However, if the interest is in the charge on the particles then the charges from micelle ionization and those from particle charging must be separated. This is traditionally done by measuring the charges in the charge director solution in the absence of particles and then subtracting that from the total charge measured for the particle dispersion. It is clear from the discussion above that this will lead to an incorrect evaluation as the level of micelle ionization in the particle dispersion is moderated by the presence of particles and will be over estimated by the measurement in the absence of particles.

A novel approach to obtaining the particle charge independent of the micelle charges was recently reported. ${ }^{4}$ This method obtains the charge on a given mass of particles by removing the micelle counter ions on the particles by filtration and having the filtrate flow into a Faraday cup. The measured current is caused only by the charges associated with the particles as the positive and negative charges caused by micelle ionization are equal in number and cancel.

Particle charge can also be obtained from measurement of the electrophoretic mobility of the particles. With the mobility and a knowledge of the particle size and fluid viscosity the particle charge can be calculated. ${ }^{5-7,23}$ However, these methods often empty electric fields 
which are well below the fields used in particle application. The mobilities measured under these conditions and the particle charge calculated from the mobility may not be representative of the real system. Methods to determine particle mobilities at higher fields have been reported. One method which utilizes electroacoustic effect (ESA) has proven particularly effective.

\section{MEASUREMENT OF LIQUID TONER PARTICLE MOBILITY USING ESA}

ESA has been used by a number of workers studying the charging mechanism of liquid electrostatic toners. ${ }^{13,17,22,24-27}$ In several cases the ESA measurement was found to correlate well with liquid toner performance. ${ }^{22,24}$

In one study it was demonstrated that the improved image quality that is produced by the addition of amino alcohol compounds to the formulation is due to a higher toner particle negative charge as measured by ESA. ${ }^{24}$ The aminoalcohols were seen to be more effective than the corresponding amines and even amines that were considerably more basic. These results conflict with a simple acid-base picture of liquid toner particle charging. It was proposed that the improved charging produced by the aminoalcohols is due to improved solubility of the counter ion in the micelle. It was also shown in a surface IR study that the effect of the amino alcohols is moderated by their absorption onto the particle surface. The effect of adsorption onto the surface of particles was also demonstrated in the case of acidic additives. ${ }^{13,22,25}$ Again ESA measurements were critical as they showed which additives were most effective in increasing the particle charge and this data was correlated to the tendency of the additives to adsorb onto the particle surface or remain in the hydrocarbon phase. In one $\operatorname{case}^{22}$ work also included a study of the effect of charge director concentration and the acid additive concentration on liquid toner conductivity and ESA mobility. These studies revealed that there is a threshold level of charge director required to obtain the maximum mobility and that the mobility of the particles is essentially independent of conductivity and charge director concentration above that threshold level.

In other studies the ESA has been used to explore the use of metal carboxylates as charge directors and aluminum complexes as charge additives to the toner particles..$^{26,27}$ 
It is clear from an examination of this work that the ESA measurement is an effective method for the assessment of liquid toner particle charge and can be used to discern the details of the charging mechanism and to facilitate the selection of materials to improve toner performance.

\section{SUMMARY}

A model for micelle ionization and particle charging was presented which shows these phenomena to be inextricably coupled. The observed increase for micelle ionization with temperature, in the absence of particles, compared to the insensitivity of particle charges to temperature is seen as support for the model. This phenomenon and the increase in particle charge with increasing field impacts the reliability of methods to evaluate particle charge. Particle charge is best measured by a method which is independent of the charged micelle activity and which is compatible with high fields. ESA mobility is such a method. A literature review of the use of ESA for the study of liquid toner particles showed that this method has been successful for determining particle mobilities.

\section{ACKNOWLEDGMENTS}

The author would like to thank Ian D. Morrison for his collaboration and many discussions on the ionization of micelles and temperature effects on particle charging. The assistance of Terri S. Robinson is also acknowledged. 


\section{REFERENCES}

1. For a review see: S. P. Schmidt, J, R. Larson, and R. Bhattacharya, "Liquid Toner Technology," in Handbook of Imaging Materials, ediced by A. Diamond, Marcel Dekker, New York, pp. 227-252, 1991.

2. P. E. Staples, S. A. Koop, Proceedings of IST's $7^{\text {th }}$ International Congress on NonImpact Printing Technologies, Vol. 1, IS\&T, Springfield, VA, pp. 561-569, 1991.

3. V. Novotny and M. L. Hair, Journal of Colloid and Interface Science, 71, pp. 273-282, 1979.

4. I. D. Morrison, A. G. Thomas, and C. J. Tarnawskyj, Langmuir, 7, pp. 2847-2852, 1991.

5. R. E. Kornbrekke, I. D. Morrison, and T. Oja, Langmuir, 8, pp. 1211-1217, 1992.

6. Y. Niv, Y. Adam, and Y. Krumberg, Abstracts of IST's $3^{\text {rd }}$ International Congress on Non-Impact Printing Technologies, IS\&T, Springfield, VA, pp. 57-60, 1986.

7. T. Oja, G. L. Peterson, and D. W. Cannon, U.S. Patent 4,497,208, 1985; R. W. O'Brien, J. Fluid Mech., 190, pp. 71-86 (1988).

8. F. M. Fowkes, H. Jinnai, M. A. Mostafa, F. W. Anderson, and R. J. Moore, Colloids and Surfaces in Reprographic Technology, M. Hair and M. D. Croucher, eds., Am. Chem. Soc., Washington, D.C., 1982.

9 M. D. Croucher, S. Drappel, J. Duff, K. Lok, and R. W. Wong, Colloids and Surfaces, 11, pp. 303-322, 1984. 
10. F. M. Fowkes, H. Jinnai, M. A. Mostafa, F. W. Anderson, and R. J. Moore, ACS Symposium Series, No. 200, pp. 282-306, 1984.

11. J. R. Larson, The $4^{\text {th }}$ International Congress on Advances in Non-Impact Printing, IS\&T, pp. 142-145, 1988.

12. G. A. Gibson and R. H. Luebbe, The $7^{\text {th }}$ International Congress on Advances in NonImpact Printing Technologies, IS\&T, pp. 533-550, 1991.

13. K. A. Pearlstine and J. R. Swanson, Journal of Colloid and Interface Science. 151, pp. 343-350, 1992.

14. T. G. Davis, G. A. Gibson, R. H. Luebbe, and K. Yu, Proceedings of the $5^{\text {th }}$ International Congress on Advances in Non-Impact Printing Technologies, IS\&T, p. 404, 1989.

15. H. F. Eicke and V. Arnold, Journal of Colloid and Interface Science, 46, p. 101, 1974.

16. Z. Randriamala, A. Denat, J. P. Gosse, and B. Gosse, Transactions on Electrical Insulation, 20, p. 167, 1985.

17. J. R. Larson, I. D. Morrison, and T. S. Robinson, The $8^{\text {th }}$ International Congress on Advances in Non-Impact Printing Technologies, IS\&T, pp. 193-197, 1992.

18. R. Kohler, D. Giglberger, and F. Bestenreiner, Society of Photographic Scientists and Engineers Journal, 22, pp. 218-227, 1978.

19. S. Stotz, Journal of Colloid and Interface Science, 65, pp. 118-130, 1978.

20. V. Novotny, Journal of Applied Physics, 50, pp. 324-332, 1979. 
21. V. Novotny, Journal of Applied Physics, 50, pp. 2782-2794, 1979.

22. J. R. Larson, W. A. Houle, K. A. Pearlstine, J. R. Swanson, L. A. Page, T. J. Trout, L. El-Sayed, The $6^{\text {th }}$ International Congress on Advances in Non-Impact Printing Technologies, IS\&T, pp. 585-597, 1990.

23. I. D. Morrison and C. J. Tarnawskyj, Langmuir, 7, pp. 2358-2361, 1991.

24. J. R. Larson, G. A. Lane, J. R. Swanson, T. J. Trout, and L. El-Sayed, Journal of Imaging Technology, 17, pp. 210-214 (1991).

25. K. A. Pearlstine and L. Page, Journal of Imaging Science, 35, pp. 55-58, (1991).

26. K. A. Pearlstine, Journal of Imaging Science, 35, pp. 326-329, (1991).

27. G. A. Lane, Hard Copy and Printing Materials, Media, and Processes, SPIE, Vol. 1253, p. 29 (1990). 


\title{
SESSION VI
}

\section{SUBGROUPS DISCUSSIONS}

\author{
AND
}

\section{IDENTIFICATION OF ISSUES}





\section{SESSION VI - SUBGROUPS DISCUSSIONS AND IDENTIFICATION OF ISSUES}

This section contains a summary of problems and research needs. Technical problems/issues were discussed and research opportunities were identified during the workshop under five major areas: 1) non-aqueous ESA measurements, 2) measurement considerations, 3) coatings, 4) coal, cements and clays, 5) fundamentals. The discussion leaders summaries are presented below.

\section{NON-AQUEOUS ESA MEASUREMENTS \\ CONDUCTIVITY $<20,000 \mathrm{ps} / \mathrm{cm}$}

Discussion Leader: J. R. Larson, Xerox Corporation

1. Calibration Standard

Dispersion of carbon black and Lubrizol charging additive in dodecane would be supplied to non-aqueous users for ESA measurement. A comparison of these results (available to all participants) would establish the reliability of the aqueous calibration (with Ludox) for non-aqueous measurements. This effort may eventually result in a primary calibration standard for non-aqueous ESA measurements.

2. Mixing Effects On ESA Measurements

It was noted that in some cases the rate of mixing during measurements effects the magnitude of the ESA signal. It is recommended that the exact mixing process be described when reporting results so that experiments could be reproduced.

\section{Needed Software Changes}

Current software is inadequate for temperature correction for viscosity and density and requires that some experiments (time-series runs) be discontinuted if recalibration is required. It was also noted that the system shuts down if a viscosity value greater than 25 cps is entered into the software.

\section{Non-aqueous Acoustosizer}

Great interest was expressed for an Acoustosizer for non-aqueous measurements. It was thought this might be available in 3-5 years. 


\section{Measurement Of Small Samples}

It was noted that small samples of $20.30 \mathrm{~cm}^{3}$ (too small for the probe) could be measured with the flow through cell.

\section{Temperature Resistant Probe}

A probe that can withstand temperature up to $150^{\circ} \mathrm{C}$ is being developed. The current probe should not be heated above $35^{\circ} \mathrm{C}$. The high temperature probe is expected to be available in 1994.

\section{MEASUREMENT CONSIDERATIONS}

Discussion Leader: V. A. Hackley, NIST

This group discussed various factors, problems, needs and limitations regarding the electroacoustic measurement of aqueous systems. The following summary is based on these discussions.

\section{A. Calibration Standard}

The general consensus of the group was that there is a great need for a generally accepted calibration standard for determining the necessary instrument constant for conversion of esa measurements in aqueous suspensions and for user related needs. The suggestion was made that possibly a primary standard could be developed by NIST, for instance, and dispersed to the instrument companies, while a secondary standard would be calibrated against the primary and dispersed for general application by end users.

Next we discussed the desired properties for a primary standard. The following is a list of these properties, where an asterisk indicates those properties also required for a secondary standard.

Spherical

Monodisperse (or relatively narrow distribution)

Non porous*

Chemical stability* (should be insoluble, surface stabilized, etc.)

Kinetic stability* (easily dispersed and not readily flocculated)

Significant density difference, $\Delta \rho^{*}$

High surface charge density*

Low inertial effect, $\mathrm{G}(\omega a / v)$ (this means that the particle size must be below about $0.5 \mu \mathrm{m}$ so that $\omega a / \nu$ is small) 
In addition to these attributes listed above, we thought that it may be desirable to have a standard that showed a well-characterized $\mathrm{pH}$ dependence, while $\mathrm{pH}$-independence is desirable for calibration of the instrument constant. Another consideration was that the standards could be chosen to be compatible with other (dilute) techniques such as microelectrophoresis. The Geltec silica currently used by Matec may be acceptable as a primary standard material, although long-term availability is a question.

\section{B. Measurement Procedures}

1. Analysis and Interpretation of Multicomponent Systems.

There appears to be a lot of uncertainty involved with the interpretation of suspensions containing more than one phase. We feel that more work is needed in this area, including a general protocol for carrying out such measurements.

\section{Aggregation Effects}

The influence of changes in the effective mass of particles due to aggregation is not clear. How might aggregation effect the ESA signal and determination of mobility or zeta potential?

\section{Temperature Effects on Phase Angle}

In the new Matec system, where the accurate determination of phase angle is necessary for measurement of size, what are the potential problems associated with temperature fluctuations?

4. Development of improved sample handling and mixing for ESA measurement of highly loaded suspensions. Some problems with mixing have been encountered. The concept of a continuous flow measurement system is also of general interest.

5. Development of on-line capabilities for quality control and intelligent processing of materials. Further developments in this area will require the design and testing of more robust measurement systems. 


\section{COATINGS}

Discussion Leader: N. Sanders, Specialty Minerals Inc.

1. Background Electrolyte

- Corrections

- Relevance to actual process

Strong (and sometimes relatively "strange") supporting electrolytes are commonplace in coating formulations. The influence of changes in these needs to be separable from changes in particulate properties.

2. Electrokinetics vs Viscosity (rheology)

- What are relationships?

We need something better than the dilute system theories (Von Smoluchovski, etc.) to tell us if electrokinetic measurements can, in fact, help us to understand complex rheological phenomena such as dilatancy in high solids coating colors.

3. Effect of shear on Electroacoustic measurements

One of the great potential benefits of the electroacoustic methods is the ability to measure charge under shear where many of the corresponding coating properties (rheology) are being assessed.

4. What is the correct particle size to use for interpreting ESA data?

- Effect on determination of IEP

Coating colors need to be evaluated not only under conditions of dispersion but also of partial or total flocculation - how does flocculation affect the results?

5. Need comparison of ESA methods and classical electrokinetics for adsorbed multilayers (of polyelectrolytes, e.g.)

It's currently uncertain whether the known effects of adsorbed layers in dilute suspensions carries over to ESA methods or not. Does binding strength of adsorbed layer enter?

6. How does ESA method handle "liquid" to "solid" transition?

- Immobilization detector? 
Does ESA method care whether a coating color is "flowable" or not (in theory)? If it does, perhaps an extremely useful new application is in determining immobilizaton conditions (critical for controlling dry coating structure).

7. How do we interpret ESA data on pigment mixtures?

Almost all real coating colors contain pigment mixtures. Ultimately the ESA method must be able to interpret mixture data: hopefully theoretical models can be generated to cover at least limiting cases.

8. How do we calibrate a dyamic coating slurry?

If the materials we must deal with are inherently unstable - what should we use to calibrate the ESA instrument to get dynamic information?

9. Need more measurements at high solids.

Most data available so far is at $10 \mathrm{vol} \%$ solids or less. Most coating colors are at $30+$ vol $\%$.

10. Need flow through cell that works.

High solids, higher viscosity slurries used for coating are difficult to handle in the static equipment. Flow cells currently available have not performed satisfactorily at high solids.

11. Work needed to define needs for on-line process ESA system.

Ultimately a need will develop for application of ESA methods on-line in coating color pigment manufacturing and/or coating color formulation/make-down. The requirements to impose on such new technologies in traditionally demanding environments must be scoped out.

12. Titration hysteresis effects.

More careful experiments need to be performed to determine where titration hysteresis is likely to be observed and why.

13. Is it possible to obtain a charge distribution from ESA data?

It is well worth some theoretical effort to see just how much data can be pulled out of ESA devices. If we can get particle size distribution, how about particle charge distribution? 


\section{COAL, CEMENTS AND CLAYS}

Discussion Leader: B. M. Moudgil, University of Florida

* Calibration Standards

- Ludox TM/Geltech $\mathrm{SiO}_{2} / \mathrm{TiO}_{2}$. Aging effects should be studied

* Mixed Components

- Size and mobility distributions are major concerns

* Correction Factors (e.g. electrolyte, particle size)

- Provide addendum to manual of all correction factors

* Response Time

- Option to have a quicker response (at a higher noise to signal ratio)

* Cell Design

- Torque meter to monitor dispersion/aggregation

* On-line Instrument

- Ruggedness

- Minimum cleaning

- Quick response to changes

- Handle wide solids loading (20-60 wt.\%)

* Upgrades/Technical Support

- Software upgrades

- Strong technical support 


\title{
FUNDAMENTALS OF ELECTROACOUSTICS
}

\author{
Discussion Leader: R. O. James, Eastman Kodak Company
}

During the workshop it was seen that the successful application of electroacoustic characterization techniques to the evaluation of the properties of colloidal dispersions depends on several factors. The first of these factors is the ability to reliably and accurately measure the ESA signal amplitude and phase shifts that arise from the interaction of colloidal particles with an electric or acoustic field. The second important factor is the availability of quantitative theoretical models to relate the raw data to dynamic electroacoustic mobility to the familiar characteristic quantities such as the (static) electrophoretic mobility, the zeta potential and the diffuse layer charge. Third, it is important to have suitable materials that could be used as model colloids for calibration purposes or to check for correlations with related experimental techniques, e.g., microelectrophoresis and dielectric spectroscopy.

In terms of the theories available to connect dynamic electroacoustic mobility to the static mobility a number of related theoretical studies have been recently published and more are in press. The modern theories begin treating the particle as a hard sphere with a smeared uniform surface charge balanced by a diffuse double layer with an equal and opposite charge density. The paper by O'Brien (J. Fluid Mech., 1988, 190, 71) provides an approximate analytical expression for the thin double layer case, i.e., $к \mathrm{a}>>1$. O'Brien's patent (US Patent 5059 909, 1991) and the study by Sawatzky and Babchin (J. Fluid Mech., 1993, 246, 321) provide the relationship for arbitrary ка and for small values of the zeta potential or surface charge for the same hard sphere, diffuse EDL model. Concurrent with developments in ESA, progress in the related field of dielectric spectroscopy of colloids has raised questions about the applicability of the smeared surface charge/diffuse EDL model to the interfacial structure in which a more detailed microscopic model of bound and mobile ions in the Stern or inner layer may have a significant influence on the comparison of experimental observations and the model predictions. More recently than the workshop, White and Mangelsdorf at the University of Melbourne reported comparison of exact and approximate theoretical computations of high frequency electrokinetics of colloidal particles (paper 44, $67^{\text {th }}$ Colloid and Surface Science Symposium, University of Toronto, 1993). In comparison with O'Brien's computations the numerical solutions showed agreement at for thin double layers (high ка) and for low zeta potentials. It was reported that the paper had been submitted to JCIS and that it built on an earlier report by DeLacey and White (JCS Faraday Trans 2 (1981) 79, 2007) via the inclusion of the particle inertial terms in the Navier-Stokes and colloidal force balance equations. For thicker double layers and high zeta potential, significant differences did occur between the "exact" and approximate methods as a function of frequency.

Thus it appears that at the time of the workshop much of the theoretical background for electroacoustic phenomena had been established. Progress continues with further attention needs to be allocated to: 
a) arbitrary ка and large zeta potential and

b) the effect of microscopic structure, ionic binding and dynamics with in the hydrodynamic shear zone.

Currently, the O'Brien model ( $к$ a $>>1$ ) is most easily applied to insulating particles (low $\epsilon$ ) where the electric field factor, $f$, in the equation

$$
\mu_{d}(\omega)=(2 \varepsilon \zeta / 3 \eta)(1+f) G\left(\omega a^{2} / v\right)
$$

is approximated to 0.5 so that $\mu_{d}(\omega)=\varepsilon \zeta / \eta \cdot G\left(\omega a^{2} / \nu\right)=\mu(0) \cdot G\left(\omega a^{2} / v\right)$.

At the workshop, Prof. R. J. Hunter agreed to develop a spreadsheet that would compute the factor $\mathrm{f}$ for various conductivities, particle sizes, and frequencies. In addition, the computation of an equivalent spherical volume diameter for use in the inertial term $\mathrm{G}\left(\omega \mathrm{a}^{2} / \nu\right)$ from a particle size distribution was another task that Prof. Hunter volunteered to cast into a spreadsheet macro. The above discussion applies to the low volume fraction range from about $\phi=0$ to 0.05 in which the ESA response is linear with $\phi$. There was some discussion of the use of cell models to help describe the phenomema at higher volume fractions. e.g., $\phi=0.1$ to 0.4 where the ESA response becomes non-linear and maxima in the signal may be seen. At this moment this area of electroacoustics needs further research and development. The major difficulty is to establish robust methods to obtain appropriate methods to relate the static and dynamic mobilities in the high $\phi$ regime.

\section{Particles with Complex Geometry and Structure}

Colloidal dispersions are prepared in a wide variety of particle geometries and interparticle structures e.g., floccs, gels, colloidal crystals etc. The particles may also have a wide range of thickness of adsorbed polymeric layers or coatings where polymer chains or other matter may penetrate the hydrodynamic shear plane. Also other particles and adsorbed layers may be in contact in the form of loose or elastic floccs of dimension much larger than the single particle or solvent displacement during ESA oscillations. Even other particles may be crystalline but have surface and internal pores. The question that arises is how we should treat these types of geometries to evaluate dynamic and static mobilities and surface charge, or, is the structure too complex for detailed analysis and description? All of the complicated geometries and surface structures and reactions provide many challenges to researchers in the field.

\section{Particle Size Distribution}

In ESA measurments and transformations, the mass or volume distribution of sizes and the relative density of particles compared to the medium determines the inertial term in the conversion to static mobilities. There was some discussion of the best suited techniques for providing this data. X-ray detection of sedimentation or centrifugation provides one direct 
route to this type of data, but other methods of detection with appropriate accuracy and correction factors, e.g., Mie theory correction for optical density or turbidity detection and other separation/detection schemes can be used.

\section{Acoustic Absorption}

Two papers at the workshop discussed acoustic absorption and acoustophoresis for evaluation of particle size and particle surface charge. This is separate but perhaps complimentary to ESA and the "Acoustosizer" apparatus that was also described during the meeting. Both electroacoustic and acoustic absorption method can yield signal amplitude and phase information about colloidal dispersions as a function of frequency and solution properties. The methods may offer some redundancy and also complementary information, but the deconvolution of the frequency dependent electroacoustic data to yield dynamic mobility and size distribution seems more robust at present.

\section{Further Research and Development}

There was some discussion about ways to promote further understanding and use of electroacoustic techniques and methods. Presently, instrument makers, university researchers and industrial researchers are involved in various aspects of the field e.g., including hardware and software system development, theoretical modelling, calibration and application of methods, etc. The question was asked whether more activity could be sponsored or encouraged through efforts by bodies such as NSF, IFPRI, AIChE and NIST. No firm recommendations were proposed, although the workshop type forum did encourage communication between numbers of researchers might otherwise meet only rarely. The workshop did previde a more public view of the achievements and challenges in the field.

\section{$\underline{\text { Reactive Sites and Kinetics }}$}

With increased experience using ESA apparatus it has become evident that not all measurements are made at equilibrium or steady state. In fact, since ESA is a fairly rapid technique, e.g., measurements on the time scale of seconds, ESA could be used to follow the slow kinetics of surface charge reactions. Most simple ionic reactions are fast compared to this, but mixing and surface re-equilibration are often slower in aqueous systems. In nonaqueous colloids with low conductivity, the surface reactions may be considerably slower.

In view of this it was recommended that users measure ESA amplitude and phase as a function of time in at least part of any epxerimental study to evaluate the importance of instrument stability or drift and to obtain some idea of rates of equilibration. 
Al Behan

UOP R\&D

Rolf Braunig

Eupenen Strasse 150

David Cannon

Matec Applied Sciences

Subhash Chander

Penn State Univ.

Hans Coll

Eastman-Kodak

David Darwin

W.R. Grace \& Co.

Nikolaas De Jaeger

Parcotec

Fred Desai

Univ. of Michigan

Andrei Dukhin

Pen Kem, Inc.

Arunava Dutta

GTE Electrical Products

Amjad Farooq

Colgate Palmolive

Ellen Forbus

Engelhard Corp.

George Gibson

Xerox
Bob Gill

Matec Applied Sciences

Philip Goetz

Pen Kem, Inc.

Vincent A. Hackley

NIST

Anne Hardy

Saint Gobain/Norton

Yan C. Huang

International Paper

Martin A. Hubbe

International Paper

Robert Hunter

Univ. of Sydney

Robert O. James

Eastman Kodak Co.

Ralph Kornbrekke

The Lubrizol Corp.

Ramasamy Krishnan

Sun Chemical

James Larson

Xerox

Roger Leung

W.R. Grace \& Co.

Jie Li

Binney \& Smith, Inc. 
Subhas G. Malghan

NIST

Berislav Markovic

Columbia University

W. Morris Mobley

NYSCC at Alfred Univ.

Ian Morrison

Xerox Corp.

Brij Moudgil

Univ. of Florida

Raymond Mountain

NIST

Tonis Oja

Matec Applied Sciences

Patrick Pei

NIST

Hemant Pendse

Univ. of Maine

Nancy Phillips

Pen Kem, Inc.

John Pollinger

Allied Signal Ceramic

Kevin J. Roth

Specialty Minerals, Inc.

Dale Russell

Hewlett Packard

Nigel Sanders

Specialty Minerals, Inc.
Trevor S.B. Sayer

ICI Paints

Richard Slepetys

Engelhard Corp.

Stuart Soled

Exxon

Krishna Kumar Subramanyan

Columbia University

Jorge Valdes

AT\&T Bell Labs.

Boudewijn Van Lent

Bayer AG

Bruce Weiner

Brookhaven Instruments 





\section{Periodical}

Journal of Research of the National Institute of Standards and Technology-Reports NIST research and development in those disciplines of the physical and engineering sciences in which the Institute is active. These include physics, chemistry, engineering, mathematics, and computer sciences. Papers cover a broad range of subjects, with major emphasis on measurement methodology and the basic technology underlying standardization. Also included from time to time are survey articles on topics closely related to the Institute's technical and scientific programs. Issued six times a year.

\section{Nonperiodicals}

Monographs-Major contributions to the technical literature on various subjects related to the Institute's scientific and technical activities.

Handbooks - Recommended codes of engineering and industrial practice (including safety codes) developed in cooperation with interested industries, professional organizations, and regulatory bodies.

Special Publications - Include proceedings of conferences sponsored by NIST, NIST annual reports, and other special publications appropriate to this grouping such as wall charts, pocket cards, and bibliographies.

Applied Mathematics Series-Mathematical tables, manuals, and studies of special interest to physicists, engineers, chemists, biologists, mathematicians, computer programmers, and others engaged in scientific and technical work.

National Standard Reference Data Series - Provides quantitative data on the physical and chemical properties of materials, compiled from the world's literature and critically evaluated. Developed under a worldwide program coordinated by NIST under the authority of the National Standard Data Act (Public Law 90-396). NOTE: The Journal of Physical and Chemical Reference Data (JPCRD) is published bimonthly for NIST by the American Chemical Society (ACS) and the American Institute of Physics (AIP). Subscriptions, reprints, and supplements are available from ACS, 1155 Sixteenth St., NW, Washington, DC 20056.

Building Science Series-Disseminates technical information developed at the Institute on building materials, components, systems, and whole structures. The series presents research results, test methods, and performance criteria related to the structural and environmental functions and the durability and safety characteristics of building elements and systems.

Technical Notes-Studies or reports which are complete in themselves but restrictive in their treatment of a subject. Analogous to monographs but not so comprehensive in scope or definitive in treatment of the subject area. Often serve as a vehicle for final reports of work performed at NIST under the sponsorship of other government agencies.

Voluntary Product Standards-Developed under procedures published by the Department of Commerce in Part 10, Title 15, of the Code of Federal Regulations. The standards establish nationally recognized requirements for products, and provide all concerned interests with a basis for common understanding of the characteristics of the products. NIST administers this program in support of the efforts of private-sector standardizing organizations.

Consumer Information Series - Practical information, based on NIST research and experience, covering areas of interest to the consumer. Easily understandable language and illustrations provide useful background knowledge for shopping in today's technological marketplace. Order the above NIST publications from: Superintendent of Documents, Government Printing Office, Washington, DC 20402.

Order the following NIST publications-FIPS and NISTIRs-from the National Technical Information Service, Springfield, VA 22161.

Federal Information Processing Standards Publications (FIPS PUB)-Publications in this series collectively constitute the Federal Information Processing Standards Register. The Register serves as the official source of information in the Federal Government regarding standards issued by NIST pursuant to the Federal Property and Administrative Services Act of 1949 as amended, Public Law 89-306 (79 Stat. 1127), and as implemented by Executive Order 11717 (38 FR 12315, dated May 11, 1973) and Part 6 of Title 15 CFR (Code of Federal Regulations).

NIST Interagency Reports (NISTIR) - A special series of interim or final reports on work performed by NIST for outside sponsors (both government and non-government). In general, initial distribution is handled by the sponsor; public distribution is by the National Technical Information Service, Springfield, VA 22161, in paper copy or microfiche form. 


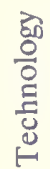

를

ขั

हื

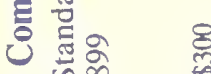

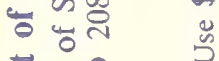

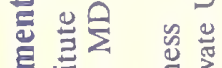

हE की

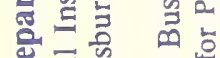

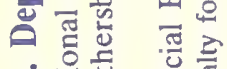

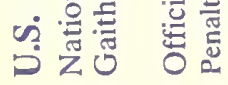

